\title{
Bacterial Communities \\ Associated with Human \\ Decomposition
}

\section{Rachel Parkinson}

\author{
A thesis submitted to the \\ Victoria University of Wellington \\ in fulfilment of the requirements for the degree of \\ Doctor of Philosophy in Science
}

Victoria University of Wellington

2009 


\section{Abstract}

Human decomposition is a little-understood process with even less currently known about the microbiology involved. The aim of this research was to investigate the bacterial community associated with exposed decomposing mammalian carcasses on soil and to determine whether changes in this community could potentially be used to determine time since death in forensic investigations. A variety of soil chemistry and molecular biology methods, including molecular profiling tools T-RFLP and DGGE were used to explore how and when bacterial communities change during the course of a decomposition event. General bacterial populations and more specific bacterial groups were examined.

Decomposition was shown to cause significant and sequential changes in the bacterial communities within the soil, and changes in the bacterial community often correlated with visual changes in the stage of decomposition. Organisms derived from the cadavers and carcasses were able to be detected, using molecular methods, in the underlying soil throughout the decomposition period studied. There was little correlation found between decomposition stage and the presence and diversity within the specific bacterial groups investigated. Organisms contributing to the changes seen in the bacterial communities using molecular profiling methods were identified using a cloning and sequencing based technique and included soil and environment-derived bacteria, as well as carcass or cadaver-derived organisms.

This research demonstrated that pig (Sus scrofa) carcass and human cadaver decomposition result in similar bacterial community changes in soil, confirming that pig carcasses are a good model for studying the microbiology of human decomposition. The inability to control for differences between donated human cadavers made understanding the human 
cadaver results difficult, whereas pig carcass study allowed many variables to be held constant while others were investigated.

The information gained from this study about the bacteria associated with a cadaver and how the community alters over the course of decomposition may, in the future, enable the development of a forensic post mortem interval estimation tool based on these changes in the bacterial community over time. The findings in this thesis suggest that high variability between human bodies and their microflora is likely to present a challenge to the development of such a tool, but further study with emerging high-throughput molecular tools may enable identification of microbial biomarkers for this purpose. 


\section{Acknowledgments}

I'd like to express my sincere thanks to all the wonderful people who have helped me get through my $\mathrm{PhD}$. It has been immensely enjoyable and I will actually be sad (on some level) to finish. Others perhaps didn’t enjoy it as much as I did......

I'd like to thank my brilliant supervisor Dr Jacqui Horswell for guiding me through this project, kick-starting me when I needed it and telling me to go home when it was beginning to seem like I lived in the lab. Thanks too to my University supervisor Dr Geoff Chambers for giving me the freedom to diverge into interesting areas and for generally keeping things on track. A huge thankyou has to go to my US based supervisor Dr Arpad Vass for allowing me to stink out his lab and office for three months and for sampling with me at the Body Farm (not the nicest of jobs!!). Thankyou so much Arpad and Vicky, for taking such good care of me during my stay and making me feel like one of the family. Thanks to the kind staff at ORNL and the University of Tennessee's Anthropology department for allowing me access to the lab and Body Farm.

Thanks also to the others in the ESR lab: Tom, Andrew, Jen, Catriona, Danny, Penny, Mel, Justine, Steph, Alice and Cliff for not complaining TOO much about the smell of my samples and helping out when my sampling regime got a bit out of hand. Thanks to Phil and Marilyn for teaching, and fixing and re-running and explaining various things over and over! Also thanks to Justine the cloning guru and Steph the general everything guru for their endless patience with my questions. We really miss you Steph and Cat!

Thanks also to Jeremy, who successfully decrypted science-geek speak into computer-geek speak and wrote super computer programmes, which made my life so much easier. 
I'd also like to thank my extended family for their support and ongoing interest in my weird choice of study topic, and my parents for helping with the pig cage building and sewing.

Thanks must go to my 10 little pigs for the ultimate sacrifice, and to the generous people of the US who donate their bodies to science. I'll never know the names in life of the bodies that were used in this research, but I respect and thank each of you for your gift.

To my amazing Reid - Thankyou for being so interested in my research, for encouraging and inspiring me through the tough times and for promising to spoil me rotten once it's all done!

Finally....thankyou to the lab fairy for visiting the others for a while and letting me finally finish my lab work......

\section{Dedication}

This thesis is dedicated to my grandfathers: Walter Chester and Murray Parkinson, who both, in their own ways, encouraged me to follow my heart......even if my heart took me in a very strange direction indeed!! I hope that I have made them both proud. 


\section{Table of Contents}
Abstract ii

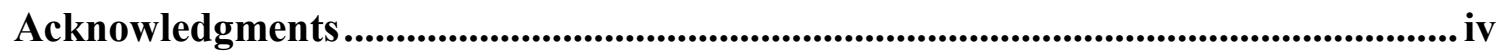

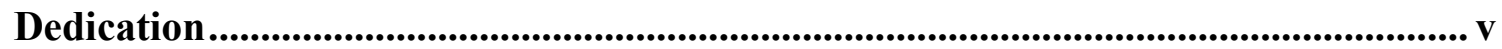

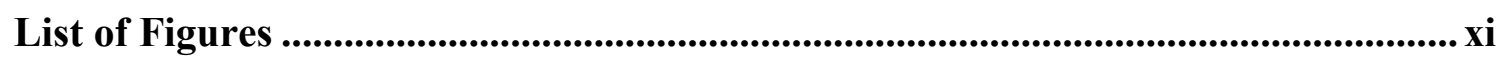

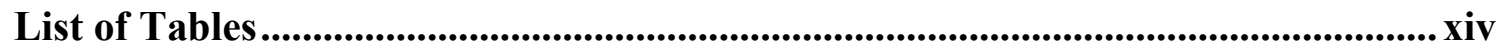

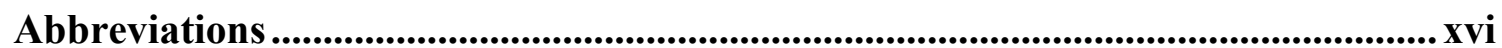

Chapter 1: Introduction and Aims.............................................................1

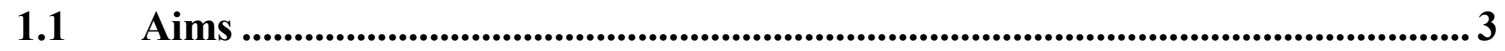

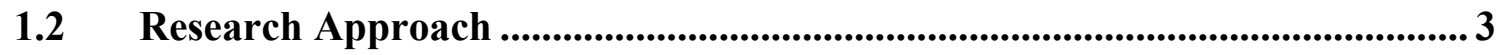

Chapter 2: Background and Literature Review ..........................................6

2.1 Human Decomposition ....................................................................................................6 6

2.1.1 Physical and Chemical Processes of Decomposition ...................................6

2.1.2 Variables Affecting Decomposition ........................................................... 8

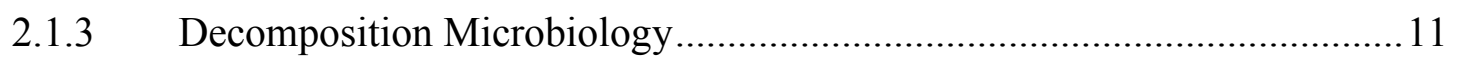

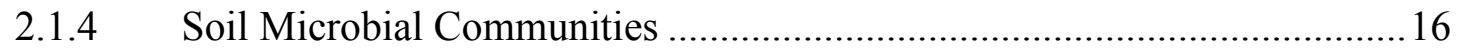

2.2 Post Mortem Interval Estimation ..................................................................... 19

2.3 Molecular Analysis of Microbial Communities ............................................... 23

2.3.1 Genomic Microbial Community Analysis..................................................24

Chapter 3: General Methods ................................................................33

3.1 Sample Characterisation............................................................................33

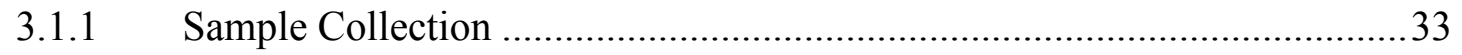

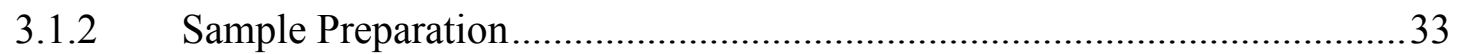

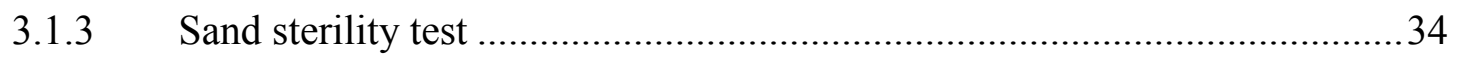

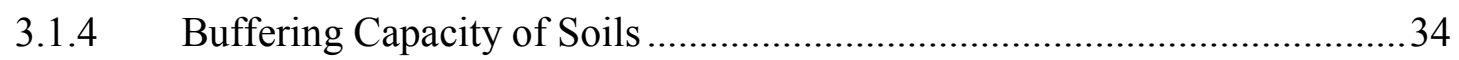

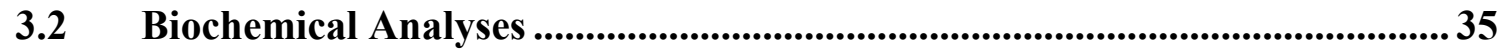

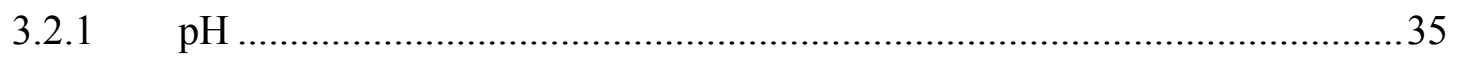

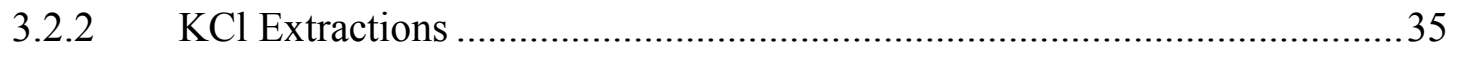

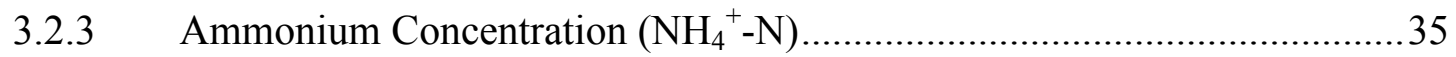

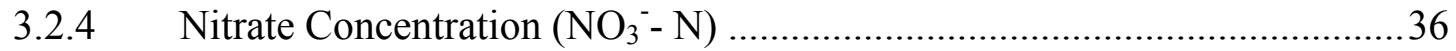

3.3 Enzyme Assays .......................................................................................................... 37

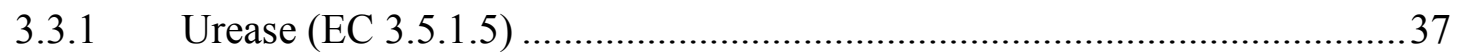




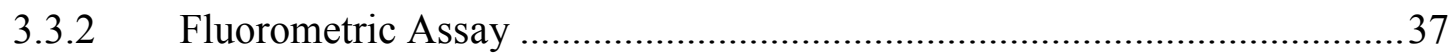

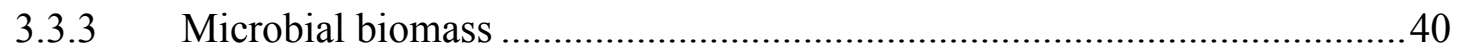

3.4 Molecular Biology Methods.................................................................................. 41

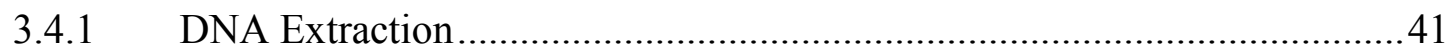

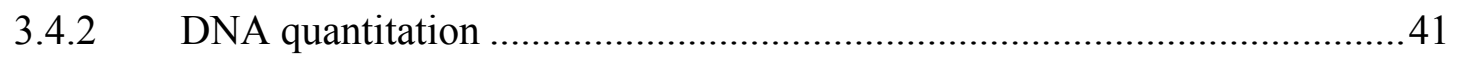

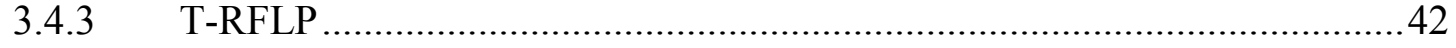

3.4.4 Denaturing Gradient Gel Electrophoresis.................................................45

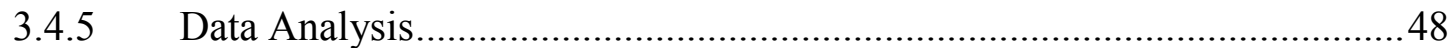

\section{Chapter 4: Pig Carcass Decomposition on Sand and the Effect of} Insect Colonisation ..........................................................................................51

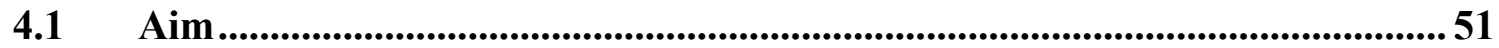

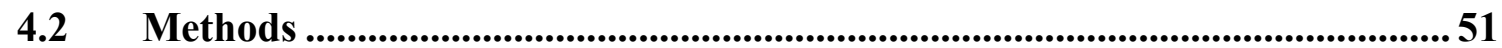

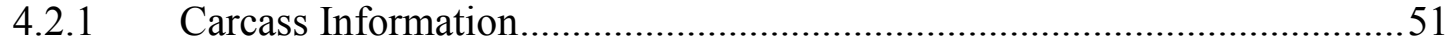

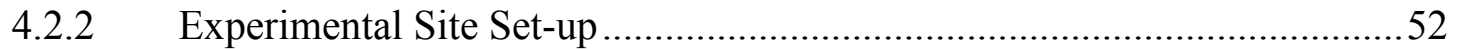

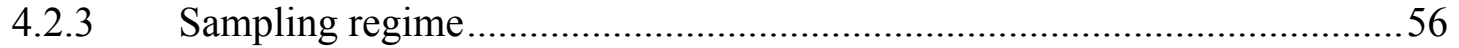

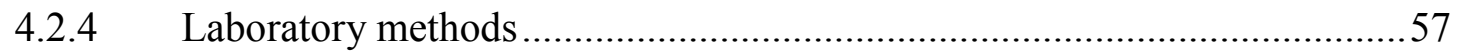

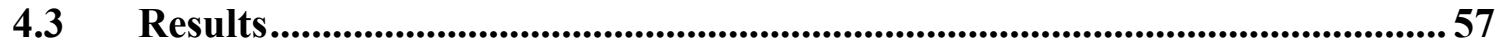

4.3.1 Documentation of Decomposition Progression...........................................57

4.3.2 Temperature, Relative Humidity \& Rainfall ..............................................6 60

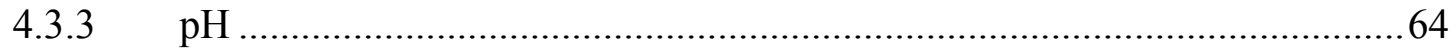

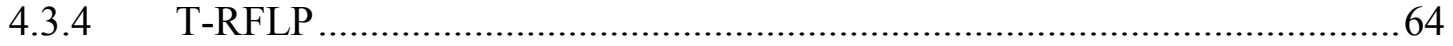

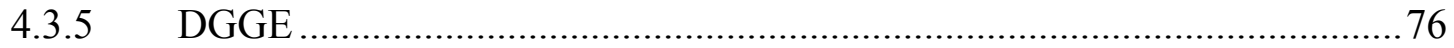

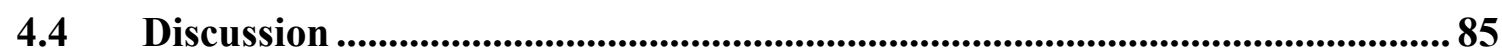

Chapter 5: Pig Carcass Decomposition on Soil and Sand .......................88

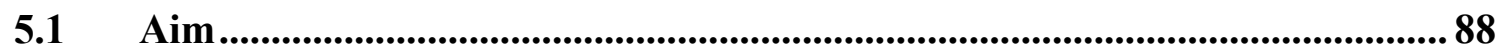

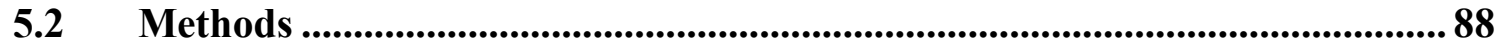

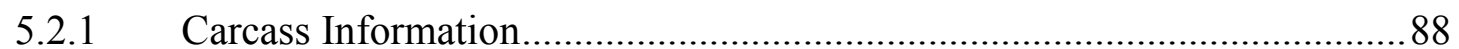

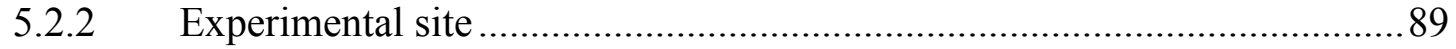

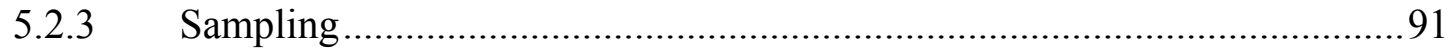

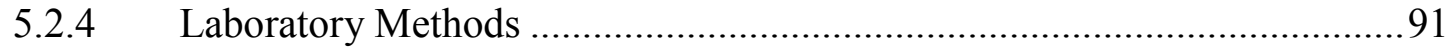

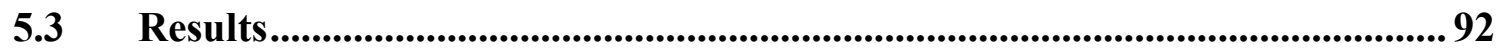

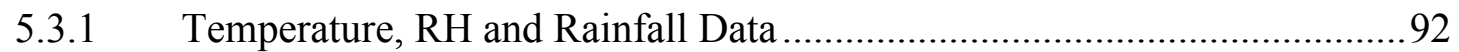

5.3.2 Documentation of Decomposition Progression..........................................95 
5.3.3 Chemistry \& Biochemistry Results .......................................................97

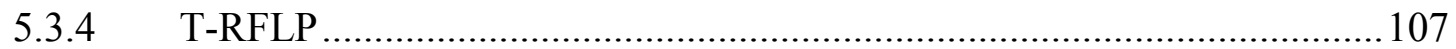

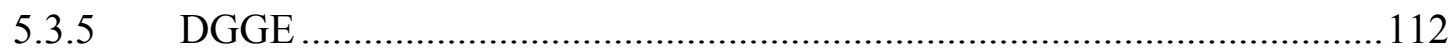

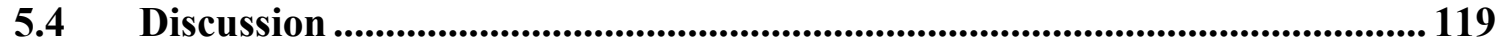

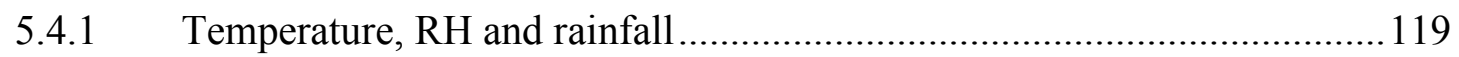

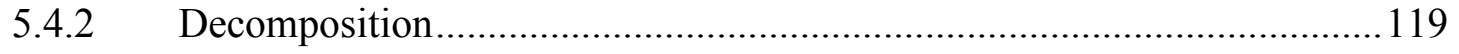

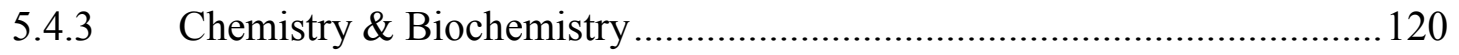

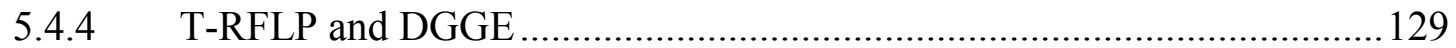

Chapter 6: Human Cadaver Decomposition on Soil and Sand...........131

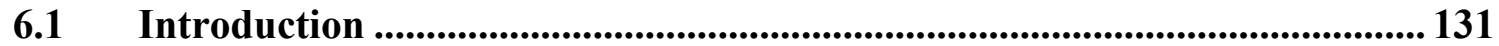

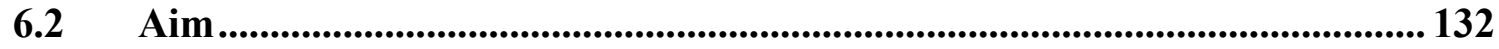

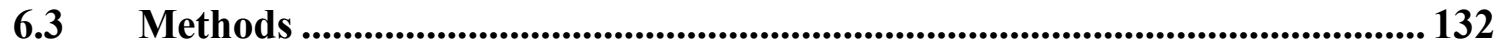

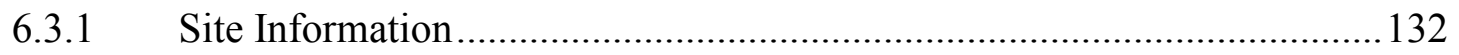

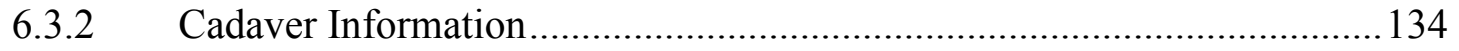

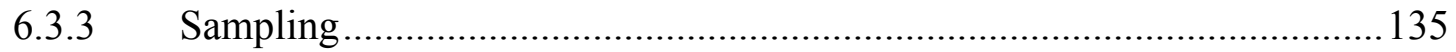

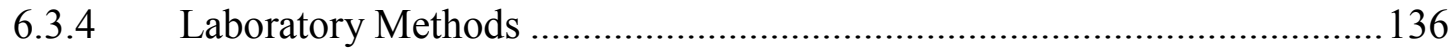

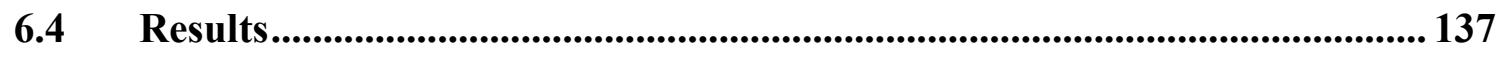

6.4.1 Decomposition Documentation .................................................................. 137

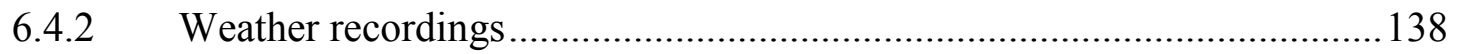

6.4.3 Buffering Capacity of Soil and Sand ..................................................... 140

6.4.4 Chemistry and Enzyme Assay Results .................................................... 142

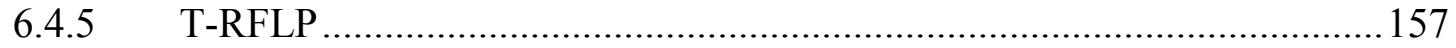

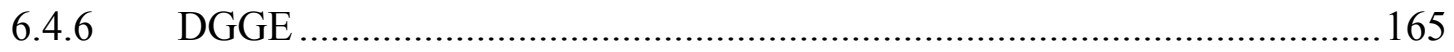

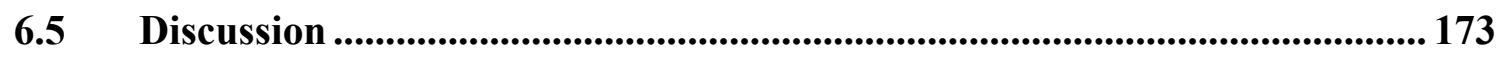

6.5.1 Observation of Decomposition Process................................................... 173

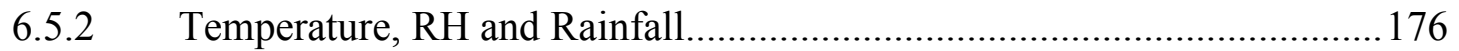

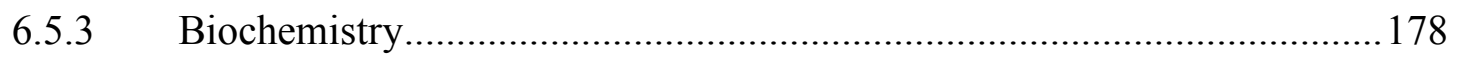

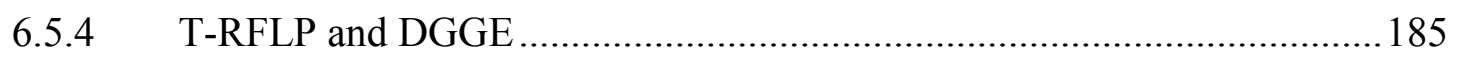

6.5.5 Concluding Comments …................................................................... 186

Chapter 7: Molecular Biological Examination of Communities ..............187

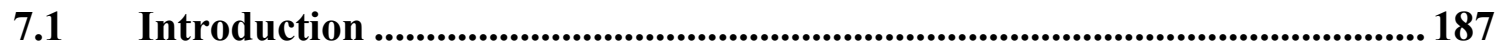

7.2 Group-Specific DGGE/T-RFLP Analysis .................................................. 189

7.2.1 Bacterial Groups Tested For................................................................ 189 
7.2.2 Presence/Absence of Specific Bacterial Groups using PCR ..................... 193

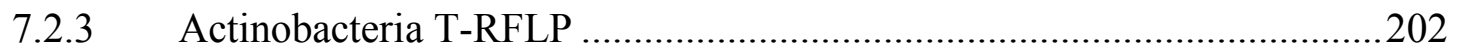

7.2.4 DGGE using Group-specific Primers .......................................................206

7.2.5 Group-Specific Analysis Discussion...................................................217

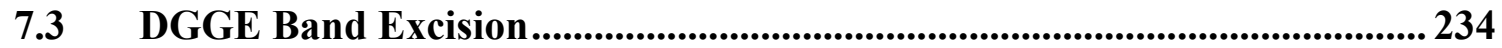

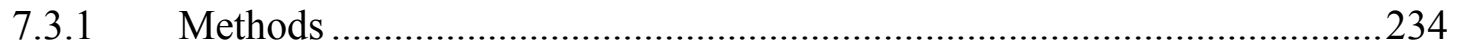

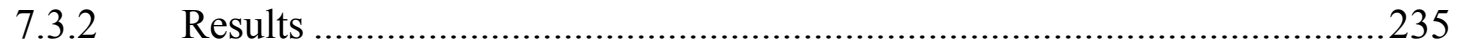

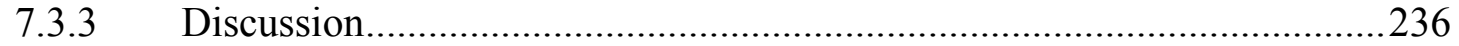

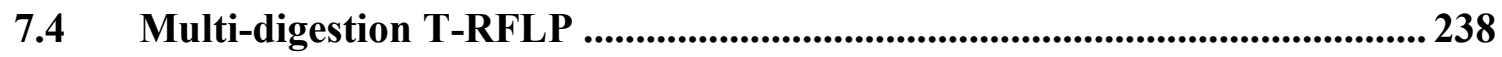

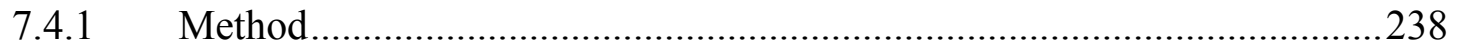

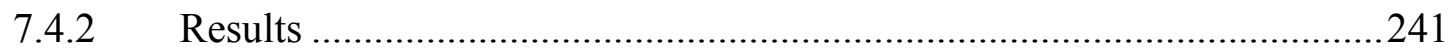

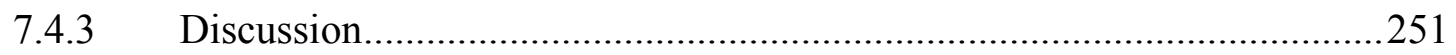

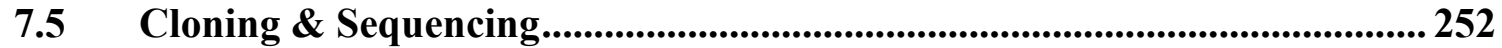

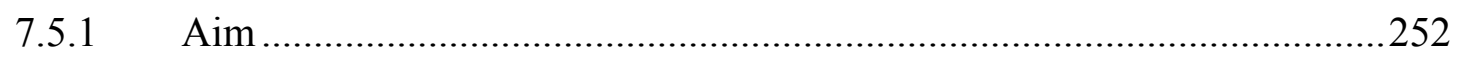

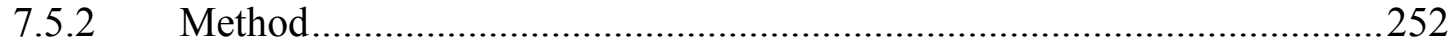

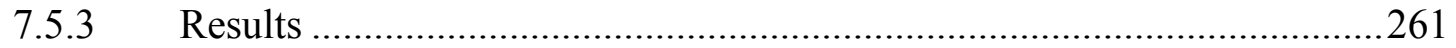

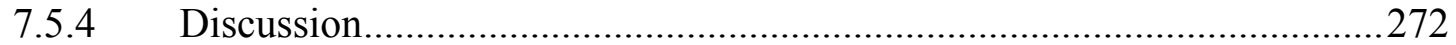

7.6 Conclusions ...................................................................................................................... 290

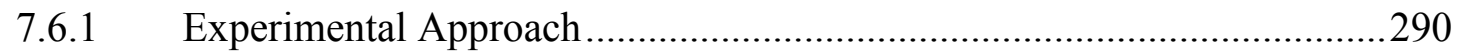

7.6.2 Insights into decomposition bacterial communities ................................292

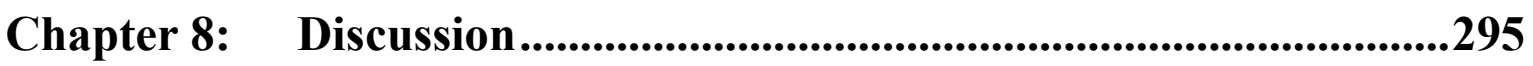

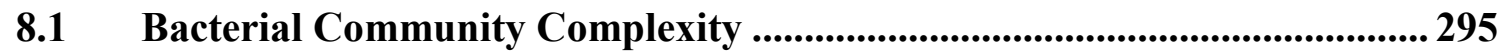

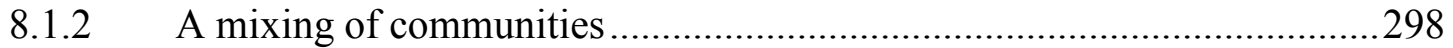

8.2 Physical Environment Changes........................................................................305

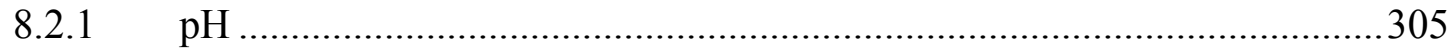

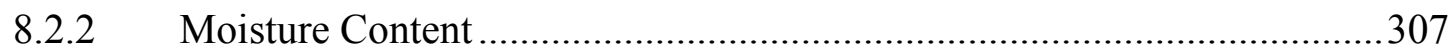

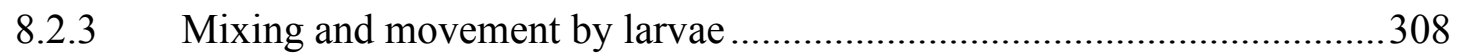

8.3 Nutritional Environment Changes..................................................................3 309

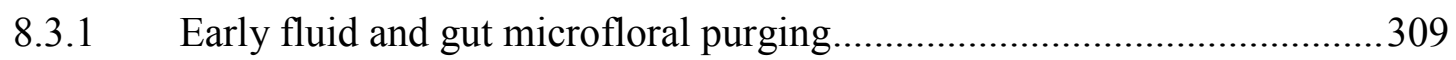

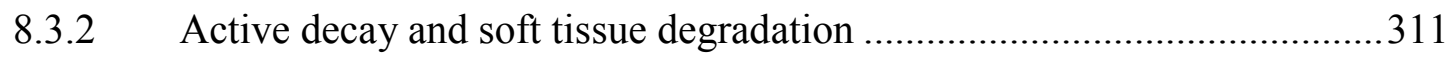

8.3.3 Insect larval growth and pupation .......................................................... 312

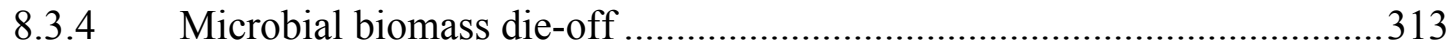

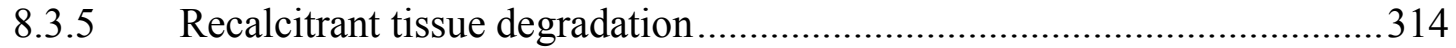




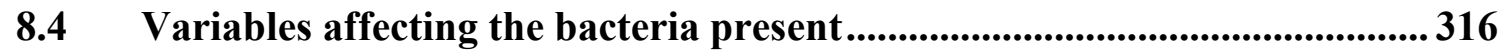

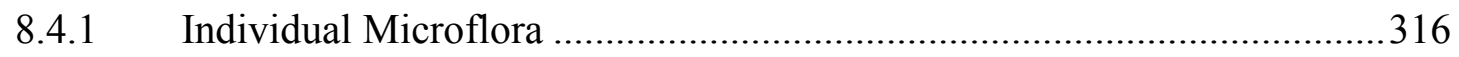

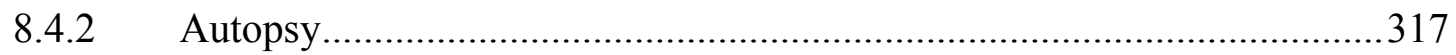

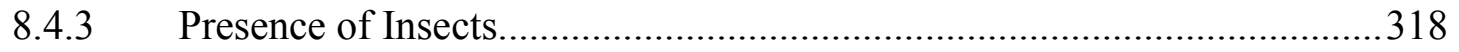

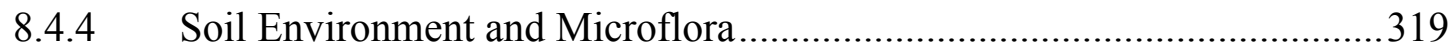

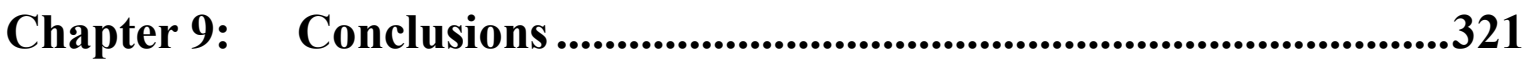

9.1 The Bacteriology of Decomposition ...........................................................321

$9.2 \quad$ Pig Carcasses as Models..................................................................................... 323

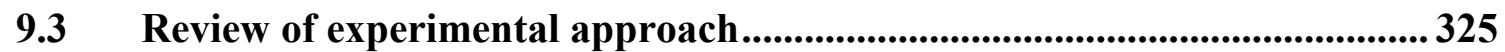

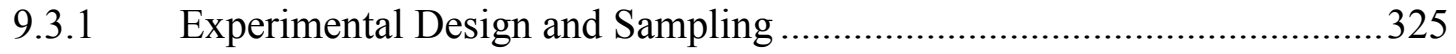

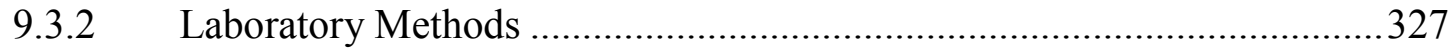

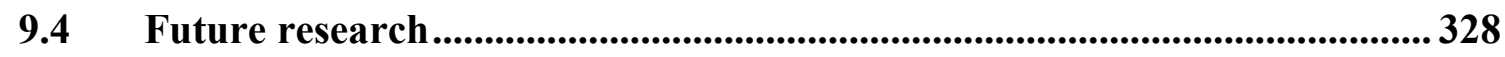

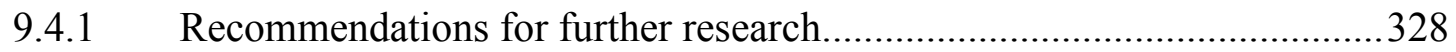

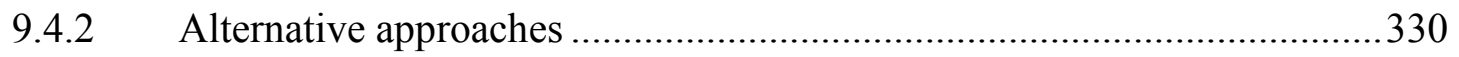

9.5 Forensic Application .............................................................................................. 332

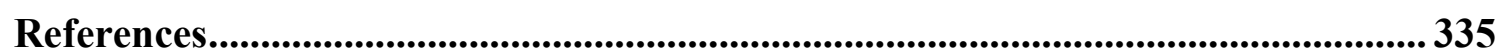

Appendices (on CD) ............................................................................................................... 362 


\section{List of Figures}

Figure 2.1The five basic stages of decomposition....................................................... 7

Figure 2.2 Flow diagram for determining time since death in cumulative degree hrs. 22

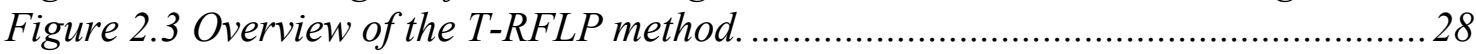

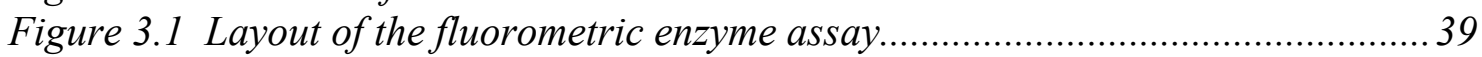

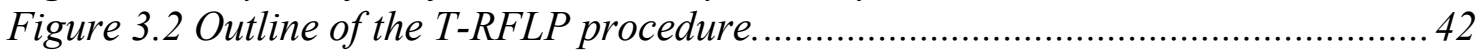

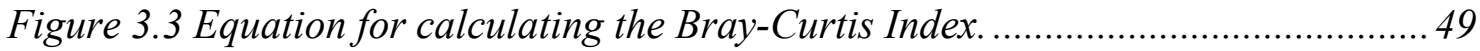

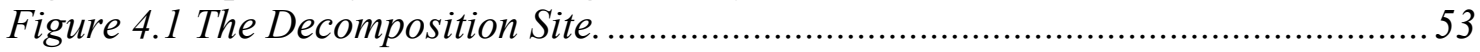

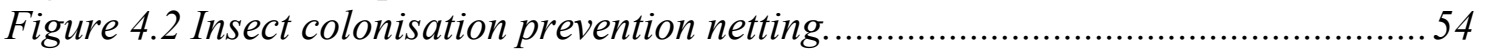

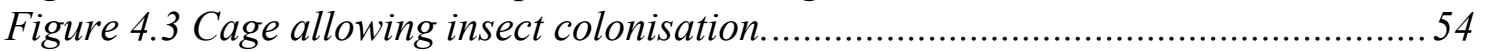

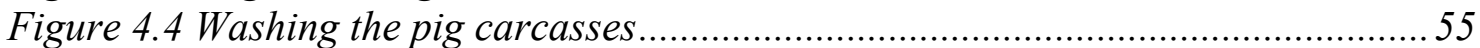

Figure 4.5 Pig lifted in netting to allow sampling of underlying soil. ...........................55

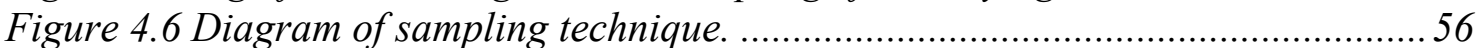

Figure 4.7 Carcass A (insect colonised) decomposition over time ................................58

Figure 4.8 Carcass $B$ (no insect colonisation) decomposition over time........................59

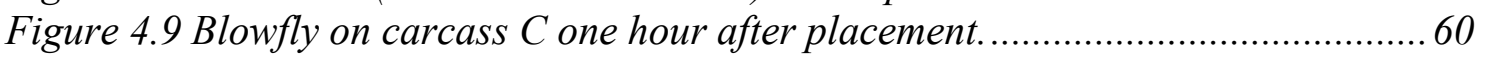

Figure 4.10 Fly eggs on the mouth of carcass within four hours placement................ 60

Figure 4.11 Mean daily ambient air temperature ....................................................6. 61

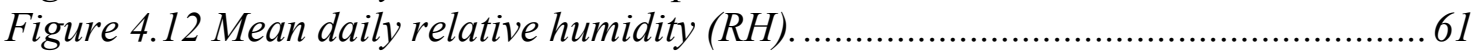

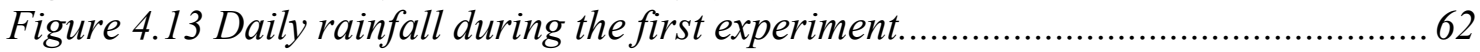

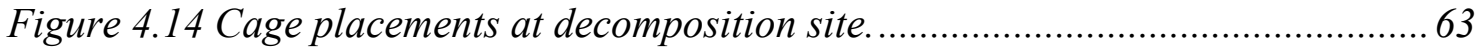

Figure 4.15 Temperature difference between cages $A$ and $D$.......................................63

Figure 4.16 Mean pH of the replicate plots with or without insect colonisation........... 64

Figure 4.17 Selected T-RFLP profiles from samples at different stages........................65

Figure 4.18 MDS analysis plot of T-RFLP data from sand from under carcass A....... 68

Figure 4.19 MDS analysis plot of T-RFLP data from sand from under carcass C. .....69

Figure 4.20 MDS analysis plot of T-RFLP data from sand from under carcass E....... 69

Figure 4.21 MDS plot of T-RFLP data from the three colonised carcass.................... 70

Figure 4.22 MDS plot of T-RFLP data from sand collected from under carcass $B$. ...71

Figure 4.23 MDS plot of T-RFLP data from sand collected from under carcass $D \ldots . . .72$

Figure 4.24 MDS plot of T-RFLP data from sand collected from under carcass $F$..... 72

Figure 4.25 MDS plot of T-RFLP data from the non-colonised carcasses.................... 73

Figure 4.26 MDS plot of T-RFLP data from sand from under all six carcasses. .......... 74

Figure 4.28 Photograph of DGGE gel analysis of carcass A.................................... 77

Figure 4.29 Photograph of DGGE gel analysis of $r$ carcass $B$...................................... 77

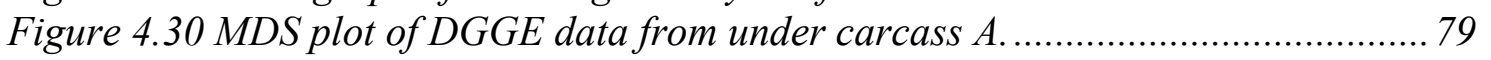

Figure 4.31 MDS plot of DGGE data from under carcass $B$..................................... 79

Figure 4.32 MDS plot of DGGE data from under carcass $C$......................................8 80

Figure 4.33 MDS plot of DGGE data from under carcass $D$...................................... 81

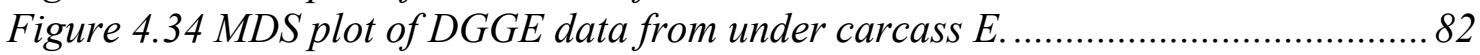

Figure 4.35 MDS plot of DGGE data from under carcass $F$................................... 82

Figure 4.36 MDS plot of DGGE data from the three colonised carcasses ................... 83

Figure 4.37 MDS plot of DGGE data from the non-colonised carcasses......................83

Figure 4.38 MDS plot of DGGE data from sand collected from under all carcasses. .. 84

Figure 5.1 Carcasses in the cages at the eastern end of the site ..................................8 89

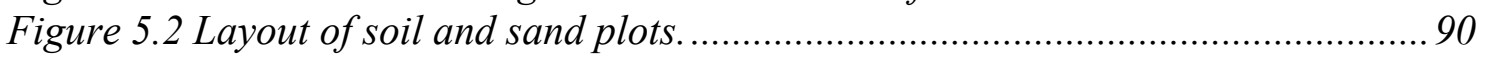

Figure 5.3 Soil pit and sand tray prior to carcass placement .......................................90 
Figure 5.4 Mean daily air, soil and sand temperatures........................................ 92

Figure 5.5 Moisture content of samples over the sampling period............................. 93

Figure 5.6 Daily rainfall at the Trentham Weather Station over the sampling period..94

Figure 5.7 Mean daily RH.................................................................................. 94

Figure 5.8 Carcass $G$ (soil) decomposition over time. ................................................95

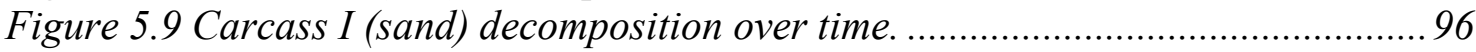

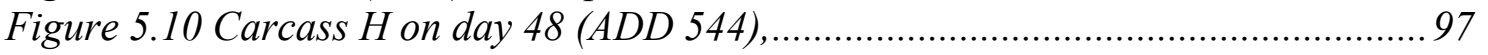

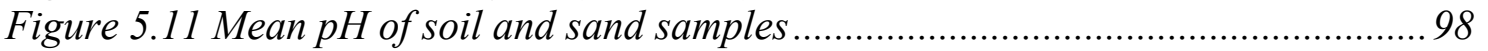

Figure $5.12 \mathrm{NH}_{4}{ }^{+}-\mathrm{N}$ concentrations in soil and sand samples ..................................99

Figure 5.13 NO3--N concentrations in soil and sand samples.................................... 100

Figure 5.14 Potential urease activity in soil and sand samples.............................. 101

Figure 5.15 Potential N-acetyl- $\beta$-D-glucosaminidase activity ................................ 102

Figure 5.16 Potential glucosidase activity in samples............................................... 103

Figure 5.17 Potential acid phosphatase activity in soil samples ................................ 104

Figure 5.18 Potential sulphatase activity in soil samples ........................................... 106

Figure 5.19 MDS plot of T-RFLP data from samples collected under carcass G...... 108

Figure 5.20 MDS plot of T-RFLP data from samples collected under carcass $H$....... 108

Figure 5.21 MDS plot of T-RFLP data from all soil samples ................................. 110

Figure 5.22 MDS plot of T-RFLP data from samples under carcasses on sand..........111

Figure 5.23 MDS plot of T-RFLP data from samples under all carcasses .................. 112

Figure 5.24 Photograph of DGGE gel analysis of carcass $G$ (on soil). ..................... 113

Figure 5.25 Photograph of DGGE gel analysis of carcass I (on sand) ..................... 113

Figure 5.26 Photograph of DGGE gel analysis of soil control samples..................... 114

Figure 5.27 MDS plot of DGGE data from samples collected from under carcass G. 115

Figure 5.28 MDS plot of DGGE data from samples collected from under carcass H. 116

Figure 5.29 MDS plot of DGGE data from samples collected from under carcass I.. 117

Figure 5.30 MDS plot of DGGE data from samples collected from under carcass J.. 118

Figure 5.31 MDS plot of DGGE data from the control soil samples.........................118

Figure 6.1 Site map of University of Tennessee Anthropological Facility. ................ 134

Figure 6.2 Sampling under cadaver P.......................................................................... 136

Figure 6.3 Daily Rainfall at the human cadaver decomposition site .......................... 139

Figure 6.4 Mean daily air, control soil and under cadaver temperatures.................. 139

Figure 6.5 Mean daily relative humidity .................................................................... 140

Figure 6.6 Mean daily barometric pressure ............................................................... 140

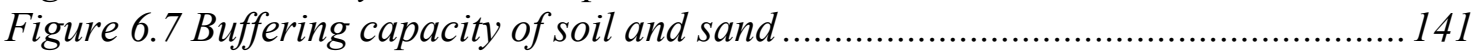

Figure 6.8 Moisture content of samples over the sampling period............................ 142

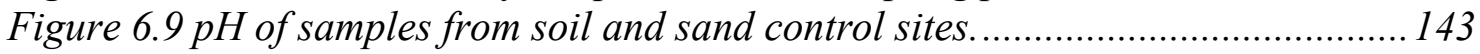

Figure 6.10 pH of samples from under the cadavers on soil .................................... 144

Figure $6.11 \mathrm{pH}$ of samples from under the cadavers on sand................................. 144

Figure $6.12 \mathrm{NH}_{4}{ }^{+}-\mathrm{N}$ concentration in soil and sand samples ................................... 145

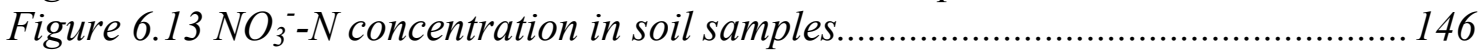

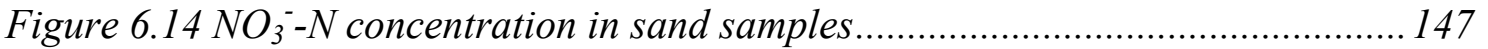

Figure 6.15 Potential urease activity in soil samples ................................................ 148

Figure 6.16 Potential urease activity in sand samples.............................................. 149

Figure 6.17 Potential N-acetyl- $\beta$-D-glucosidase activity in soil samples................... 150

Figure 6.18 Potential $N$-acetyl- $\beta$-D-glucosidase activity in sand samples .................. 150

Figure 6.19 Potential glucosidase activity in soil samples ..................................... 151

Figure 6.20 Potential glucosidase activity in sand samples ...................................... 152

Figure 6.21 Potential acid phosphatase activity in soil samples ............................... 153

Figure 6.22 Potential acid phosphatase activity in sand samples ........................... 153

Figure 6.23 Potential alanine aminopeptidase activity......................................... 154 


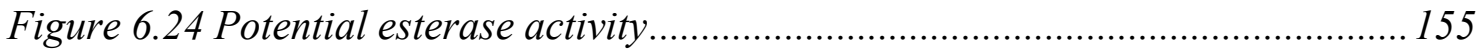

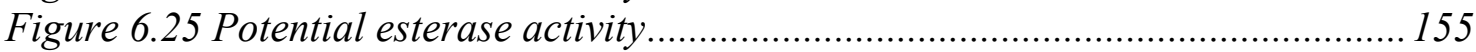

Figure 6.26 Potential esterase activity ....................................................................... 156

Figure 6.27 Potential esterase activity s ................................................................. 157

Figure 6.28 MDS plot of T-RFLP profiles from five replicate samples ...................... 158

Figure 6.29 MDS plot of T-RFLP data from samples collected under cadaver P...... 159

Figure 6.30 MDS plot of T-RFLP data from samples collected under cadaver R ...... 160

Figure 6.31 MDS plot of T-RFLP data from samples collected under cadaver T...... 161

Figure 6.32 MDS plot of T-RFLP data from samples collected under cadaver V....... 162

Figure 6.33 MDS plot of T-RFLP data from samples collected under cadaver X...... 163

Figure $6.34 \mathrm{MDS}$ plot of T-RFLP data from samples collected under cadaver Z...... 163

Figure 6.35 MDS plot of T-RFLP data from all soil samples .................................... 164

Figure 6.36 MDS plot of T-RFLP data from soil control samples.............................. 165

Figure 6.37 Photograph of DGGE gel analysis of cadaver R.................................... 166

Figure 6.38 Photograph of DGGE gel analysis of cadaver T................................... 166

Figure 6.39 Photograph of DGGE gel analysis of cadaver V......................................167

Figure 6.40 Photograph of DGGE gel analysis of soil control samples...................... 167

Figure 6.41 MDS plot of DGGE data from samples collected under cadaver P........ 169

Figure 6.42 MDS plot of DGGE data from samples collected under cadaver R........ 169

Figure 6.43 MDS plot of DGGE data from samples collected under cadaver T........ 170

Figure 6.44 MDS plot of DGGE data from all samples collected under cadaver V.... 171

Figure 6.45 MDS plot of DGGE with V4 excluded............................................... 171

Figure 6.46 MDS plot of DGGE data from cadaver X............................................. 172

Figure 6.47 MDS plot of DGGE data from cadaver $Z$............................................ 172

Figure 6.48 MDS plot of DGGE data from soil control samples................................. 173

Figure 7.1 T-RFLP profiles for Actinobacteria specific primers for cadaver P .........203

Figure 7.2 T-RFLP profiles for Actinobacteria specific primers for cadaver R........ 203

Figure 7.3 MDS plot of Actinobacterial group-specific T-RFLP data.......................205

Figure 7.4 Photograph of the Atopobium group specific DGGE gel .........................208

Figure 7.5 MDS plot of the Atopobia group specific DGGE profile data...................209

Figure 7.6 Photograph of the Bacteroides fragilis group specific DGGE gel............209

Figure 7.7 MDS plot of the Bacteroides fragilis group specific DGGE profile data .. 210

Figure 7.8 Photograph of DGGE gel analysis of Bifidobacterium communities........ 211

Figure 7.9 Photograph of the Clostridium coccoides group DGGE gel....................2212

Figure 7.10 MDS plot of the Clostridium coccoides group specific DGGE ...............2 213

Figure 7.11 Photograph of the Clostridium perfringens group DGGE gel ................213

Figure 7.12 MDS plot of the Clostridium perfringensc DGGE profile data...............2 214

Figure 7.13 Photograph of the Enterococcus group-specific DGGE gel ...................2 215

Figure 7.14 Photograph of DGGE gel of Lactobacilli...........................................2. 216

Figure 7.15 MDS analysis of DGGE profile data from the Lactobacillus group ....... 217

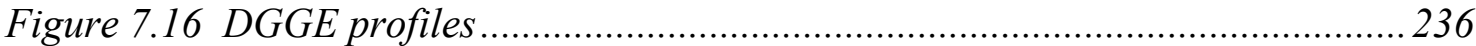

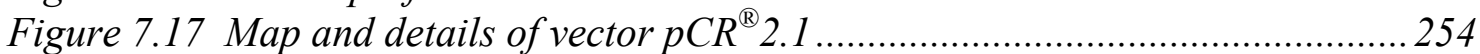

Figure 7.18 Pooled samples were created for each row and column .........................256

Figure 7.19 The well from which particular clone T-RFs originated .......................258

Figure 7.20 Relative frequencies of the different orders represented in V12 library. 268

Figure 7.21 Relative frequencies of the different orders represented in Z11 library.269

Figure 8.1 Time-line of events during decomposition of cadaver $P$...........................300

Figure 8.3 Time-line of events during decomposition of carcass $G$ (on soil)............. 303

Figure 8.4 Time-line of events during decomposition of cadaver T............................304 


\section{List of Tables}

Table 2.1 Variables affecting the decay rate of a human body .................................... 9

Table 2.2 Predominant organisms of the body sites colonised by microflora ............... 15

Table 3.1 Fluorometric substrates (Sigma) used and the enzymes which act on them... 38

Table 3.2 T-RFLP PCR recipe. ..................................................................................... 43

Table 3.3 T-RFLP PCR thermocycling regime. .......................................................... 43

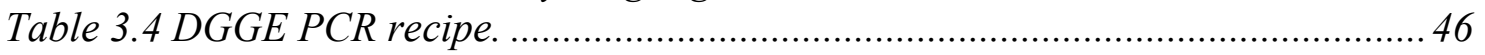

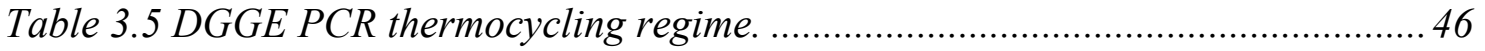

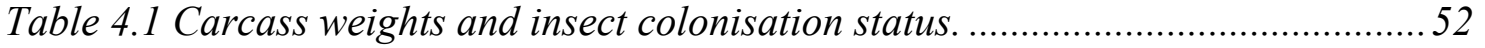

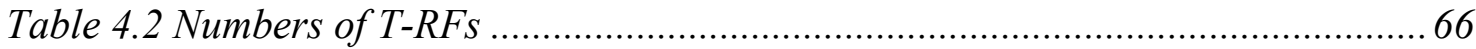

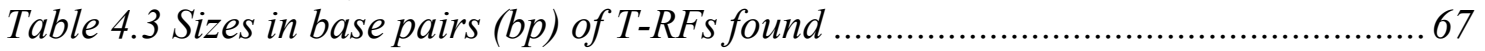

Table 4.4 Mean similarity between consecutive profiles for each decomposition plot. 75

Table 4.5 Number of bands present in profiles from each carcass............................... 78

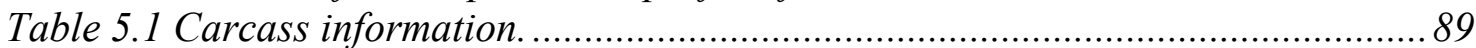

Table 5.2 Number of T-RFs present in profiles from each carcass........................... 107

Table 5.3 Number of bands present in profiles from each carcass. .......................... 115

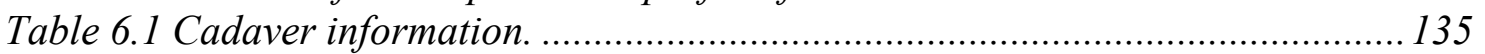

Table 6.2 Mean number of T-RFs included in analysis from each cadaver............... 158

Table 6.3 Number of bands present in DGGE profiles from under each cadaver...... 168

Table 7.1 16S primers used for group specific DGGE and T-RFLP analyses........... 194

Table 7.2 Recipe for group specific PCR. ….............................................................. 195

Table 7.3 Thermocycling regime for group specific PCRs....................................... 195

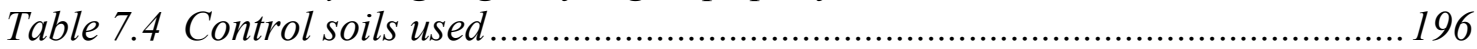

Table 7.5 Presence/absence of specific bacterial groups under cadaver P............... 197

Table 7.6 Presencelabsence of specific bacterial groups under cadaver X................198

Table 7.7 Presencelabsence of specific bacterial groups under cadaver R................ 198

Table 7.8 Presencelabsence of specific bacterial groups under cadaver T............... 199

Table 7.9 Presencelabsence of specific bacterial groups under cadaver V................ 199

Table 7.10 Presencelabsence of specific bacterial groups under cadaver Z ..............200

Table 7.11 Presencelabsence of specific bacterial groups in control soil samples .... 200

Table 7.12 Samples chosen for T-RFLP analysis of the Actinobacteria population...202

Table 7.13 Number of T-RFs generated from each sample......................................2. 204

Table 7.14 T-RFs specific to a substrate or treatment...........................................2. 205

Table 7.15 Acrylamide percentage and gradients used in g-s DGGE analysis..........206

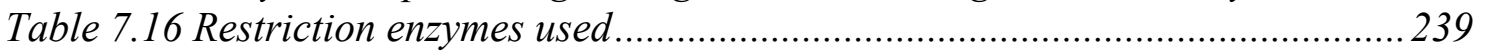

Table 7.17 Samples chosen for multi-digestion T-RFLP analysis. ...........................239

Table 7.18 The ten highest T-RFs in each of the 30 T-RFLP profiles........................ 242

Table 7.19 Organisms identified as potentially contributing to the selected T-RFs...243

Table 7.20 Potentially contributing bacterial orders $50-200 \mathrm{bp}$............................244

Table 7.21 Potentially contributing acterial orders between 200 and $300 \mathrm{bp}$............245

Table 7.22 Potentially contributing bacterial orders for T-RFs 300-552bp .............. 246

Table 7.23 Bacterial orders under pig carcasses and cadavers on soil and sand.......247

Table 7.24 Bacterial orders under human cadavers and pig carcasses. ...................248

Table 7.25 Bacterial orders that were found under cadavers on soil.........................248

Table 7.26 Bacterial orders from samples under pig carcasses. ................................249

Table 7.27 Bacterial orders that were found to be contributors to the T-RFs............249

Table 7.28 PCR recipe for clone insert creation.......................................................... 253

Table 7.29 PCR thermocycling regime for clone insert creation..............................253 


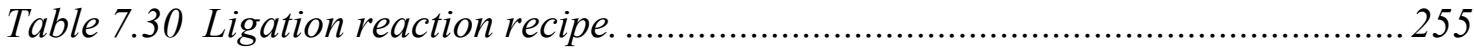

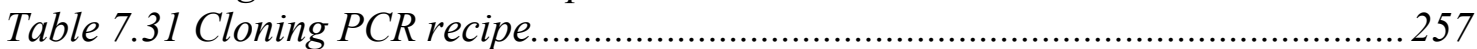

Table 7.32 PCR protocol for clone pool T-RFLP analysis. .......................................2257

Table 7.33 Bacteria identified as closest matches to cloned sequences.....................2.265

Table 7.34 Bacterial orders identified as being present in the bacterial community. 266

Table 7.35 Bacterial orders identified as being present in the bacterial community. 267

Table 7.36 Bacterial orders identified by sequencing up to $200 \mathrm{bp}$.........................2.270

Table 7.37 Bacterial orders identified by sequencing between 200 and 300 bp........2.271

Table 7.38 Bacterial orders identified by sequencing greater than $300 \mathrm{bp}$. 


\section{Abbreviations}

ADDs - Accumulative Degree Days

AMC - 7-Amino-4-methylcoumarin (fluorogenic substrate)

BSA - Bovine Serum Albumin

CDHs - Cumulative Degree Hours

DGGE - Denaturing Gradient Gel Electrophoresis

DNA - Deoxyribonucleic Acid

dsDNA - Double Stranded DNA

ESR Ltd - Institute of Environmental Science \& Research Ltd.

FAM - 6-Carboxyfluorescein

FAME - Fatty Acid Methyl Ester analysis

GIT - Gastrointestinal Tract

HEX - 6-Carboxyhexafluorescein

MES - 2-(4-Morpholino) ethanesulphonic Acid Monohydrate

MUB - Methylumbelliferone (fluorogenic substrate)

ORNL - Oak Ridge National Laboratory

PCA- Principle Component Analysis

PCR - Polymerase Chain Reaction

PMI - Post Mortem Interval

rDNA - Ribosomal Deoxyribonucleic Acid

RFU - Relative Fluorescence Units

RNA - Ribonucleic Acid

rRNA - Ribosomal Ribonucleic Acid

ssDNA - Single Stranded DNA

T-RF - Terminal Restriction Fragment

T-RFLP - Terminal Restriction Fragment Length Polymorphism

TSD - Time Since Death

VFA - Volatile Fatty Acid 


\section{Chapter 1: Introduction and Aims}

The processes by which human bodies decompose after death are, largely, unknown. The study of human decomposition is a relatively recent field of research, and until recently, very few organisations around the world devote resources to this topic. Forensic anthropologist, Dr William Bass recognised this lack of knowledge of the decomposition process in the late 1970 's which led to the development of the world's first centre for the study of human decomposition, The University of Tennessee's Forensic Anthropology Facility (Bass and Jefferson, 2003). The centre has since gone on to become the main authority in forensic death research worldwide.

Forensic decomposition research has, until recently, concentrated on physical anthropology, entomology and taphonomy, and only fragmented data exist on the actual biochemical and microbiological processes that occur during body decomposition. This is because forensic requirements focus mainly on identification of remains and on crime scene interpretation, and hence most research efforts and resources have been put into these areas. The estimation of time since death, or the post mortem interval (PMI), however, has recently become an area of growing interest. Accurately determining the PMI is now an essential part of any death investigation (Henssge and Madea, 2007). The techniques currently employed for PMI determination, while useful, are not always able to give an accurate time of death (Swift et al., 2001). Research into application of innovative techniques and ideas is required for continued development of forensic science and to help law enforcement improve the resolution rate of crimes. Accurately determining time elapsed since death provides clearly defined timeframes for investigators to work within and can also lead to more rapid identification of the deceased and quicker resolution of investigations (Gerberth, 1996). 
Decomposition of organic life-forms is facilitated by two main groups of organisms: insects and microbes (Carter and Tibbett, 2006). Insects have been well studied because of their known use as post mortem interval bio-indicators (Payne, 1965; Campobasso et al., 2001; Lopes de Carvalho and Linhares, 2001), but the microbiology associated with human decomposition is largely unexplored. This is, in part, likely to be due to cultural taboos that surround death and decomposition but may also be due to the perceived complexity of such a diverse microbial community. The advent of molecular ecology tools which allow researchers to study changes in microbial community structures (as well as molecular biology tools such as cloning and sequencing) have opened a pathway for studying complex microbial communities that bypasses the laborious and limiting culturing-based microbiological methods.

The microbial communities involved in decomposition of a human body are thought to be highly diverse (Vass, 2001). Complex interactions and successions of species ensure complete and efficient break down of the body tissues and use of the derived resources for growth. While environmental factors can greatly influence the decomposition process, tissues and organs typically break-down in a characteristic and sequential manner (Gill-King, 1997). It is also known that individual insect species will colonise a body at a particular stage of decomposition that provides for optimal nutritional benefit to that organism, thus causing an ordered succession of species to inhabit the carcass (Anderson, 2001). Microbes also exploit favourable niches, replicating quickly when nutritional and environmental conditions are most suitable, and are quickly out-competed by better-adapted species when conditions change. The complex and dynamic decomposition process is likely to provide a continuously changing environment for microbial species, offering the opportunity for succession to occur. The putrefactive or active decay stage of decomposition is primarily driven by anaerobic organisms from within the carcass itself (Forbes, 2008). The human body is believed to carry 
more bacterial cells than human cells (Tannock, 1994), providing a profusion of organisms awaiting an opportunity to exploit the lack of regulation that occurs upon death of the human cells. While the innate microflora of mammalian bodies is still not well described, a growing body of evidence suggests that these microbial communities are diverse, and provide essential services to their mammalian host (Amor and Vaughan, 2006). It is known that individual hosts have unique combinations of microbial species, but tend to have stable and remarkably similar gut flora composition at higher taxonomic levels (Guarner and Malagelada, 2003). There are also key members of the human microbiota that seem to be common to all humans (Wilson, 2005). It is also, then, likely that the same main microbial groups will participate in degradation of the host tissue in different mammalian hosts.

Gaining a greater understanding of the human decomposition process, in particular of the microbiology associated with decomposition is likely to enable development of innovative PMI estimation methods and is a step towards better time since death estimation in forensic death investigations.

\subsection{Aims}

The overall aim of this research is to investigate the bacterial community associated with exposed decomposing mammalian carcasses on soil and to assess whether changes in this community have the potential to be used in the development of a time since death tool for use in forensic investigations.

\subsection{Research Approach}

A combination of chemistry, biochemistry and molecular biology techniques were employed in this study with the aim of gaining a more complete picture of the microbial processes associated with decomposition. The molecular biology tools used include: denaturing 
gradient gel electrophoresis (DGGE), terminal restriction fragment length polymorphism (TRFLP) analysis and cloning and sequencing. These are used extensively in ecological studies to monitor and characterise changes in complex bacterial communities. The chemistry and biochemistry methods used include measurement of $\mathrm{pH}$, nitrate and ammonium ion concentrations and various enzyme activity assays. These methods are used routinely in soil ecology for monitoring the impacts of land treatments, such as the application of sewage biosolids to soils (Speir et al., 2003). This is the first study to combine these tools to examine the processes that occur in soil in response to decomposition of a human or mammalian carcass.

The experimental approach was to begin by using a model animal system (pig carcasses) to investigate the bacterial communities associated with decomposition in two experiments in New Zealand, followed by a human cadaver decomposition study performed in the United States. The first experiment studied a simplified bacterial community, by allowing pig carcasses to decompose on sterile sand. The sand acted as a matrix to catch the decomposition products in the same way that soil would, but without the complication of the existing microbial community. The effect of insect colonisation on the bacterial community was also examined, with some carcasses being protected from normal colonisation. The second experiment involved the introduction of soil as the underlying matrix, again using pig carcasses, and with all carcasses allowed to be colonised normally by insects. The third experiment used human cadavers placed on both soil and sterile sand, again all exposed to insect colonisation. Although it was impossible to control many of the variables associated with using human cadavers, the information about the bacterial populations associated with human decomposition was compared with the pig decomposition data, to determine whether the pig (Sus scrofa) is suitable as a model for studying human decomposition microbiology. The advantage of running three successive experiments is that it allowed analytical methods 
and experimental setup to be altered and fine-tuned to accommodate the difficulties and peculiarities of decomposition research. Some methods were found to be unsuitable for use with decomposition derived samples, and others generated unexpectedly interesting results.

The laboratory strategy used was to screen almost all soil and sand samples using T-RFLP to characterise the bacterial community at each time point, followed by more in-depth investigation of selected samples using other molecular techniques. Cloning and sequencing of DNA derived from samples from particular time points allowed identification of a number of key organisms. 


\section{Chapter 2: Background and Literature Review}

\subsection{Human Decomposition}

Remarkably little is known about the process of human decomposition (Mann et al., 1990). It is a complex process affected by a myriad of environmental factors as well as the physical characteristics of the individual. The rate at which a body decomposes is primarily determined by the environment in which that body lies, although in many cases, the body moves through a predictable degradation process regardless.

\subsubsection{Physical and Chemical Processes of Decomposition}

Human decomposition begins as soon as four minutes after death. Characteristic changes in the early post mortem period include the cooling of the body to ambient temperatures (algor mortis), the stiffening of muscles (rigor mortis) and the settling of blood (livor mortis) due to gravity (Clark et al., 1997). As cells are starved of the oxygen they require to live, carbon dioxide builds up, $\mathrm{pH}$ decreases, wastes accumulate and cells rupture through a process called autolysis (Vass, 2001). This process begins in tissues with high enzyme activity such as the brain and liver and proceeds to all tissues of the body. The release of nutrient-rich cellular fluids during cell lysis promotes the anaerobic process of putrefaction where the body's natural microflora gain access to the internal tissues and begin to break them down, releasing gases as by-products of metabolism (Clark et al., 1997). These collect within the abdominal cavity, bloating the body. Gas and fluid accumulation purge from natural body openings or burst the skin, releasing decomposition compounds into the external environment (Vass, 2001). Active decay follows, and is characterised by heavy aerobic microbial and insect activity. By the end of this stage only approximately 10 percent by weight of the corpse remains. The skeleton and any remaining tissues continue to dry, and bone is slowly degraded 
through a process known as diagenesis (Galloway, 1997). The progression of decomposition can be divided into a rough sequence of stages (Goff, 2000) as seen in Figure 2.1.
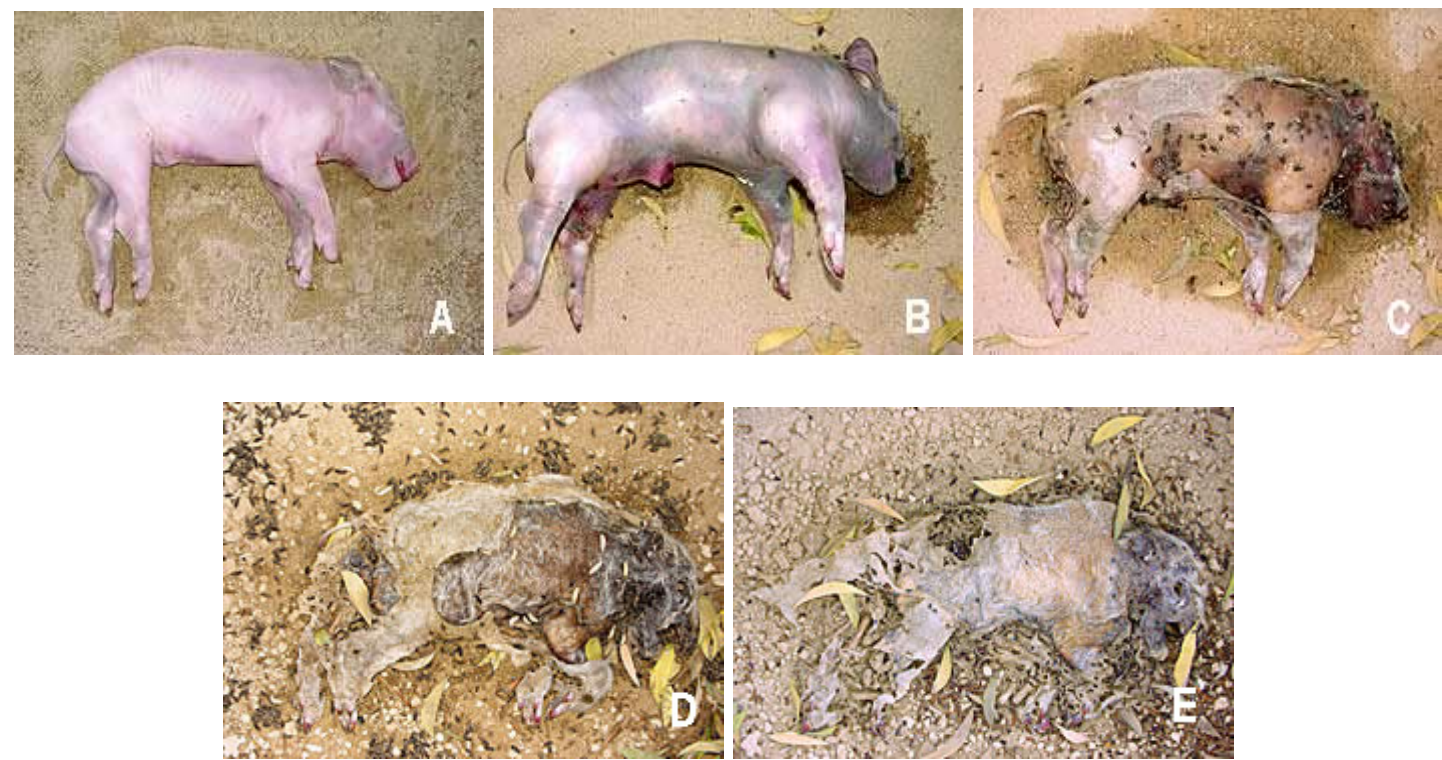

Figure 2.1 The five basic stages of decomposition : (A) fresh, (B) bloat, (C) active decay, (D) post decay or dry and (E) skeletonisation (Australian Museum, 2003)

Under certain conditions, a substance known as adipocere can form. This is a soft, greyish white, cream-like substance that is often referred to as grave wax (Fiedler and Graw, 2003). It is most commonly encountered in burials and submerged bodies, as it tends to form in warm, damp environments where oxygen is limited (Forbes et al., 2005), but can also be associated with warm, dry conditions (Janaway, 1996). The degree of adipocere formation can vary from a small amount only found in body areas rich in adipose tissue, through to most of the body. Preservation of soft tissues, as well as eyes, genitals and internal organs has also been observed in some cases (O'Brien and Kuehner, 2007). Triglycerides in the soft tissues are hydrolysed by bacterial enzymes which causes a mixture of free fatty acids to form. As decomposition proceeds, the quantity of free fatty acids in the tissue increases while the amount of triglycerides decreases. Hydrogenation of the unsaturated fatty acids occurs to yield saturated fatty acids such as myristic, palmitic and stearic acids (Forbes et al., 2004) which form the adipocere mass. An obligate anaerobe, Clostridium perfringens has been 
identified as playing a role in the hydrogenation of fatty acids. This process creates unfavourable growth conditions for most micro-organisms, reducing the degree of bacterial decomposition and causing preservation of the tissues (O'Brien and Kuehner, 2007).

\subsubsection{Variables Affecting Decomposition}

Mann et al. (1990) concluded that "variability in the decay rate of the human body is the rule" because of the huge influence that a wide array of factors can have on the decomposition process. Environmental conditions such as temperature, humidity and rainfall have a significant influence on decomposition rate, as do factors such as the surface the body is placed on, clothing present and extent of pre-existing trauma to the body. The characteristics of the body itself also influence the process and rate at which it decays. Mann et al. (1990) identified many of the known variables, and their estimated degree of effect (Table 2.1).

Temperature has the greatest impact because of its effect on the live participants in the decomposition process: the insects and micro-organisms. If the temperature is low, the whole decomposition process is slowed, and may be completely arrested if the body is near freezing. Similarly, if the temperature is high, the process can be very fast due to an increase in biological activity and chemical reaction rates (Carter and Tibbett, 2006). If the humidity is low, however, such as occurs in a desert environment, a body will largely desiccate and mummify (Galloway, 1997). 


\begin{tabular}{|c|c|}
\hline Variable & Effect on Decay Rate* \\
\hline Temperature & 5 \\
\hline Access by insects & 5 \\
\hline Burial and depth & 5 \\
\hline Carnivores/rodents & 4 \\
\hline Trauma (penetrating/crushing) & 4 \\
\hline Humidity/aridity & 4 \\
\hline Rainfall & 3 \\
\hline Body size and weight & 3 \\
\hline Embalming & 3 \\
\hline Clothing & 2 \\
\hline Surface placed on & 1 \\
\hline Soil pH & unknown \\
\hline
\end{tabular}

Table 2.1 Variables affecting the decay rate of a human body (from Mann et al., 1990). *Subjective criteria based on a five-point scale, 5 being the most influential.

Insect access to the body also determines the way in which a body will decompose. If access is restricted or prevented completely, decomposition proceeds with the microbes alone, making for a much slower process. Similarly, trauma to the body can speed decomposition as insects preferentially colonise natural and injury-derived orifices (Mann et al., 1990).

Mann et al. (1990) identify burial as a major factor influencing the rate of decomposition. Whether a body is buried, submerged in water or left on the soil surface will in turn influence other factors such as temperature, insect and scavenger access to the body and oxygen availability and so also greatly affect the rate of decomposition. Processes occurring within the burial environment seem to have been more comprehensively studied than surface decomposition, although much of the present data are observational rather than experimental (Carter and Tibbett, 2008).

Other environmental conditions such as exposure to sunlight, scavengers, rainfall, and humidity also have a major effect on the decomposition process. Geographic location will influence many of these conditions. For instance, a deep gully will provide a very different 
decomposition environment to an exposed hillside site. Different locations can also have distinct insect and scavenger populations. Introduced species such as dogs, cats, rodents and possums, and native birds such as hawks are the most likely scavengers to be attracted to a corpse in New Zealand. Because of New Zealand's geographic isolation and unique fauna, the insect species involved in corpse decomposition differ from those in other countries (Smeeton et al., 1984). High humidity and rainfall can affect the rate of cadaver breakdown by saturating the tissues with water, preventing aerobic degradation (Campobasso et al., 2001). Humidity is positively correlated with insect activity while rainfall has little effect on the insects (Mann et al., 1990).

Physical differences between individual human bodies may also influence the decomposition process. Mann et al. (1990), observed that heavier individuals decompose more quickly and that there appears to be a "melting away" of the excess body fat. They cite experimental observations where large individuals $(>110 \mathrm{~kg})$ were placed alongside smaller individuals ( $\sim 65 \mathrm{~kg})$. The larger individuals displayed much faster progression through the stages of decomposition. No difference in decomposition between the sexes has been documented beyond the size and body fat effects.

Mann et al. (1990) also noted that bodies placed on concrete decayed more slowly and became mummified faster than bodies placed on soil. This difference could be related to temperature differences, but could also be caused by soil microbes participating in the decomposition process of bodies. Soil properties such as $\mathrm{pH}$ and texture are likely to affect decomposition rate and processes. Carter and Tibbett (2008) speculate that decomposition may be expected to be slower in acidic soils. Coarse-textured (sandy) soil with low moisture content frequently promotes desiccation (Fiedler and Graw, 2003), which is likely to be related to a higher rate of gas and water vapour diffusion through the soil matrix (Carter and Tibbett, 2008). Finetextured (clay) soils have been associated with retarded decomposition in a burial environment 
(Turner and Wiltshire, 1999; Hopkins et al., 2000) due to lower gas diffusivity, leading to development of reducing conditions and slow, anaerobic degradation (Carter and Tibbett, 2008). Carter and Tibbett (2002) also propose that the soil microbial population can adapt in response to previous decomposition events, resulting in more rapid decomposition rates of subsequent cadaveric material.

\subsubsection{Decomposition Microbiology}

Limited information exists on which microbial species might play a role in the decomposition process (Janaway, 1996; Clark et al., 1997; Gill-King, 1997; Carter and Tibbett, 2008; Hopkins, 2008). Current knowledge is based mainly on observational studies and to date, there have been no comprehensive surveys of the microbial ecology of human decomposition. This is likely to be due to the restrictions of classical microbiological methods and the number of microbial species expected to be involved in the decomposition process (Vass, 2001).

\subsubsection{Human Body Microflora}

The human body contains ten times as many microbial cells as mammalian cells (Wilson, 2005). This equates to each of us carrying around approximately $1.25 \mathrm{~kg}$ of microbes. It is estimated that each person has at least 1,500 different microbial taxa resident on and in them (Wilson, 2005). Humankind has co-evolved with microbial life-forms over many millennia to develop a delicately balanced symbiosis of mutual benefit to both.

The human microbiota consists of a number of microbial communities, each with a composition characteristic of a particular body site. By far the most densely populated region is the gastrointestinal tract (GIT) with $10^{14}$ bacterial cells resident in the average adult (Wilson et al., 2002). The oral cavity is also densely populated and has very different species colonising different regions (e.g. the teeth, tongue and gingival crevice). The vagina flora is 
less diverse but still densely populated and is dominated by lactobacilli (Vitali et al., 2007). The skin, urinary tract, upper respiratory tract and eyes also have their own characteristic resident microflora. Although most of the body surfaces exposed to the external environment are colonised by microbes, some, such as the lungs, are not (Kim and Weiser, 1999).

The resident microflora of an individual is greatly influenced by age, gender and genotype, as well as environmental factors such as diet, disease and medications (Guarner and Malagelada, 2003). Humans begin life without a microbiota but are quickly colonised during and after birth. In breast-fed children prior to weaning, the faecal microbiota, for example, are dominated by Bifidobacterium spp., whereas in adults Bacteroides spp. predominate (McCartney and Gibson, 2006). In the elderly, differences to younger adults arise due to a weaker immune system, poorer nutrition and dysfunction of organs (Hebuterne, 2003; Bartosch et al., 2004; Woodmansey et al., 2004). The host genotype is also thought to have a strong influence on their resident microbial population, but few studies have explored this relationship to date, except in relation to disease susceptibility (Zoetendal et al., 2001). Diet has a huge effect on the innate microbiota, in particular on those of the gastrointestinal tract (Drasar et al., 1973). Medications, such as antibiotics, can also have a massive effect on microfloral composition (Jernberg et al., 2005). Antibiotics such as ampicillin, clindamycin and cefoperazone, for example, have dramatic effects on the microbiota of the colon, whereas others such as chloramphenicol, metronidazole and sulphonamides appear to have little overall effect (Bartosch et al., 2004; Wilson, 2005). Emotional stress has also been shown to affect an individual's microfloral composition (Holdeman et al., 1976).

\subsection{The Gastrointestinal Tract (GIT) Microflora}

The GIT consists of a series of different anatomical sites (the oesophagus, stomach, duodenum, jejunum, ileum and colon), each having a different microbial community and 
population density. The stomach, duodenum and ileum have relatively low numbers of bacteria, whereas the jejunum, caecum and colon are densely populated. The stomach, because of its low $\mathrm{pH}$ and the rapid transit of material through it, has only an estimated $10^{3}$ $\mathrm{cfu} / \mathrm{ml}$ of viable microbes (Wilson, 2005). Species frequently isolated are acid-tolerant streptococci and lactobacilli. The upper region of the small intestine (duodenum and jejunum) also has a low $\mathrm{pH}$ and rapid peristalsis, and has a range of secretions which prevent heavy microbial colonisation (Hao and Lee, 2004). Like the stomach, most of the species isolated from the small intestine are acid-tolerant, although others such as Bacteroides spp., enterobacteria, Bifidobacterium spp. and Veillonella spp. have been isolated. In the ileum, peristalsis is slower and the $\mathrm{pH}$ is also closer to neutral. The luminal contents of the ileum can contain between $10^{6}$ and $10^{8} \mathrm{cfu} / \mathrm{mL}$, and the microbiota is dominated by facultative anaerobes (Wilson, 2005). The mucosal surface of the ileum, however, has many obligate anaerobes. Streptococci are the most frequently isolated group, followed by the enterococci, enterobacteria, Veillonella spp., Clostridium spp., lactobacilli and Bacteroides spp. (Wang et al., 2003; Wang et al., 2005). The large intestine (colon) is the most densely populated site of the human body. Each gram of faeces contains approximately $10^{8}$ facultative anaerobes and $10^{11}$ obligate anaerobes. More than 400 species from more than 190 genera have been isolated from the colon, but this is likely to be a gross underestimate of the true number, as culturing techniques only capture between $15-58 \%$ of the total species, depending on the culturing method (Wilson, 2005).

\subsection{The Skin Microflora}

After the GIT, skin harbours the second largest number of bacterial residents, primarily because it is the largest organ of the human body. Human skin has a complex microbial ecosystem with both transient residents, and long term resident organisms (Gao et al., 2007). Normal skin flora includes Staphylococcus, Micrococcus, Corynebacterium, Brevibacteria, 
Propionibacteria, and Acinetobacter (Roth and James, 1988). Gao et al. (2007) recently completed a survey of bacterial species associated with the forearm skin of six healthy volunteers using $16 \mathrm{~S}$ rDNA sequencing and cloning, and discovered that each individual had a community composition unique to themselves that is relatively stable over time. Of the five bacterial phyla found on other body sites (Actinobacteria, Firmicutes, Proteobacteria, Bacteroidetes and Fusobacteria) all except Fusobacteria are present on the skin. Skin, however, is the only mucosal surface on which Actinobacteria predominate (Gao et al., 2007).

\subsection{Microflora of Other Body Sites}

More than 200 bacterial species have been cultured from the oral cavity of humans, and between 400 and 500 additional taxa detected by $16 \mathrm{~S}$ rRNA gene analysis. Of the possible

microbes present, however, a more limited number, perhaps up to 75 are present in the oral cavity of a healthy adult at any given time (Wilson, 2005). The most frequently detected genera are listed in Table 2.2.

The respiratory system includes the nose and pharynx (upper respiratory tract) together with the larynx, trachea, bronchi and lungs (lower respiratory tract). While the upper respiratory tract is usually colonised, the bronchi and lungs are generally sterile (Kim and Weiser, 1999). One of the roles of the upper respiratory tract is to filter particulate matter from inhaled air and to provide antimicrobial defence for the lungs. This involves secretion of mucous, the composition of which differs throughout the respiratory tract. Mucous contains a number of antimicrobial substances and prevents adhesion of many organisms to the tract surface. The most frequently detected species of the respiratory tract are shown in Table 2.2. 


\begin{tabular}{|c|c|}
\hline Body Site & Predominant Organisms \\
\hline Gastrointestinal Tract & $\begin{array}{c}\text { Bacteroides, Eubacterium, Clostridium, } \\
\text { Bifidobacterium, Streptococcus, Lactobacillus, } \\
\text { Peptostreptococcus, Peptococcus, Ruminococcus, } \\
\text { Fusobacterium, Veillonella, Enterococcus, } \\
\text { Propionibacterium, Actinomyces, } \\
\text { Methanobrevibacter, Desulphovibrio, } \\
\text { Helicobacter, Porphyromonas, Prevotella, } \\
\text { Escherichia, Enterobacter, Citrobacter, Serratia, } \\
\text { Candida, Gemella, Proteus }\end{array}$ \\
\hline Skin & $\begin{array}{l}\text { Corynebacterium, Staphylococcus, } \\
\text { Propionibacterium, Micrococcus, Malassezia, } \\
\text { Brevibacterium, Dermabacter, Acinetobacter }\end{array}$ \\
\hline Oral Cavity & $\begin{array}{c}\text { Streptococcus, Actinomyces, Veillonella, } \\
\text { Fusobacterium, Porphyromonas, Prevotella, } \\
\text { Treponema, Neisseria, Haemophilus, } \\
\text { Eubacterium, Lactobacillus, Bifidobacterium, } \\
\text { Capnocytophaga, Eikenella, Leptotrichia, } \\
\text { Peptostreptococcus, Staphylococcus, } \\
\text { Propionibacterium }\end{array}$ \\
\hline Respiratory Tract & $\begin{array}{c}\text { viridans streptococci, Strep. pyogenes, Strep. } \\
\text { pneumoniae, Nesseria spp., Haemophilus spp., } \\
\text { Moraxella spp., Staph. aureus, coagulase-negative } \\
\text { staphylococci, Corynebacterium spp., } \\
\text { Propionibacterium spp. Prevotella spp., } \\
\text { Mollicutes, Porphyromonas spp. }\end{array}$ \\
\hline Female Reproductive System & $\begin{array}{l}\text { Lactobacillus spp., Staphylococcus spp., } \\
\text { Corynebacterium spp., Streptococcus spp., } \\
\text { Enterococcus spp., Can. albicans, Bifidobacterium } \\
\text { spp., Gardnerella vaginalis, Propionibacterium } \\
\text { spp., Gram-positive anaerobic cocci, Bacteroides } \\
\text { spp., Porphyromonas spp., Prevotella spp., } \\
\text { Clostridium spp., Fusobacterium spp., Veillonella } \\
\text { spp., Ureaplasma } \text { spp. Mycoplasma spp. }\end{array}$ \\
\hline Male Urinary Tract & $\begin{array}{l}\text { Coagulase-negative staphylococci, } \\
\text { Corynebacterium spp., viridans streptococci, } \\
\text { Gram-positive anaerobic cocci, Mollicutes }\end{array}$ \\
\hline Female Urinary Tract & $\begin{array}{c}\text { Lactobacillus spp., coagulase-negative } \\
\text { staphylococci, Corynebacterium spp., viridans } \\
\text { streptococci, Bacteroides spp., Gram-positive } \\
\text { anaerobic cocci, Mollicutes }\end{array}$ \\
\hline
\end{tabular}

Table 2.2 Predominant organisms of the body sites colonised by microflora (Wilson, 2005).

Of the parts of the female reproductive system, only the vagina, vulva and cervix are normally colonised by microbes. The microbial communities of these areas are dominated by lactobacilli. The average number of lactobacilli in the vagina of a post-menarchal, pre- 
menopausal woman is between $10^{8}$ and $10^{9}$, and in most women, only a single Lactobacillus species is present (Pascual et al., 2006). Other organisms are often present (see Table 2.2), but these tend to vary widely between hosts (Larsen and Monif, 2001). The urethra is the only area of the urinary system colonised by microbes in healthy individuals and the predominant organisms differ between males and females (see Table 2.2).

\subsubsection{The Role of Body Microflora in Decomposition}

Very little is known or understood about the roles that the microflora plays in decomposition of a human body. The natural body microflora is thought to play a central role in the anaerobic putrefaction stage of decomposition (Gill-King, 1997; Forbes, 2008) primarily because this process occurs deep within the body before insect activity becomes well established. In a normal adult colon, the bacterial community consists of $96-99 \%$ anaerobes e.g., Bacteroides spp., anaerobic lactobacilli, clostridia, and anaerobic streptococci, and only 1-4 \% aerobes e.g., Gram negative coliforms, enterococci, and small numbers of Proteus spp., Pseudomonas spp. and others (Jawetz et al., 1982). It is believed that the enteric anaerobes gain access to other tissues post-mortem via the lymphatic and artero-vascular system (Janaway, 1996). It is likely that other organisms such as the aerobic skin flora also begin to degrade their associated host tissue during this time.

\subsubsection{Soil Microbial Communities}

Human and mammalian decomposition are likely to involve not only the microbes in and on the body, but also the bacterial communities in the environment, including if the body is on soil, from the soil as well. Putrefaction is thought to be primarily driven by the body's own flora, but environmental microbes are likely to be heavily involved in the aerobic active decay phase. 
The organisms that live in soil range from the microscopic flora (bacteria and fungi), through to the macrofauna (earthworms, millipedes, spiders, insects and molluscs). Bacteria are the most numerous component of the soil microbial population. It has been estimated that there may be as many as $10^{9}$ bacterial cells per gram of soil (Harris, 1994) and it is widely accepted that the majority of soil bacteria, possibly as many as $99 \%$, cannot be cultured using traditional laboratory media based techniques (Amann et al., 1995). This is due to the complex nature of the environments that soil provides, which can not be simulated in the laboratory. The most common culturable soil bacterial genera are Arthrobacter, Pseudomonas and Bacillus (Killham, 1994).

Soil bacteria often show morphological and physiological adaptations that allow them to utilise the soil habitat effectively. Many soil bacteria also exhibit the ability to slow their metabolism in response to low levels of available carbon and nutrients, and then increase it when nutrient levels rise (Wood, 1995). Metabolic flexibility allows the persistence of microbial species over time, as soil is nutritionally poor most of the time (Stotzky, 1997).

Soil microbes can be divided into two distinct classes in terms of their response to nutrients. This idea was first proposed by Winogradsky (1949) who described the classes as autochthonous and zymogenous. Autochthonous microbial populations are indigenous soil organisms that 'tick over' and are most competitive at low substrate concentrations. Their populations persist actively in the soil for long periods of time and are present at relatively constant levels. They use soil carbon sources that are relatively resistant to degradation, such as complex organic matter. The genus Arthrobacter is an example of autochthonous soil microbes that are able to maintain their presence in soil, even when readily available carbon is limiting. The zymogenous soil microbial populations are not a significant component of the soil microbial community under normal conditions, but proliferate when substrates such as plant or animal residues are introduced into the soil. They are capable of rapid multiplication 
and can often revert to resistant spore structures once the substrate is exhausted. Some saprophytic (decomposer) fungi are zymogenous, particularly those known as the "sugar fungi", which can only utilise simple sugars, and are not able to degrade more complex organic compounds (Thorn, 1997). Competition between soil organisms for nutrients is likely to lead to specialisation in terms of rate of growth and substrate utilisation. The zymogenous population may respond to readily available substrates such as simple sugars, while the autochthonous may use the more complex, less readily available substrates such as cellulose.

Actinomycetes are a component of the soil microbial community that are bacteria, but have a mycelial morphology, resembling fungi. Actinomycetes have the ability to degrade an enormous array of carbonaceous substrates including recalcitrant polymers such as chitin, celluloses and hemicelluloses (Wood, 1995).

\subsubsection{The Role of Soil Microflora in Decomposition}

A number of recent studies have looked into the effect of decomposition on the soil microbial community. The decomposition of any mammalian cadaver creates a localised patch of nutrients in the environment, which has been described by Carter et al. (2007) as a Cadaver Decomposition Island (CDI). Carbon is the most abundant element in the body, on a dry mass basis, comprising around $18 \%$ of the human body by mass (Chang, 2006). The human body is also estimated to contain approximately $32 \mathrm{~g} / \mathrm{kg} \mathrm{N}, 10 \mathrm{~g} / \mathrm{kg} \mathrm{P}, 4 \mathrm{~g} / \mathrm{kg} \mathrm{K}$ and $1 \mathrm{~g} / \mathrm{kg} \mathrm{Mg}$ (Tortora and Grabowski, 2000), which are released during active decay into the surrounding local environment. These nutrients are likely to be used to support the growth of some soil microflora, promoting changes in the composition of the soil microbial community. These changes can be long-term and may become more significant as decomposition progresses (Goff, 2000). The microbial species associated with decomposition may be present for months or even years after death, depending on local conditions. Carter and Tibbett (2002) 
documented an increased rate of decomposition in soils that had previously been exposed to soft-tissue due to an adaptation of the microbial community, although there was no associated change in microbial activity or biomass. Hopkins (2008) suggests that soil microorganisms are likely to have a relatively minor role in cadaveric decay, with the enteric community primarily responsible for biodegradation of body tissue.

Hopkins et al. (2000) investigated the microbiological activity in soil from pig graves 430 days after burial. The grave soils contained more total carbon, microbial biomass carbon and total nitrogen, and showed increased rates of respiration and nitrogen mineralisation compared with control soils. The grave soils also had higher amino acid and $\mathrm{NH}_{4}^{+}$concentrations, and reducing conditions. In contrast, Fiedler (2004) reported a decrease in soil microbial biomass, but speculate that this is due to the inert nature of adipocere which was present within the graves studied. Stokes et al. (2009) recorded increases in microbial respiration, ammonium, nitrate, lipid-phosphate and phosphate concentrations in three Australian grave soils containing juvenile mouse carcasses. No significant increase in total carbon in the grave soil was noted in this study, and the authors hypothesize that this was due to loss of carbon from the soil as carbon dioxide (Stokes et al., 2009).

\subsection{Post Mortem Interval Estimation}

Determination of time since death, or the post-mortem interval (PMI) is often essential in forensic cases, particularly homicides. It is, however, almost always a difficult task. The more recent the death, the more accurate is the PMI prediction, as estimation can be narrowed to a fairly limited time range. A variety of tools have been used to estimate the PMI, depending on the stage of decomposition of the body. Physical changes in the body itself can be used early in decomposition, and for later stages, changes to the surrounding environment, 
such as vegetation growth, or the study of the insects which colonise the body (forensic entomology) can be useful.

The physiological and biochemical changes that occur to a body after death can be reliably used to estimate the PMI for up to 24 hours following death. Early post mortem changes that can be used include body temperature, degree of onset of rigor mortis and livor mortis and biochemical changes within the body (Svensson et al., 1981). Examining the state of the gastric contents of a body can determine how much time elapsed between the last meal and the time of death, and if the time of the last meal is known, this can be used to estimate the time since death.

Forensic entomology is a commonly used method for estimating the post mortem interval, and one that can be used from the time of first insect colonisation until after active decay is finished. The succession of insects that invade corpses has been widely studied. Necrophilous insects arrive at an exposed body quickly and in a relatively predictable sequence (Goff, 2000). Knowledge of the lifecycles of body-colonising insects can be used by experienced entomologists to estimate time since death (Pickering and Bachman 1996), although insect species involved in decomposition can vary widely depending on location, and local knowledge is essential.

The state of vegetation found under and around a body can also be used as an indicator of how long a body has been exposed. Plants go through a seasonally determined growth cycle, so those growing in or around a skeleton can indicate how long it may have been there, as can the state of plant matter under a body. Plants in flower may be buried under a body, indicating the time of year of burial based on knowledge of when particular plant species flower at that site. Growth comparisons can also be made with similar plants in the vicinity (Svensson et al., 1981). Forensic botany, however, is not a commonly used tool due to the low frequency in which suitable cases occur (Bock and Norris, 1997). 
Various innovative techniques have been investigated in recent years to aid in post mortem interval estimation and research continues into gaining a better understanding of these existing tools. Forensic entomology remains the focus of much PMI estimation research with many studies undertaken worldwide (Malgorn and Coquoz, 1999; Amendt et al., 2000; Arnaldos et al., 2001; Myskowiak and Doums, 2002). Studies of biochemical and physical changes in the post mortem body are also continuing, often with the more traditional tools being improved by the application of newer technology. For example, determination of electrolyte concentrations (mainly potassium) in the vitreous humour of the human eye is one tool currently being upgraded. Bocaz-Beneventi et al. (2002) have applied new electrolyte detection techniques including capillary zone electrophoresis (CZE) to quantify these post mortem changes more accurately. Querido $(1998 ; 1999)$ investigated the potential of using electrical impedance of lateral abdominal skinfolds and scalps on rat cadavers as a PMI estimation tool, but found that individual variation made it unsuitable. Babapulle and Jayasundera (1993) found that the degeneration of white blood cells in cadavers could be used to estimate the PMI reasonably accurately up to 84 hours after death.

Volatile fatty acids, which are microbially produced degradation products of soft tissue, and various anions and cations were investigated by Vass et al. (1992) to estimate PMI for preskeletonised remains. Concentrations of these decomposition products were determined in soil samples taken from underneath bodies over time. The ratios of specific fatty acids were found to be predictive of the stage of decomposition with a reported accuracy of \pm 2 days. More recently, Vass et al. (2002) surveyed biomarkers in various organs of the body to develop a PMI estimation tool that is more accurate. Biomarkers such as amino acids, neurotransmitters and decomposition by-products from various organs were studied and it was shown that each biomarker is found in a tissue for a specific period of time. By examining relative concentration ratios between different markers, the number of cumulative degree 
hours (CDHs) since death can be calculated Figure 2.2. Cumulative degree hours are calculated by summing the average temperature $\left({ }^{\circ} \mathrm{C}\right)$ for each twelve hour period since death. The $\mathrm{CDH}$ gives a measure of the stage of decomposition irrespective of climatic conditions. By comparing data from a number of tissues, the time range could be narrowed to as little as 5 CDHs (up to $1000 \mathrm{CDHs}$ or 3 weeks after death). The significant advantage of this technique is that variables such as the victim's weight do not need to be taken into consideration because the method is based on ratios between the biomarkers and not absolute values.

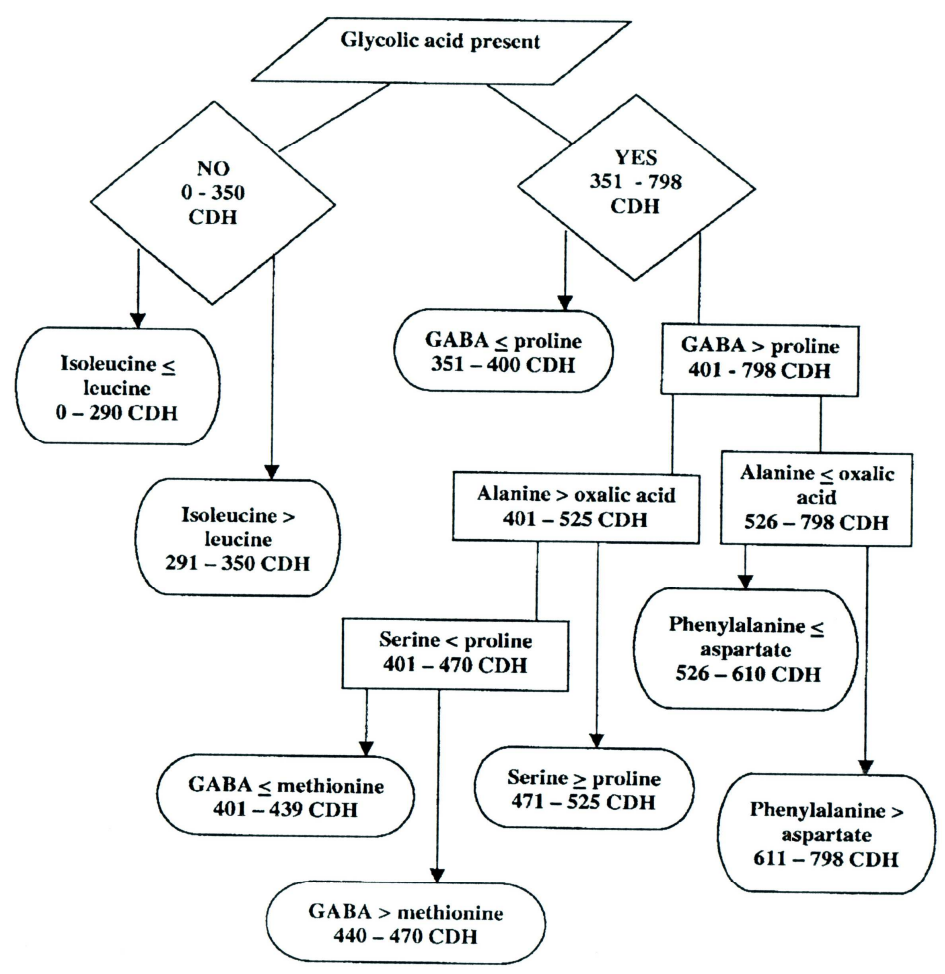

Figure 2.2 Flow diagram for determining time since death in cumulative degree hours (CDH) using the ratios of biochemical biomarkers in muscle tissue (Vass et al., 2002).

Carter and Tibbett (2003) recognised that certain fungal species that are associated with decomposition could be used as a PMI estimation tool. Known as post-putrefaction fungi, these species are commonly found in association with graves. The authors speculate that with better understanding of the fungal succession associated with cadavers, a PMI estimation tool could be developed. 
For longer term PMI estimation, studies have included determining the decay rate of DNA in ribs (Perry et al., 1988) and using bone lipid, cholesterol and protein forming multiple regression equations for dating bones (Castellano et al., 1984). More recently it has been shown that certain radionuclides and trace elements in bones could potentially be used as a means of PMI estimation for skeletal remains (Swift et al., 2001).

Vass et al. (2004) built a database of odours that emanate from a decomposing body, and have developed from this a hand-held odour-detector called LABRADOR (light-weight analyzer for buried remains and decomposition odor recognition). This technology could also be applied to determining the stage of decomposition, and time since death, in the field. Determining the volatile compounds that cadaver seeking dogs recognise may also help with more effective location of clandestine graves.

While techniques from many branches of science have been investigated for their potential in aiding time since death estimation, relatively few are routinely used in forensic death investigations. This is primarily because the methods have not, as yet, been developed to a standard for use in the criminal courts, or because of the requirement for specialist expertise which is not always available to the death investigators. Most methods are restricted to being useful in only a limited number of cases, and often have considerable costs associated with them. Continued research into more effective time since death estimation tools is required.

\subsection{Molecular Analysis of Microbial Communities}

In recent years, many different approaches to studying complex microbial communities have evolved. These include DNA-based genomic methods, RNA-based transcriptomic methods and protein based proteomic methods. Because of the relative ease of working with DNA as opposed to RNA and proteins, a great deal of the microbial ecology literature is based around genomic methods (Nakatsu, 2007). 


\subsubsection{Genomic Microbial Community Analysis}

Large-scale sequencing of the variants of a particular gene present has long been the goldstandard for assessing diversity and identifying community members using DNA. This, however, can be prohibitively expensive and labour intensive and is usually limited to, at most, a few hundred sequences per sample (Gans et al., 2005). The very recent advent of high-throughput pyrosequencing technology (Ronaghi, 2001) has revolutionised the study of complex microbial communities, allowing the number of sequence reads from a sample to be orders of magnitude greater than with traditional sequencing methods (Roesch et al., 2007). Complex communities including soil- (Roesch et al., 2007) and gut-microflora (Andersson et al., 2008) have recently been examined using pyrosequencing techniques. This technique looks set to revolutionise microbial ecology in coming years.

Because of the limitations to date of the traditional sequencing methods, alternative approaches have been popular. Various molecular "profiling" techniques have been developed which resolve the diversity of certain genes within a sample depending on their sizes or sequences. These techniques employ a PCR step to amplify the gene of interest for easier detection. The most commonly used methods include denaturing gradient gel electrophoresis (DGGE) analysis (Muyzer et al., 1993), terminal restriction fragment length polymorphism (T-RFLP) analysis (Liu et al., 1997), single strand conformational polymorphism (SSCP) analysis (Schwieger and Tebbe, 1998), length heterogeneity PCR analysis (Suzuki et al., 1998) and ribosomal intergenic spacer analysis (Ranjard et al., 2000; Cardinale et al., 2004). The most commonly targeted genes are the rRNA genes. These are common to all known bacteria and are useful because they contain highly conserved sections and also regions of high diversity.

Methods which do not include a PCR step, but analyse nucleic acids directly have also been used, including DNA:DNA reassociation kinetics (Torsvik et al., 1990), nucleic acid 
hybridisation (Buckley et al., 1998), fluorescent in situ hybridisation (Christensen et al., 1999), microarrays (Yergeau et al., 2009). More recently, meta-genomics, or a wider survey of all genes present has risen in popularity for understanding the functionality of a complex community as opposed to simply detecting the organisms present (Jarvie and Harkins, 2007; Kowalchuk et al., 2007; Schmeisser et al., 2007).

\subsubsection{Advantages and disadvantages of genomic methods}

The use of DNA in microbial community studies offers some great advantages to the researcher, but also has some potential pitfalls. The greatest advantage of genomic methods is the ability to detect and characterise a vast diversity of microbes previously undiscovered using culture-based methods. An estimated $90-99 \%$ of soil microbes, for example, are as yet undescribed and are known only from molecular-based studies (Thies, 2007b).

Every physical, chemical and biological step in the molecular analysis of an environmental sample is a source of bias which will lead to a distorted view of the "real world" (von Witzengerode et al., 1997). Bias can be introduced during the retrieval of nucleic acids from environmental samples, depending on the method used. Many methods have been used to extract nucleic acids, but it is unlikely that any method recovers all of the naturally occurring DNA (Ward et al., 1992). Some procedures, particularly enzymatic lysis methods, are biased towards recovery of DNA from Gram-negative bacteria, which have a thinner cell wall than their Gram-positive counterparts. Mechanical lysis is more rigorous and is considered to give a more representative sample, although lower molecular weight DNA is recovered this way due to shearing of the DNA molecules (Ward et al., 1992). Fragmented nucleic acids are a source of artefacts in PCR amplification and may contribute to the formation of chimeric molecules (Liesack et al., 1991). Conversely, DNA liberated from easily lysed organisms can become adsorbed to soil colloids, especially in clay soils, skewing the recovered DNA profile towards Gram-positive bacterial species (Frostegard et al., 1999). The size of cells may also 
affect DNA extraction efficiency. More et al. (1994) showed that small bacterial cells (1.2 to $0.3 \mu \mathrm{m})$ are more difficult to lyse than larger bacteria; 50 to $70 \%$ of bacteria in nonrhizosphere soil are less that $0.5 \mu \mathrm{m}$ in size. Extracellular DNA in soil may also contribute to the total DNA extracted, as it can remain adsorbed to soil particles for long periods after the cell is lysed (Paget et al., 1992). Frostegard (1999), however, found that only $6 \%$ of extracellular DNA added to soil before DNA extraction could be recovered, suggesting that the effect of extracellular DNA is minimal.

The PCR step is also a potential source of bias. Bacterial community $16 \mathrm{~S}$ rRNA analysis is widely regarded as a qualitative rather than quantitative technique, because PCR can result in differential amplification of community rDNAs, distorting the final template ratios away from the initial template ratios. This can be caused by a number of mechanisms including preferential denaturation of low GC containing sequences, template reannealing inhibition (Reysenbach et al., 1992), low template concentrations (Chandler et al., 1997), and reannealing of single-stranded template molecules (Suzuki and Giovannoni, 1996). Another problem that has been reported is the formation of chimeric DNA molecules (recombinant DNA sequences of two or more different parent sequences in the sample). Chimeric sequences are thought to be caused by reannealing of the $3^{\prime}$ end of an incompletely synthesised rDNA product to a different template, which is synthesised to completion (Qiu et al., 2001). Kopczynski et al. (1994), identified chimeric 16S rRNA products from PCR and cautioned that their detection is important when sequencing genes, as erroneous sequences may be entered into ribosomal databases, giving a false estimate of diversity. Anomalous sequence data is proving to be a problem in public-access databases, where an estimated one out of every twenty sequences could contain chimeras or other anomalies (Ashelford et al., 2005). 
Although not an actual PCR bias, operon heterogeneity and gene clusters complicate the interpretation of environmental genetic data (Hugenholtz and Goebel, 2001). The copy number of the $16 \mathrm{~S}$ gene can range between 1 and 14 in different bacterial species (Cole and Girons, 1994). These copies can have some variation between them, and this may artificially increase the diversity seen in a profile (Clayton et al., 1995). Coenye and Vandamme (2003), have shown that this variation is low in the 55 bacterial genomes they studied, with similarity between copies being between 100 and $98.74 \%$. Actinomycetes may have higher intragenomic variability, with differences of up to $6.5 \%$ being reported (Yap et al., 1999). Alternatively, a single sequence may represent more than one cellular lineage or species due to the highly conserved nature of the 16S rDNA (Stackebrandt and Goebel, 1994).

The aim of many molecular community analyses is to describe population diversity by calculating taxon richness and evenness. Due to bias in DNA extraction and PCR amplification, it is difficult, if not impossible to assess the true abundance of different taxa using this approach (Thies, 2007b). Molecular methods, however, do offer rapid, reproducible and high-throughput advantages that can offer significant insights into microbial ecology if the limitations of the approach are taken into consideration.

\subsubsection{T-RFLP Analysis}

Terminal Restriction Fragment Length Polymorphism (T-RFLP) analysis is one fingerprinting methodology that can incorporate automated genotyping systems for laser detection of fluorescently-labelled DNA fragments. The T-RFLP analysis technique involves the amplification of a gene of interest using fluorescent primers, followed by restriction endonuclease digestion, and automated analysis of the end or terminal restriction fragments. Because the 16S rRNA genes from all of the bacteria in the soil community are amplified, the resulting PCR products are gene copies of a similar length but with different internal 
sequences. The PCR products are cleaved with a restriction enzyme that recognises and cleaves DNA at particular sequences. In the variable regions of the $16 \mathrm{~S}$ gene, restriction sites occur in different places resulting in different length fragments. The more diverse the bacterial community in a sample, the greater the range of resulting fragments. The end, or terminal fragments are analysed and the size and frequency of each fragment assessed to produce a profile or 'fingerprint' of the whole bacterial community in a soil sample.

The T-RFLP method was originally developed by Avaniss-Aghajani et al. (1994) to identify Mycobacteria, but its potential to analyse variation between genes from a mixture of bacteria was first shown by Liu et al. (1997). Since then, T-RFLP analysis has been used in numerous studies to look at bacterial (Liu et al., 1998), archaeal (van der Maarel et al., 1998) and eukaryotic (Marsh et al., 1998) populations in many different substrates, and has been identified as a reproducible and accurate tool for community fingerprinting (Egert and Friedrich, 2003).

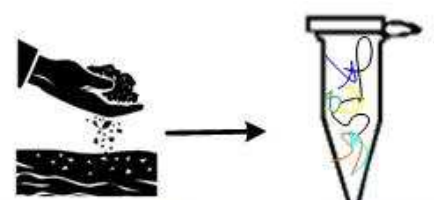

Whole microbial community DNA is extracted from the soil

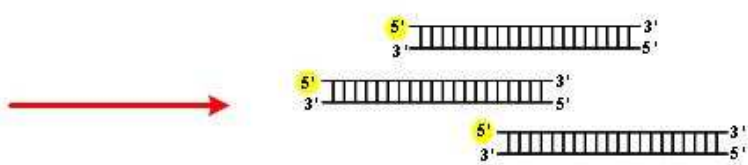

PCR with fluorescently labelled primers to amplify a region of the gene of interest

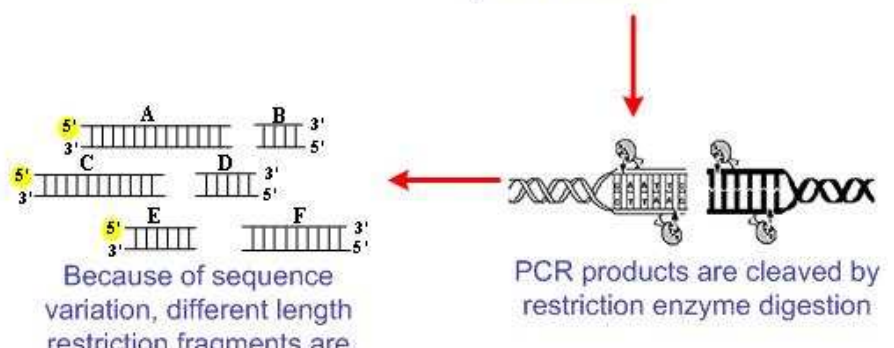

The fluorescently labelled end fragments are separated and detected using the $A B \mid 310$ Genetic Analyser and an electropherogram generated

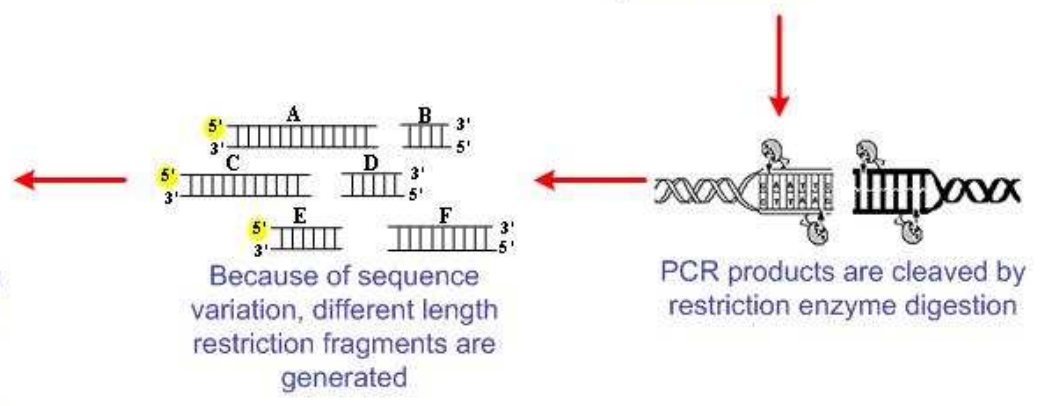
generated

Figure 2.3 Overview of the T-RFLP method.

Because it is a PCR-based method, T-RFLP is subject to all of the potential pitfalls of PCR (von Witzengerode et al., 1997). The validity of using T-RFLP to quantify bacterial 
ribotypes, or fragments, giving an idea of the importance of that species in the community is also disputed. Factors such as rRNA operon copy number and the generation of multiple TRFs by a single isolate (Avis et al., 2006) affect quantitation. Lueders and Friedrich (2003) maintain that quantitation is valid, as accurate template to product ratios have been found using T-RFLP analysis. Hartmann and Widmer (2008) agree that T-RFLP is able to accurately demonstrate relative changes in the microbial community, as does Schmidt (2007). In contrast, other studies have documented unpredicted results from mixed populations (Frey et al., 2006) and cautioned against interpreting T-RFLP results quantitatively.

Since T-RFLP relies on restriction sites, which can be located in the same place in the gene in more than one species, it has limited resolution for identifying specific phylogenetic groups within a complex community (Lee et al., 1996; Dunbar et al., 2001). Ideally, a terminal restriction fragment (T-RF) would be representative of a single species, but this is highly unlikely, even with the use of multiple restriction enzymes.

Another problem to be considered when performing T-RFLP is the formation of pseudoterminal restriction fragments (pseudo-T-RFs). Egert and Friedrich (2003) investigated the occurrence of pseudo T-RFs. These are restriction fragments that are present in a T-RFLP profile, but are caused by single-stranded amplicons, and cannot be digested by restriction endonucleases, giving false T-RFs. The height and area of the pseudo-T-RFs increases with the number of PCR cycles, indicating that they are a PCR artefact. Extra T-RFs can occur because of partial digestion of the PCR products, but this can be overcome by increasing the amount of restriction enzyme. Egert and Friedrich (2003), hypothesize that single-stranded amplicons are formed by intrastrand annealing under the same conditions as template reannealing. These structures may prevent correct amplification by polymerases, leaving single stranded amplicons. To further complicate the problem, single stranded sequences can form local palindromic secondary structures that allow restriction enzymes to digest them. 
Restricting the number of PCR cycles to the minimum required will reduce the effect of pseudo-T-RFs, but is unlikely to eliminate them completely. Hence the presence of these TRFs should be considered when evaluating species diversity in a mixed community using TRFLP. Inaccuracies during the sizing of T-RFs due to differences in purine content of the amplicons has also been reported (Kitts, 2001; Kaplan and Kitts, 2003). Marsh (2005) warns that mis-calls in T-RF length could be up to $7 \mathrm{bp}$, a factor which must be considered when analysing T-RFLP data.

There are clear advantages for using T-RFLP over many of the other molecular techniques presently in use, including the fact that direct reference to sequence databases can be used to derive phylogenetic inferences (Kitts, 2001). By using online tools such as the Microbial

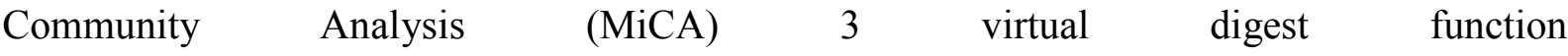
(http://mica.ibest.uidaho.edu/news.php) predicted T-RF sizes for a given primer set and combination of restriction enzymes can be made for comparison with actual T-RF data (Shyu et al., 2007). Some studies have included development of a specific database for identification of T-RFs (Fey and Conrad, 2000; Dickie and Fitzjohn, 2007).

Other variants of T-RFLP that allow sequence information to be recovered directly have been developed, including an adaptor-ligation step that allows fragment size selection in clones (Widmer et al., 2006) and a method using biotinylated primers and standard agarose gel to allow nucleic acid recovery (Blackwood and Buyer, 2007).

The DNA sequencing technology usually employed with T-RFLP analysis also has considerably higher resolution than electrophoretic systems, and the output is digital, allowing for easy data handling (Marsh, 1999). This contributes to the method's high-throughput potential, making it a logical approach when ecological questions requiring extensive sampling across large temporal or spatial ranges are addressed (Marsh, 2005). The T-RFLP method has recently been successfully multiplexed (Singh et al., 2006), allowing a number of 
different groups of organisms to be studied simultaneously, further increasing the method's value.

Despite the potential biases inherent in the T-RFLP method, it has become a popular technique for bacterial community analysis. As long as these potential areas of concern are taken into consideration when assessing communities quantitatively, T-RFLP is a simple, reproducible and useful technique (Marsh, 1999).

\subsubsection{Denaturing Gradient Gel Electrophoresis}

Denaturing gradient gel electrophoresis (DGGE) was first used for identifying gene mutations (Borresen et al., 1988). Muyzer et al. (Muyzer et al., 1993) adapted the protocol for use in microbial community analysis. DGGE has since been used to assess community changes in a wide range of simple and more complex matrices including foods (Florez and Mayo, 2006; Li et al., 2006; Hovda et al., 2007), hot springs (Ferris and Ward, 1997), deep-sea hydrothermal vents (Muyzer et al., 1995), artworks (Mohlenhoff et al., 2001), sewage sludge (Choi et al., 2007), intestinal and faecal samples (Simpson et al., 1999; Wang et al., 2007) and soils (Heuer et al., 1997; Kowalchuk et al., 1997; Duineveld et al., 2001; Lerner et al., 2006). A common approach is to use DGGE to assess specific populations within complex communities such as in soil. Groups studied using DGGE have included: actinomycetes (Heuer et al., 1997), ammonia-oxidising bacteria (Yergeau et al., 2005; Hornek et al., 2006), methanotrophs (Jensen et al., 1998), cyanobacteria (Boutte et al., 2006) and sulphate-reducing bacteria (Teske et al., 1996).

The DGGE method involves amplification of a target gene by PCR, producing DNA fragments of about the same length, but different sequence. A gel composed of a linear concentration gradient of denaturant (usually urea and formamide) is used to separate the PCR products. Differential migration occurs under electrophoresis because more denaturant is 
required to separate sequences with higher guanine $(\mathrm{G})$ and cytosine $(\mathrm{C})$ content due to differences in the number of $\mathrm{H}$ bonds between complementary nucleotides holding DNA strands together (Nakatsu, 2007). A GC-rich sequence (GC clamp) is added to the end of one primer to prevent complete strand separation (Sheffield et al., 1989). The PCR products with differing sequences separate based on their GC contents to give a genetic profile or "fingerprint" of the bacterial population present in that sample.

Because the first step of DGGE is the PCR, this technique is also affected by the biases inherent with PCR (section 2.3.1.1). Being a gel-based method, the greatest limitation of DGGE is in the reproducibility of gradient gels. Even with experience it is very difficult to exactly reproduce gel gradients, leading to different migration of bands on replicate gels. This affects the ability to perform between-gel comparisons. Another limitation of DGGE is that co-migration of unrelated DNA sequences can occur, due to them having a similar CG content (Yang and Crowley, 2000). If a community is very complex, it is also possible that rather than distinct, identifiable bands, a smear of bands results (Nakatsu, 2007). Single isolates have also been shown to produce multiple bands (Nubel et al., 1996; Satokari et al., 2001) complicating interpretation of banding patterns.

Perhaps the greatest advantage of DGGE over other bacterial community profiling methods, is the ability to excise individual bands from the gel for DNA sequence analysis (Ovreas et al., 1997). DGGE can also be linked with other methods such as Stable Isotope Probing (SIP) to enable phylogenetic identification of communities responsible for utilisation of a substrate (Dumont and Murrell, 2005). 


\section{Chapter 3: General Methods}

Three separate experiments were performed to collect samples for analysis. The first two were carried out in New Zealand with pig carcasses (Chapters 4 and 5). The third was performed at the University of Tennessee's Forensic Anthropology Research Facility (Chapter 6) using donated human cadavers. General methods that were used in two or more of the experiments are described in this chapter. The experimental set-up, sample collection and analytical methods specific to each experiment are presented in their respective chapters (Chapters 4, 5 and 6). The molecular methods used for microbial species identification using selected samples from each of the three decomposition experiments are detailed in Chapter 7.

\subsection{Sample Characterisation}

\subsubsection{Sample Collection}

The sample collection protocol differed slightly for each experiment and is outlined in the experimental chapters 4,5 and 6 .

\subsubsection{Sample Preparation}

Following collection, all soil and sand samples were transported directly to the laboratory and either processed immediately (for the second pig experiment, Chapter 5 and human cadaver experiment, Chapter 6) or frozen at $-20^{\circ} \mathrm{C}$ for later processing (first pig experiment, Chapter 4). Insect larvae and any other contaminants such as plant matter or small stones were removed from each sample. The entire sample was then homogenised by thorough mixing with a sterile spatula. 


\subsubsection{Sand sterility test}

Prior to use, all batches of sand used were tested for sterility. The sand used in the New Zealand based experiments (Chapters 4 and 5) was acid washed prior to sterility testing (section 4.2.2). The sand used in the US (Chapter 6) was not acid washed but was considerably cleaner than the NZ sand when purchased. One in 10 suspensions $(1 \mathrm{~g}$ sand to $10 \mathrm{~mL}$ sterile $\mathrm{dH}_{2} \mathrm{O}$ ) were made of sand from each of the $10 \mathrm{~kg}$ bags purchased. Aliquots of 1 $\mathrm{mL}$ were plated on tryptic soy agar and incubated for 48 hours at $37^{\circ} \mathrm{C}$ and $23^{\circ} \mathrm{C}$ in an aerobic environment. Colonies were counted following incubation.

Total DNA extractions were also performed on three $300 \mathrm{mg}$ samples from each $10 \mathrm{~kg}$ bag of sand, using the method outlined in section 3.4.1. The amount of DNA extracted from each sample was measured using the pico green assay method outlined in section 3.4.2.

\subsubsection{Buffering Capacity of Soils}

The buffering capacities of the soils used were determined using a method based on that outlined by Weaver et al. (2004). Buffer curves were established for each of the soils by titrating $25 \mathrm{~g}$ soil suspended in $\mathrm{dH}_{2} \mathrm{O}(1: 10)$ with $1 \mathrm{~mL}$ inputs of $0.022 \mathrm{M} \mathrm{Ca}(\mathrm{OH})_{2}$. The $\mathrm{pH}$ was measured after each $1 \mathrm{~mL}$ input using a standard $\mathrm{pH}$ meter, and was recorded after the solution had had time to equilibrate and there had been no further change in $\mathrm{pH}$ for the last 10 seconds. 


\subsection{Biochemical Analyses}

\subsection{1 pH}

Four grams of soil or sand from each experimental plot were weighed out into $25 \mathrm{~mL}$ plastic

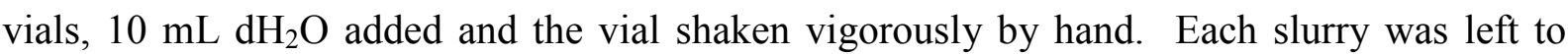
settle overnight, and the $\mathrm{pH}$ of the liquid phase read the following day using a standard $\mathrm{pH}$ meter (Blakemore et al., 1987).

\subsubsection{KCl Extractions}

Filtered $\mathrm{KCl}$ extracts prepared from soil samples were required for ammonium and nitrate concentration measurements. This is a standard solvent extraction method for ions in soil (Blakemore et al., 1987). From each sample, either $3.5 \mathrm{~g}$ or $7 \mathrm{~g}$ soil was weighed out into a 50 $\mathrm{mL}$ plastic centrifuge tube and $35 \mathrm{~mL} 1 \mathrm{M} \mathrm{KCl}$ added. The tubes were shaken on a rotary shaker for 1 hour before centrifugation for 15 minutes at $2,500 \mathrm{~g}$ at $15^{\circ} \mathrm{C}$, and the supernatants filtered through GFC filter paper (Whatman). Soil extracts were stored at $-20^{\circ} \mathrm{C}$ until analysis. For control soils and for the cleaner samples collected at the beginning of each experiment, $7 \mathrm{~g}$ was used, but this was reduced to $3.5 \mathrm{~g}$ for the samples heavily loaded with decomposition products, as supernatants recovered when using the greater amount proved difficult to filter adequately.

\subsubsection{Ammonium Concentration $\left(\mathrm{NH}_{4}{ }^{+}-\mathrm{N}\right)$}

Analysis of $\mathrm{NH}_{4}{ }^{+}-\mathrm{N}$ levels in soil and sand samples was performed because large amounts of ammonia are liberated from decomposing corpses (Vass et al., 1992; Hopkins et al., 2000). The method used is a modified version of the Searle and Speir (1976) method. The detailed laboratory protocol is included in Appendix A2. This method uses the Berthelot or 
indophenol reaction, which is the name given to the reaction between $\mathrm{NH}_{4}{ }^{+}-\mathrm{N}$ and a phenolic compound, which under suitable oxidising conditions results in the formation of a bright blue indophenol dye (Searle, 1984).

Filtered extracts (section 3.2.2) were analysed in batches of 40-42. These were thawed at room temperature and $0.5 \mathrm{~mL}$ of each were transferred to plastic vials for analysis. To each, 4 $\mathrm{mL}$ of complexing agent was added, quickly followed by $6 \mathrm{~mL}$ sodium salicylate solution, 3 $\mathrm{mL}$ sodium hypochlorite solution and $1 \mathrm{~mL}$ sodium nitroprusside solution, in that order (See Appendix A2 for detailed method). Vials were capped and shaken and left to develop for 30 minutes. The absorbance of the solution was read on a spectrophotometer at $660 \mathrm{~nm}$. Standards containing $32 \mathrm{mg} / \mathrm{L}, 24 \mathrm{mg} / \mathrm{L}, 16 \mathrm{mg} / \mathrm{L}, 8 \mathrm{mg} / \mathrm{L}, 4 \mathrm{mg} / \mathrm{L}$ and $0 \mathrm{mg} / \mathrm{L}$ of ammonium sulphate $\left(\left(\mathrm{NH}_{4}\right)_{2} \mathrm{SO}_{4}\right)$ were analysed each time and a standard curve constructed. Some extract filtrates required dilution and re-analysis to fall within the standard curve range.

\subsubsection{Nitrate Concentration $\left(\mathrm{NO}_{3}{ }^{-}-\mathrm{N}\right)$}

The filtered soil $\mathrm{KCl}$ extract (section 3.2.2) was also used for $\mathrm{NO}_{3}{ }^{-}-\mathrm{N}$ analysis. The method used is based on that described by Blakemore et al. (1987) and is described in more detail in Appendix A3. Hydrazine sulphate was used to reduce nitrate to nitrite, which was then determined colorimetrically using a diazotization coupling reaction, reading the absorbance spectrophotometrically at $540 \mathrm{~nm}$. Standard solutions containing $0 \mathrm{mg} / \mathrm{L}, 1 \mathrm{mg} / \mathrm{L}, 2 \mathrm{mg} / \mathrm{L}, 3$ $\mathrm{mg} / \mathrm{L}, 4 \mathrm{mg} / \mathrm{L}$. and $5 \mathrm{mg} / \mathrm{L}$ of potassium nitrate $\left(\mathrm{KNO}_{3}\right)$ were analysed each time soil or sand filtrates were analysed to construct a standard curve. Some filtrates required dilution and reanalysis in order to fall within the standard curve range. A detailed method and solution recipes are included in Appendix A3. 


\subsection{Enzyme Assays}

\subsubsection{Urease (EC 3.5.1.5)}

The method used for potential urease activity was adapted from the Searle and Speir (1976) method. In this method, the urea added to the sample is converted to $\mathrm{NH}_{4}{ }^{+} \mathrm{N}$ and carbon dioxide by the urease enzyme, if present in the sample. The amount of $\mathrm{NH}_{4}{ }^{+}-\mathrm{N}$ is then determined using the Berthelot Reaction based assay described in section 3.2.3. Controls are used to determine the concentration of $\mathrm{NH}_{4}{ }^{+}-\mathrm{N}$ already present in the soil sample.

From each sample, four $0.5 \mathrm{~g}$ sub-samples were weighed out into glass vials. To each vial, 1.6 $\mathrm{mL}$ phosphate buffer (Appendix A4) was added and the soil dispersed in the buffer using a glass rod. Quickly, $0.4 \mathrm{~mL} 1 \mathrm{M}$ urea in phosphate buffer was added to three of the four vials and all four were capped and incubated at $30^{\circ} \mathrm{C}$ for $4 \mathrm{~h}$. The vials without urea were used as controls. After incubation, $0.4 \mathrm{~mL} 1 \mathrm{M}$ urea in phosphate buffer was added to the control vial, and $8 \mathrm{~mL} 2 \mathrm{M} \mathrm{KCl}$ was added to all four vials. The extracts were filtered through $90 \mathrm{~mm}$ Whatman No. 1 filter papers into plastic storage vials. If any turbidity was evident in the filtrate, the extracts were re-filtered. Filtrates were stored at $-20^{\circ} \mathrm{C}$ until analysis using the $\mathrm{NH}_{4}{ }^{+}-\mathrm{N}$ assay (section 3.2.3). A detailed method is included in Appendix A4. Enzyme

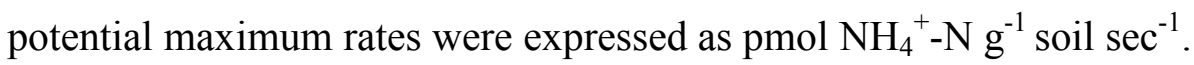

\subsubsection{Fluorometric Assay}

The potential activity of a number of soil enzymes was analysed using fluorometric substrates. The selection of enzymes analysed differ between the experiments, because those that had low activity in the pig experiments were dropped and other enzymes were added in for the human cadaver experiment. The details of these individual analyses are given in the relevant chapter. Table 3.1 lists all of the substrates used and their related enzymes (Miller et al., 1998; Niemi and Vepsalainen, 2005). 


\begin{tabular}{|c|c|}
\hline Substrate & Enzyme \\
\hline 4-MUB- $\beta$-D-N-acetyl-glucosamine \\
4-MUB- $\beta$-D-glucoside & Chitinase (EC 3.2.1.14) \\
4-MUB sulphate & $\beta$-glucosidase (EC 3.2.1.21) \\
4-MUB-phosphate & Arylsulphatase (EC 3.1.6.1) \\
4-MUB acetate & Acid phosphatase (EC 3.1.3.2) \\
4-MUB-butyrate & Esterase (EC 3.1.1.6) \\
4-MUB-palmitate & Esterase (EC 3.1.1.6) \\
L-alanine-7-AMC & Esterase (EC 3.1.1.6) \\
\hline
\end{tabular}

Table 3.1 Fluorometric substrates (Sigma) used and the enzymes which act on them (4-MUB=4methylumbelliferone, 7-AMC =7-amino-4-methylcoumarin).

All enzyme assays using fluorometric substrates were performed in a 96-well plate format (Marx et al., 2001). The enzyme substrates and standards were dissolved in $1 \mathrm{~mL}$ methylcellosolve (due to the low water solubility of some of the compounds), and further diluted with $0.1 \mathrm{M}$ 2-( $N$-morpholino)ethanesulfonic acid (MES) buffer to a final working concentration of $800 \mu \mathrm{M}$, except for 4-MUB butyrate and 4-MUB palmitate, which had low solubility at $800 \mu \mathrm{M}$ and were diluted to $400 \mu \mathrm{M}$. A series of standards were made using 7AMC and 4-MUB to the concentrations of: $0,10,20,30,40,50,60$ and $70 \mu \mathrm{M}$ (for more detailed method see Appendix A5).

A soil suspension was prepared from each sample by diluting $0.5 \mathrm{~g}$ soil in $100 \mathrm{~mL} \mathrm{dH}_{2} \mathrm{O}$ and shaken to disperse the soil particles. In New Zealand, the samples were briefly sonicated for 120 seconds at energy output of $50 \mathrm{~J} / \mathrm{s}$ using a probe sonicator. This was not possible for the human cadaver experiment samples (Chapter 6) which were processed in the US lab, so these suspensions were agitated vigorously on a vortex mixer for 120 seconds followed by shaking on a flatbed mixer for 5 minutes. 
An example of one of the 96-well plate layouts used is shown in Figure 3.1. This layout shows two soil samples being analysed per plate, for all enzyme activities. A different layout was used for the second pig experiment (Chapter 5) when the activity of only four enzymes was tested and four samples could be analysed on a single 96-well plate. A $100 \mu \mathrm{L}$ volume of each substrate was added to three wells containing $100 \mu \mathrm{L}$ soil suspension, and to one control

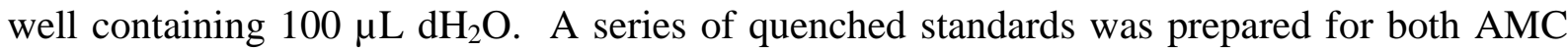
and MUB by adding $100 \mu \mathrm{L}$ of each standard to wells containing $100 \mu \mathrm{L}$ of the soil suspension (Freeman et al., 1995). The plate was incubated at $30^{\circ} \mathrm{C}$ for 1 hour before $50 \mu \mathrm{L}$ $0.5 \mathrm{M} \mathrm{NaOH}$ was added to each well to raise the $\mathrm{pH}$ and stop the reaction. The fluorescent output from each well was read using a fluorescent plate reader with 365/460 nm (excitation/emission) filters.
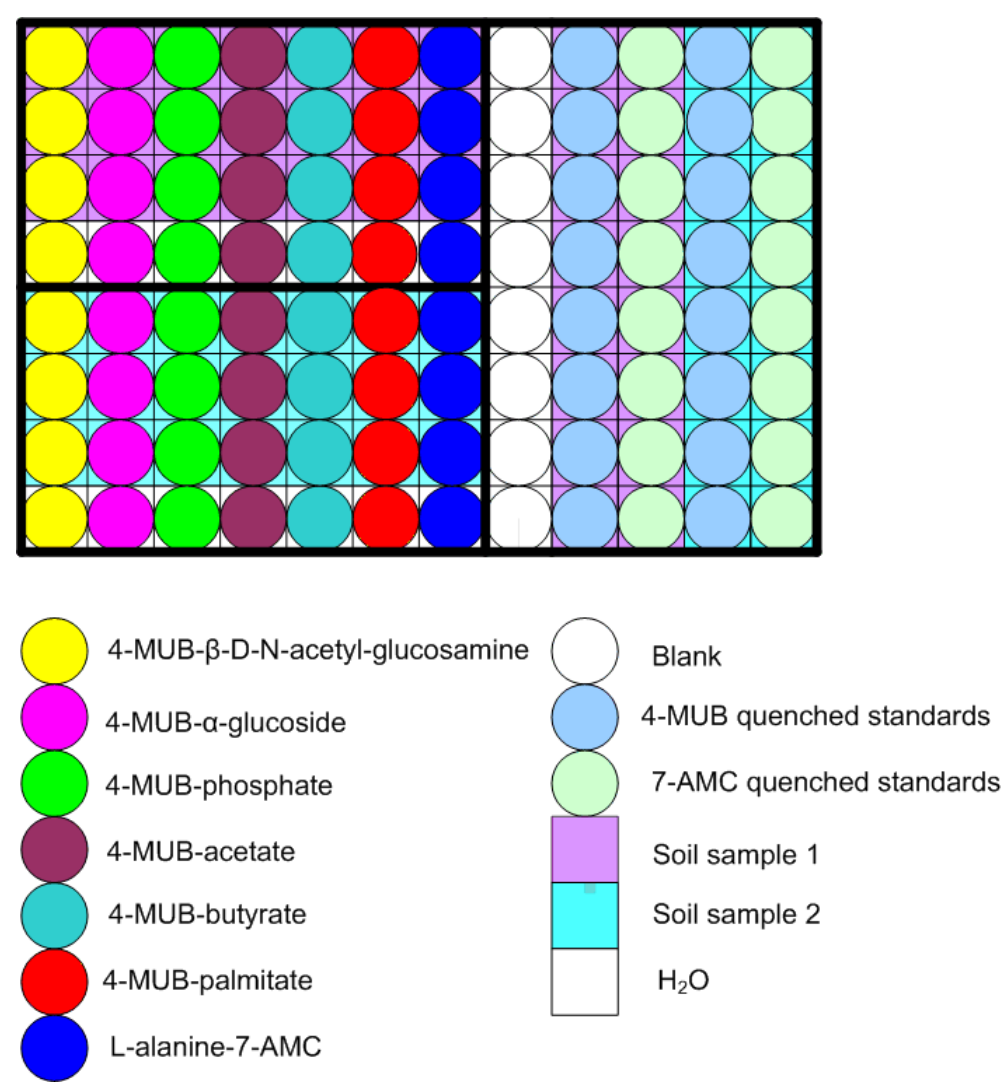
The gravimetric moisture content of each soil sample was calculated (Appendix A6) as part of the enzyme assays by determining the percentage of moisture lost from $5 \mathrm{~g}$ of soil sample after drying overnight in a $100^{\circ} \mathrm{C}$ oven (Blakemore et al., 1987).

Enzyme potential maximum rates were expressed as pmol MUB/AMC g $\mathrm{g}^{-1} \mathrm{soil} \mathrm{sec}^{-1}$.

\subsubsection{Microbial biomass}

The microbial biomass of samples was originally going to be analysed using a modified chloroform fumigation method (Jörgensen, 1995). All samples were processed using this method and the extracts frozen for later analysis.

For each sample, $0.5 \mathrm{~g}$ was weighed out into six vials. Three vials (controls) were placed in a closed glass fumigation tank and stored overnight. The other three vials were placed in a desiccator with $50 \mathrm{ml}$ chloroform in a beaker. If the $\mathrm{pH}$ of the samples was above 6 , a small beaker of soda lime was also placed in the desiccator. A vacuum source was applied to evacuate the air from the desiccator, and this was left overnight for the samples to fumigate with chloroform vapour. Next morning, the chloroform vapour was evacuated from the desiccator using the vacuum source, air was readmitted and the lid removed. To each vial (fumigated and controls), $10 \mathrm{~mL} 0.01 \mathrm{M} \mathrm{CaCl}_{2}$ was added, the vials capped and shaken gently on a rotary shaker for 1 hour. Extracts were filtered through $90 \mathrm{~mm}$ Whatman no. 1 filter papers into plastic vials and filtrates stored frozen at $-20^{\circ} \mathrm{C}$. These were thawed and refrigerated at $4^{\circ} \mathrm{C}$ for one day before total carbon analysis. Total carbon readings were performed by LandCare Research in Palmerston North.

Unfortunately, because of highly variable results seen for the first set of replicate extracts analysed, and the relatively high costs associated with total carbon analysis, this method was 
discontinued. It is likely that this method was not suitable for the decomposition derived samples, which were often very wet, making effective fumigation difficult.

\subsection{Molecular Biology Methods}

\subsubsection{DNA Extraction}

Microbial DNA was extracted from $0.3 \mathrm{~g}$ soil or sand sub-samples using the FastDNA SPIN kit for Soil (QBIOgene) following the manufacturer's protocol with the addition of a DNAzol cleanup step (Appendix A7).

\subsubsection{DNA quantitation}

The amount of DNA extracted from each soil sample was measured using a PicoGreen dsDNA Quantitation Kit (Molecular Probes Inc.) as per the manufacturer's directions. An assay was performed using $1 \mu \mathrm{L}$ of the extracted genomic DNA solution, and the amount of DNA present estimated using a standard curve (Appendix A8). The pico green reagent is a dye, that, upon binding to double stranded DNA, exhibits a $>1000$-fold fluorescence enhancement. The fluorescence generated by this reaction was measured using a FLUOstar fluorescence plate reader and associated software (BMG LabTechnologies). The concentration of DNA present was determined prior to any PCR, and also after each cleanup step in the T-RFLP method. 


\subsubsection{T-RFLP}

The terminal restriction fragment length polymorphism analysis method used was originally designed by Osborn et al. (2000) and adapted for New Zealand soil analysis by Horswell et al. (2002). The basic method was expanded and modified for use with decomposition derived samples by Parkinson (2005). The steps involved are shown in Figure 3.2.

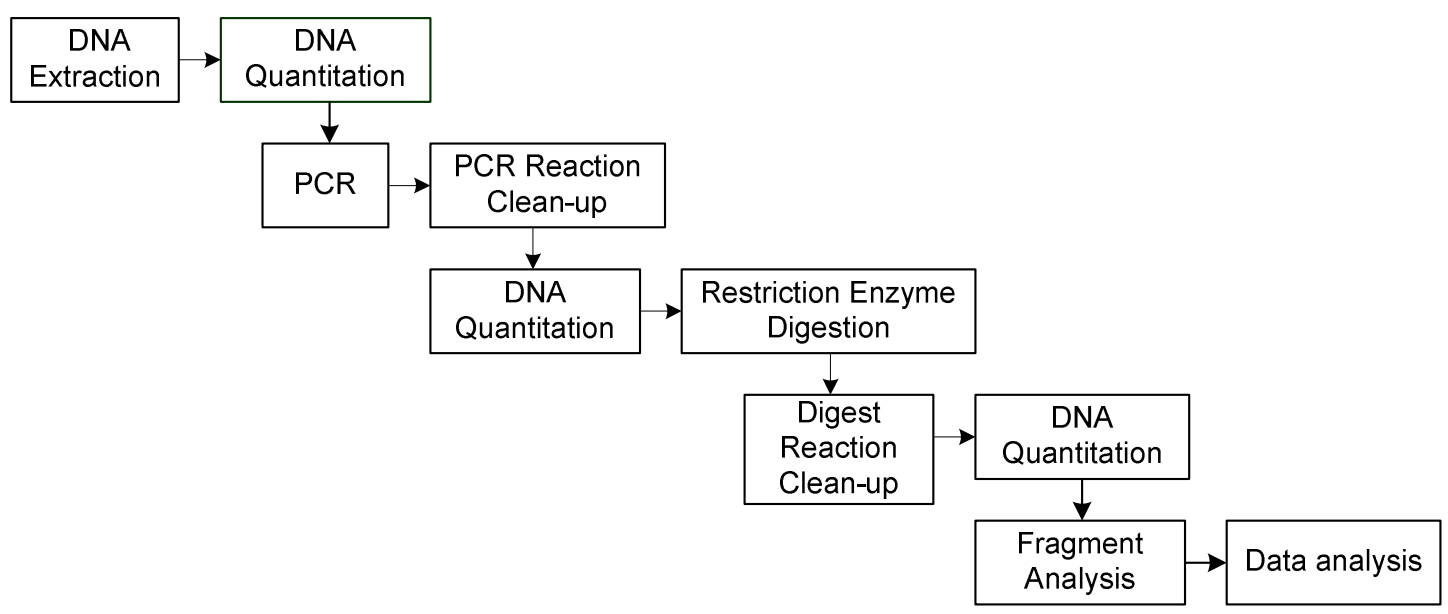

Figure 3.2 Outline of the T-RFLP procedure.

Fluorescently labelled synthetic oligonucleotide primers were used to facilitate automated detection of a $1 \mathrm{~kb}$ region of amplified bacterial $16 \mathrm{~S}$ rRNA genes. Primer pair F63 (5' CAGGCCTAACACATGCAAGTC 3') and R1087 (5' CTCGTTGCGGGACTTAACCC 3') were used as they allow amplification of most of the 16S rRNA (Van de Peer et al., 1996). Using the MiCA primer sequence prevalence analysis tool (http://mica.ibest.uidaho.edu/primer.php), this primer pair was shown to theoretically be able to amplify DNA from a large number of organisms in the database. Both primers were labelled with fluorescent dyes: F63 with FAM and R1087 with HEX. The PCR recipe is shown in Table 3.2 and the PCR thermocycling regime is shown in Table 3.3. Qiagen Taq Core PCR Kits were used, and provided the Taq polymerase, PCR buffer, $\mathrm{MgCl}_{2}$ solution, and dNTPs mixture. A Palmcycler (Corbett Life Science) thermocycler was used for all target amplifications. 


\begin{tabular}{|c|c|}
\hline Component & Amount/Final Concentration \\
\hline Qiagen Taq Polymerase & $1.25 \mathrm{U}$ \\
PCR buffer & $1 \mathrm{x}$ \\
$\mathrm{MgCl}_{2}$ & $2 \mathrm{mM}$ \\
dNTPs & $200 \mu \mathrm{M}$ of each dNTP \\
FAM-F63 & $200 \mathrm{nM}$ \\
R1087-HEX & $200 \mathrm{nM}$ \\
Template DNA & $20 \mathrm{ng}$ \\
DNase free $\mathrm{H}_{2} \mathrm{O}$ & to total volume of $50 \mu \mathrm{L}$ \\
\hline
\end{tabular}

Table 3.2 T-RFLP PCR recipe.

\begin{tabular}{|c|c|c|}
\hline Step & Time & Temperature $\left({ }^{\circ} \mathbf{C}\right)$ \\
\hline Initial Denaturation & $3 \mathrm{~min}$ & 94 \\
\hline 3 Step Cycling (30x) & & \\
Denaturation: & $0.5 \mathrm{~min}$ & 94 \\
Annealing: & $0.5 \mathrm{~min}$ & 55 \\
Extension: & $1 \mathrm{~min}$ & 72 \\
\hline Final Extension & $20 \mathrm{~min}$ & 72 \\
\hline
\end{tabular}

Table 3.3 T-RFLP PCR thermocycling regime.

A $4 \mu \mathrm{L}$ sample from each PCR reaction was electrophoresed on a $2 \%$ agarose gel with $100 \mathrm{bp}$ ladder (Invitrogen) to check for successful amplification of the target DNA (see Appendix A9). Gels were stained with ethidium bromide $(10 \mathrm{mg} / \mathrm{mL})$ and visualised using ultra-violet (UV) light. A digital photograph was taken of each gel using a Gel-Doc System (Bio-Rad).

A purification step was required after the PCR to remove any unused primers and other PCR components that might interfere with subsequent detection of the DNA fragments and profile generation. For this purpose, the QIAquick PCR Purification Kit (Qiagen) procedure was 
followed (Appendix A10). Amplified products were cleaned and eluted in $30 \mu \mathrm{L}$ of elution buffer (10 mM Tris-Cl, $\mathrm{pH} 8.5)$ and stored frozen at $-20^{\circ} \mathrm{C}$.

Following the above purification step, the PCR products were digested using the restriction enzyme MspI (Roche Molecular Biochemicals). This enzyme was chosen because it was found to provide good discriminatory power in preliminary studies performed at ESR (Dr. Catriona Macdonald, ESR Molecular Ecologist, unpublished data, 2005) and is cost effective for large scale work. A standard amount of each PCR product DNA (300 ng) was digested with $2 \mu \mathrm{L}$ (20 units) MspI in $3 \mu \mathrm{L}$ buffer A (10x stock supplied with MspI) and sterile distilled $\mathrm{H}_{2} \mathrm{O}$ to a total volume of $30 \mu \mathrm{L}$. Digests were incubated at $37^{\circ} \mathrm{C}$ for 3 hours, followed by enzyme inactivation through heating to $65^{\circ} \mathrm{C}$ for 20 minutes. The MinElute Enzymatic Reaction Cleanup Kit (Qiagen) was used to remove components of the enzymatic reaction prior to fragment analysis as per the manufacturer's instructions (Appendix A11).

The resulting fluorescent DNA 5' and $3^{\prime}$ end fragments of the 16S rRNA gene or terminal restriction fragments (T-RFs) were then analysed by the Allan Wilson Centre for Biodiversity (Palmerston North) using an ABI3700 Genetic Analyser (Applied Biosystems). The T-RFLP profile data were collated and analysed using Genemapper v.4 software (Applied Biosystems). This software takes the raw data generated by the ABI13700 Genetic Analyser and determines the size of the fluorescently-labelled DNA fragments by comparing them to a size standard. It also provides semi-quantitation of the fragments at each size by measuring the amount of fluorescence emitted. T-RFs which fell outside the calibration range of the size standards (50$500 \mathrm{bp}$ ) were excluded from analysis. All T-RFs of less than 100 fluorescence units were also excluded, as were any minor T-RFs that contributed less than $1 \%$ of the total fluorescence. A perl script (Appendix C1) was used to detect and eliminate such T-RFs. This procedure serves to eliminate noise from profiles with very high fluorescence, without eliminating small T-RFs in profiles with low overall fluorescence. Profiles that subsequently had less than 1500 
units of total fluorescence or fewer than $10 \mathrm{~T}-\mathrm{RFs}$ in each dye channel were excluded from further analysis. Most samples whose profile failed to meet these criteria were sent for reanalysis, but if they failed a second time, they were simply excluded from analysis.

\subsubsection{Denaturing Gradient Gel Electrophoresis}

Denaturing Gradient Gel Electrophoresis (DGGE) was used as an additional means of studying the composition of the bacterial communities. Universal primers were used to study the whole bacterial community, and a series of group- or genera-specific primers were used to study more specific groups of bacteria within the greater community. The universal primers were used with samples from each of the three experiments, whereas the group-specific primer analysis was only used with samples from the human decomposition experiment (Chapter 6). Further details of the group-specific DGGE methods are given in section 7.3. All DGGE gels were prepared, run and stained as per the silver staining method described in detail in Appendix A13.

\subsubsection{Whole Community Analysis Using Universal Primers}

Polymerase chain reactions were performed using universal bacterial $16 \mathrm{~S}$ primers: F341 (5' CCTACGGGAGGCAGCAG 3') and R534 (5' ATTACCGCGGCTGCTGG 3') (Muyzer et al., 1993). The forward primer (F341) had a GC clamp (5' CGCCCGCCGCGCGCGGCGGGCGGGGCGGGGGCACGGGGGG 3') attached to prevent complete separation of the DNA strands during denaturing gradient electrophoresis.

The PCR recipe and thermocycler protocol are shown in Tables 3.4 and 3.5. A long final extension time of 20 minutes was used, as this procedure has been shown to eliminate a common artefact of DGGE known as “double-banding” (Janse et al., 2004). 


\begin{tabular}{|c|c|}
\hline Component & Amount/ Final Concentration \\
\hline Qiagen Taq Polymerase & $1.25 \mathrm{U}$ \\
PCR buffer & $1 \mathrm{x}$ \\
$\mathrm{MgCl}_{2}$ & $2 \mathrm{mM}$ \\
dNTPs & $200 \mu \mathrm{M}$ of each dNTP \\
F341-GC & $200 \mathrm{nM}$ \\
R534 & $200 \mathrm{nM}$ \\
Template DNA & $20 \mathrm{ng}$ \\
$\mathrm{H}_{2} \mathrm{O}$ & to total volume of $50 \mu \mathrm{L}$ \\
\hline
\end{tabular}

Table 3.4 DGGE PCR recipe.

\begin{tabular}{|c|c|c|}
\hline Step & Time & Temperature \\
\hline Initial Denaturation & $3 \mathrm{~min}$ & $94^{\circ} \mathrm{C}$ \\
\hline 3 Step Cycling (30x) & & \\
Denaturation: & $0.5 \mathrm{~min}$ & $94^{\circ} \mathrm{C}$ \\
Annealing: & $0.5 \mathrm{~min}$ & $56^{\circ} \mathrm{C}$ \\
Extension: & $1 \mathrm{~min}$ & $72^{\circ} \mathrm{C}$ \\
\hline Final Extension & $20 \mathrm{~min}$ & $72^{\circ} \mathrm{C}$ \\
\hline
\end{tabular}

Table 3.5 DGGE PCR thermocycling regime.

The PCR products were cleaned using the Qiagen QIAquick PCR cleanup kit as per the manufacturer's instructions (Appendix A10) to remove excess primers and polymerases which can cause artefacts on the gels. The DNA eluted was quantified using the pico green assay (section 3.4.2) and $300 \mathrm{ng}$ loaded onto the gel.

An initial screening DGGE gel was run for each pig carcass or human cadaver, with a maximum of 16 DNA extracts selected from each set of collections (see corresponding experimental chapters for extracts chosen). Gels were cast to have a gradient of $40-60 \%$ denaturant, as this gradient provided the greatest concentration of bands visible when PCR products created using the GC-F341 and R534 primer pair were run on 0-90\% gradient gels. 
The DGGE patterns obtained from four of the human cadavers did not show many bands and had weak banding patterns when using a 40-60\% gradient, therefore extra gels with a 15-55 \% denaturant gradient were also run for each set of cadaver sample sets (Muyzer et al., 1993). A control extract was run on each of the gels used for the human cadaver experiment (Chapter 6) to allow for between-gel analysis. All DGGE gels were stained with silver stain using a modified version of the method described by Muyzer et al.(2004) - see Appendix A13. Each gel also had three size standard DNA ladder lanes (100 kb, Invitrogen) so that any distortion across the gels and differences in run distance between gels could be corrected for.

Many of the DGGE gels exhibited a series of reddish smears apparent after silver staining, which masked some of the authentic DNA bands. Simpson et al. (1999) suggest this may be caused by single stranded DNA (ss-DNA). These smears were reduced by purification of the DNA using the MinElute PCR Cleanup Kit (Qiagen) prior to loading on the gel, but they were not completely eliminated. Digestion with mung bean nuclease was found to eliminate these smears, so all gels were re-run with the PCR reactions treated with mung bean nuclease. The reaction mixture contained $15 \mu \mathrm{L}$ PCR product (containing 300 ng DNA), $3 \mu \mathrm{L} 10 \mathrm{x}$ mung bean buffer, $1.5 \mu \mathrm{L}$ mung bean nuclease (diluted 1:50 in nuclease dilution buffer), and

DNase/RNase free $\mathrm{H}_{2} \mathrm{O}$ to $30 \mu \mathrm{L}$. The mixture was then incubated at $38^{\circ} \mathrm{C}$ for $10 \mathrm{~min}$. After incubation, $10 \mu \mathrm{L}$ of DGGE gel loading buffer $(0.05 \%$ bromophenol blue, $0.05 \%$ xylene cyanol, by $70 \%$ glycerol w/v in $\mathrm{H}_{2} \mathrm{O}$ ) was added to stop the reaction (Simpson et al., 1999).

\subsubsection{Data Acquisition from DGGE Gels}

Gels were scanned using a GS-800 BioRad Imaging Densitometer. For each gel, an image was captured and imported into the Quantity One 4.4 software package (BioRad). Each image was saved as a high-resolution jpeg file and reopened using Bionumerics (Applied Maths). General background colouration from the silver stain was subtracted. Lanes and bands were autodetected by the software, and manually edited to ensure gel artefacts were not being 
erroneously detected as bands. Any distortion across the gel was amended by normalisation against the three replicate DNA ladder lanes. The relative front of each band was determined by the software by measuring the distance from the anchor point divided by the total length of the lane. The relative front position and band intensity data were exported in text files and converted to .csv files for further analysis. All bands detected were included in subsequent analysis and relative front positions were rounded to whole numbers.

\subsubsection{Data Analysis}

Data from both T-RFLP and DGGE were analysed using PRIMER 6 (Plymouth Marine Laboratory), Excel 2003 (Microsoft) and SPSS 15 (SPSS) statistical software packages.

Datasets were checked for distribution normality and equality of variances before appropriate statistical tests were chosen to examine the data (see experimental chapters for details). Excel was used to find the skew statistic of each dataset and to calculate the standard error. Datasets were taken to be non-normally distributed if their skewness statistic was more than two times the standard error (Leech et al., 2005). If data were found to be normally distributed, standard t-tests and analysis of variance (ANOVA) were used to determine statistical significance. If data were non-normally distributed, ANOSIM analysis was performed (section 3.4.5.1).

\subsubsection{Analysis of Similarity (ANOSIM)}

Analysis of similarity (ANOSIM) is a non-parametric, multivariate analogue of the analysis of variance (ANOVA) test (Clarke and Gorley, 2006). When datasets were found to be nonnormally distributed and thus did not meet the requirements for analysis using the parametric ANOVA test, the ANOSIM routine was used. This was performed using PRIMER 6 (Clarke, 1993). This method produces a test statistic (R) that ranges between -1 and 1 depending on whether the average rank similarity between samples within a group of samples is more similar than the average rank similarity between samples from different groups. A value close 
to 0 indicates little or no separation between the tested groups, whereas a value close to 1 indicates complete separation (Rees et al., 2004). A level of significance is also assigned to the result.

\subsubsection{Multi-Dimensional Scaling (MDS) Analysis}

The perl script described in section 3.4.3 was used to prepare datasets for Multi-Dimensional Scaling (MDS). This method was used to visualise the differences between the sample profiles. The script discarded T-RFs outside of the accurate sizing range (50-500 bp). It also discarded any T-RFs with fluorescence units of less than 100 to minimise the effect of artefacts and any T-RFs that contributed less than $1 \%$ of total fluorescence after exclusion of those under 100 fluorescence units. All DGGE bands were included in MDS analysis. Datasets were exported as .csv files.

Multi-dimensional Scaling analysis was performed using PRIMER 6 software (Clarke, 1993). Data were imported from .csv files and square root transformed. A Bray-Curtis Similarity matrix was constructed for each dataset and the MDS plot generated from this, using 50 restarts to ensure the best solution, was presented. The equation for calculating the BrayCurtis index between two profiles is shown in Figure 3.3.

$$
B C=100 \cdot \frac{\sum_{i} \min \left\{y_{i 1}, y_{i 2}\right\}}{\left(\sum_{i} y_{i 1}+\sum_{i} y_{i 2}\right) / 2}
$$

Figure 3.3 Equation for calculating the Bray-Curtis Index.

\subsubsection{CLUSTER analysis}

Cluster analysis was performed on the T-RFLP and MDS data to identify any statistically significant clusters of samples. This was performed using the CLUSTER routine included in 
the PRIMER 6 software package. Significance testing is performed by a routine associated with CLUSTER, the SIMPROF test (Clarke and Gorley, 2006).

\subsubsection{RELATE analysis}

The RELATE function of PRIMER 6 allows the similarity matrices underlying MDS plots from two different datasets to be tested for correlation. The two similarity matrices are then compared and a Spearman rank correlation coefficient $(\rho)$ is generated. To determine statistical significance, a simple permutation test is used, with the null hypothesis that there is no relationship between the two similarity matrices. If the null hypothesis is found to be true, a $\rho$ value of approximately zero is returned. The null distribution can be obtained by permuting sample labels at random 999 times and recalculating $\rho$ to build up a frequency histogram with which the true value of $\rho$ can be compared. RELATE was performed to test for correlation between datasets from replicate pig carcasses (sections 4.3 .4 and 5.4.4). This routine requires the sample labels to be the same in the two matrices tested. This prevented replicate cadaver datasets being compared using this routine because sampling dates and ADD values differed between the cadaver sites (section 6.4.5). Datasets produced from the same samples but using different profiling methods (i.e. DGGE and T-RFLP) were also compared using RELATE. 


\section{Chapter 4: Pig Carcass Decomposition on Sand and the Effect of Insect Colonisation}

\subsection{Aim}

The aim of this experiment was to characterise the temporal profiles of the bacteria that leach from pig carcasses over time. Acid-washed sand was used as a substrate to collect the decomposition products and microflora released. It was hypothesized that as decomposition progressed, the bacterial community in the sand underlying the carcass would change, because of the sequential nature of decomposition and the changing environment under the carcass. The effect of insect colonisation of the carcasses on the resultant bacterial community was also investigated by excluding insects from some carcasses and allowing colonisation of others. This experiment was performed as a first step to look at the carcass microflora, and the species contributed by colonising insects without the presence of a complex soil bacterial community.

\subsection{Methods}

The laboratory methods used in this experiment are described in Chapter 3. The experimental set-up is described below.

\subsubsection{Carcass Information}

Carcasses of six organically raised pigs were obtained from a free-range organic farm in the central North Island. Organically raised pigs were used because conventional piggeries routinely administer antibiotic prophylaxis treatment. Antibiotics are known to affect the gut microfloral composition of pigs (Davis et al., 2007), which is undesirable for this study. Each pig weighed approximately $24 \mathrm{~kg}$ (Table 4.1). This weight is commonly used for forensic 
entomological studies as the carcasses are easily manoeuvrable and using larger carcasses does not seem to increase similarity to human decomposition (Haskell et al., 2001).

The pigs were euthanised by the farmer prior to purchase in the manner regularly used in daily farming practice. This consisted of sedation using $5 \mathrm{mg}$ of Stresnil (azaperone) and a headshot using a .22 rifle. The carcasses were weighed and put into large plastic bags which were sealed using packaging tape to prevent insect colonisation during transportation. The carcasses were labelled A to F. Carcass weights and subsequent insect colonisation statuses are shown in Table 4.1.

\begin{tabular}{|c|c|c|}
\hline Carcass ID & Weight (kg) & Insects? \\
\hline A & 22.9 & Yes \\
\hline B & 24.5 & No \\
\hline C & 24.4 & Yes \\
\hline D & 29.6 & No \\
\hline E & 22.5 & Yes \\
\hline F & 24.7 & No \\
\hline
\end{tabular}

Table 4.1 Carcass weights and insect colonisation status.

\subsubsection{Experimental Site Set-up}

The site used for the experiment was a secluded area at the Wallaceville Animal Research Facility in Wallaceville, Wellington. The experiment was run from summer through to winter, from 28 January 2005 to 2 August 2005. The area was secure with no public access permitted. The site was surrounded by fencing on all sides and thick native bush surrounded the fence on three sides. Sheep were occasionally grazed in the area to maintain the grass length. The site had a long, permanent table covered with a v-shaped corrugated iron roof 
(Figure 4.1). This experiment was set up under the roof adjacent to the table, to prevent flooding of the trays in which the pig carcasses were placed, in the event of heavy rainfall. The area was also sheltered and shaded by a large tree (Figure 4.1).

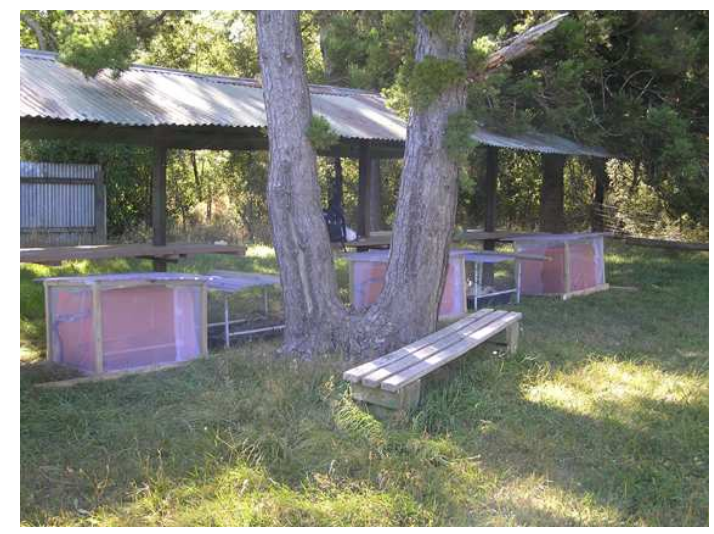

Figure 4.1 The Decomposition Site.

Although there was no public access to the site, it was possible that stray farm dogs or wildlife such as possums or birds might disturb the carcasses, so they were placed in individual chicken-mesh cages (Figure 4.3) to prevent interference by scavengers. Three cages were designed to prevent colonisation of the carcasses by insects (Figure 4.2), and three allowed normal colonisation. The carcasses that were protected from colonisation by insects were surrounded by a fine nylon mesh around a steel frame, with sleeved openings to allow sampling access (Figure 4.2). A larger timber framed cage with chicken wire sides and a plastic corrugated roof was placed over the nylon mesh cage. The cages allowing insect colonisation consisted of a steel frame with chicken wire sides and a plastic corrugated roof (Figure 4.3). The roof could be lifted off and one side opened to allow access for sampling. 


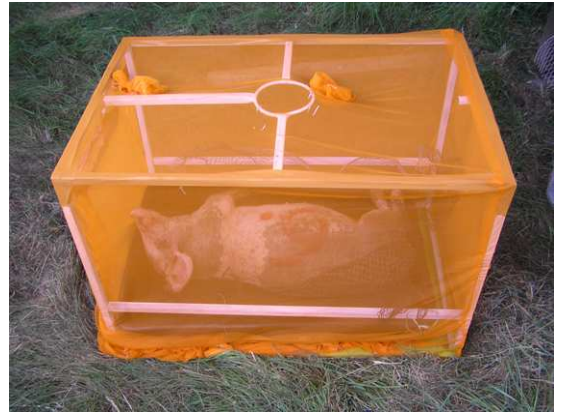

Figure 4.2 Insect colonisation prevention netting.

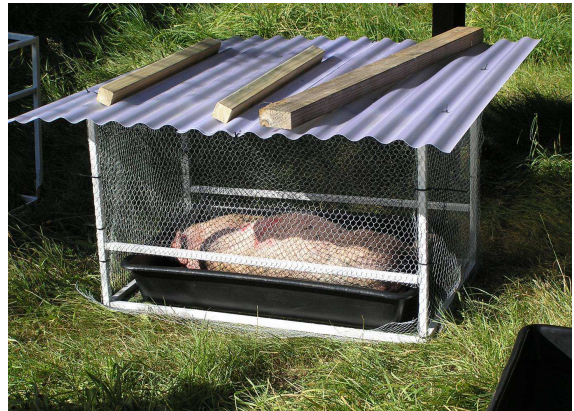

Figure 4.3 Cage allowing insect colonisation.

Clean dune sand was purchased from Ablaze, Grenada North, and placed in black plastic trays (885 $\mathrm{mm} \times 612 \mathrm{~mm} \times 155 \mathrm{~mm}$ ) to a depth of $10 \mathrm{~cm}$. The sand was rinsed thoroughly with running tap water to remove any silt contamination, followed by an acid wash to remove contaminating microbes and residual DNA (Dr Gupta Vadakattu, CSIRO Australia, pers. comm.). Approximately $20 \mathrm{~L}$ of $0.1 \mathrm{~N} \mathrm{HCl}$ was added to the plastic trays containing the sand and left for 24 hours, with occasional mixing. The $\mathrm{HCl}$ was then decanted and the sand thoroughly rinsed ten times with distilled water. The sand was loosely covered with paper towels and air dried in the laboratory for approximately a week. Three $0.5 \mathrm{~g}$ samples of sand were taken from each tray, and DNA extractions were performed on each sample to determine the background level of DNA (section 3.4.1). Viable bacterial counts were also determined using the method described in section 3.1.3. The DNA extractions yielded negligible quantities of DNA (as detected using the pico green DNA quantitation method, section 3.4.2), and the viable bacterial count was $4 \pm 2$ colonies per $5 \mathrm{~g}$ sand sample. This was deemed to be as close as reasonably possible to sterility for the volume of sand used in this experiment.

Before placement on the sand, the carcasses were washed briefly with tap water (Figure 4.4) to remove excess soil and any insect eggs that had already been laid. The carcasses were randomly assigned to a treatment: three were protected from insect colonisation, and three 
exposed to allow insect colonisation (Table 4.1). Control sand samples were collected from each tray before the carcass was placed. A smaller tray of sand with no pig carcass was also used as a control, and samples were collected from this at each sampling date.

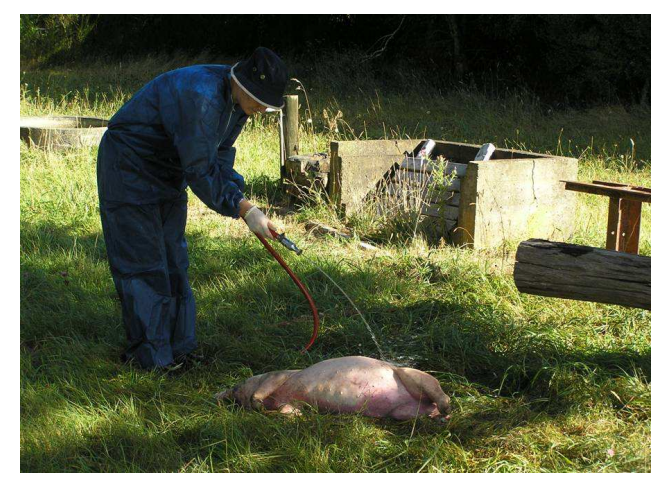

Figure 4.4 Washing the pig carcasses to remove insect eggs and adhering soil before placement in the cages.

To enable sampling under the carcass when decomposition became more advanced, a length of plastic nylon netting was placed on top of the sand before carcass placement, creating a type of hammock in which the carcass could be lifted. The hammock was temporarily suspended from the cage frame using steel hooks whilst sampling (Figure 4.5).

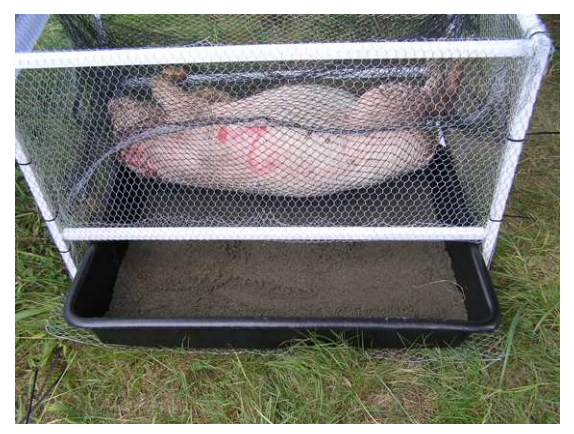

Figure 4.5 Pig lifted in netting to allow sampling of underlying soil.

Temperature and relative humidity $(\mathrm{RH})$ readings were collected hourly using a data-logger attached to one of the cages. Rainfall data collected at a weather station situated at the Seddon Rifle Range in Trentham were downloaded from the web after completion of the experiment (http://www.wmac.org.nz/trenthamweather). This was the closest weather station from which data are freely available, and is located approximately $1 \mathrm{~km}$ from the experimental site. 


\subsubsection{Sampling regime}

Samples were collected daily for the first 26 days until active decay was complete for the insect colonised carcasses (A, C and E). Samples were then collected every other day until day 60. Decomposition progressed much more slowly in the absence of insect colonisation, so carcasses B, D and F were sampled only every second day through to day 60 . From day 60 onwards, when the colonised carcasses had reached skeletonisation, samples were collected from under all carcasses approximately once every two weeks until day 155.

The trays were sampled by taking a $50 \mathrm{~g}$ surface scraping of the sand over the entire surface area by scooping up the soil using a serpentine motion, starting from one corner and finishing at its diagonal opposite (Figure 4.6). This method was used in order to reduce the effect of localised differences under the carcass, and also to prevent major disruption to the surface interaction between the carcass and sand. Samples were collected in plastic $50 \mathrm{ml}$ sterile screw-top disposable centrifuge tubes and stored at $-20^{\circ} \mathrm{C}$ on return to the laboratory. Photos were taken of each carcass on each sampling date to document the general progression of decomposition.

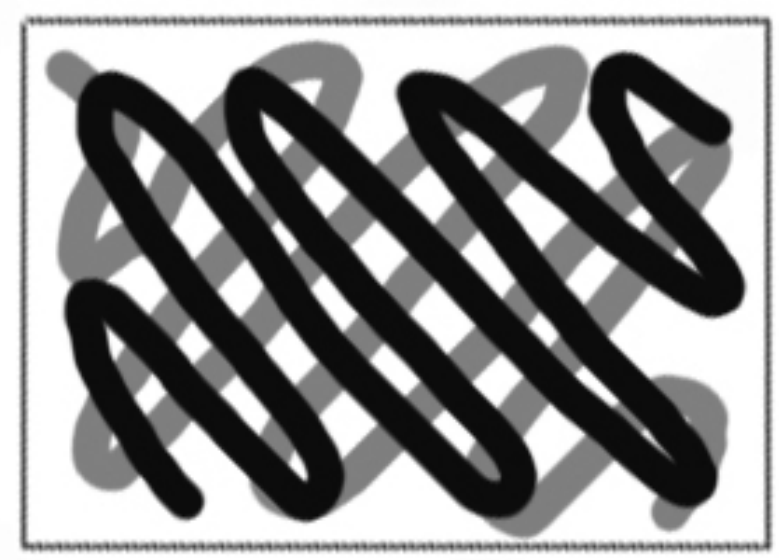

Figure 4.6 Diagram of sampling technique. 


\subsubsection{Laboratory methods}

For this experiment, the $\mathrm{pH}$ of each sample was determined (section 3.2.1) and DNA was extracted from a sub-sample of each (section 3.4.1) for molecular analysis. The molecular methods used were T-RFLP (section 3.4.3), and DGGE using universal 16S rRNA primers (section 3.4.4.1).

Accumulative degree day (ADD) values were calculated for each sampling time by summing the average hourly temperature $\left({ }^{\circ} \mathrm{C}\right)$ for each twenty-four hour period since death. Using ADDs instead of days as a measure of the degree of decomposition takes into consideration the fact that temperature strongly affects the rate of decomposition.

\subsection{Results}

\subsubsection{Documentation of Decomposition Progression}

A selection of photographs of carcasses A (with insect colonisation) and B (without insect colonisation), which demonstrate the progression of decomposition, are shown in Figures 4.7 and 4.8. Photos of the other four carcasses are included in Appendix E. Figures 4.7 and 4.8 clearly show the difference in the decomposition process between the carcasses that were allowed to be colonised by insects (carcass A, 4.7) and those protected from insect colonisation (carcass $\mathrm{B}, 4.8$ ). The colonised carcasses progressed rapidly through the typical stages of decomposition (section 2.1.1) and had all reached the dry or skeletonisation stage by the end of the 155 day sampling period. The insect-free carcasses slowly mummified over time with decomposition fluids purging from the orifices, but with the skin remaining relatively intact throughout the 155 days. The insect exclusion netting successfully prevented insect colonisation until day 155, when very small (3 $\mathrm{mm}$ long) Diptera (flies) were found under carcass $\mathrm{F}$. 
Carcass A displayed the most rapid decomposition of all six carcasses. Possible reasons for this are discussed in section 4.3.2. This carcass clearly displayed all the documented stages of decomposition, with the fresh, bloat, active decay and post decay stages clearly recognisable (4.7). The skeletonisation stage tends to be defined as comprising remains older than 1285 ADD in humans (Vass et al., 1992) but this carcass was largely skeletonised by around 750 ADD. Because the carcasses were not covered by clothing or other materials, the maggot mass did not degrade the exposed abdominal skin surface. This often occurs in uncovered remains, as the maggots leave a protective layer against the sun and predators such as birds (Bass, 1997). This leathery sheet of skin could be lifted to expose disarticulated bones at day 50 (ADD 758). Carcass A demonstrated a build up of gases in the gastrointestinal tract, with the skin perforating at the abdomen, but the peritoneum remaining intact and ballooning out through the skin (4.7, ADD 114). Carcass F also exhibited a skin rupture, through which the intestines had ballooned (Appendix E). The other carcasses also displayed bloating, but without skin ruptures. The decomposition gases could be heard escaping from existing orifices when the carcasses were moved for sampling during the bloat stage.

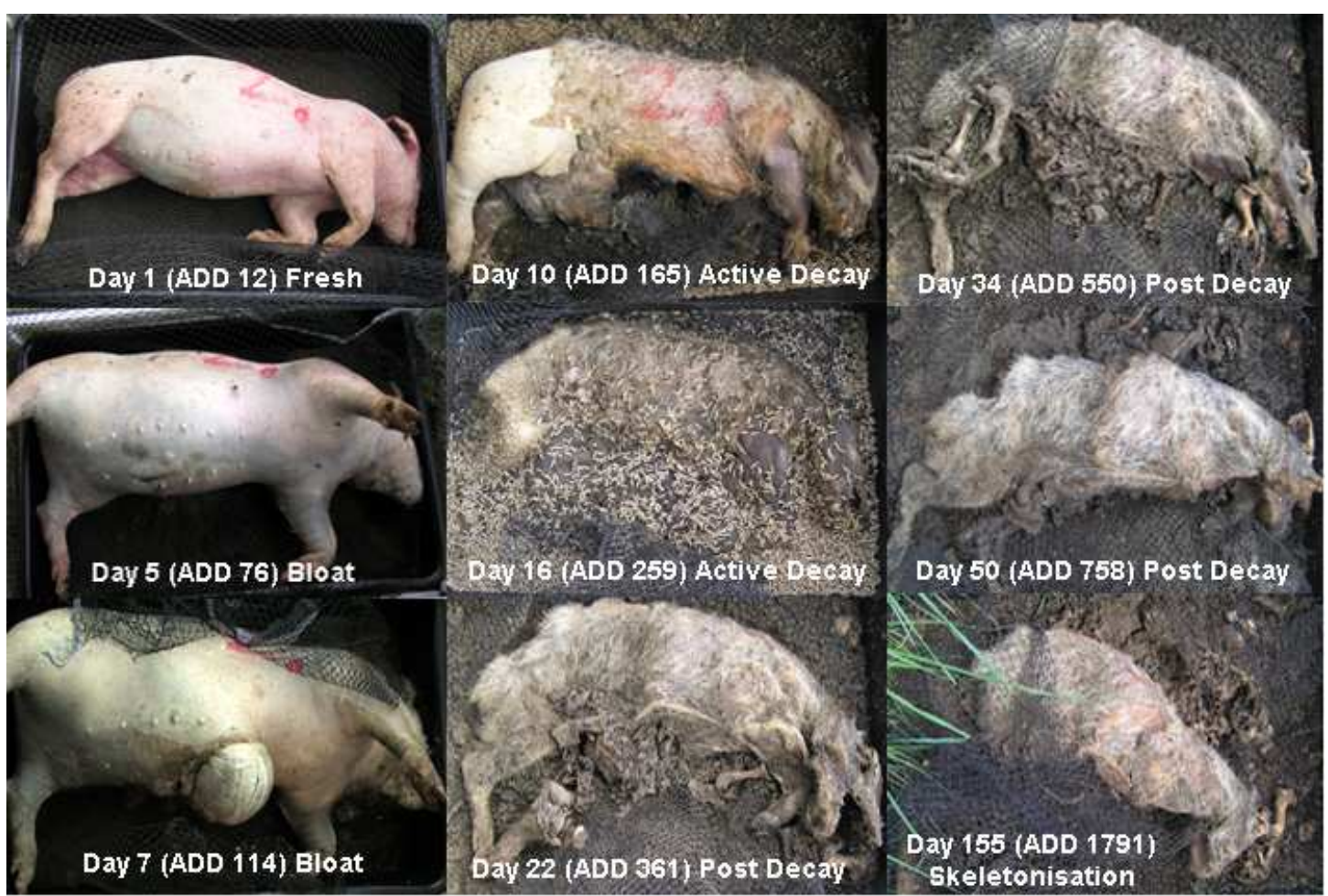

Figure 4.7 Carcass A (insect colonised) decomposition over time. 


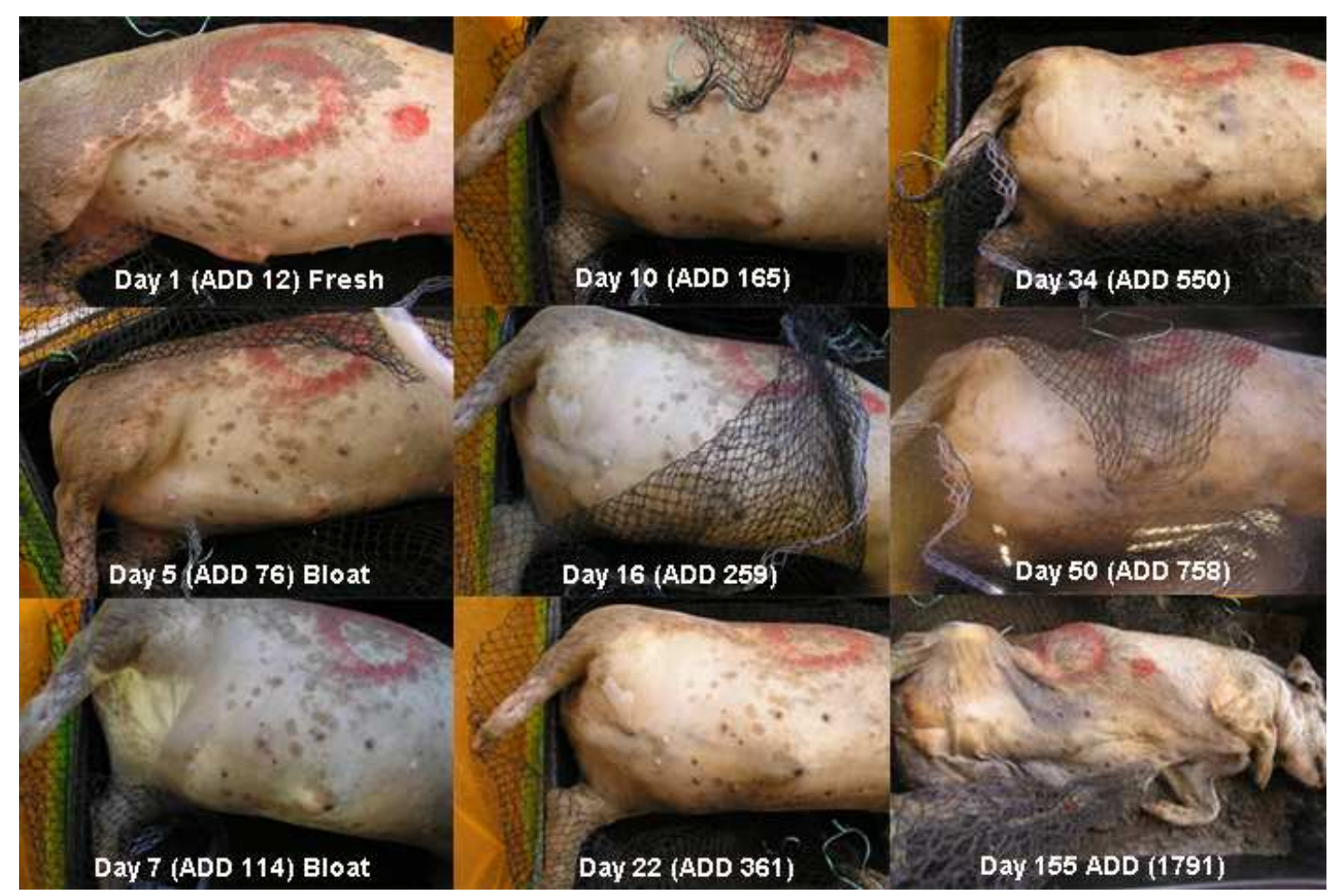

Figure 4.8 Carcass B (no insect colonisation) decomposition over time.

Carcasses $\mathrm{C}$ and $\mathrm{E}$ (both colonised by insects) progressed through decomposition in a similar manner to carcass A, albeit slightly more slowly. This could be due to differences in exposure to direct sunlight on the carcasses (section 4.3.2), or it may be related to the skin rupture in carcass A, allowing easier access for insects to the internal tissues, resulting in more rapid advancement through active decay. Carcasses B, D and F (no insect colonisation) displayed similar decomposition progression to each other at each sampling date, with the exception of the skin rupture that occurred in carcass F (Appendix E). The typical fresh and bloat stages were identifiable for the non-colonised carcasses, but because active decay tends to begin when the insect larvae become active, these carcasses did not go through the typical later stages of decay and skeletonisation. A slight increase in the volume of purge fluid was noted at around day 9 (ADD 148) onwards. Fluids had previously purged from the natural openings prior to this day, but had remained as small pools around the orifices. From day 9 onwards 
the fluids permeated much further into the sand, creating a large pool around the whole carcass.

Insect colonisation of carcasses $\mathrm{A}, \mathrm{C}$ and $\mathrm{E}$ occurred almost immediately after placement at the site, with flies landing on them within minutes (Figure 4.9), and eggs appearing on the carcasses within four hours (Figure 4.10).

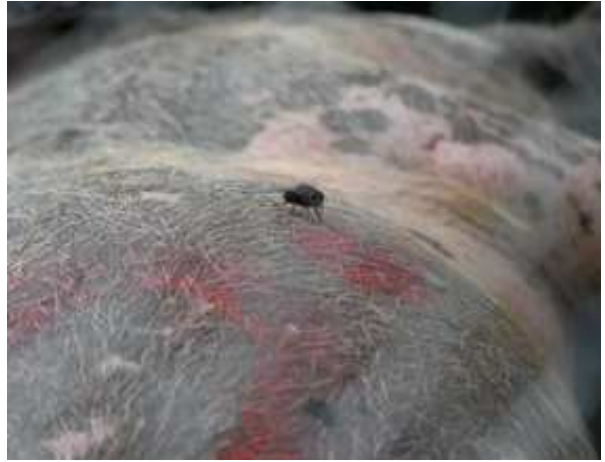

Figure 4.9 Blowfly on carcass $C$ one hour after placement.

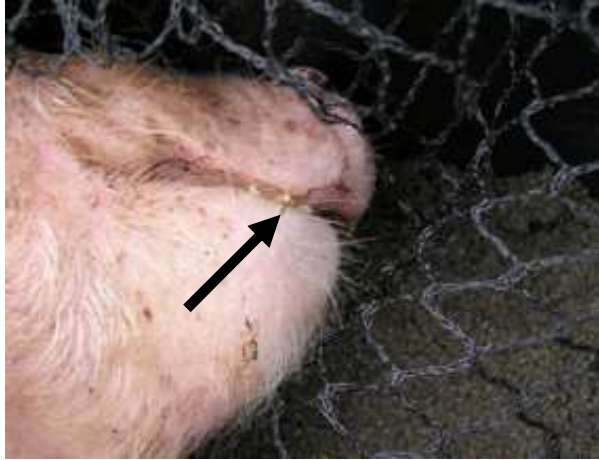

Figure 4.10 Fly eggs on the mouth of carcass within four hours of placement.

\subsubsection{Temperature, Relative Humidity \& Rainfall}

The mean temperature over the sampling period was $11.5^{\circ} \mathrm{C}$ with a mean relative humidity $(\mathrm{RH})$ of $86.7 \%$. The mean daily rainfall was $2.81 \mathrm{~mm}$ with a total rainfall of $436 \mathrm{~mm}$ over the 155 days. The carcasses were partially sheltered from the rain by the roofing material on the cages, but this was not totally effective at preventing rain entering the cages. The mean daily temperature data for the site, calculated by finding the mean of the 24 hourly readings, are shown in Figure 4.11 and the mean daily relative humidity data in Figure 4.12. The daily rainfall for the sampling period is shown in Figure 4.13. 


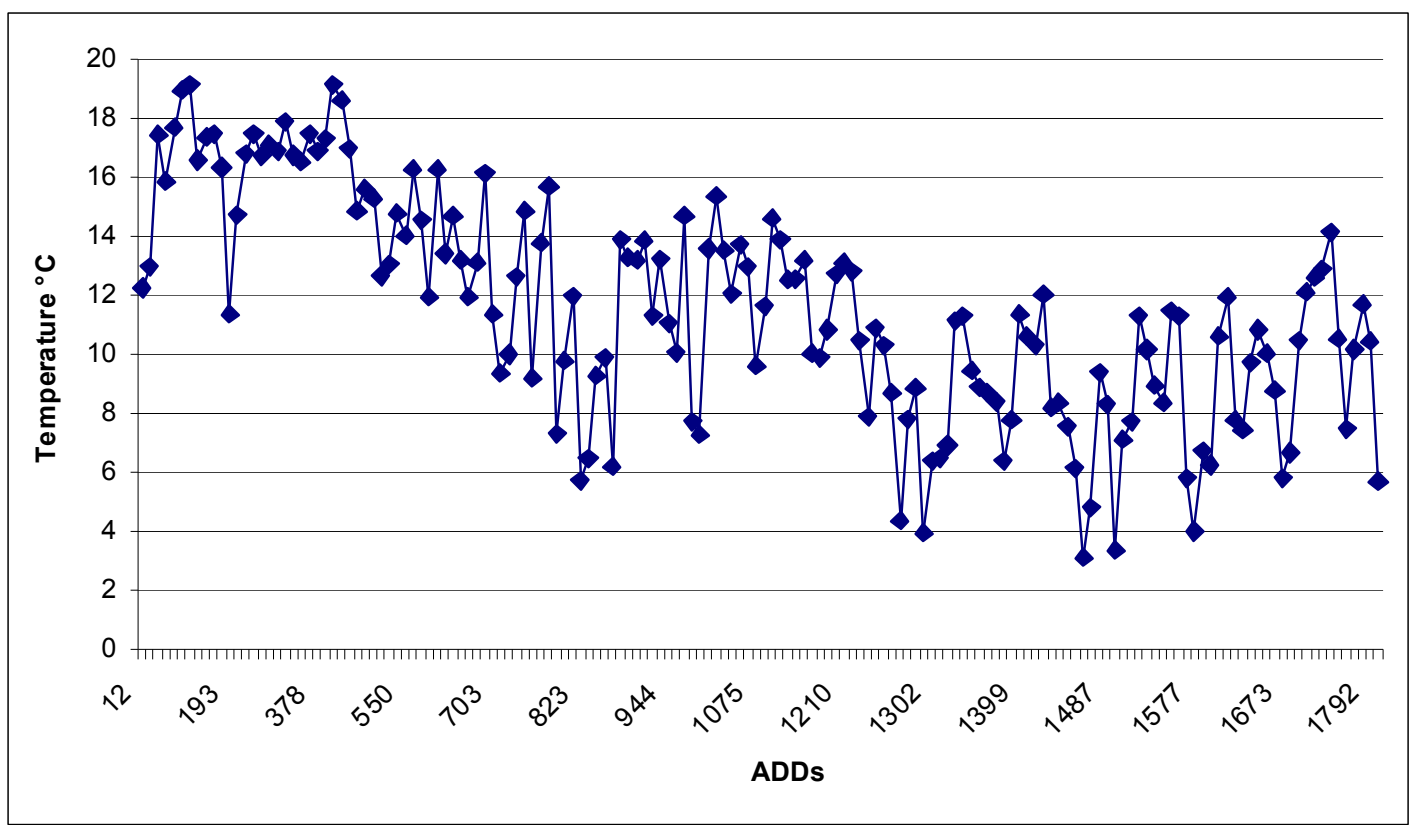

Figure 4.11 Mean daily ambient air temperature.

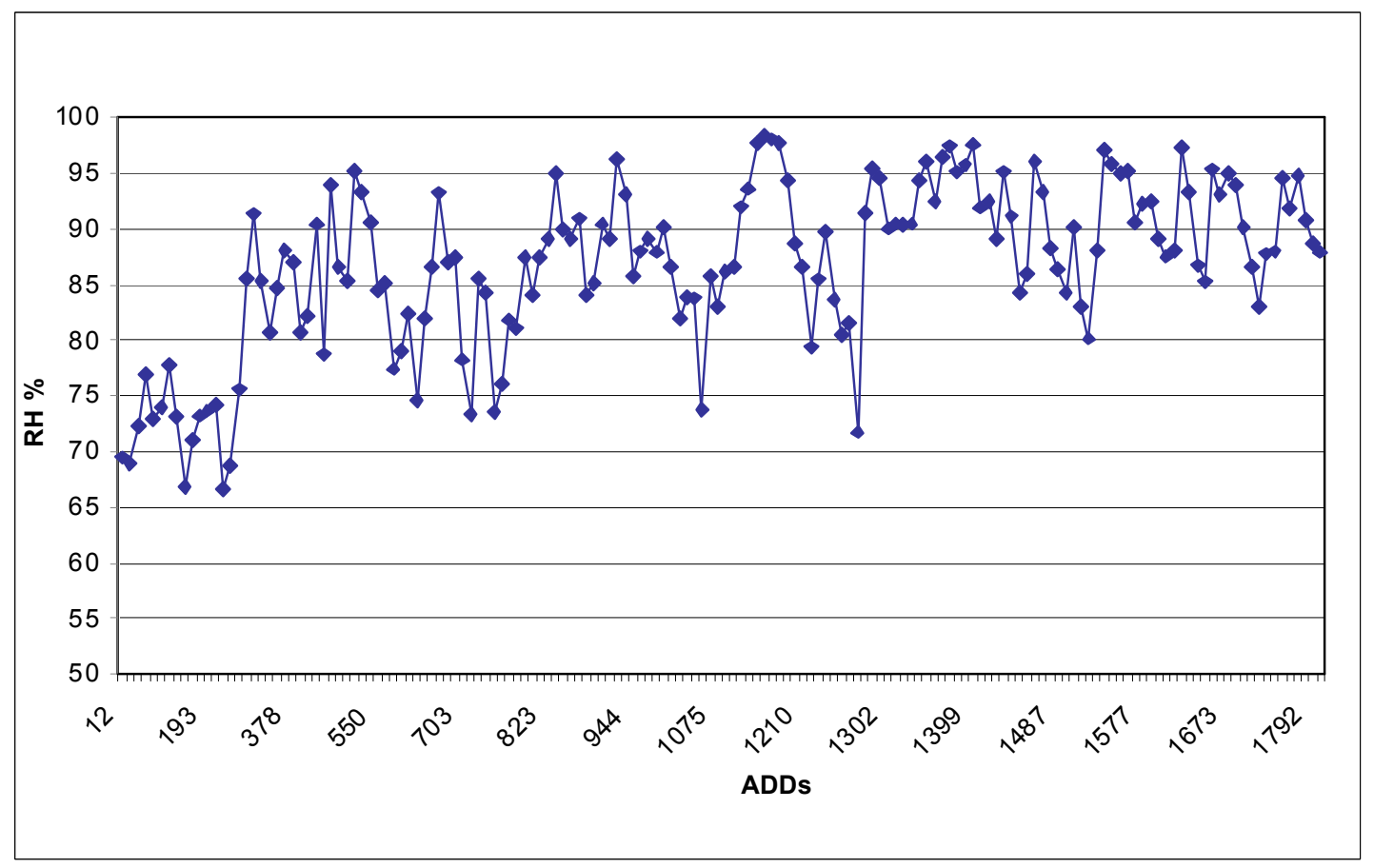

Figure 4.12 Mean daily relative humidity ( $\mathrm{RH})$.

61 


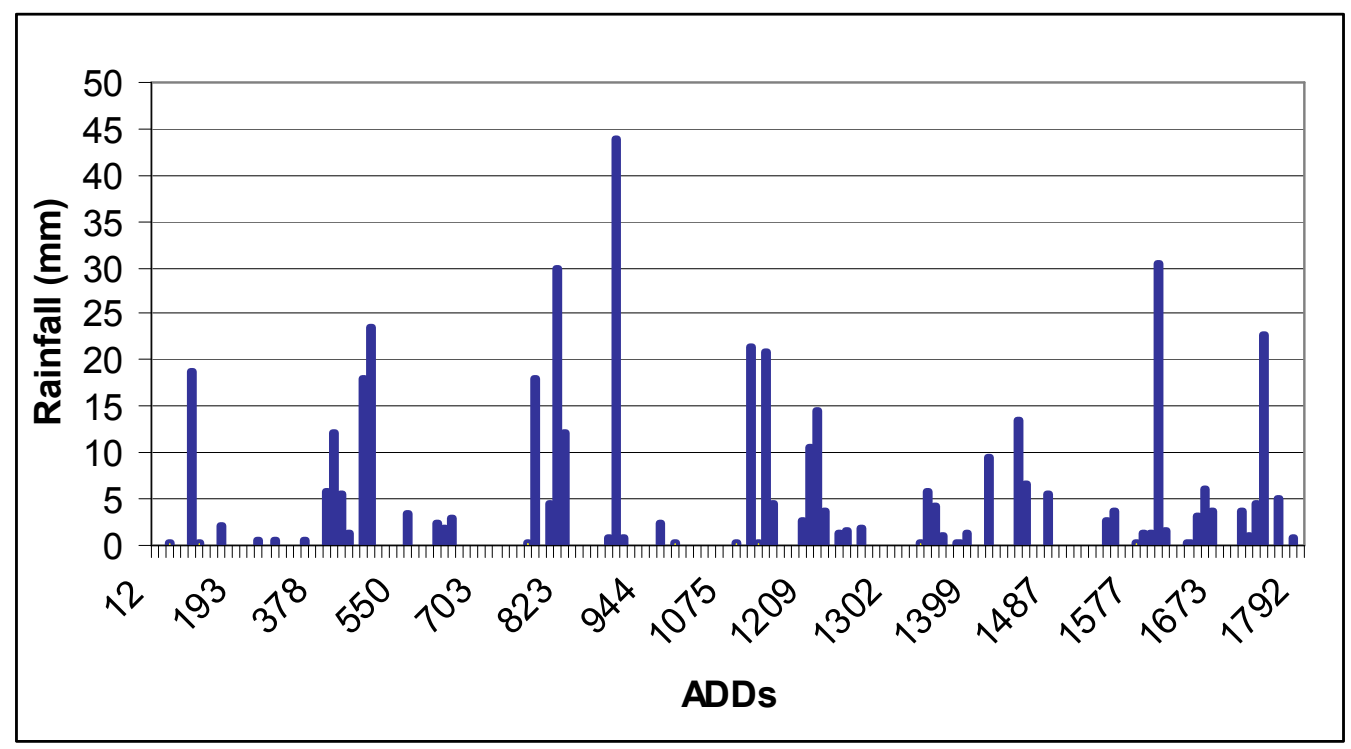

Figure 4.13 Daily rainfall during the first experiment.

The positioning of the cages led to different amounts of sunlight exposure for each carcass, which is largely unavoidable in a natural situation. Due to the position of tall trees and the covered table structure, carcass A received approximately 90 minutes more sunlight each day than the other carcasses, although this altered throughout the duration of the experiment with the changing angle of the sun. Carcasses $\mathrm{B}$ and $\mathrm{C}$ did not receive this extra direct sunlight due to shading by a nearby tree (see Figure 4.14). As carcass A appeared to decompose at a slightly faster rate, an additional data logger was positioned in its cage to compare with the data collected by the logger positioned on the cage of carcass D (Figure 4.14). Data were collected using the second logger over a period of one week (7 March 2005 to 14 March 2005 which corresponds to days 7 to 14 , or ADDs 114 to 225). The temperature differences are shown in Figure 4.15. Increased temperatures are known to stimulate maggot activity and development (Greenberg and Kunich, 2002) and different insect species are attracted to shaded carcasses versus those in direct sunlight (Anderson, 2001) which may also have potentially played a role. Greenberg (1991) noted in one study using rabbit carcasses, that while the average air temperature was $11 \%$ higher in the sun than in the shade, the 
accumulative degree hours (ADHs) of carcasses in the sun were $28-48 \%$ higher than those in the shade.

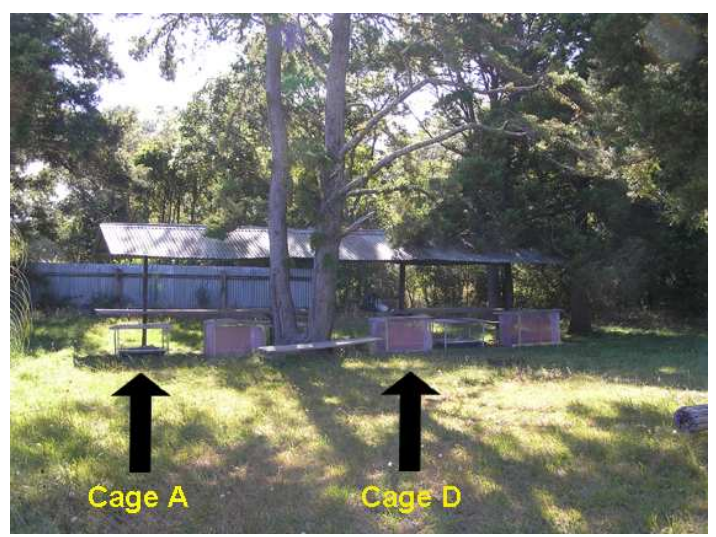

Figure 4.14 Cage placements at the decomposition site.

Cages are arranged in alphabetical order, A through F. Cage C is obscured by the tree trunk.

The mean daily temperature difference was only $0.31^{\circ} \mathrm{C}$, and when the differences are taken into consideration they do not cause any difference in overall ADD calculations for that carcass. As decomposition progressed, the changing angle of the sun meant that the temperature effect is likely to have reduced with time.

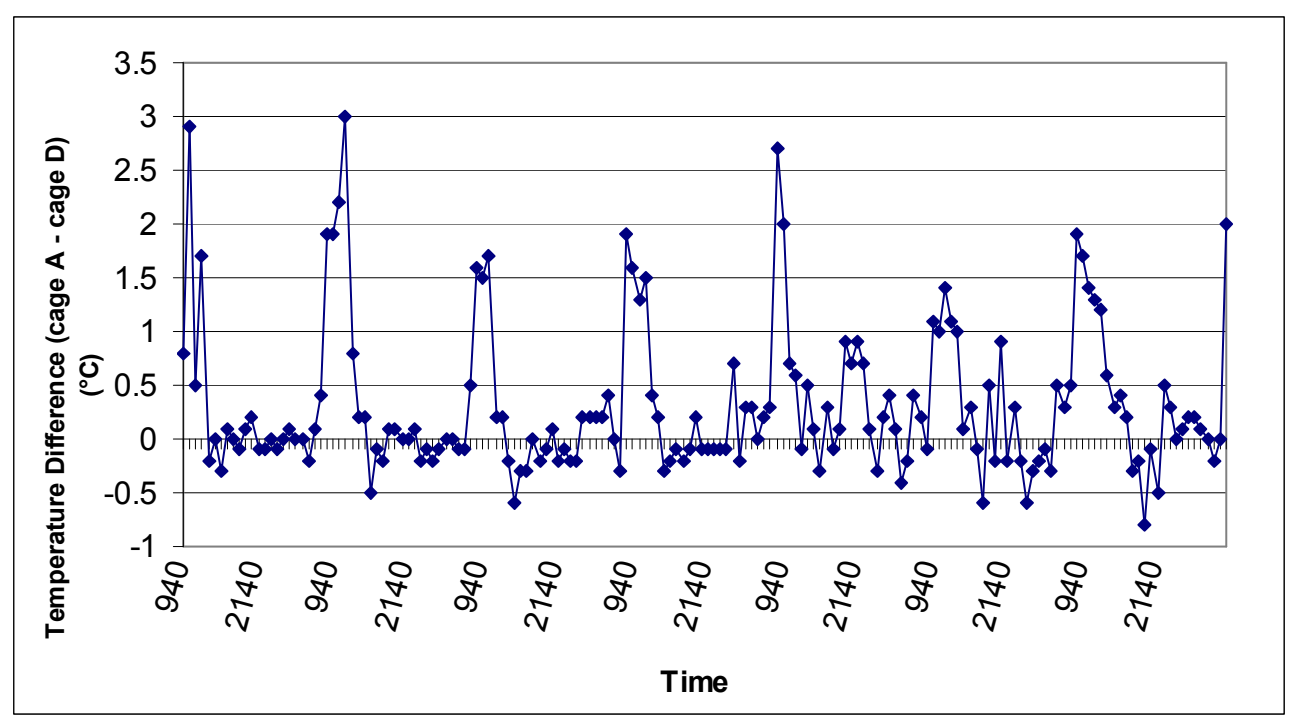

Figure 4.15 Temperature difference between cages $A$ and D over the course of one week. Readings were taken every hour beginning at 9.40 am on $7^{\text {th }}$ March until 9.40 am on $14^{\text {th }}$ March 2005. 


\subsection{3 $\mathrm{pH}$}

The mean $\mathrm{pH}$ of the sand under the carcasses at each sampling (with and without insect colonisation) are shown in Figure 4.16.

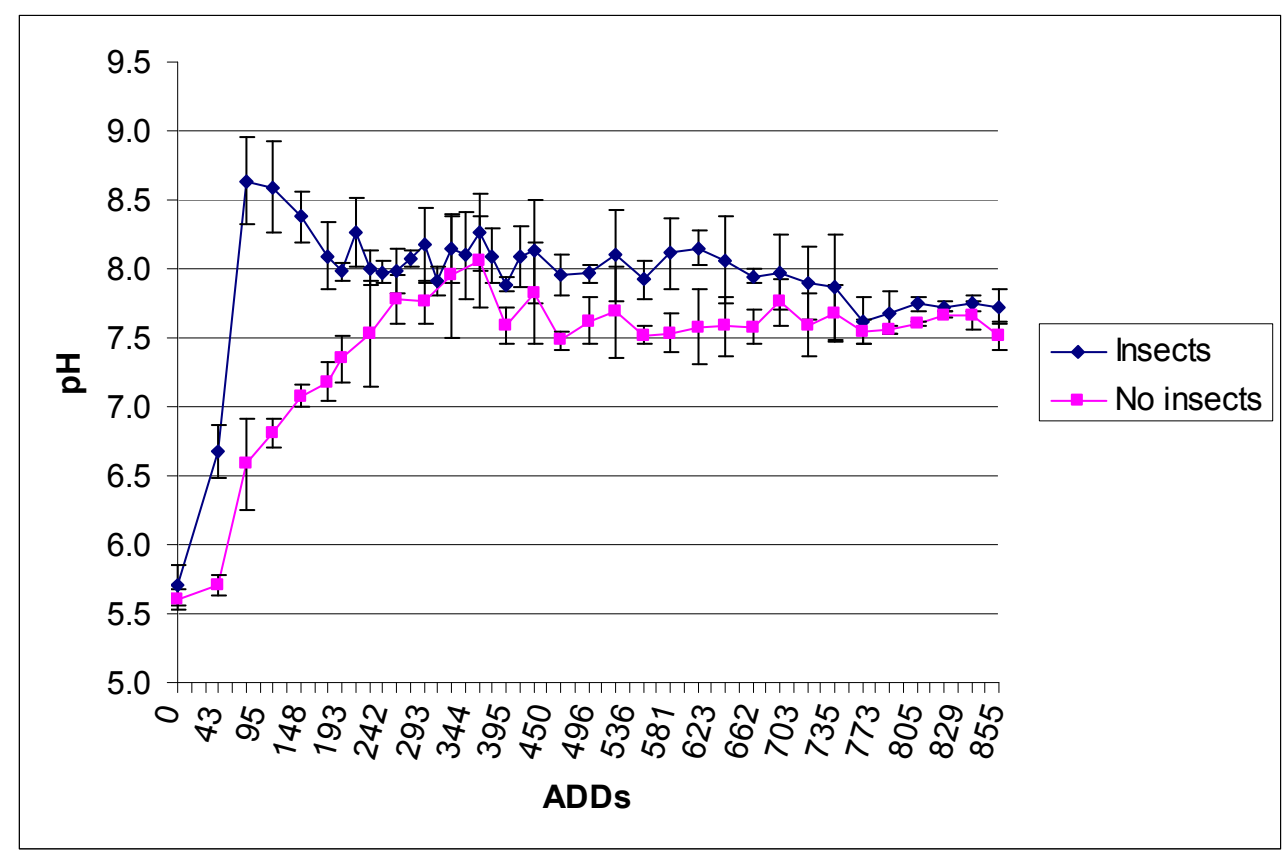

Figure 4.16 Mean pH of the replicate plots with or without insect colonisation. Error bars represent the standard error.

The sand under the carcasses colonised by insects displayed a dramatic rise in $\mathrm{pH}$ early in decomposition from $\mathrm{pH} 5.6$ to around $\mathrm{pH} 8.6$ by ADD 76, which was 5 days after death. After this time, the $\mathrm{pH}$ stabilised to between 7.5 and 8.5. The sand under the carcasses not colonised by insects showed a slower $\mathrm{pH}$ rise, reaching its highest mean value of 8.05 at day 22 (ADD 361), and then stabilised to between 7.5 and 8 .

\subsubsection{T-RFLP}

Bacterial community profiles were generated using the T-RFLP analysis method described in section 3.4.3. Reasonable quality T-RFLP profiles were generated for all samples collected in this experiment. Profiles were not able to be generated from any control sand samples, as negligible amounts of DNA were recovered from each. Similarly, no DNA could be extracted 
from early samples collected under the carcasses, on day 3 (ADD 43) for all carcasses and also on day 5 (ADD 76) for carcasses B, D and E. Selected sample profiles from carcass A are displayed in Figure 4.17.
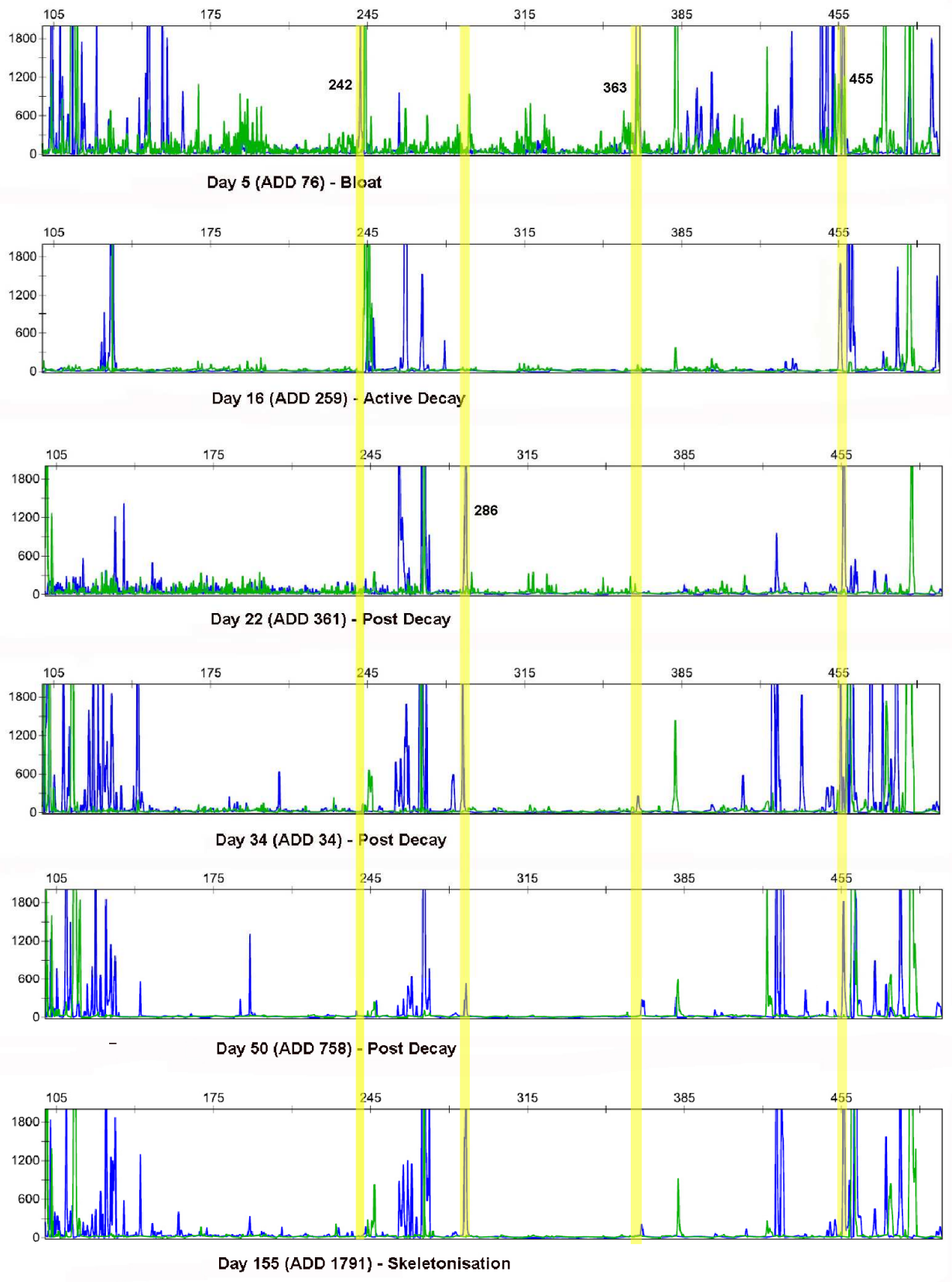

Figure 4.17 Selected T-RFLP profiles from samples collected at different stages of decomposition from decomposition plot A (insect colonised). Some T-RFs of interest are highlighted.

The selected T-RFLP profiles from the carcass A sand samples (Figure 4.17) depict the changes that occur in the microbial community as the carcass progresses through the stages of 
decomposition. It is readily apparent that some T-RFs, representing specific bacterial phylotypes, are present from early in decomposition right through to skeletonisation (for example, peak 455, Figure 4.17). Other phylotypes (e.g. peak 286 in Figure 4.17) appear during the decay stage and persist until the final sampling date. A number of T-RFs present in the early stage profiles do not appear in any subsequent profiles (e.g. T-RFs 242 and 363, Figure 4.17) and there are numerous other examples of more transient phylotypes that come and go throughout the decomposition process.

A total of 137 different T-RFs were identified from all six carcasses, with 85 of these from the $5^{\prime}$ end of the gene fragment, and 52 from the $3^{\prime}$ end. Each carcass had a number of T-RFs exclusively associated with it (Table 4.2), as did each treatment (insect colonised/noncolonised). No single peak was present in every profile from a carcass, although many T-RFs were present in at least 10 profiles from each carcass (see Table 4.3).

\begin{tabular}{|c|c|c|c|c|c|c|}
\hline & \multicolumn{3}{|c|}{$\begin{array}{c}\text { Colonised by } \\
\text { insects }\end{array}$} & \multicolumn{3}{|c|}{$\begin{array}{c}\text { Not colonised by } \\
\text { insects }\end{array}$} \\
\hline & $\mathbf{A}$ & $\mathbf{C}$ & $\mathbf{E}$ & B & D & $\mathbf{F}$ \\
\hline Total number of T-RFs & \multicolumn{6}{|c|}{137} \\
\hline Number of T-RFs from $5^{\prime}$ end & \multicolumn{6}{|c|}{85} \\
\hline Number of T-RFs from $3^{\prime}$ end & \multicolumn{6}{|c|}{52} \\
\hline No of T-RFs from each carcass & 45 & 61 & 46 & 41 & 48 & 37 \\
\hline No of T-RFs specific to each carcass & 9 & 5 & 5 & 8 & 6 & 7 \\
\hline No. of T-RFs common to all carcasses & \multicolumn{6}{|c|}{23} \\
\hline No of T-RFs exclusive to treatment & \multicolumn{3}{|c|}{16} & \multicolumn{3}{|c|}{12} \\
\hline $\begin{array}{l}\text { No of T-RFs exclusive to treatment but } \\
\text { common to all } 3 \text { carcasses }\end{array}$ & \multicolumn{3}{|c|}{6} & \multicolumn{3}{|c|}{11} \\
\hline
\end{tabular}




\begin{tabular}{|c|c|c|c|c|c|c|}
\hline & A & B & C & D & E & F \\
\hline 5' gene fragment & 102 & 111 & 131 & 111 & 131 & 129 \\
& 127 & 129 & 261 & 261 & 261 & 262 \\
& 131 & 262 & 460 & 262 & 449 & 364 \\
& 269 & 364 & 481 & 364 & 460 & 457 \\
& 424 & & & 450 & 481 & 458 \\
& 460 & & & & & \\
& 481 & & & & & \\
\hline 3' gene fragment & 103 & 113 & 113 & 113 & 244 & 113 \\
& 113 & 246 & 244 & 244 & 246 & 475 \\
& 459 & 475 & 247 & 246 & 247 & 485 \\
& 486 & 483 & 380 & 475 & 486 & 486 \\
& & 485 & 486 & 485 & & \\
\hline
\end{tabular}

Table 4.3 Sizes in base pairs (bp) of T-RFs found in more than 10 profiles from each pig.

\subsubsection{Multi-Dimensional Scaling (MDS) Analysis}

Multi-Dimensional Scaling (MDS) analysis was used to determine when notable changes were occurring in the bacterial communities during the decomposition process.

The MDS plot of the carcass A T-RFLP data (Figure 4.18) shows three separate groups at the $20 \%$ similarity threshold, with clusters a and b showing less than $20 \%$ similarity to each other and to the remaining samples. At the $40 \%$ similarity level this large group (clusters c-i) is split into two further groups (clusters $\mathrm{c}, \mathrm{d}$ and $\mathrm{e}$ together and clusters $\mathrm{f}$ to $\mathrm{i}$ together). At the $60 \%$ similarity level, the samples are further split into smaller groups which closely match the statistically significant clusterings. This suggests that the bacterial community undergoes a series of major composition shifts, particularly at the A7-A9 (ADD 114-148) and A16-A18 (ADD 259-293) time-points. A series of more gradual changes seem to be occurring between these times. When the T-RFLP profiles are examined, the more dramatic shifts are associated with changes in the dominant T-RFs, while the smaller shifts seem to be caused largely by changes in T-RF intensity. 


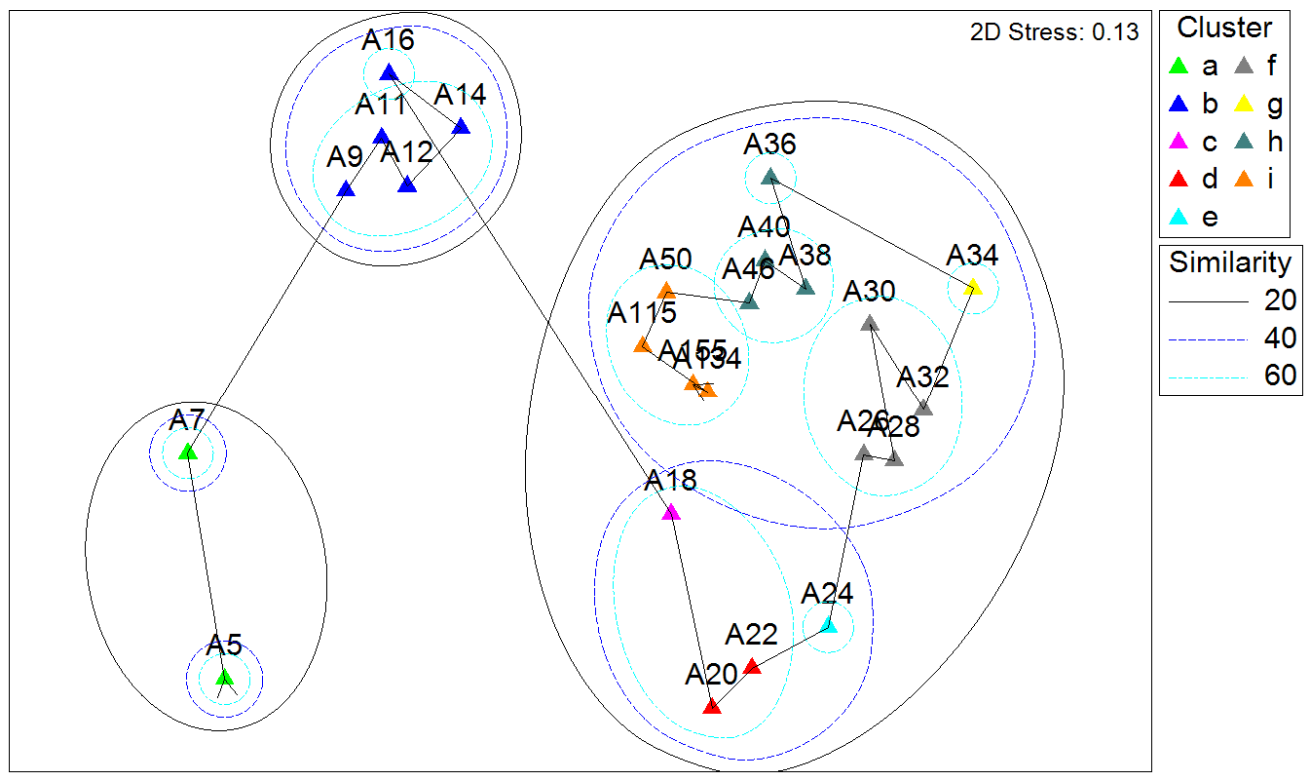

Figure 4.18 MDS analysis plot of T-RFLP data from sand collected from under carcass A. Nine statistically significant clusters were distinguished using CLUSTER. The Bray-Curtis similarity index was used on square root transformed data. Samples are denoted by the number of calendar days post-death.

The separate clustering of the fresh/bloat sample profiles (cluster a) from profile A9 onwards corresponds with a visual change in the decomposition stage of the carcass, from bloat to active decay. There was a sudden increase in the volume of purge fluids at this time, and this is likely to correspond to a release of different bacterial species from the carcass, as different tissues began to degrade. Additionally, from day 9 (ADD 148) onwards, the insect larvae began to hatch and rapidly break down the tissues.

The separation of cluster $b$ from later samples also corresponds to visual changes seen in the carcasses. From around A18 (ADD 259) onwards, the maggot mass was beginning to move away from the carcass to pupate and the sand and remaining tissues began to look drier, with the carcasses advancing into the post-decay stage.

The samples from carcass $\mathrm{C}$ also separate into three groups at the $20 \%$ similarity threshold (Figure 4.19), but it is the skeletonisation/dry samples, C115 to C155 (ADD 1440-1791) that cluster away from the main group, rather than the early active decay samples, as was seen for carcass A (Figure 4.18). The carcass E samples separate into four groups at the $20 \%$ 
similarity threshold (Figure 4.20), with both the early active decay (E9 to E16) samples and skeletonisation/dry samples (E115 to E155) forming separate clusters.

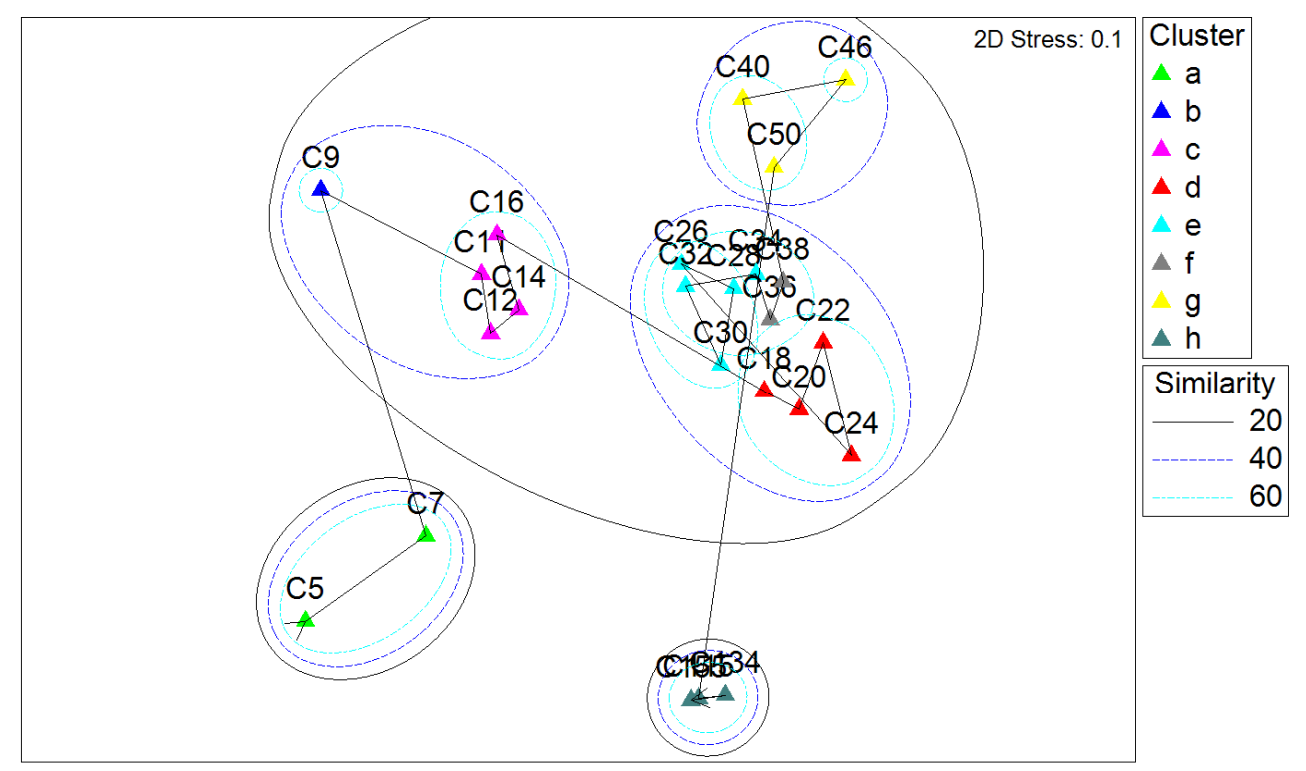

Figure 4.19 MDS analysis plot of T-RFLP data from sand collected from under carcass $C$. The Bray-Curtis similarity index was used on square root transformed data. Eight statistically significant clusters were distinguished using CLUSTER. The Bray-Curtis similarity index was used on square root transformed data. Samples are denoted by the number of calendar days post-death.

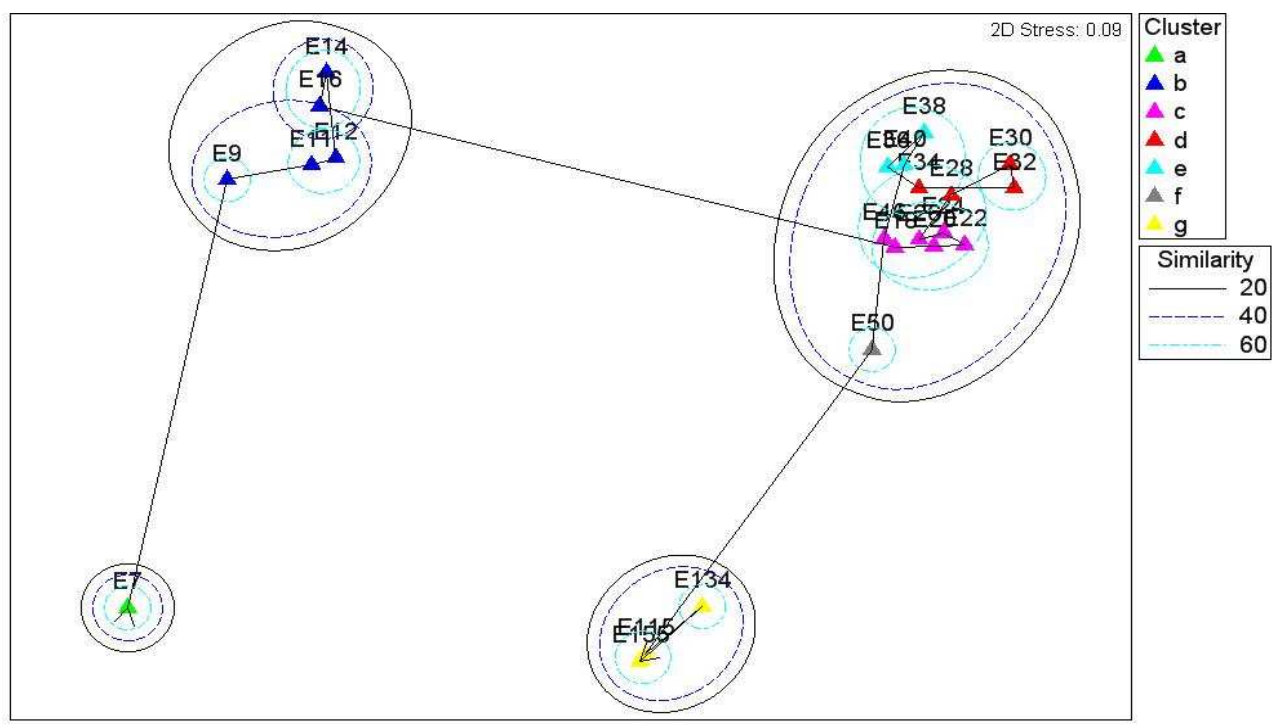

Figure 4.20 MDS analysis plot of T-RFLP data from sand collected from under carcass $\boldsymbol{E}$. No DNA could be extracted from sample E5, so this is not plotted. Seven statistically significant clusters were distinguished using CLUSTER. The Bray-Curtis similarity index was used on square root transformed data. Samples are denoted by the number of calendar days post-death. 
When samples from the three carcasses that were colonised by insects are plotted together using MDS (Figure 4.21), four distinct clusters are evident and these are separated at the $20 \%$ similarity level. The fresh/bloat stage samples for all three carcasses (A5, A7, E7, C5, C7) cluster together, as do many of the early active decay samples for carcasses C and E (E9, E11, E14, E16, C11, C20). The carcass A samples from the early active decay stage do not fall within the same cluster as the early active decay samples from carcasses $\mathrm{C}$ and $\mathrm{E}$, but cluster with the later decay stage samples from those carcasses. The carcass A samples from A18 onwards cluster predominantly with the skeletonisation/dry stage samples from carcasses $\mathrm{C}$ and E. This suggests that the bacterial community associated with carcass A underwent a composition shift during the decay stage, whereas those from carcasses $\mathrm{C}$ and $\mathrm{E}$ did not undergo this shift until the carcasses reached skeletonisation. This could be because carcass A was exposed to slightly more direct sunlight and was slightly warmer than the other carcasses, even though the ADD calculations themselves were not affected by these increases.

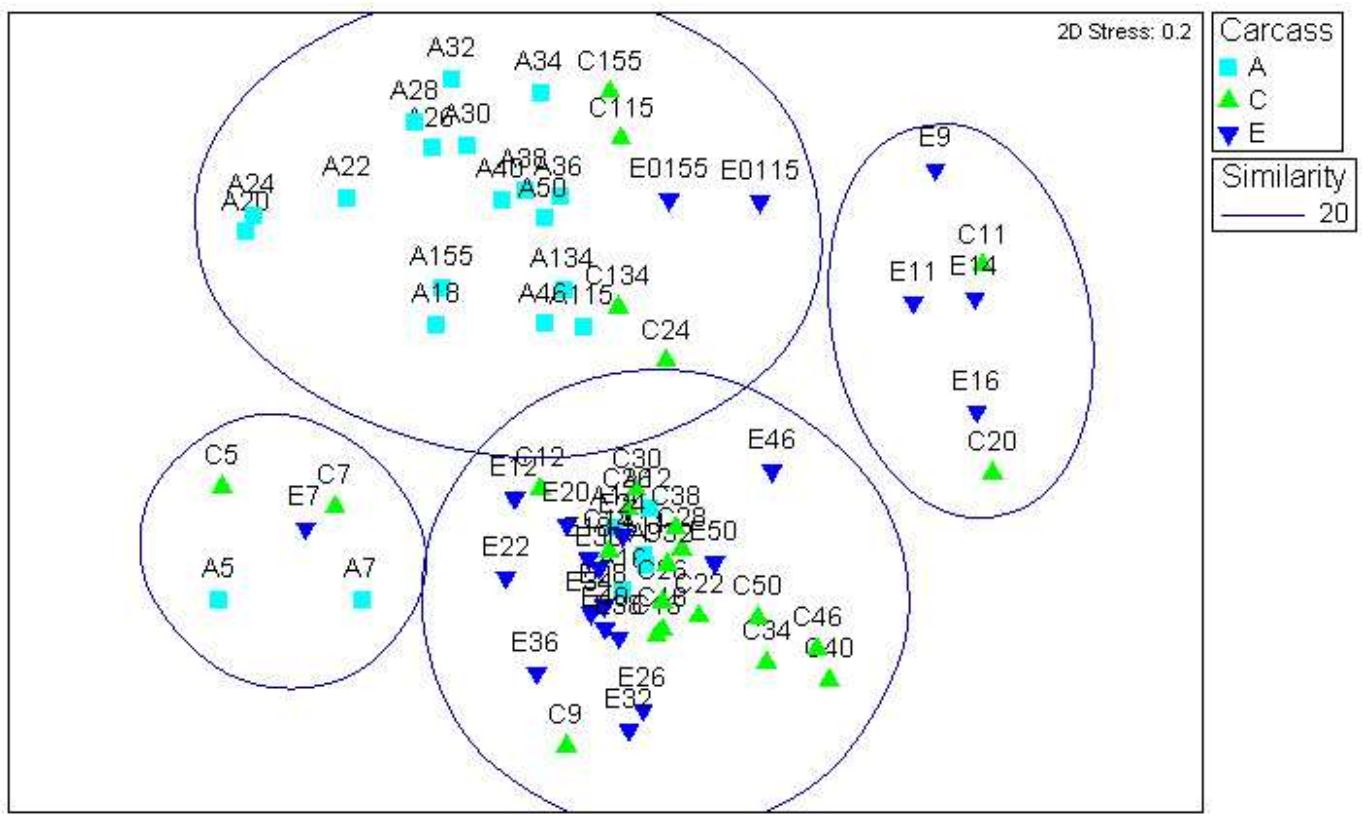

Figure 4.21 MDS plot of T-RFLP data from sand collected from under the three carcass allowed to be colonised by insects (A, $\boldsymbol{C}$ and $\boldsymbol{E})$. The Bray-Curtis similarity index was used on square root transformed data. Samples are denoted by the number of calendar days post-death. 
Figures 4.22, 4.23 and 4.24 show individual MDS plots of sample profiles for each of the non-colonised carcasses, B, D and F. As for the insect colonised carcasses, those not colonised also show separation of the fresh/bloat stage samples (B7, D7, F5, F7) from the later samples. All three also show separation of the skeletonisation/dry samples and some separation between early decay and later decay, although the time-point at which this separation occurs differs between the carcasses.

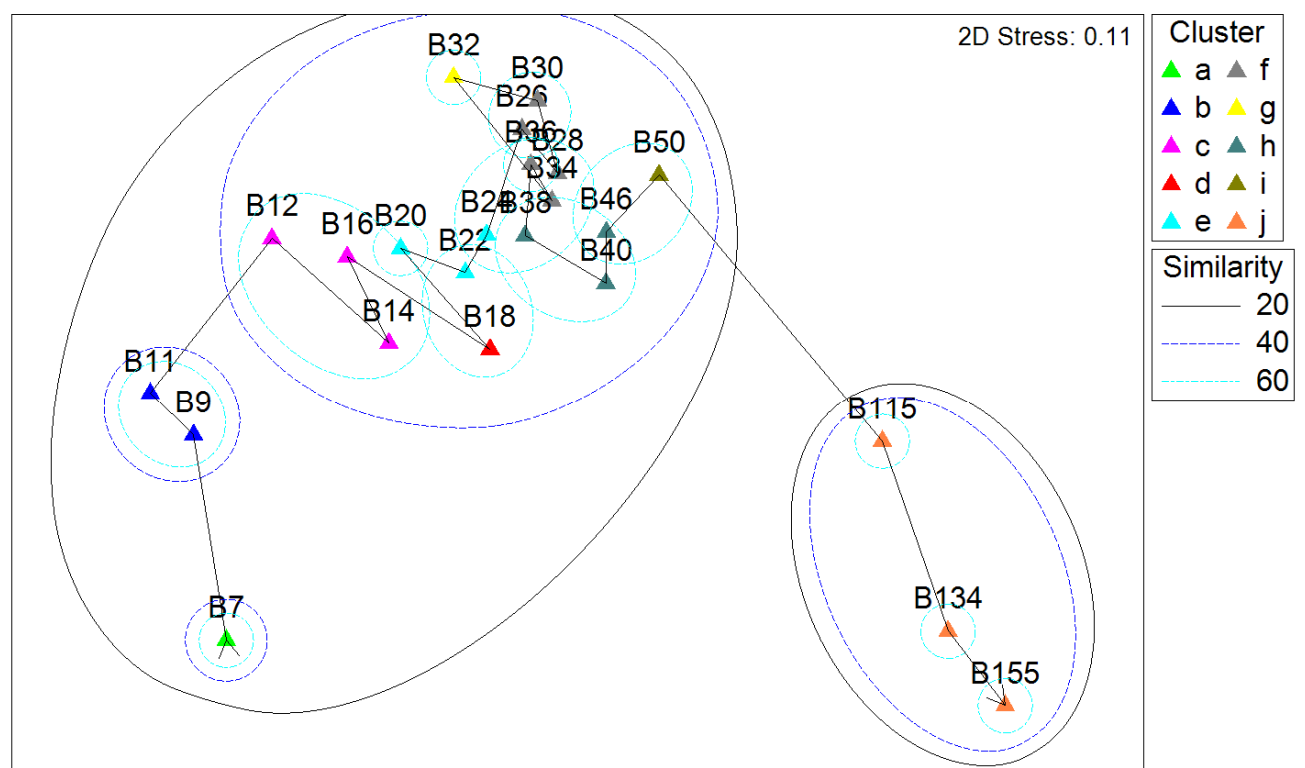

Figure 4.22 MDS plot of T-RFLP data from sand collected from under carcass B. The Bray-Curtis similarity index was used on square root transformed data. Ten statistically significant clusters were distinguished using CLUSTER. The Bray-Curtis similarity index was used on square root transformed data. Samples are denoted by the number of calendar days post-death. 


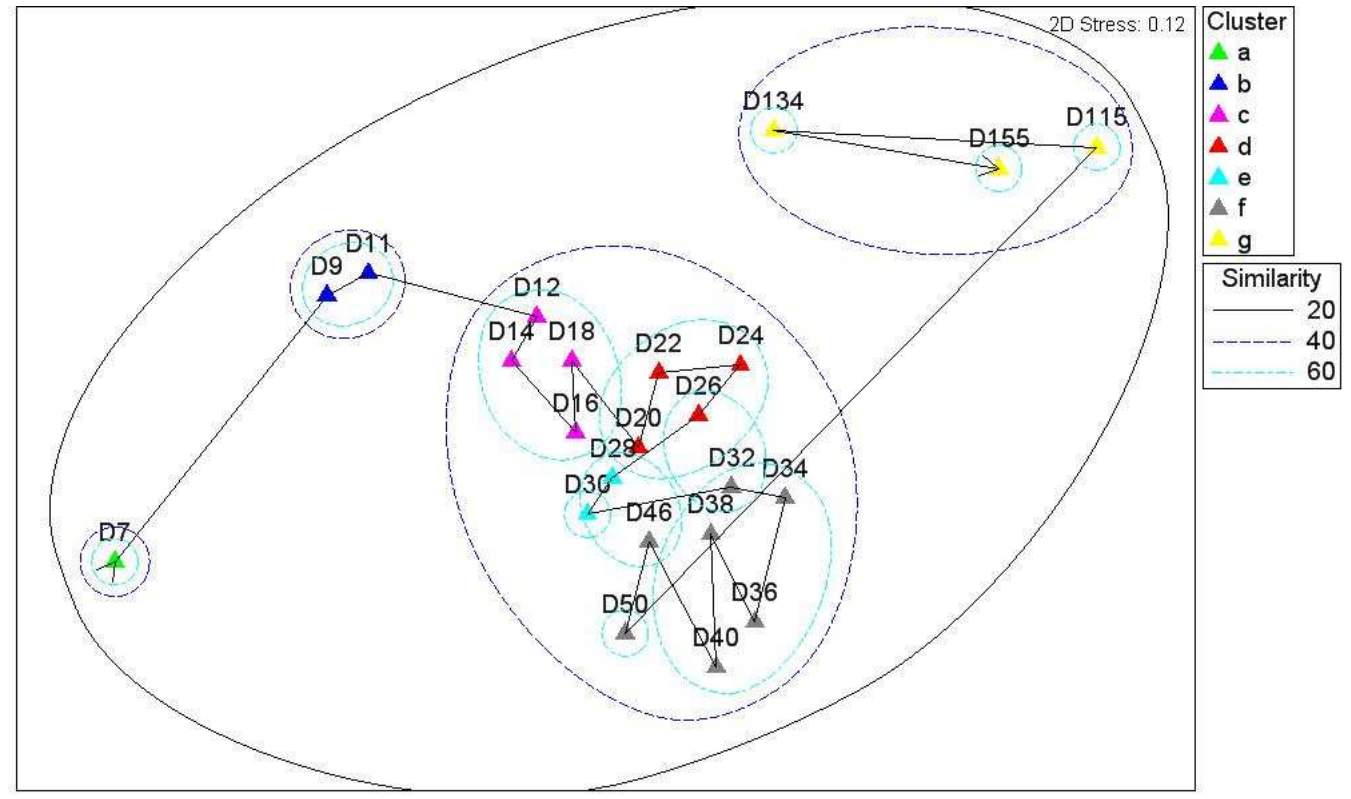

Figure 4.23 MDS plot of T-RFLP data from sand collected from under carcass D. The Bray-Curtis similarity index was used on square root transformed data. Seven statistically significant clusters were distinguished using CLUSTER. The Bray-Curtis similarity index was used on square root transformed data.

Samples are denoted by the number of calendar days post-death.

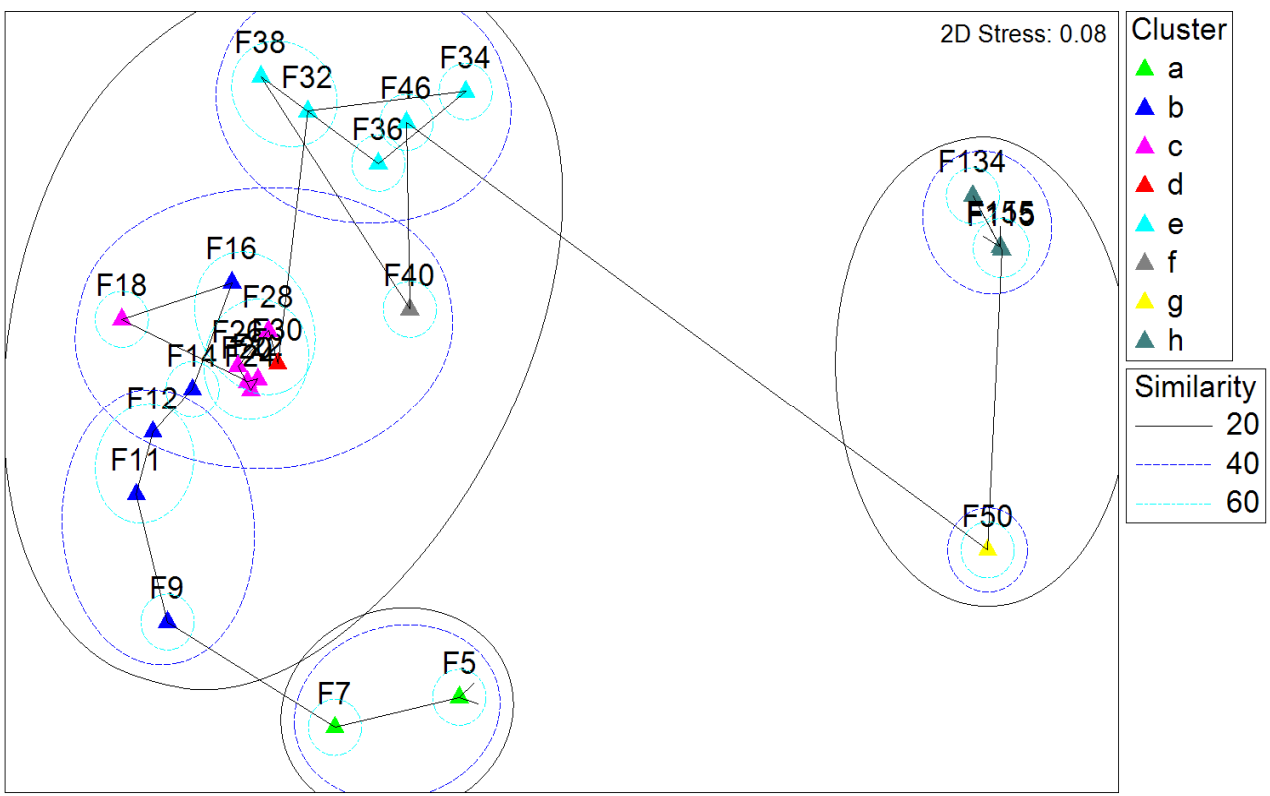

Figure 4.24 MDS plot of T-RFLP data from sand collected from under carcass $\boldsymbol{F}$. The Bray-Curtis similarity index was used on square root transformed data. Eight statistically significant clusters were distinguished using CLUSTER. The Bray-Curtis similarity index was used on square root transformed data. Samples are denoted by the number of calendar days post-death. 
When all three non-colonised carcasses are plotted together using MDS (Figure 4.25), three clusters are evident at the $20 \%$ similarity threshold. The fresh/bloat samples cluster away from the later samples, but the skeletonisation/dry samples do not cluster separately, grouping instead with the early decay stage samples.

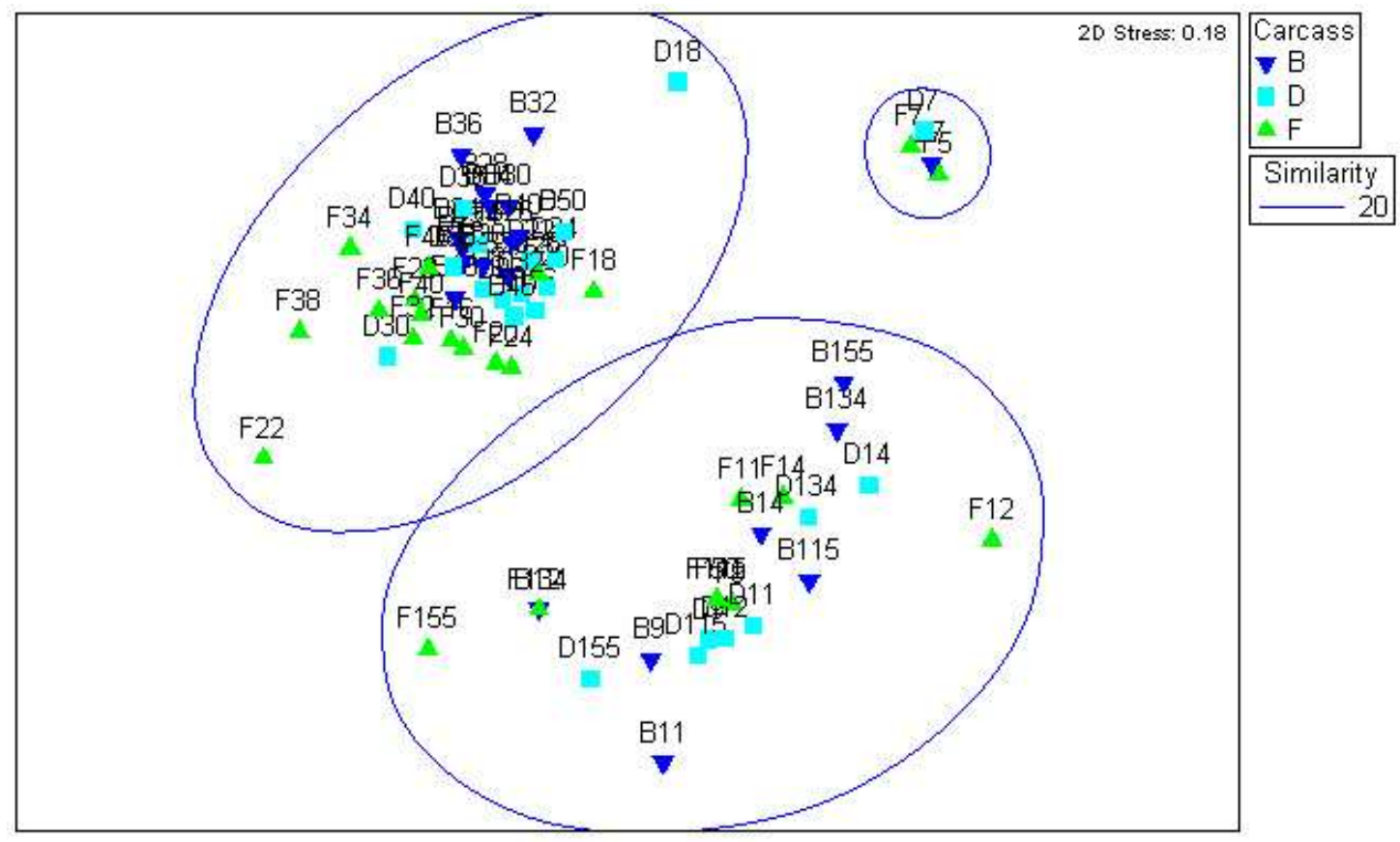

Figure 4.25 MDS plot of T-RFLP data from sand collected from under the three carcasses which were not allowed to be colonised by insects (B, D and F). The Bray-Curtis similarity index was used on square root transformed data. Samples are denoted by the number of calendar days post-death.

The MDS analysis with all six datasets plotted (Figure 4.26) shows a separation between the samples from carcasses that were colonised by insects and those that were not, suggesting that the composition of the bacterial community associated with a decomposing pig carcass is affected by insect colonisation. The samples from the fresh/bloat stage of all six carcasses cluster reasonably closely, with the colonised carcass samples then displaying more variability, clustering more loosely than the non-colonised samples, which cluster near to the fresh/bloat stage samples. 


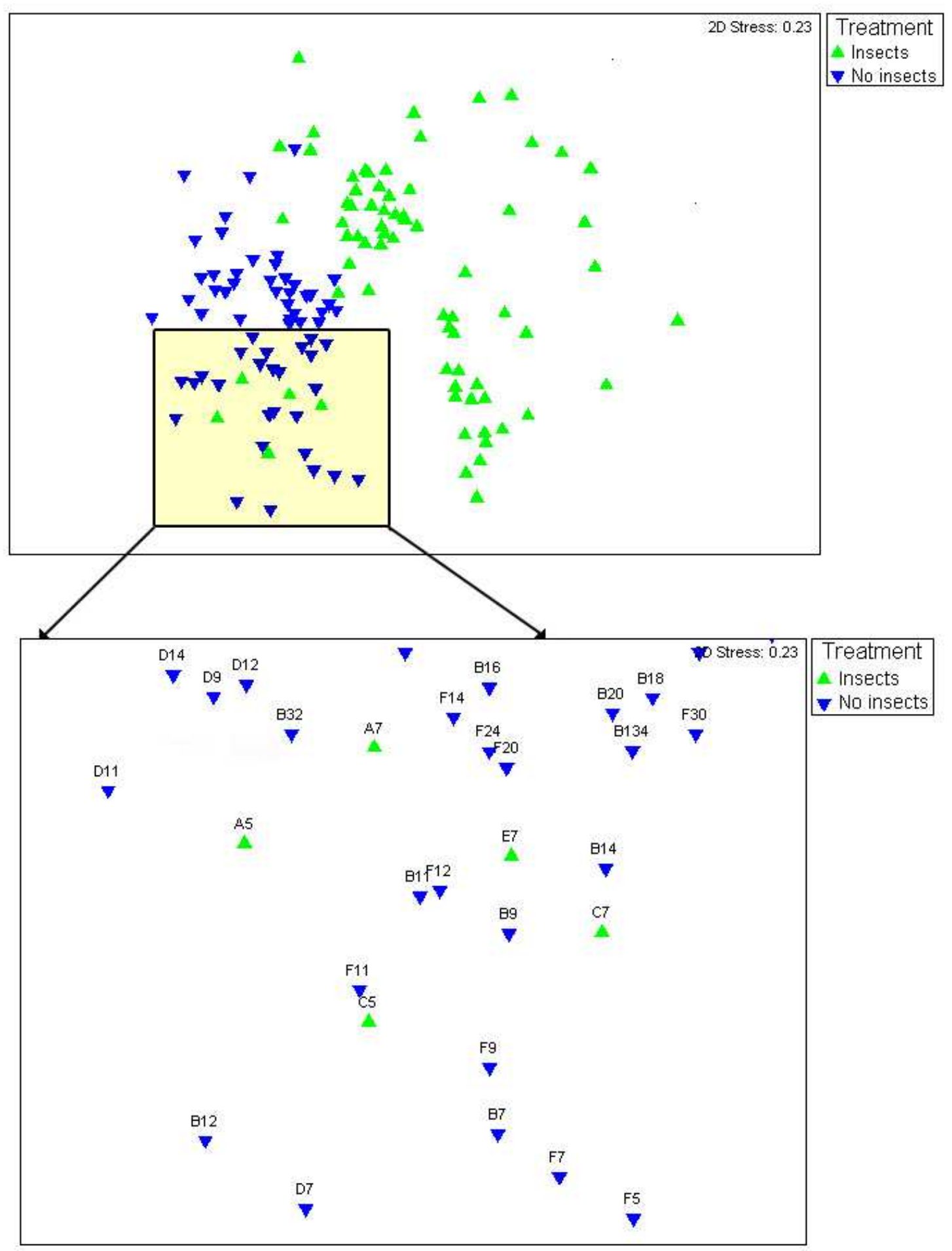

Figure 4.26 MDS plot of T-RFLP data from sand from under all six carcasses. The Bray-Curtis similarity index was used on square root transformed data. The enlargement shows samples from early on in decomposition. Samples are denoted by the number of calendar days post-death. 


\subsubsection{RELATE analysis of T-RFLP-derived similarity matrices}

The similarity matrices which underlie the MDS analyses for each carcass were compared using RELATE to determine how similar the T-RFLP MDS patterns were. Most of the six matrices showed a degree of similarity to each other (with $\rho$ values ranging from 0.273 to 0.787 and $\mathrm{p}<0.05$ ), with the exception of matrices $A$ and $F$ which showed no similarity ( $\rho$ $=0.06, \mathrm{p}=0.297)$. This means that there was a similar pattern of change under all six carcasses over time and that the changes were not random fluctuations. It did not appear that the MDS patterns for the carcasses held under the same conditions (i.e. insects or no insects) were any more similar to each other than to the patterns of the other treatment. This suggests that, while the make-up of the bacterial communities may have differed between the treatments, the pattern of change was similar for all decomposition events.

\subsubsection{T-RFLP Similarity Index Data Analysis}

The similarity between T-RFLP profiles was determined using the Bray Curtis Similarity Index (section 3.4.5.2). The mean similarity between profiles for consecutively collected samples for each carcass is shown in Table 4.4.

\begin{tabular}{|l|l|}
\hline$A$ & $0.63 \pm 0.24$ \\
\hline$B$ & $0.49 \pm 0.19$ \\
\hline$C$ & $0.49 \pm 0.31$ \\
\hline$D$ & $0.57 \pm 0.24$ \\
\hline$E$ & $0.51 \pm 0.27$ \\
\hline$F$ & $0.44 \pm 0.23$ \\
\hline
\end{tabular}

Table 4.4 Mean similarity between consecutive profiles for each decomposition plot.

The profiles from the replicate carcasses (colonised or non-colonised) were compared against each other using the CAI, and then compared against the other treatment. The difference between the colonised carcasses and the non-colonised carcass samples was significant at the $95 \%$ confidence level $(\mathrm{p}=0.018)$, with the colonised carcasses showing a higher mean 
similarity. The range however, was very similar for each group. The between treatment comparisons were also significantly lower than the same treatment replicate comparisons $(\mathrm{p}<0.001)$, confirming that the replicates were more similar to each other than to the nonreplicates, and that the colonised and non-colonised groups are significantly different.

If the treatments were compared at the different stages of decomposition: fresh/bloat (ADD 76 to 114), active decay (ADD 148 to 293), post decay (ADD 327 to 759) and skeletonisation/dry (ADD 1440 to 1791) it appears that there was no significant difference between the colonised and non-colonised carcasses at the fresh/bloat stage $(p=0.26)$, but that they are significantly different at each of the subsequent stages at the $95 \%$ confidence level.

\subsubsection{DGGE}

Seventeen selected samples from under each carcass were run on DGGE gels as described in section 3.4.4. Initial DGGE gels had a problem with red smears (see Figure 4.27) so DGGE was repeated with the mung bean nuclease treatment step included (section 3.4.4.1). The samples chosen spanned the entire decomposition period. The DGGE gels of samples from carcasses A and B are shown in Figures 4.28 and 4.29 respectively. The profiles obtained on each gel were very high quality with a mean of 32 bands per profile (Table 4.5).

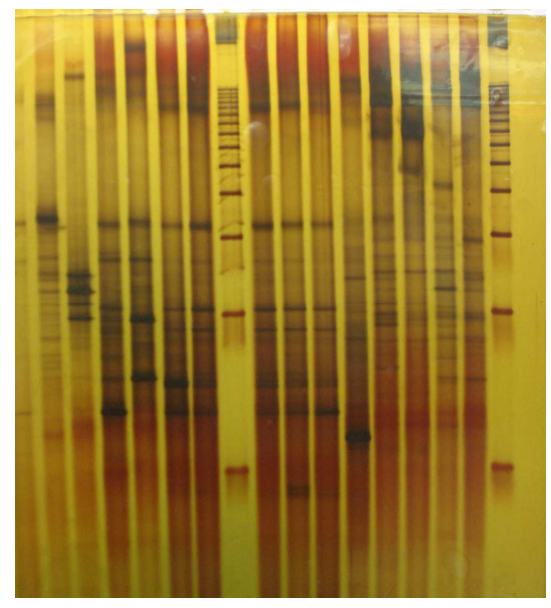

Figure 4.27 Red ssDNA smears on a DGGE gel. 
Figure 4.28 Photograph of DGGE gel analysis of bacterial communities from selected sand samples collected under carcass $A$. This carcass was allowed to be colonised by insects.
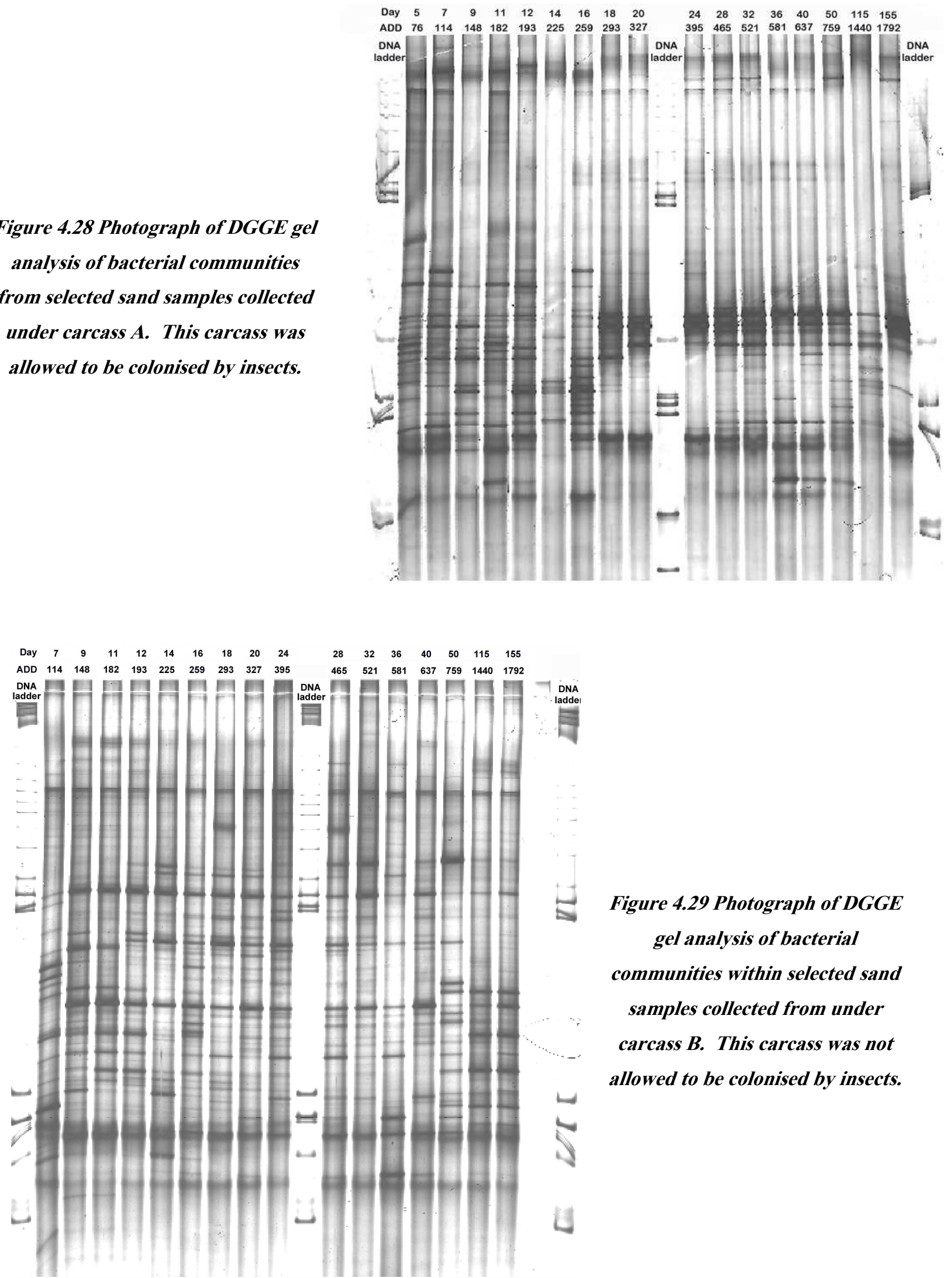

Figure 4.29 Photograph of DGGE gel analysis of bacterial communities within selected sand samples collected from under carcass $B$. This carcass was not allowed to be colonised by insects. 


\begin{tabular}{|c|c|c|c|c|c|c|}
\hline & \multicolumn{3}{|c|}{$\begin{array}{c}\text { Colonised by } \\
\text { insects }\end{array}$} & \multicolumn{3}{c|}{$\begin{array}{c}\text { Not colonised by } \\
\text { insects }\end{array}$} \\
\hline & A & C & E & B & D & F \\
\hline Mean number of bands in each profile & 32 & 31 & 27 & 46 & 22 & 31 \\
\cline { 2 - 8 } Total number of different bands per gel & 53 & 75 & 61 & 56 & 56 & 54 \\
\hline
\end{tabular}

Table 4.5 Number of bands present in profiles from each carcass.

It was noted during collation of the data, that there was considerable variation in band migration between the six gels despite considerable care and effort being applied to align the gels using the marker lanes run in triplicate on every gel. This is widely recognised as a methodological limitation of DGGE (Nakatsu, 2007). Reproducing gel gradients can be very difficult given that they are cast by hand, and although the Bionumerics software allows for adjustments and modifications of the gel photographs based on the running of the marker samples (section 3.4.4.2), it is difficult to ensure that localised areas of the gel have not been affected by uneven gradient formation. For this reason, comparisons between gels have not been made for this experiment. Data from the gels were, however, used for MDS analysis (section 4.3.5.1) to demonstrate the effect of the inter-gel variability on the results.

\subsubsection{Multi-Dimensional Scaling Analysis}

Figures 4.30 and 4.31 show the DGGE data from carcasses A and B plotted using MDS. At the $40 \%$ similarity level, the early carcass A samples form six clusters. This is a different clustering pattern to that found using the T-RFLP data (Figure 4.18), where the fresh/bloat samples A5 and A7 were separated from the decay samples (A9 onwards). The skeletonisation/dry samples also cluster separately using DGGE, whereas this was not the case for T-RFLP. The carcass B samples also form six clusters at the 40\% similarity level (4.31), and the DGGE and T-RFLP data show more similar clustering patterns for carcass B than they did for carcass A. When compared to their respective T-RFLP patterns using RELATE, $\rho$ 
values of $0.381(p<0.001)$ and $0.51(p<0.001)$ were found. This suggests that there was some similarity in the sequence of community change detected using both molecular profiling methods when using these sample sets.

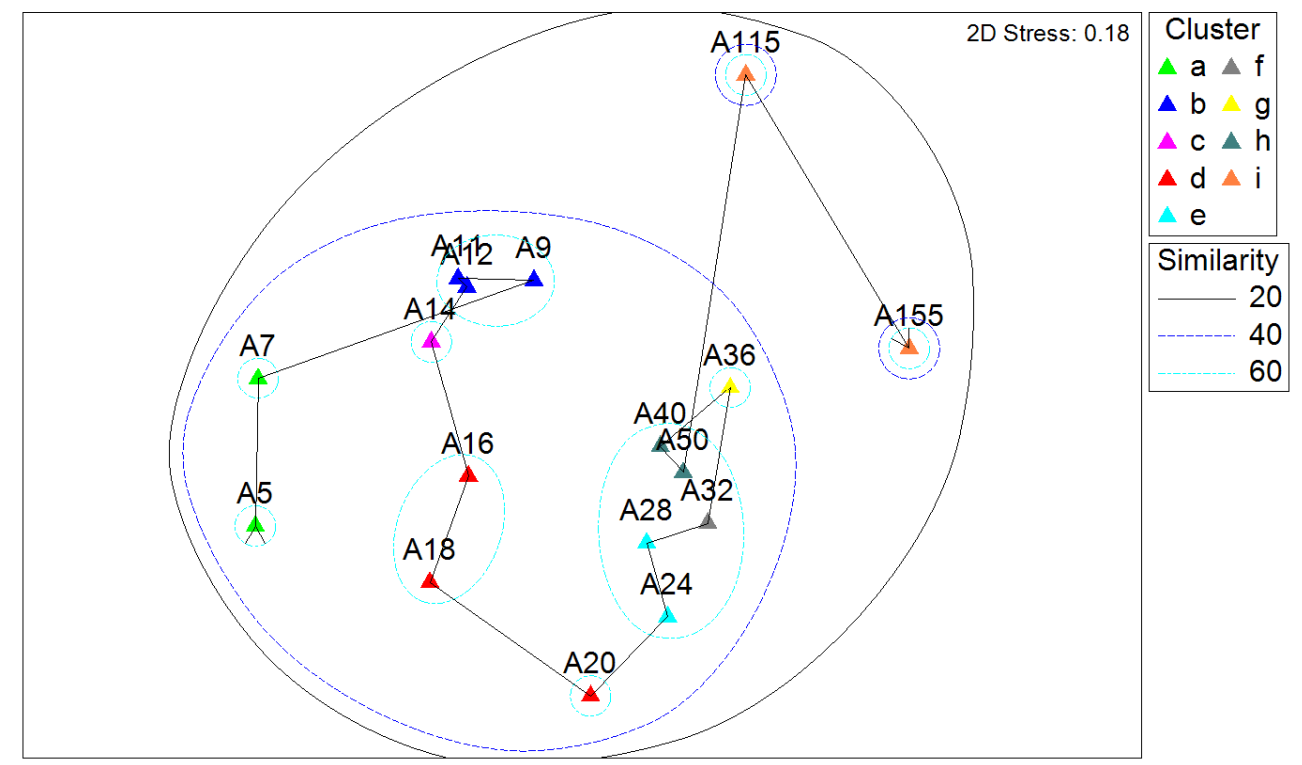

Figure 4.30 MDS plot of DGGE data from sand samples collected from under carcass $A$. This carcass was allowed to be colonised by insects. Nine statistically significant clusters were distinguished using CLUSTER. The Bray-Curtis similarity index was used on square root transformed data. Samples are denoted by the number of calendar days post-death.

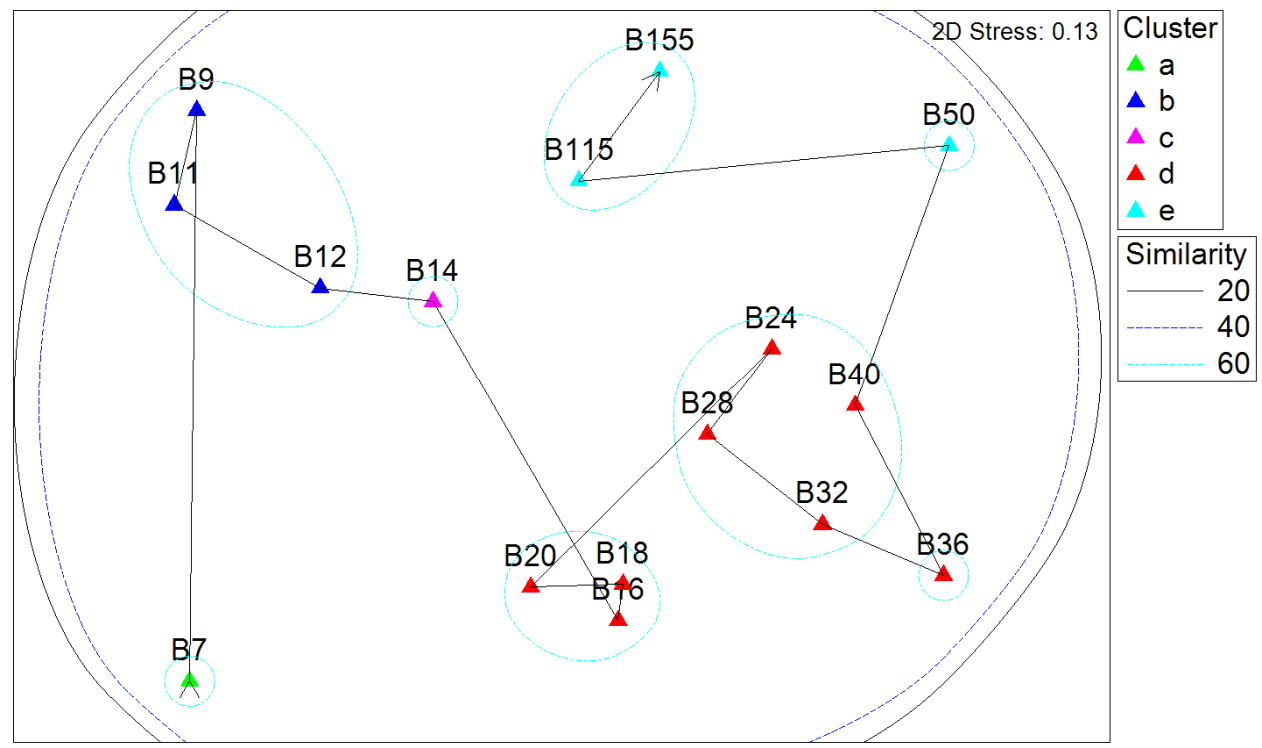

Figure 4.31 MDS plot of DGGE data from sand samples collected from under carcass B. This carcass was not allowed to be colonised by insects. Five statistically significant clusters were distinguished using CLUSTER. The Bray-Curtis similarity index was used on square root transformed data. Samples are denoted by the number of calendar days post-death. 
Figures 4.32 and 4.33 show the DGGE data from carcasses C and D plotted using MDS. The carcass D samples (Figure 4.33) are quite spread out with no tight clustering of samples and more of a step-wise pattern of change than was demonstrated in the T-RFLP MDS for these samples. When compared to their respective T-RFLP patterns using RELATE, $\rho$ values of $0.36(p<0.01)$ and $0.726(p<0.001)$ were found, suggesting that the two molecular profiling methods (T-RFLP and DGGE) detected similar patterns of change.

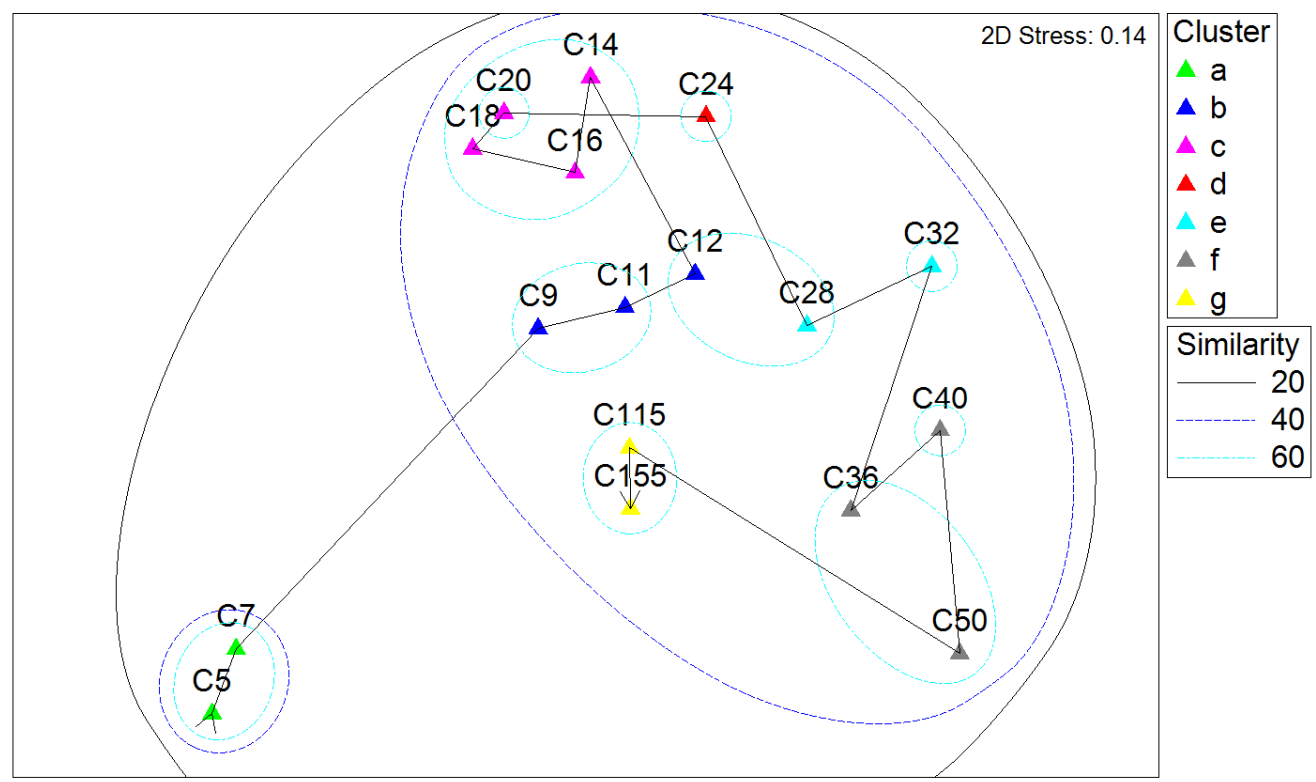

Figure 4.32 MDS plot of DGGE data from sand samples collected from under carcass C. This carcass was allowed to be colonised by insects. Seven statistically significant clusters were distinguished using CLUSTER. The Bray-Curtis similarity index was used on square root transformed data. Samples are denoted by the number of calendar days post-death. 


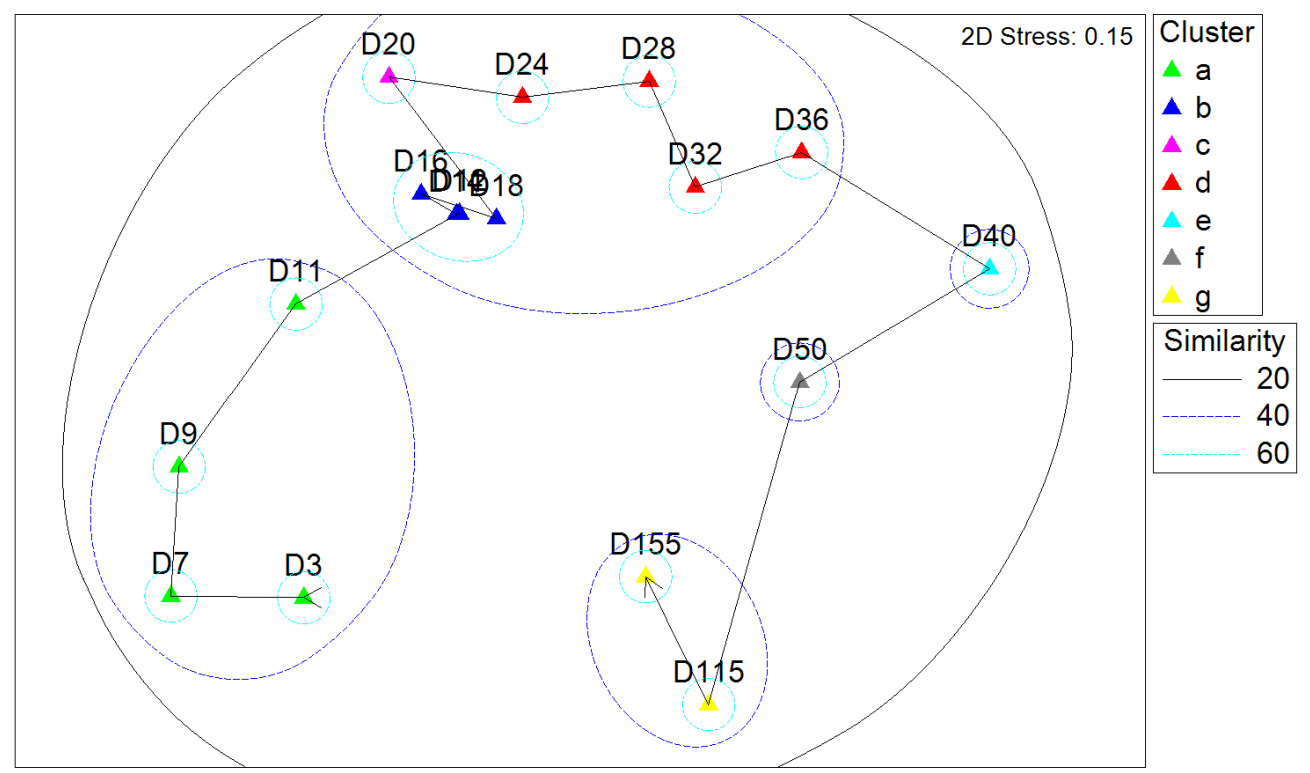

Figure 4.33 MDS plot of DGGE data from sand samples collected from under carcass D. This carcass was not allowed to be colonised by insects. Seven statistically significant clusters were distinguished using CLUSTER. The Bray-Curtis similarity index was used on square root transformed data. Samples are denoted by the number of calendar days post-death.

Figures 4.34 and 4.35 show the DGGE data from carcasses E and F plotted using MDS. Sample F155 plotted some distance from the other samples (Figure 4.35). This sample did not produce a good DGGE profile due to having very faint bands in comparison to the other profiles on the gel. It is possible that slightly less DNA was loaded on to the gel due to an error in DNA quantification or a gel loading error. When compared to their respective TRFLP patterns using RELATE, $\rho$ values of $0.338(p<0.01)$ and $0.632(p<0.001)$. 


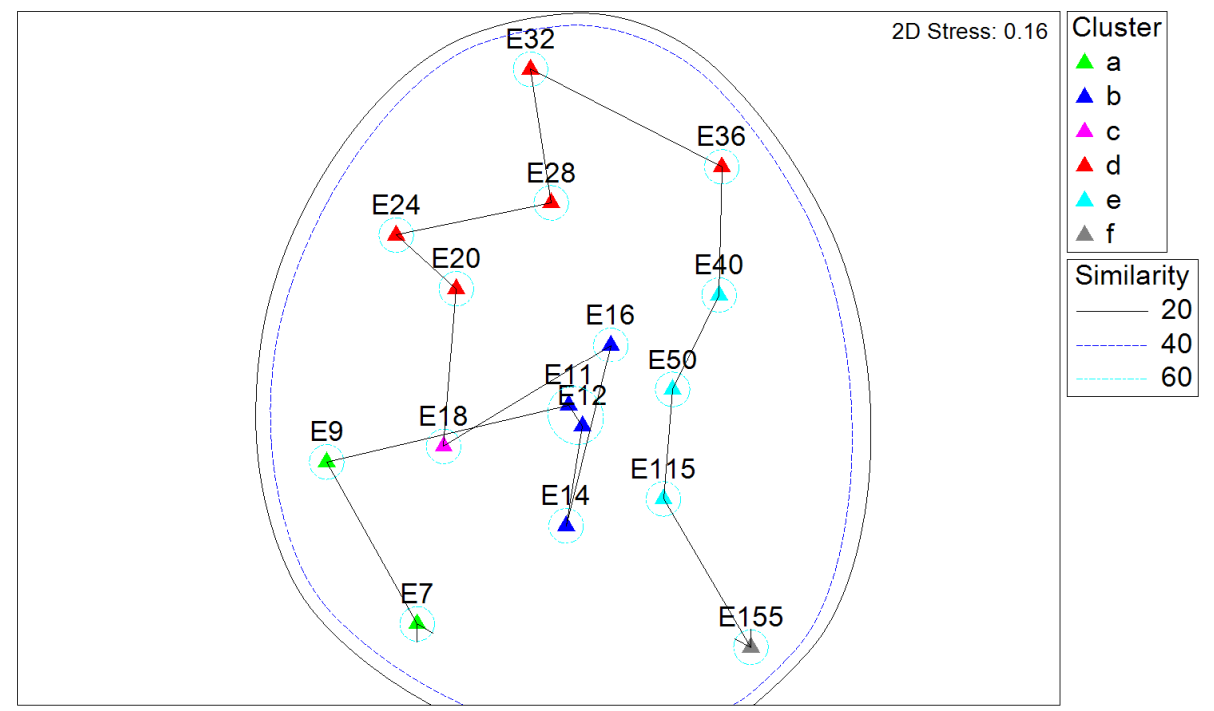

Figure 4.34 MDS plot of DGGE data from sand samples collected from under carcass $\boldsymbol{E}$. This carcass was allowed to be colonised by insects. Six statistically significant clusters were distinguished using CLUSTER. The Bray-Curtis similarity index was used on square root transformed data. Samples are denoted by the number of calendar days post-death.

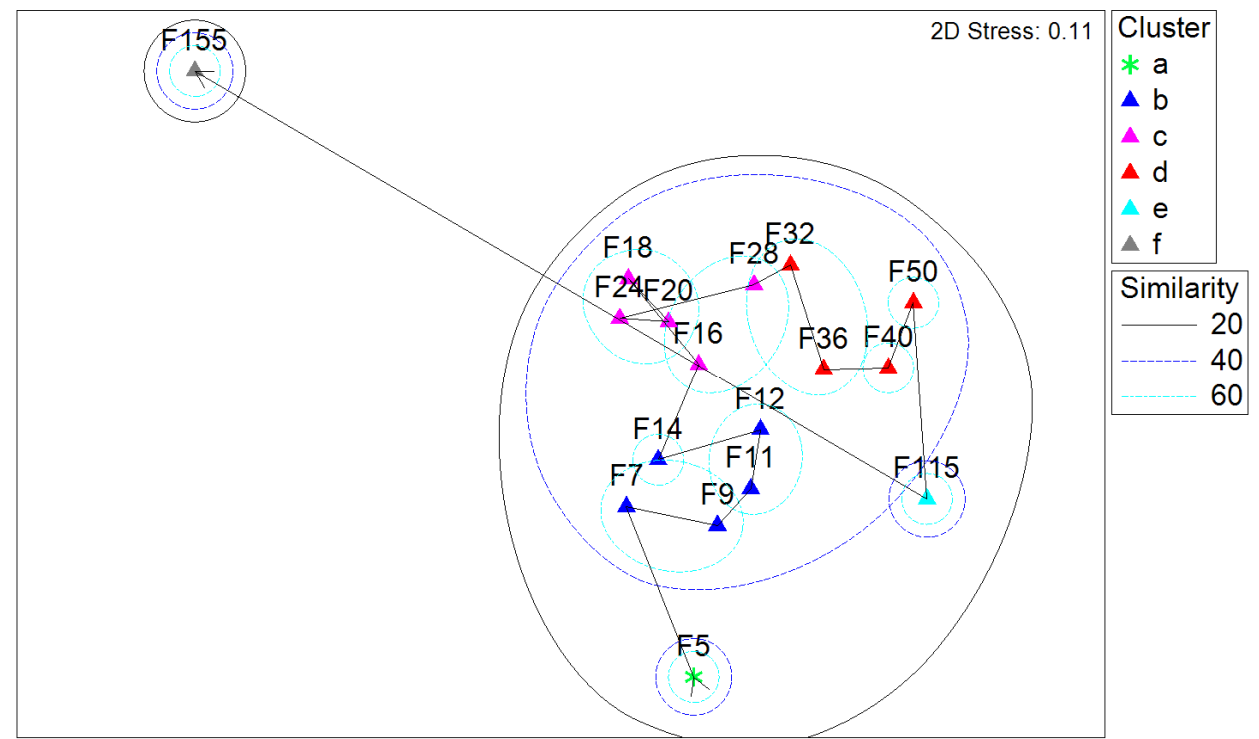

Figure 4.35 MDS plot of DGGE data from sand samples collected from under carcass $\boldsymbol{F}$. This carcass was not allowed to be colonised by insects. Six statistically significant clusters were distinguished using CLUSTER. The Bray-Curtis similarity index was used on square root transformed data. Samples are denoted by the number of calendar days post-death.

When the DGGE sample data from the replicate carcasses for each treatment (colonised or non-colonised) are plotted together using MDS (Figures 4.36 and 4.37), samples tend to group according to the carcass they originated from. This is in contrast to the MDS plots of 
the T-RFLP data for the same samples (Figures 4.21 and 4.25), where samples tended to group according to sampling date. This is likely to be caused by variation between the gels affecting the migration distance of bands on individual gels, as discussed in section 4.3.5.

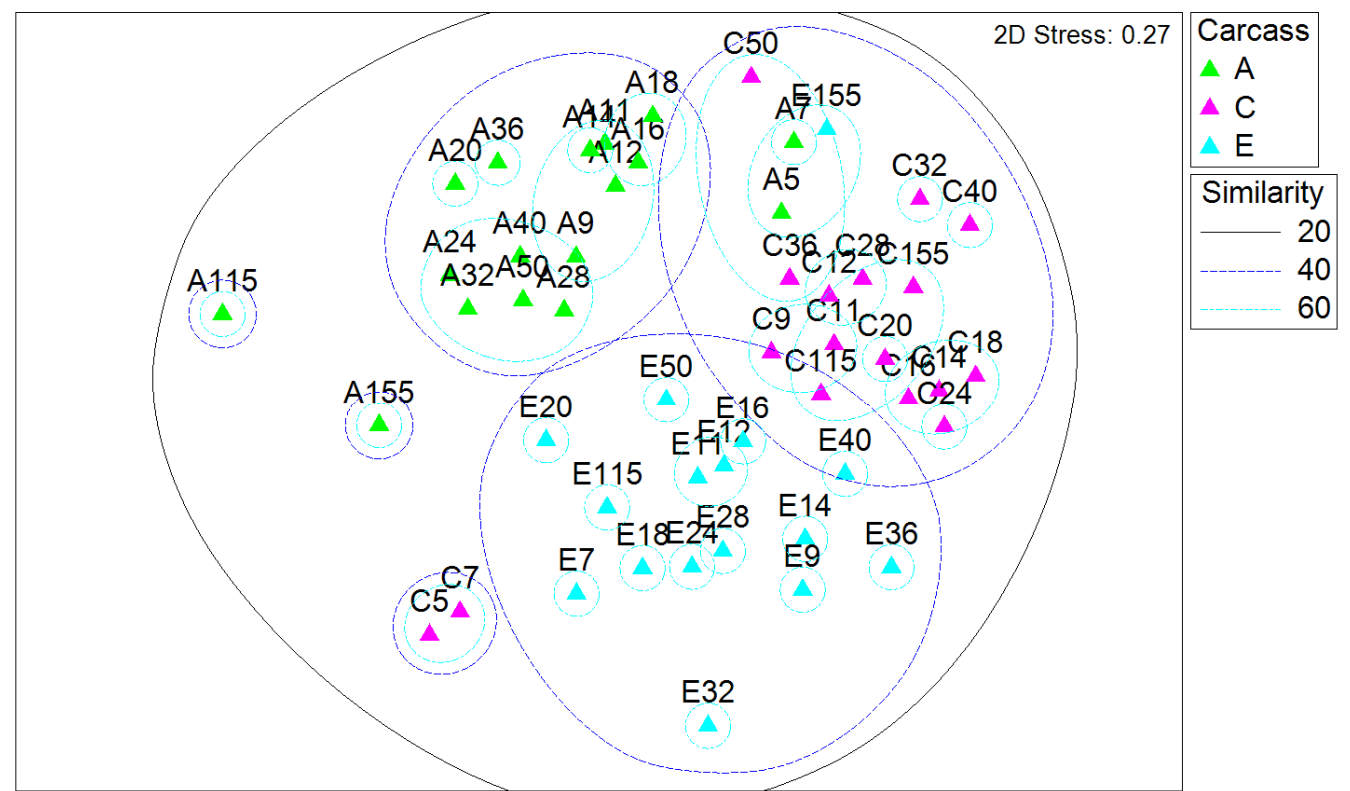

Figure 4.36 MDS plot of DGGE data from sand samples collected from under the three carcasses that were allowed to be colonised by insects ( $A, C$ and $E$ ).

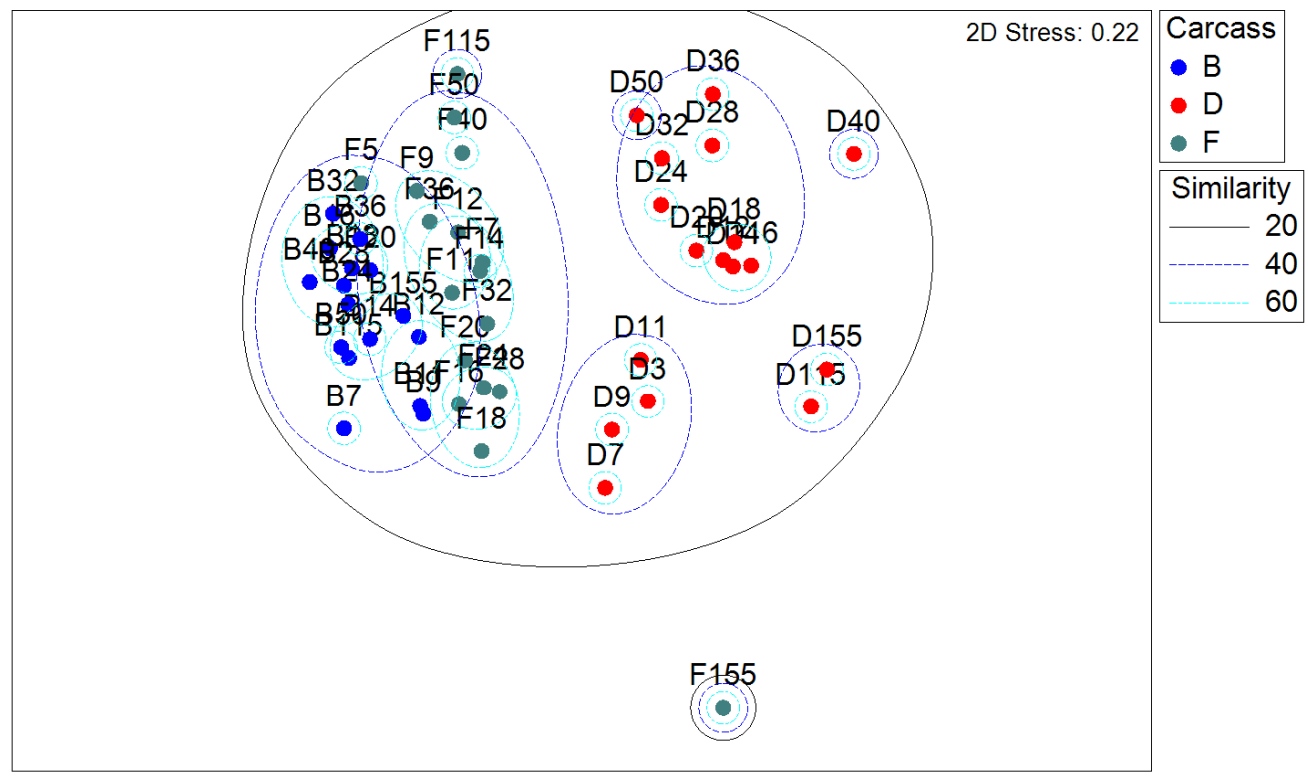

Figure 4.37 MDS plot of DGGE data from sand samples collected from under the three carcasses that were not allowed to be colonised by insects ( $B, D$ and $F$ ). 
When the DGGE data from all six carcasses are plotted together (Figures 4.38) the carcass profile sets tend to cluster together. Carcass D samples were much less tightly clustered and these plotted away from the other carcass samples. This, again, is likely to be caused by the inability to standardise migration distances between gels and so should not be over interpreted.

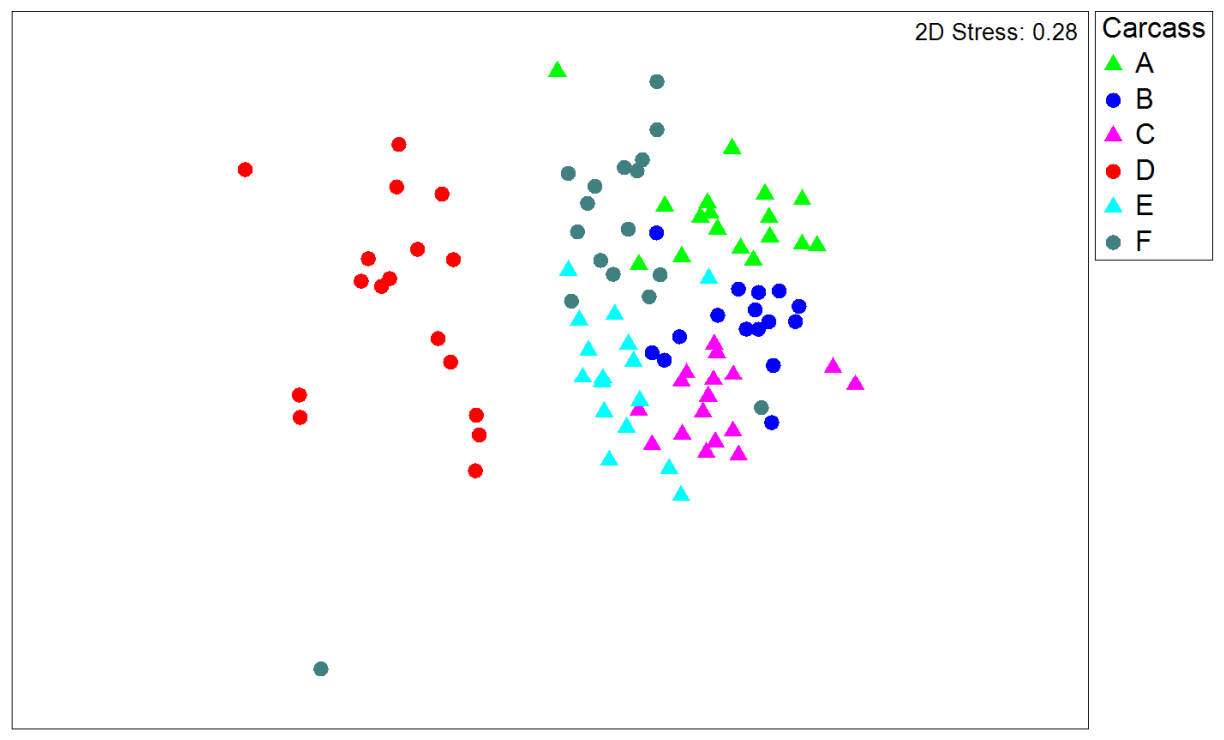

Figure 4.38 MDS plot of DGGE data from sand collected from under all six carcasses. The carcasses with insect colonisation are represented by triangles and those without are represented by circles.

\subsubsection{RELATE analysis of DGGE-derived similarity matrices}

The similarity matrices which underlie the MDS analyses for each carcass were compared using RELATE to determine how similar the MDS patterns were. All six matrices showed a degree of similarity to each other with $\rho$ values ranging from 0.252 to 0.554 . The results were all significant, with $p<0.05$. This means that there was a similar pattern of change under all six carcasses over time and that the changes were not random fluctuations. It did not appear that the MDS patterns for the carcasses held under the same conditions (i.e. insects or no insects) were any more similar to each other than to the patterns of the other treatment, suggesting that while the make-up of the bacterial communities may have differed between the treatments, the pattern of change was similar for all decomposition events. 


\subsection{Discussion}

The first pig experiment proved very useful for examining the decomposition process, for determining the most important time points for sampling, and for fine-tuning the experimental set-up. The experimental set-up worked well, with the cages designed to prevent insect colonisation working effectively for two of the carcasses, and colonisation of the other carcass occurring only at the very end of the experiment. Collecting the samples and taking photographs inside the fine netting without admitting the very persistent insects proved challenging, but was successfully achieved. The cages designed to prevent disturbance by scavengers also worked well, with none of the carcasses being disturbed. The orientation of the cages could have been planned better to prevent the cages receiving differing levels of sun exposure. At the time, the main concern was heavy rainfall events, but these did not eventuate and the sand trays did not collect much rainfall.

Using both T-RFLP and DGGE for examining the change in the bacterial communities over time allowed comparison of the pattern of change observed by the two methods. Unfortunately, the ability to compare DGGE data for samples from different carcasses was affected by inter-gel variation. This is a common problem with DGGE (Nakatsu, 2007), that can be ameliorated to some extent by running control samples and then adjusting the results using the Bionumerics software. However, it was evident that the DNA ladders used were not adequate for this purpose. This restricted the statistical comparisons that could be performed. Both profiling methods demonstrated that the bacterial communities derived from the sand under the decomposing carcasses clearly changed as decomposition progressed.

The T-RFLP clustering patterns seen (Figures 4.18 - 4.20 and $4.22-4.24$ ) suggest that both colonised and non-colonised carcass bacterial communities underwent an early compositional shift, at the onset of active decay, followed by a short period of less change, before another 
shift later on in active decay. From the clustering shown in 4.25 , it appears that the noncolonised carcass communities then underwent another shift when skeletonisation occurred, back to a composition more similar to the early decay community, which was somewhat unexpected. At this stage, the $\mathrm{pH}$ was still high, although the sand was visually drier looking, which is likely to influence which bacterial species can survive in the sand.

The T-RFLP MDS plots for the individual carcasses show some clustering of consecutive samples, with the fresh/bloat stage samples (days 5 and 7) consistently plotting away from later, active decay stage samples, suggesting a major bacterial community composition shift between days 7 and 9 (ADD 114 and 148). This change in community structure correlates closely with the first appearance of insect larvae on the colonised carcasses, and with an increase in the amount of fluid purging from the non-colonised carcasses. It is possible that during the fresh/bloat stage, the bacteria being detected were derived from the skin, or from the mouth/other orifices, and that once putrefaction began and gas build-up forced more fluid from the carcass, the gut microflora began to be released. It appears that a second community composition shift occurred around the day 16 or 18 (ADD 259/283) mark for the colonised carcasses. This roughly correlates with the beginning of insect larvae migration away to pupate, and when the sand began to dry. A third shift is evident for most of the carcasses as they became skeletonised (colonised) or mummified (non-colonised), somewhere between days 50 and 115 (ADD 758 and 1440). When the six T-RFLP datasets were plotted together using MDS, the treatments (colonised or not) cluster distinctly, with the exception of the fresh/bloat stage samples from the colonised carcasses, which fall within the non-colonised carcass samples cluster. This suggests that during the fresh/bloat stages, before insect larvae hatch, the carcasses produce similar bacterial communities, but once the insect larvae begin to decompose tissues of the colonised carcasses, the associated bacterial communities diverge and remain significantly different from those associated with the non-colonised carcasses 
throughout the remainder of the decomposition process. The samples from the non-colonised carcasses also cluster quite closely suggesting a steady, gradual community change, whereas those from the colonised carcasses display a greater spread, suggest overall more variability, with punctuated changes in the community over the course of decomposition. This fits in with the more punctuated physical changes occurring in the colonised carcass decomposition process compared with the slower, more gradual decomposition of the non-colonised carcasses.

The colonised carcasses had a number of different T-RFs or phylotypes (60) that were not detected at all for the non-colonised carcasses (Table 4.2), suggesting that some bacterial species may be derived from the colonising insects or the surrounding environment, although an almost equal number (58) were found exclusively in the non-colonised carcass profiles. This suggests that instead of the insects bringing novel bacterial species to the carcass, insect colonisation may simply change the decomposition environment in some way, leading to different carcass-derived bacterial species proliferating. Selected samples from this experiment were used to generate $16 \mathrm{~S}$ clone libraries for sequencing and identification (section 7.5) in an attempt to determine the likely origin of some of the phylotypes specific to either treatment. A number of phylotypes were also determined to be unique to each carcass (Table 4.2), which is likely to be caused by differences in the microflora associated with each pig. 


\section{Chapter 5: Pig Carcass Decomposition on Soil and Sand}

\subsection{Aim}

The aim of this experiment was to compare the bacterial communities associated with pig carcass decomposition on soil and sterile sand. Sand was used in this experiment to monitor only the bacterial community associated with the carcass itself, while soil was used to examine how the existing bacterial community responded to the carcass microflora and decomposition products.

\subsection{Methods}

The laboratory based methods used in this experiment are described in Chapter 3. The experimental set-up and sampling regime used specifically for this experiment are described in this chapter. This experiment was started 14 months after the first pig experiment (9 April 2006) and was run for 60 days (to 18 June 2006). This time period was deemed sufficient for the purpose of this experiment, as the colonised pig carcasses in the first experiment had reached the skeletonisation phase of decomposition by the 60 day point.

\subsubsection{Carcass Information}

Four pig carcasses were obtained from the same farm as in the previous experiment (section 4.2.1). The carcasses were slightly heavier than those used in the first experiment, which averaged $24.7 \mathrm{~kg}$ (see Table 5.1). The average weight for the carcasses in this experiment was $29.8 \mathrm{~kg}$. A control plot of soil or sand was also set up for each carcass (see Table 5.2). The carcasses were transported and washed as in the previous experiment (section 4.2.2). 


\begin{tabular}{|c|c|c|c|}
\hline Carcass & Weight (kg) & Treatment & Control name \\
\hline G & 26.0 & Soil & K \\
\hline H & 31.5 & Soil & L \\
\hline I & 30.8 & Sand & M \\
\hline J & 30.8 & Sand & N \\
\hline
\end{tabular}

Table 5.1 Carcass information.

\subsubsection{Experimental site}

The same experimental site was used as in the previous experiment (Chapter 4), and the same cages were used to prevent scavenger interference. The cages were, however, moved to the eastern end of the area near to the bush-line (Figure 5.1) to prevent the differing sunlight exposure between the carcasses that occurred in the previous experiment (section 4.3.2). The pig carcasses were placed in the four central cages, with the four control plots on the outside (Figure 5.2).

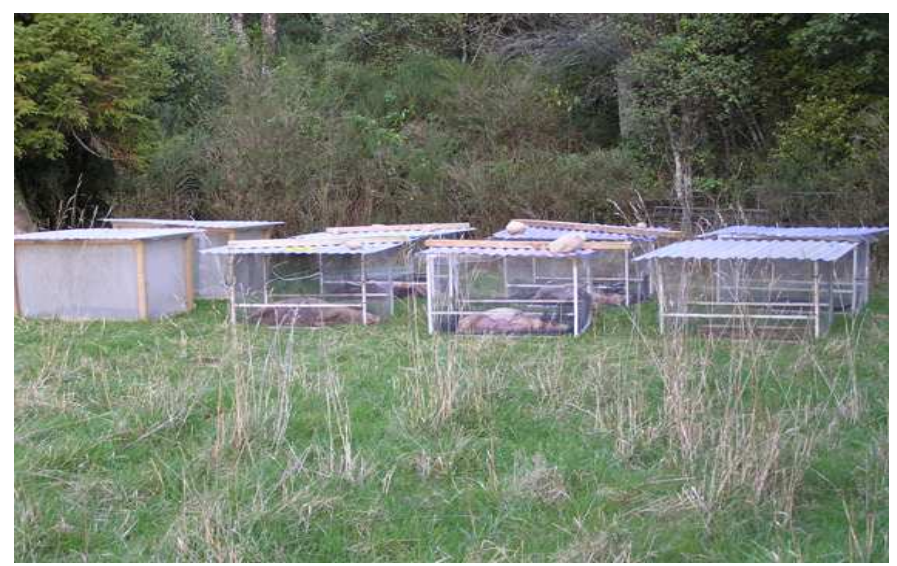

Figure 5.1 Carcasses in the cages at the eastern end of the site. 


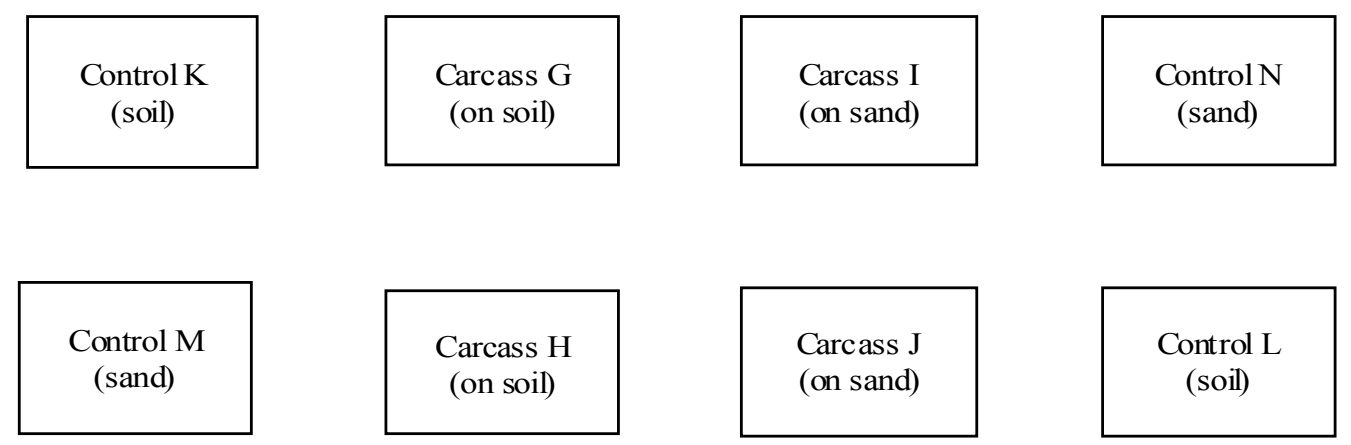

Figure 5.2 Layout of soil and sand plots.

Eight pits, each $40 \mathrm{~cm}$ x $60 \mathrm{~cm}$ x $20 \mathrm{~cm}$ deep were dug, with $50 \mathrm{~cm}$ between each. The soil was taken back to the lab and sieved through a $4 \mathrm{~mm}$ soil sieve and homogenised by mixing. Sieved soil was replaced in four of the pits three weeks prior to the start of the experiment. Sand was acid-washed in the same manner as in the previous experiment (section 4.2.2). Two $2 \mathrm{~cm}$ diameter drainage holes were drilled in the bottom of four plastic tubs used in the previous experiment, and these were placed in the remaining four pits, and filled with sand to a depth of $20 \mathrm{~cm}$ (Figure 5.3).
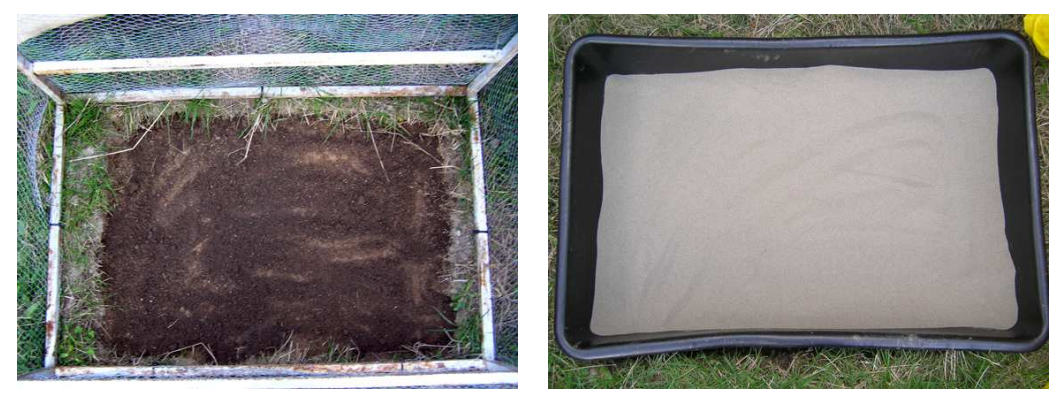

Figure 5.3 Soil pit and sand tray prior to carcass placement.

Insects were allowed to colonise all four carcasses. The controls had plastic bags containing $10 \mathrm{~kg}$ sand placed on the surface of the substrate to replicate the environmental conditions the weight of the pig carcass alone may produce. For example, the moisture loss from soil would be less in the shade of a sandbag than if direct sunlight could reach the soil surface, altering the bacterial community structure. The carcasses were placed on black plastic netting to allow them to be lifted for sampling, as was used in the previous experiment. 


\subsubsection{Sampling}

Samples were collected every two days for the first 40 days and then every 4 days until day 60 , using the same sampling method as in the previous experiment (section 4.2.3). At each sampling date, samples were collected from under the four carcasses ( $\mathrm{G}, \mathrm{H}, \mathrm{I}$ and $\mathrm{J})$ and from the four control plots $(\mathrm{K}, \mathrm{L}, \mathrm{M}$ and $\mathrm{N})$. Time zero control samples were collected prior to carcass placement.

Temperature and RH readings were collected hourly using a data-logger attached to one of the cages. Rainfall data collected at a weather station situated at the Seddon Rifle Range in Trentham were downloaded from the web after completion of the experiment (http://www.wmac.org.nz/trenthamweather). Due to a malfunction of the RH reader during this experiment (section 5.3.2), RH data was also subsequently downloaded from the Trentham weather station site. Single-use, temperature-only data-loggers (Tiny Tag SOLOS) were placed under each carcass and under the sand-bags of one soil control site $(\mathrm{K})$ and one sand control site (M). The data-logger casings were sealed closed using a silicone sealant to prevent rain or decomposition fluids from corroding the logger.

\subsubsection{Laboratory Methods}

The samples collected from this study were analysed for $\mathrm{pH}$; ammonium and nitrate concentration; urease, acid phosphatase, sulphatase, N-acetyl- $\beta$-D-glucosaminidase and glucopyranosidase enzyme activity; microbial biomass; and DNA was extracted for molecular analysis with T-RFLP, DGGE and sequencing technologies. 


\subsection{Results}

\subsubsection{Temperature, RH and Rainfall Data}

The period of time over which this experiment was run was slightly colder and much wetter than when the previous experiment was run. The mean daily air temperature was $10.5^{\circ} \mathrm{C}$ and the mean daily rainfall was $4.37 \mathrm{~mm}$ with four days that had over $40 \mathrm{~mm}$ rainfall. The total rainfall over the 60 day sampling period was $262 \mathrm{~mm}$. This was almost double the mean daily rainfall in the previous experiment $(2.81 \mathrm{~mm})$. The mean daily air temperature in the first pig experiment was $11.5^{\circ} \mathrm{C}$.

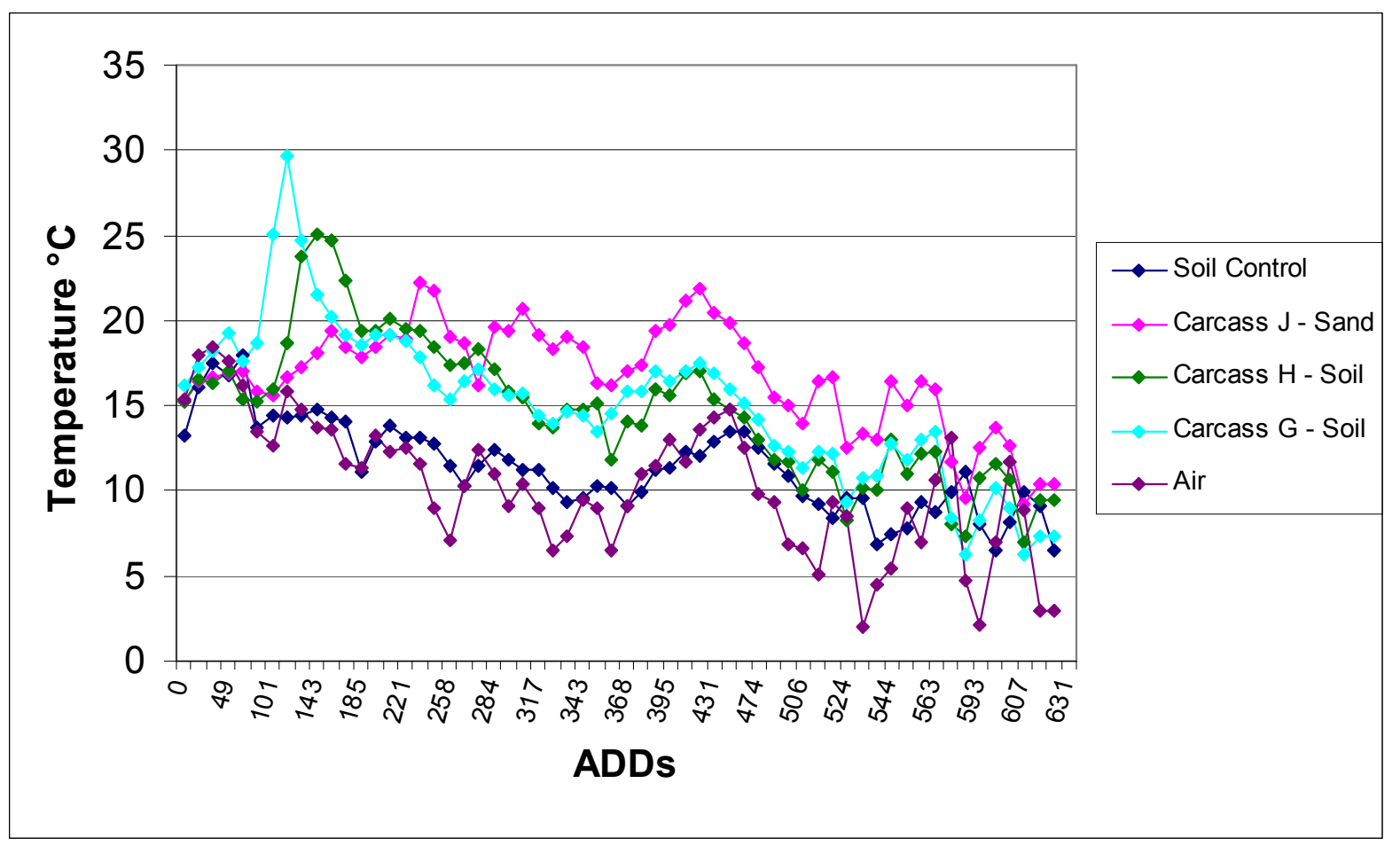

Figure 5.4 Mean daily air, soil and sand temperatures.

Figure 5.4 shows the mean daily ambient air temperature, the mean daily temperature under carcasses $\mathrm{J}$ (sand), $\mathrm{H}$ and $\mathrm{G}$ (both soil), and in the control soil $\mathrm{K}$. The data-logger placed under carcass I (sand) suffered a malfunction and the data could not be recovered. 
In this experiment, the cages suffered more wind damage than in the previous experiment, partly because of being positioned at the eastern end of the site rather than under the sheltered northern end, but also because of a series of storms with high winds. The lids of the cages occasionally blew off, leaving the experiment exposed to the high rainfall, which led to the pits becoming waterlogged at times (Figure 5.5). The soil gravimetric moisture content of each sample was calculated as part of the enzyme activity method (section 3.3.2) and the results shown in Figure 5.5. Soil gravimetric moisture content is calculated as a ratio of the dry mass of solid to the mass of liquid. The daily rainfall, as measured at the Trentham weather station is shown in Figure 5.6. The sand decomposition plot moisture content readings were not particularly representative of the actual effect of rainfall on the plot. The moisture content appeared to increase steadily over the course of the sampling period, but was lower than the soil moisture content values. This is because the sand tended to settle at the bottom of the flooded tubs, and sampling of this resulted in the wet sand being collected, but not the flood water above it.

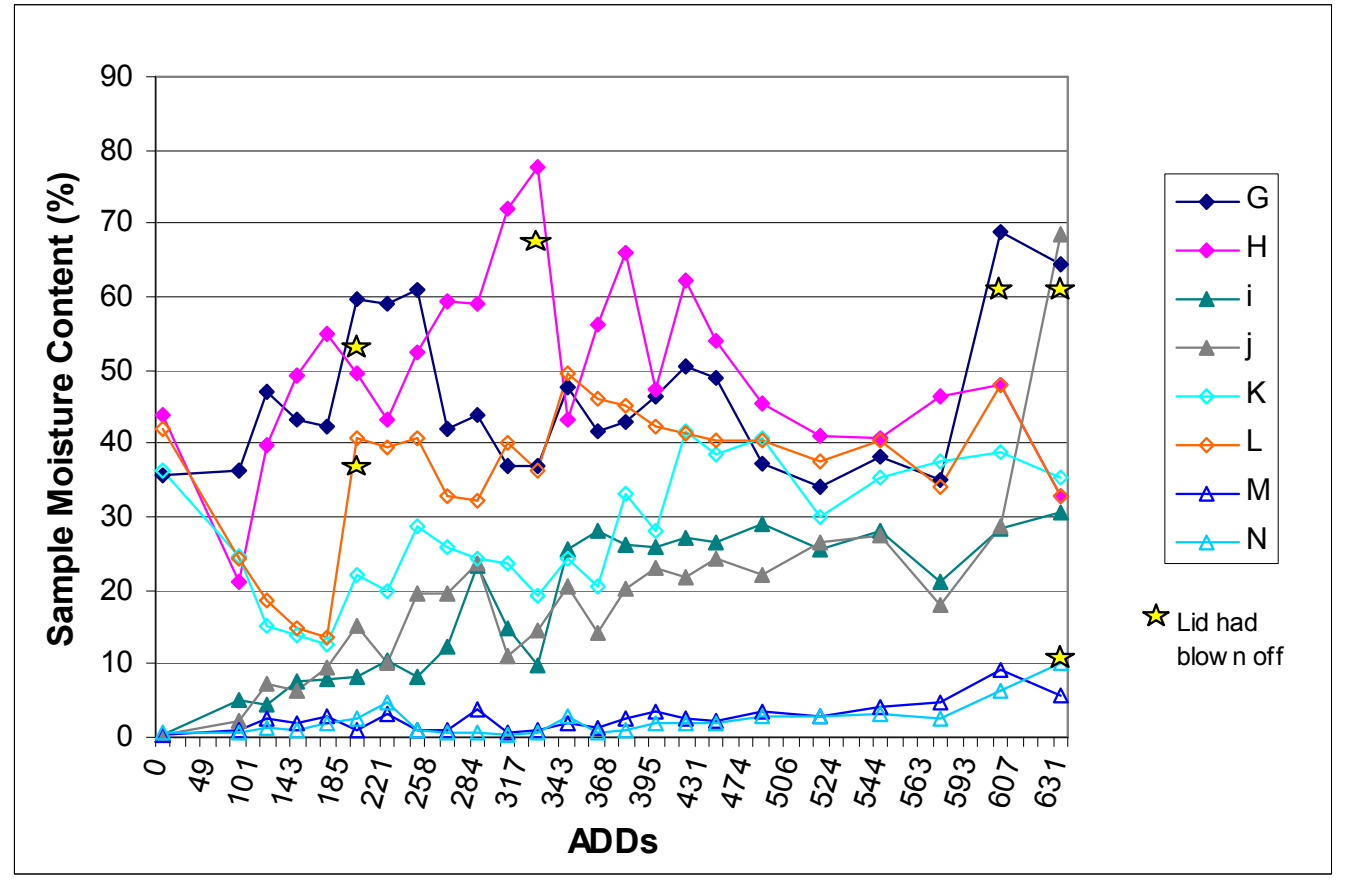

Figure 5.5 Moisture content of samples over the sampling period. Days where the cage lids had blown open are indicated with a star. 


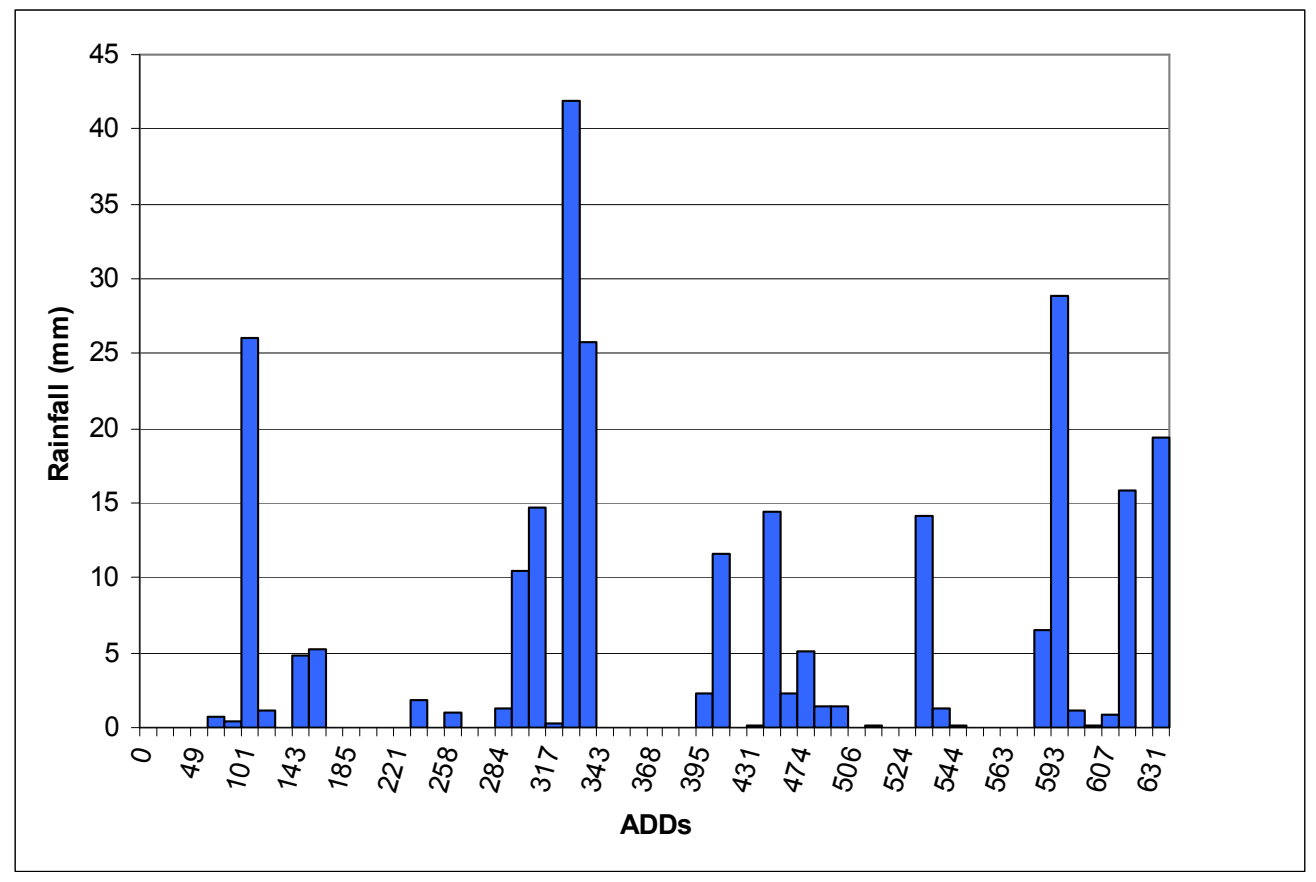

Figure 5.6 Daily rainfall at the Trentham Weather Station over the sampling period.

The mean daily RH data for the experiment time period from the Trentham weather station are shown in Figure 5.7.

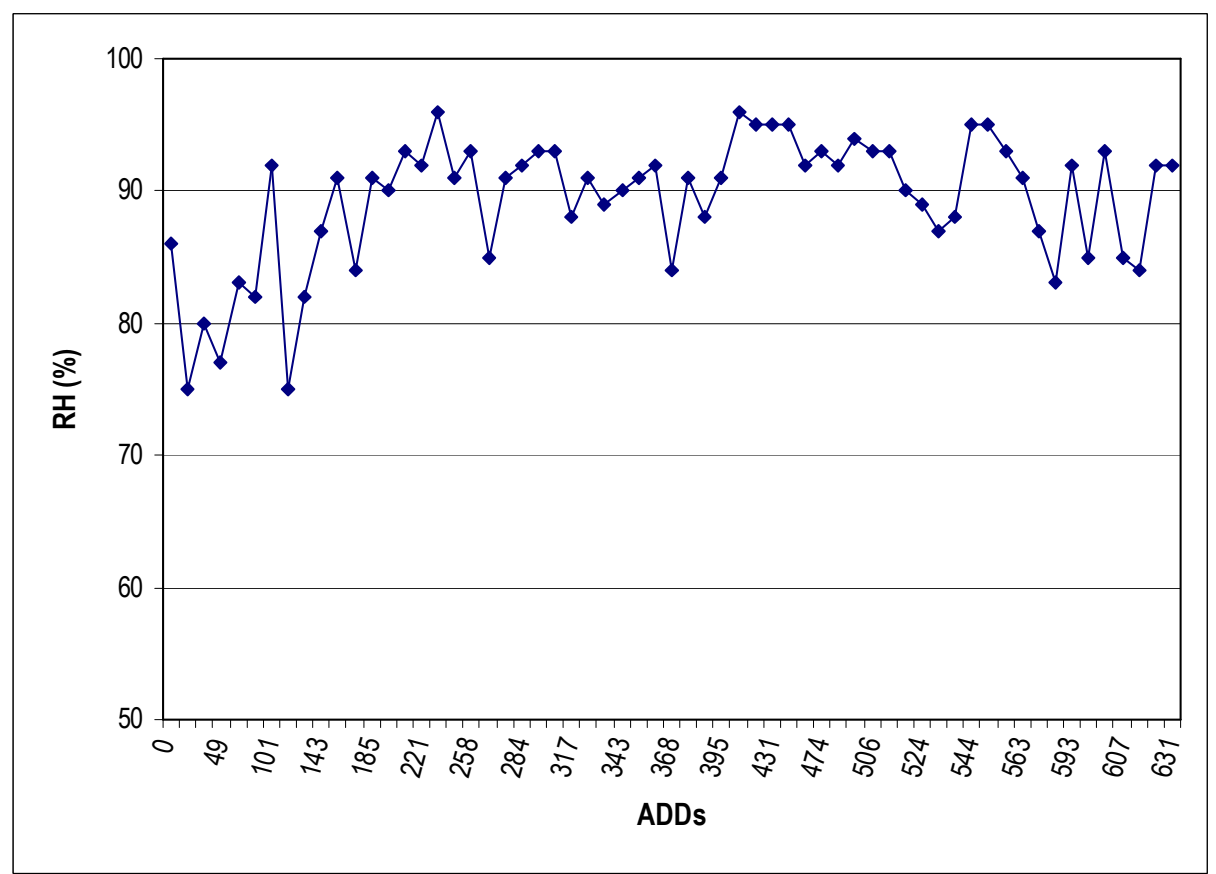

Figure 5.7 Mean daily RH at the Trentham Weather Station over the sampling period. 


\subsubsection{Documentation of Decomposition Progression}

Photos were taken at each sampling to document the stage of decomposition for comparison with the previous experiment. Photos carcass G (on soil) and carcass I (on sand) which show the progression of decomposition are shown in Figures 5.8 and 5.9. Photos of the other carcasses are included in Appendix B.

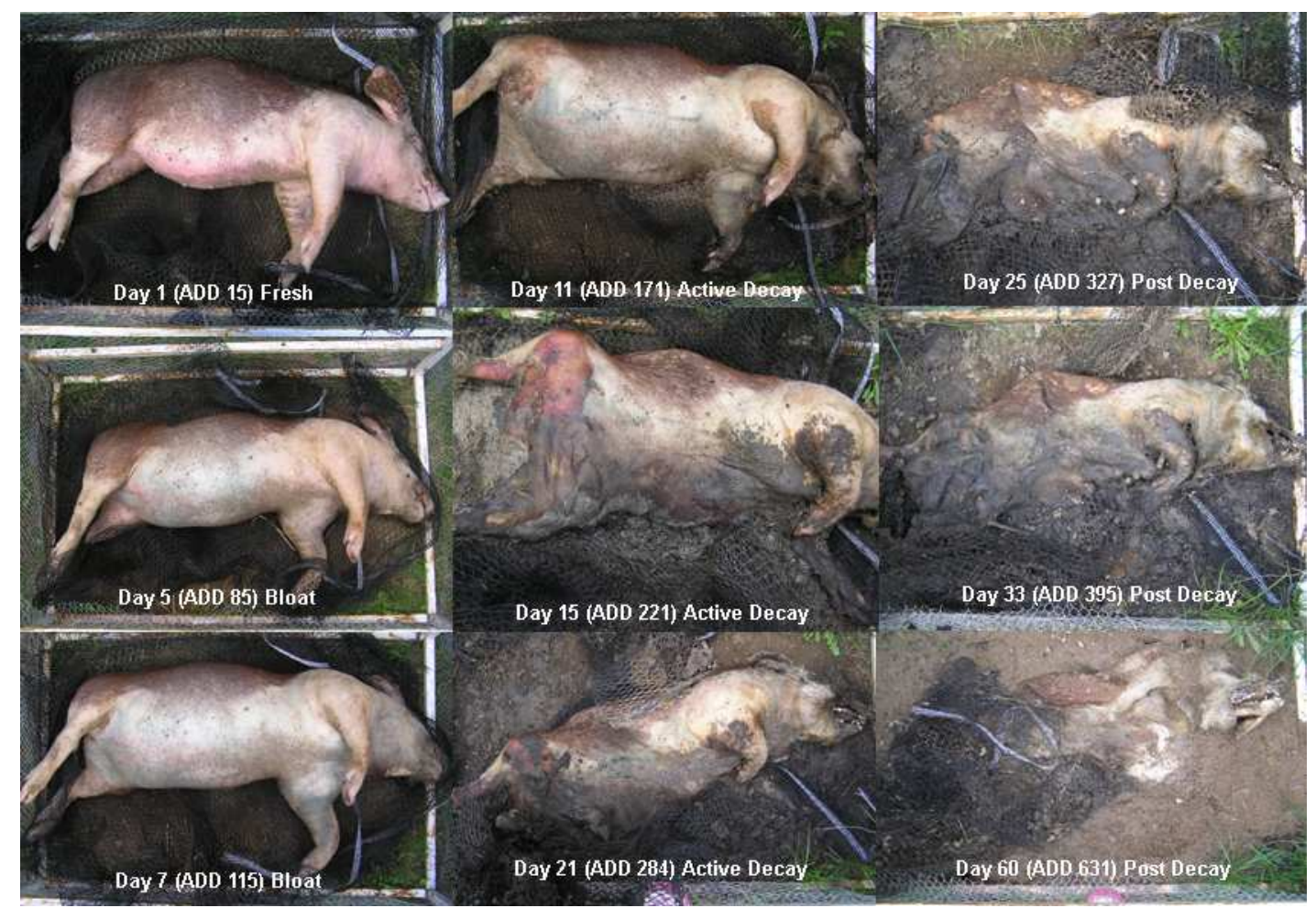

Figure 5.8 Carcass $G$ (soil) decomposition over time. 


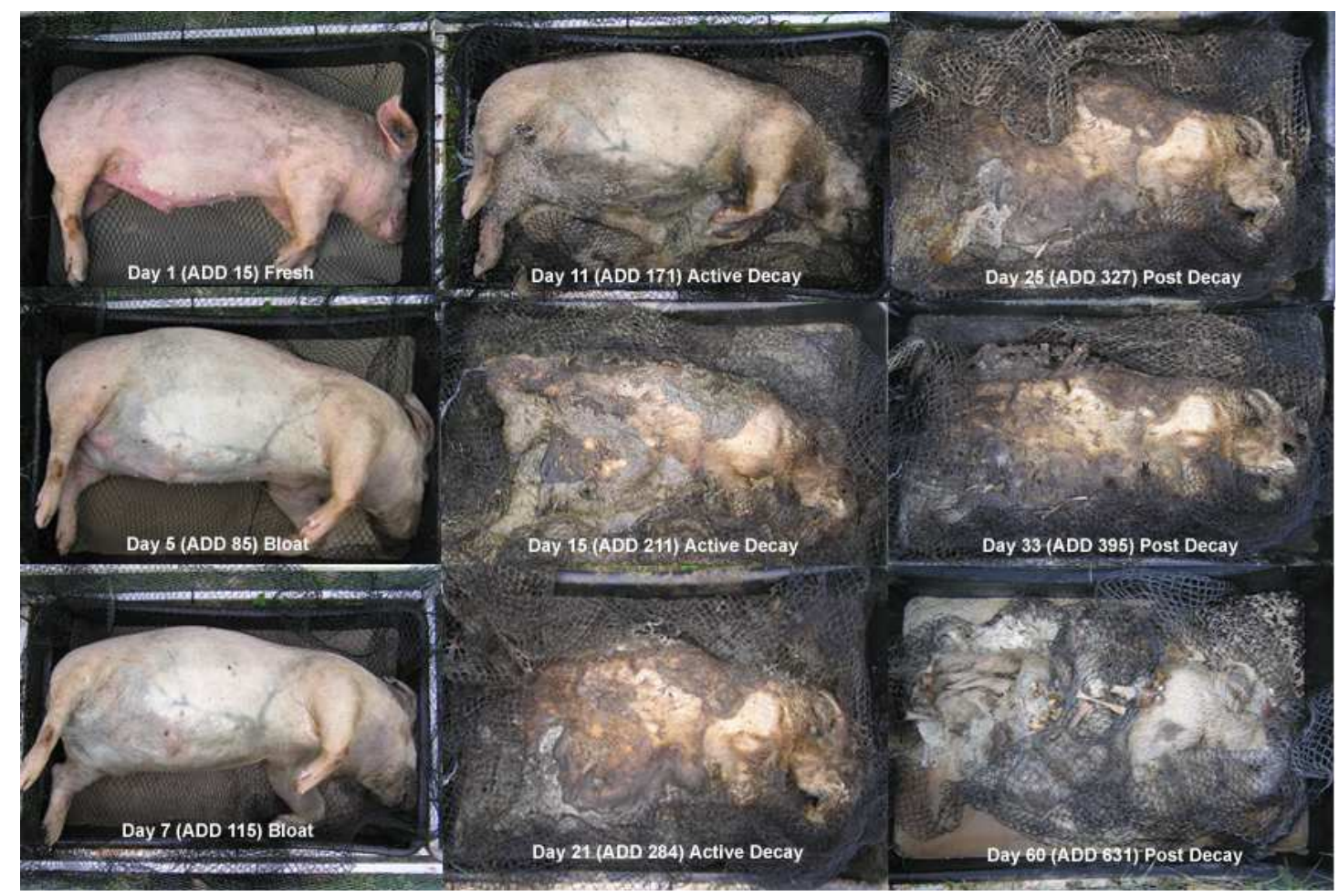

Figure 5.9 Carcass I (sand) decomposition over time.

The two pig carcasses on soil decomposed in a very similar way to those from the previous experiment (section 4.3.1) with the exception that the active decay stage was slightly extended by comparison in this experiment (Figure 5.8). The two carcasses on sand also differently to those from the previous experiment, as the tubs holding the sand became water-logged on a few occasions (Figure 5.9). The tubs had four large drainage holes, but the heavy rainfall coupled with the cage lids occasionally blowing open resulted in large volumes of water entering the tubs, and the drainage system could not cope. These plots had a noticeably different odour, which may have been indicative of a more anaerobic decomposition. The maggot masses seen in this experiment were not as large as those observed in the previous one, particularly on the carcasses on sand, as the maggots were almost all drowned on at least one occasion. The carcasses on soil were disturbed on a couple of occasions by an unknown animal burrowing into the plot, but there was no evidence of interference with the carcass itself, rather just disturbance of the soil (Figure 5.10). The cage was not damaged, so it appears that the plot was burrowed into under the netting. 


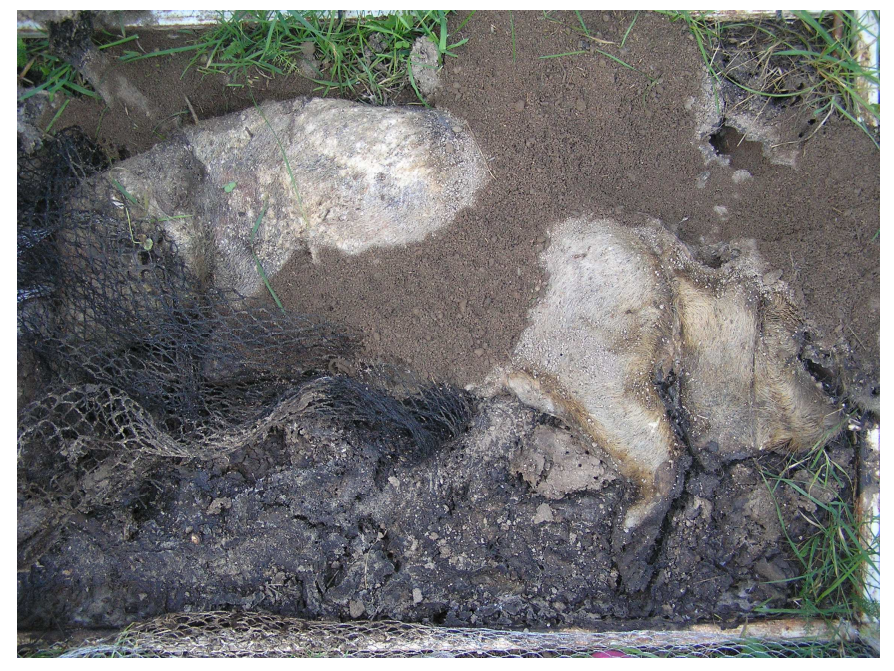

Figure 5.10 Carcass H on day 48 (ADD 544), showing disturbance of the soil, with a burrow evident in the top right-hand corner.

\subsubsection{Chemistry \& Biochemistry Results}

\subsubsection{1 pH}

The mean $\mathrm{pH}$ for the replicate carcasses (sand and soil, decomposition and control) at each sampling is shown in Figure 5.11.

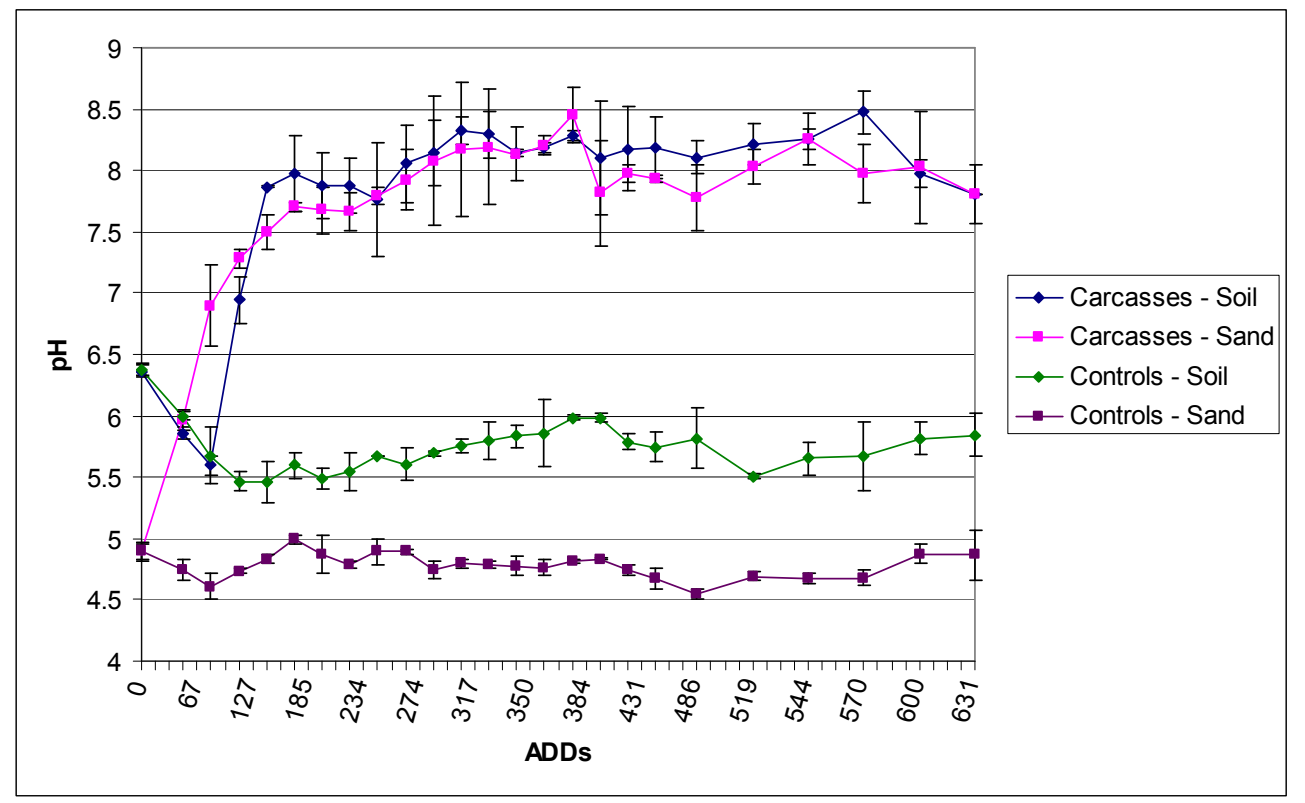




\section{Error bars represent the standard error.}

The $\mathrm{pH}$ of the sand and soil samples from under all the carcasses displayed rapid increased in $\mathrm{pH}$, very similar to those seen in the previous experiment (section 4.3.3). The $\mathrm{pH}$ rise was fastest on sand, with the soil samples' $\mathrm{pH}$ initially dropping in unison with the soil control samples, before rapidly rising to around the same point as the sand (around 8.0). This is similar to the change seen for the carcasses with insect colonisation on sand for the previous experiment, although there was no initial peak before a drop to the plateau in this experiment, just a fairly steady increase before the plateau. The initial $\mathrm{pH}$ of the sand in this experiment was lower than in the first pig experiment (4.89 compared with 5.7), possibly due to natural variations in the sand or in the washing technique (section 4.2.2), although the same method was used, and the same brand of sand was purchased each time. Measuring the $\mathrm{pH}$ of an inert matrix such as sand in water, however, is not as accurate as measuring the $\mathrm{pH}$ of a buffered solution such as soil, so this difference could be an artefact of the method. The initial soil $\mathrm{pH}$ was 6.4 , but it dropped to 5.5 in the first week before rising to around 6.0. The duplicate treatment samples showed no significant differences, but the decomposition and control samples were significantly different $(\mathrm{p}<0.01)$. If the ADD 0 samples (prior to carcass placement controls) are excluded, the sand and soil $\mathrm{pH}$ values are not significantly different $(\mathrm{p}=0.32)$, suggesting that the presence of a body may raise the $\mathrm{pH}$ to around 8 regardless of the original soil $\mathrm{pH}$. 


\subsubsection{Ammonium $\left(\mathrm{NH}_{4}^{+}-\mathrm{N}\right)$}

The ammonium concentration in samples at each sampling date is shown in Figure 5.12.

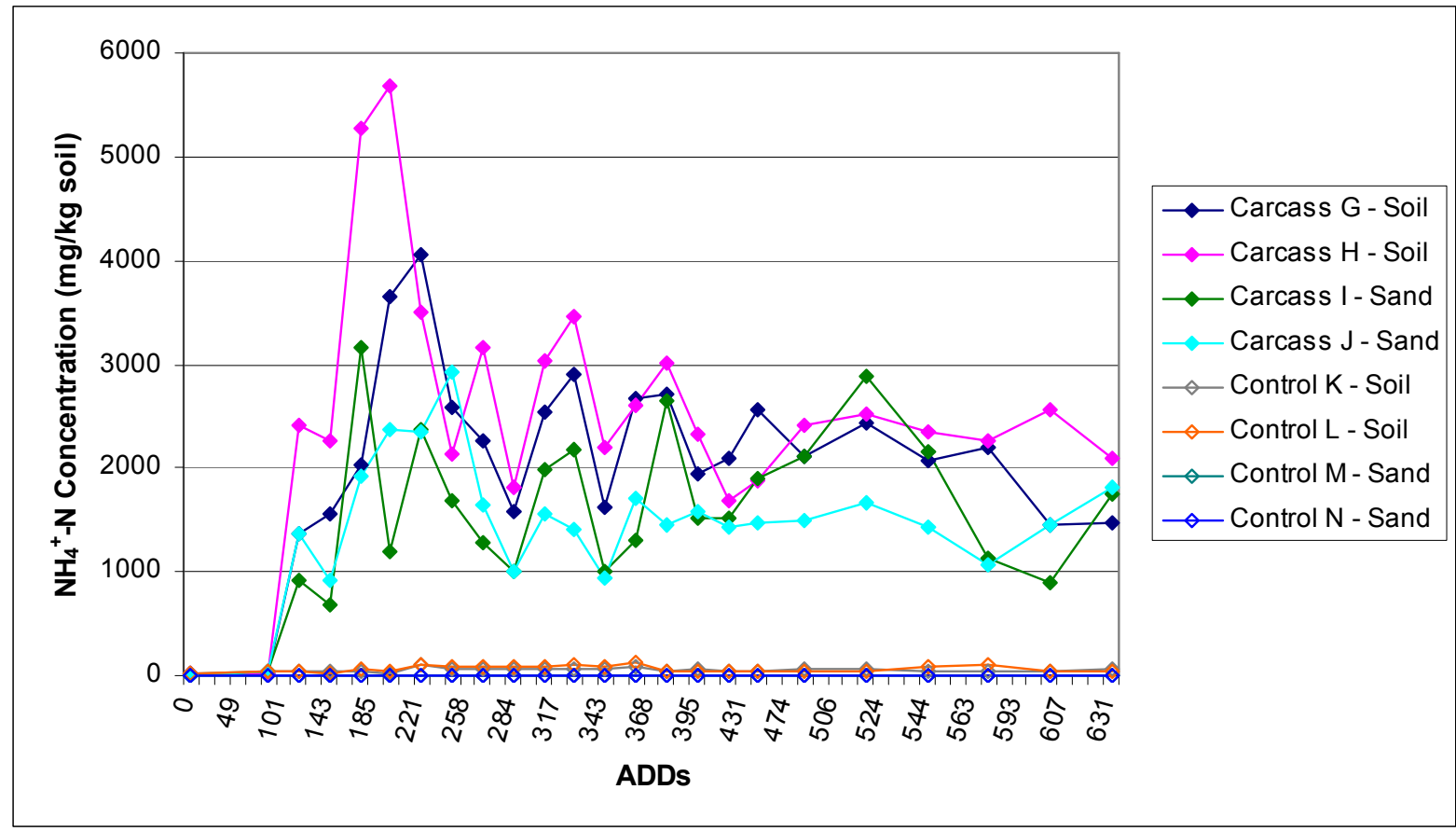

Figure $5.12 \mathrm{NH}_{4}^{+}-\mathrm{N}$ concentrations in soil and sand samples collected from under carcasses and from soil and sand controls.

The concentration of $\mathrm{NH}_{4}{ }^{+}-\mathrm{N}$ under each of the carcasses increases from baseline levels beginning on day 5 (ADD 67). The concentration appeared to reach a peak (5682 $\mathrm{mg} / \mathrm{kg}$ for pig $\mathrm{H}$ ) between ADD 185 and 234 before oscillating between 1000 and $3000 \mathrm{mg} / \mathrm{kg}$ soil until the conclusion of sampling at ADD 628. The soil controls were, by comparison, very consistent, ranging between 24.78 and 119.19 (with a mean of $59.48 \pm 25.10 \mathrm{mg} / \mathrm{kg}$ ). The sand controls were also very consistent, ranging between 0 and $2.06 \mathrm{mg} / \mathrm{kg}$ (with a mean of $0.86 \pm 0.61$ ). There were no significant differences between the treatment replicates, but the differences between the pig and control samples were significant for both soil and sand $(\mathrm{p}<0.01)$. The difference between the soil and sand decomposition samples was also significant $(\mathrm{p}<0.01)$. 


\subsubsection{Nitrate $\left(\mathrm{NO}_{3}^{-}-\mathrm{N}\right)$}

The concentration of $\mathrm{NO}_{3}{ }^{-} \mathrm{N}$ in the soil and sand samples at each sampling date is shown in Figure 5.13.

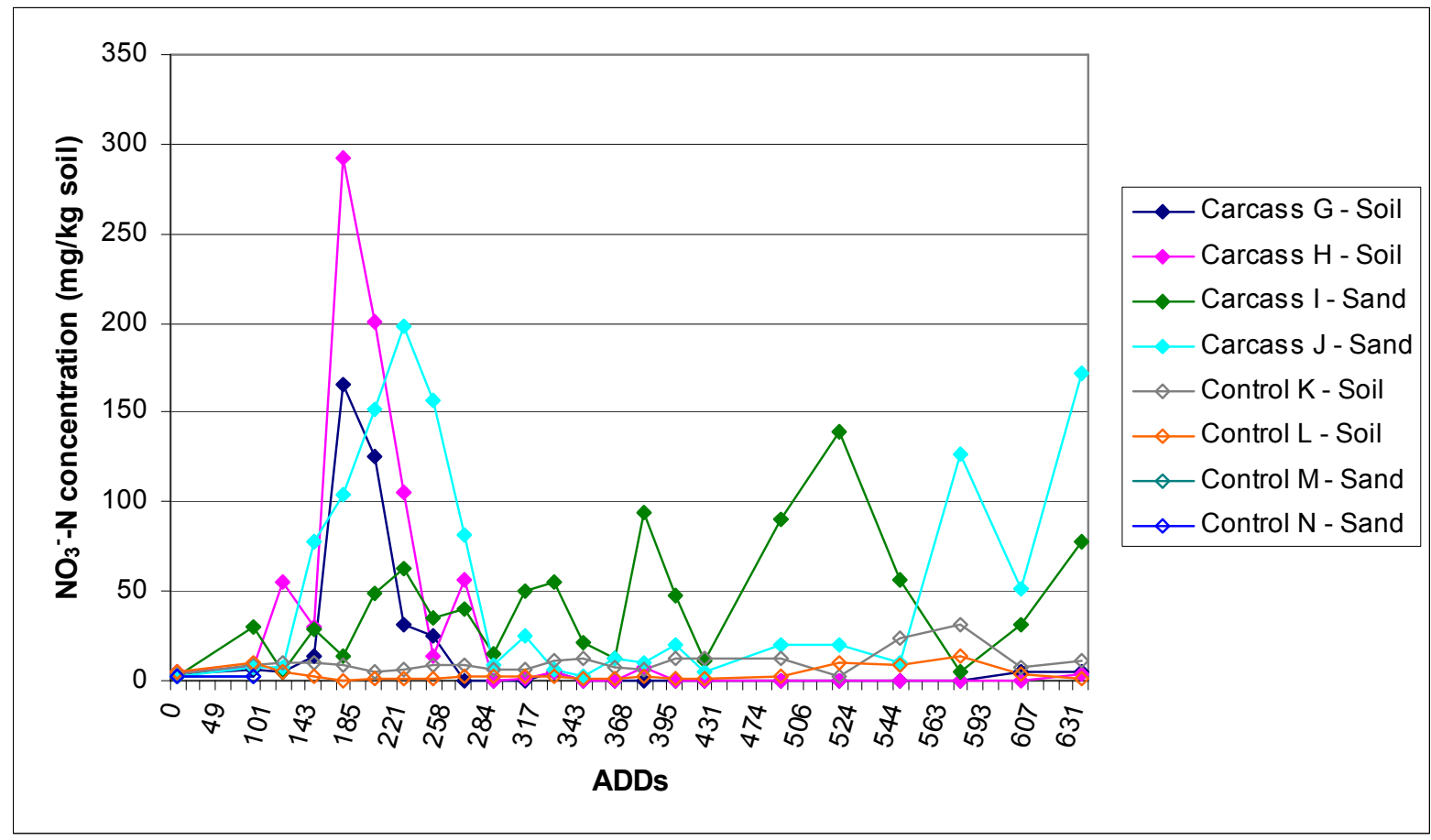

Figure 5.13 NO3 -N concentrations in soil and sand samples collected from under carcasses and from soil and sand controls.

The nitrate concentrations from samples collected under the carcasses on soil (carcasses $\mathrm{G}$ and H) showed a dramatic increase between ADDs 143 and 171 before returning to near baseline levels. Carcass J (on sand) showed a similar peak at ADD 221, but the replicate carcass on sand (carcass I) had a smaller peak. The sand decomposition samples both had quite variable $\mathrm{NO}_{3}{ }^{-}-\mathrm{N}$ concentrations throughout the time sampled. The replicate soil decomposition samples showed a significant difference $(p<0.05)$, but there is no significant difference between the other replicates. There is also no significant difference between the soil and sand decomposition samples, but the differences between the decomposition and control samples for both soil and sand are significant $(\mathrm{p}<0.01)$. The nitrate concentration seems to peak just before the ammonium concentration does for each carcass (with the exception of carcass I). 


\subsubsection{Urease (EC 3.5.1.5)}

Potential urease activity in the soil and sand samples over the course of the decomposition experiment is shown in Figure 5.14.

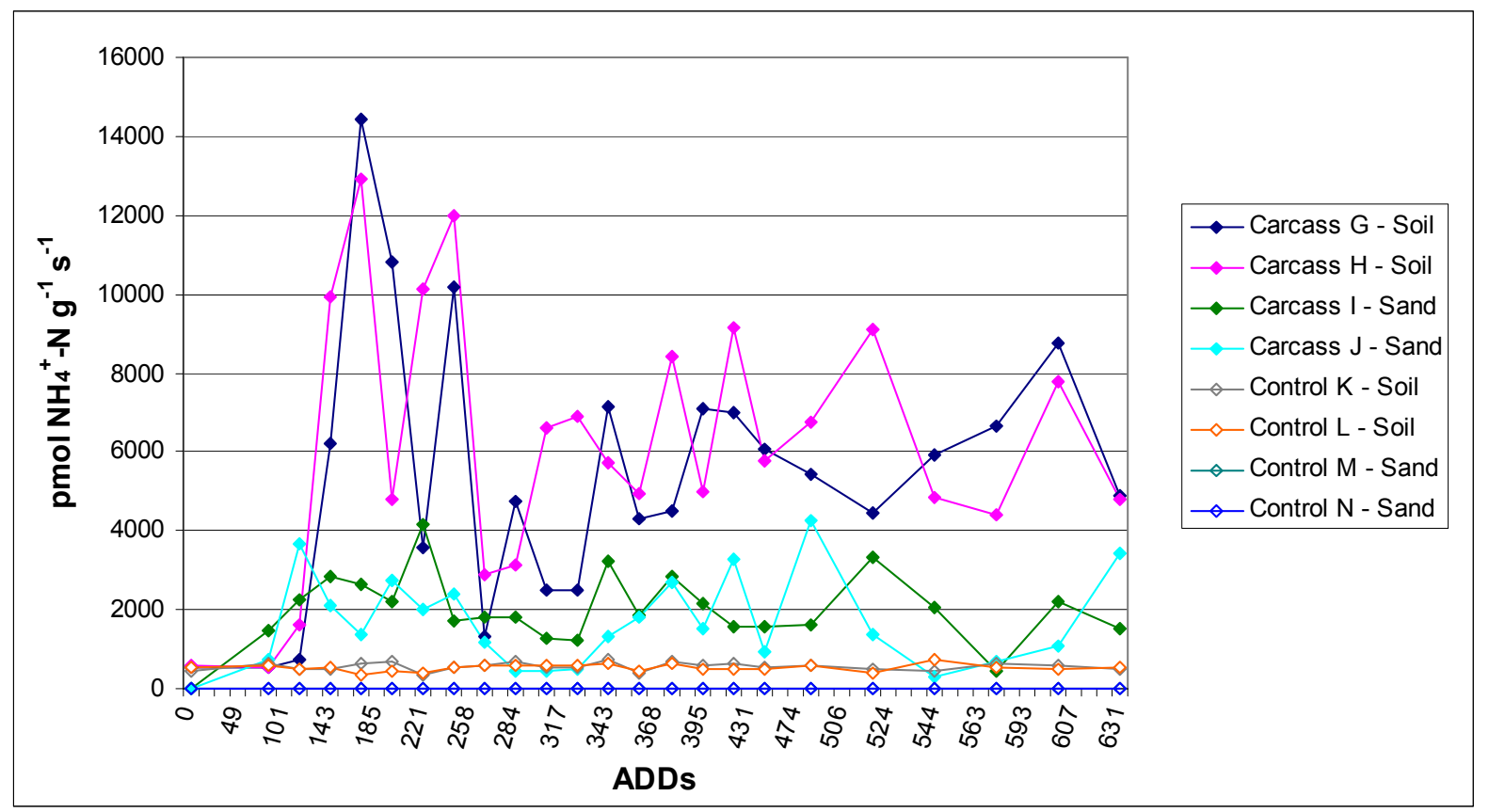

Figure 5.14 Potential urease activity in soil and sand samples collected from under carcasses and from soil and sand controls.

The soil decomposition samples displayed a large increase in potential urease activity to 14414 and $12908 \mathrm{pmol} \mathrm{g}^{-1} \mathrm{~s}^{-1}$ (carcasses $\mathrm{G}$ and $\mathrm{H}$ respectively) followed by a temporary drop to close to $4000 \mathrm{pmol} \mathrm{g}^{-1} \mathrm{~s}^{-1}$, before a second spike in potential activity. Following this spike, activity in samples from under both carcasses dropped to its lowest point before oscillating between 4000 and $9000 \mathrm{pmol} \mathrm{g}^{-1} \mathrm{~s}^{-1}$ until the end of the sampling period. The sand decomposition samples showed much lower potential activity (between 400 and $4000 \mathrm{pmol} \mathrm{g}^{-1}$ $\mathrm{s}^{-1}$ ) which varied over the sampling period but with no discernible trend common to both sand and soil samples. There were no significant differences between the duplicate treatment samples, but the difference between the decomposition and control samples, and the soil and sand samples were significant $(\mathrm{p}<0.01)$. The soil controls were consistently around $500 \mathrm{pmol}$ 
$\mathrm{NH}_{4}{ }^{+}-\mathrm{N} \mathrm{g}^{-1} \mathrm{~s}^{-1}$ and the sand controls were very close to zero activity throughout the sampling period.

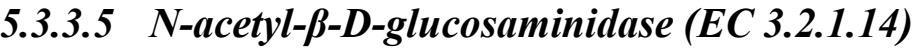

Potential N-acetyl- $\beta$-D-glucosaminidase activity in the soil and sand samples over the course of the decomposition experiment is shown in Figure 5.15.

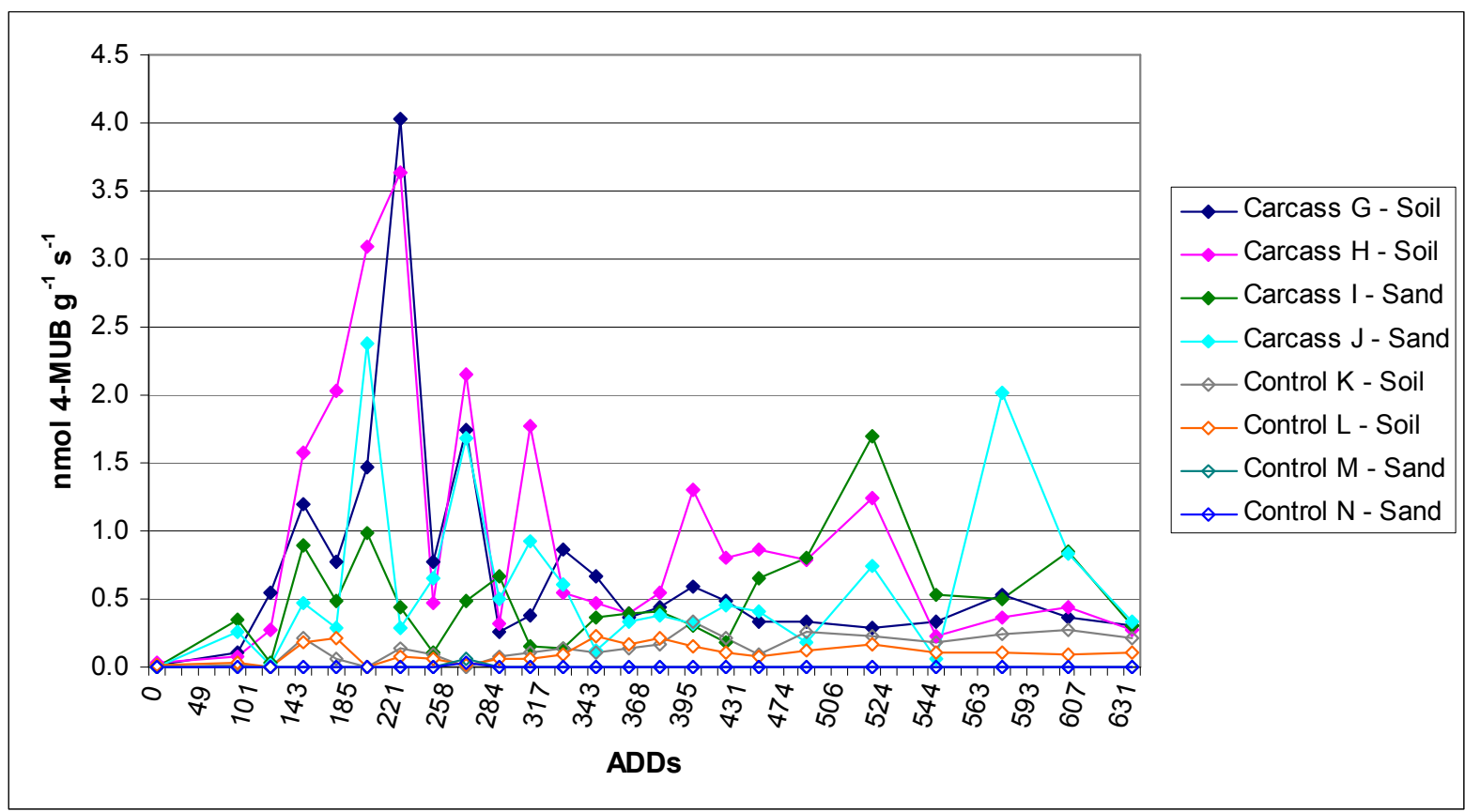

Figure 5.15 Potential $N$-acetyl- $\beta$-D-glucosaminidase activity in soil and sand samples collected

from under carcasses and from soil and sand controls.

The potential activity of enzyme N-acetyl- $\beta$-D-glucosaminidase in the soil decomposition samples peaked at around ADD 234. One of the sand decomposition samples gave a high peak at a similar stage but it was not as high. The sand decomposition samples seemed to show a higher level of potential activity than the soil decomposition samples towards the end of the sampling period. The soil controls tended to show small variations over the course of the sampling period, but the sand samples consistently returned no potential activity. At the $95 \%$ level, the replicate carcasses on soil showed significant differences. The sand replicates showed no significant differences throughout the sampling period. The decomposition 
samples were overall significantly different from their respective controls $(p<0.01)$ and the sand and soil decomposition samples were also significantly different $(\mathrm{p}<0.01)$.

\subsubsection{Glucosidase (EC 3.2.1.21)}

Potential glucosidase activity in the soil and sand samples over the course of the decomposition experiment is shown in Figure 5.16.

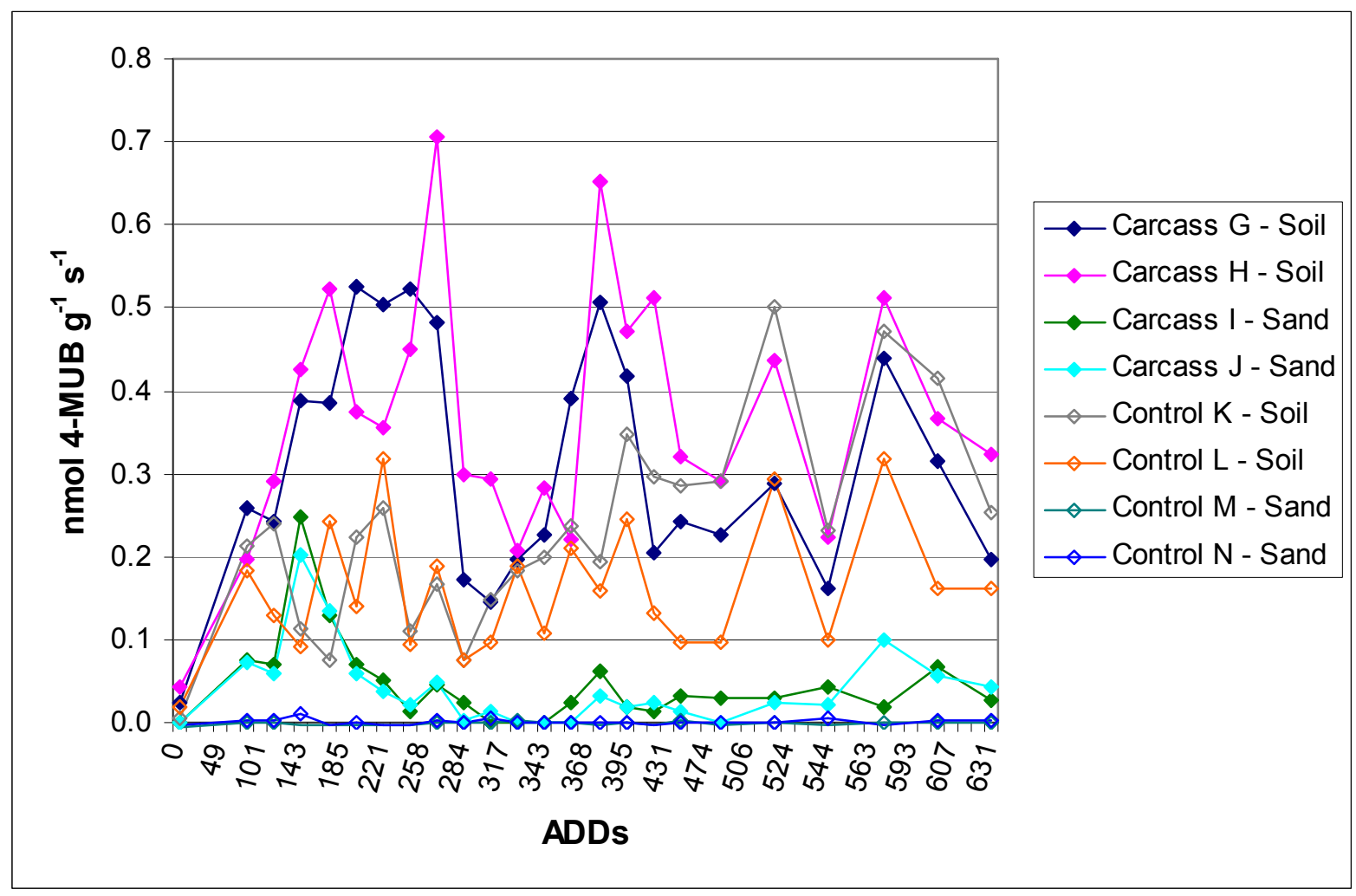

Figure 5.16 Potential glucosidase activity in samples collected from under carcasses and from controls.

The potential glucopyranosidase activity was low throughout the experimental period and the soil data are very noisy. The soil decomposition samples showed an immediate rise in activity as soon as the carcasses were placed, reaching a peak at around ADD 267 for carcass $\mathrm{H}$ and slightly earlier at ADD 197 for carcass G. Samples from both carcasses then displayed a drop and period of lower activity after ADD 267, followed by an increase to almost the previous peak at ADD 375. The soil control data were also very noisy and oscillated between 0.1 and 0.3 nmol 4-MUB g ${ }^{-1} \mathrm{~s}^{-1}$ until around ADD 395 when the control L activity began to rise. The 
sand control samples showed negligible activity throughout the experimental period, and the sand decomposition samples showed a small spike of activity at ADD 143 before returning to close to control levels. The replicate carcasses on sand were not significantly different but the replicate carcasses on soil were significantly different at the $95 \%$ level $(p<0.05)$. The control soil samples were also significantly different $(\mathrm{p}<0.01)$, most likely due to the rise in activity seen in control $\mathrm{K}$ samples towards the end of the experimental period. The decomposition samples were significantly different from the relevant controls $(p<0.01)$ and the sand and soil sample activities were also significantly different $(\mathrm{p}<0.01)$.

\subsubsection{Acid Phosphatase (EC 3.1.3.2)}

Potential acid phosphatase activity in the soil and sand samples over the course of the decomposition experiment is shown in Figure 5.17.

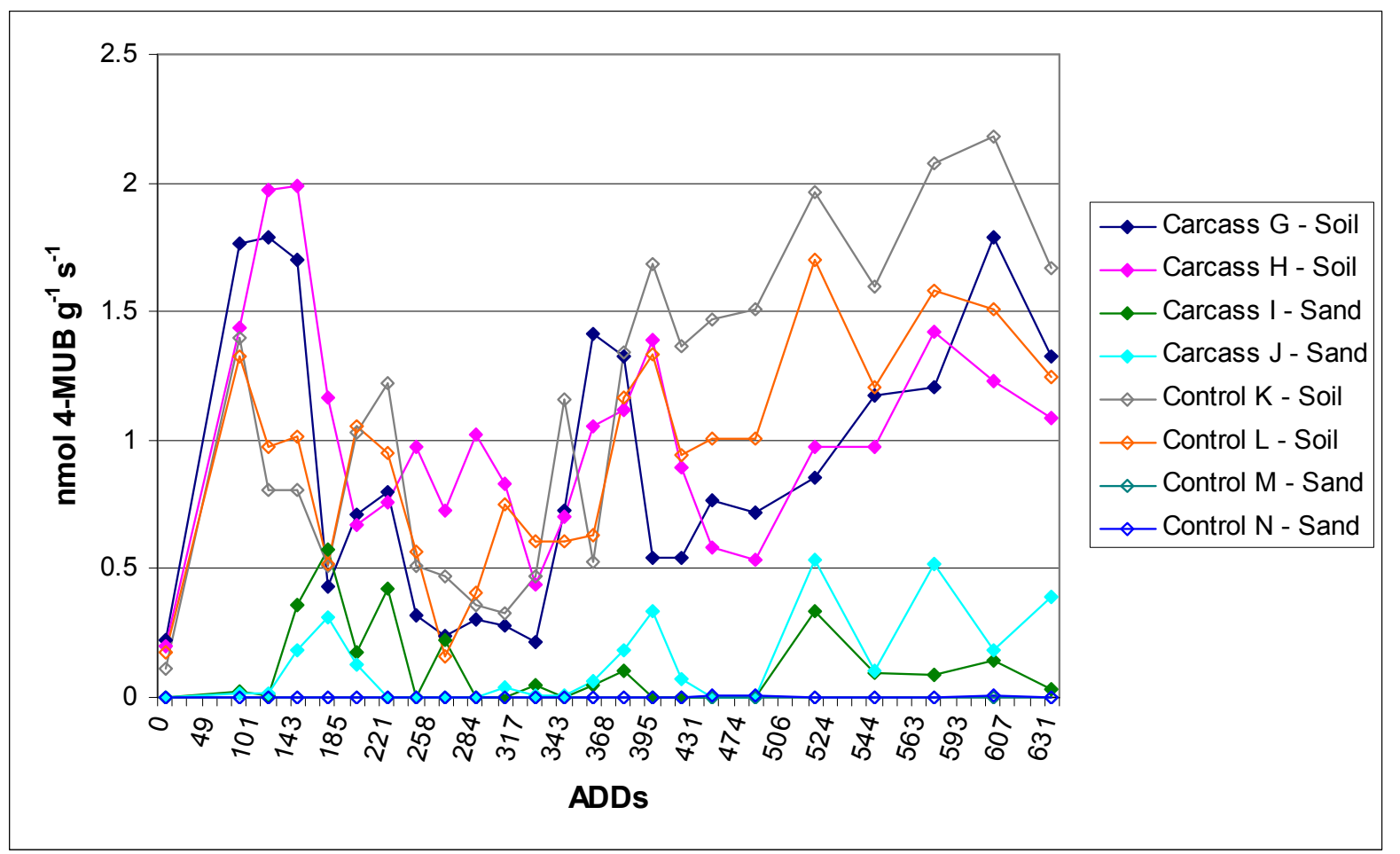

Figure 5.17 Potential acid phosphatase activity in soil samples collected from under carcasses and from soil and sand controls. 
The potential acid phosphatase activity in both the soil and sand samples was very changeable with erratic fluctuations between consecutive samplings. There was no significant difference detected between the soil decomposition and control samples overall $(p=0.155)$. The difference between the sand decomposition and control samples was significant, but the potential activity in the decomposition samples showed no clear trend. The soil samples all showed an initial spike in potential activity, followed by a drop and then steady increase over time until sampling ceased at ADD 631. The only time when there was a significant difference $(\mathrm{p}<0.01)$ between the soil decomposition and control samples was at the time of the initial spike (from ADD 85-197), where the decomposition samples temporarily showed higher potential activity than the controls. The overall variability may have been a response by the soil population to the disruption of the soil by sieving, which masked any increase in potential activity caused by decomposition. 


\subsubsection{Sulphatase (EC 3.1.6.1)}

Potential sulphatase activity in the soil and sand samples over the course of the decomposition experiment is shown in Figure 5.18.

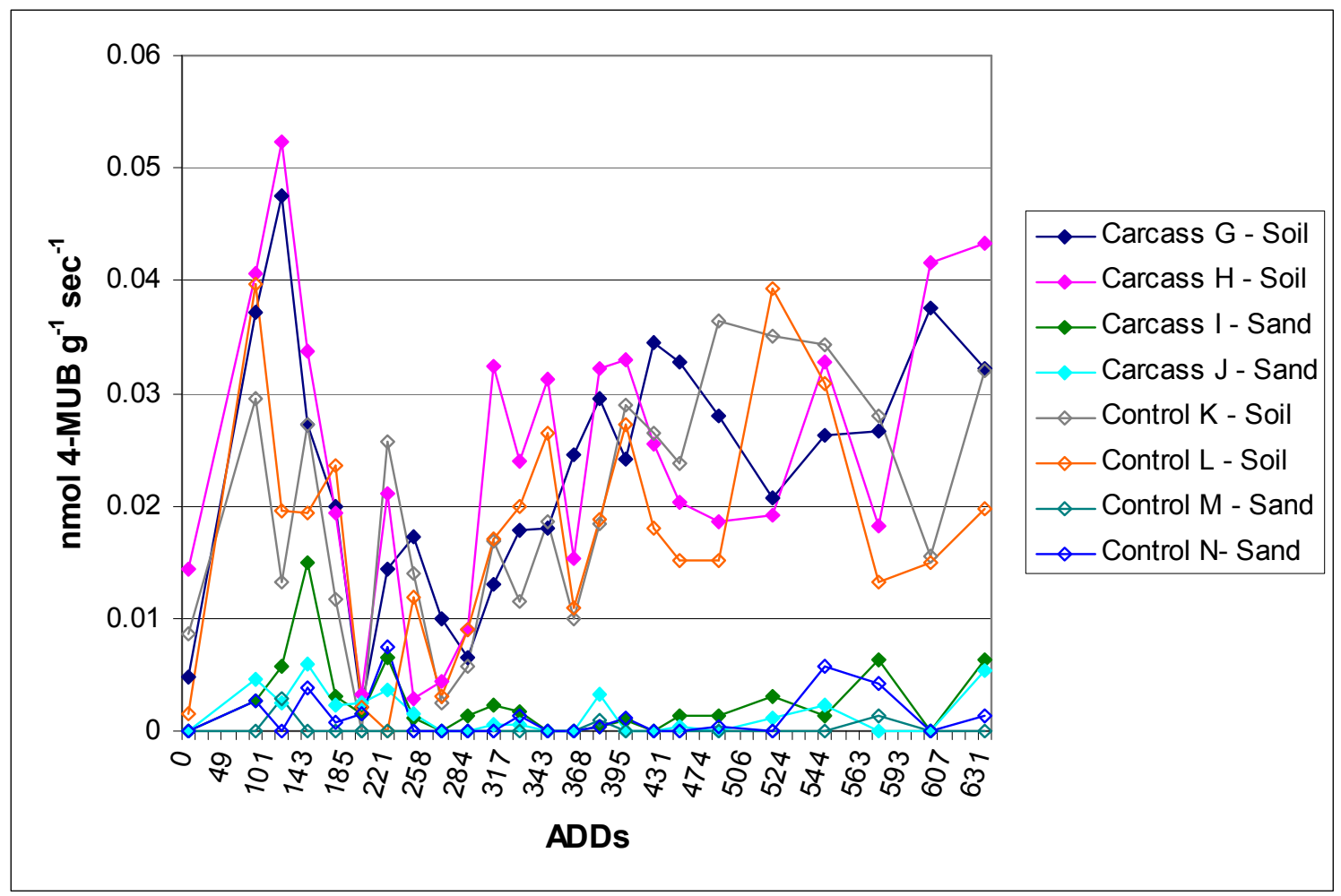

Figure 5.18 Potential sulphatase activity in soil samples collected from under carcasses and from soil and sand controls.

Like the acid phosphatase activity (section 5.3.3.7), the potential sulphatase activity in the soil samples was very variable over time. There was, however, a significant increase in potential activity in the sand decomposition samples compared with the sand controls $(p<0.01)$. The difference between the soil decomposition samples and the soil controls was also significant $(\mathrm{p}<0.01)$, although visually these look very similar. As with the acid phosphatase, it appears that both the soil controls and decomposition samples underwent an initial increase, followed by a drop, and then a steady increase towards the end of the sampling period. The difference between the decomposition soil and sand samples, however, was significant $(p<0.01)$, with carcass I samples showing an initial peak three-fold lower than the initial peaks in activity in the soil samples. Because the acid phosphatase soil samples results also appeared to show the 
same trend of increasing towards the end of the sampling period, it is possible that this is simply an artefact of sampling and the heterogeneous nature of soil. The units shown on the graph are also small, making the data appear very noisy although the variations are small. The samples from the duplicate carcasses on soil and sand showed no significant differences, but the duplicate sand controls showed a difference significant at the $95 \%$ level.

\subsubsection{T-RFLP}

Sample profiles were generated using the T-RFLP analysis method described in section 3.4.3. Reasonable quality T-RFLP profiles were generated for all samples collected in this experiment. No DNA could be extracted from any control sand samples so no T-RFLP profiles could be generated for these. Similarly, no DNA could be extracted from the sand samples from under carcasses I and $\mathrm{J}$ at day 3 (ADD 49). DNA was able to be extracted from sand samples from day 5 onwards in this experiment, while many day 5 samples from the previous experiment yielded no detectable DNA. . The mean number of T-RFs present in each profile, and the total number per carcass profile dataset are shown in Table 5.2.

\begin{tabular}{|c|c|c|c|c|c|}
\hline & \multicolumn{3}{|c|}{ Soil } & \multicolumn{2}{c|}{ Sand } \\
\cline { 2 - 7 } & G & H & Controls & I & J \\
\hline Mean number of T-RFs in each profile & 38 & 41 & 58 & 30 & 32 \\
\hline Total number of T-RFs per carcass dataset & 68 & 76 & 66 & 51 & 50 \\
\hline
\end{tabular}

Table 5.2 Number of T-RFs present in profiles from each carcass.

\subsubsection{Multi-Dimensional Scaling (MDS) Analysis}

Figures 5.19 and 5.20 show sample profile data from carcasses $\mathrm{G}$ and $\mathrm{H}$ (on soil) respectively, plotted using MDS using the parameters described in section 3.4.5.2. Figure 5.21 shows 
sample profile data from both carcasses $\mathrm{G}$ and $\mathrm{H}$, and the soil control samples $\mathrm{K}$ and $\mathrm{L}$ and in the inset graph, the soil control samples and early decomposition samples that clustered with the controls. The sample profile data for the carcasses on sand (I and J) are plotted using MDS in Figure 5.22 and data from all the samples collected during this experiment are plotted in Figure 5.23.

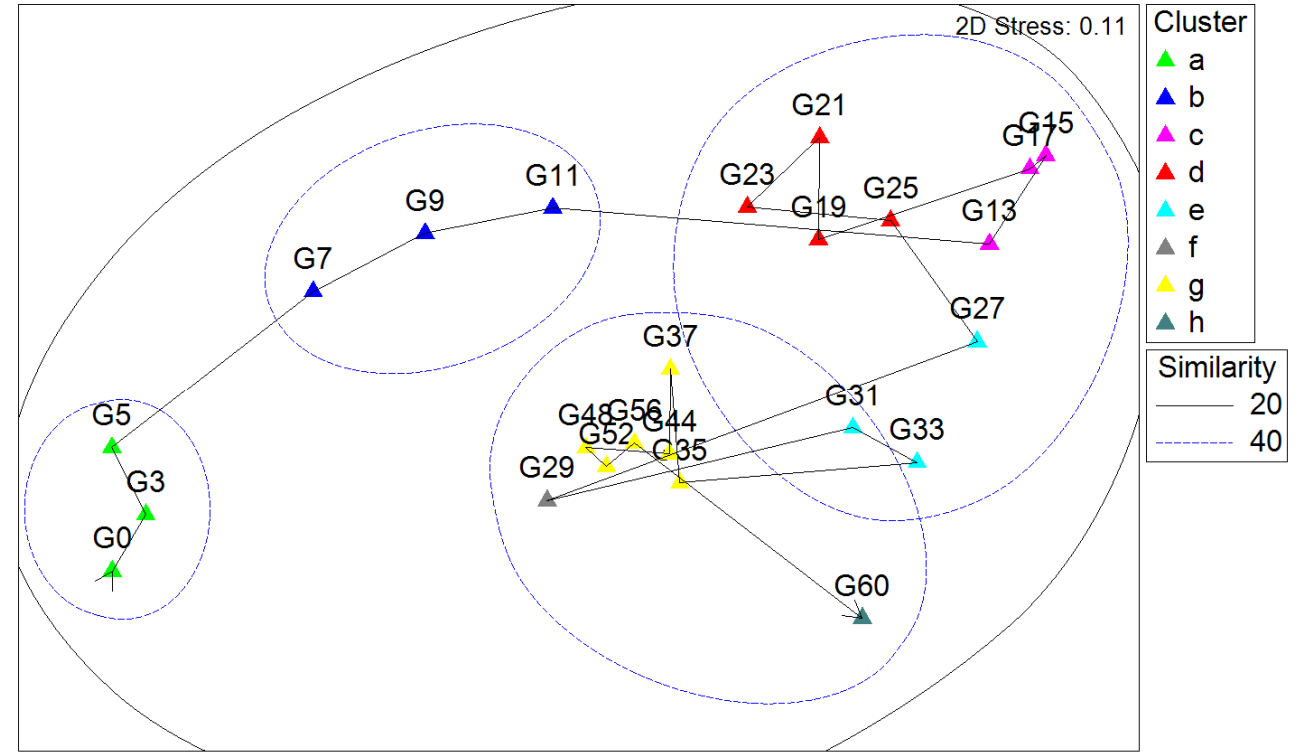

Figure 5.19 MDS plot of T-RFLP data from samples collected under carcass $G$ (on soil). Eight statistically significant clusters were distinguished using CLUSTER. The Bray-Curtis similarity index was used on square root transformed data. Samples are denoted by the number of calendar days post-death.

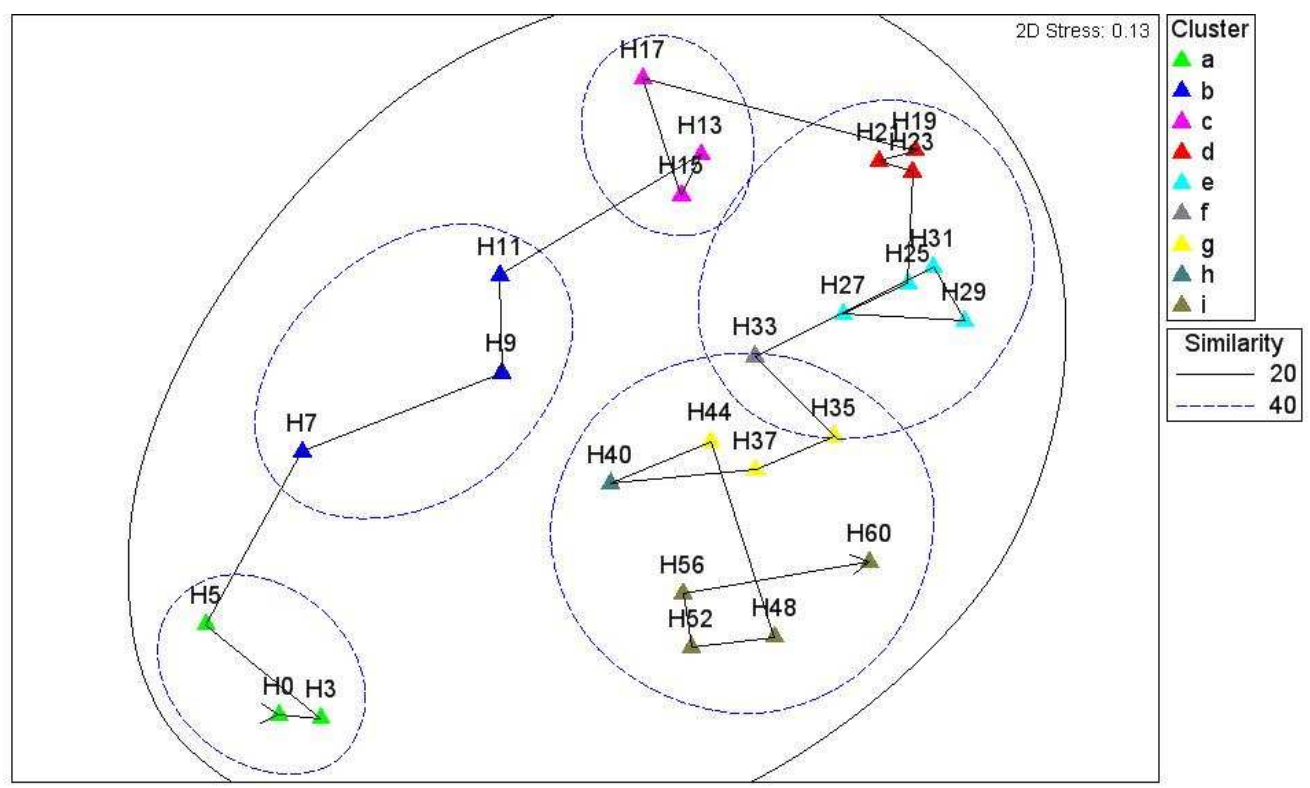

Figure 5.20 MDS plot of T-RFLP data from samples collected under carcass $\boldsymbol{H}$ (on soil). Nine statistically significant clusters were distinguished using CLUSTER. The Bray-Curtis similarity index was used on square root transformed data. Samples are denoted by the number of calendar days post-death. 
When plotted using MDS, the T-RFLP profile data generated from carcass G samples showed a separation of samples into five clusters at the $40 \%$ similarity level. The control soil sample (G0), which was collected prior to carcass placement fell within a $40 \%$ similarity cluster with samples collected on days 3 and 5 (ADDs 49 and 85), suggesting that the soil bacterial community changed little in response to decomposition within this time period. The bacterial community appeared to undergo a shift between days 5 and 7 (ADDs 85 and 115), with a second cluster containing samples collected on days 7, 9 and 11 (ADDs 115 to 171) falling within this cluster. The community appeared to shift again between days 11 and 13 (ADDs 171 and 197). Samples from day 13 (ADD 197) to day 31 (ADD 375) clustered together at the $40 \%$ level, but there was some overlap with the next group of samples, which also clustered at the $40 \%$ similarity level. The carcass $\mathrm{H}$ samples showed a very similar clustering pattern, with the main difference being more of a separation of the day 13 to 17 (ADDs 197 to 246) samples, which clustered slightly away from the later samples. The RELATE $\rho$ value for the two patterns of change reflects the visual similarity, as it was 0.798 with $p<0.01$.

An important point to note is that in both the carcass G and carcass H MDS plots (Figures 5.19 and 5.20), a lag is noticeable between the change in visual decomposition stage and a shift in the bacterial community. Samples from days 0, 3 and 5 (ADDs 0 to 85) clustered together in both data sets, although at day 5 the carcasses had moved from the fresh to bloat stage. This was followed by a shift in the bacterial community between days 5 and 7 (ADDs 85 and 115). Similarly, days 7, 9 and 11 (ADDs 115 to 171) clustered together, although by day 11 the carcasses were undergoing active decay. A shift in the bacterial community followed between days 11 and 13 (ADDs 171 and 197). It appears that after these initial bacterial community shifts, the changes between successive samplings were more gradual. 
The control soil samples clustered quite closely, with all samples clustering within the $60 \%$ similarity level (Figure 5.21). The early samples from under the carcasses (days 0, 3 and 5 ADDs 0 to 85 ) also fell within this cluster.

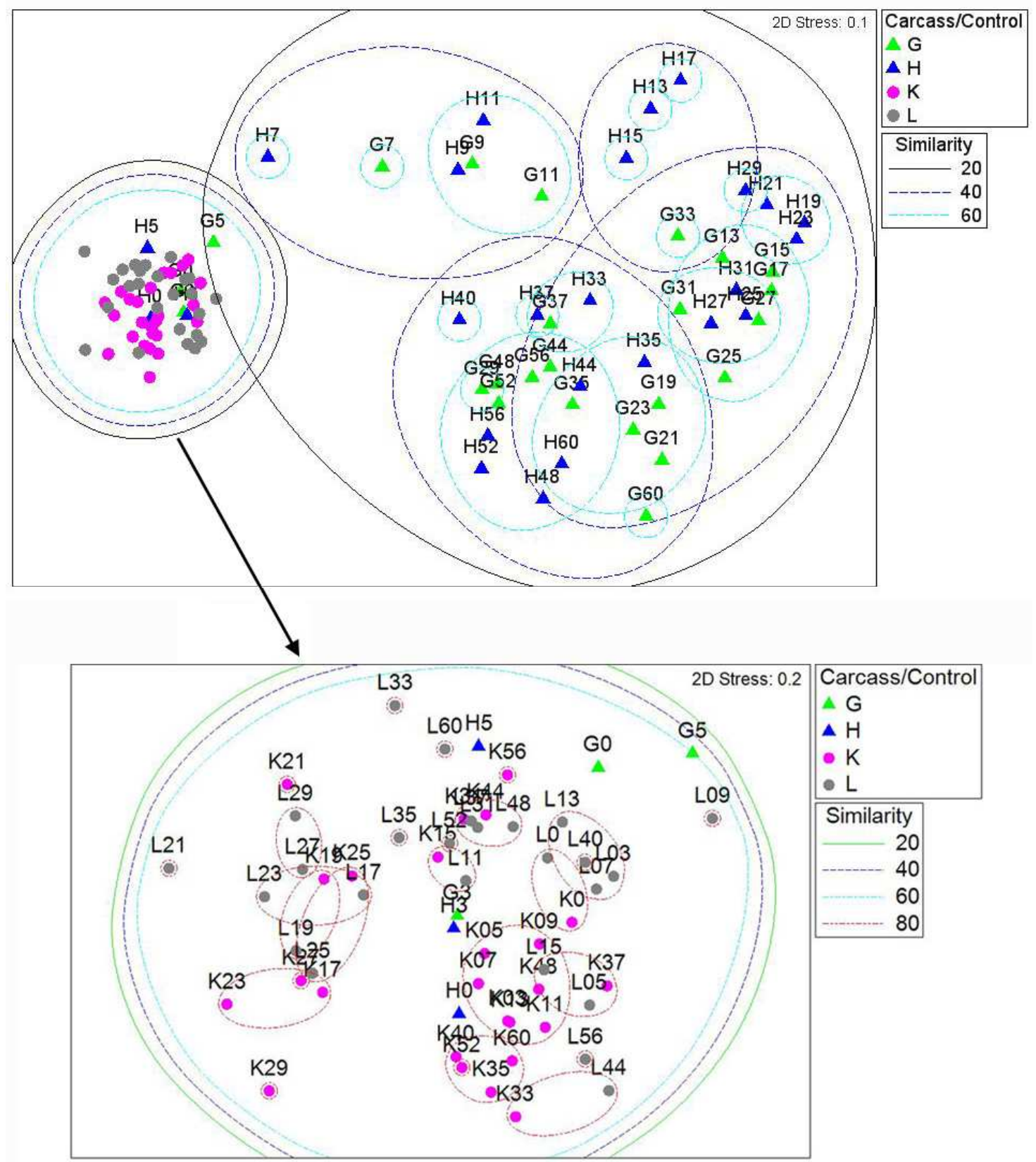

Figure 5.21 MDS plot of T-RFLP data from all soil samples (from under carcasses $H$ and $G$ and controls $K$ and $L$ ) and of T-RFLP data taken from the control sample cluster.

The samples collected from under the carcasses on sand showed a different clustering pattern to the soil samples, with three distinct clusters forming (Figure 5.22). The first included the 
earliest samples, days 5 and 7 (ADDs 85 and 115), followed by a second cluster that encompasses samples from day 11 to day 37 (ADDs 171 to 445 ) and the third, of days 40 to 60 (ADDs 486 to 631). As with the soil plots, the replicate sand plots showed highly similar clustering patterns (with a RELATE $\rho$ value of $0.848, \mathrm{p}<0.01$ ). Figure 5.23 shows that there was a clear difference between the bacterial communities from the soil and sand plots, with samples from under carcasses I and $\mathrm{J}$ (sand) plotting mostly away from the samples from under carcasses $\mathrm{G}$ and $\mathrm{H}$ (soil). When the sand and soil plots were compared using RELATE, $\rho$ values of between 0.497 and $0.572(\mathrm{p}<0.05)$ were obtained, suggesting that there was a reasonable degree of similarity between the patterns regardless of substrate.

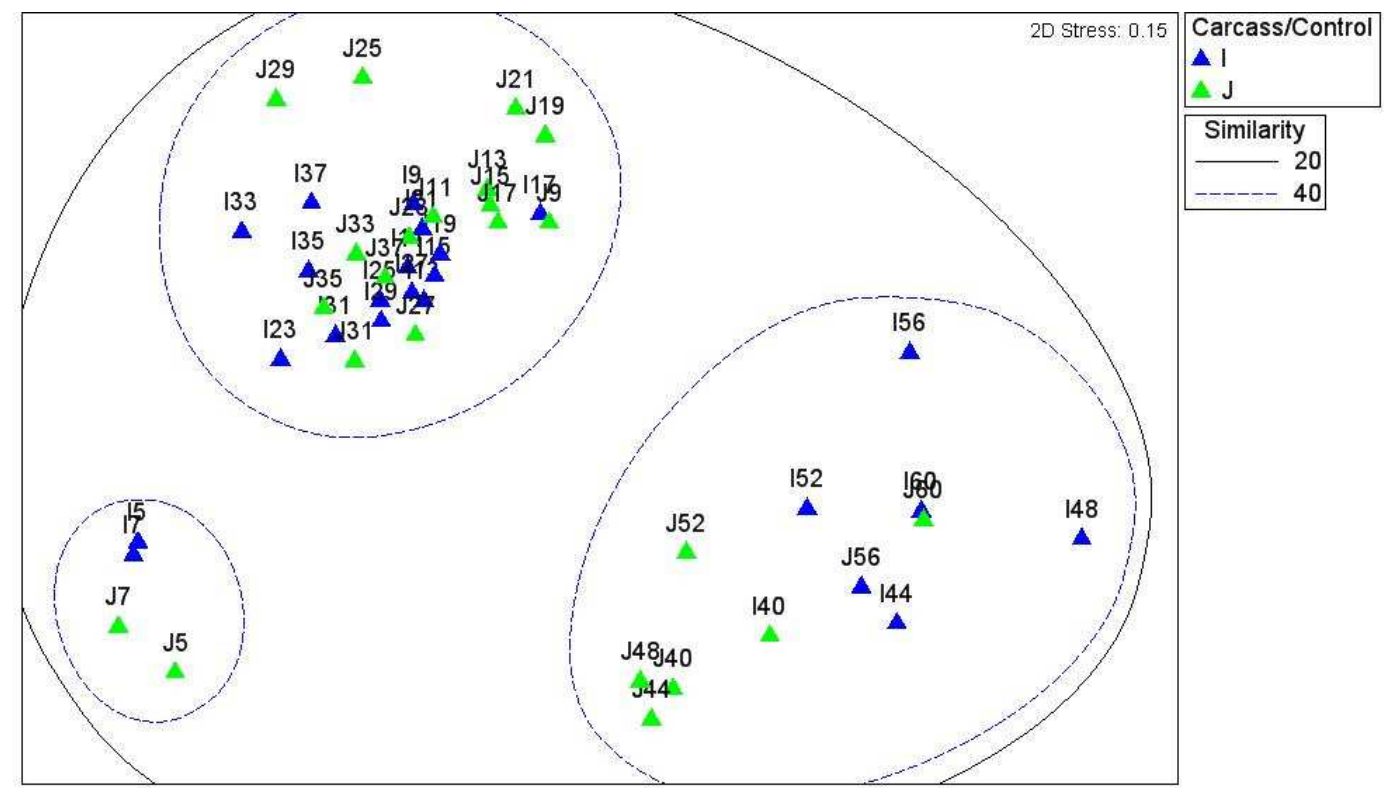

Figure 5.22 MDS plot of T-RFLP data from samples collected under the two carcasses on sand (I and J). The Bray-Curtis similarity index was used on square root transformed data. Samples are denoted by the number of calendar days post-death. 


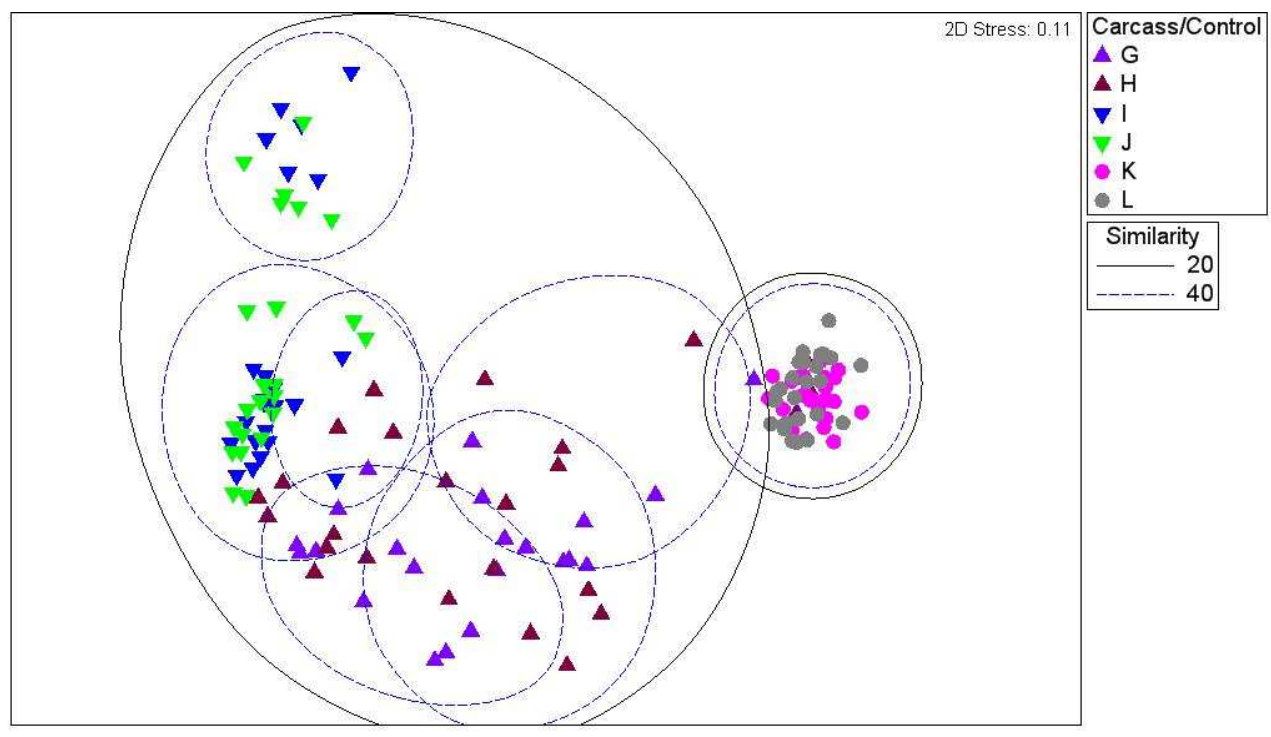

Figure 5.23 MDS plot of T-RFLP data from samples collected under the four carcasses (on soil and sand) and the soil control samples.

\subsubsection{DGGE}

Seventeen selected samples from under each carcass were run on DGGE gels as per the method described in section 3.4.4. The samples were chosen to cover the entire decomposition period sampled. Figures 5.24 and 5.25 show gel photographs of the DGGE gels for samples from carcasses G and J. An additional control sample (G0) was run on every gel for this experiment to help alignment of gels during data analysis, and a different DNA ladder which gave more bands (1 kb DNA ladder, Invitrogen) was used for the three marker lanes (the $100 \mathrm{bp}$ ladder was used in the previous experiment). Sixteen control soil samples were selected (seven each from $\mathrm{K}$ and $\mathrm{L}$ and the time 0 control samples from $\mathrm{G}$ and $\mathrm{H}$ prior to carcass placement) and were run on a single DGGE gel (Figure 5.26). 


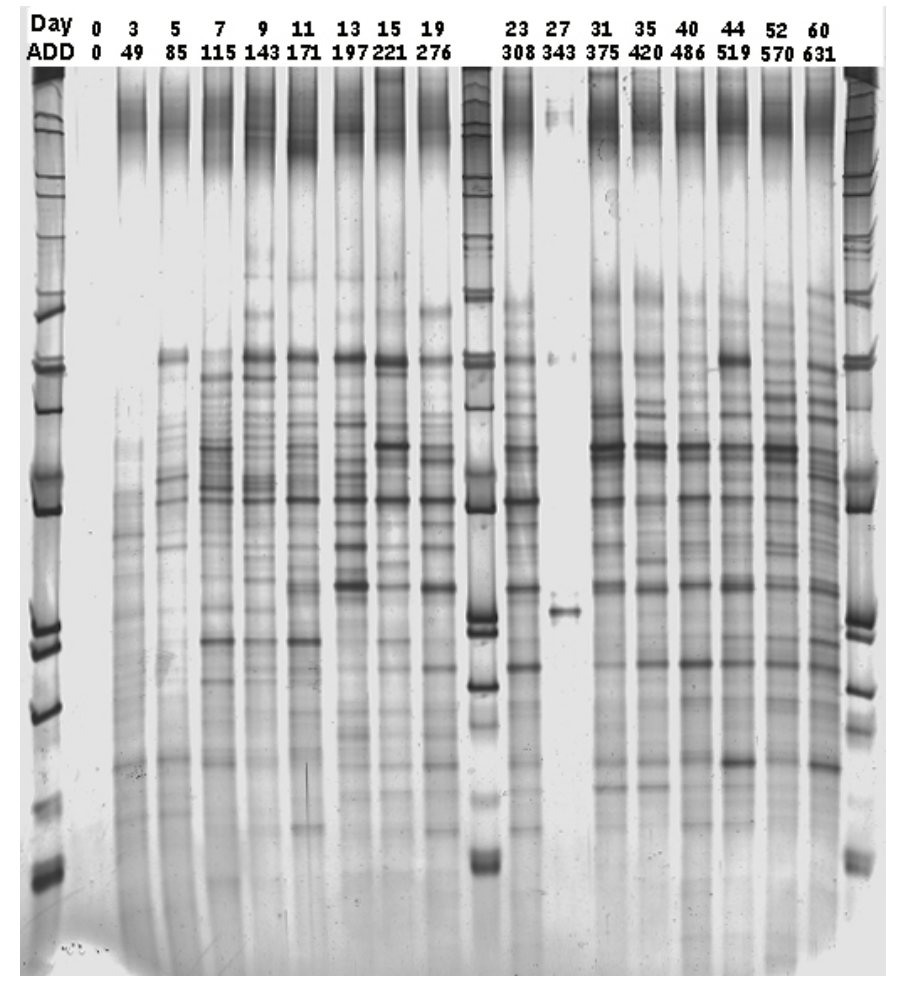

Figure 5.24 Photograph of DGGE gel analysisof bacterial communities from selected samples collected under carcass $G$ (on soil).

Figure 5.25 Photograph of DGGE gel analysis of bacterial communities from selected samples collected under carcass I (on sand).

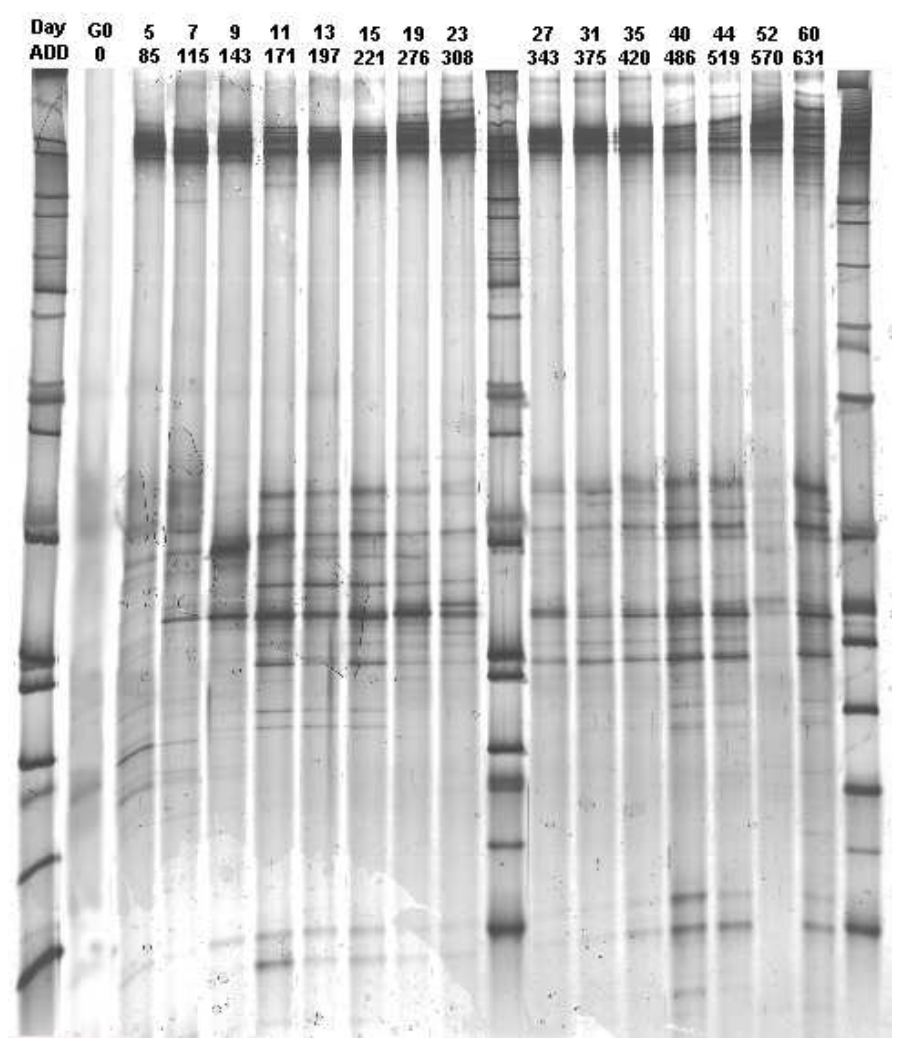




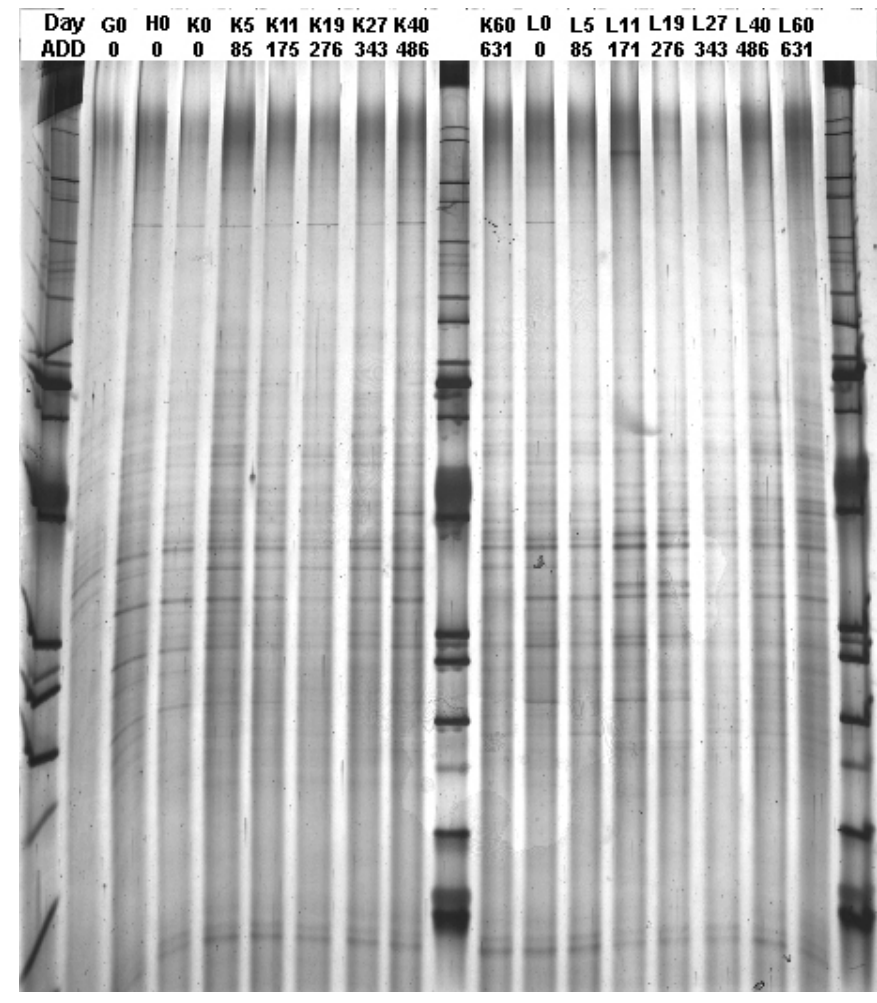

Figure 5.26 Photograph of DGGE gel analysis of bacterial communities from soil control samples.

The soil samples collected early in decomposition (up until around day 7) show fainter patterns than the samples collected later in decomposition. The same amount of DNA was loaded into each well, and exactly the same conditions were used for pouring and running all the gels, so it is likely that the original soil community was much more diverse prior to decomposition fluid purging, exhibiting more numerous, but fainter bands. This can be also seen in the control soil sample gel (Figure 5.26) where the bands are much finer and fainter than in the decomposition gels run under exactly the same conditions. There are also more, but fainter bands in the control sample profiles than in those collected during active decomposition. Unfortunately, a soil control sample, G0, was arbitrarily chosen as the one sample loaded onto every gel to aid in alignment of lanes from different gels. Because of the very faint banding in this sample compared with the decomposition samples, this lane did not show up well when stained, making inter-gel variation difficult. No clear banding pattern was visible for this sample on the carcass G sample gel (Figure 5.24) so this lane was excluded from further data analysis. 


\begin{tabular}{|c|c|c|c|c|c|}
\hline & \multicolumn{3}{|c|}{ Soil } & \multicolumn{3}{c|}{ Sand } \\
\cline { 2 - 7 } & G & H & Controls & I & J \\
\hline Mean number of bands in each profile & 23 & 22 & 33 & 17 & 22 \\
\hline Total number of different bands per gel & 56 & 48 & 40 & 37 & 42 \\
\hline
\end{tabular}

Table 5.3 Number of bands present in profiles from each carcass.

\subsubsection{Multi-Dimensional Scaling (MDS) Analysis}

The DGGE data from each carcass were analysed using MDS as described in section 3.4.5.2. Figures 5.28 and 5.29 shows the MDS plots of the DGGE profile data from samples collected under carcasses $\mathrm{G}$ and $\mathrm{H}$ respectively. Figures 5.30 and 5.31 show the MDS plot for samples from carcasses I and J, and the selected soil control data are plotted in Figure 5.32.

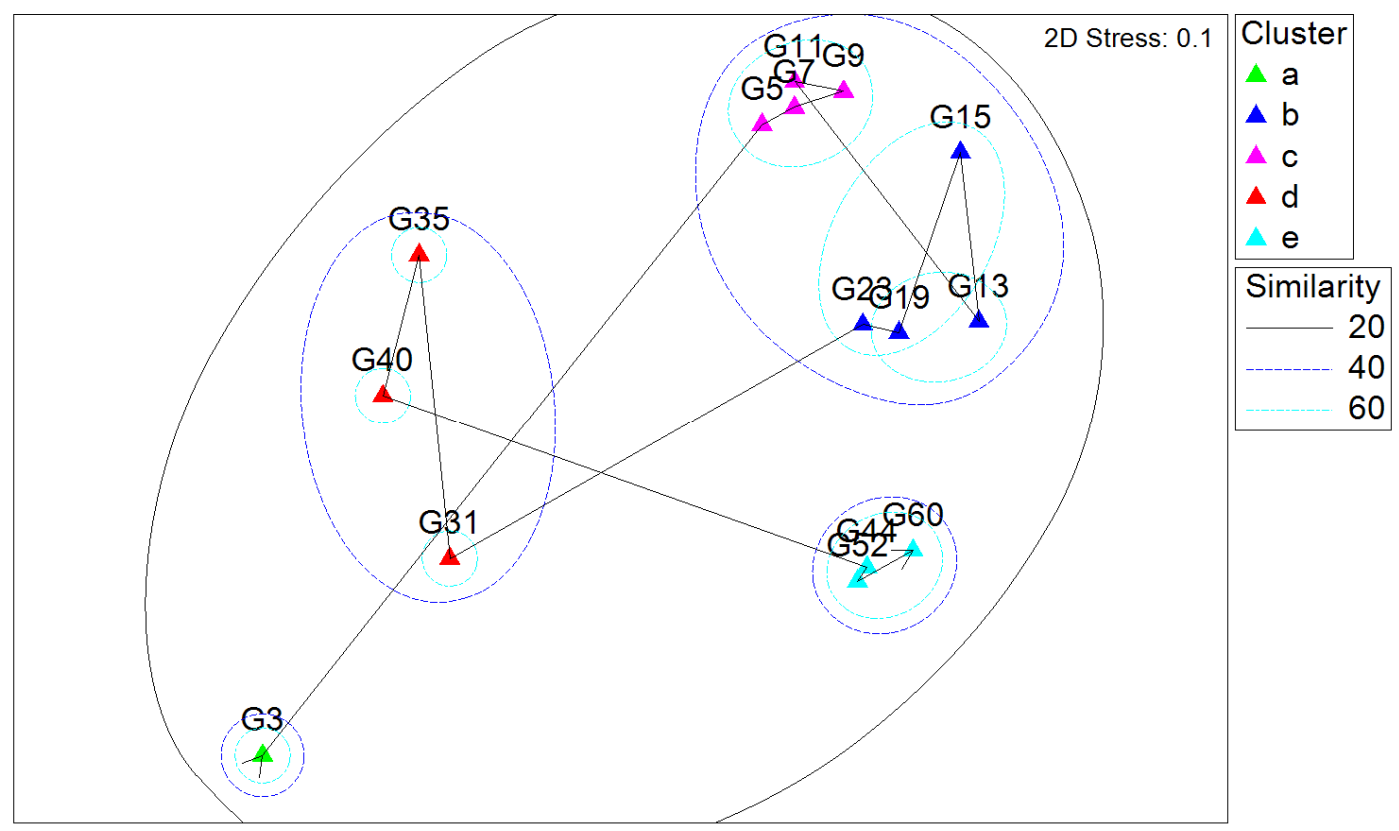

Figure 5.27 MDS plot of DGGE data from samples collected from under carcass $G$ (on soil). Five statistically significant clusters were distinguished using CLUSTER. The Bray-Curtis similarity index was used on square root transformed data. Samples are denoted by the number of calendar days post-death. 


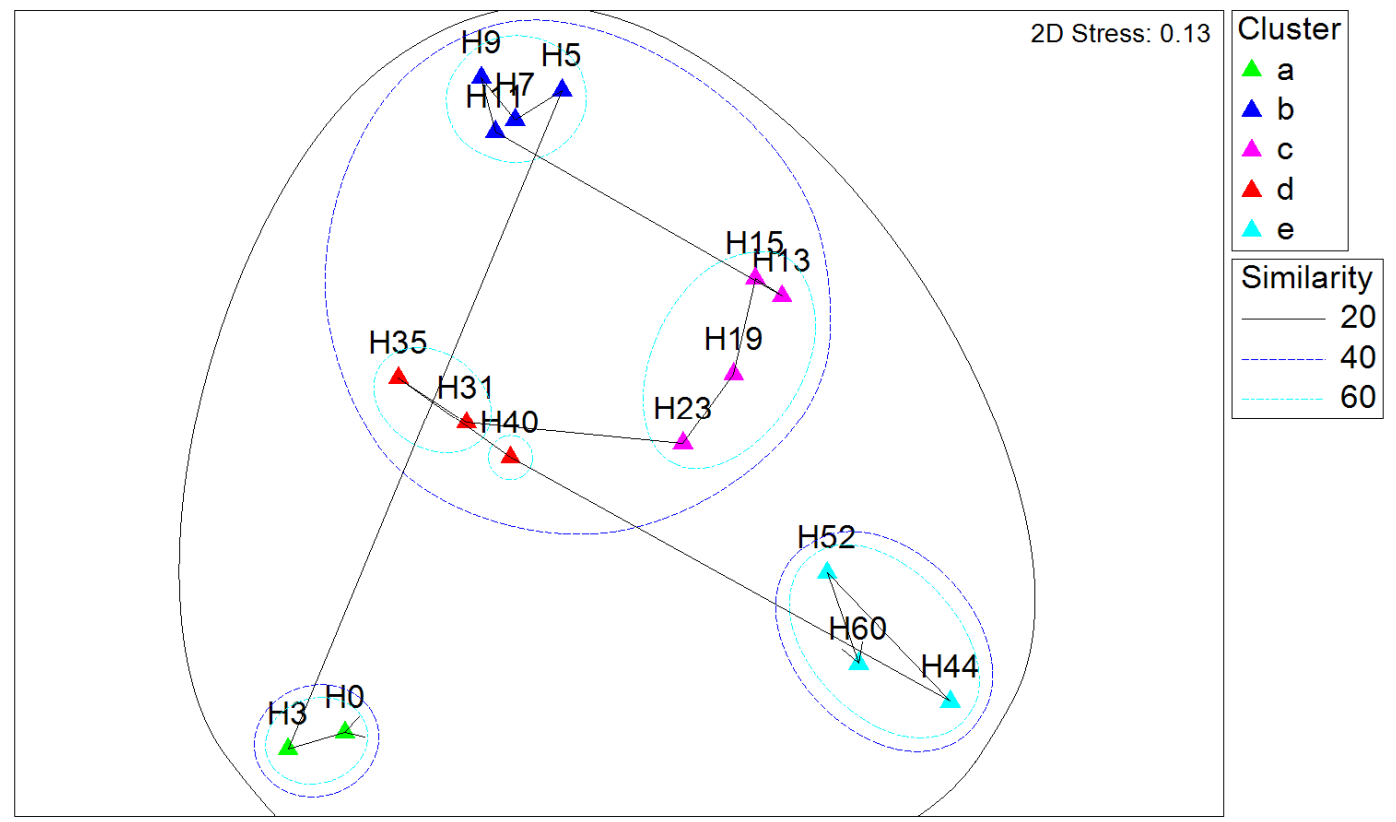

Figure 5.28 MDS plot of DGGE data from samples collected from under carcass $\mathrm{H}$ (on soil). Five statistically significant clusters were distinguished using CLUSTER. The Bray-Curtis similarity index was used on square root transformed data. Samples are denoted by the number of calendar days post-death.

The DGGE analysis of the samples from the soil decomposition plots plotted slightly differently, using MDS, to the T-RFLP analysis results, although the replicate carcasses gave very similar DGGE MDS plots with high pattern similarity $(\rho=0.877, p<0.1)$. The day 5 (ADD 85) sample plotted closely with later samples from days 7, 9 and 11 (ADDs 115 to 171) rather than with the early day 0 and 3 (ADDs 0 and 49) samples. There also seems to be more defined clustering of samples using the DGGE data, with days 13 to 23 (ADDs 197 to 308), 35 to 40 (ADDs 420 to 486 ) and 44 to 60 (ADDs 519 to 631) forming distinct clusters for both carcasses on soil. 


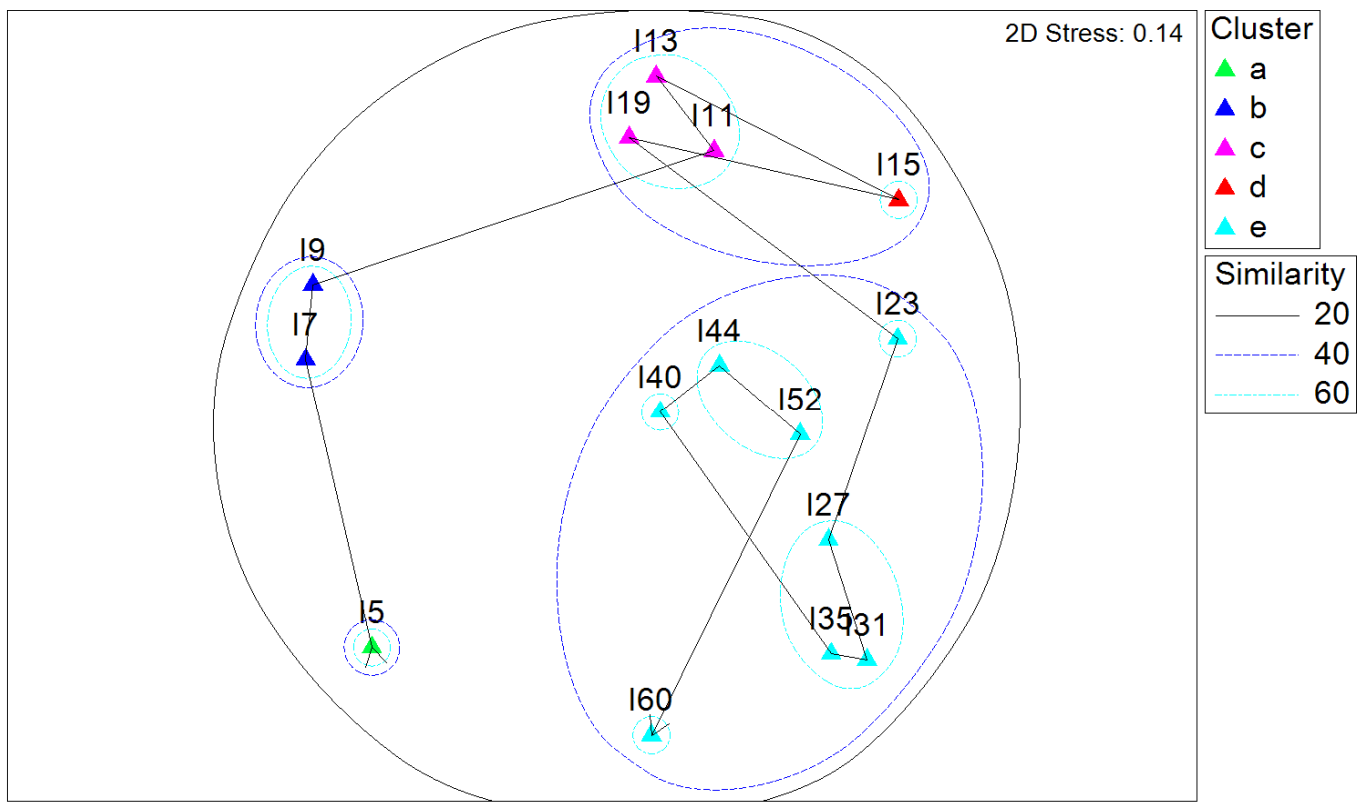

Figure 5.29 MDS plot of DGGE data from samples collected from under carcass I (on sand). Five statistically significant clusters were distinguished using CLUSTER. The Bray-Curtis similarity index was used on square root transformed data. Samples are denoted by the number of calendar days post-death.

The samples from under the carcasses on sand also showed different clustering patterns using DGGE compared with T-RFLP (Figures 5.23). The initial samples, days 5 and 7 (ADDs 85 and 115) clustered away from the later samples, as was seen with the T-RFLP plot, but for the DGGE profiles, the samples from day 9 (ADD 143) also groups with these earlier samples. The T-RFLP data showed two large clusters of days 11 to 37 (ADDs 171 to 445) and days 40 to 60 (ADDs 486 to 631), whereas the DGGE data shows clusters of days 11 to 19 (ADDs 171 to 267 ) and 23 to 60 (ADDs 308 to 631) for carcass I samples, and clusters of days 11 to 27 (ADDs 171 to 343), 31 to 44 (ADDs 375 to 519) and 52 to 60 (ADDs 570 to 631) for carcass J. These two plots showed less similarity in their overall patterns, with a RELATE $\rho$ value of $0.329, \mathrm{p}<0.01$. 


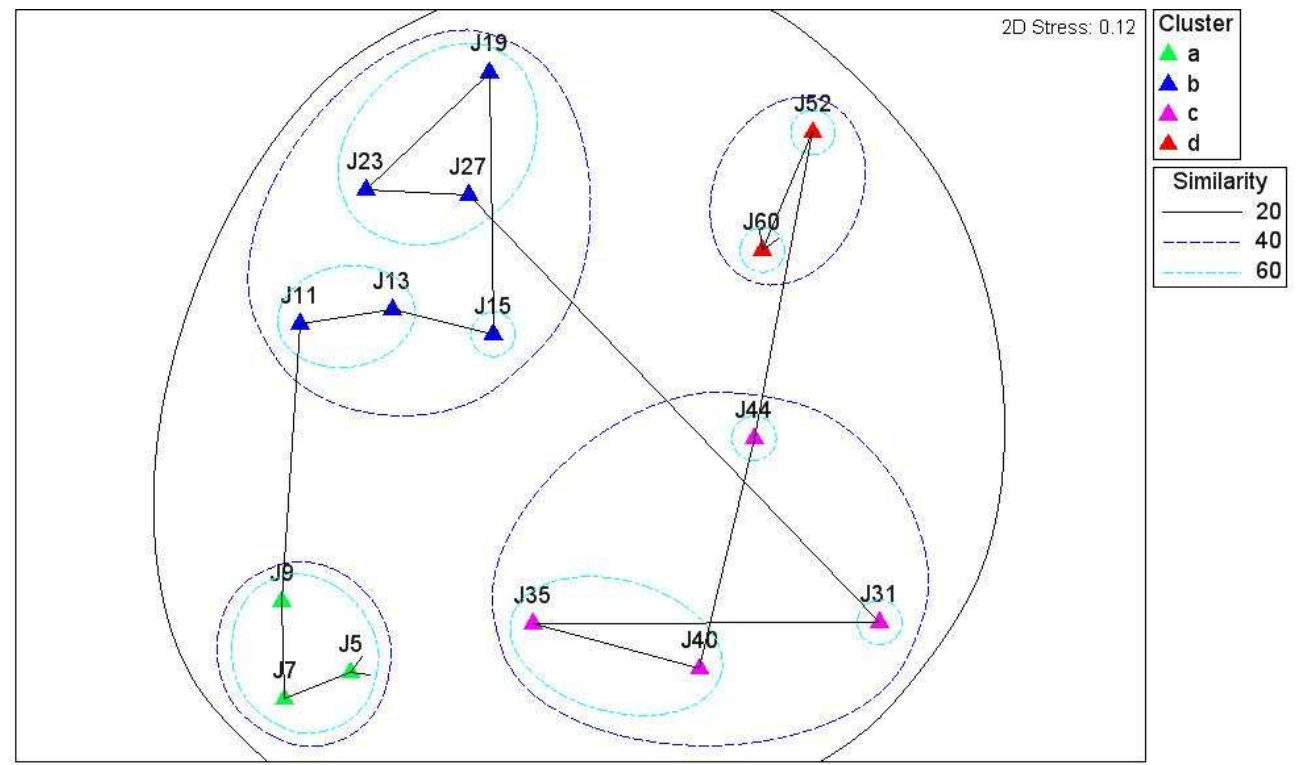

Figure 5.30 MDS plot of DGGE data from samples collected from under carcass $J$ (on sand). Four statistically significant clusters were distinguished using CLUSTER. The Bray-Curtis similarity index was used on square root transformed data. Samples are denoted by the number of calendar days post-death.

The soil control samples clustered closely, within the $80 \%$ similarity threshold using the DGGE analysis. This is closer similarity than was seen for the same samples using T-RFLP (Figure 5.22).

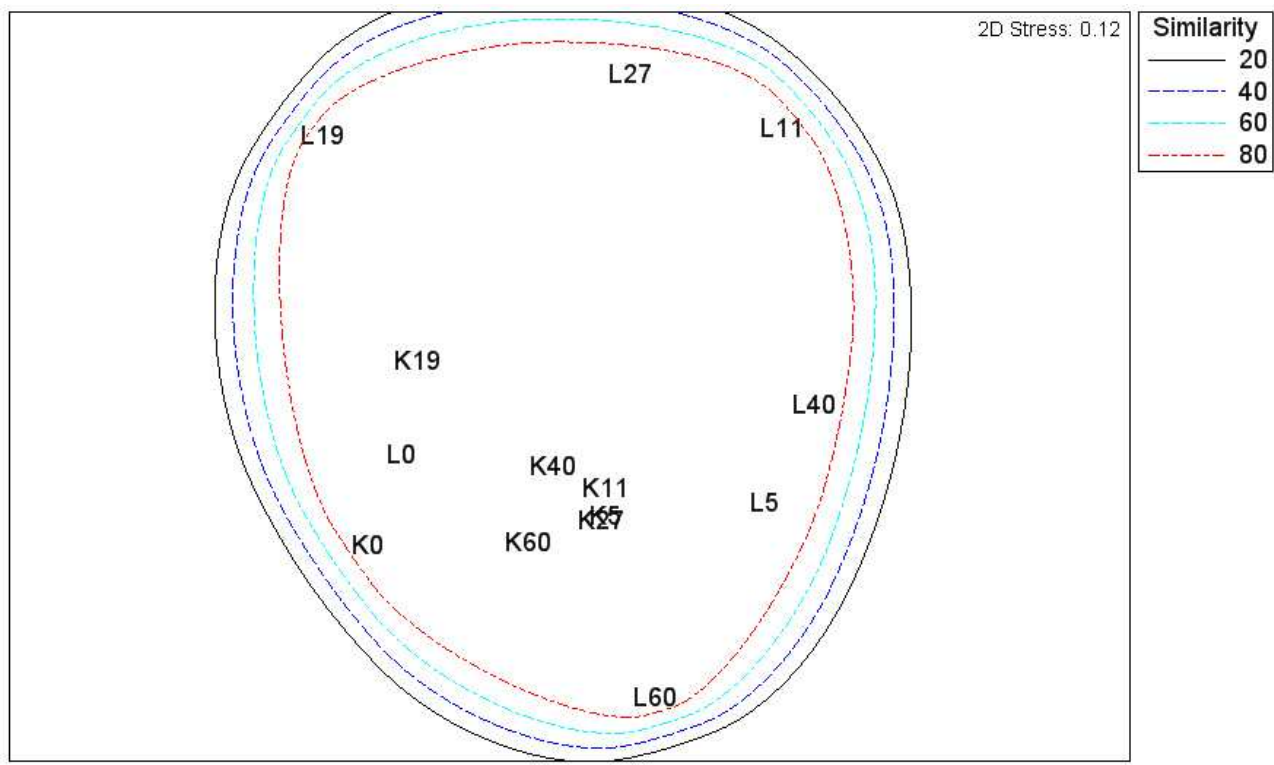

Figure 5.31 MDS plot of DGGE data from the control soil samples. The Bray-Curtis similarity index was used on square root transformed data. Samples are denoted by the number of calendar days post-death. 


\subsection{Discussion}

\subsubsection{Temperature, $\mathrm{RH}$ and rainfall}

The mean daily air temperature for this experiment was slightly lower than in the previous experiment $\left(10.5^{\circ} \mathrm{C}\right.$ compared with $\left.11.5^{\circ} \mathrm{C}\right)$. The mean daily rainfall for this experiment, however, was close to double that recorded during the previous experiment $(4.37 \mathrm{~mm}$ compared with $2.81 \mathrm{~mm}$ ). This is compounded by the placement of the cages at the eastern end of the site, where the cages were more exposed and not sheltered from heavy rainfall by the table roofing as they were in the previous experiment. High winds during the course of the experiment meant that the cage lids were occasionally blown off, despite efforts to weigh down and also tie down the lids, leading to water-logging of the area surrounding the carcasses. This was a particular problem with the sand trays, but the soil plots also experienced some water-logging.

\subsubsection{Decomposition}

The carcasses on soil in this experiment decomposed in a similar manner to those in the previous experiment (Chapter 4), although those carcasses were placed on sand. The carcasses on sand in this experiment, however, exhibited a decreased rate of decomposition, most likely due to the waterlogging of the experiment which would have reduced oxygen availability in the plots substantially, affecting any aerobic microbial activities. A notable difference was the lack of substantial maggot masses on the four carcasses in this experiment, on both sand and soil. The maggot masses on the carcasses on sand were drowned on a few occasions due to the water-logging, but the soil plot maggot masses were decreased in size also. This could in part be due to the higher rainfall and humidity experienced during the second experimental period which can affect insect activity (Lopes de Carvalho and Linhares, 
2001). The duplicate carcasses displayed very similar decomposition with both carcasses on each substrate moving through the visual stages of decomposition at the same times.

\subsubsection{Chemistry \& Biochemistry}

\subsubsection{1 pH/Ammonium/Nitrate/Urease}

The $\mathrm{pH}$, ammonium and nitrate concentrations and potential urease activity are intrinsically linked through their being part of the nitrogen cycle. For this reason, the urease enzyme assay results are discussed here with the other nitrogen cycle chemistry.

The $\mathrm{pH}$ of an environment has a strong effect on what types of micro-organisms can inhabit it. In general, most bacterial and fungal groups prefer a neutral $\mathrm{pH}$, but their tolerances can vary widely. Bacteria tend to predominate in soils with a $\mathrm{pH}$ slightly above 7 , whereas in soils with a $\mathrm{pH}$ below 5.5, fungi predominate due to the lack of bacterial competition (Stotzky, 1997). Some bacterial species, however, prefer a low pH environment, including the Acidobacteria (Jones et al., 2009) and many Actinobacterial species (Niva et al., 2006).

As with the previous experiment, the $\mathrm{pH}$ of both the sand and soil plots increased quickly in response to the decomposition events. This is thought to be a result of ammonification of proteins and other organic nitrogen sources from the carcass (Vass et al., 1992; Haslam and Tibbett, 2009). Microbes have been shown to invest energy into the synthesis of enzymes to acquire the nutrients they need when degrading low quality substrates (with a high carbon:nitrogen ratio). If the nutrients are readily available from the detritus, as would be the case in carcass decomposition, the excess nitrogen is mineralised to ammonium. This is known as ammonification and is performed by many microbial species that use amino acids as a carbon source. Much of the ammonia is volatilised and returned to the atmosphere and the rest is retained in the soil system as ammonium ions. A higher $\mathrm{pH}$ increases the amount of ammonia that is volatilised. 
The ammonium ion data for the carcasses both on soil and sand show a big increase in the concentration of ammonium ions in the underlying substrate, beginning to rise at day 5 (ADD 67) when the carcasses were still in the bloat stage, and peaking between days 12 and 16 (ADDs 185 and 234). This is when the maggot masses were at their greatest, at the beginning of the active decay stage and muscle was rapidly being broken down by the insect larvae and microbial species. The ammonium levels remained elevated until the end of the sampling period, as did the $\mathrm{pH}$. The results from the previous experiment (Chapter 4) suggest that the $\mathrm{pH}$ of the sand plots could be expected to remain high for some time after a decomposition event, as the $\mathrm{pH}$ had not begun to decrease after 155 days (ADD 1285). The soil plots may be expected to show a decrease earlier than the sand plots due to the presence of the soil microbial community, but this was not seen in the 60 day sampling period used in this experiment. In Vass et al.'s (1992) study, the pH began to decrease as early as ADD 750, eventually dropping to below the soil's initial $\mathrm{pH}$ in an acidic shift. This was not observed in this study due to the shortened sampling period used.

Urease is the enzyme that hydrolyses urea to yield ammonia and carbon dioxide (Mobley and Hausinger, 1989). At physiological $\mathrm{pH}$, the carbonic acid proton dissociates and the ammonia molecules equilibrate with water to become protonated, resulting in a net increase in $\mathrm{pH}$. Mammals excrete up to 20 pounds of nitrogenous waste as urea each year, to avoid build-up of toxic ammonia within the body (Burne and Chen, 2000). Most urea created in the liver is excreted via the kidneys, but up to $20-25 \%$ also enters the gastrointestinal tract where it is used by the gut microflora as a nitrogen source and mucosal secretions also contain some urea. Saliva for example can contain between 1-10 mM urea (Burne and Chen, 2000).

Urease activity is found in a wide range of bacterial species from many divisions, including those found in soil and in human and porcine gastrointestinal tracts (Mobley and Hausinger, 1989). Urease is particularly important in the ruminant gut, as it enables breakdown of urea to 
ammonia which provides the animal with its main nitrogen source. The most commonly isolated culturable ureolytic bacteria in the pig gut is Streptococcus (Varel et al., 1987). Peptostreptococcus productus is the most common ureolytic organism found in human faeces (Wozny et al., 1977).

Maggots (Lucilia sericata) have been shown to contain urease activity, but those raised aseptically exhibited reduced levels of the enzyme, suggesting that the endosymbiotic microbial species are largely responsible for this activity (Robinson and Baker, 1939).

In this experiment, there was a spike of potential urease activity in the soil decomposition plots which was not seen in the sand plots. The sand plots did have significantly higher potential urease activity than the controls, but it was more constant with no activity spike as was seen in the soil plots. This suggests that urease activity could have been contributed to the plots by the gastrointestinal microbiota, the maggots and the maggot-associated microorganisms. There was also an obvious up-regulation of urease activity within the existing soil microbial population as well, around the ADD 185 and 250 times.

Whilst urease in soil is mostly of bacterial origin, it also exists freely in soil, either adsorbed to clay particles or free in solution. If adsorbed to soil particles, the enzyme is afforded some protection from protease degradation, and the structure of urease may further prevent degradation (Davet, 2004). Urease-like activity has been demonstrated in Alaskan soils frozen for over 9000 years (Skujins and McLaren, 1969), in peat-land soils after 6000 years (Speir and Ross, 1990).

Aerobic nitrification can occur (ammonium to nitrite to nitrate) via two steps carried out by different groups of soil bacteria (e.g. Nitrosomonas ammonia to nitrite and Nitrobacter nitrite to nitrate) although archaea are thought to also play a major role in ammonia oxidation in soil (Leininger et al., 2006). Nitrite ammonification can return the nitrite to ammonium ions and 
this can be performed by Clostridium species. Denitrification converts nitrite to molecular nitrogen $\left(\mathrm{N}_{2}\right)$. A number of species, including Pseudomonas, Moraxella, Spirillum, Thiobacillus and Bacillus are capable of denitrification. Denitrification accounts for $10-15 \%$ of nitric nitrogen production, but in anoxic conditions (such as after heavy rain) this can be much higher (Davet, 2004). Nitrate is often leached to ground and surface waters, but can also be converted back to ammonium ions via assimilatory nitrate reduction.

Ammonia is a strong inhibitor of Nitrobacter which is responsible for the transformation of nitrites to nitrates. Most fungi are also adversely affected by high ammonia concentrations and by high $\mathrm{pH}$. Additionally, nitrification is an aerobic process, so if oxygen under a carcass were to become limited, this process is likely to cease. Nitrifiers are also more susceptible to unfavourable conditions than ammonifiers, so re-establishment of the initial equilibrium can be slow (Davet, 2004). Nitrifying bacteria, however, are quite sensitive to environmental pH with the two groups preferring a $\mathrm{pH}$ of 5-8 and 7-9 respectively (Forbes, 2008).

In favourable conditions, ammonia is rapidly converted to nitrite, then nitrate. Denitrification is the regulatory mechanism that stops the nitrate from building up. Under the carcasses, the ammonium concentration rapidly increases along with the $\mathrm{pH}$ early in decomposition. The ammonium concentration quickly drops after the initial spike and steadies around $2000 \mathrm{mg} / \mathrm{kg}$. This coincides with the time when the maggot mass is at its greatest. The $\mathrm{pH}$ however stays high throughout the decomposition period studied. The nitrate peaks and drops to around baselines for the soil samples at around the same time as when the ammonium peaks. The sand nitrate fluctuates more, perhaps because these samples lack the denitrifying soil species. This suggests that the amino acid degradation to ammonia is mediated partly by body microflora. Nitrification is performed by aerobic chemoautotrophs in soil, but nitrification by some heterotrophic bacteria fungi has also been demonstrated (Castignetti and Hollocher, 
1984). Without measuring each of the nitrogenous compounds in the samples, it is difficult to determine the processes occurring within the nitrogen cycle.

\subsubsection{Enzymes}

Enzyme activity in soil has long been studied as a means of understanding the functioning of soil microbial communities (Gianfreda, 2006). Enzymes in soil can come from other sources such as plant and animal cells, but because of the considerable biomass that microbes contribute to soil, most soil enzyme activity derives from microbial sources (Nannipieri et al., 2002). Whilst most enzymes originate from inside live microbial cells, some enzymes are thought to persist for some time extra-cellularly in the soil environment. This is disputed by some who claim that free proteins are unlikely to persist for long in an environment as inhospitable as soil (Gianfreda, 2006), even if trapped by humic molecules or adsorbed by clays.

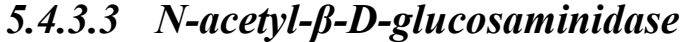

$\mathrm{N}$-acetyl- $\beta$-D-glucosaminidase is a chitinase which hydrolyses terminal, non-reducing 2acetamido-2-deoxy- $\beta$-D-glucose residues in chitin. Chitin is the second most abundant polymer on earth after cellulose (Duo-Chuan, 2006), forming the structural component of the cell wall in fungi and in the exo-skeletons and pupae of insects. Chitin is hydrolysed to Nacetyl-glucosamine (GlcNAc) by chitinase enzymes. Many bacterial and fungal species, as well as mammals, have been shown to possess chitinolytic activity. One of the best studied groups capable of GlcNAc hydrolysis is the Actinomycetes (Metcalfe et al., 2002), although it is argued that the majority of chitinase activity in soil may come from fungi (Miller et al., 1998). Chitinase activity is often used as an indicator of fungal activity for this reason (Miller et al., 1998). 
It was originally hypothesized, in the planning of this experiment, that there may be an increase in potential chitinase activity following pupation of the insect species due to an upregulation of chitin-degrading microbial species in the soil when the more readily utilisable decomposition products had already been degraded but when the more recalcitrant compounds, such as pupal casings remained. Within the decomposition plots, however, the potential chitinase activity appeared to reach a peak at around ADD 200-234 for both soil and sand, much earlier than when the larvae were seem to enter pupation. When compared with the documentation of decomposition progress (Figures 5.4 and 5.5) this appeared to coincide with when the maggot mass was at its greatest for three of the four pigs, and also when the carcasses were going through the stage of active decay. The sand samples did not show as high a potential activity as the soil samples, but both were significantly higher than the controls, suggesting that at least some chitinase activity was derived from the body microflora, the body itself, or the insect larvae. Two variants of human derived chitinases have been isolated: one, chitotriosidase, from epithelial cells and macrophages of mucus containing organs (particularly eyes, intestines and lungs) and is thought to play a role in protection against fungal and insect pathogens (Overdijk et al., 1996; Tjoelker et al., 2000; Boot et al., 2001) and another, acidic mammalian chitinase (AMCase), which is present in the lung and gastrointestinal tract (Bussink et al., 2007). This may also be the case for pigs. Some insect larvae, including those of the common housefly (Musca domestica) have also been shown to contain chitinolytic enzymes (Singh and Vardanis, 1984; Kramer and Muthukrishnan, 1997) which are expressed particularly during moulting and pupation. A number of entomopathogenic nematode species have been found to produce chitinases which helps them penetrate and colonise their host (Wu et al., 1996). Plants are also known to produce chitinases to protect themselves against fungal attack (Schlumbaum et al., 1986). It is unknown how many of the many variants of chitinase enzymes are able to cleave the assay substrate 4-MUB- $\beta$-D-N-acetyl-glucosamide, but it appears likely, given the increase in 
potential chitinase activity in the sand decomposition plots, that either non-soil derived microbial species or human, pig or insect enzymes are also detected. As with urease, it seems unlikely that the chitinase enzyme could persist free for long in the harsh decomposition environment, rendering the pig carcass itself unlikely as a source of the activity seen. Because the highest levels of potential activity were seen when the maggot mass was at it's greatest, it is possible that insect derived chitinases were detected, or it may be that because active decay was also at its peak at around this time, the body microfloral species may also have contributed.

\subsubsection{4 $\beta$-glucosidase}

$\beta$-glucosidases are widely distributed among animals, plants and micro-organisms, in particular in fungi (Hayano and Katami, 1977). They constitute a group of enzymes which catalyse the hydrolysis of non-reducing $\beta$-D-glucose residues with the release of $\beta$-D-glucose. $\beta$-glucosidases are commonly found in soil as they catalyse the breakdown of cellulose, which is the structural component of plant cell walls (Hayano and Tubaki, 1985). This makes them an important part of the soil carbon cycle.

Mammalian carcasses do not contain cellulose, and therefore it was not expected that there would be any significant change in glucosidase potential activity in response to mammalian decomposition event. This assay was, however, included in this study as it is routinely performed in the ESR Ltd laboratory and was able to be performed simultaneously with the other fluorometric assays at no extra cost. Glucosidases are found widely in mammalian tissues, which could explain the small peak in potential activity seen in the sand samples from under the decomposing carcasses. The spike in activity occurred around ADD 143, which was around the time when active decay began in the carcasses on sand. Glucosidases have been found in the salivary glands and guts of termites and other plant parasites (Tokuda et al., 
2007), but are not likely to be present in the larvae or adult forms of the insects colonising a decomposing pig carcass.

The soil samples from under cadavers $\mathrm{G}$ and $\mathrm{H}$ displayed a rise in potential activity, followed by a period of lower activity before a second rise. No satisfactory explanation for this activity has been found at this time.

\subsubsection{Acid Phosphatase}

Phosphatases are enzymes which remove the phosphate molecule from organic compounds such as phospholipids and nucleic acids. In soil, phosphatases often have two $\mathrm{pH}$ optima, one acid and one alkali. The potential activity of the acid phosphatases was measured in this study. Soil acid phosphatases catalyze the hydrolysis of organic phosphate esters to orthophosphate, and thus constitute an important link between biologically unavailable and bioavailable P pools in the soil (Speir and Ross, 1978). Phosphatase is produced by numerous species of microorganisms in response to low levels of inorganic $\mathrm{P}$, and its production and activity are inhibited by elevated levels of inorganic P (Speir and Ross, 1978). Human tissues contain acid phosphatases, but in relatively small quantities (Bull et al., 2002). Plants also produce phosphatases (Yin, 2006) as do insect larvae (Jones and Bowen, 1993).

The acid phosphatase assay results were not particularly informative as the soil control and decomposition data were not significantly different overall. All four soil plots showed a steady increase in potential activity towards the end of the experiment, suggesting involvement of some other factor that may well have masked any real differences. The sand control and decomposition data were significantly different overall, but showed no clear trends or identifiable patterns in potential activity. 


\subsubsection{Sulphatase}

Arylsulphatase plays an important part in sulphur cycling by catalysing the hydrolysis of organic sulphate esters. This assay was included because sulphur is an essential component of the human body, constituting $0.25 \%$ by weight (Haneklaus et al., 2007). Also, many sulphur containing compounds are produced during decomposition (Vass et al., 2008), which suggested that an insight into the sulphur cycling that occurs during decomposition may be informative. Arylsulphatase mainly originates from fungi and bacteria, although plants and animals produce this enzyme too ( $\mathrm{Li}$ and Sarah, 2003). Thirteen different sulphatase enzymes have been identified in humans, and in a number of other mammals (Sardellio et al., 2005). Insect sulphatases have been identified through genome searches (Sardellio et al., 2005).

Like the acid phosphatase assay, the sulphatase assay was not particularly informative. After a period of increased activity early on in the decomposition plots, all soils exhibited a steady increase in activity, including the control soils. Initially, this was thought to be contamination of the fluorometric substrate stocks, leading to premature cleavage of the substrate, but the rise in potential activity was not seen in the sand plots and any contamination should have been controlled for by using a range of controls including quenched soil standards. The small spike in activity in one of the sand decomposition plots was significant, indicating that at least some of the sulphatase activity seen in the soil plot activity spikes was derived from non-soil sources such as the carcass itself or the colonising insects.

When reflecting on the enzyme activity results, the potential effect of the rise in $\mathrm{pH}$ in the decomposition plots should also be taken into consideration. Each enzyme exhibits a characteristic $\mathrm{pH}$ profile, an optimum $\mathrm{pH}$ for its maximum activity and a specific stability which is related to soil $\mathrm{pH}$ (Gianfreda and Bollag, 1996). Often, enzyme activities of soils increase with an increase in $\mathrm{pH}$, which could be occurring to some degree in the decomposition plots, although the potential activity of most of the enzymes (particularly 
chitinase) decreased after its initial spike, despite the $\mathrm{pH}$ remaining high. The fluorometric assay method involves addition of $0.5 \mathrm{M} \mathrm{NaOH}$ to increase the $\mathrm{pH}$ after incubation for a maximum potential reading and to attempt to prevent differences in activity between samples from being caused by $\mathrm{pH}$ differences.

The four fluorometric substrates used in this experiment are thought to be stable over most of the $\mathrm{pH}$ range, although the chitinase substrate 4-MUB- $\beta-\mathrm{D}-\mathrm{N}$-acetyl-glucosamide becomes unstable at high pH (Niemi and Vepsalainen, 2005).

\subsubsection{T-RFLP and DGGE}

The MDS plots showing the relationship between samples over time demonstrated a definite succession occurring within the bacterial community under each carcass. The patterns were similar between replicate carcasses on both sand and soil, as shown by correlations being detected between each pair of replicates using the RELATE function of the PRIMER software. For both the T-RFLP and DGGE datasets, the MDS pattern of the samples from under carcass $\mathrm{H}$ (soil) also showed a correlation with the patterns generated from carcass I and J samples (sand), suggesting that the temporal changes occurring within the bacterial communities may be strongly mediated by the body-derived bacteria rather than being a response by the pre-existing soil organisms.

As with the previous experiment (Chapter 4), the T-RFLP and DGGE results, as displayed using MDS, visually looked to be quite different. Some of the temporal patterns displayed on the T-RFLP MDS plots (for carcasses $\mathrm{H}$ and J), however, did display some correlation with the patterns on the DGGE MDS plots. This indicates that while the two profiling techniques produce similar MDS plots with different clusterings, the overall sequence and direction of change is similar. The T-RFLP profile clusters seemed to match quite closely with changes in 
the stage of decomposition, whereas the DGGE clusters often had a lag evident, with the first samples from a new visual stage clustering with the previous stage's samples. The same samples were used to generate both the T-RFLP and DGGE profiles, so this is an unexpected result.

Unfortunately, once again, the DGGE results from different gels could not be directly compared because of a lack of a clear control. This was due to a poor random choice of a control. The soil control samples are thought to have generated more diffuse DGGE patterns with a greater number of faintly stained bands, due to the bacterial communities within them being more diverse. This could mean that the decomposition event is causing a general reduction in diversity in the soil, with greater proliferation of fewer species tolerant of the changed environment. This was echoed by the T-RFLP results which showed a greater diversity of T-RFs in the control soil profiles than in and of the samples collected during the decomposition event. Greater numbers of bands/T-RFs were seen in the soil profiles than in the sand profiles which suggests that many soil derived phylotypes are being detected as well as the body derived organisms. Because DNA has been known to persist in the soil environment for some time following cell death (England and Trevors, 2003; Levy-Booth et al., 2007), it is not possible to draw conclusions from this experiment on whether the soil bacterial species are surviving during the decomposition event or if extracellular DNA is being detected. DNA is not particularly stable in unfavourable conditions and is less likely to bind to clay and humic substances in soil at higher pH (Levy-Booth et al., 2007) so it is unlikely that the DNA would persist for the entire 60 day period in which greater numbers of T-RFs were being detected for soil compared with the sand samples. 


\section{Chapter 6: Human Cadaver Decomposition on Soil and Sand}

\subsection{Introduction}

The University of Tennessee's Forensic Anthropological Research Facility is currently one of only two facilities in the world that are able to use donated human cadavers for forensic decomposition and anthropology research. It has a reputation for quality research and is heavily used as a training facility for law enforcement. The facility consists of a scrubby section of approximately $10,000 \mathrm{~m}^{2}$ adjacent to the University of Tennessee Medical Centre car park, and is bordered on one side by the Tennessee River. The facility is roughly half flat grassy terrain with moderate tree cover, and half on a slope, with slighter more dense tree cover. It is fenced to prevent access by unauthorized people and larger scavengers such as dogs, but is accessible by other wildlife including birds, cats, rodents, raccoons, reptiles and insects. Cadavers are delivered to the facility as they become available through donation by next-of-kin. Cadavers can come from any of the states immediately surrounding Tennessee and may be recent deaths, bodies that have partially decomposed, or bodies that have been held in morgues. Many of the bodies have been autopsied, but others, whose cause of death is known, have not. Cadavers are often frozen until required, but no freezer space was available at the time this experiment was run, so cadavers were placed at the experimental sites as they became available. This was also desirable as freezing is likely to have a major effect on the microbial community associated with the cadaver. Micozzi (1986) demonstrated that freezing also alters the rate and pattern of decay. 
The experiment was run over the period of 22nd August 2006 to 30th October 2006, during the US autumn. Laboratory work was performed at Oak Ridge National Laboratories in Oak Ridge, Tennessee as facilities were not available on site.

\subsection{Aim}

The aim of this experiment was to examine the bacterial communities associated with human cadaver decomposition. The experiment was designed to repeat those performed in New Zealand, using human cadavers instead of pig carcasses. This was to allow comparison of the results from carcasses and cadavers and to evaluate the validity of using pig carcasses as models for human decomposition.

\subsection{Methods}

The laboratory methods used in this experiment are as described in Chapter 3. Different laboratories were used, but the same methodological procedures were performed. The experimental set-up and sampling regime methods specific for this experiment are described below.

\subsubsection{Site Information}

Suitable sites were identified within the facility (see Figure 6.1). These sites were all along an internal fence-line and were chosen based on the fact that there had been no previous decomposition events on that site. The fence-line location also meant that the experiment would not interfere with the forensic training role of the facility. One cadaver was placed on a lower site due to its large size and difficulty in moving it to a fence-line location. The flattest possible sites were selected, but because the previously unused area was on a moderate slope, a totally flat site was not available. All reasonable attempts were made to 
level the sites used but all had a small degree of slope to them. Leaf litter and growing plant material was removed from each site by raking. An area of soil approximately $1 \mathrm{~m} \mathrm{x}$ $60 \mathrm{~cm}$ was dug to a depth of $10 \mathrm{~cm}$ and homogenised by raking and mixing. Root material and any small stones were removed by hand. Sieving of the soil (as was performed in the previous experiments) was not an option due to the short time period available before the first cadaver was available. Five time-zero control samples from different parts of each site were collected for soil DNA analysis and assessment of the effectiveness of the homogenisation. The soil was very friable and easily broken up and mixed. A control area $(\sim 20 \times 20 \mathrm{~cm})$ upslope from the cadaver site was also prepared in this manner.

Three cadavers were placed on soil, and three on sand. Clean, commercial-use sand was purchased and used in place of dune sand (from previous experiments) due to availability. This sand was pre-washed and did not require further washing as the silt content was very low. Acid washing of the sand was not an option due to time and laboratory limitations, but DNA extractions from the sand yielded undetectable amounts of DNA and bacterial counts from plating serial dilutions were negligible (section 3.1.2).

For the cadavers placed on sand, a pit $\sim 10 \mathrm{~cm}$ deep was dug and loose soil removed. A black polythene sheet was laid along the bottom of the pit and sand was placed in the pit to a depth of $\sim 8 \mathrm{~cm}$. A control area $(\sim 20 \times 20 \mathrm{~cm})$ upslope from each cadaver was also prepared in this manner.

All cadavers were covered with a black polythene sheet to shield the experiments from onlookers from outside the facility, and to promote complete decomposition. The primary goal of the facility is to allow skeletonisation of cadavers for use in anthropological studies. Leaving the cadavers uncovered during summer months often results in desiccation and mummification rather than complete decomposition. 


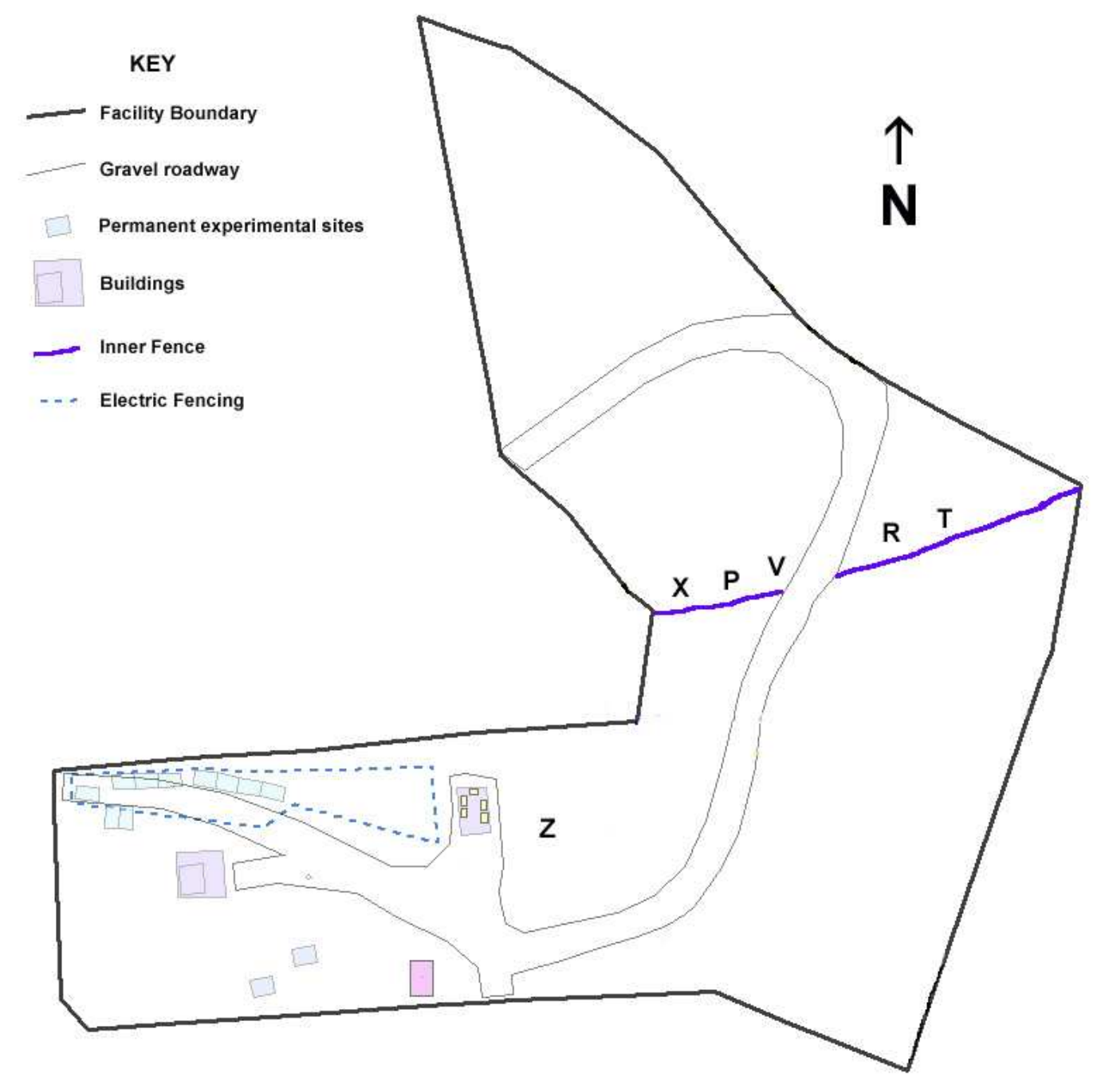

Figure 6.1 Site map of University of Tennessee Anthropological Facility with cadaver placement marked. Sites where cadavers were placed are marked with ID letters (Table 6.1).

\subsubsection{Cadaver Information}

Six cadavers became available for use during the time period available. Each cadaver was placed as part of the experiment as soon as it was delivered to the facility. This meant that cadavers were placed on different days within a 6-week period, except for cadavers V and $\mathrm{X}$, which were placed on the same day. Ideally, non-autopsied bodies would have been used, but three autopsied individuals were accepted for this study because of time restrictions and the unavailability of other cadavers. Internal organs had been replaced in the body cavity of autopsied individuals but the $\mathrm{Y}$ incisions were not closed. All cadavers 
were placed naked and facedown. Table 6.1 gives the information available about each cadaver. All six were Caucasian.

\begin{tabular}{|c|c|c|c|c|c|c|c|c|c|c|}
\hline ID & Age & Sex & $\begin{array}{c}\text { Weight } \\
\text { (kg) }\end{array}$ & $\begin{array}{c}\text { Height } \\
(\mathbf{c m})\end{array}$ & DOD & COD & Autopsy? & Substrate & $\begin{array}{c}\text { Control } \\
\text { Name }\end{array}$ & $\begin{array}{c}\text { Date } \\
\text { placed }\end{array}$ \\
\hline P & 69 & M & 159 & 184 & $20-08-06$ & Natural & No & Soil & O & $22-08-06$ \\
\hline R & 62 & F & 40 & 162.5 & $09-09-06$ & $\begin{array}{c}\text { Renal } \\
\text { Failure }\end{array}$ & No & Soil & Q & $11-09-06$ \\
\hline T & 43 & M & 59 & 169 & $28-08-06$ & Gunshot & Yes & Sand & S & $29-08-06$ \\
\hline V & 54 & F & 55 & 165.1 & $04-09-06$ & $\begin{array}{c}\text { Lung } \\
\text { cancer }\end{array}$ & No & Sand & U & $14-09-06$ \\
\hline X & 42 & M & 59 & 168 & $19-08-06$ & Hanging & Yes & Soil & W & $14-09-06$ \\
\hline Z & 42 & F & 100 & 168 & $17-09-06$ & Unknown & Yes & Sand & Y & $22-09-06$ \\
\hline
\end{tabular}

Table 6.1 Cadaver information. Each cadaver had a corresponding control site that was sampled in tandem with the body. COD = cause of death, DOD= date of death, PM=Post mortem.

\subsubsection{Sampling}

Soil and sand samples were collected from under each cadaver using a similar method to that used in the pig carcass experiments (sections 4.2.2 and 5.2.2). Rather than using a hammock method for lifting the cadaver, however, plastic mesh sheeting was placed under each cadaver, which allowed them to be tilted away from the underlying substrate for sampling underneath (see Figure 6.2). Sampling required two people due to the size of some of the cadavers. This permitted unhindered sampling. Cadavers were held away from the substrate only long enough to permit sampling, and were then replaced as close to their original position as possible. Photographs were taken of each cadaver to record decomposition progress and the black plastic covering replaced. 


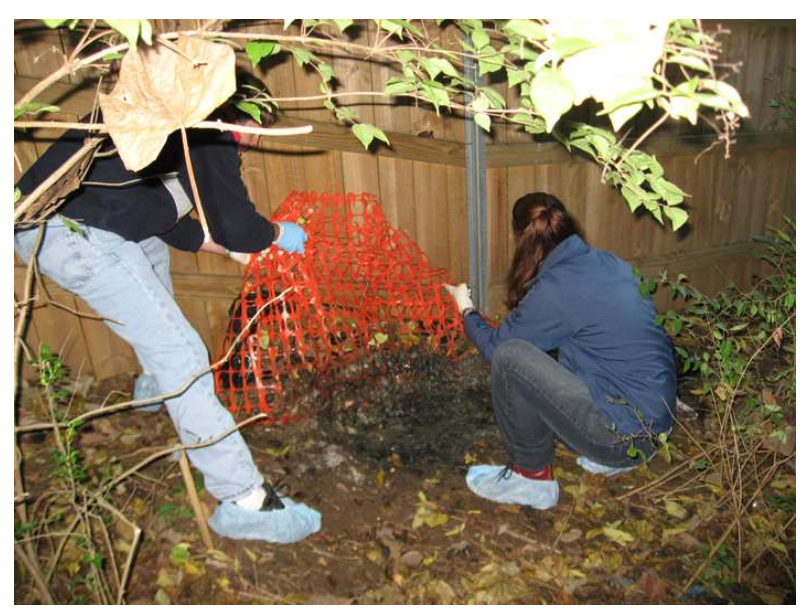

Figure 6.2 Sampling under cadaver $P$ using the plastic mesh to tilt the cadaver away from the substrate.

Ambient air and soil temperature (at $15.2 \mathrm{~cm}$ depth), barometric pressure and relative humidity $(\mathrm{RH})$ readings were collected by a permanent weather station at the facility and data were downloaded after the completion of the experiment. Temperature data-loggers were placed under cadavers $\mathrm{R}, \mathrm{T}$ and $\mathrm{X}$ at the time of cadaver placement.

\subsubsection{Laboratory Methods}

The same soil biochemical and enzymatic analyses were performed as for the second pig carcass experiment (sections 3.3.2 and 5.3.3) except that the sulphatase fluorometric assay was eliminated and esterase ( 3 substrates) and alanine aminopeptidase assays were added (section 3.3.2). A slight methodological modification was made to the fluorometric enzyme assay whereby all substrate solutions were freshly prepared every three days to reduce the possibility of contamination or of long-term stability issues (Roy, 1980) which may have contributed to the erratic results seen in the previous experiment (section 5.3.3). The T-RFLP and DGGE analyses were performed as outlined in section 3.4. 


\subsection{Results}

\subsubsection{Decomposition Documentation}

Photos were taken of each cadaver at each sampling to document the progression of decomposition and to help relate analytical results to cadaveric changes. Due to the sensitive nature of these photographs, however, and the potential for identification of the individuals from them, the photographs are not included in this thesis. Photographs may be made available as a restricted electronic resource on application to the author.

The progression of decomposition differed greatly between the cadavers. Those that were autopsied (T, X and Z) showed slightly accelerated decomposition compared with two of the non-autopsied cadavers ( $\mathrm{R}$ and $\mathrm{V}$ ), but one non-autopsied cadaver, Cadaver $\mathrm{P}$ showed remarkably rapid decomposition, being largely skeletonised by day 10 (ADD 286) and completely skeletonised by the next sampling on day 14 (ADD 376).

Cadaver T (autopsied) was the first cadaver placed on sand, one week after cadaver P. This cadaver progressed through the stages quickly and was almost skeletonised after 16 days (ADD 382). The upper body skeletonised slightly more quickly than the lower body, probably due to the autopsy wounds to the upper body.

Cadaver R (non-autopsied), was placed on soil, decomposed very slowly. This cadaver did not exhibit noticeable bloating or active decay around the abdominal region at any time, but did show active decay around the face and fungus growth on the trunk and limbs. At the cessation of sampling at day 49 (ADD 797), cadaver R was still largely intact.

Cadavers $\mathrm{X}$ and $\mathrm{V}$ were placed on the same day (but had died on different days - see Table 6.1). Cadaver $\mathrm{X}$ was autopsied and placed on soil and cadaver $\mathrm{V}$ was not autopsied and was placed on sand. These cadavers displayed a similar overall rate of decomposition with 
neither reaching skeletonisation by the cessation of sampling (day 46 ADD 731). Cadaver $\mathrm{V}$ exhibited faster decomposition around the upper torso region and head than the lower body. The upper body was favoured by the maggot mass and was largely skeletonised by day 26 (ADD 499). The lower body appeared to have areas of adipocere formation. Cadaver $\mathrm{X}$ displayed a more even decomposition, although the lower extremities were closer to skeletonisation at cessation of sampling than the upper torso. There was no evidence of adipocere formation in this cadaver.

Cadaver $\mathrm{Z}$ was the last to be placed at the facility and samples were collected until day 38 (ADD 574). By the cessation of sampling, cadaver $Z$ was in the active decay stage, but did not have a large associated maggot mass and had not lost much body mass.

\subsubsection{Weather recordings}

The mean daily rainfall over the sampling period was high, at $9.3 \mathrm{~mm}$. There was one day (1/10/2006) with very heavy rainfall associated with severe thunderstorms (Figure 6.3). The mean daily air temperature was $20.4^{\circ} \mathrm{C}$, although there was a discernable seasonal change over the sampling period, with the mean daily temperature ranging from $27.2^{\circ} \mathrm{C}$ on the first day to $5^{\circ} \mathrm{C}$ towards the end of the period (Figure 6.4). The mean daily soil temperature was more stable than air temperature, but the decrease in temperature as the season changed can clearly be seen in both. In contrast to the pig carcass experiment (section 5.3.1), the mean daily soil temperature was almost always higher than the mean daily air temperature. With the exception of two early time periods, the mean daily soil temperature (at a depth of $15.2 \mathrm{~cm}$ ) was also higher than at the soil surface under the three cadavers. The readings from under the cadavers tended to track the mean daily air temperature. The mean daily relative humidity is shown in Figure 6.5 and the mean daily barometric pressure is shown in Figure 6.6. 


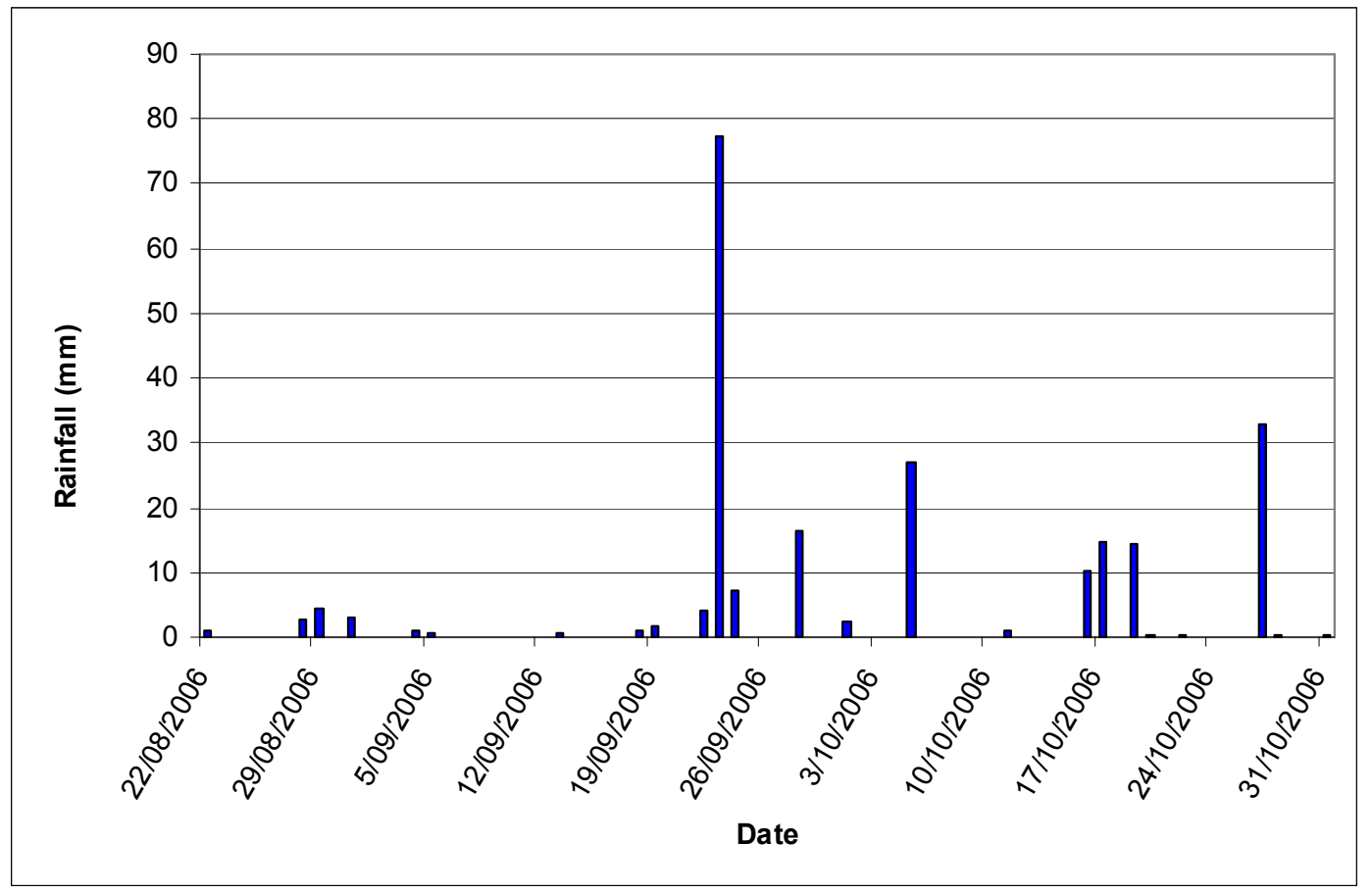

Figure 6.3 Daily Rainfall at the human cadaver decomposition site during the experimental period.

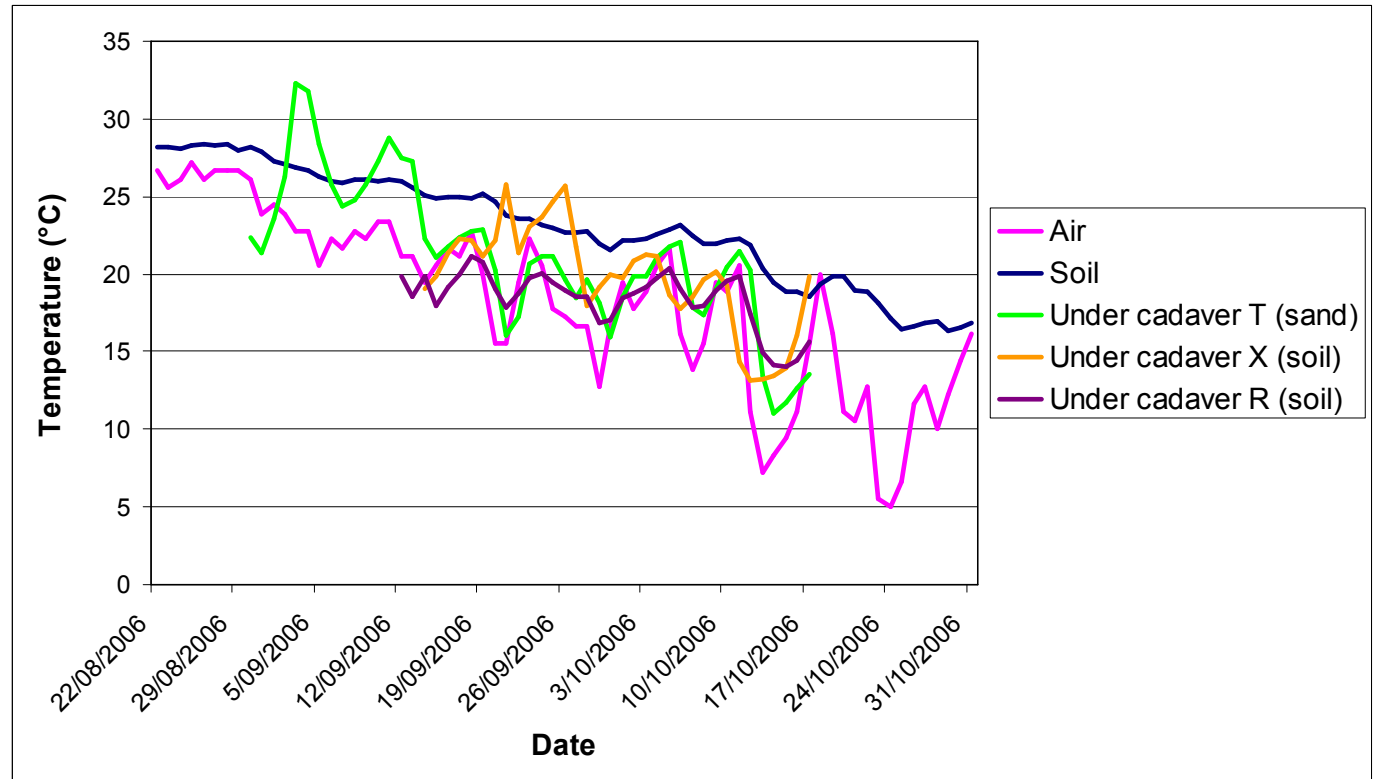

Figure 6.4 Mean daily air, control soil and under cadaver temperatures. 


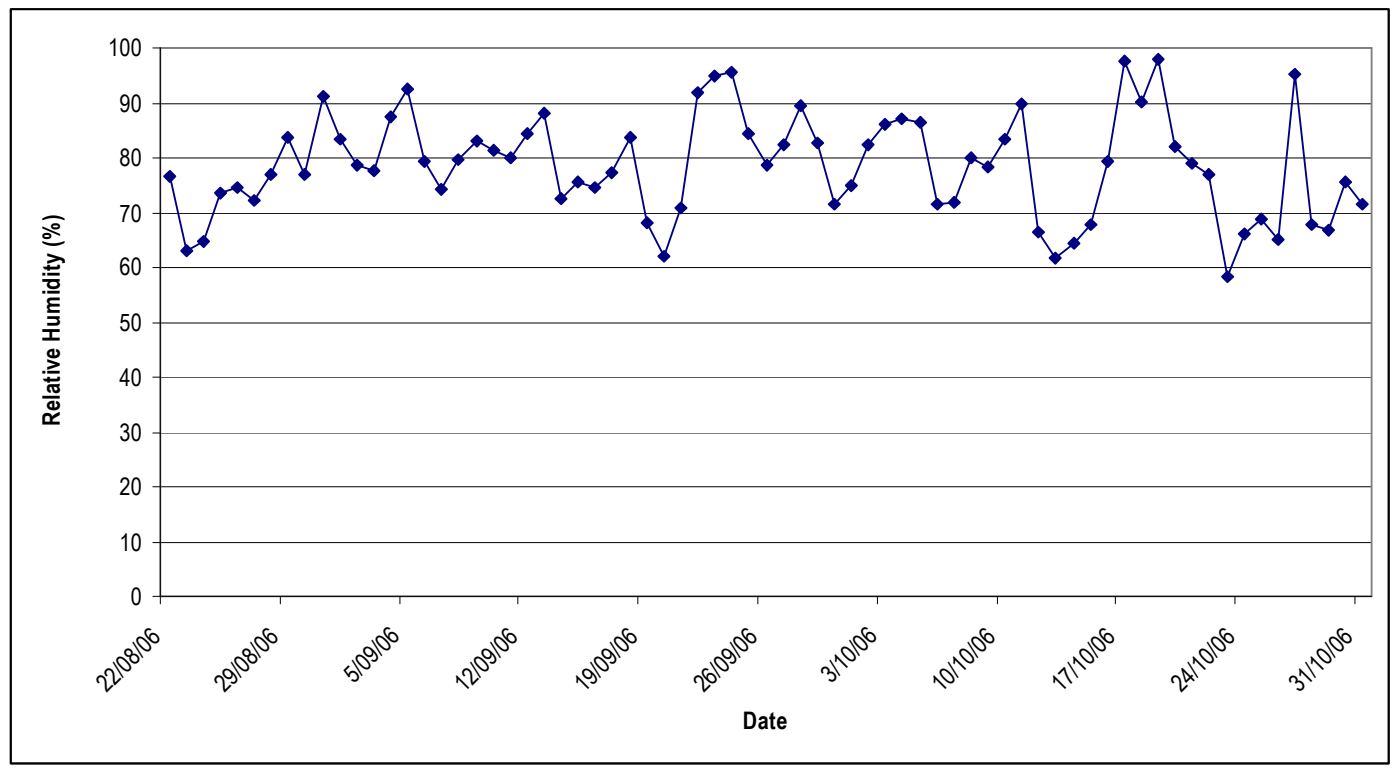

Figure 6.5 Mean daily relative humidity at the decomposition site during the experimental period.

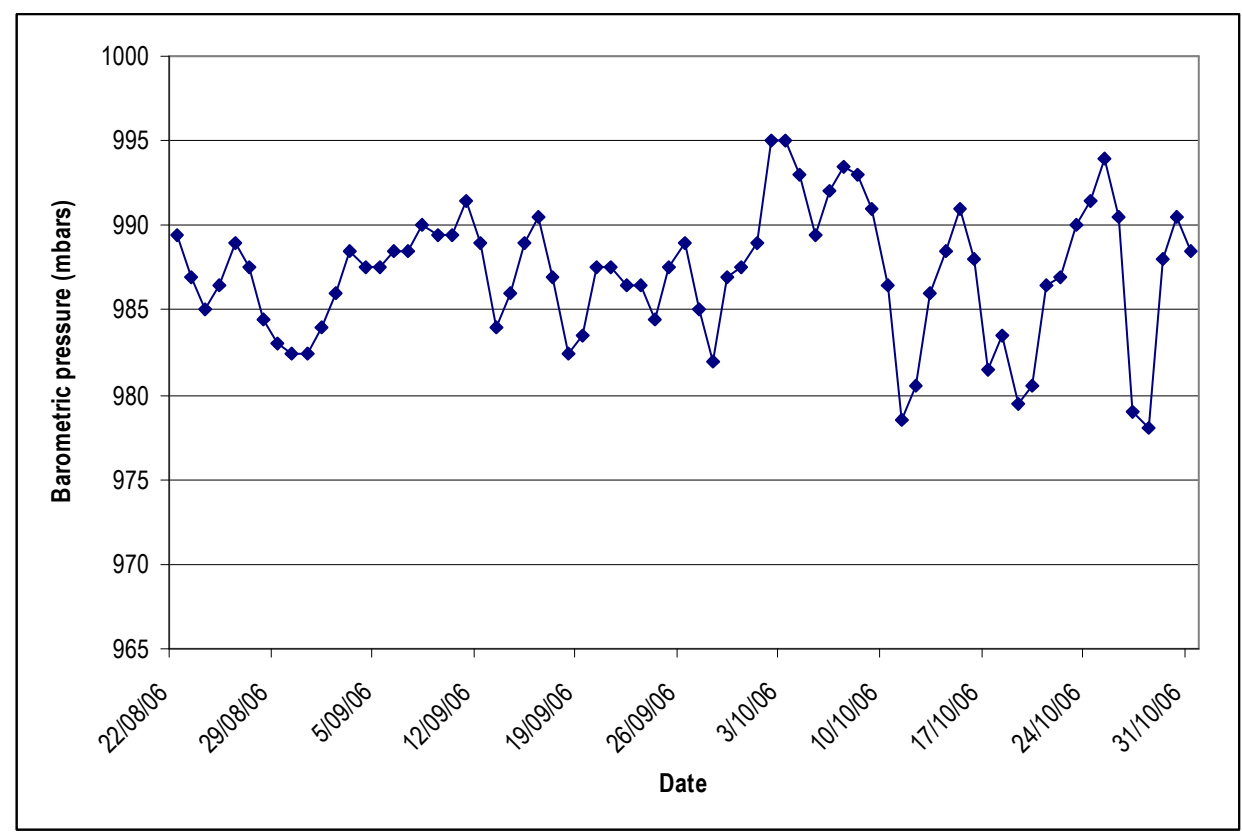

Figure 6.6 Mean daily barometric pressure at the decomposition site during the experimental period.

\subsubsection{Buffering Capacity of Soil and Sand}

To allow comparison of the $\mathrm{pH}$ changes between the soils and sands within and between the three experiments, the buffering capacity of each was determined (section 3.1.3). The buffering ability of a soil is determined primarily by three properties: organic content, clay mineral content and the types of clay minerals (Weaver et al., 2004). The soil from the pig 
carcass experimental site at Wallaceville in New Zealand was classified as Heretaunga silt loam (Typic Firm Brown soil) and the soil from the Anthropology Facility in the USA was classified as a fine, mixed, thermic Typic Paleudalf according to US Soil Taxonomy (Vass et al., 1992). The buffering capacity of soil from each of the control sites is shown in Figure 6.7.

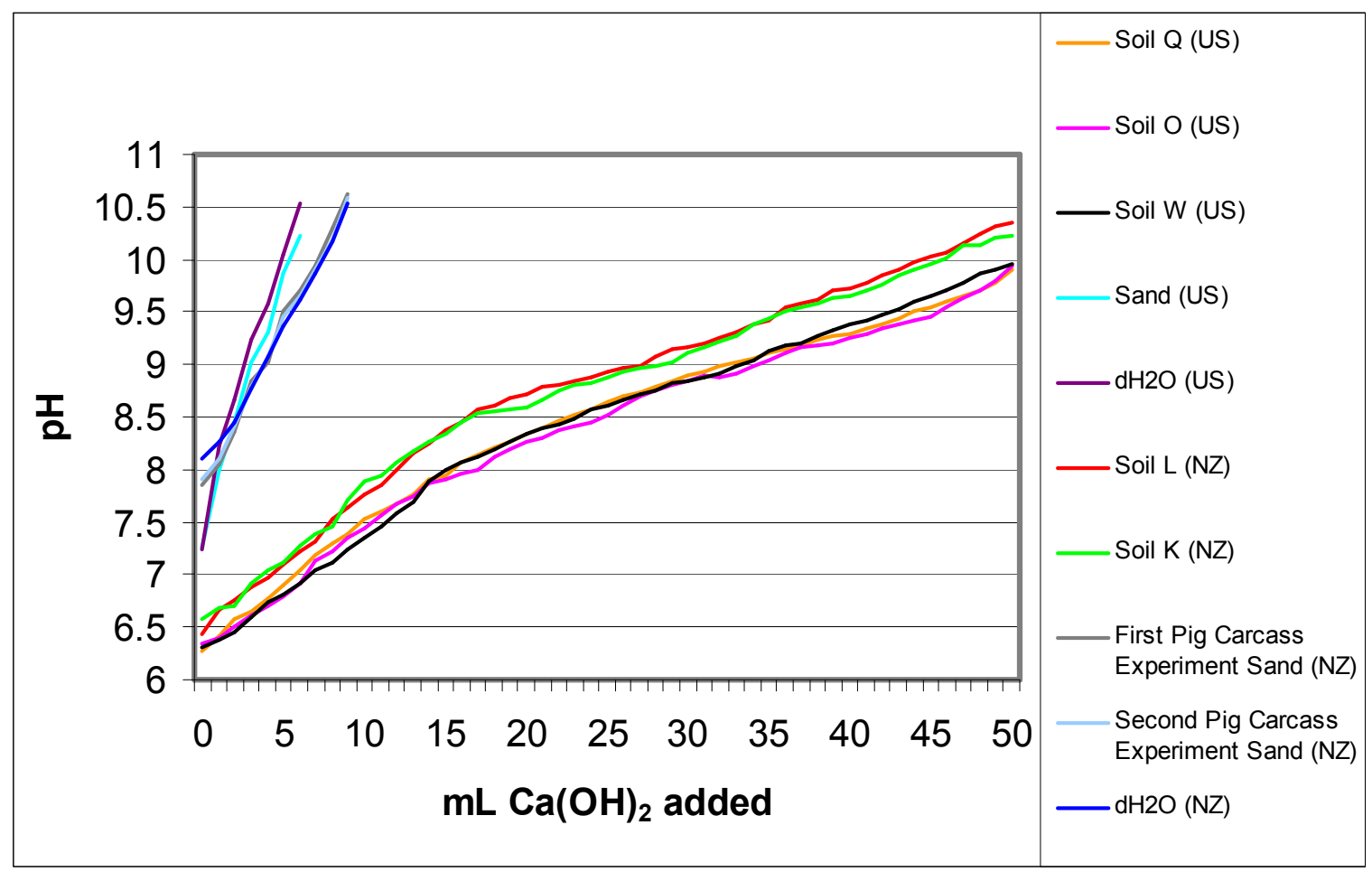

Figure 6.7 Buffering capacity of soil and sand from each of the US and NZ control sites, and of the distilled $\mathrm{H}_{2} \mathrm{O}$ used.

As expected, all the sand controls showed little to no buffering capacity, and exhibited similar $\mathrm{pH}$ changes as were seen for the related $\mathrm{dH}_{2} \mathrm{O}$ sample. The initial $\mathrm{pH}$ of the $\mathrm{dH}_{2} \mathrm{O}$ in the New Zealand laboratory (used for the pig carcass experiments) was considerably higher at 8.1 than the $\mathrm{dH}_{2} \mathrm{O}$ in the laboratory at Oak Ridge National Laboratory, which was 7.25. This, however, is unlikely to have an effect on the buffering results or the $\mathrm{pH}$ measurements from under the bodies as $\mathrm{H}_{2} \mathrm{O}$ has no buffering capacity on its own. The New Zealand soils showed very similar buffering capacity to the US samples given the

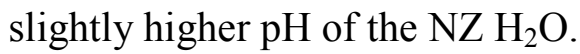




\subsubsection{Chemistry and Enzyme Assay Results}

The chemistry and enzyme assay datasets were tested for normality by determining the standard error and skewness using Excel (section 3.4.5). Datasets were taken to be nonnormally distributed if their skewness statistic was more than 2.5 times the standard error (Leech et al., 2005). Seven of the ten chemistry and biochemistry datasets were found to be non-normally distributed, so the non-parametric ANOSIM test (Clarke, 1993) was used to test for differences between sample groups (section 3.4.5).

\subsubsection{Moisture Content}

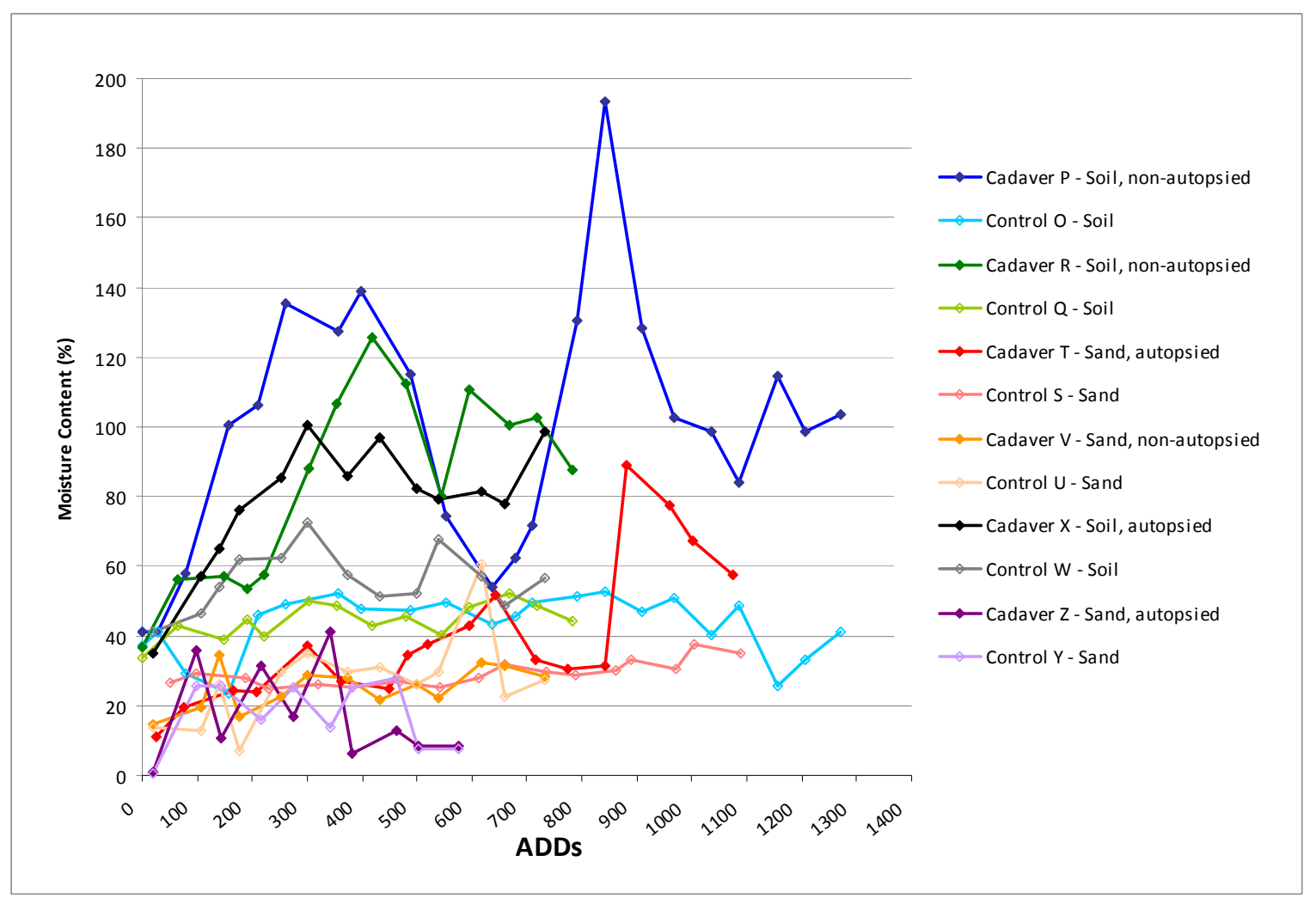

Figure 6.8 Moisture content of samples over the sampling period. 
The moisture content of the sand and soil was determined for each sampling date. The cadaver sites showed higher overall moisture content than the associated control sites, with dramatic increases in moisture content in the cadaver $\mathrm{P}, \mathrm{T}$ and $\mathrm{R}$ sites that correspond to the day of heavy rainfall $(23 / 09 / 2006)$.

\subsubsection{2 $\mathrm{pH}$}

The $\mathrm{pH}$ values for samples collected from control sites are shown in Figure 6.9. The values for samples from under the three cadavers on soil are shown in Figure 6.10 and those from under the cadavers on sand, in Figure 6.11. The initial $\mathrm{pH}$ for the sand was 7.4 and around 6.7 for the soil. The $\mathrm{pH}$ of the control samples changed very little over the sampling period (Figure 6.9).

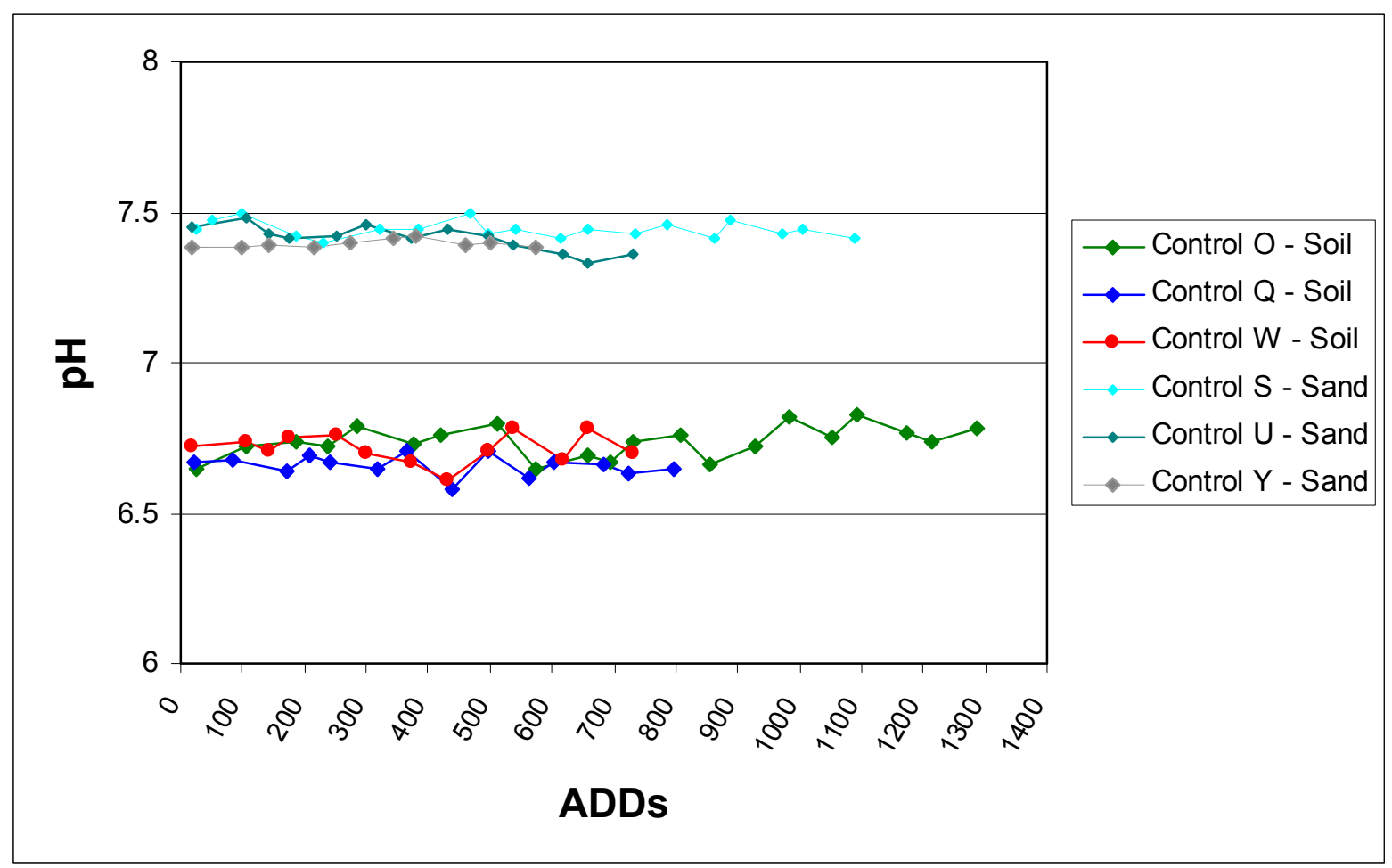

Figure $6.9 \mathrm{pH}$ of samples from soil and sand control sites. 


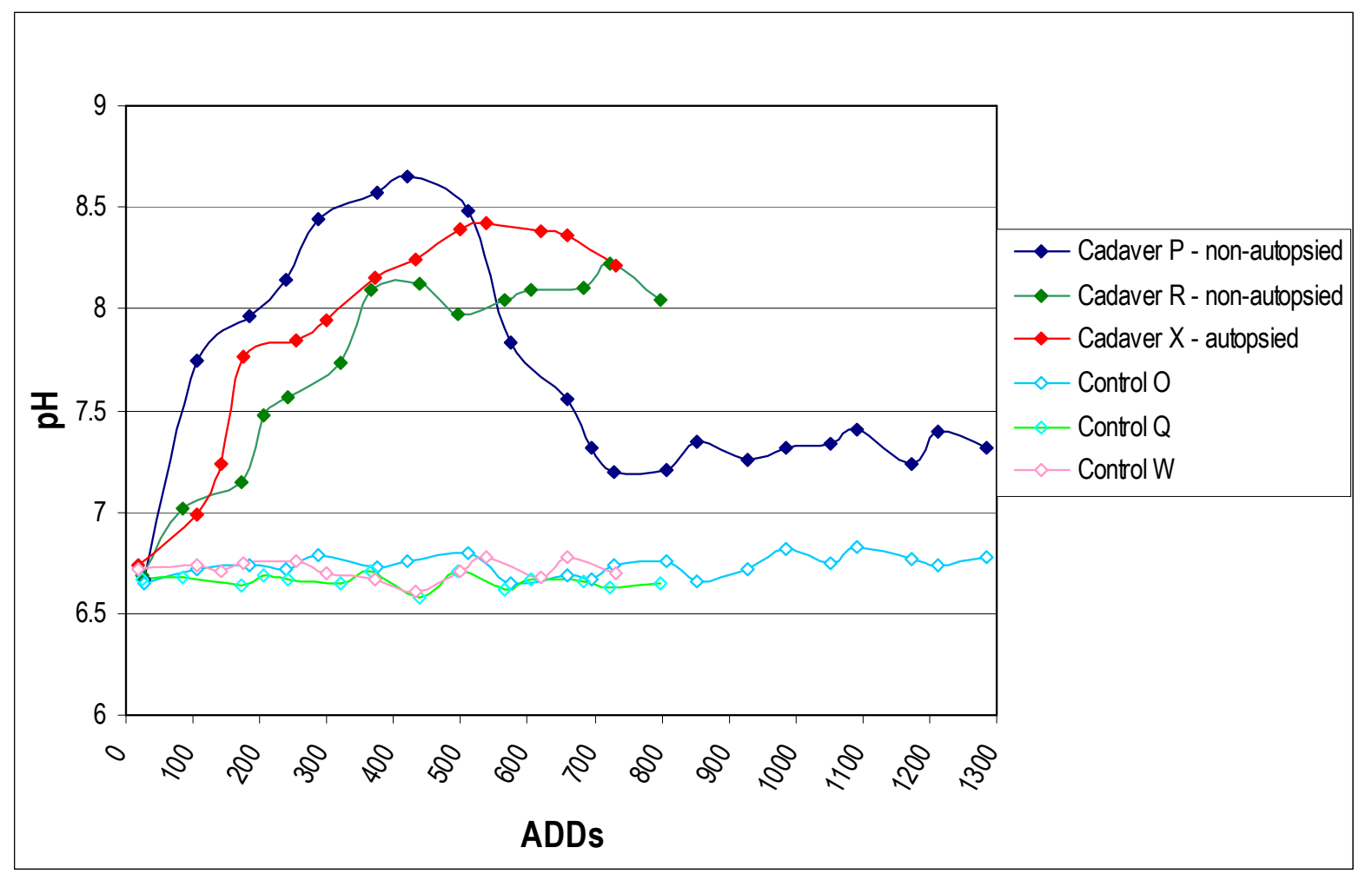

Figure 6.10 pH of samples from under the cadavers on soil and the associated controls.

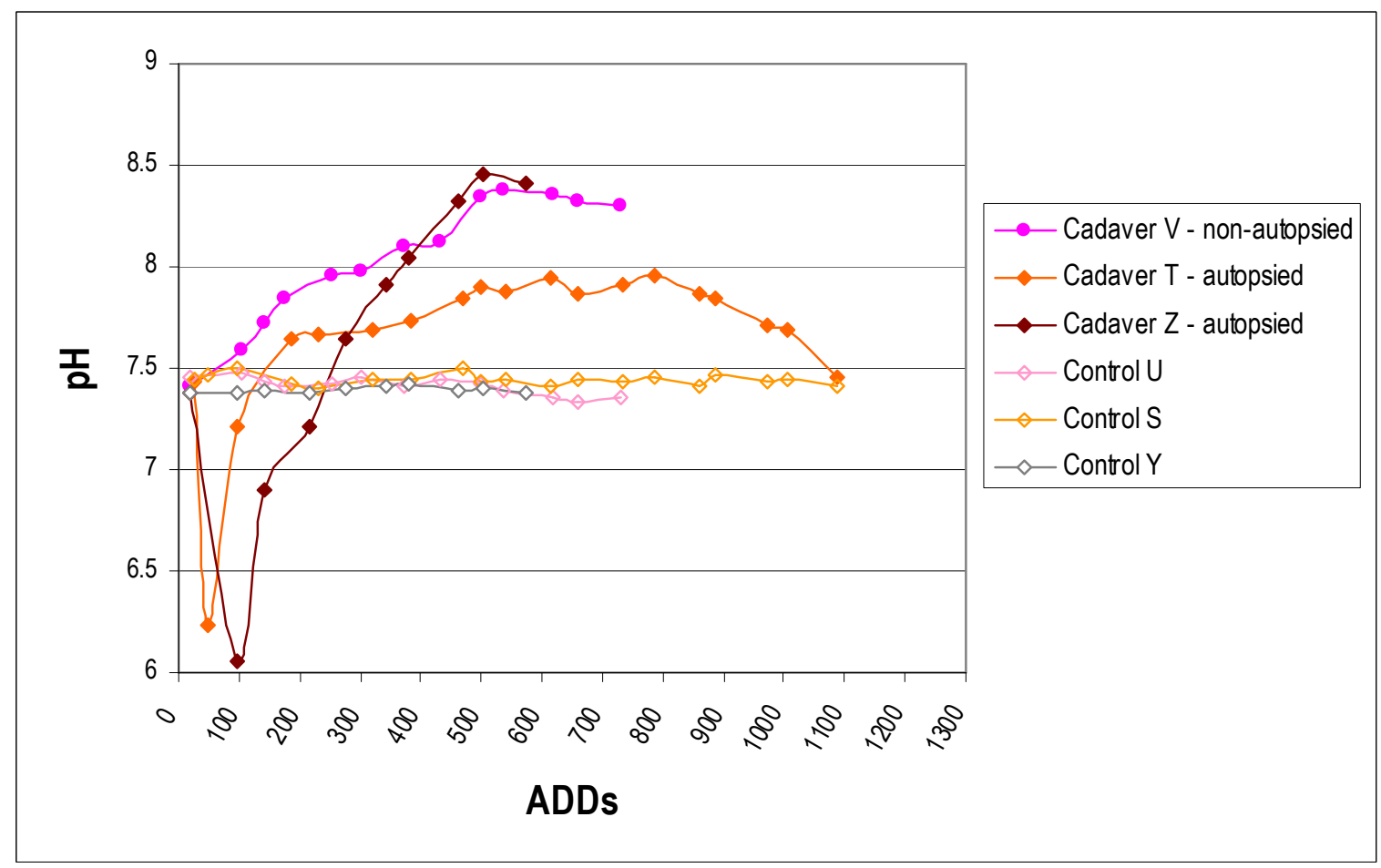

Figure 6.11 pH of samples from under the cadavers on sand and associated controls. A soil control and sand control are also shown for comparison. 
The series of samples collected from under the cadavers (decomposition sites) all showed a rise in $\mathrm{pH}$ over time, but two, $\mathrm{T}$ and $\mathrm{Z}$, showed a rapid decrease from the initial $\mathrm{pH}$ to around 6 , before rapidly rising to above the initial $\mathrm{pH}$. Both of these cadavers were autopsied, and were on sand. The autopsied cadaver on soil, X, and the other cadaver on sand, $\mathrm{V}$, did not show a decrease in $\mathrm{pH}$ at any stage.

Samples from under only one non-autopsied cadaver (P) exhibited a decrease in $\mathrm{pH}$ after the rise from day 16 (ADD 420) onwards (Figure 6.11), whereas those from all three autopsied cadavers exhibited some decrease (Figure 6.10). This occurred from day 38 (ADD 787) for cadaver $\mathrm{T}$ on sand, from day 26 (ADD 499) for cadaver $\mathrm{X}$ on soil and day 31 (ADD 502) for cadaver $\mathrm{Z}$ on sand. The $\mathrm{pH}$ under cadaver $\mathrm{P}$ reached a plateau at around 7.25 , half a $\mathrm{pH}$ unit higher than the control soil values.

\subsubsection{Ammonium $\left(\mathrm{NH}_{4}^{+}-\mathrm{N}\right)$}

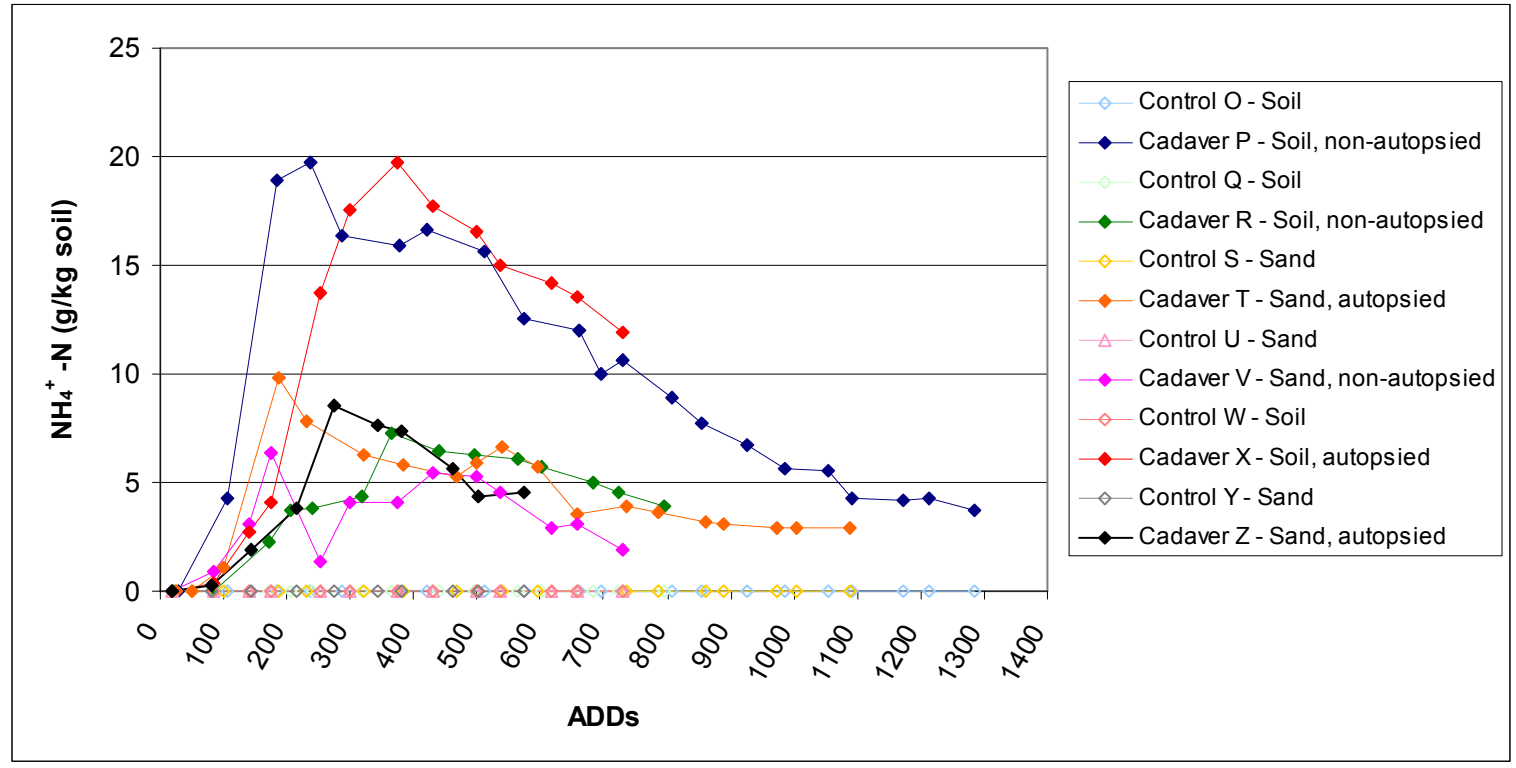

Figure $6.12 \mathrm{NH}_{4}^{+}-\mathrm{N}$ concentration in soil and sand samples collected from decomposition and control sites. 
As in the previous experiments using pig carcasses, a dramatic increase in $\mathrm{NH}_{4}{ }^{+}-\mathrm{N}$ concentration was seen in all decomposition sites, both soil and sand. The soil under cadavers $\mathrm{P}$ and $\mathrm{X}$ showed the greatest increase in $\mathrm{NH}_{4}{ }^{+}-\mathrm{N}$ concentration, both reaching $19.7 \mathrm{~g} / \mathrm{kg}$. The other decomposition sites peaked at between 5 and $10 \mathrm{~g} / \mathrm{kg}$. The $\mathrm{NH}_{4}{ }^{+}-\mathrm{N}$ concentration increased rapidly from baseline levels at day zero, with all decomposition sites showing an increase by ADD 175. The cadaver P site exhibited the earliest increase with a rise to $4.2 \mathrm{~g} / \mathrm{kg}$ by day 3 (ADD 106), reaching its maximum concentration at day 8 (ADD 238). Maximum $\mathrm{NH}_{4}{ }^{+}-\mathrm{N}$ concentrations were reached at around ADD 180 in two of the sand decomposition sites, whilst in the third (cadaver Z), the maximum was reached slightly later at ADD 274. For the other two cadavers on soil (X and R), maximum concentrations were reached at ADDs 366 and 373. The soil and sand controls showed very little change over the period sampled.

\subsubsection{Nitrate $\left(\mathrm{NO}_{3}^{-}-\mathrm{N}\right)$}

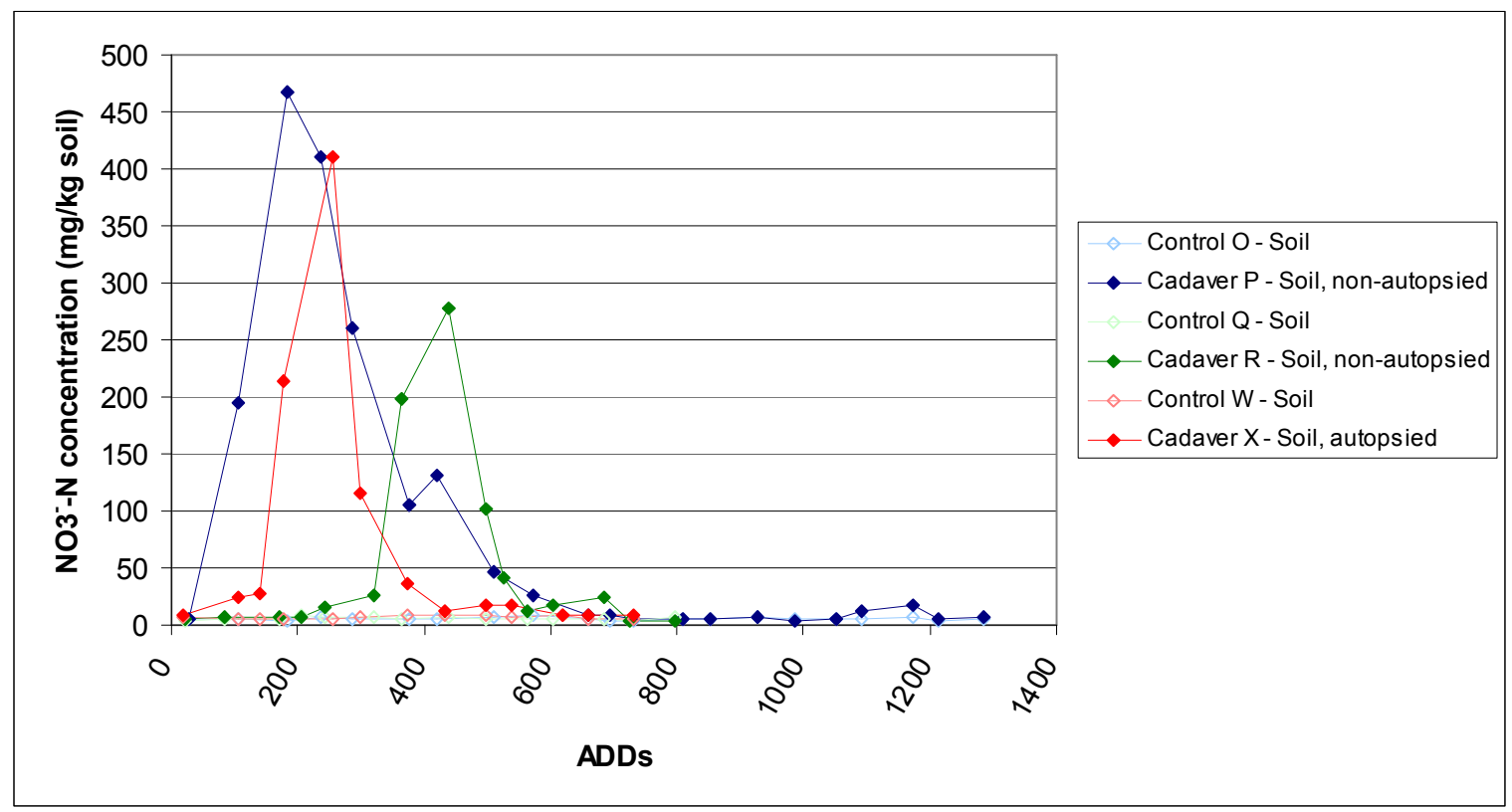

Figure 6.13 $\mathrm{NO}_{3}^{-}-\mathrm{N}$ concentration in soil samples collected from decomposition and control sites. 
The soil decomposition sites each showed a dramatic, but transient, increase in nitrate concentration during the early stages of decomposition (Figure 6.13). Nitrate concentration increased quickly under cadaver $\mathrm{P}$, reaching a maximum concentration of $467 \mathrm{mg} / \mathrm{kg}$ at day 6 (ADD 185) before rapidly returning to baseline levels. The cadaver X site showed a similar increase, but this occurred slightly later, with the nitrate concentration beginning to rise after day 6 (ADD 174) and peaking at $410 \mathrm{mg} / \mathrm{kg}$ at day 11 (ADD 254). The peak occurred even later under cadaver $\mathrm{R}$, with the rise in concentration not beginning until day 18 (ADD 366), peaking at $277 \mathrm{mg} / \mathrm{kg}$ day 22 (ADD 438). The sand decomposition sites did not show such dramatic spikes in nitrate concentration (Figure 6.14), but tended to fluctuate throughout the sampling period, reaching maximum concentrations of 163,121 and $114 \mathrm{mg} / \mathrm{kg}$ under cadavers $\mathrm{T}, \mathrm{V}$ and $\mathrm{Z}$ respectively.

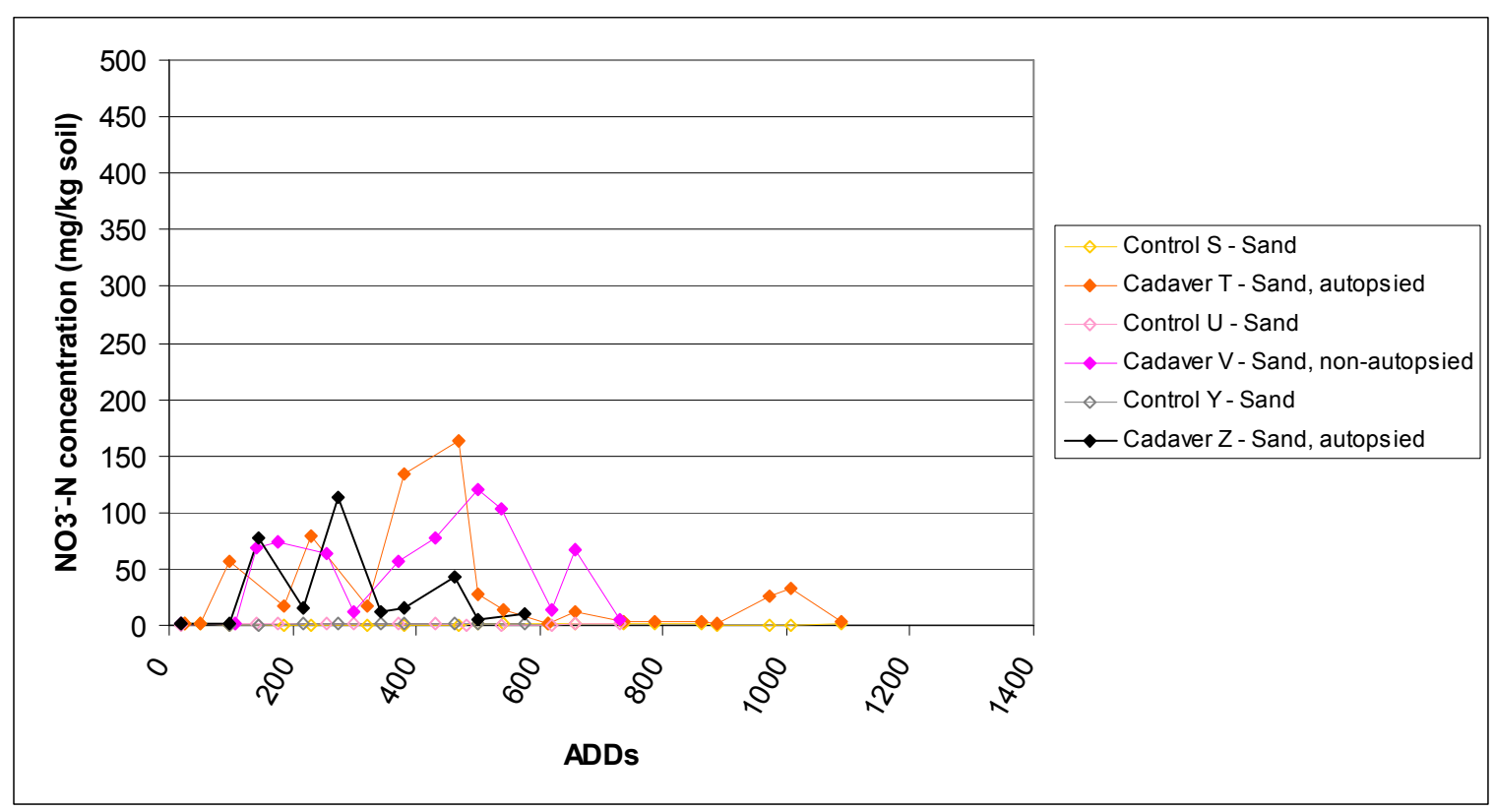

Figure 6.14 $\mathrm{NO}_{3}^{-}-\mathrm{N}$ concentration in sand samples collected from decomposition and control sites.

\subsubsection{Urease (EC 3.5.1.5)}

A dramatic increase in potential urease activity occurred in response to the decomposition of cadavers on soil (Figure 6.15). The decomposition sites for cadavers $\mathrm{P}$ and $\mathrm{X}$ showed 
similar patterns of activity over time, reaching a peak between ADD 100 and 200, followed by a slight decrease before a second spike in activity at ADD 420 and 373 respectively. Activity then decreased to between 3 and $6 \mathrm{nmol} \mathrm{g}^{-1} \mathrm{~s}^{-1}$ for the rest of the sampling time. Cadaver R's site showed a slower and smaller rise in urease activity, beginning after ADD 171, peaking at $7.8 \mathrm{nmol} \mathrm{g} \mathrm{s}^{-1}$ at ADD 319 before dropping back to plateau at 2 and 4 $\mathrm{nmol} \mathrm{g} \mathrm{g}^{-1} \mathrm{~s}^{-1}$. The sand decomposition sites showed lower overall potential activity (Figure 6.16), which tended to fluctuate between baseline levels and up to $8 \mathrm{nmol} \mathrm{g}^{-1} \mathrm{~s}^{-1}$ throughout the sampling period.

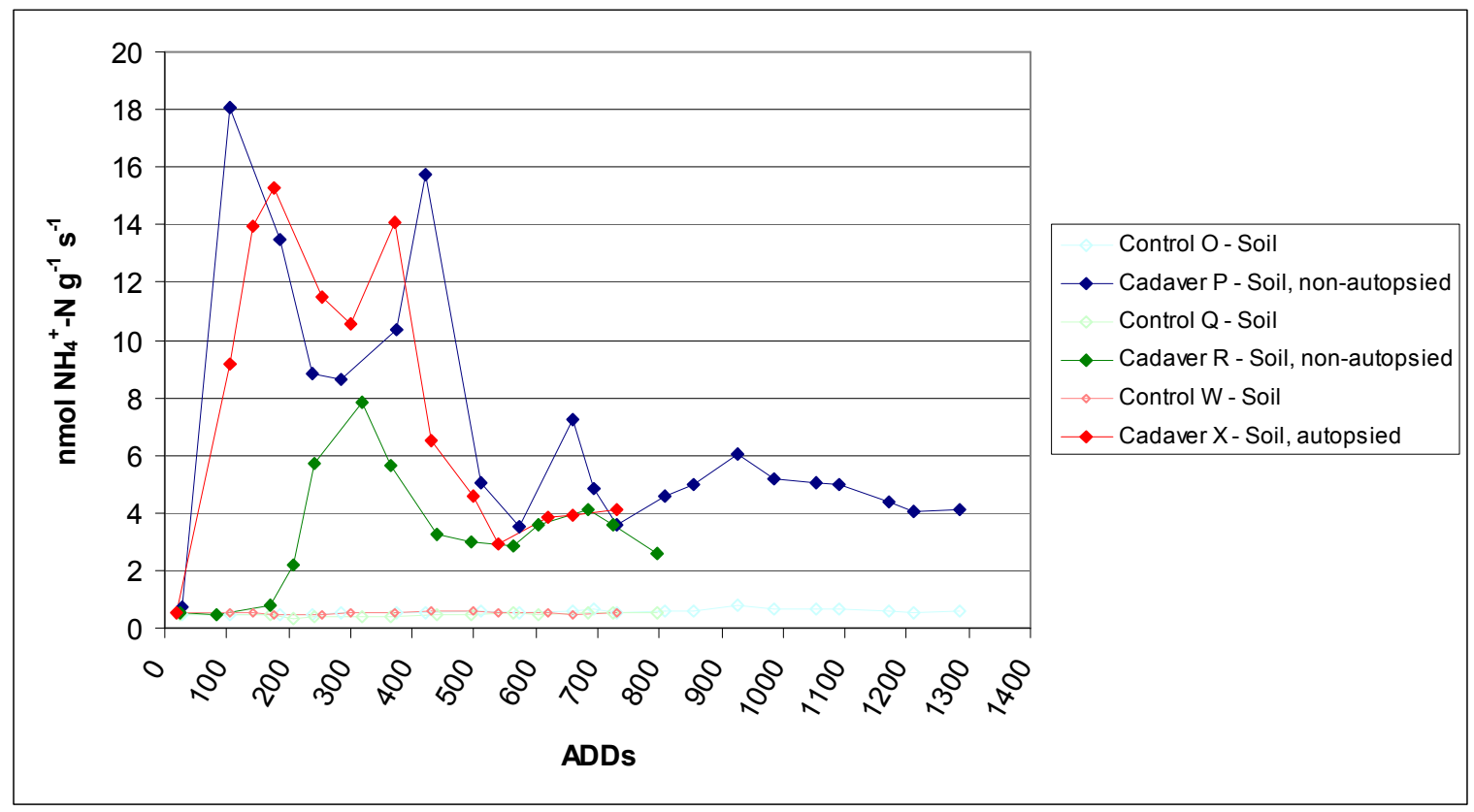

Figure 6.15 Potential urease activity in soil samples collected from decomposition and control sites. 


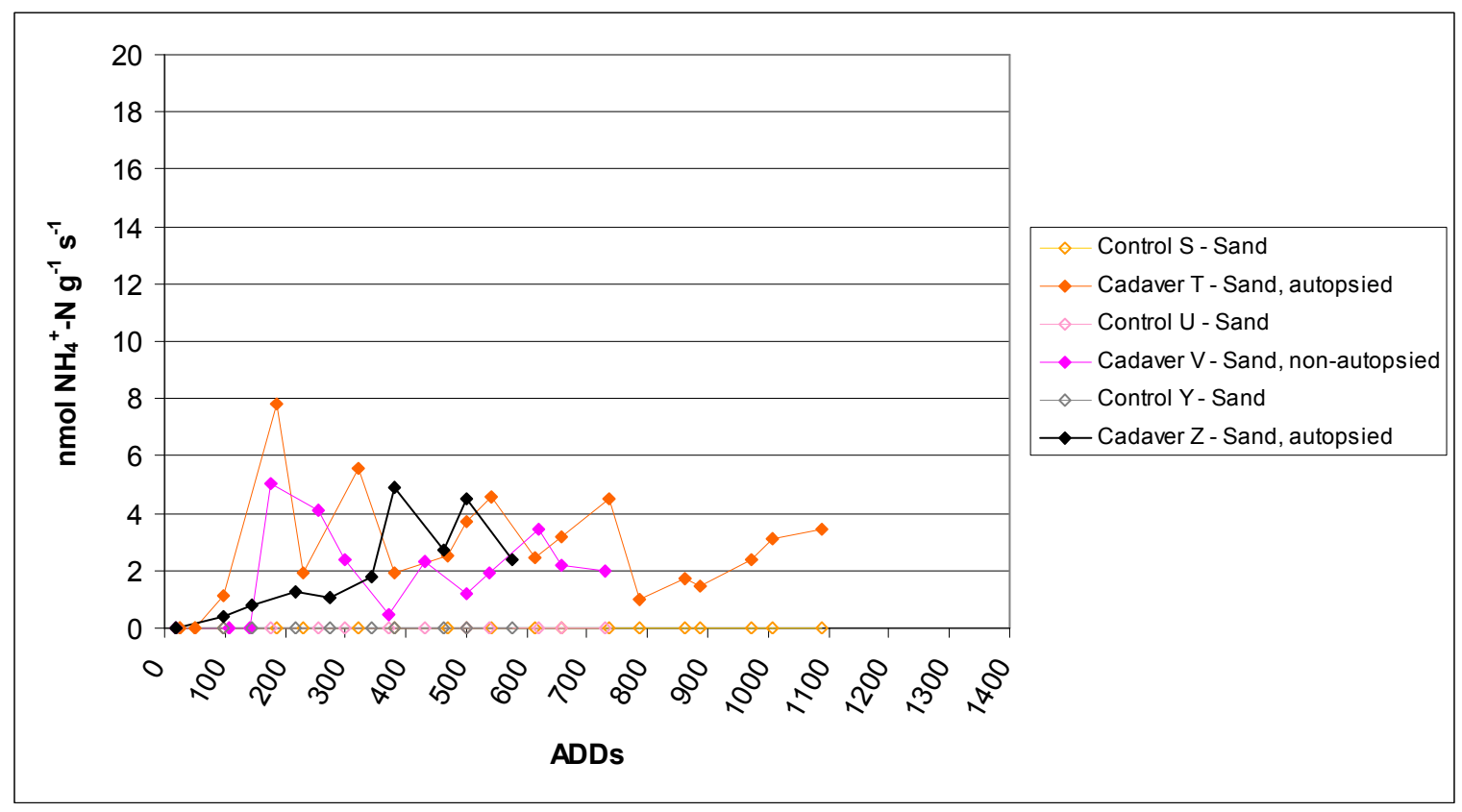

Figure 6.16 Potential urease activity in sand samples collected from decomposition and control sites.

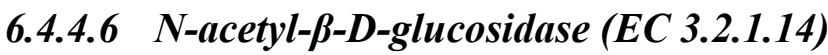

The enzyme $\mathrm{N}$-acetyl- $\beta$-D-glucosidase showed an increase in potential activity at all six decomposition sites. The soil sites showed the greatest increase (Figure 6.17), which began from ADD 0 under cadaver $\mathrm{P}$ and from ADD 141 under cadaver $\mathrm{X}$. The cadaver $\mathrm{R}$ site did not begin to increase until after ADD 242, but showed a similar pattern of activity as the other two soil decomposition sites, but at later ADDs and at a much reduced level. All three soil decomposition sites showed an initial increase, followed by fluctuations between 1 and $4.2 \mathrm{nmol} 4-\mathrm{MUB} \mathrm{g}^{-1} \mathrm{~s}^{-1}$ throughout the sampling period. The cadaver $\mathrm{P}$ site showed a decrease in potential activity beginning from ADD 730 and levelling off around $0.5 \mathrm{nmol} 4-\mathrm{MUB} \mathrm{g}{ }^{-1} \mathrm{~s}^{-1}$ until the end of sampling. This trend was not observed in any of the other decomposition sites but these were not sampled past ADD 800. 


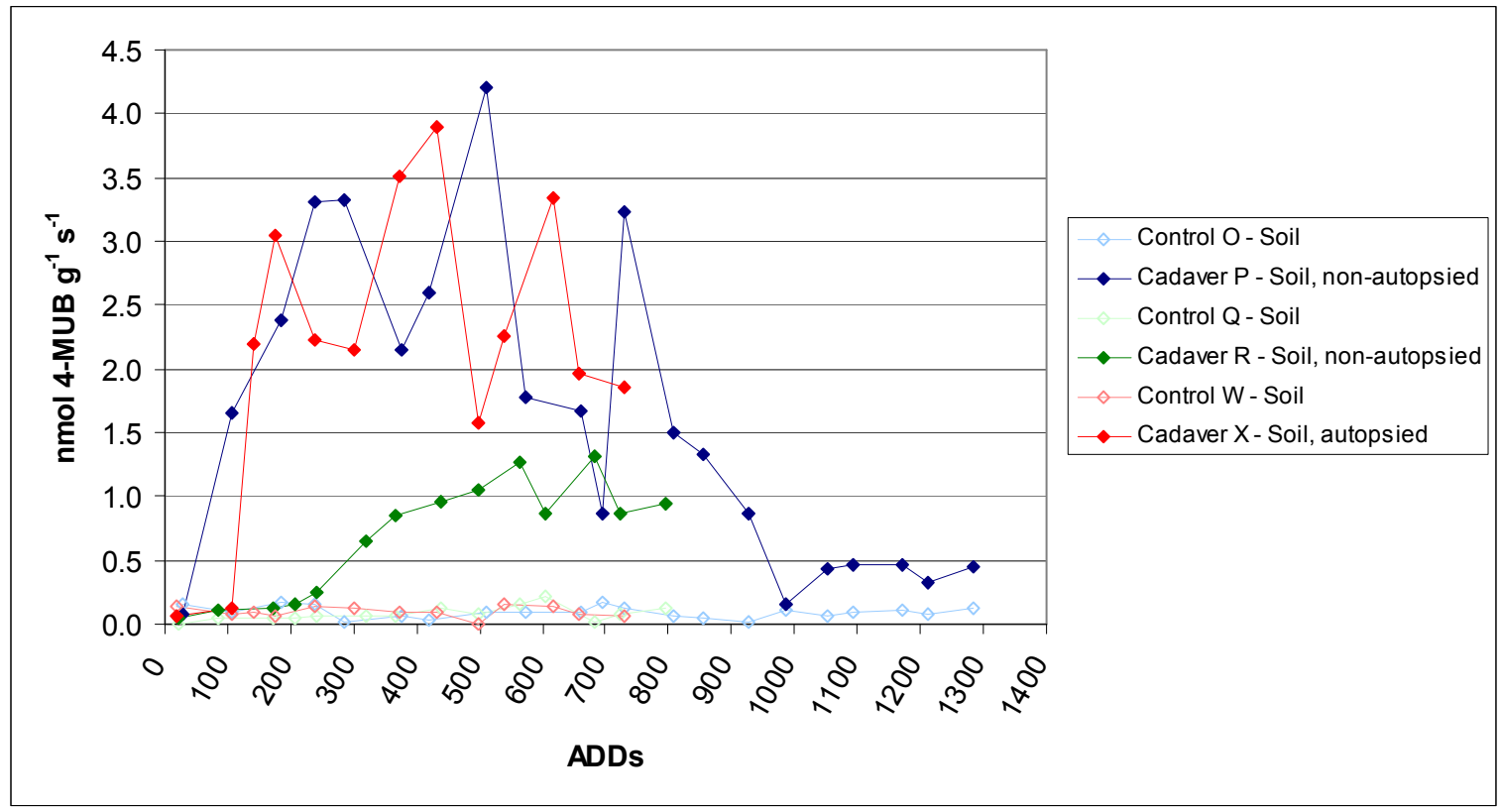

Figure 6.17 Potential N-acetyl- $\beta$-D-glucosidase activity in soil samples collected from decomposition and control sites.

The sand decomposition sites also showed an increase in potential activity (Figure 6.18), although not as much as the soil sites. Enzyme activity fluctuated between baseline levels and $1.5 \mathrm{nmol} 4-\mathrm{MUB} \mathrm{g}^{-1} \mathrm{~s}^{-1}$ for all three sand decomposition sites.

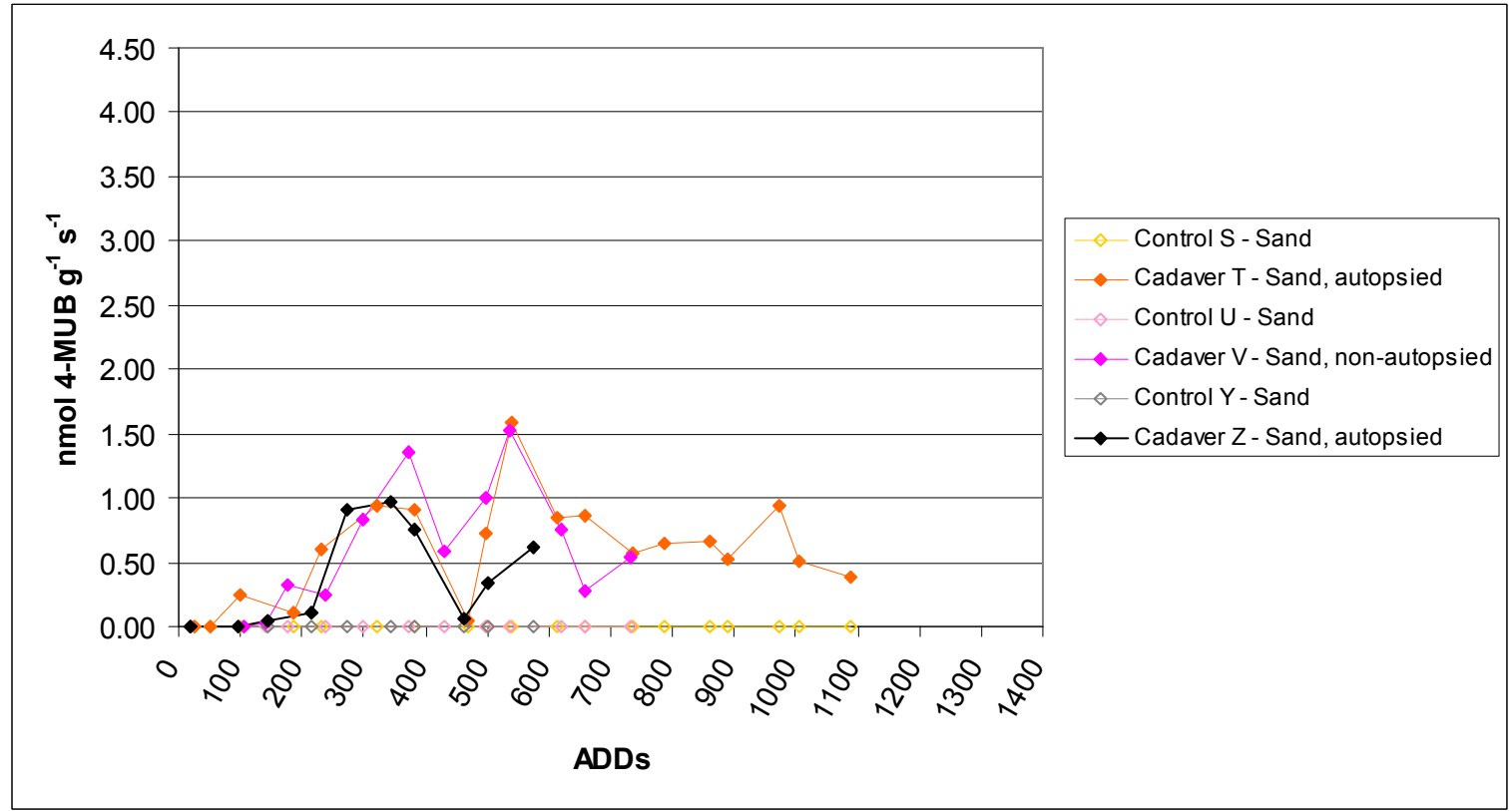

Figure 6.18 Potential N-acetyl- $\beta$-D-glucosidase activity in sand samples collected from decomposition and control sites. 


\subsubsection{Glucosidase (EC 3.2.1.21)}

The enzyme glucosidase showed an increase in potential activity in all three of the soil decomposition sites (Figure 6.19). The cadaver P site showed an immediate increase to 1.6 nmol 4-MUB $\mathrm{g}^{-1} \mathrm{~s}^{-1}$ at ADD 106 before rapidly dropping again to near baseline levels, followed by a fluctuating but overall decreasing level of potential activity. The cadaver X site showed a single spike of activity, peaking at $0.93 \mathrm{nmol} 4-\mathrm{MUB} \mathrm{g}^{-1} \mathrm{~s}^{-1}$ at ADD 176 followed by a return to baseline levels. The cadaver $\mathrm{R}$ site showed a later and more gradual increase in activity, peaking at $0.6 \mathrm{nmol} 4-\mathrm{MUB} \mathrm{g}^{-1} \mathrm{~s}^{-1}$ at ADD 438. Very little potential glucosidase activity was detected in the sand decomposition sites (Figure 6.20), although there was a small spike in activity under cadaver V at ADD 618.

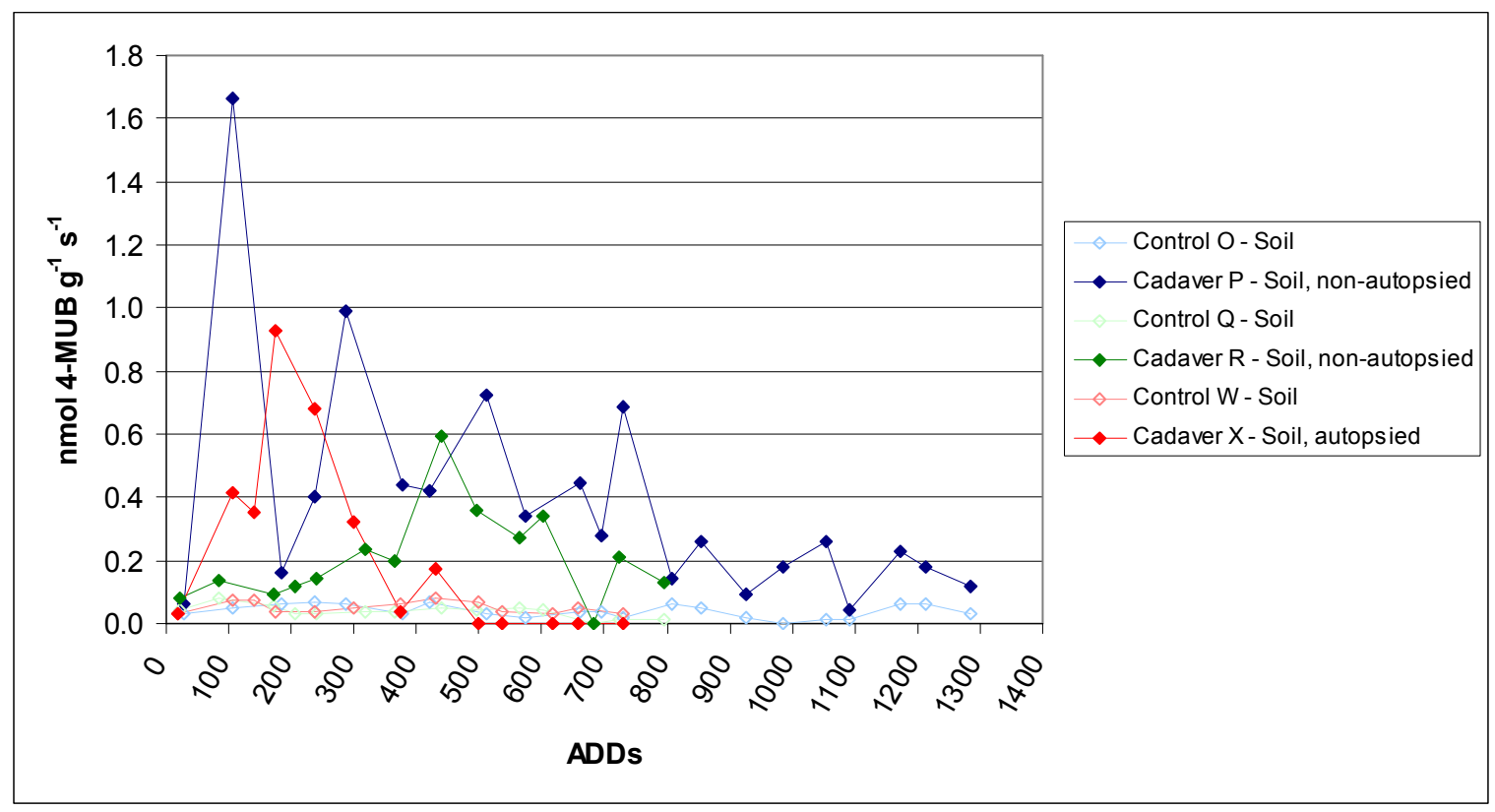

Figure 6.19 Potential glucosidase activity in soil samples collected from decomposition and control sites. 


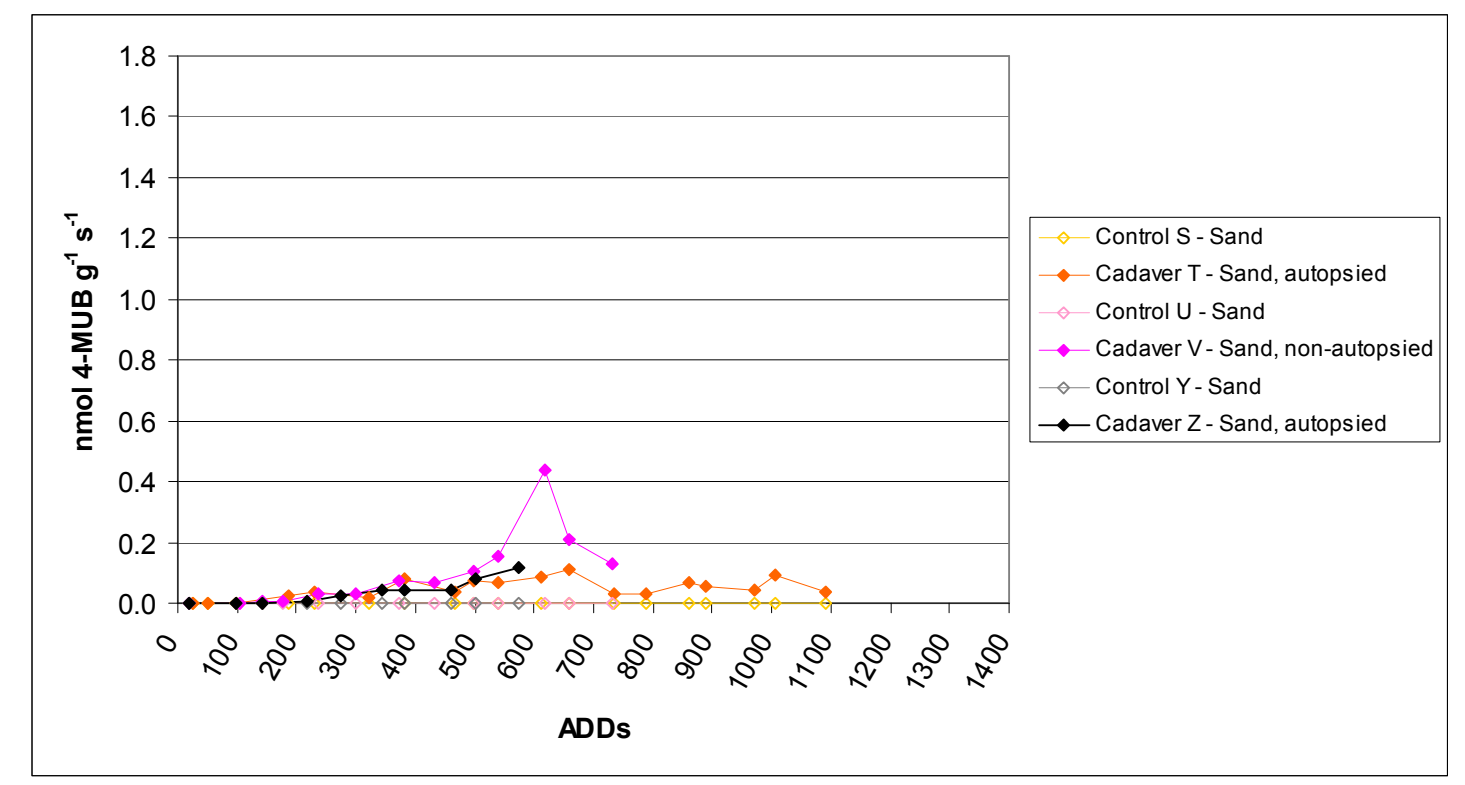

Figure 6.20 Potential glucosidase activity in sand samples collected from decomposition and control sites.

\subsubsection{Acid Phosphatase (EC 3.1.3.2)}

The potential acid phosphatase activity in five of the six cadaver sites showed two distinct peaks, the first early in decomposition, around ADD 200, and the second at around ADD 300 (Figures 6.21 and 6.22). All five showed a decrease to almost baseline levels in between the two peaks. The cadaver R site exhibited only one spike in potential enzyme activity, at ADD 438. Because of cadaver R's slower decomposition, this spike occurs at a similar stage of decomposition to the first spike for the other cadavers. The sand decomposition sites showed slightly lower overall potential activity than the soil sites, with the highest activity of the sand sites being $3.5 \mathrm{nmol} 4-\mathrm{MUB} \mathrm{g}^{-1} \mathrm{~s}^{-1}$ at ADD 468 for the cadaver T site. 


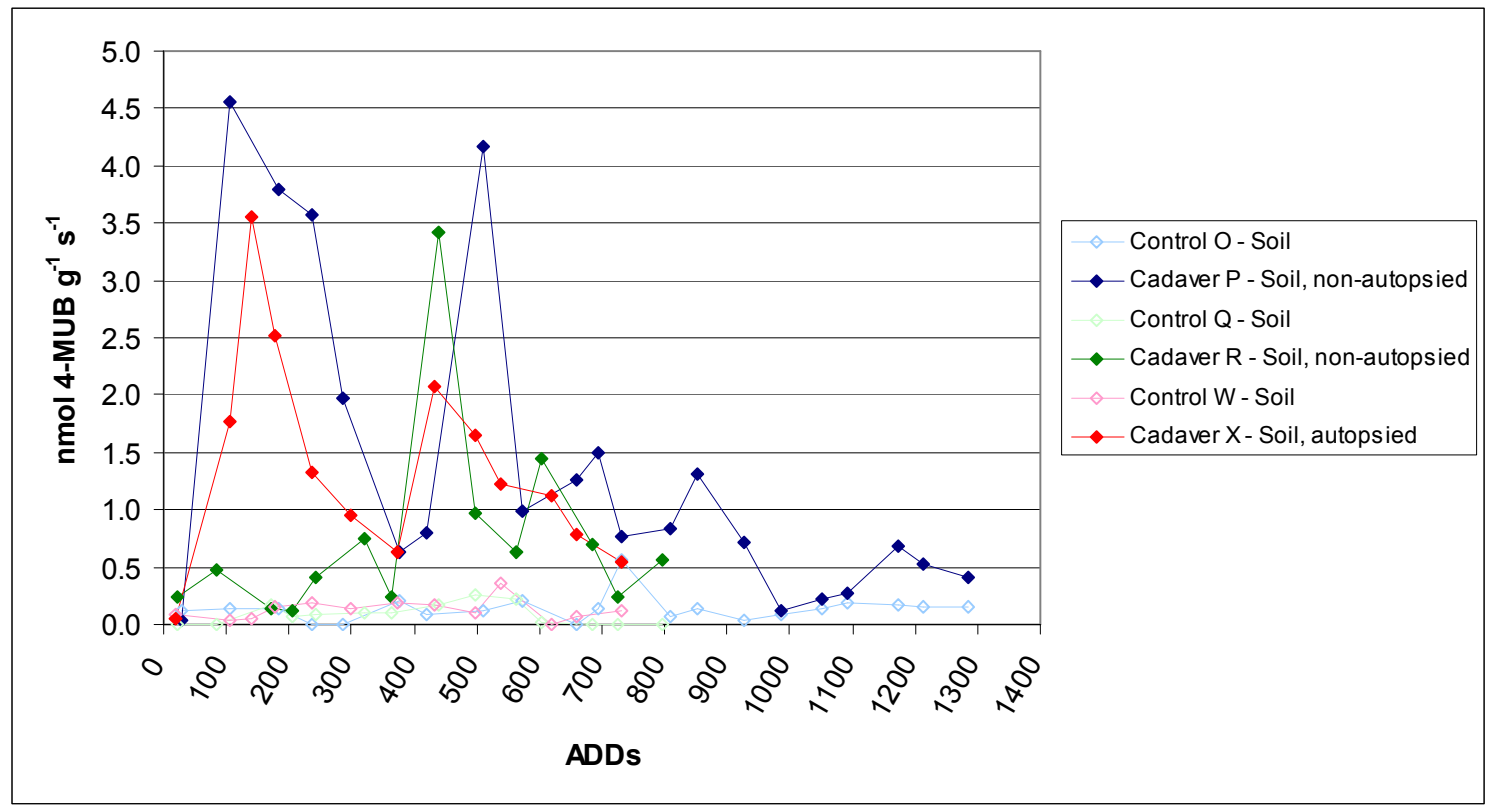

Figure 6.21 Potential acid phosphatase activity in soil samples collected from decomposition and control sites.

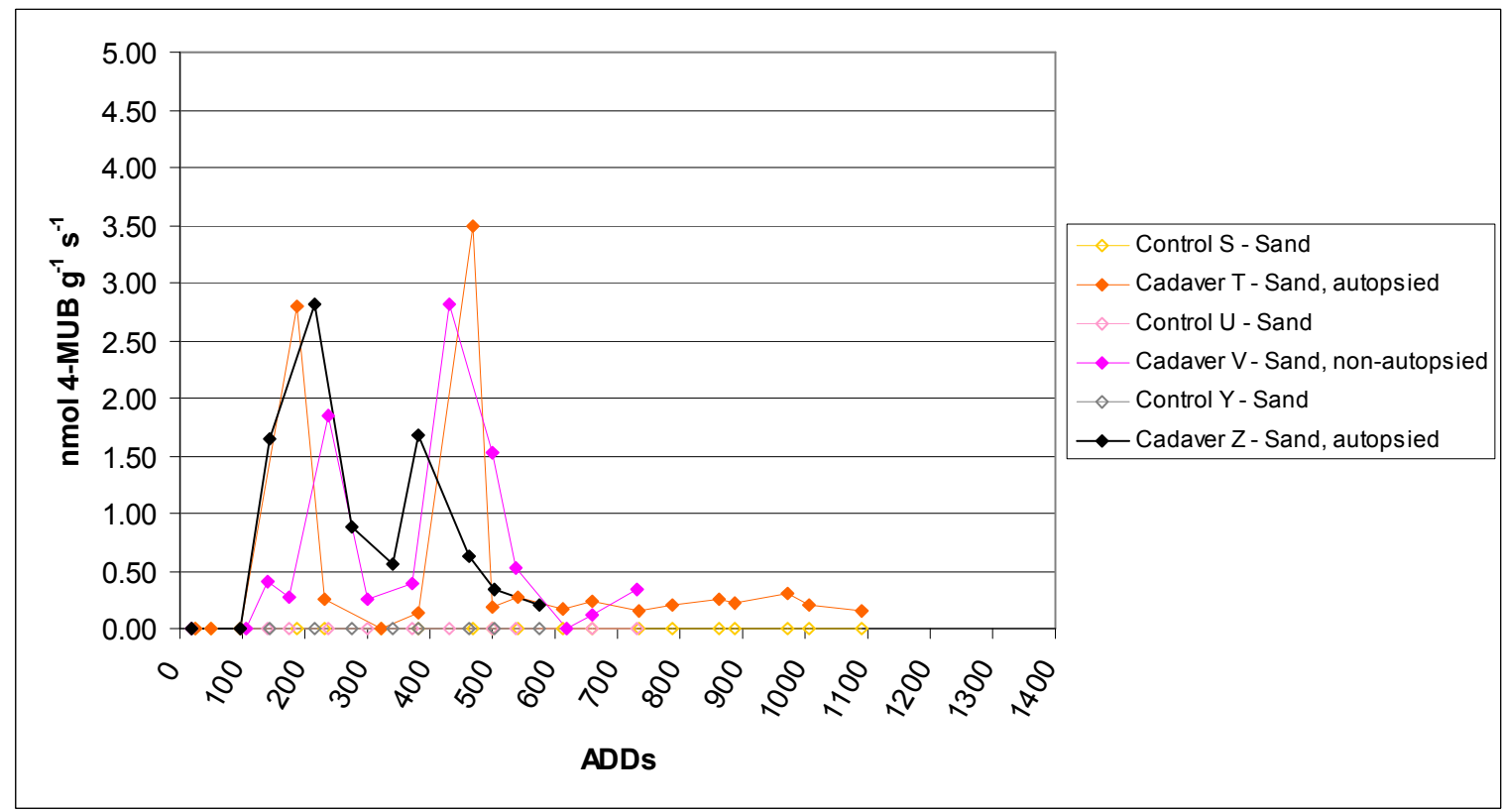

Figure 6.22 Potential acid phosphatase activity in sand samples collected from decomposition and control sites.

\subsubsection{Alanine Aminopeptidase (EC 3.4.11.2)}

Very little alanine aminopeptidase activity was seen throughout the decomposition experiment with the exception of a transient spike of potential activity at around ADD 300 
for two of the soil decomposition sites ( $\mathrm{P}$ and $\mathrm{X}$ ) and a spike at around ADD 650 for the cadaver R soil site (Figure 6.23). Because of the slow decomposition exhibited by cadaver $\mathrm{R}$, this spike could be occurring at a similar stage of decomposition as those seen for those in the cadaver $\mathrm{P}$ and $\mathrm{X}$ sites. No spikes in activity were observed in any of the sand decomposition plots, but small fluctuations above baseline levels were detected (Figure $6.23)$.

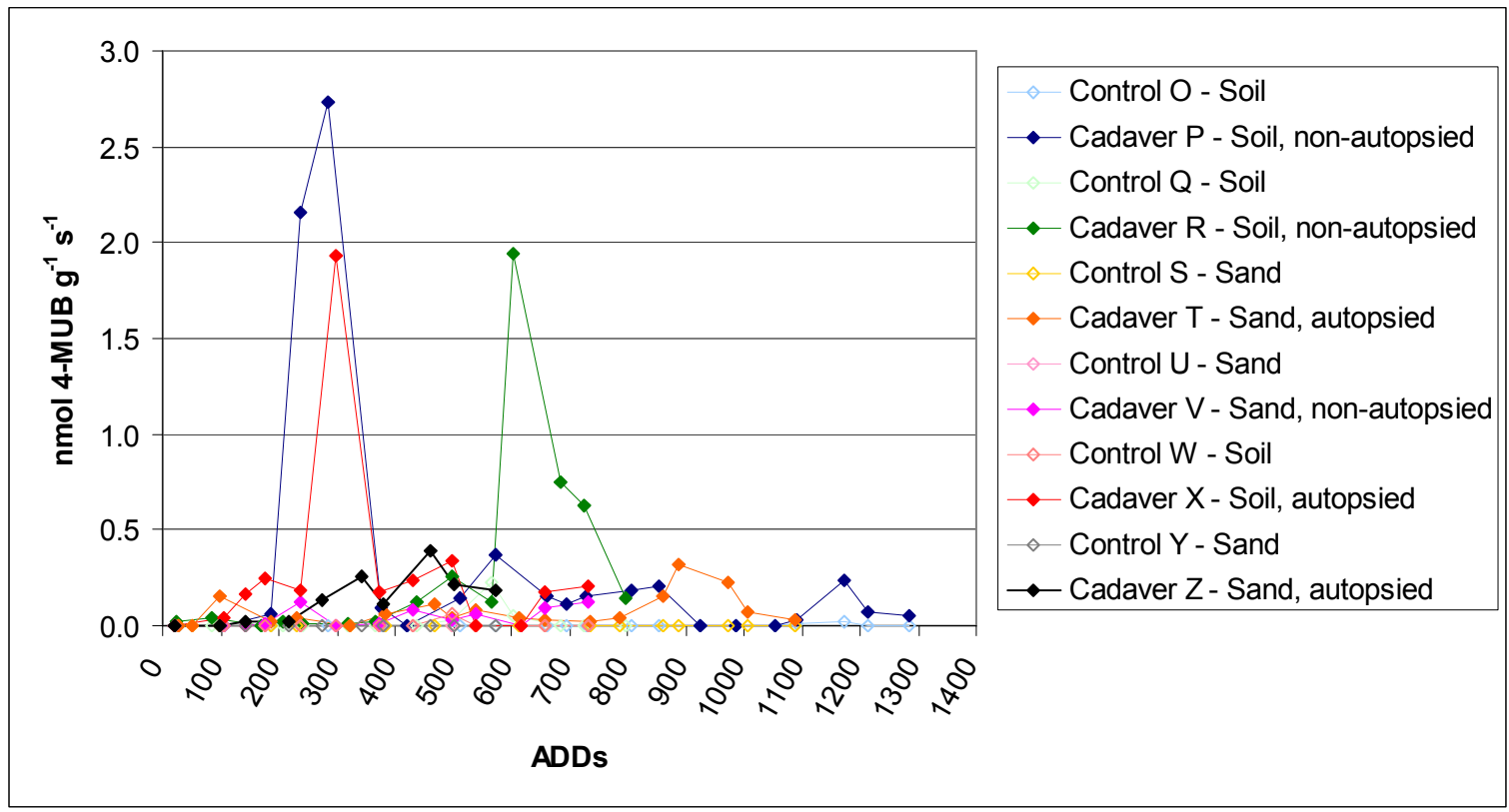

Figure 6.23 Potential alanine aminopeptidase activity in soil and sand samples collected from decomposition and control sites.

\subsubsection{Esterase (3.1.1.6) - 4-MUB acetate}

The potential esterase activity measured using 4-MUB acetate showed slightly different patterns for each soil decomposition site, but all showed an increased level of activity at some time (Figure 6.24). The cadaver P site showed an increase in activity immediately following cadaver placement, followed by some sharp fluctuations, reaching baseline activity levels on some occasions before returning to higher levels. Three major peaks in activity were apparent at ADDs 286, 512 and 854, and all were above 20 nmol 4-MUB g ${ }^{-1}$ $\mathrm{s}^{-1}$. The cadaver $\mathrm{X}$ site also showed an early increase, but reached only one peak above 20 
nmol 4-MUB g ${ }^{-1} \mathrm{~s}^{-1}$ at ADD 254, followed by a rapid decrease in activity to around $3 \mathrm{nmol}$ 4-MUB $\mathrm{g}^{-1} \mathrm{~s}^{-1}$. The cadaver $\mathrm{R}$ site showed a delayed increase in activity compared with the other two soil decomposition sites, beginning from about ADD 242 onwards and reaching a peak of $18.5 \mathrm{nmol} 4-\mathrm{MUB} \mathrm{g}^{-1} \mathrm{~s}^{-1}$ at ADD 438. After decreasing to 8.3, this site then showed another rise in activity to $13.9 \mathrm{nmol} 4-\mathrm{MUB} \mathrm{g}^{-1} \mathrm{~s}^{-1}$.

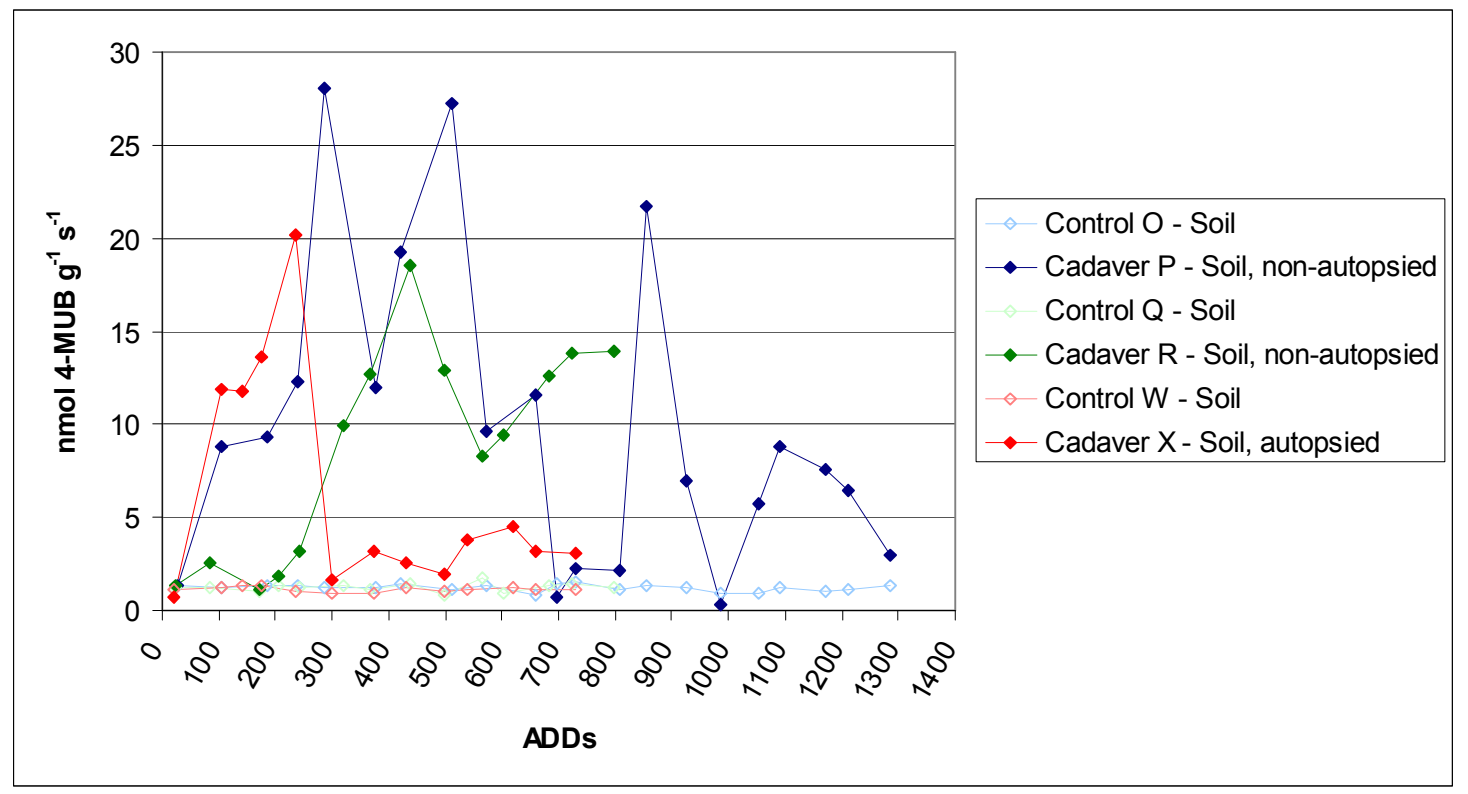

Figure 6.24 Potential esterase activity measured (using the substrate4-MUB acetate) in soil samples collected from decomposition and control sites.

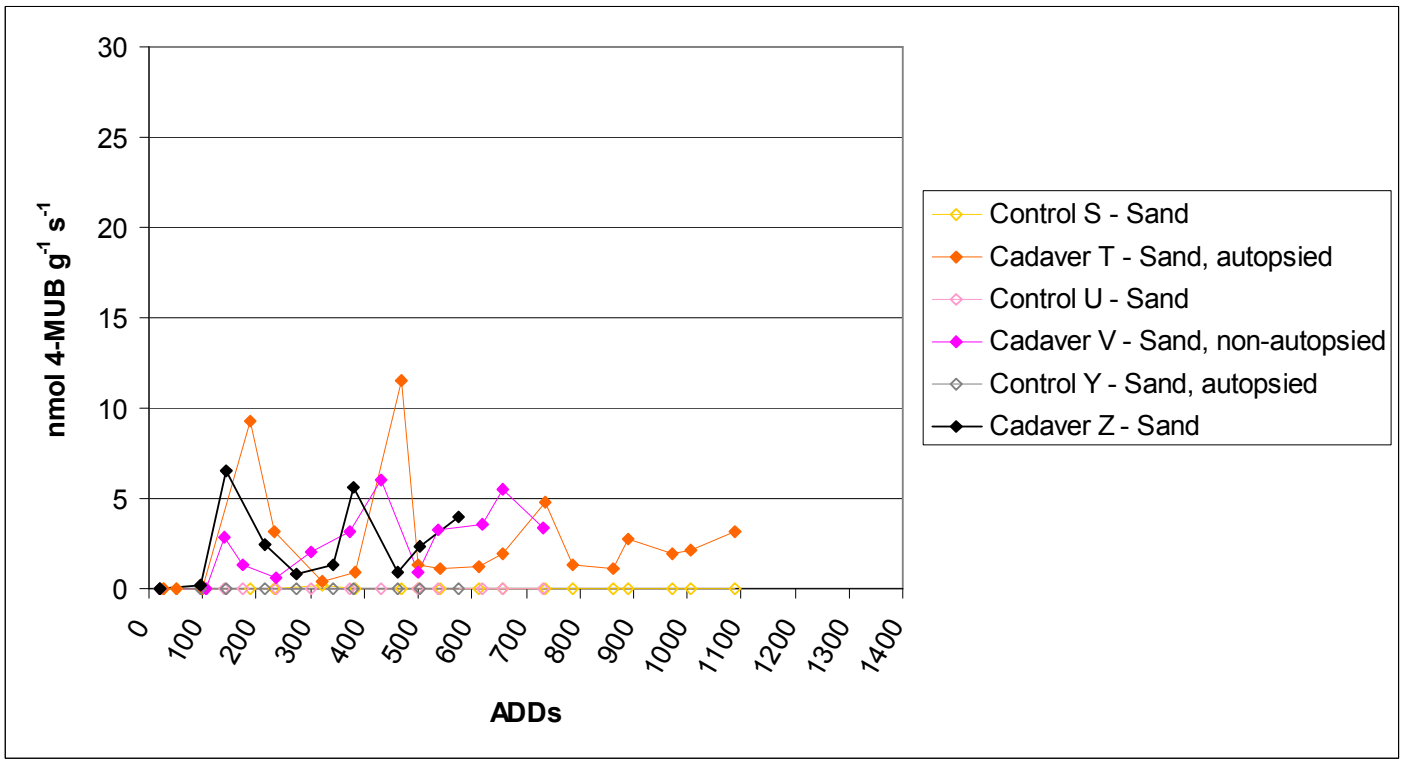

Figure 6.25 Potential esterase activity measured (using the substrate4-MUB acetate) in sand samples collected from decomposition and control sites. 
The sand decomposition sites also showed some increase in potential activity (Figure 6.25), but the highest measured was $11.5 \mathrm{nmol} 4-\mathrm{MUB} \mathrm{g}^{-1} \mathrm{~s}^{-1}$ at ADD 468 for the cadaver T site. All three sites showed an increase from about ADD 100 onwards, peaking and then dropping away again until between ADD 300 and 400 when all three showed another rise in potential activity, followed by fluctuating activity until the end of the sampling period.

\subsubsection{Esterase (EC 3.1.1.6) - 4-MUB butyrate}

The potential esterase activity (Figures 6.26 and 6.27) measured using 4-MUB butyrate very closely mirrored that when using 4-MUB acetate (section 6.4.4.10) with spikes in activity occurring at similar time-points under most of the cadavers.

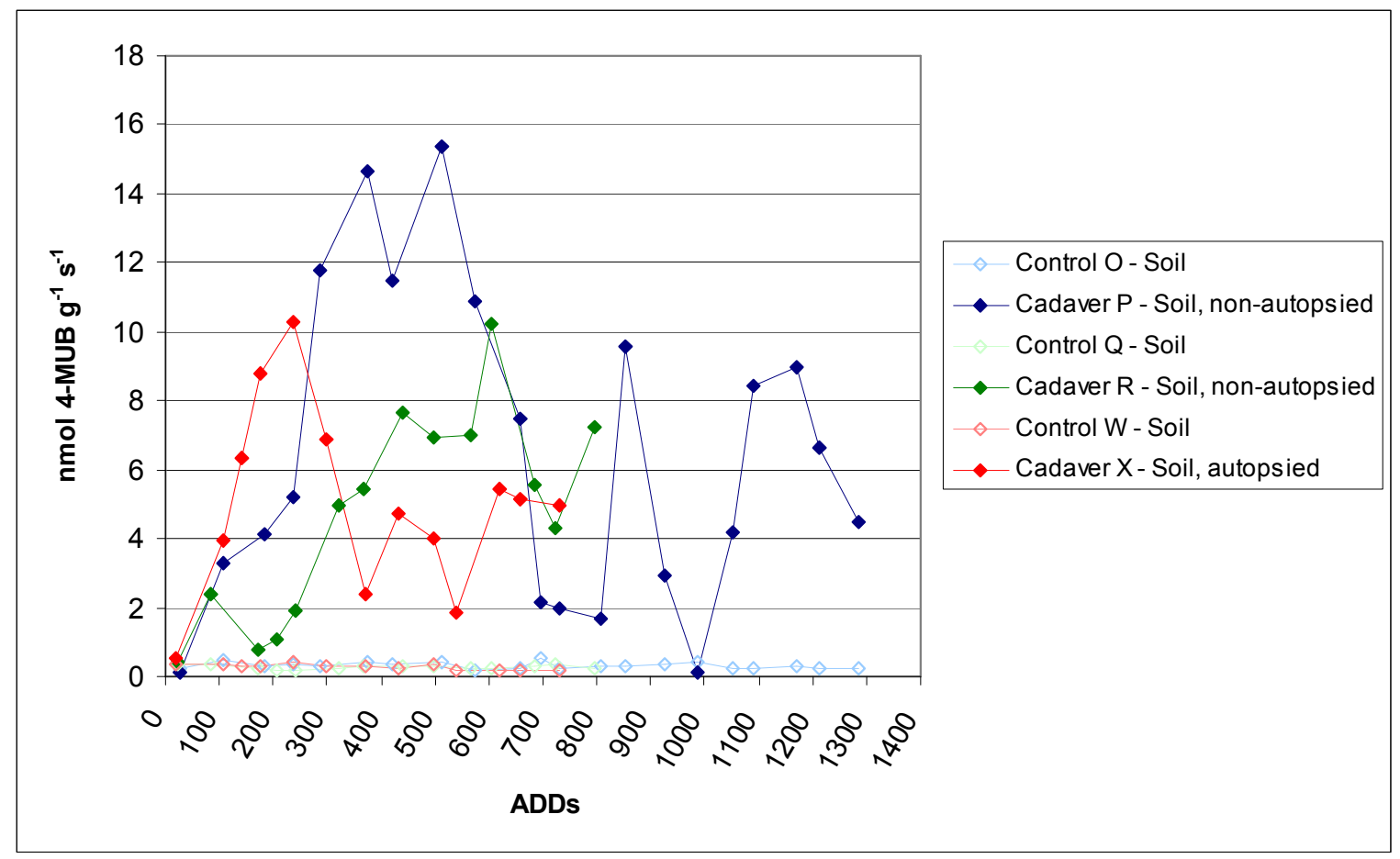

Figure 6.26 Potential esterase activity measured (using the substrate 4-MUB butyrate) in soil samples collected from decomposition and control sites. 


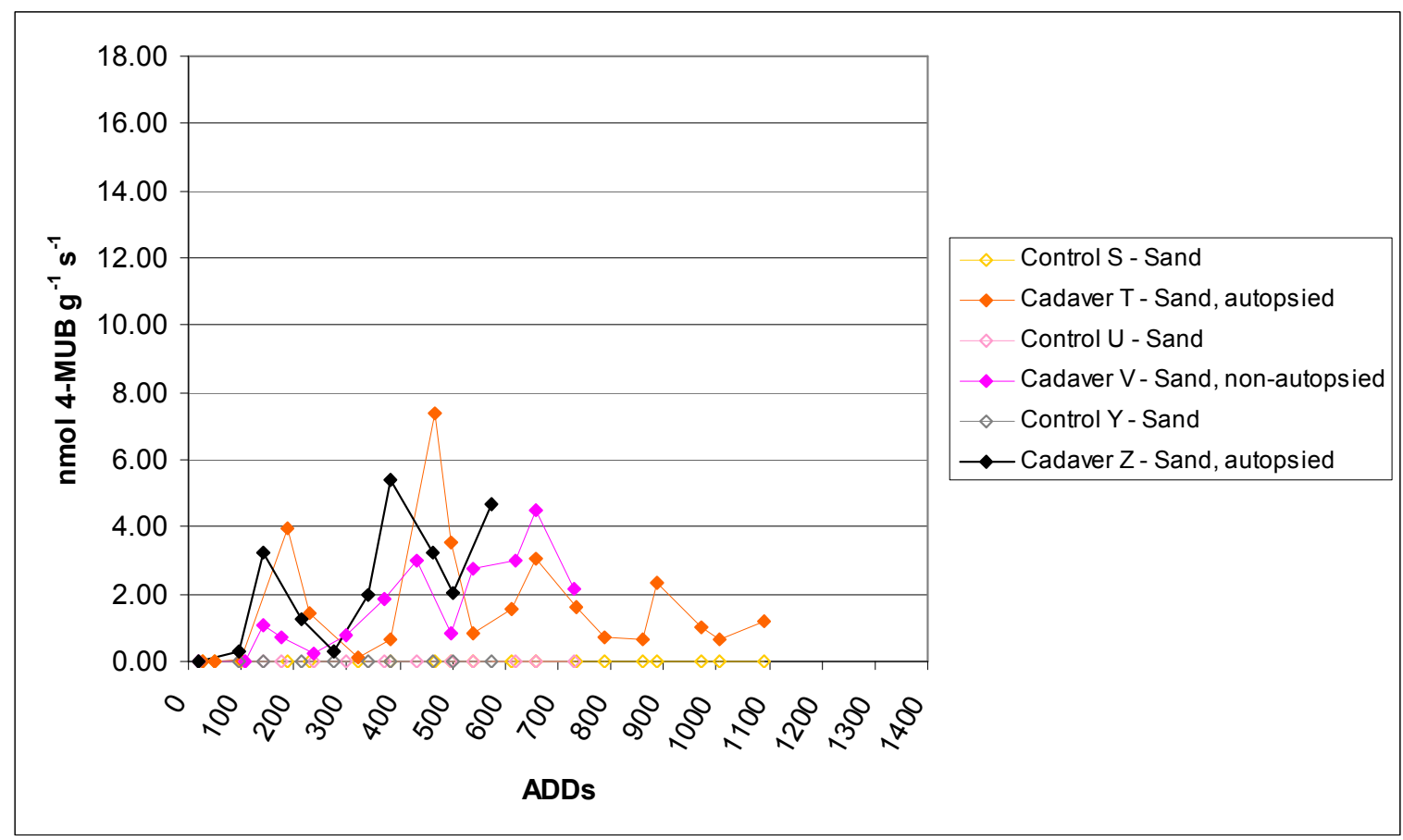

Figure 6.27 Potential esterase activity measured (using the substrate4-MUB butyrate) in sand samples collected from decomposition and control sites.

\subsubsection{Esterase (EC 3.1.1.6) - 4-MUB palmitate}

The esterase assay using the long-chain ester substrate 4-MUB palmitate did not yield any useable data. The control wells in the plate-based assay consistently gave higher fluorescence values than the actual samples which led to negative potential enzyme values. This may have been a result of the substrate being unstable at the $\mathrm{pH}$ used, or some kind of quenching interaction with the soil. This substrate also had low solubility (section 3.3.2), and had to be diluted to $400 \mu \mathrm{M}$ rather than the $800 \mu \mathrm{M}$ at which most of the other substrates were used.

\subsubsection{T-RFLP}

Good quality T-RFLP profiles were generated for all samples collected in this experiment. The mean number of T-RFs in profiles from each cadaver are shown in Table 6.2. The five time-zero control samples collected from each decomposition and control site prior to 
cadaver placement showed very high similarity to each other (Figure 6.28), suggesting that attempts to homogenise the soil were reasonably effective and that most of the variation seen in the samples collected over time was due to temporal changes rather than spatial differences.

\begin{tabular}{|c|c|c|c|c|c|c|c|c|c|}
\hline & \multicolumn{3}{|c|}{$\begin{array}{c}\text { Soil } \\
\text { Decomposition }\end{array}$} & \multicolumn{3}{c|}{ Soil Controls } & \multicolumn{3}{c|}{ Sand Decomposition } \\
\cline { 2 - 10 } & $\begin{array}{c}\mathrm{P} \\
\text { (NA) }\end{array}$ & $\begin{array}{c}\mathrm{R} \\
\mathrm{NA})\end{array}$ & $\begin{array}{c}\mathrm{X} \\
(\mathrm{A})\end{array}$ & $\mathrm{O}$ & $\mathrm{Q}$ & $\mathrm{W}$ & $\begin{array}{c}\mathrm{T} \\
(\mathrm{A})\end{array}$ & $\begin{array}{c}\mathrm{V} \\
(\mathrm{NA})\end{array}$ & $\begin{array}{c}\mathrm{Z} \\
(\mathrm{A})\end{array}$ \\
\hline $\begin{array}{c}\text { Mean number of } \\
\text { T-RFs per cadaver/control }\end{array}$ & 26 & 34 & 28 & 39 & 41 & 39 & 22 & 23 & 25 \\
\hline Mean number of T-RFs per substrate & \multicolumn{3}{|c|}{30} & \multicolumn{3}{|c|}{40} & \multicolumn{4}{|c|}{23} \\
\hline
\end{tabular}

Table 6.2 Mean number of T-RFs included in analysis from each cadaver.

$$
A=\text { autopsied }, N A=\text { not autopsied } .
$$

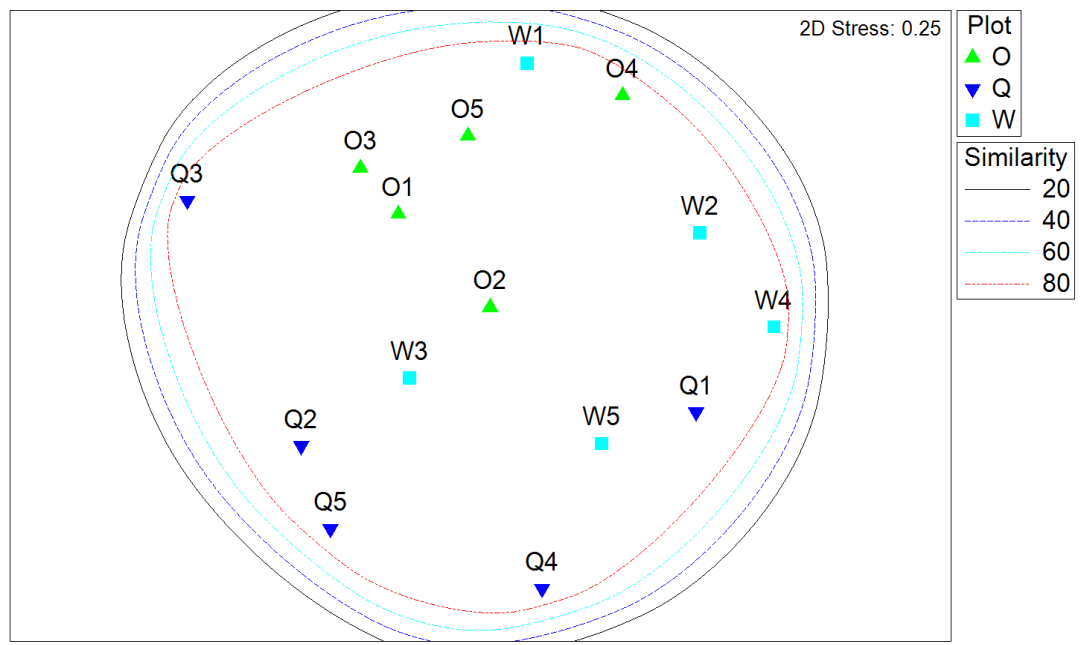

Figure 6.28 MDS plot of T-RFLP profiles from five replicate samples collected from each soil site prior to cadaver placement. The Bray-Curtis similarity index was used on square root transformed data.

\subsubsection{Multi-Dimensional Scaling (MDS) analysis}

Figures 6.29 to 6.34 show sample T-RFLP profile data from the six cadavers plotted using MDS as described in section 3.4.5.2. The RELATE function of the PRIMER software (section 3.4.5) could not be used to compare the patterns generated by the MDS analyses of 
these datasets due to the different frequency and total number of samplings for each cadaver.

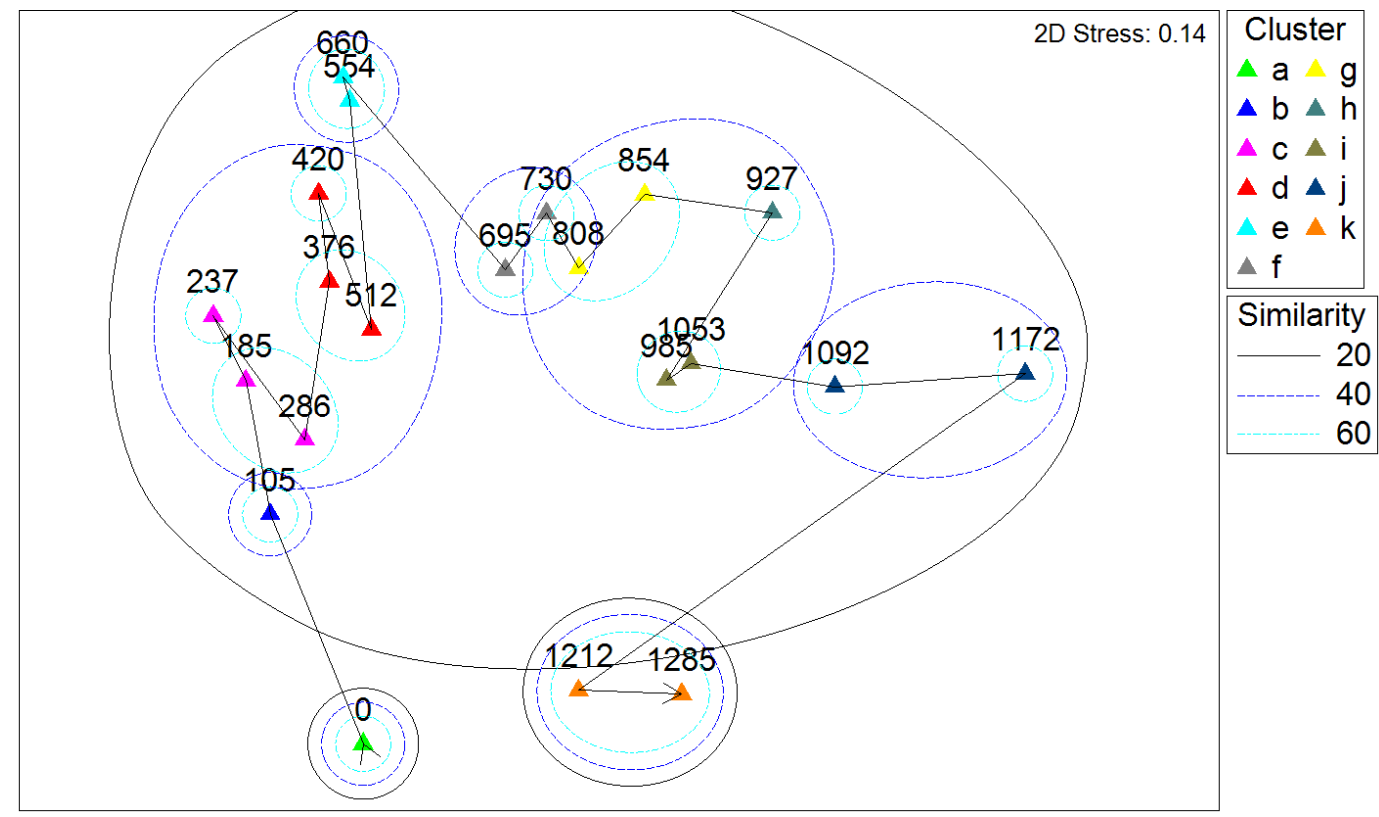

Figure 6.29 MDS plot of T-RFLP data from samples collected under cadaver P (non-autopsied on soil). Eleven statistically significant clusters were distinguished using CLUSTER. The Bray-Curtis similarity index was used on square root transformed data. Samples are denoted by their ADD values.

When plotted using MDS, the T-RFLP profile data generated from cadaver $\mathrm{P}$ samples shows a separation into eleven statistically significant clusters (Figure 6.29). All except two of those clusters show at least $20 \%$ similarity, with the time zero control sample and the two last samples at ADD 1212 and 1285 having less than $20 \%$ similarity to all the other sample profiles. The profile data plot chronologically, with a clearly sequential pattern evident. There is a large shift between the time zero sample and ADD 105 followed by less dramatic changes until a big shift in community composition appears to occur between ADD 1172 and ADD 1212.

The T-RFLP profile data from cadaver R (Figure 6.30) shows a less sequential pattern than for cadaver P, with some scattering of samples within clusters rather than a chronological succession. Five statistically significant clusters were found using CLUSTER. Of note is 
the ADD 207 profile, which clusters with the time zero profile. It appears that the bacterial community underwent a composition shift resulting in the two profiles in cluster $b$, and then underwent a shift back towards the original composition, before continuing to change to the composition which gave cluster c. There is a large shift between ADD 319 and 366, resulting in another cluster of seven samples before a final shift between ADD 724 and 797.

Cadaver T's T-RFLP profile data show a chronological pattern not unlike that of cadaver P (Figure 6.29), with the exception that the ultimate profile ADD 1089 does not cluster near the first sample, but further away again (Figure 6.31). One difference was that this cadaver was placed on sand rather than soil, so there was not a pre-existing bacterial community. Eight statistically significant clusters were detected using CLUSTER. All sample profiles have more than $20 \%$ similarity, except the time zero control.

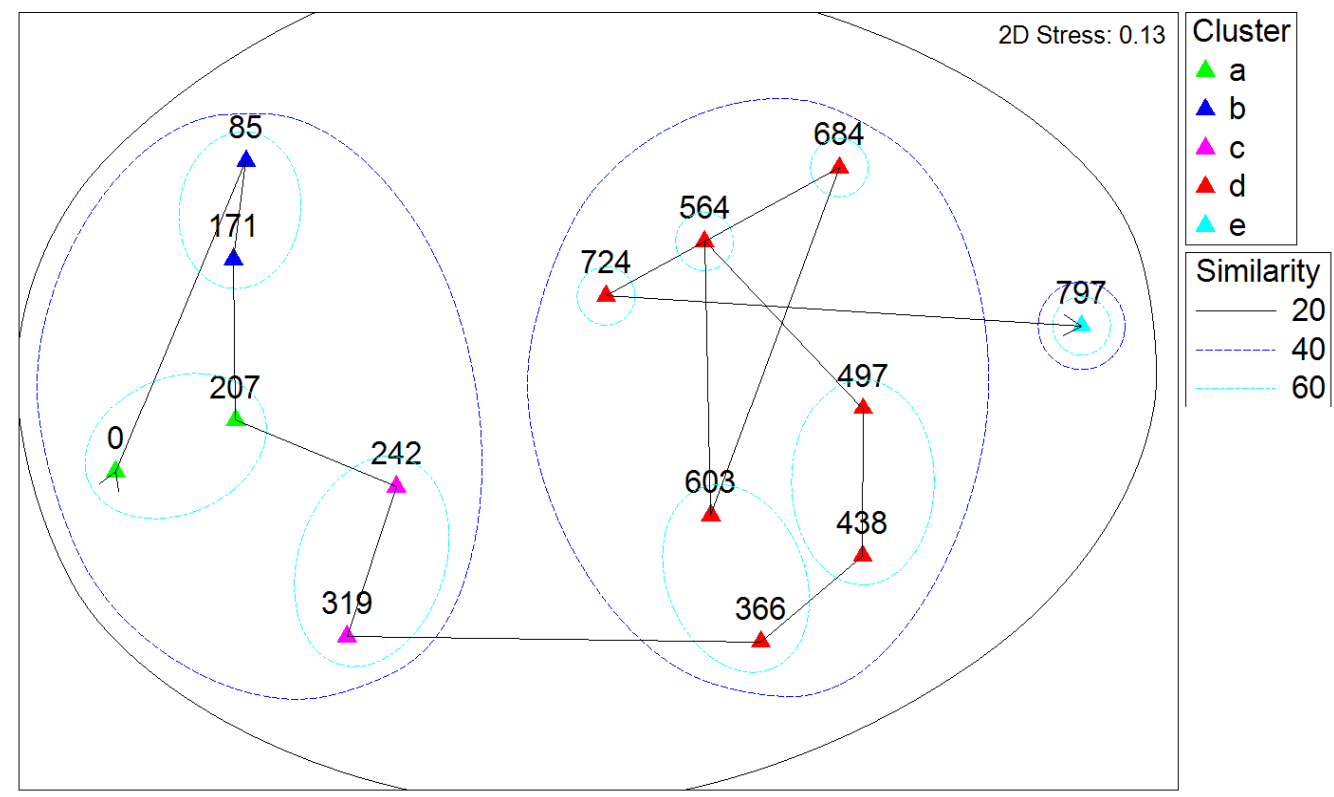

Figure 6.30 MDS plot of T-RFLP data from samples collected under cadaver R (non-autopsied on

soil). Five statistically significant clusters were distinguished using CLUSTER. The Bray-Curtis similarity index was used on square root transformed data. Samples are denoted by their ADD values. 


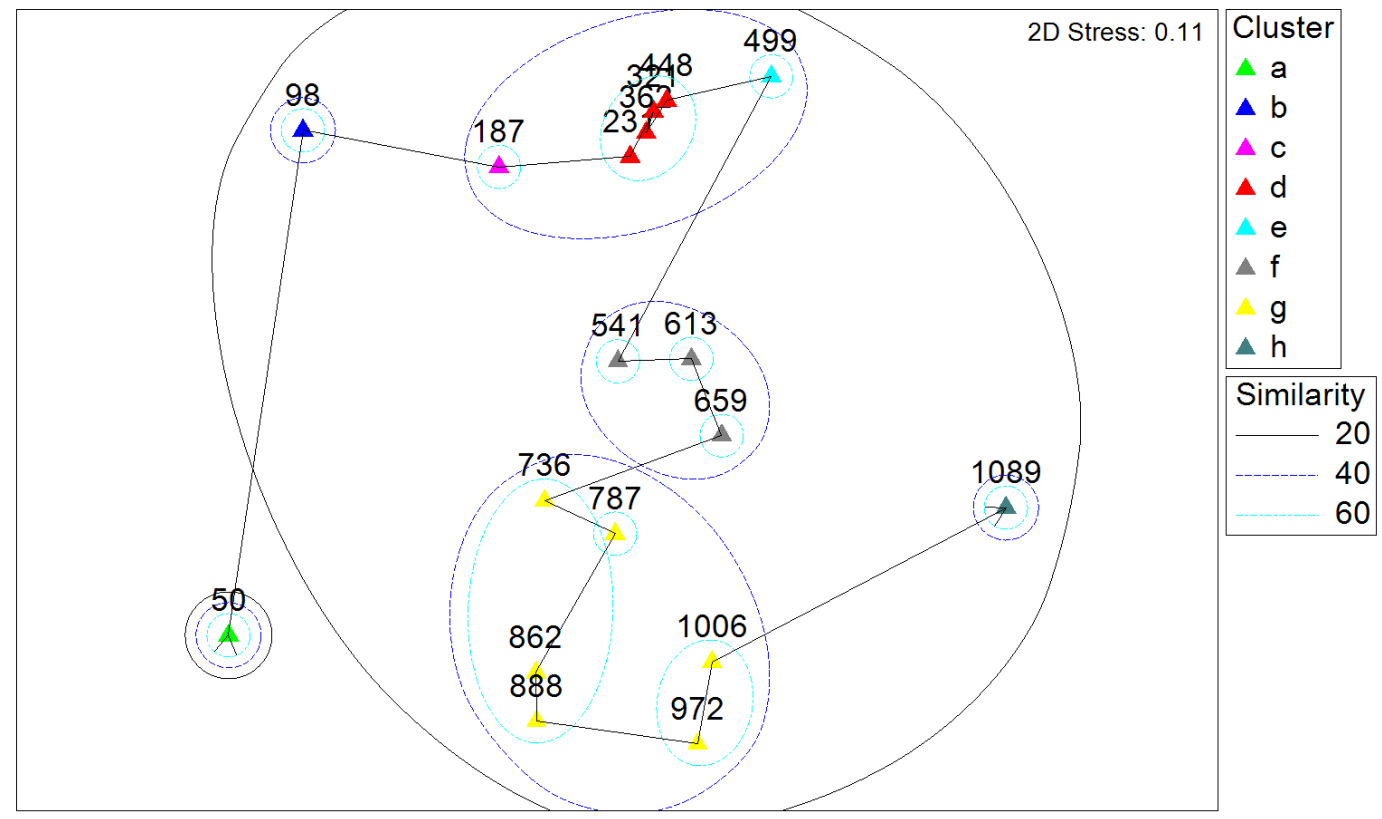

Figure 6.31 MDS plot of T-RFLP data from samples collected under cadaver T (autopsied on sand). Eight statistically significant clusters were distinguished using CLUSTER. The Bray-Curtis similarity index was used on square root transformed data. Samples are denoted by their ADD values.

The T-RFLP profile data from the cadaver V samples (Figure 6.32) forms a very similar pattern to those seen for cadaver T (Figure 6.31), although the ADD values are shifted along the pattern. The first two samples collected from under cadaver $\mathrm{V}$ cluster together, before there is a big shift to ADD 176, not unlike the shift between ADD 50 and 98 seen for cadaver $\mathrm{T}$ (Figure 6.31). This is followed by another shift creating three significant clusters at the $60 \%$ similarity level. Only six statistically significant clusters were detected for the cadaver $\mathrm{V}$ data. 


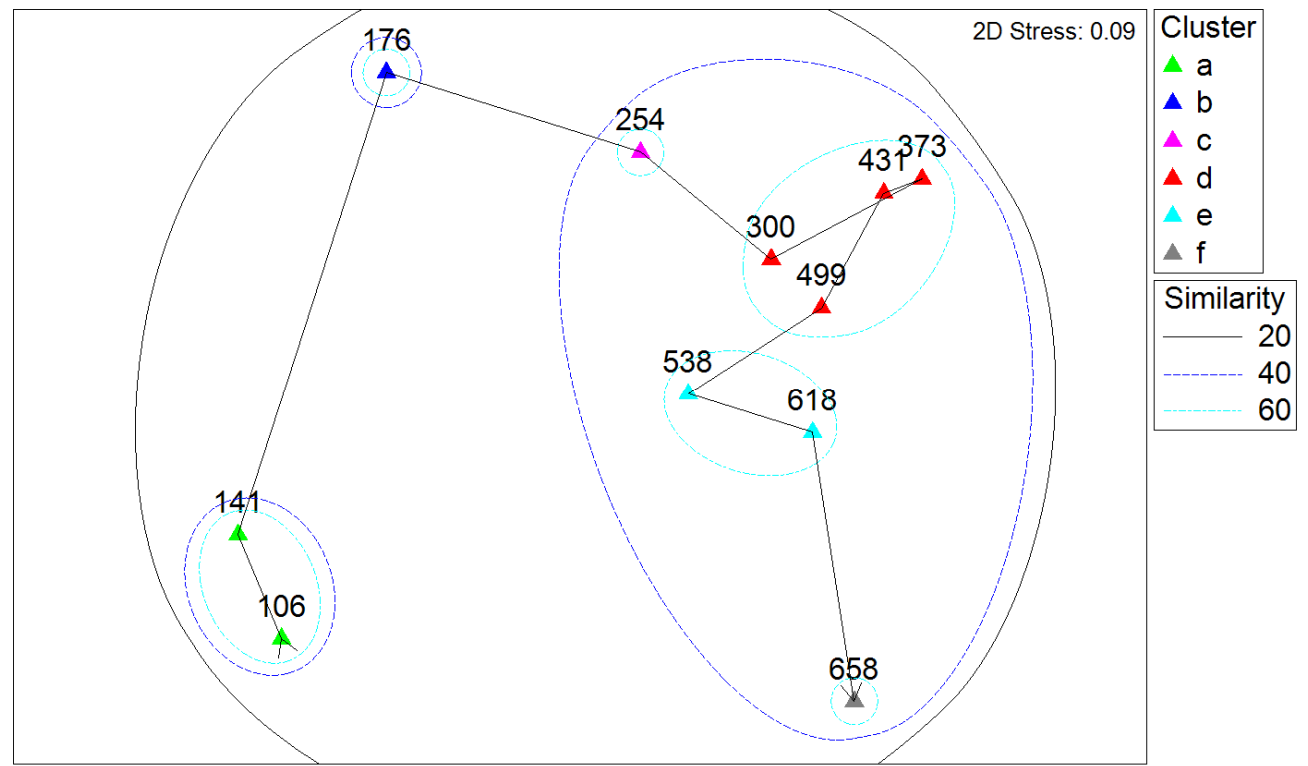

Figure 6.32 MDS plot of T-RFLP data from samples collected under cadaver V (non-autopsied on sand). Six statistically significant clusters were distinguished using CLUSTER. The Bray-Curtis similarity index was used on square root transformed data. Samples are denoted by their ADD values.

The T-RFLP profile data from the cadaver $\mathrm{X}$ samples clustered into seven statistically significant groups (Figure 6.33). These are divided into two larger clusters at the $20 \%$ similarity level, with the first four sample profiles grouped together and the remaining nine profiles together in another group. The pattern generated by the MDS profiling is reminiscent of that for one of the other cadavers on soil, cadaver R. Cadaver Z (Figure 6.34) shows a simple MDS plot with only four statistically significant clusters, because sampling had to cease at ADD 574. At the $20 \%$ similarity level, there were two distinct groups and the four significant clusterings also cluster at the $40 \%$ similarity level. 


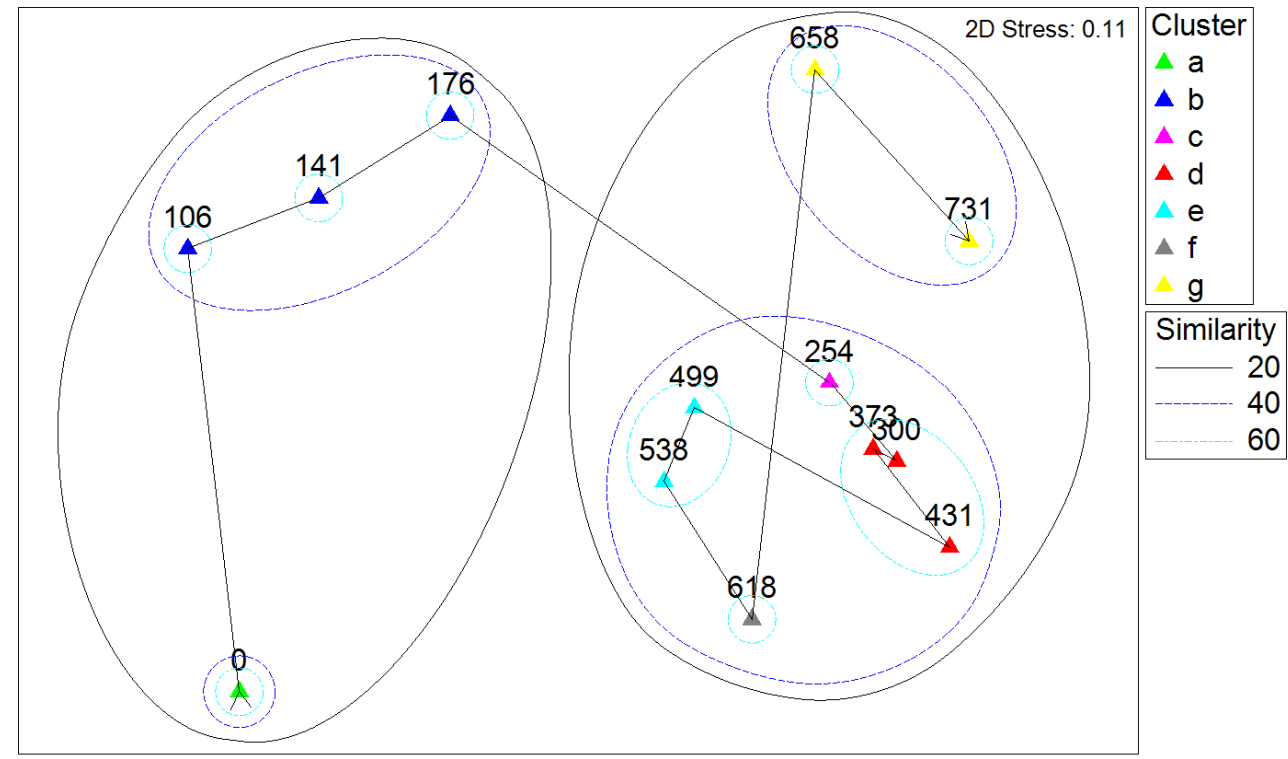

Figure 6.33 MDS plot of T-RFLP data from samples collected under cadaver X (autopsied on soil).

Seven statistically significant clusters were distinguished using CLUSTER. The Bray-Curtis similarity index was used on square root transformed data. Samples are denoted by their ADD values.

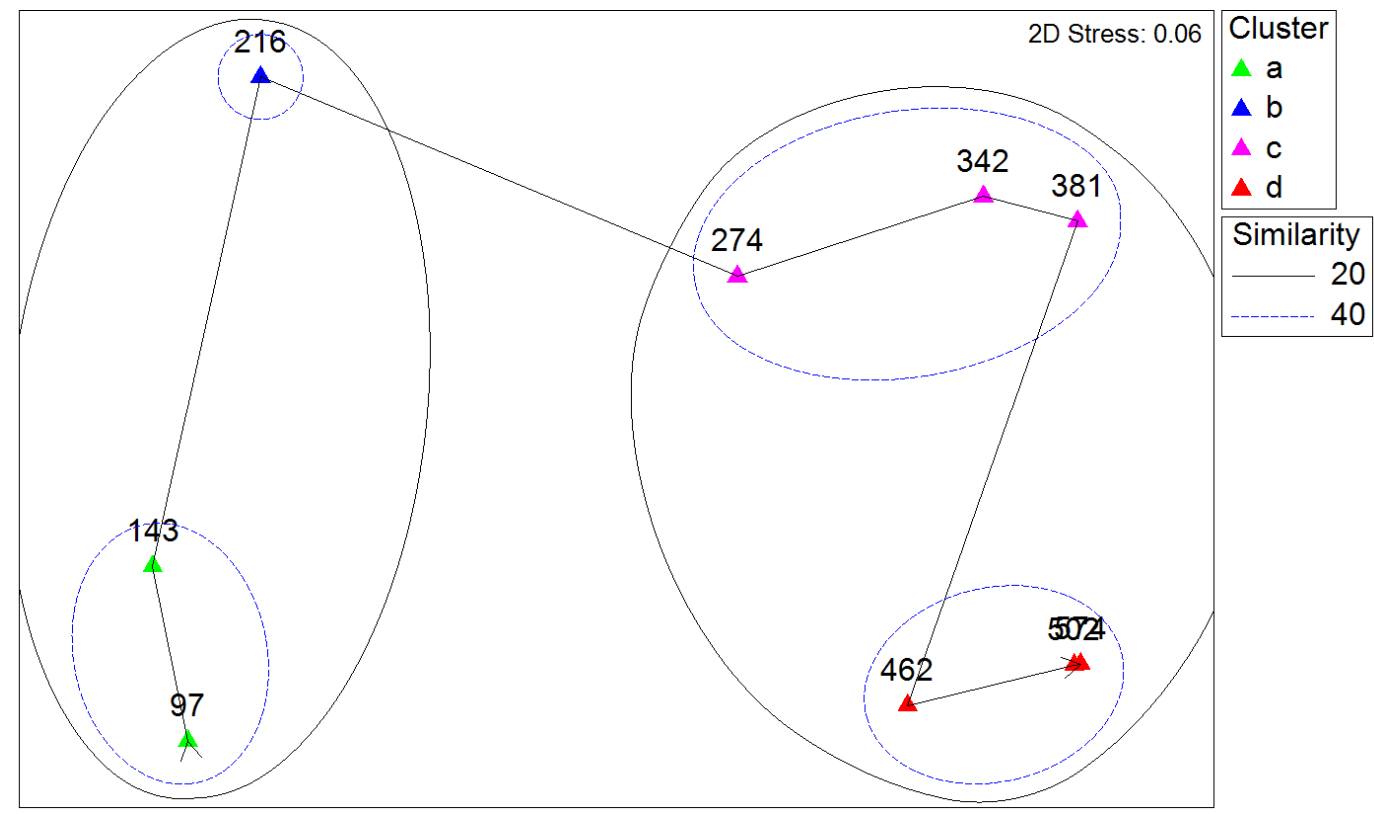

Figure 6.34 MDS plot of T-RFLP data from samples collected under cadaver Z (autopsied on sand). Four statistically significant clusters were distinguished using CLUSTER. The Bray-Curtis similarity index was used on square root transformed data. Samples are denoted by their ADD values.

Figure 6.35 shows all the soil samples, both control samples and those from under cadavers plotted together. This clearly shows that the control samples cluster more closely than 
those from under cadavers, and also shows the early samples from under each cadaver clustering with the controls.

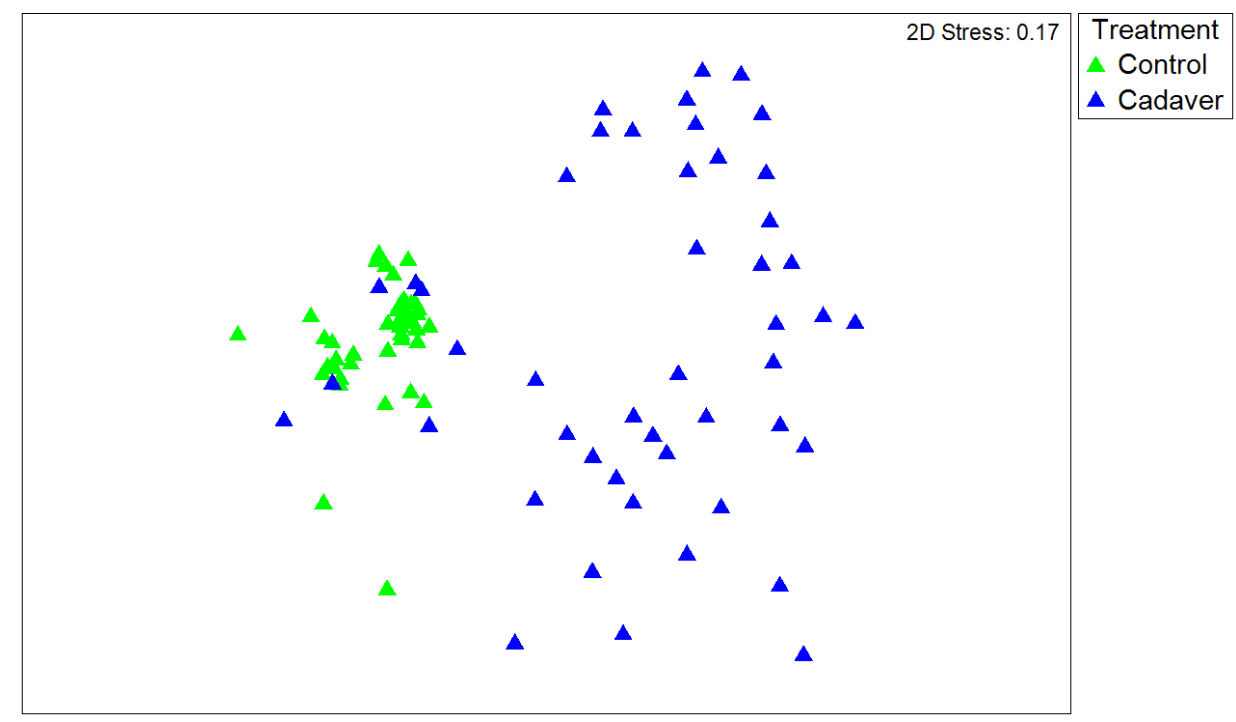

Figure 6.35 MDS plot of T-RFLP data from all soil samples, those collected from control sites and those from under cadavers. The Bray-Curtis similarity index was used on square root transformed data.

The control soil samples from the different sites were reasonably similar to each other (Figure 6.36), with all having $60 \%$ or greater similarity to each other. The time-zero samples from under the cadavers each fell within the clusters of their corresponding control site samples. At the $70 \%$ similarity level, the control site Q largely separated into a different cluster. This is likely to be caused by spatial heterogeneity in the soil, as the $\mathrm{O} / \mathrm{P}$ and W/X sites were approximately 4 metres apart but on the same side of the gravel road, whereas the $\mathrm{Q} / \mathrm{R}$ site was further away, on the eastern side of the road (Figure 6.1). 


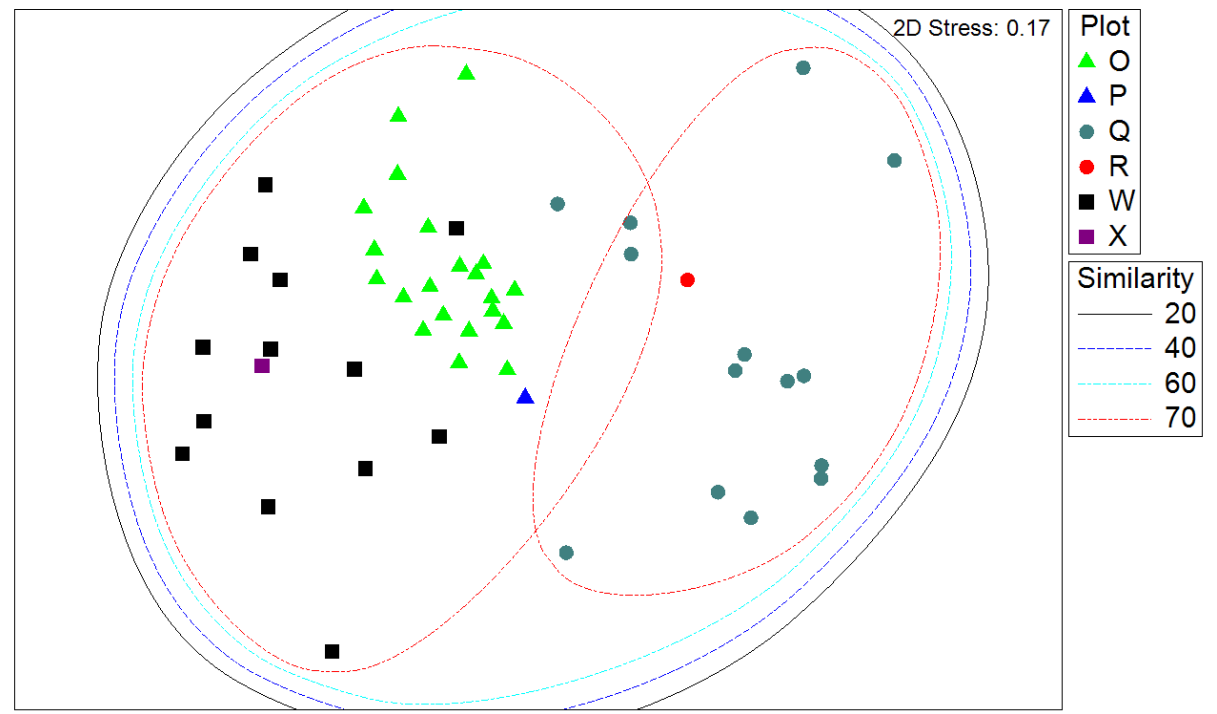

Figure 6.36 MDS plot of T-RFLP data from soil control samples from the control sites $(O, Q$ and $W)$ and the $\boldsymbol{t}=\boldsymbol{0}$ sample from under each cadaver $(\boldsymbol{P}, \boldsymbol{R}$ and $\boldsymbol{X})$. The Bray-Curtis similarity index was used on square root transformed data.

\subsubsection{DGGE}

Samples collected from under the cadavers were analysed using DGGE as per the method described in section 3.4.4. All the samples collected were analysed for cadavers R, T, V, X and $\mathrm{Z}$ as fewer than 16 samples were collected for each of these. Cadaver $\mathrm{P}$, however, had a sample set of 21 , so 14 samples that spanned the collection period were chosen for analysis. A separate gel was run for samples from each cadaver. Figures 6.37, 6.38 and 6.39 show photographs of the DGGE gels for samples from cadavers $\mathrm{R}, \mathrm{T}$ and $\mathrm{V}$ respectively. Fifteen control soil samples were selected (four each from soil control sites $\mathrm{O}, \mathrm{W}$ and $\mathrm{Q}$ and the time 0 control samples from $\mathrm{P}, \mathrm{X}$ and $\mathrm{R}$ prior to cadaver placement) and were run on a single DGGE gel (Figure 6.40). 

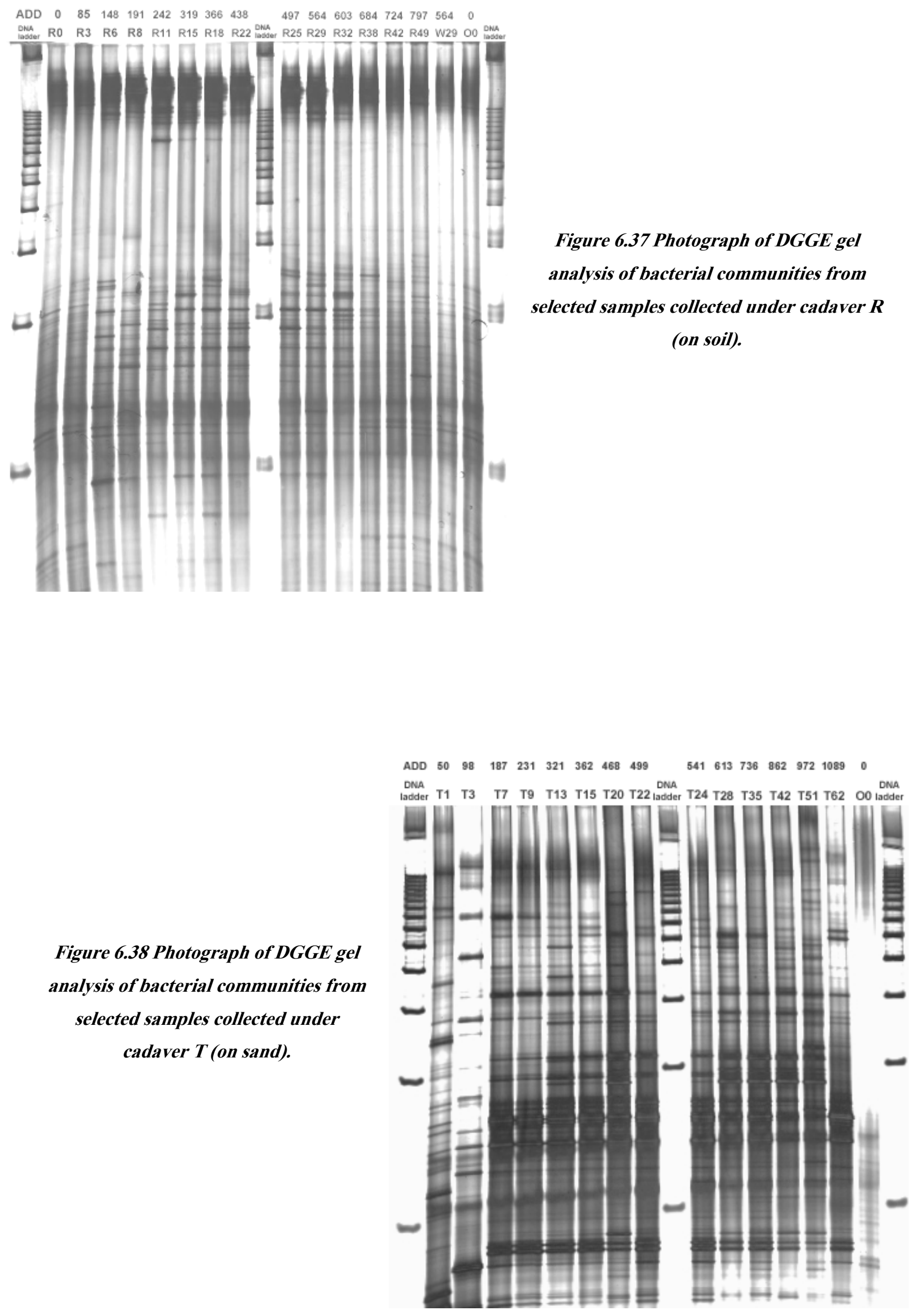


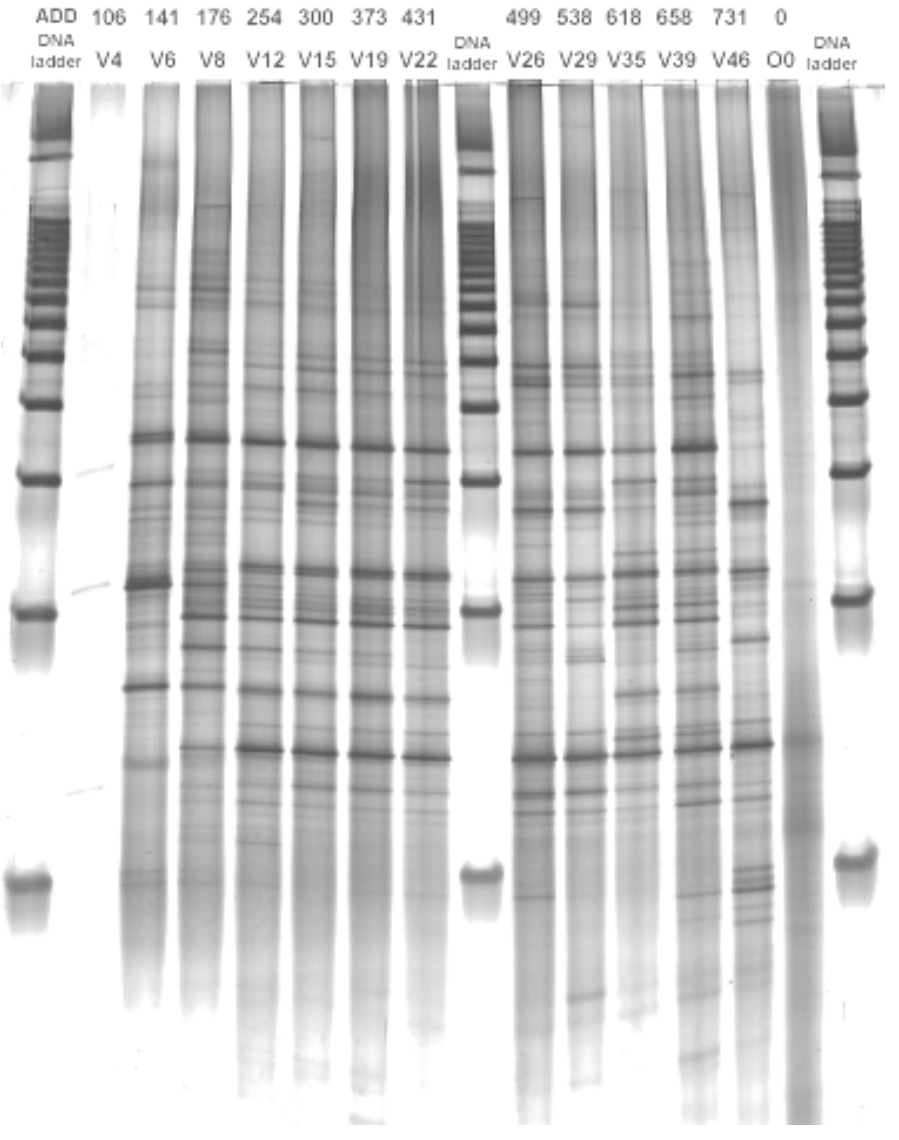

Figure 6.39 Photograph of DGGE gel analysis of bacterial communities from selected samples collected under cadaver V(on sand).

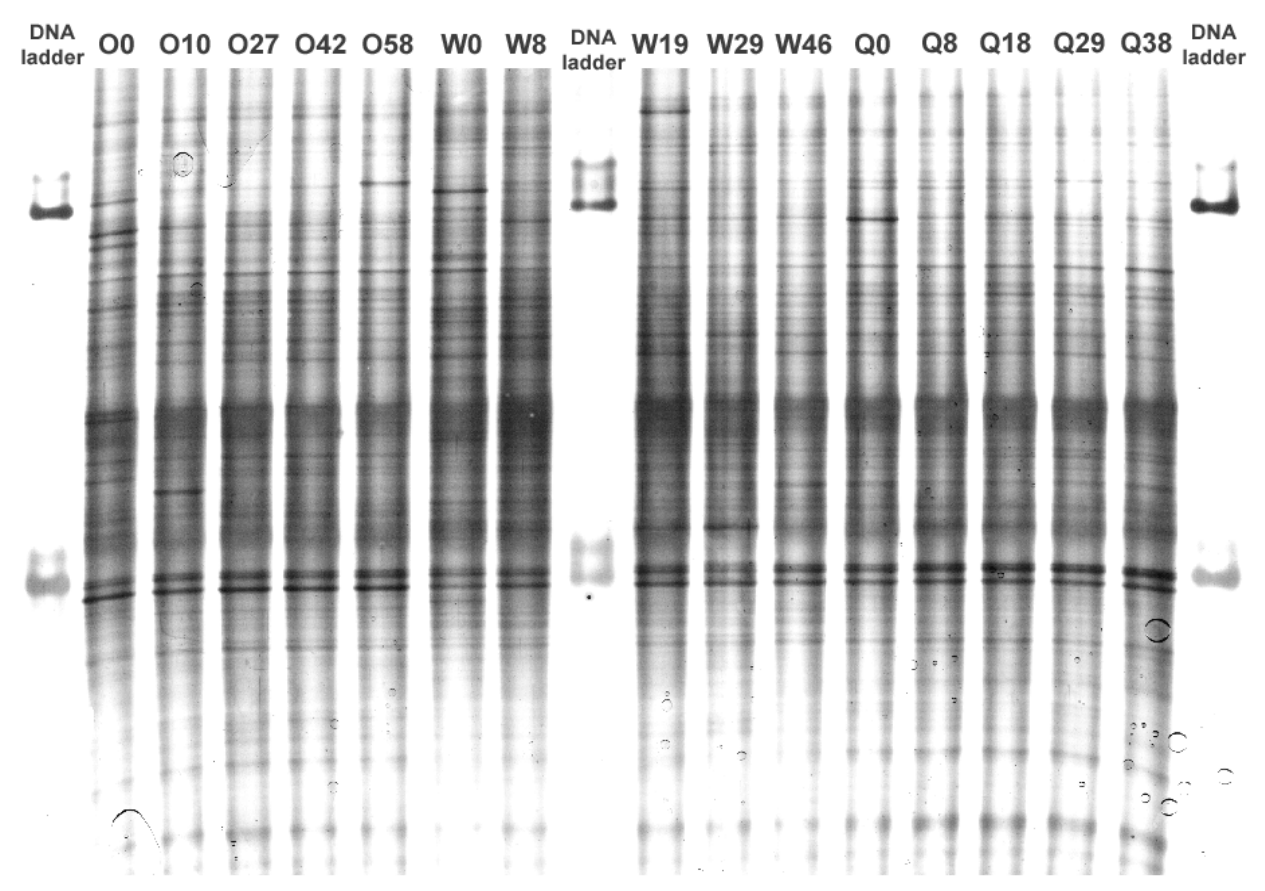

Figure 6.40 Photograph of DGGE gel analysis of bacterial communities from selected soil control samples. 
The DGGE gels generated were all of good quality with a high number of bands evident for most samples (Table 6.3). As with the previous experiment (chapter 5), the control soil samples generated a greater number of fainter bands than the decomposition derived samples. The sample chosen to be run on each of the gels to allow inter-gel comparisons was a control soil sample, which meant, like in the previous experiment, few bands were evident. This meant that inter-gel comparisons were not made.

\begin{tabular}{|c|c|c|c|c|c|c|c|}
\hline & \multicolumn{4}{|c|}{ Soil } & \multicolumn{3}{c|}{ Sand } \\
\cline { 2 - 9 } & P & R & X & Controls & T & V & Z \\
\hline Mean number of bands per profile & 21 & 26 & 18 & 50 & 40 & 31 & 37 \\
\hline Mean number of bands per substrate & \multicolumn{3}{|c|}{22} & & \multicolumn{3}{|c|}{36} \\
\hline
\end{tabular}

Table 6.3 Number of bands present in DGGE profiles from under each cadaver.

\subsubsection{Multi-Dimensional Scaling (MDS) analysis}

The DGGE data from each carcass were analysed using MDS as described in section 3.4.5.2. Figures 6.41 to 6.47 show the MDS plots of the DGGE profile data from samples collected under the six cadavers. Figure 6.48 shows the DGGE profile data from selected soil control samples. When compared to their respective T-RFLP MDS plots using RELATE, the cadaver P and R DGGE MDS plots returned $\rho$ values of $0.616(\mathrm{p}<0.001)$ and $0.433(\mathrm{p}<0.01)$ respectively. This suggests that for both cadavers, the T-RFLP and DGGE similarity matrixes underlying the MDS plots are significantly correlated. 


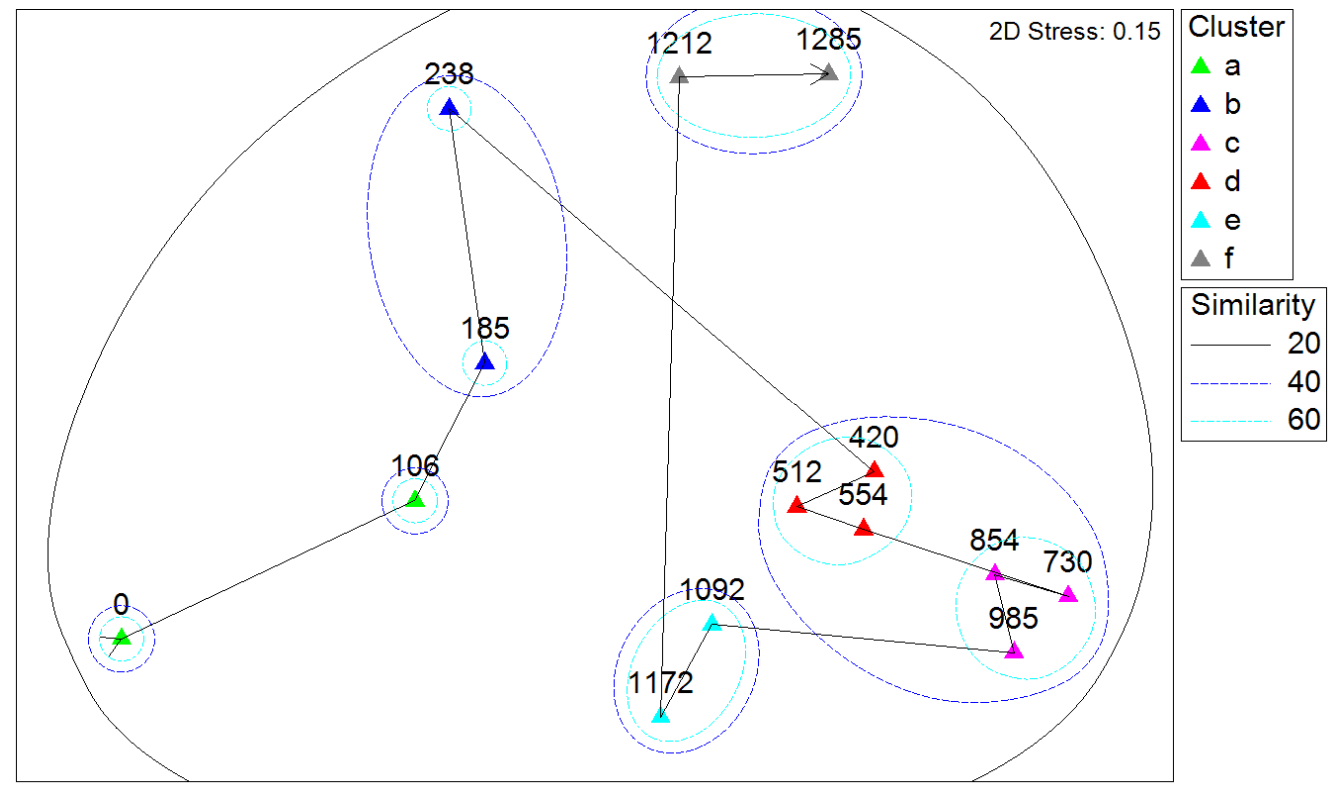

Figure 6.41 MDS plot of DGGE data from samples collected under cadaver P (non-autopsied on soil). Six statistically significant clusters were distinguished using CLUSTER. The Bray-Curtis similarity index was used on square root transformed data. Samples are denoted by their ADD values.

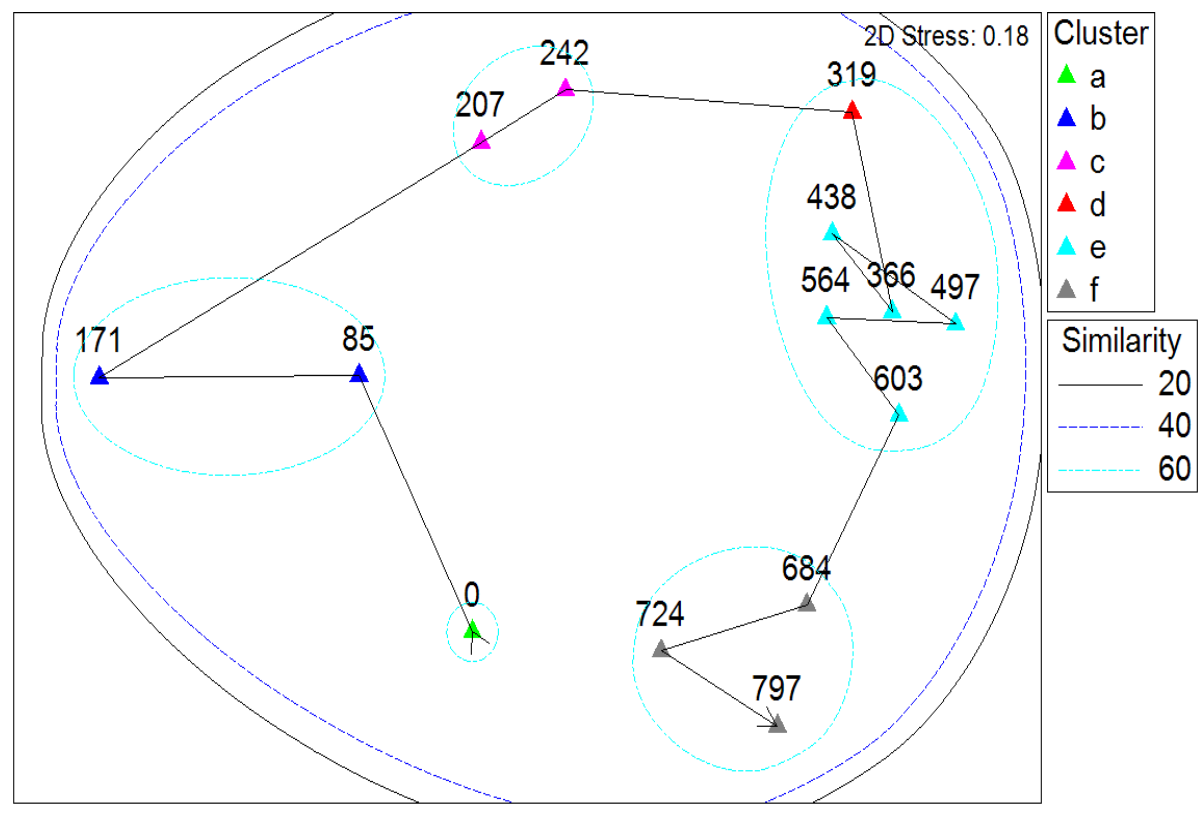

Figure 6.42 MDS plot of DGGE data from samples collected under cadaver R (non-autopsied on soil).

Six statistically significant clusters were distinguished using CLUSTER. The Bray-Curtis similarity index was used on square root transformed data. Samples are denoted by their ADD values. 


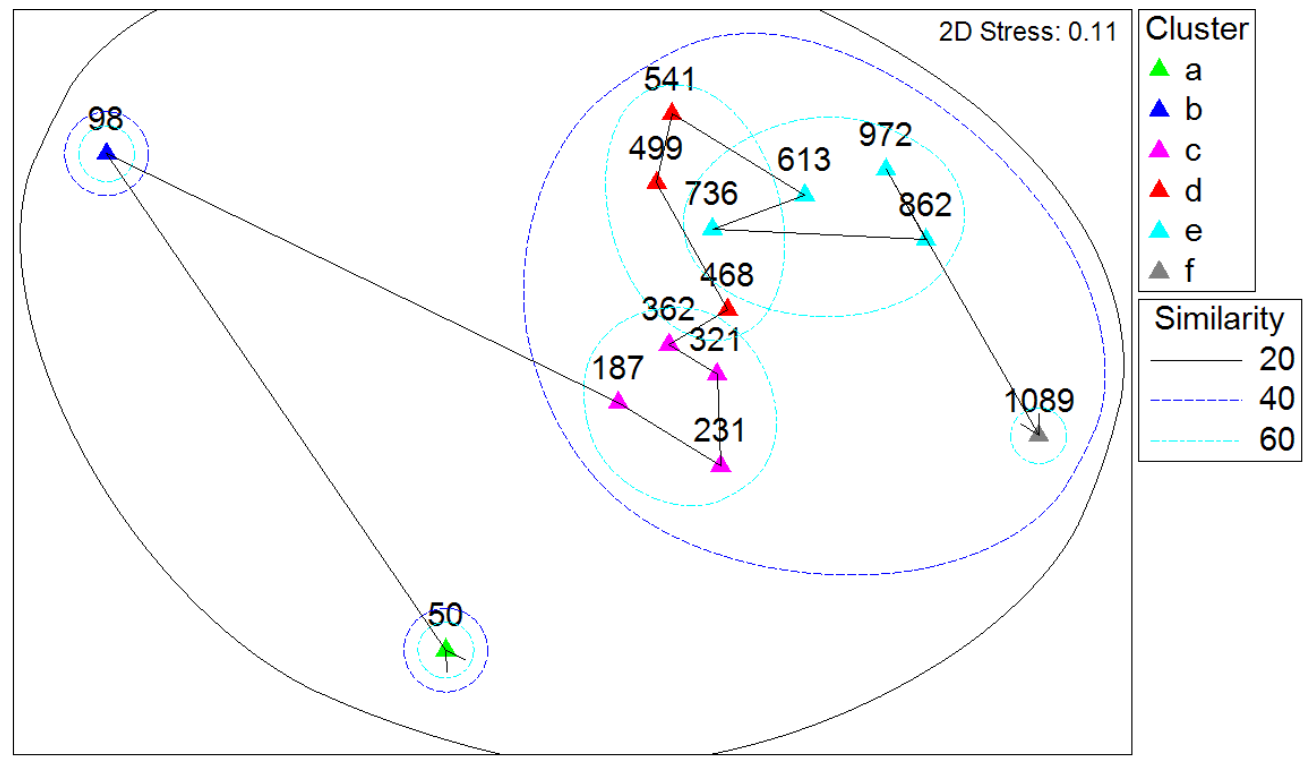

Figure 6.43 MDS plot of DGGE data from samples collected under cadaver T (autopsied on sand). Six statistically significant clusters were distinguished using CLUSTER. The Bray-Curtis similarity index was used on square root transformed data. Samples are denoted by their ADD values.

Sample V4 (ADD 106) did not produce a good DGGE profile, with only three bands discernible. It was initially included in the MDS analysis (Figure 6.44) but was excluded from a subsequent MDS analysis (Figure 6.45) to allow a more meaningful representation to be created. Three statistically significant clusters were detected. As with cadaver T, the initial sample (ADD 141) clustered away from the rest of the samples. Because the very first sample V4 was not included in the analysis, the ADD 141 timepoint is similar to the ADD 98 sample from cadaver T. A similar successive pattern was seen from this timepoint onwards. When compared with their T-RFLP MDS patterns using RELATE, the cadaver T and V DGGE MDS pattern gave $\rho$ values of 0.288 and 0.064 respectively with significance levels of $\mathrm{p}=0.042$ and 0.322 . This indicates that the two cadaver T MDS similarity matrices show some similarities and this is a significant result at the $95 \%$ level. The two plots for cadaver $\mathrm{V}$, however, gave a very low correlation, even with the low quality V4 (ADD 106) DGGE profile removed from analysis. 
Cadaver X (Figure 6.46) displayed a pattern very similar to those seen for cadavers $\mathrm{T}$ (Figure 6.43), V (Figure 6.45) and R (Figure 6.41). When compared to its T-RFLP pattern using RELATE, a $\rho$ value of 0.641 resulted $(p=0.023)$. This suggests the two similarity matrixes underlying the MDS plots are significantly similar. Cadaver Z's two matrices gave slightly higher similarity again with a $\rho$ value of 0.659 and $p=0.009$.

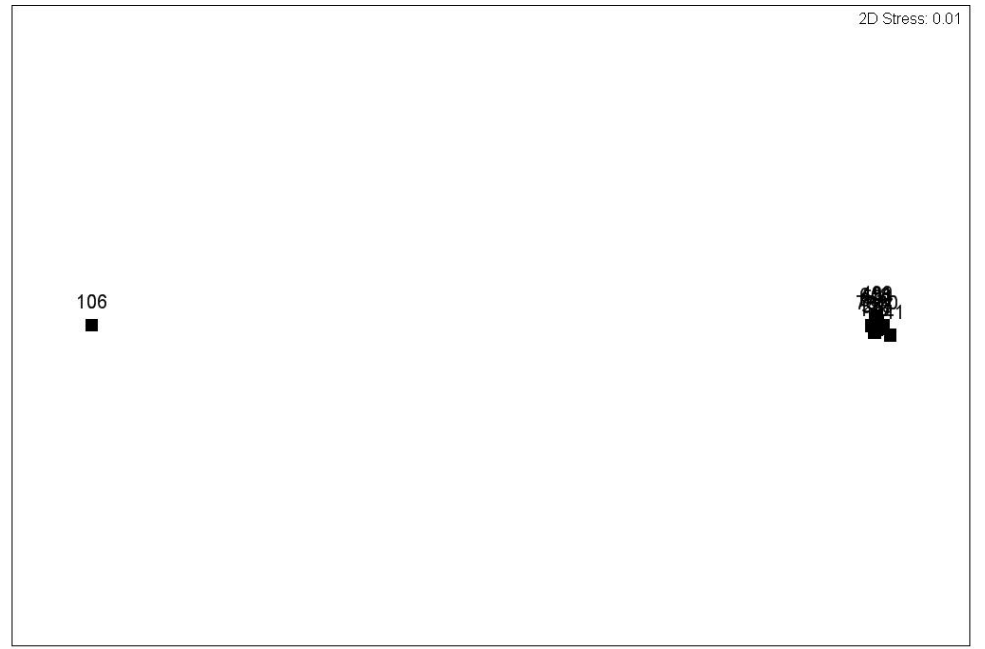

Figure 6.44 MDS plot of DGGE data from all samples collected under cadaver V (non-autopsied on sand). The Bray-Curtis similarity index was used on square root transformed data.

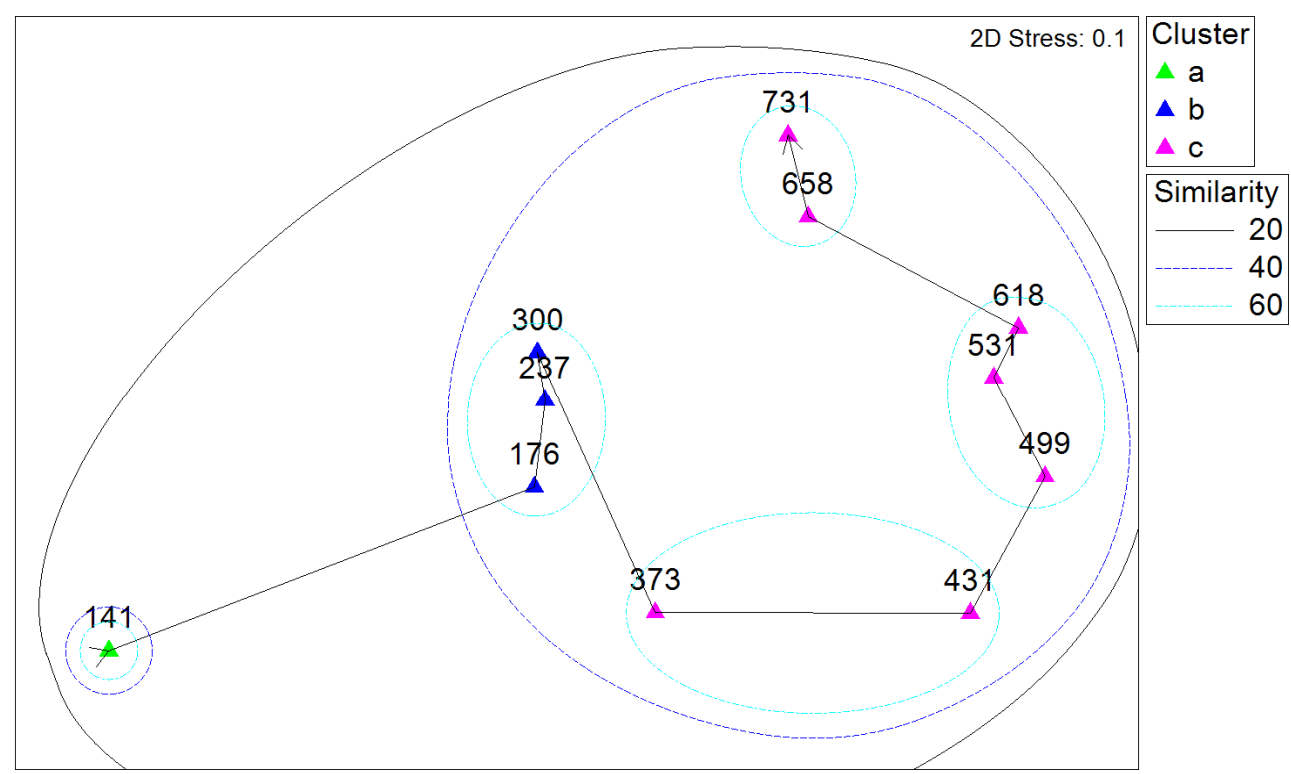

Figure 6.45 MDS plot of DGGE data from samples collected under cadaver V with sample V4 (ADD 106) excluded. Three statistically significant clusters were distinguished using CLUSTER. The Bray-Curtis similarity index was used on square root transformed data. Samples are denoted by their ADD values. 


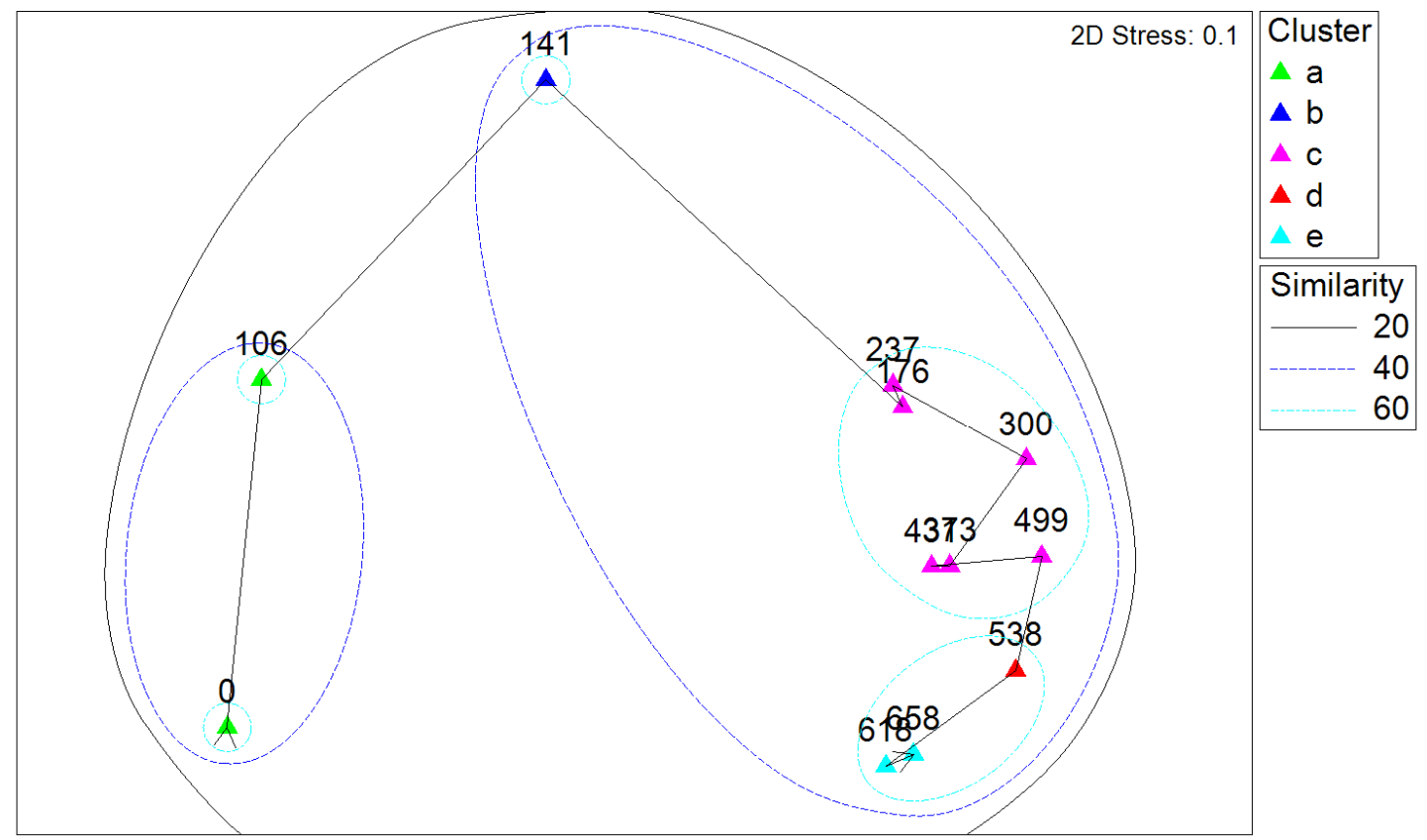

Figure 6.46 MDS plot of DGGE data from samples collected under cadaver X (autopsied on soil). Five statistically significant clusters were distinguished using CLUSTER. The Bray-Curtis similarity index was used on square root transformed data. Samples are denoted by their ADD values.

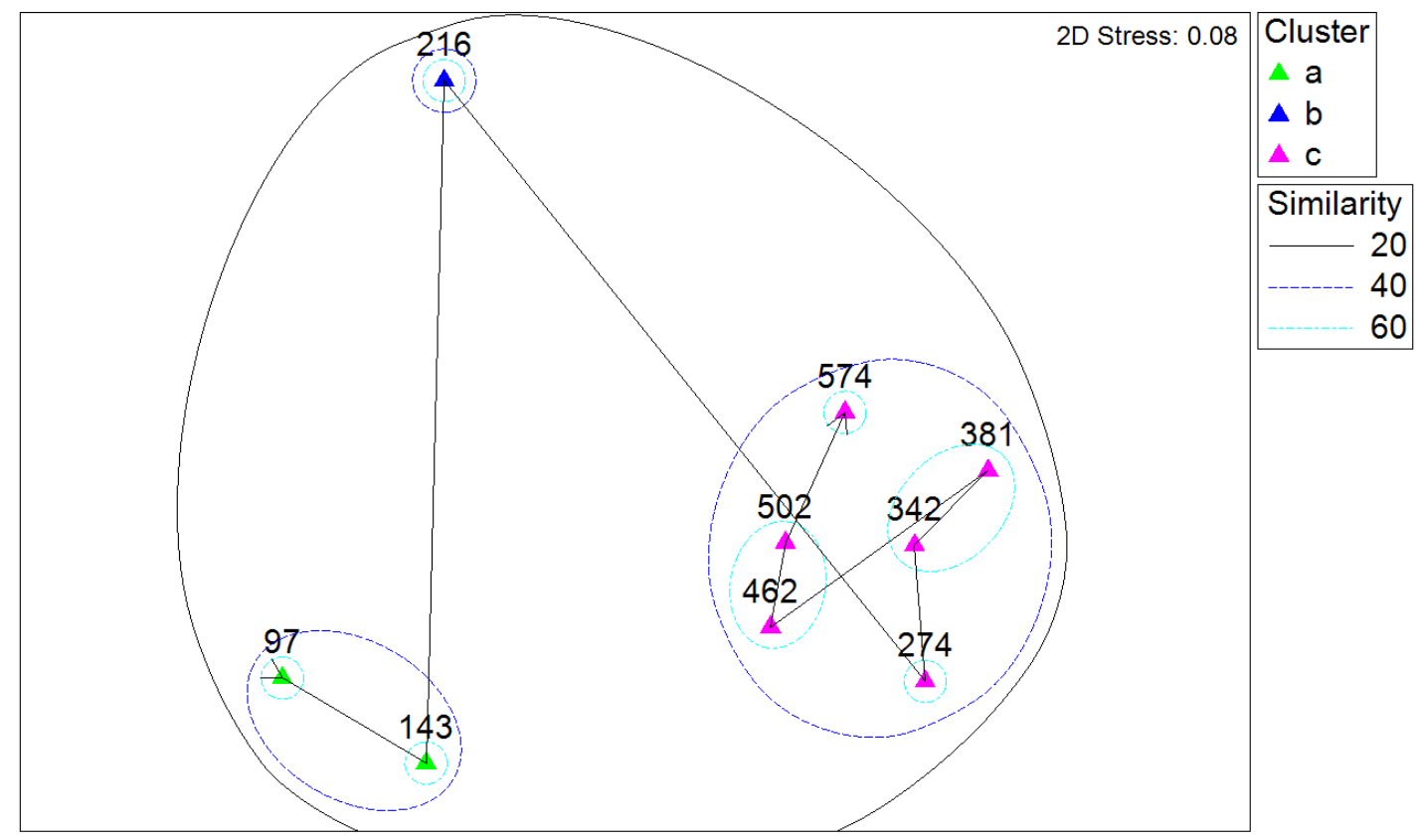

Figure 6.47 MDS plot of DGGE data from samples collected under cadaver Z (autopsied on sand).

Three statistically significant clusters were distinguished using CLUSTER. The Bray-Curtis similarity index was used on square root transformed data. Samples are denoted by their ADD values. 


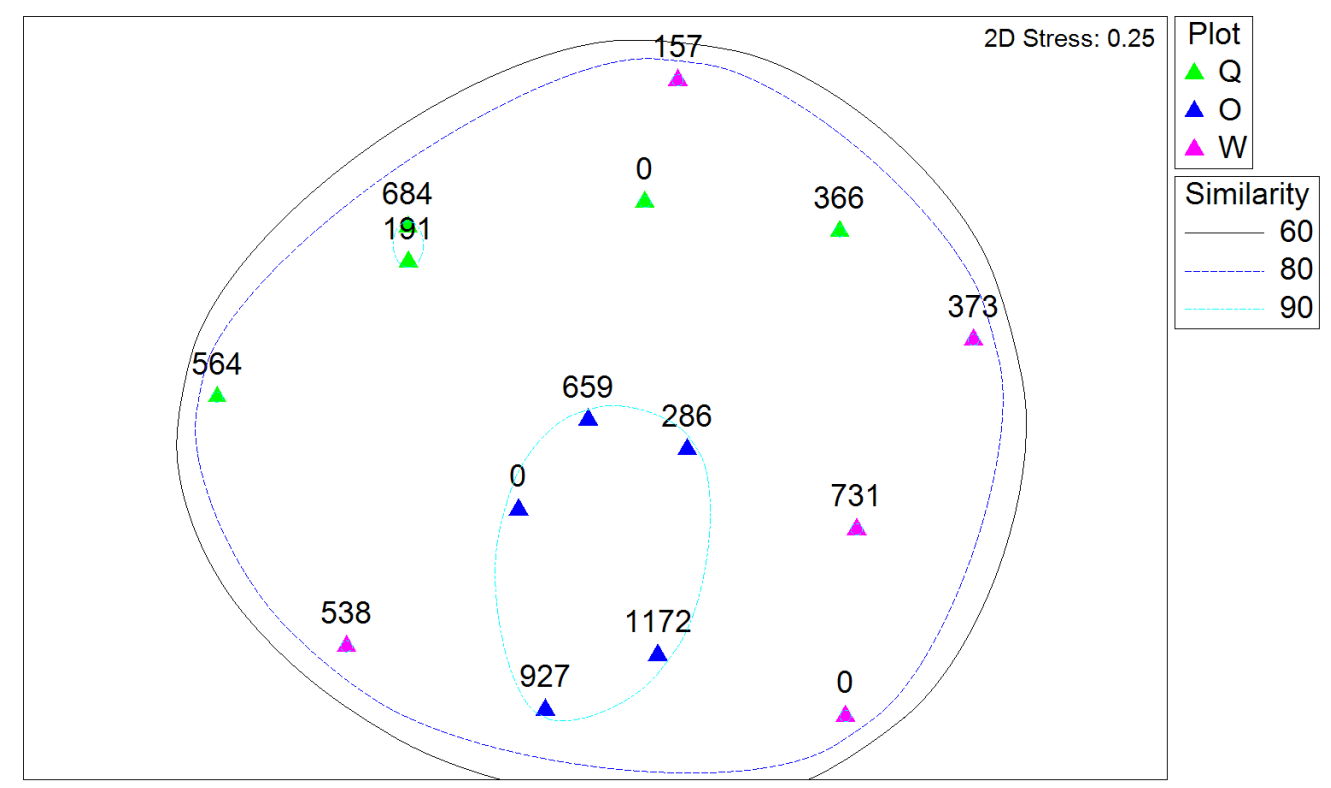

Figure 6.48 MDS plot of DGGE data from soil control samples from the control sites $(O, Q$ and $W)$.

The Bray-Curtis similarity index was used on square root transformed data.

The MDS plot of the control sample data (Figure 6.48) shows a high level of similarity between the three sites and also within each site over time. Site O showed the highest similarity with all five samples over time being over $90 \%$ similar to each other. The other two sites show slightly more variability but all were still over $80 \%$ similar to each other.

\subsection{Discussion}

\subsubsection{Observation of Decomposition Process}

A great deal of variability was seen in the decomposition rates and processes undergone by the six cadavers. Cadaver $\mathrm{P}$ (non-autopsied on soil) exhibited rapid progression through the bloat and active decay stages. Bass (1997) noted that in Tennessee it is not unusual for a cadaver to be reduced to a skeleton in about two weeks during the peak of summer and refers to a case where a missing teenager was almost a complete skeleton after 10 days. Mann et al. (1990) also document the skeletonisation of a female cadaver in seven days after autopsy and in an open body bag. Cadaver $\mathrm{P}$ was largely skeletonised by day 10 
(ADD 286) and was completely skeletonised by the next sampling on day 14 (ADD 376), which suggests it was comparatively rapid. As this person died from "natural causes" and was not autopsied, no information regarding the cause of death or medical conditions was available, giving no clues as to possible causes for this accelerated decomposition, other than their large body mass. Cases of accelerated maggot growth have been documented on cadavers where large doses of certain drugs were ingested shortly before death (Goff and Lord, 2000). Goff et al. (1989) reared maggots on the carcasses of rabbits that had received known dosages of cocaine. Lethal and twice-lethal doses caused maggots to develop more quickly and pupate earlier than those fed on carcasses with sub-lethal does and control carcasses. Goff et al. (1991) conducted a similar study using heroin and found that this drug produced larger maggots that underwent a longer pupation period. To rule this out as a possible cause, larvae samples were collected by a forensic entomologist affiliated with the Facility for drugs testing, but none of the drugs screened for (cocaine, heroin, methamphetamine) were detected (Arpad Vass, pers. comm.)

In contrast to cadaver P's rapid decomposition, cadaver R (non-autopsied on soil) exhibited slow decomposition. This cadaver did not exhibit noticeable bloating or active decay around the abdominal region at any time, but did show active decay around the face and fungus growth on the trunk and limbs. At the cessation of sampling at day 49 (ADD 797), cadaver R was still largely intact. The cause of death for this person was listed as renal failure and at death she weighed only $40 \mathrm{~kg}$, suggesting a lengthy period of illness before death. Renal failure can also cause accumulation of various toxins in the body (Schrier et al., 2004), which would also affect the microflora. Medical records were not available to check, but it is likely that this individual was consuming various prescription pharmaceuticals prior to death, and these may have included antimicrobial agents which would have considerably disrupted the natural body microflora. The observed fungal 
activity is also suggestive of reduced bacterial activity because in complex microbial communities such as soil, fungal activity is often kept in check by the more diverse bacterial community, but in the absence of a strong bacterial community, certain fungal species are able to proliferate (Mille-Lindblom et al., 2006). The gut microflora may also have been further affected by restricted dietary intake prior to death. The retarded decomposition is unexpected, however, as early and rapid putrefaction is thought to be associated with persons who have wasted-away before death (Campobasso et al., 2001).

Cadavers V and Z (both on sand, non-autopsied and autopsied respectively) both exhibited signs of possible adipocere formation, or at least different rates of decomposition in different areas of the body, with the legs in particular showing slower decomposition. Adipocere forms from fat deposits, meaning females are more likely to develop adipocere, particularly around the buttocks and thighs (Fiedler and Graw, 2003). Because of the limited time frame of this study it was not possible to determine how decomposition eventuated for these tissues.

With the exception of cadaver $\mathrm{P}$, the autopsied bodies ( $\mathrm{T}, \mathrm{X}$ and $\mathrm{Z}$ ) decomposed faster than those not autopsied ( $\mathrm{R}$ and $\mathrm{V}$ ), which was expected because of the large wounds allowing insects unimpeded access to internal tissues (Campobasso et al., 2001). The substrate the cadavers were placed on did not seem to have a noticeable effect on decomposition rate. There did appear to be a slight difference between the male and female cadavers, but this is likely to be a due to a combination of factors including cause of death, weight at death and autopsy effects. The male cadaver $\mathrm{T}$ (on sand) skeletonised quickly, whereas the two females on sand were slower, even when taking temperature differences into account using ADD calculations. The female on soil (cadaver R) exhibited the slowest decomposition overall. It was interesting to note that of the three female cadavers, none were completely 
skeletonised by the end of the experimental period, whereas all three of the male cadavers were skeletonised or very nearly skeletonised.

Mann et al. (1990) concluded that "variability in the decay rate of the human body is the rule", which is supported by this study. Many variables affect human decomposition and unfortunately few of these could be controlled for in this study. Some cadavers decomposed very rapidly, while others, very slowly. Differences in temperature could not account for all of the variability as the decomposition processes were still remarkably different between cadavers even when ADDs were calculated and taken into consideration.

The inclusion of autopsied bodies in this study was not ideal, but necessary to increase the number of cadavers studied. Similarly, the extreme differences in size between the cadavers could not be avoided. It must also be noted that for some of the cadavers used there was a delay of many days between death and placement. The cadavers would have been refrigerated in the mortuary for most of this time. This may have had some effect on the microbial communities. Micozzi (1986) found that freeze-thawed animal carcasses decomposed in a different way to fresh carcasses, with the freeze-thawed appearing to have reduced enteric micro-organism mediated putrefaction. Freezing is likely to have a much more severe effect on the microbes than refrigeration, but this factor should still be considered.

\subsubsection{Temperature, RH and Rainfall}

Because the experimental sampling period spanned a rapid change in season (from summer to autumn), there was a decrease in mean daily temperature during this period. Accumulated degree days (ADDs) were used to compare decomposition progression rather than calendar days, but the cadavers placed at a later date (cadavers $\mathrm{X}, \mathrm{V}$ and $\mathrm{Z}$ ) seemed to undergo a slower decomposition rate than those placed earlier on (cadavers $\mathrm{P}$ and $\mathrm{T}$ ). It is 
likely that some variable other than temperature associated with the change in season was affecting the rate of decay. Relative humidity was one factor that was considered (Figure 6.5), as was barometric pressure (Figure 6.6). The relative humidity did not change much during the sampling period, so is unlikely to have had a great effect. The barometric pressure did show greater fluctuations from around the beginning of October (approximately half way through sampling) onwards. Barometric pressure is known to affect insect behaviour (Flitters, 1963), including reduced oviposition when barometric pressure is falling (Roitberg et al., 1993) and different flight behaviours (Fournier et al., 2005) in fluctuating pressure. The fluctuating barometric pressure observed towards the end of the study period may have affected insect oviposition or activity on the cadavers placed during this time, thus affecting the rate at which the cadavers decayed.

In this experiment, the recorded soil temperature was consistently higher than the ambient air temperature and except for a couple of timepoints, also higher than the temperatures recorded under the cadavers (Figure 6.4). This is in contrast to the temperatures recorded in the pig carcass experiment (section 5.4) where the soil temperature closely followed the air temperature and the soil under the carcasses had consistently higher temperatures than both the soil and air temperatures. It is not unusual for the soil to be warmer than the air in summer and vice versa in winter (Gehrig-Fasel et al., 2008), but the cooler temperatures exhibited under the cadavers is unexpected. This difference is also probably caused by the soil temperature being recorded at a control site which was covered by a "control" carcass (a sand-filled sack) for the pig carcass experiment (chapter 5), while the soil temperature was recorded in an uncovered location at the University of Tennessee Facility at a depth of $15.2 \mathrm{~cm}$. Regardless, a brief increase in temperature was detected under two of the cadavers monitored ( $\mathrm{T}$ and $\mathrm{X}$ ), while this did not appear to occur under cadaver R. This is 
most likely to be due to the retarded decomposition exhibited by cadaver $\mathrm{R}$ and the lack of a definite active decay phase (section 6.5.1).

All enzyme and chemistry data was examined for anomalies around the 23rd October 2006, when the lone heavy rainfall event occurred. None were found, so it appeared that the rainfall did not have any major effect on decomposition rates or results in this experiment.

\subsubsection{Biochemistry}

\subsubsection{1 $\mathrm{pH}$}

The buffering capacity of the soils from the US human cadaver experiment site and the New Zealand pig carcass experiment site were very similar, suggesting that the $\mathrm{pH}$ response to a decomposition event is likely to be similar between the two soil types. The initial $\mathrm{pH}$ of the $\mathrm{NZ}$ soil was 6.4 and the US soil, 6.7, which are reasonably similar. The $\mathrm{pH}$ of the $\mathrm{dH}_{2} \mathrm{O}$ from the two labs was quite different (8.1 in NZ compared with 7.25 in the US) but this is unlikely to affect the $\mathrm{pH}$ measurements.

Autopsy strongly affected the $\mathrm{pH}$ response to the decomposition event on sand, with the autopsied cadavers causing a rapid drop in $\mathrm{pH}$ before the expected increase. This was only seen with the cadavers on sand, not on soil, which could be due to the higher buffering ability of the soil over sand masking this effect. The sand samples from these early timepoints had a distinct red colouring, suggesting that there was a high blood content in the sand. The $\mathrm{pH}$ of blood in a living human, is usually in the range of 7.35 to 7.45 , but this is known to decrease rapidly post-mortem. This is due to carbon dioxide build-up in the initial post-mortem phase followed by metabolism of glucose with resulting lactic acid and phosphoric acid accumulation (Sawyer et al., 1988), as well as carbon dioxide accumulation (Clark et al., 1997). This influx of acidified blood is the likely cause of the initial drop in $\mathrm{pH}$ observed. 
The pattern of $\mathrm{pH}$ change over the course of decomposition varied between cadavers. The highest $\mathrm{pH}$ reached was 8.46 at ADD 502 for cadaver $\mathrm{Z}$ (autopsied and placed on sand). There did not appear to be any correlation between autopsy, sex or substrate (soil or sand) on the $\mathrm{pH}$ reached. There did appear to be some correlation between weight at death and the magnitude of $\mathrm{pH}$ rise, with the heaviest individuals producing the greatest $\mathrm{pH}$ increases, although the lightest individuals did not necessarily cause the smallest $\mathrm{pH}$ increase. This is not unexpected, as bigger individuals would be expected to have a greater muscle mass and thus be expected to produce more $\mathrm{NH}_{4}{ }^{+}$as a by-product of muscle degradation (Vass et al., 1992). Results were not adjusted for pre-death weight (for example, as was performed by Vass et al., (1992)) as the correlation between weight and $\mathrm{pH}$ shift did not hold for all the cadavers, and even though the cadavers were vastly different in terms of weight, the $\mathrm{pH}$ changes were not so different.

In contrast to the results seen using pig carcasses, the sand sites did not seem to undergo a more rapid $\mathrm{pH}$ increase than the soil sites. The autopsied human cadavers on sand did show an immediate decrease in $\mathrm{pH}$, but the non-autopsied cadaver on sand $(\mathrm{V})$ produced a slower rise in $\mathrm{pH}$ than the cadavers on soil. A more rapid and greater overall rise in $\mathrm{pH}$ was expected at the sand sites as compared with the soil sites due to the buffering of the $\mathrm{pH}$ effect that soil has. This was not seen in the previous pig carcass experiment or in this experiment. It appears that decomposition of pig carcasses and cadavers of varying size cause a basic shift, with all the carcasses and cadavers studied reaching a maximum $\mathrm{pH}$ between 7.5 and 8.7. Vass et al. (1992) found that the $\mathrm{pH}$ increased (up to $\mathrm{pH}$ 9) until soft tissue decomposition was complete at around 1285 AD. This was followed by a gradual decrease in $\mathrm{pH}$ to below the initial value for the substrate over the longer term (ADD >4500). Compared with the findings of Vass et al. (1992), cadaver P samples exhibited an early decrease in $\mathrm{pH}$, but this cadaver did exhibit unusually rapid decomposition (section 
6.3.1), and soft tissue decomposition was largely complete at around the time the $\mathrm{pH}$ began to drop. The other cadavers did not reach skeletonisation as the available timeframe did not allow for sampling through until ADD 1285, meaning that the expected acidic shift was not documented.

\subsubsection{2 $\mathrm{NH}_{4}^{+}-\mathrm{N}$ and $\mathrm{NO}_{3}^{-}-\mathrm{N}$}

As with the $\mathrm{pH}$ results, the ammonium concentrations were not standardised according to the pre-mortem weight of the cadavers even though this variable is likely to influence the quantity of ammonium ions released by the cadaver. The decomposition sites that experienced the highest ammonium increases were not those that held the heaviest individuals. Cadaver $\mathrm{P}$ was the heaviest $(159 \mathrm{~kg})$ and produced a substantial increase in ammonium concentration, but cadaver $X(59 \mathrm{~kg})$ caused a very similar pattern of change in ammonium concentration over time, increasing rapidly and then decreasing steadily, although weighing $100 \mathrm{~kg}$ less. Both of these cadavers were placed on soil although cadaver $\mathrm{P}$ was not autopsied and cadaver $\mathrm{X}$ was. The remaining cadavers all produced smaller increases in ammonium concentration but all produced similar patterns to each other, and decreased at similar rates. The increase in ammonium concentration is likely to be caused primarily by protein degradation which in turn causes the $\mathrm{pH}$ increase observed (section 6.5.3.1). The rise in both $\mathrm{pH}$ and ammonium concentration began early in the decomposition process, essentially as fluids began to purge from the cadavers and began to decrease while the cadavers were still in active decay, but when much of the muscle tissue appeared to have been degraded. The exception to this was for cadaver R, which did not have a clearly defined active decay stage and appeared to still retain a great deal of muscle mass even when the ammonium concentration appeared to be decreasing. 
The observed change in the $\mathrm{pH}$, ammonium and nitrate concentration results were very similar to those from the pig carcass experiment. The ammonium and nitrate concentrations peak at around the same time, with the nitrate dropping away very quickly and the ammonium also decreasing. The ammonium concentrations did, however, decrease more slowly under the cadavers than under the pig carcasses. Discussion of nitrogen cycling and processes that might occur are discussed in section 5.4.3.1. As with the pig carcass experiment (Chapter 5), some nitrate was detected in the sand sites suggesting that some body derived bacteria are capable of nitrification (conversion of ammonium to nitrate), although the soil sites showed higher nitrate concentrations, due to the nitrifying activity of soil derived organisms.

\subsubsection{Enzymes}

The urease assay for the human cadaver experiment gave very similar results to those from the pig carcass experiment (Chapter 5). The soil sites for both gave a dual-peak rise in activity early in the decomposition process, although the timing for when these occurred was slightly different. The increase in potential urease activity began immediately for the human cadavers on soil (with the exception of cadaver R) and did not decrease to a constant (but elevated above baseline) activity until around ADD 500. The increase began later for the pig carcasses (around ADD 100), but decreased earlier, at around ADD 260. In both experiments, the sand sites had fluctuating levels of activity, suggesting that pockets of organisms capable of ureolytic activity were being detected sporadically due to the sampling method.

The N-acetyl- $\beta$-D-glucosaminidase showed a much more prolonged rise in potential activity in soil under the human cadavers than in the pig carcass experiment (chapter 5). A distinct spike in activity was evident in the soil under the pig carcasses, whereas an 
increase followed by a period of fluctuating, but raised activity was evident under all three cadavers on soil. This effect is likely to be related to the smaller maggot masses and less obvious maggot migrations away from the cadavers as compared to the carcasses. The pig carcasses tended to have a clear migration over about a two day period, whereas the cadavers seemed to have more continuous larval activity. A decrease in activity was evident under cadaver P after ADD 730, although similar decreases were not seen under the other cadavers because these were not sampled past the ADD 700 timepoint. The decrease in activity corresponds closely to the time when insect larvae activity appeared to decrease on cadaver P. Cadaver R, had little insect activity throughout the sampling period and showed reduced potential N-acetyl- $\beta$-D-glucosaminidase activity compared with the other cadavers on soil. The samples from under the cadavers on sand, like those from the pig carcasses on sand, showed slightly elevated potential activity, suggesting that some Nacetyl- $\beta$-D-glucosaminidase was contributed either from the cadaver itself or from colonising insects.

$\beta$-glucosidase activity was higher in the human cadaver sites than under the pig carcasses. As with the data from the pig carcass experiment, the cadaver data were again quite noisy with wide fluctuations occurring, although an overall trend of a rapid increase followed by a decrease over time was evident. Very little activity was seen in the sand sites, although a small peak in potential activity occurred under cadaver $\mathrm{V}$, suggesting a contribution of organisms or extracellular enzyme from an external source although a likely source has not been identified. In the soil sites, it is possible that the increased activity seen in the soil is a result of the homogenisation of the soil just prior to the cadavers being placed, which mixed cellulose-containing plant material into the soil, promoting an increase in organisms capable of degrading cellulose. It is also likely that cellulose-containing material 
continued to enter the decomposition sites during the decomposition process as the sites were exposed to normal weather and environmental conditions such as rain and wind.

In contrast to the pig carcass experiment, clear increases in acid phosphatase activity were seen in response to the cadaver decomposition events on soil. No significant difference was seen between the soil controls and the decomposition events for the pig carcasses, but the increase in activity under the cadavers on sand and soil was significant. Both substrates showed two clear peaks of increased potential activity, one around ADD 200 and one around ADD 500. There is no obvious correlation between these particular timepoints and a visual change in decomposition although the first activity spike was around the time when body fluids began to purge from the cadavers. The second spike does not clearly correlate with any change in insect activity, fluid quantity or physical change in the cadavers based on the photographs taken to document decomposition progression.

Very little alanine aminopeptidase activity was seen, although a single small spike in activity was apparent in each of the soil sites. This occurred at around ADD 300 for cadavers $\mathrm{P}$ and $\mathrm{X}$ but later at ADD 600 for cadaver R. This is likely to be because cadaver $\mathrm{R}$ experienced retarded decomposition compared to the other cadavers. This minimal activity was unexpected, as human decomposition causes a massive influx of protein into the soil system. Vepsalainen et al. (2001) tested alanine aminopeptidase activity in soil simultaneously with other aminopeptidases and found alanine aminopeptidase to have the highest activity. Human alanine aminopeptidase is produced many tissues including the liver and the intestine, where it is involved in protein degradation (McClellan and Garner, 1980). Deficiency in human alanine aminopeptidase is associated with malabsorption (Gravinghoff and Hutter, 1977) although most of the aminopeptidase activity found in the human intestine is bacterially derived (Macfarlane et al., 1988). It is unlikely that the spike in activity is cadaver derived because this increased activity was only evident under the 
cadavers on soil, not on sand. It appears that soil organisms with alanine aminopeptidase activity respond briefly to the decomposition event during active decay. L-alanine aminopeptidase is only produced by Gram-negative bacterial species so presence of this enzyme is often used to distinguish Gram-positive and negative organisms (Anderson et al., 2008).

The esterase assays using different substrates gave similar results due to cross-over in activity of enzymes. It is likely that there are many esterases and related enzymes that have the ability to cleave both 4-MUB butyrate and 4-MUB acetate (Wittmann et al., 2000). Both of these substrates have been found to be unstable at high $\mathrm{pH}$ (Niemi and Vepsalainen, 2005), but both have been used to assess esterase activity in a variety of environments for some time (Roy, 1980; Wittmann et al., 2000). The substrate 4-MUB palmitate is also cleaved by lipolytic enzymes and has also been used to measure esterase activity (Roy, 1980). Studies have been performed to determine the effect of $\mathrm{pH}$ on many of the fluorescent substrates used, but little seems to have been published about the stability of 4-MUB palmitate as a substrate for fluorometric assays. Roy et al. (1980) note that long chain acyl esters of 4-methylumbelliferone (such as 4-MUB palmitate) may be unstable when diluted. This is likely to be a cause of the failure of this substrate to yield any useable data. Palmroth et al. (2005) used it when studying the effect of diesel fuel on the soil microbiota, but measured so little activity that kinetic parameters could not be determined. Wittman et al. (2000) had some success but noted that lipolytic activity towards 4-MUB palmitate was only $1 / 10$ of that observed for the related substrates 4-MUB acetate and 4-MUB butyrate.

Overall, the enzyme data was better than those obtained as part of the pig carcass experiment (section 5.3.3) with less seemingly random variability. This is likely due to the slight change in methodology which saw the fluorometric assay substrate solutions being 
prepared more regularly to avoid potential stability or contamination issues over time (Roy, 1980).

\subsubsection{T-RFLP and DGGE}

As with the pig carcass experiments, the cadaver sample derived T-RFLP and DGGE MDS results showed some clear successional changes occurring within the bacterial communities. Almost all MDS plots demonstrated stepwise changes with each subsequent profile plotting in a successive manner, rather than in random or erratic movements. The overall direction or pattern generated tended to differ between cadavers but some trends did become obvious such as the first one or two sample profiles plotting relatively closely followed by a big shift to a cluster of one or two further samples, followed by another big shift in community composition. Change after this point seemed to be less dramatic and varied more between the cadavers. Patterns did tend to be similar on both sand and soil, again suggesting that the shifts may be mediated largely by the body-derived microbes themselves rather than an environmental organism response to nutrient influx. The TRFLP and DGGE patterns from a given cadaver tended to be reasonably similar, with most of the big community shifts occurring between the same sampling times. There were occasional differences with the DGGE profile not reflecting a change in the community seen in the T-RFLP data until the following sampling A couple of examples of this were also seen in the pig carcass experiment (section 5.4.4). The RELATE function which tests for correlation between the similarity matrices underlying the MDS plots found some degree of correlation between the T-RFLP and DGGE results for all but one cadaver sample set. These correlations were not very strong but suggested that both profiling methods gave reasonably similar overall results in this experiment. The T-RFLP and DGGE methods gave conflicting results on the diversity within the profiles. For T-RFLP, 
the soil-derived profiles had a greater mean number of T-RFs than the sand-derived profiles (soil 30 vs. sand 23, see Table 6.2), whereas the DGGE profiles from sand had a greater mean number than the soil profiles (soil 22 vs. sand 36, see Table 6.3). This result reflects the differing methods by which the T-RFs and bands are generated (restriction sites/overall CG content). Both methods showed that the control soil samples had greater diversity than the soil decomposition samples.

\subsubsection{Concluding Comments}

This experiment clearly highlights some of the biggest problems encountered using human cadavers for replicated research. First, there are few facilities world-wide that enable human decomposition research to be performed, restricting research to particular environmental and geographic locations. The researcher also has little or no control over many of the variables that may strongly influence the decomposition process. Animal models such as pigs offer a strong advantage in this situation as can be seen by the much less variable results seen overall in the previous chapters. This experiment did, however, confirm that many of the results obtained using the pig carcasses were very similar to those obtained when using human cadavers. This suggests that the use of pig carcasses as models for human decomposition is valid, particularly when specific variables that cannot be held constant in cadaver experiments are being investigated. 


\section{Chapter 7: Molecular Biological Examination of Bacterial Communities}

\subsection{Introduction}

Profiling tools such as T-RFLP and DGGE are effective at characterising changes in wider complex bacterial communities, however, they offer little information on the actual composition of the community, on functional groups or on individual species.

The previous chapters in this thesis discuss analysis of the greater bacterial communities in soil associated with decomposition events and the sequential changes that occur within them during the process. This chapter discusses a series of experimental approaches that were used in conjunction with the community profile data to try to gain an in-depth understanding of the process. Analyses were also performed with the aim of identifying the bacterial species contributing to the peaks/bands seen in the profiling methods, in the hope that specific bacterial biomarkers for time since death could be identified.

Four different approaches were used: 1) profiling using group-specific primers (T-RFLP and DGGE); 2) excision and DNA sequencing of bands from DGGE gels; 3) the use of multiple restriction enzyme digests of $16 \mathrm{~S}$ fragments with online databases to make predictions about the bacterial orders represented by T-RFs; and 4) selective cloning and sequencing of $16 \mathrm{~S}$ gene fragments for database-aided identification.

Bacterial groups were chosen for T-RFLP and DGGE profiling based on their potential for being involved in the decomposition process (section 7.2). These included common, known soil organisms and body-derived enteric organisms. 
One advantage of the DGGE method is that bands of interest can be excised from the gel and purified for DNA sequencing and subsequent identification of the contributing organism (section 7.3). This procedure was attempted for bands from gels generated using the universal F341 and R534 primers (section 3.4.4.1) and the group-specific primer sets (section 7.2).

In a separate exercise, a selection of samples chosen based on the presence of key T-RFs were profiled using three different restriction enzymes. By generating multiple T-RFLP profiles for each DNA extract using different restriction enzymes and comparing these data with predicted fragment lengths for sequences in the Ribosomal Database Project (RDP) online database (Maidak et al., 1999), predictions of potential taxonomic groups that could create T-RFs of interest were made (section 7.4).

Cloning and sequencing of $16 \mathrm{~S}$ gene fragments is a commonly employed technique for identification of species present within a mixed community (Zwolinski, 2007). Unfortunately, standard cloning and sequencing methods are time consuming and costly and are not ideal for screening large numbers of samples. In this chapter, a method was used which combines T-RFLP with cloning and sequencing (Grant and Ogilvie, 2004) to reduce the number of clones sequenced (section 7.5). The understanding of complex microbial communities can be greatly enriched by combining T-RFLP with morphological, biochemical or sequence analyses (Thies, 2007a). A standard cloning method was used to generate $16 \mathrm{~S}$ clone libraries from selected samples. Three samples originating from soil or sand under cadaver and pig carcass were chosen to enable comparison of early, mid and late decomposition stages. By screening the libraries using T-RFLP, clones containing sequences that generated peaks of interest in the original, whole community T-RFLP profiles were able to be identified and the sequence of the inserts determined. The online 
RDP database (Maidak et al., 1999), was used to find the closest match to known sequences.

\subsection{Group-Specific DGGE/T-RFLP Analysis}

A number of bacterial groups of potential interest were investigated using PCR with groupspecific bacterial primers followed by DGGE and T-RFLP profiling. Bacterial groups commonly found in soil and/or as part of the normal human microflora were targeted and assessed for their presence or absence in each sample using PCR. The community composition of these groups in selected positive samples was further investigated using DGGE or T-RFLP.

\subsubsection{Bacterial Groups Tested For}

\subsubsection{Actinobacteria}

Actinobacteria are a phylum of mostly Gram positive aerobes, although some species are anaerobic. Actinobacteria are found in many environments including most soils (Piao et al., 2008), human gut (Andersson et al., 2008), and human skin (Gao et al., 2007) . Actinobacteria constitute one of the two main groups of Gram positive bacteria (the other being the Firmicutes) and exhibit the greatest variety of growth forms of all bacterial groups, from single-celled rods to cocci and mycelial structures (Prosser and Tough, 1991). Actinobacteria were chosen for investigation because they are found in both soil and the gastrointestinal system and some species produce chitinase, which was assayed for (sections 5.3.3.5 and 6.4.4.6). Actinobacteria were reported to be the second most abundant phylum in the human colon after the Firmicutes in a recent study (Andersson et al., 2008) and are a large and diverse group (Matsuki et al., 2004; Rinttila et al., 2004; Ventura et al., 2007). 


\subsubsection{Atopobium group}

Atopobium is a genus of obligately anaerobic Gram positive bacteria. This genus is a member of the Actinobacteria phylum but were not expected to be amplified by the primer pair used in the Actinobacteria-specific PCR. The primer pair used to detect this group are also known to amplify related organisms within the Coriobacteriaceae family, including common faecal flora genera Eggerthella, Olsenella, Collinsella, Slackia and Denitrobacterium (Matsuki et al., 2004; Rinttila et al., 2004). The Atopobium group is thought to be one of the predominant groups of microbes within the human intestine and present within the majority of adults (Matsuki et al., 2004; Rinttila et al., 2004) and was chosen for investigation for these reasons.

\subsubsection{Bacteroides fragilis group}

Bacteroides are anaerobic Gram-negative pleomorphic rods that are members of the Bacteroidetes phylum. They are amongst the most aerotolerant of all anaerobes. The Bacteroides genus is the most abundant in the gastrointestinal system, is the most commonly isolated genus from human faeces and constitutes $20-30 \%$ of the cultivable microbiota (Wilson, 2005). The combinations of Bacteroides species which inhabit individuals are extremely diverse, even amongst closely related individuals (Li et al., 2009). Ammonia, which is produced during decomposition, is the primary source of nitrogen for Bacteroides species. Most species can utilise a variety of carbohydrates as energy and carbon sources and can degrade a wide range of polysaccharides and mucins. Some species are proteolytic. Bacteroides are often used as indicators of faecal contamination (Bernhard and Field, 2000; Dick and Field, 2004) and are not thought to be common soil inhabitants (Okabe and Shimazu, 2007). 


\subsubsection{Bifidobacterium group}

The species of the Bifidobacterium genus are Gram positive obligate anaerobes. This genus belongs to the Actinobacteria phylum, and are a subset of the species that could be detected using the Actinobacteria group-specific primers. There are currently 32 described species in the genus, and the majority of these are found in mammalian guts (Klijn et al., 2005). They have been well studied because of their perceived benefits to human gut health and are the main candidates for gastrointestinal probiotics (Wilson, 2005). This group was chosen for analysis because of its association with the gut and its likely absence in soil.

\subsubsection{Clostridium groups}

Clostridium species are obligately anaerobic Gram positive rods that fall within the Firmicutes phylum. The Clostridium genus is very large and contains more than 100 species, most of which are fermentative and/or proteolytic (Wilson, 2005). The genus is genetically very diverse, meaning that group specific primers can amplify targets from only parts of the group. For this study, two major clusters, the Clostridium perfringens (Cluster I) and the Clostridium coccoides (Cluster XIV) were looked at using group specific primers. Clostridium species from both groups are widely distributed in the environment (Matches et al., 1974; Wiegel et al., 2006) and are also found as part of the normal human gut flora (Wilson, 2005).

\subsubsection{Enterococcus group}

Enterococci are Gram positive facultative anaerobes that are members of the Firmicutes phylum. The genus consists of 17 species but only two (E. faecalis and E. faecium) are regularly found in the human gastrointestinal (GI) tract (Wilson, 2005). Enterococci are 
very hardy organisms and can tolerate a wide variety of growth conditions, including temperatures of $10-45^{\circ} \mathrm{C}$, as well as acidic, alkaline, hypotonic or hypertonic environments. They are also resistant to bile salts, desiccation, detergents and many antimicrobial agents (Wilson, 2005). Enterococci are a commonly used indicator of faecal contamination of water, but they have also been found in soils (Aarestrup et al., 2002; Hardina and Fujioka, 2006) and can survive for prolonged periods in association with sludge and slurry application to soils (Cools et al., 2001).

\subsubsection{Lactobacillus group}

Lactobacilli are Gram positive, mostly facultative anaerobes that fall within the Firmicutes phylum. They are common members of the gut microflora, and are also found in the vaginal tract of human females. They are not, however, always detectable in adult human faeces, being absent from $27 \%$ of the adult US population (McCartney et al., 1996). At least 34 species are currently recognised. Although they are not considered to be a major component of the soil microflora, Chen et al (2005) isolated and identified Lactobacillus species from a number of Taiwanese soils. They were primarily found in regions of high nutrient enrichment, such as under fruit trees and on farms, so their wider prevalence in soil with fewer nutrients is unknown. Despite comprising only a small part of the total gut microflora, lactobacilli are perhaps the best studied of all the intestinal inhabitants (Walter, 2008). 


\subsubsection{Presence/Absence of Specific Bacterial Groups using PCR}

\subsubsection{Methods}

Primers specific for each of the candidate bacterial groups (sections 7.2.1.1-7.2.1.7) were used to amplify target sequences from genomic DNA extracted from all the soil samples collected under all six cadavers (Chapter 6). All primer sequences were taken from previously published papers (Table 7.1). The Actinobacteria group forward primer was labelled with a PET fluorophore and was profiled using T-RFLP. The forward primer of each of the other primer pairs had a GC clamp (5' CGC CCG CCG CGC GCG GCG GGC GGG GCG GGG GCA CGG GGG G 3') added to the 5' end for DGGE analysis. Annealing temperatures used for each primer pair are listed in Table 7.1. The PCR recipe is shown in Table 7.2 and the PCR thermocycling regime in Table 7.3. A long final extension time (20 minutes) was used to reduce the formation of artifactual "double bands" in DGGE gel analysis (Janse et al., 2004) see section 3.4.4.1. Five samples were selected from each of the control soil sample sets $(\mathrm{O}, \mathrm{Q}$ and $\mathrm{W})$ from the human cadaver experiment (Chapter 6). These control samples were chosen to provide a control samples set that spanned the experimental time-frame and were from all three control sites. 


\begin{tabular}{|c|c|c|c|c|c|}
\hline $\begin{array}{c}\text { Target } \\
\text { bacterial } \\
\text { group } \\
\end{array}$ & Sequence & $\begin{array}{l}\text { Product } \\
\text { size (bp) }\end{array}$ & Reference & $\begin{array}{l}\text { Annealing } \\
\text { Temp }\left({ }^{\circ} \mathrm{C}\right)\end{array}$ & $\begin{array}{l}\text { Positive Control } \\
\text { Species }\end{array}$ \\
\hline $\begin{array}{l}\text { Bacteroides } \\
\text { fragilis group }\end{array}$ & $\begin{array}{l}\text { 5' ATAGCCTTTCGAAAGRAAGAT 3' } \\
\text { 5' CCAGTATCAACTGCAATTTTA 3' }\end{array}$ & $\sim 501$ & (Matsuki et al., 2002) & 50 & Bacteroides fragilis \\
\hline $\begin{array}{l}\text { Clostridium } \\
\text { coccoides } \\
\text { group }\end{array}$ & $\begin{array}{l}\text { 5' CGGTACCTGACTAAGAAGC 3' } \\
\text { 5' AGTTTYATTCTTGCGAACG 3' }\end{array}$ & $438-441$ & (Matsuki et al., 2002) & 55 & Clostridium sphenoides \\
\hline $\begin{array}{l}\text { Clostridium } \\
\text { perfringens } \\
\text { group }\end{array}$ & $\begin{array}{l}\text { 5' ATGCAAGTCGAGCGAKG 3' } \\
\text { 5' TATGCGGTATTAATCTYCCTTT 3' }\end{array}$ & $\sim 120$ & (Rinttila et al., 2004) & 55 & Clostridium perfringens \\
\hline Enterococcus & $\begin{array}{l}\text { 5' CCCTTATTGTTAGTTGCCATCATT 3' } \\
\text { 5' ACTCGTTGTACTTCCCATTGT 3' }\end{array}$ & $\sim 144$ & (Rinttila et al., 2004) & 61 & Enterococcus faecalis \\
\hline Atopobia & $\begin{array}{c}\text { 5' ACCGCTTTCAGCAGGGA 3' } \\
\text { 5' ACGCCCAATGAATCCGGAT 3' }\end{array}$ & $\sim 120$ & (Rinttila et al., 2004) & 55 & Eggerthella lenta \\
\hline Bifidobacteria & $\begin{array}{l}\text { 5' TCGCGTCYGGTGTGAAAG 3' } \\
\text { 5' CCACATCCAGCRTCCAC 3' }\end{array}$ & $549-563$ & (Rinttila et al., 2004) & 58 & Bifidobacterium longum \\
\hline $\begin{array}{l}\text { Actinobacteria } \\
\text { T-RFLP (PET } \\
\text { labelled) }\end{array}$ & $\begin{array}{l}\text { 5' GGATGAGCCCGCGGCCTA 3' } \\
\text { 5' TCCGAGTTRACCCCGGC 3' }\end{array}$ & $\sim 916$ & $\begin{array}{c}\text { (Thirup et al., 2001; } \\
\text { Blackwood et al., 2005) }\end{array}$ & 59 & Cultured soil isolate \\
\hline Lactobacilli & $\begin{array}{l}\text { 5' CACCGCTACACATGGAG 3' } \\
\text { 5' AGCAGTAGGGAATCTTCCA 3' }\end{array}$ & $\sim 341$ & $\begin{array}{l}\text { (Walter et al., 2001; Heilig } \\
\text { et al., 2002; Rinttila et al., } \\
\text { 2004) }\end{array}$ & 56 & Lactobacilli gasseri \\
\hline
\end{tabular}

Table 7.1 16S primers used for group specific DGGE and T-RFLP analyses. 


\begin{tabular}{|c|c|}
\hline Component & Volume/Amount \\
\hline Qiagen Taq Polymerase & $1.25 \mathrm{U}$ \\
PCR buffer & $1 \mathrm{x}$ \\
$\mathrm{MgCl}_{2}$ & $2 \mathrm{mM}$ \\
dNTP mix & $200 \mu \mathrm{M}$ of each dNTP \\
Forward primer & $200 \mathrm{nM}$ \\
Reverse primer & $200 \mathrm{nM}$ \\
Template DNA & $20 \mathrm{ng}$ \\
DNAse free $\mathrm{H}_{2} \mathrm{O}$ & To a total volume of $50 \mu \mathrm{L}$ \\
\hline
\end{tabular}

Table 7.2 Recipe for group specific PCR.

\begin{tabular}{|c|c|c|}
\hline & Time & Temperature \\
\hline Initial Denaturation & $3 \mathrm{~min}$ & $94^{\circ} \mathrm{C}$ \\
\hline 3 Step Cycling (x30) & & \\
Denaturation: & $0.5 \mathrm{~min}$ & $94^{\circ} \mathrm{C}$ \\
Annealing: & $0.5 \mathrm{~min}$ & Variable (see Table 7.3) \\
Extension: & $1 \mathrm{~min}$ & $72^{\circ} \mathrm{C}$ \\
\hline Final Extension & $20 \mathrm{~min}$ & $72^{\circ} \mathrm{C}$ \\
\hline
\end{tabular}

Table 7.3 Thermocycling regime for group specific PCRs.

Positive and negative controls were run with each batch of PCR reactions. The positive controls were $\sim 20$ ng DNA extracted from a pure culture of a selected organism within each target group. The species used are shown in Table 7.1. All cultures were purchased from the New Zealand National Reference Laboratory and were grown using supplied instructions. The positive control for the Actinobacteria group was an unidentified, cultured isolate from soil, identified as most likely to be an Actinomycete based on colony morphology. Genomic DNA was extracted from colonies of each control species using the same protocol as used for extracting genomic DNA from soil samples (Appendix A7). 
A further set of control soil samples, collected from different locations around the Wellington region (Table 7.4), was also tested to determine the presence or absence of these bacterial groups in soil that had not been exposed to a known decomposition event. These samples were collected as part of another, larger project (Macdonald et al., 2009) and were selected to represent a wide geographic range and different soil types.

\begin{tabular}{|c|c|}
\hline SAMPLE NAME & LOCATION \\
\hline SC1 & Kaitoke Regional Park \\
SC2 & Judgeford \\
SC3 & Walker Estate, Porirua \\
SC4 & Whitireia Park, Porirua \\
SC5 & Belmont Regional Park \\
SC6 & Taita Cemetery \\
SC7 & Rimutaka Regional Park \\
SC8 & East Harbour Regional Park \\
SC9 & Wellington Botanical Gardens \\
SC10 & Otari-Wilton Bush Reserve \\
\hline
\end{tabular}

Table 7.4 Control soils used to determine the naturally occurring prevalence of the target bacterial groups in soils not exposed to a decomposition event. SC $=$ soil control.

Genomic DNA was extracted from these control soil samples using the method described in section 3.4.1. The extracted DNA was stored at $-20^{\circ} \mathrm{C}$ until use. The DNA concentration in each extract was determined using the pico green method (section 3.4.2) and $20 \mathrm{ng}$ was used as a template for PCR reactions as per the method outlined in Tables 7.1 and 7.3. A nested PCR was also used for the control soils, to determine whether any groups were present, but in quantities below the detection threshold of the standard PCR. The same PCR recipe and thermocycling regimes were used as for the original community T-RFLP PCRs (Tables 3.2 and 3.3) but the template used was $1 \mu$ PCR product from a 16S amplification using unlabelled primers F63 and R1087 (section 3.4.3), with an annealing temperature of $55^{\circ} \mathrm{C}$. 
The bacterial species expected to be amplified using each primer pair are listed in Appendix D1. These were deduced using the probe search function at the Ribosomal Database Project (RDP) website (http://rdp.cme.msu.edu) for each primer and comparing the resulting hits for the forward and reverse primers.

\subsubsection{Results}

The PCR reactions were run on agarose gels (Appendix A9) and were scored as positive or negative for the presence of each bacterial group in each sample as shown in Tables 7.5 to 7.11. No DNA was able to be extracted from the sand control samples.

\begin{tabular}{|llllllllll|}
\hline Sample (ADD) & E & Bi & Ba & Cc & Cp & At & Ac & L \\
\hline P0 $(0)$ & - & - & + & + & - & - & + & - \\
P3 $(106)$ & - & - & + & + & - & - & + & + \\
P6 $(185)$ & - & - & - & + & - & - & + & - \\
P8 $(238)$ & - & - & + & + & - & + & + & - \\
P10 $(286)$ & - & - & + & + & - & + & + & - \\
P14 $(376)$ & - & - & + & + & - & + & + & + \\
P16 $(420)$ & - & - & + & + & - & + & + & - \\
P20 $(512)$ & - & - & + & + & - & + & + & - \\
P22 $(554)$ & - & - & + & + & - & - & + & - \\
P27 $(659)$ & - & - & + & + & - & - & + & + \\
P29 $(695)$ & - & - & + & + & - & + & + & + \\
P31 (730) & - & - & - & + & - & - & + & - \\
P35 $(808)$ & - & - & - & - & - & - & + & + \\
P38 $(854)$ & - & - & + & - & - & - & + & - \\
P42 $(927)$ & - & - & + & + & - & + & + & + \\
P45 $(985)$ & - & - & + & + & - & + & + & - \\
P49 $(1053)$ & - & - & + & + & - & - & + & - \\
P52 $(1092)$ & - & - & - & - & - & - & + & - \\
P58 $(1172)$ & - & - & - & - & - & - & + & - \\
P62 (1212) & - & - & + & + & - & + & + & - \\
P69 (1285) & - & - & + & + & - & + & + & - \\
\hline O0 (0) & - & - & - & + & + & - & + & - \\
O10 (286) & - & - & - & + & + & - & + & - \\
O27 (659) & - & - & - & + & + & - & + & - \\
O42 (927) & - & - & - & + & + & - & + & - \\
O58 (1172) & - & - & - & + & - & - & + & - \\
\hline
\end{tabular}

Table 7.5 Presence/absence of specific bacterial groups within samples collected from under cadaver P and at associated control site $O$. Cadaver $P$ was not autopsied and was placed on soil. $(E=$ Enterobacteria, $B i=$ Bifidobacteria, $B a=$ Bacteroides, $C p=$ Clostridium perfringens group, $C c=$ Clostridium coccoides group, At = Atopobium, Ac $=$ Actinobacteria, $L=$ Lactobacilli). 


\begin{tabular}{|llllllllll|}
\hline Sample (ADD) & E & Bi & Ba & Cc & Cp & At & Ac & L \\
\hline X0 $(0)$ & - & - & - & - & - & - & + & - \\
X4 (106) & + & + & + & + & + & + & + & + \\
X6 $(141)$ & - & - & + & + & - & + & + & + \\
X8 $(176)$ & - & - & + & + & + & + & + & + \\
X12 $(254)$ & - & - & + & + & + & + & + & + \\
X15 $(300)$ & - & - & + & + & - & + & + & + \\
X19 $(373)$ & - & - & + & + & + & + & + & + \\
X22 (431) & - & - & + & + & + & + & + & + \\
X26 (499) & - & - & + & + & + & + & + & + \\
X29 (538) & - & - & + & + & + & + & + & - \\
X35 (618) & - & - & + & + & - & + & + & + \\
X39 (658) & - & - & + & + & - & + & + & + \\
X46 (731) & - & - & + & + & - & + & + & + \\
\hline W0 (0) & - & - & - & + & + & - & + & - \\
W8 (176) & - & - & - & + & + & - & + & - \\
W19 (373) & - & - & - & + & + & - & + & - \\
W29 (538) & - & - & - & + & + & - & + & - \\
W46 (731) & - & - & - & + & + & - & + & - \\
\hline
\end{tabular}

Table 7.6 Presence/absence of specific bacterial groups within samples collected from under cadaver $X$ and at associated control site $\boldsymbol{W}$. Cadaver $\boldsymbol{X}$ was autopsied and was placed on soil. ( $E=$ Enterobacteria, $B i=$ Bifidobacteria, $B a=$ Bacteroides, $C p=$ Clostridium perfringens group, $C c=$ Clostridium coccoides group, At $=$ Atopobium, Ac $=$ Actinobacteria, $L=$ Lactobacilli).

\begin{tabular}{|c|c|c|c|c|c|c|c|c|}
\hline Sample ( & $\mathbf{E}$ & $\overline{\mathbf{B i}}$ & $\mathbf{B a}$ & $\overline{\mathrm{Cc}}$ & $\overline{C p}$ & $\overline{\text { At }}$ & $\overline{\text { Ac }}$ & $\mathbf{L}$ \\
\hline $\begin{array}{ll}\text { R0 } & (0)\end{array}$ & & - & - & + & + & 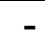 & + & \\
\hline R3 (85) & & - & - & - & - & - & + & - \\
\hline R7 (171) & & - & - & + & - & - & + & - \\
\hline R9 (107 & & - & - & + & + & - & + & 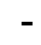 \\
\hline R11 $(24$ & & - & - & + & - & - & + & 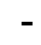 \\
\hline R15 (31 & & - & - & + & - & - & + & 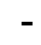 \\
\hline $18(36$ & & - & - & + & - & - & + & - \\
\hline R22 (438) & & - & + & + & + & - & + & 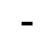 \\
\hline R25 (497) & - & - & + & + & + & - & + & - \\
\hline R29 (564) & & - & - & + & + & - & + & - \\
\hline R32 (603) & 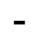 & - & - & + & + & - & + & 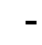 \\
\hline R38 (684 & - & - & + & + & + & - & + & \\
\hline R42 (724 & - & - & - & + & + & - & + & \\
\hline R49 (797) & $=$ & - & + & + & + & - & + & \\
\hline Q0 (0) & & - & - & + & - & - & + & \\
\hline Q8 (19 & & - & - & + & + & - & + & - \\
\hline Q18 (366 & & - & - & + & + & - & + & \\
\hline Q29 (56 & & - & - & + & + & - & + & \\
\hline Q38 (684) & - & - & - & + & + & - & + & \\
\hline
\end{tabular}

Table 7.7 Presence/absence of specific bacterial groups within samples collected from under cadaver $R$ and at associated control site $Q$. Cadaver $R$ was not autopsied and was placed on soil. 


\begin{tabular}{|llllllllll|}
\hline Sample (ADD) & E & Bi & Ba & Cc & Cp & At & Ac & L \\
\hline T1 & $(50)$ & + & + & + & + & - & + & + & + \\
T3 $(98)$ & - & - & + & + & + & + & + & + \\
T7 $(187)$ & + & - & + & + & - & + & + & + \\
T9 $(231)$ & + & - & + & + & + & + & + & + \\
T13 $(321)$ & - & + & + & + & + & + & + & + \\
T15 (362) & - & - & + & + & - & + & + & + \\
T20 (468) & + & - & + & + & + & - & + & + \\
T22 (499) & + & - & + & + & + & - & + & + \\
T24 (541) & + & - & + & + & - & + & + & + \\
T28 (613) & - & - & + & + & + & + & + & + \\
T31 (659) & - & - & + & + & + & + & + & + \\
T35 (736) & + & - & + & + & + & + & + & + \\
T38 (787) & - & - & + & + & + & + & + & + \\
T42 (862) & - & - & + & + & + & + & + & + \\
T45 (888) & - & - & + & + & + & + & + & + \\
T51 (972) & + & - & + & + & + & + & + & + \\
T55 (1006) & - & - & + & + & - & - & + & - \\
T62 (1089) & - & - & + & + & - & - & + & - \\
\hline
\end{tabular}

Table 7.8 Presence/absence of specific bacterial groups within samples collected from under cadaver $T$ which was autopsied and placed on sand. (E = Enterobacteria, Bi = Bifidobacteria, Ba = Bacteroides, $C p$ $=$ Clostridium perfringens group, $C c=$ Clostridium coccoides group, $A t=$ Atopobium, $A c=$ Actinobacteria,

$$
L=\text { Lactobacilli). }
$$

\begin{tabular}{|llllllllll|}
\hline Sample (ADD) & E & Bi & Ba & Cc & Cp & At & Ac & L \\
\hline V4 (106) & + & - & + & + & + & + & + & + \\
V6 (141) & + & - & + & + & + & + & + & + \\
V8 (176) & - & - & + & + & + & + & + & + \\
V12 (254) & - & - & + & + & + & + & + & + \\
V15 (300) & - & - & + & + & + & + & + & + \\
V19 (373) & - & - & + & + & + & + & + & + \\
V22 (431) & - & - & + & + & + & + & + & + \\
V26 (499) & - & - & + & + & + & + & + & + \\
V29 (538) & - & - & + & + & + & + & + & + \\
V35 (618) & - & - & + & + & - & + & + & + \\
V39 (658) & - & - & + & + & + & + & + & + \\
V46 (731) & - & - & + & + & - & + & + & + \\
\hline
\end{tabular}

Table 7.9 Presence/absence of specific bacterial groups within samples collected from under cadaver $V$ which was not autopsied and was placed on sand. 


\begin{tabular}{|llcccccccc|}
\hline \multicolumn{2}{|c|}{ Sample (ADD) } & E & Bi & Ba & Cc & Cp & At & Ac & L \\
\hline Z4 & $(97)$ & + & - & + & + & + & + & + & + \\
$Z 7$ & $(143)$ & + & + & + & + & + & + & + & + \\
$Z 11(216)$ & - & - & + & + & - & - & + & - \\
$Z 14(274)$ & + & - & + & + & - & - & + & + \\
$Z 18(342)$ & - & - & + & + & - & + & + & + \\
$Z 21$ & $(381)$ & - & - & + & + & - & - & + & + \\
$Z 27(462)$ & - & - & + & + & - & - & + & + \\
$Z 31(502)$ & - & - & + & + & - & - & + & + \\
$Z 38(574)$ & - & - & + & + & - & - & + & + \\
\hline
\end{tabular}

Table 7.10 Presence/absence of specific bacterial groups within samples collected from under cadaver Z which was autopsied and placed on sand. ( $E=$ Enterobacteria, Bi = Bifidobacteria, Ba =Bacteroides, $C p$ = Clostridium perfringens group, $C c=$ Clostridium coccoides group, At $=$ Atopobium, Ac $=$ Actinobacteria,

$$
L=\text { Lactobacilli). }
$$

\begin{tabular}{|lllllllllllllllll|}
\hline Sample & E & \multicolumn{1}{l}{ Bi } & \multicolumn{1}{l}{ Ba } & Cc & Cp & At & Ac & L \\
\hline & S & N & S & N & S & N & S & N & S & N & S & N & S & N & S & N \\
\hline SC1 & - & - & - & - & - & - & - & - & - & - & - & - & + & + & - & + \\
SC2 & - & - & - & - & - & - & - & + & - & + & - & - & + & + & - & - \\
SC3 & - & - & - & - & - & - & - & + & - & + & - & - & + & + & - & - \\
SC4 & - & - & - & - & - & - & + & + & - & + & - & - & + & + & - & - \\
SC5 & - & - & - & - & - & + & + & + & - & + & - & - & + & + & - & + \\
SC6 & - & - & - & - & - & - & - & + & - & + & - & - & + & + & - & - \\
SC7 & - & - & - & - & - & - & - & - & - & - & - & - & + & + & - & - \\
SC8 & - & - & - & - & - & - & - & + & - & + & - & - & + & + & - & - \\
SC9 & - & - & - & - & - & - & - & + & - & + & - & - & + & + & - & - \\
SC10 & - & - & - & - & - & + & + & + & - & + & - & - & + & + & - & + \\
\hline
\end{tabular}

Table 7.11 Presence/absence of specific bacterial groups within control soil samples collected from around the Wellington region. $S=$ standard $P C R$ results, $N=$ nested $P C R$ results.

All of the samples tested were found to be positive for the presence of members of the Actinobacteria group, including the soil control samples and the sand decomposition samples.

The Atopobium group was detected in all samples from cadavers X (autopsied on soil) and V (non-autopsied on sand), 11 of the 21 samples from cadaver P (non-autopsied on soil) and 14 out of 18 from cadaver $\mathrm{T}$ (autopsied on sand). Three from cadaver Z (autopsied on 
sand) were positive but this group was not detected in any samples from under cadaver $\mathrm{R}$ (non-autopsied on soil) or in any soil control samples.

The Bifidobacterium group was detected in one sample from under cadaver X (autopsied on soil), two samples from cadaver $\mathrm{T}$ (autopsied on sand) and one sample from under cadaver Z (autopsied on sand). All three of these cadavers were autopsied. The positive samples were from early on in decomposition and no positive reactions were observed for any control samples.

The Bacteroides group was detected in almost all decomposition samples, both soil and sand and were found in only two control soil samples (both NZ soils). Similarly, both Clostridium groups were detected in most decomposition derived samples. Both Clostridium groups were detected in eight of the ten control soil samples using the nested PCR.

The Enterococcus group was detected in only one soil decomposition sample, from cadaver X (autopsied on soil) at ADD 106. The group was detected in more of the sand decomposition samples, with eight samples from under cadaver T (autopsied on sand), two from cadaver V (non-autopsied on sand) and three from cadaver Z (autopsied on sand). The positive samples from cadavers $\mathrm{V}$ and $\mathrm{Z}$ were early in the decomposition process. Enterococci were not detected in any soil control samples.

Lactobacilli were detected in almost all samples from cadavers $\mathrm{X}$ (autopsied on soil), $\mathrm{V}$ (non-autopsied on sand), $\mathrm{T}$ and $\mathrm{Z}$ (both autopsied on sand), but were only detected in six samples from cadaver P (non-autopsied on soil) and none from cadaver R (non-autopsied on soil). Three control soil samples had very weakly positive results for the nested PCR. 


\subsubsection{Actinobacteria T-RFLP}

\subsubsection{PCR amplifications that indicated positive results for the Actinobacteria group (section 7.2.2.2) were selected for T-RFLP analysis.}

\subsubsection{Method}

Twenty-one Actinobacteria group specific PCR products (Table 7.12) were processed as per the universal T-RFLP analysis method (section 3.4.3) using the restriction enzyme MspI. Amplification products were selected by choosing those derived from samples from under each cadaver that were as close as possible to ADD timepoints 100, 500 and 1000 (if sampling extended to this timepoint).

\begin{tabular}{|c|c|}
\hline Experimental plot & Sample names (ADD) \\
\hline P (non-autopsied, soil) & P0, P3, P20, P45 (0, 106, 512 and 985) \\
R (non-autopsied, soil) & R0, R3, R25 ( 0, 85 and 497) \\
T (autopsied, sand) & T3, T22, T55 (98, 499 and 1006) \\
V (non-autopsied, sand) & V4, V26 (106 and 499) \\
X (autopsied, soil) & X0, X4, X26 (0, 106 and 499) \\
Z (autopsied, sand) & Z4, Z31 (97 and 502) \\
O (soil control) & O0, O42 (0, 926) \\
Q (soil control) & Q0, Q8 (0, 191) \\
\hline
\end{tabular}

Table 7.12 Samples chosen for T-RFLP analysis of the Actinobacteria population.

\subsubsection{Results}

Examples of the T-RFLP profiles generated using Actinobacteria specific primers are shown in Figures 7.1 and 7.2. The results are discussed in section 7.2.6 along with the group-specific DGGE results. 


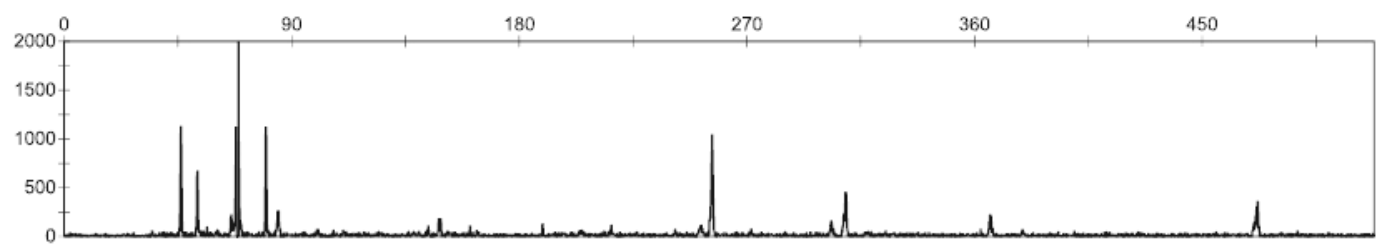

PO (ADD 0)

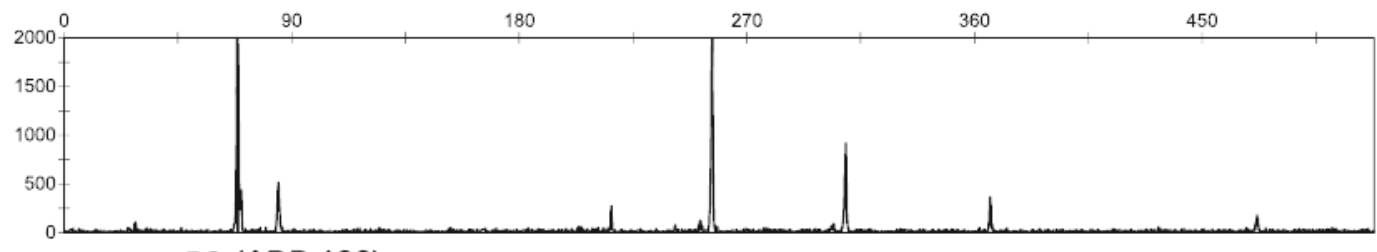

P3 (ADD 106)

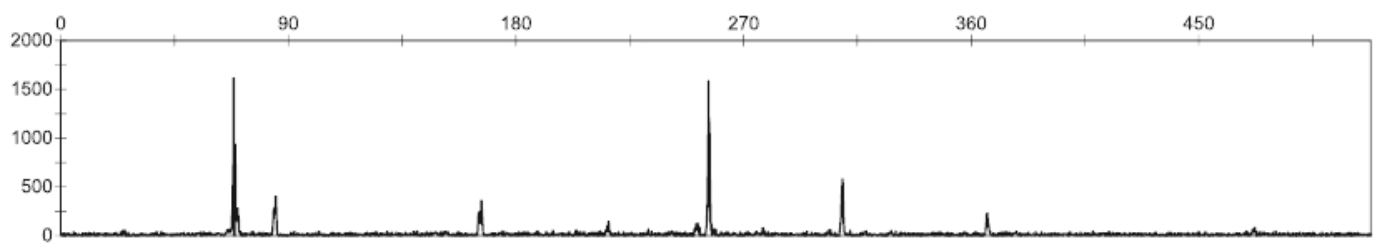

P20 (ADD 512)

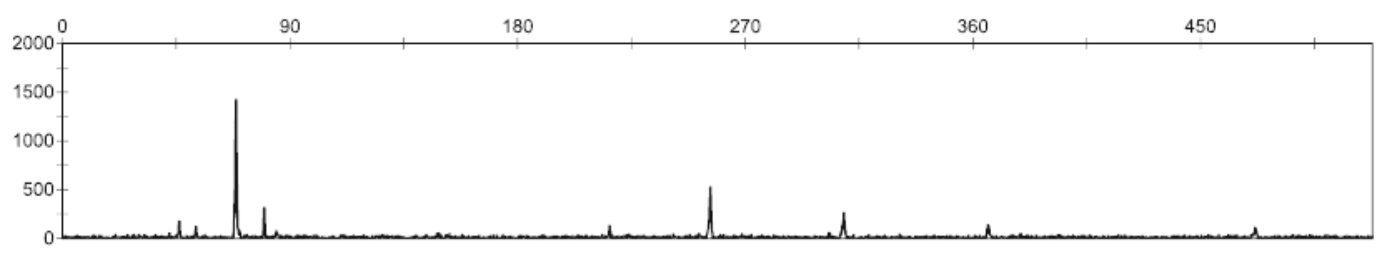

P45 (ADD 985)

Figure 7.1 T-RFLP profiles generated using Actinobacteria specific primers for cadaver P samples (non-autopsied, on soil).
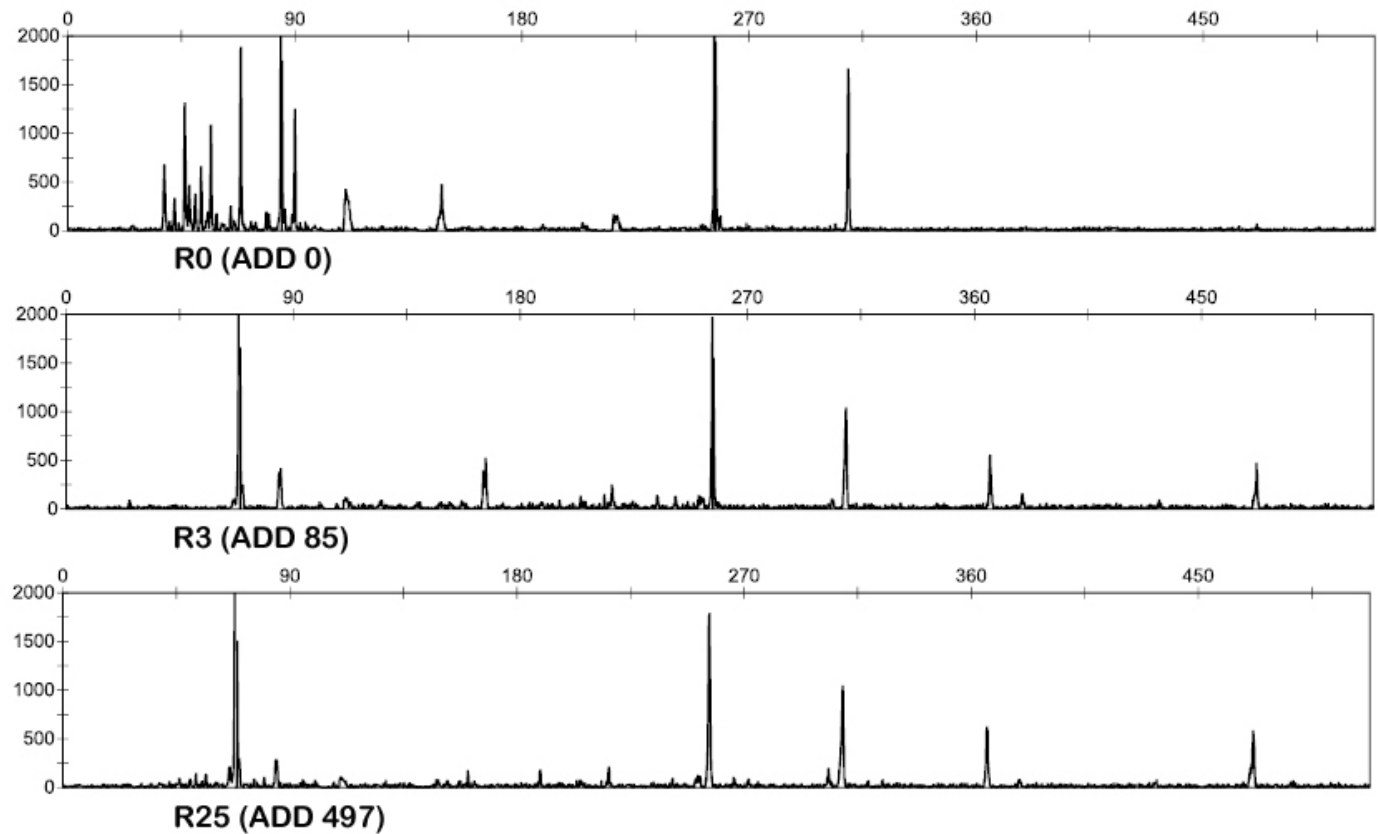

Figure 7.2 T-RFLP profiles generated using Actinobacteria specific primers for cadaver $R$ samples (non-autopsied, on soil). 


\begin{tabular}{|c|c|c|}
\hline Plot & Substrate & $\begin{array}{c}\text { Mean number of T-RFs } \\
\text { in profiles }\end{array}$ \\
\hline P (non-autopsied, soil) & Soil & 11 \\
R (non-autopsied, soil) & Soil & 17 \\
X (autopsied, soil) & Soil & 13 \\
Controls (soil) & Soil & 25 \\
T (autopsied, sand) & Sand & 9 \\
V (non-autopsied, sand) & Sand & 7 \\
Z (autopsied, sand) & Sand & 7 \\
\hline
\end{tabular}

Table 7.13 Number of T-RFs generated from each sample using Actinobacteria specific primers.

The profiles generated using the Actinobacterial primers had between 7 and 25 T-RFs above the 100 fluorescence units and $1 \%$ total fluorescence threshold (Table 7.13). The soil control plot samples and time zero control samples generated a significantly greater number of T-RFs $(\mathrm{p}<0.05)$ than soil samples collected during the decomposition phase, with a mean of $25 \mathrm{~T}$-RFs in the control sample profiles and a mean of 14 in the decomposition sample profiles. There was also a significant difference $(p<0.05)$ in the number of T-RFs between the soil and sand decomposition-derived samples, with a mean of only 7 T-RFs in the sand samples.

An MDS analysis plot of all the profiles is shown in Figure 7.3. The control soil profiles cluster quite closely with the decomposition samples separating based on the substrate the cadaver was placed on. The difference between the soil and sand sample profiles overall was significant, as was that between the decomposition-derived sample profiles and the control profiles (both $\mathrm{p}<0.01$ ). Because of the limited number of profiles in each group, only three paired groups showed significant differences at the $90 \%$ level (cadavers P and $\mathrm{T} ; \mathrm{X}$ and $\mathrm{T}$; and $\mathrm{T}$ and $\mathrm{V}$ ). There was no significant difference between the autopsied and non-autopsied cadaver sample groups. 


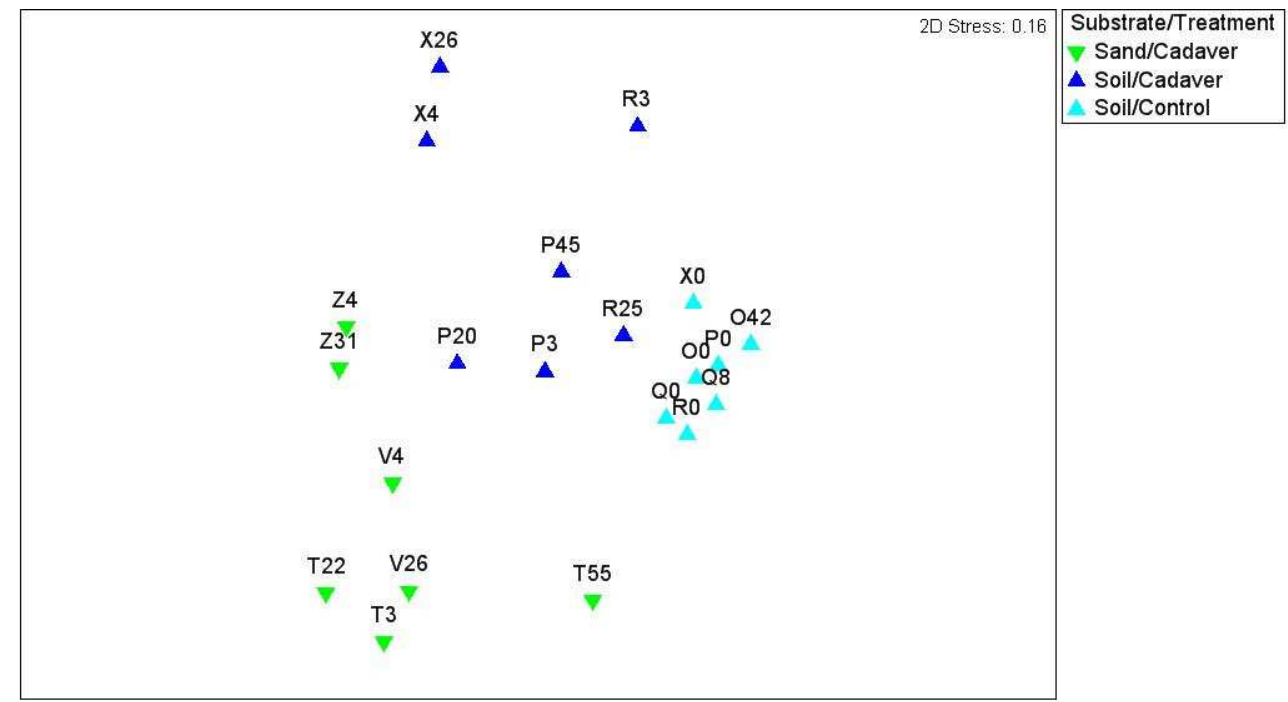

Figure 7.3 MDS plot of Actinobacterial group-specific T-RFLP data. The Bray-Curtis similarity index was used on square root transformed data.

When the individual T-RFs were examined, there appeared to be a few that were present only in the control plots, or were specific to the sand or soil plots (Table 7.14). There were also two cadaver-specific T-RFs. One T-RF ( $85 \mathrm{bp}$ ) that was present in most samples had much higher abundances in samples taken from under cadaver Z (autopsied, sand) than in any other sample. There were no T-RFs which were present consistently in both soil and sand decomposition sample, but not in the soil control samples. There were no T-RFs unique to a particular timepoint (ADD 100, 500 or 1000).

\begin{tabular}{|l|c|}
\hline & T-RF size (bp) \\
\hline Substrate & $216,252,366,472$ \\
Soil & $98,259,370$ \\
Sand & - \\
\hline Treatment & 111,161 \\
Decomposition (soil) & 165 \\
Control (soil) & 312 \\
\hline Cadaver & $69,256,309$ \\
R & \\
T & \\
\hline Common to all profiles & . \\
\hline
\end{tabular}

Table 7.14 T-RFs specific to a substrate or treatment. 


\subsubsection{DGGE using Group-specific Primers}

PCR amplifications that indicated positive results for the specific groups (section 7.2.2.2) were selected for used for subsequent DGGE analysis.

\subsubsection{Methods}

A separate DGGE gel was run for each of the specific bacterial groups (section 7.2.2.2). Up to 14 samples from each groups that yielded positive PCR results were selected for further analysis. These were chosen to give a range of time-points and from a range of cadavers. If fewer than 14 samples were positive, then all positive samples were used. These PCR products were first run on $0-90 \%$ denaturant DGGE gels to determine the region with greatest diversity, followed by running new gels with appropriate gradients for examining these regions (see Table 7.15). The same DGGE preparation and running methods were used as for the universal primer DGGE gels (section 3.4.4). The positive control species used for each presence/absence PCR were also run.

\begin{tabular}{|c|c|}
\hline Bacterial Group & DGGE Gel Conditions \\
\hline Atopobia & $10 \%$ Acrylamide, \\
& $40-60 \%$ gradient \\
\hline \multirow{2}{*}{ Bacteroides fragilis group } & $6 \%$ acrylamide, \\
& $30-50 \%$ gradient \\
\hline \multirow{2}{*}{ Clostridium coccoides group } & $6 \%$ acrylamide, \\
\hline Clostridium perfringens & $30-50 \%$ gradient \\
\hline group & $10 \%$ acrylamide, \\
\hline \multirow{2}{*}{ Enterococcus } & $30-50 \%$ gradient \\
\hline Bifidobacteria & $10 \%$ acrylamide, \\
& $40-60 \%$ gradient \\
\hline \multirow{2}{*}{ Lactobacilli } & $6 \%$ acrylamide, \\
& $30-50 \%$ gradient \\
\hline
\end{tabular}

Table 7.15 Acrylamide percentage and gradients used in group-specific DGGE analysis. 


\subsubsection{Results}

Profiles were successfully produced for most of the samples chosen for analysis using group-specific primer DGGE. A number of gels did not produce clearly defined banding patterns on the first attempt so were repeated until a gel of acceptable quality was obtained for each group. Because these profiles were expected to be simpler (contain fewer bands) than the universal primer profiles (section 3.4.4), the amount of DNA loaded into each well was decreased to $300 \mathrm{ng}$. Some profiles were very simple with only three or four bands per profile while others were slightly more complex. Photographs of the gels and MDS analysis of the resulting profiles are shown. Gels were originally scanned and analysed using the method outlined in section 3.4.4, but due to equipment failure and loss of the original scans, were re-scanned at a later date for inclusion in this thesis. Due to drying of the gels over time, the quality was not as high in these photographs.

\subsection{The Atopobium group}

The gel that the Atopobium group-specific profiles showed clearly discernible, but few and poorly separated bands using the $40-60 \%$ gradient (Figure 7.4). The DGGE PCR products were subsequently run on a gel with a more focussed gradient $(45-55 \%)$ but the resolution was very poor and the bands were not able to be analysed. The profiles shown in Figure 7.4 were very simple with between two and eight bands in each. The control, Eggerthella lenta, generated two bands which both appeared to also be present in four samples: P6 (non-autopsied on soil), V8, V29 and V45 (non-autopsied, sand). No bands were common to all the profiles. One band was common to but not exclusive to the sand profiles and one band was common to, but not exclusive to all the soil profiles. Two bands were found exclusively associated with a particular cadaver, but these bands were not present at all timepoints. 
The MDS plot of the DGGE profile data (Figure 7.5) showed a separation of three of the four samples derived from cadaver $\mathrm{P}$ (non-autopsied, on soil) and of sample Z18 (autopsied, sand) from the other samples. The other samples grouped loosely according to the cadaver they derived from and did not show any pattern suggestive of time dependant changes to the Atopobium community.

There was a significant difference between the sand and soil plots overall $(\mathrm{p}<0.01)$ and between the different cadaver sample groups overall $(\mathrm{p}<0.01)$ but because of the limited number of profiles in each group, only three paired groups showed significant differences at the $90 \%$ level (cadavers $\mathrm{P}$ (non-autopsied on soil) and $\mathrm{T}$ (autopsied on sand); $\mathrm{X}$ (autopsied on soil) and T; V (non-autopsied on sand) and T). There was no significant difference overall between the autopsied and non-autopsied cadaver sample groups.

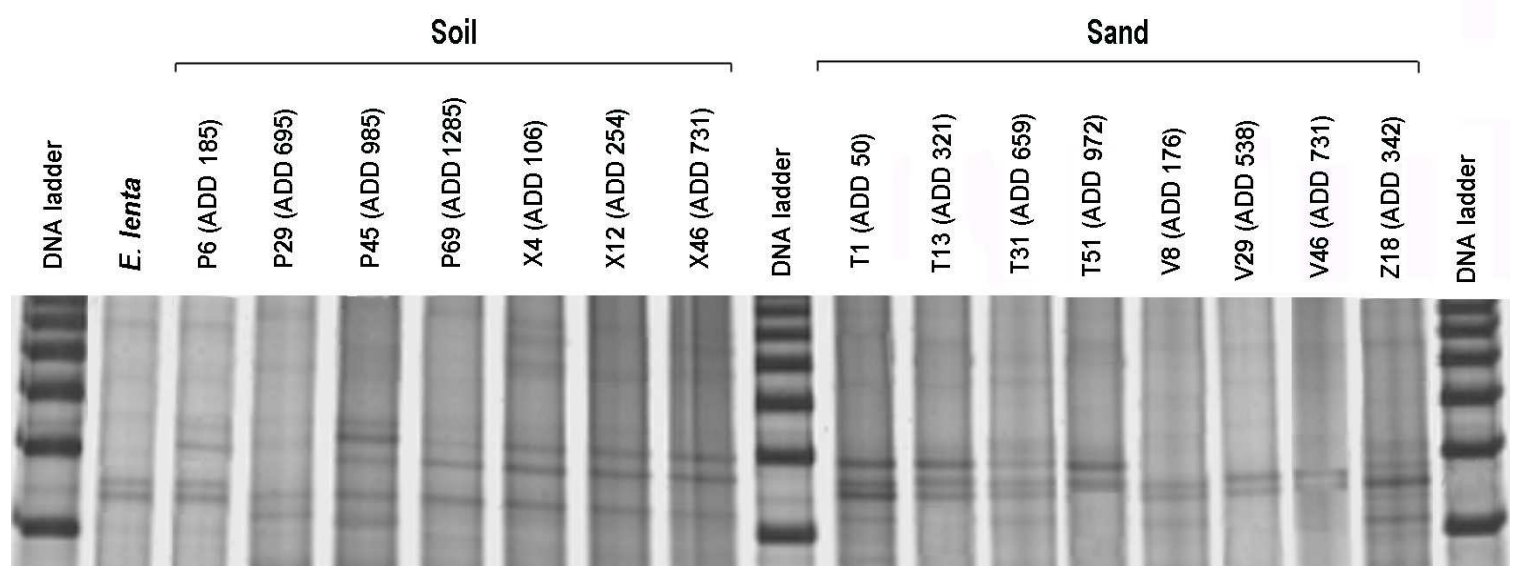

Figure 7.4 Photograph of the Atopobium group specific DGGE gel of profiles from selected sand and soil samples. 


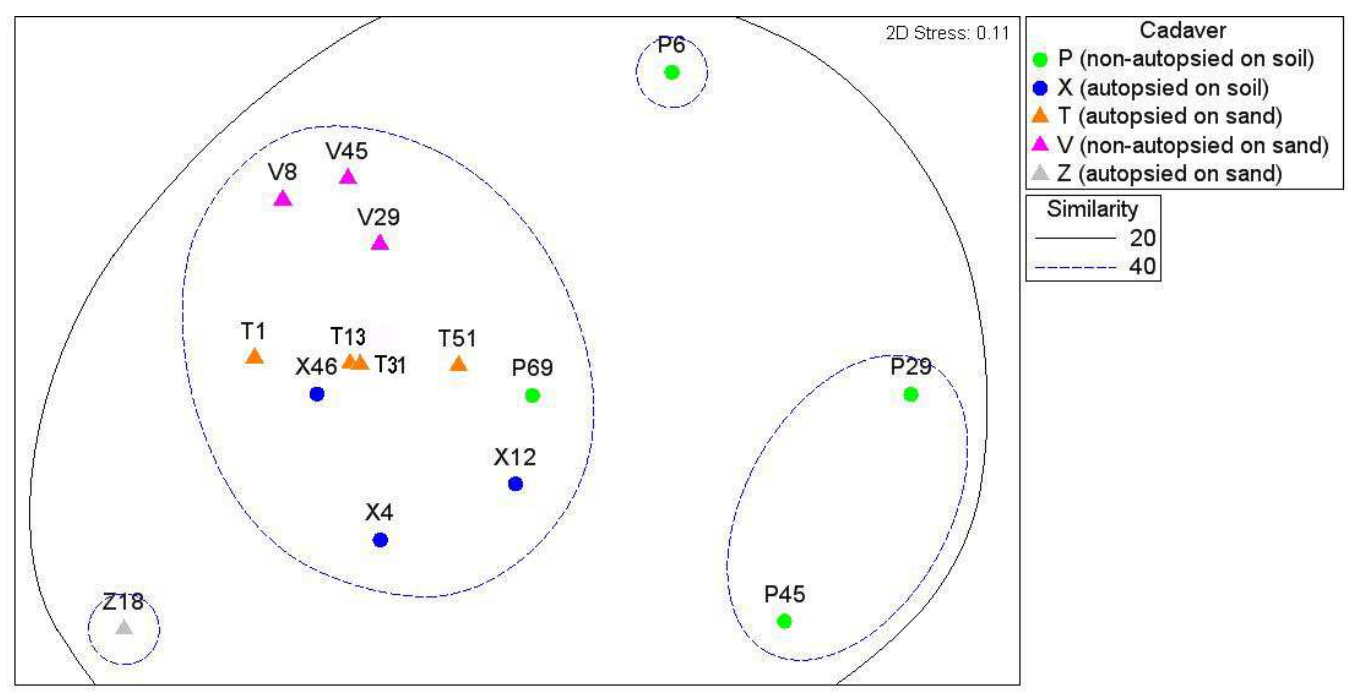

Figure 7.5 MDS plot of the Atopobia group specific DGGE profile data from selected soil and sand samples collected from under various cadavers. The Bray-Curtis similarity index was used on square root transformed data.

\subsection{The Bacteroides fragilis group}

The Bacteroides fragilis group gel gradient exhibited some warping on one edge (Figure 7.6) but a better quality gel could not be obtained despite repeated attempts. The profiles contain a number of discernible bands, ranging from 9 to 16 . The control profile, Bacteroides fragilis, showed one strong band which was also present in all of the sample profiles.

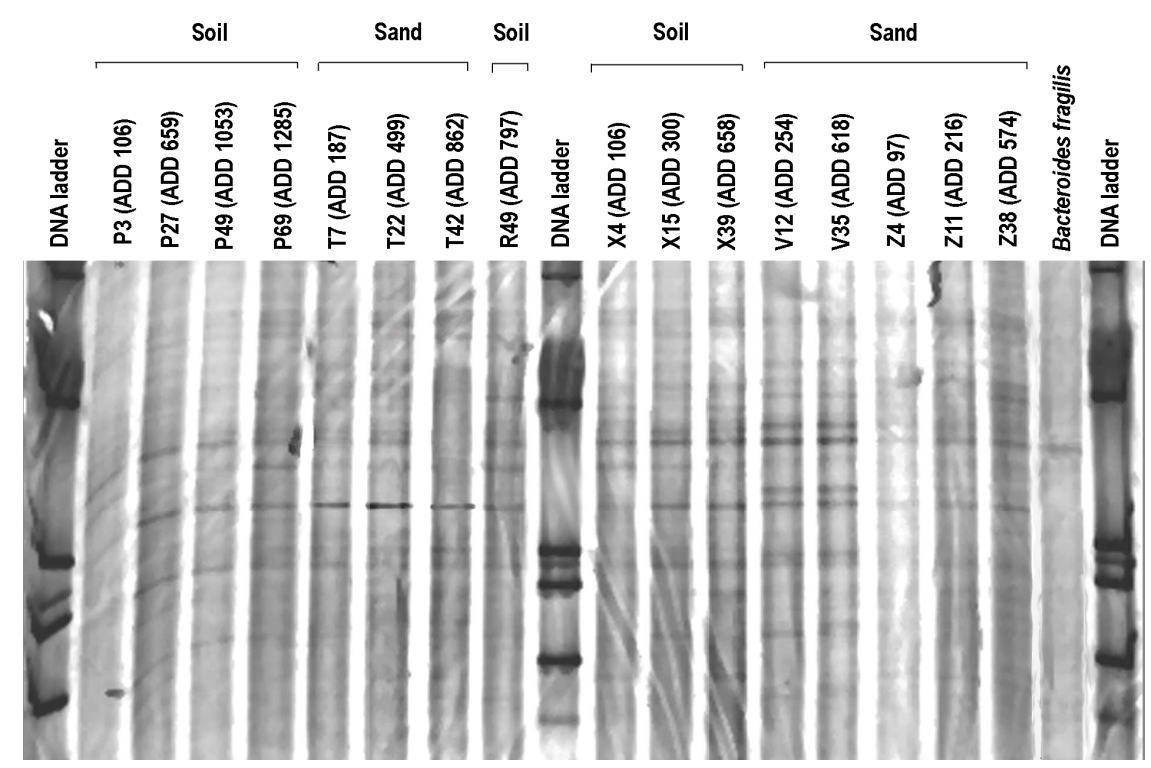

Figure 7.6 Photograph of the Bacteroides fragilis group specific DGGE gel of profiles from selected sand and soil samples. 
The MDS plot (Figure 7.7) showed some clustering of sample profiles from cadavers rather than any trend associated with stage of decomposition. There did not appear to be any strong distinction between profiles obtained from soil and sand or between profiles from autopsied/non-autopsied cadavers. There were a number of bands which were specific to a particular sample or cadaver, but there were none that appeared specifically associated with either autopsied or non-autopsied cadavers, sand or soil samples or a particular stage of decomposition.

The soil and sand sample profiles were significantly different overall $(\mathrm{p}<0.01)$ due to differences in the intensity of bands rather than their presence or absence. The profiles from the different cadavers were also significantly different $(\mathrm{p}<0.01)$ but due to the limited number of samples in some groups, the only groups which were significantly different $(\mathrm{p}<0.1)$ were from cadavers $\mathrm{P}$ (non-autopsied on soil) and Z (autopsied on sand). There was no overall significant difference associated with whether a cadaver was autopsied or not.

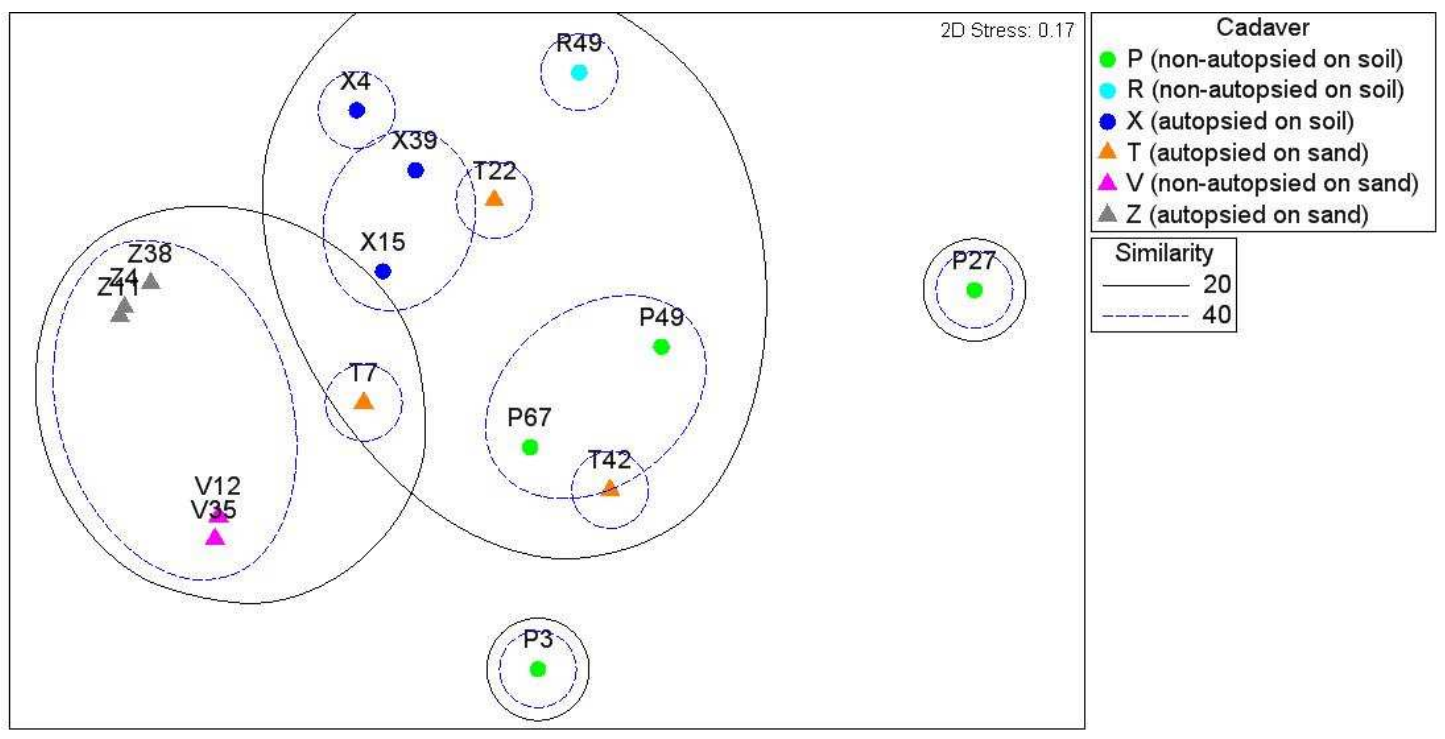

Figure 7.7 MDS plot of the Bacteroides fragilis group specific DGGE profile data from selected soil and sand samples collected from under various cadavers. The Bray-Curtis similarity index was used on square root transformed data. 


\subsection{The Bifidobacterium group}

Only four PCR reactions out of 112 tests were strongly positive for the presence of the Bifidobacterium group. These four, plus the control, Bifidobacterium longum were run on a DGGE gel, shown in Figure 7.8. The profiles were very simple with between three and five bands per sample profile. The Bifidobacterium longum profile contained only one band. Two samples which both derived from autopsied cadavers on sand, T1 and Z7 were very similar, with the same three bands present. Sample X4 (autopsied on soil) had the same three present but with an extra two bands, and sample T13 with an extra one.

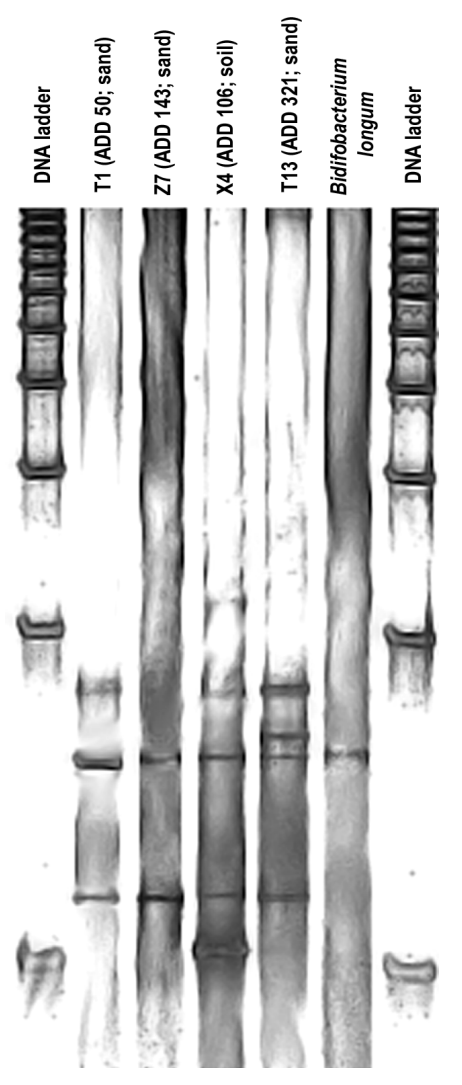

Figure 7.8 Photograph of DGGE gel analysis of Bifidobacterium communities from selected samples from under various cadavers.

\subsection{The Clostridium coccoides group}

Multiple attempts were made to generate a good quality gel showing the Clostridium coccoides group specific profiles. Due to problems with inconsistent running of bands in the outer lanes, the number of samples analysed were reduced to nine, plus the control to 
avoid using the outer two lanes on the gel. The best quality gel obtained is shown in Figure 7.9. The darkly-stained region with relatively ill-defined bands at the top of the profiles was excluded and only bands in the centre part of the gel (as marked with a red box) were analysed. The number of bands in this section of the gel ranged from none (in the C. coccoides control profile) to nine (in the T35 profile). The MDS plot (Figure 7.10) shows clustering of the profile data according to the cadaver they were derived from with separation between those on soil and sand. There were a number of bands which were specific to a particular sample or cadaver, but there were none that appeared specifically associated with either autopsied/non-autopsied cadavers, sand/soil samples, or a particular stage of decomposition. The soil and sand samples were significantly different overall $(\mathrm{p}<0.01)$ but the differences between the cadaver groups and between control/decomposition samples were not significant, probably due to the small number of samples. There was no significant overall difference between profiles derived from autopsied/non-autopsied cadavers.

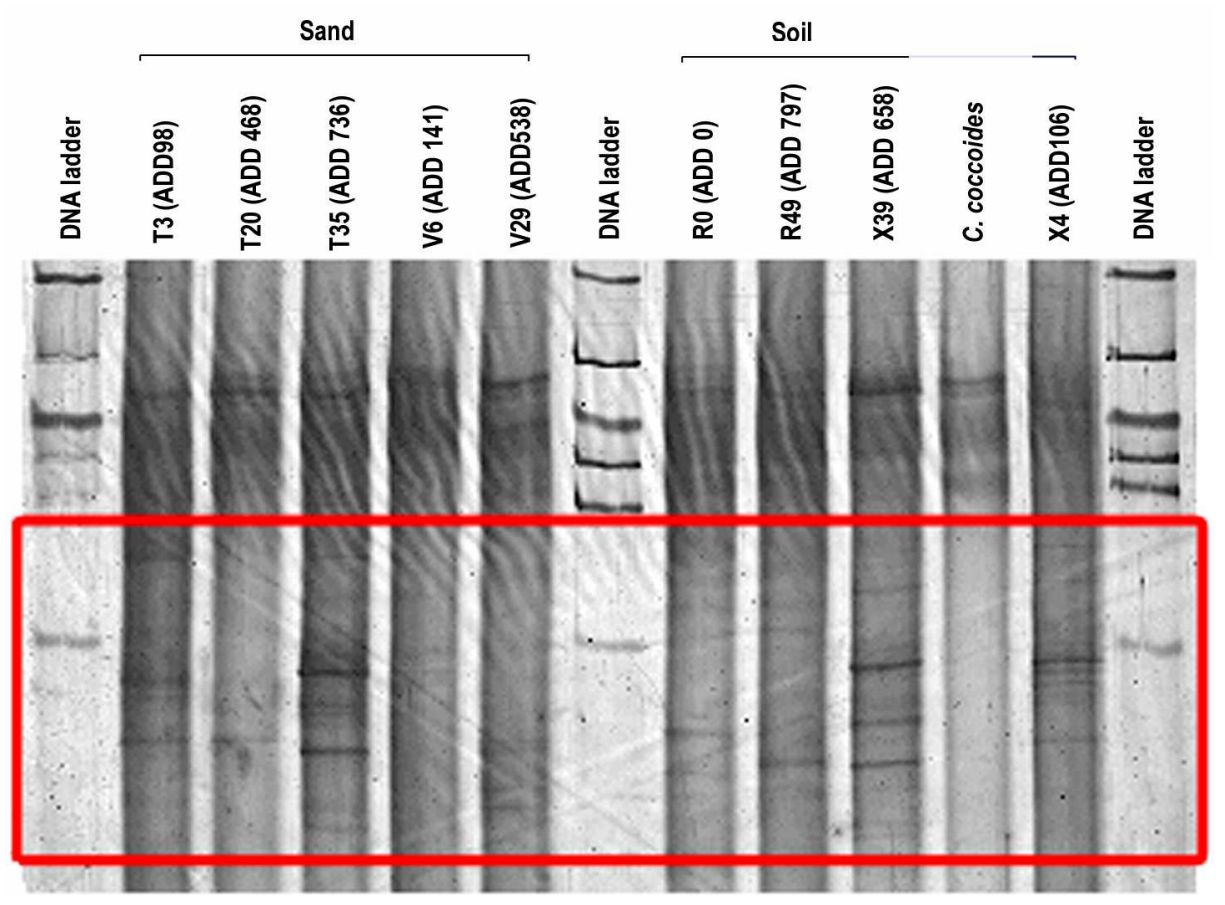

Figure 7.9 Photograph of the Clostridium coccoides group DGGE gel of profiles from selected sand and soil samples. The area of the gel analysed is indicated. 


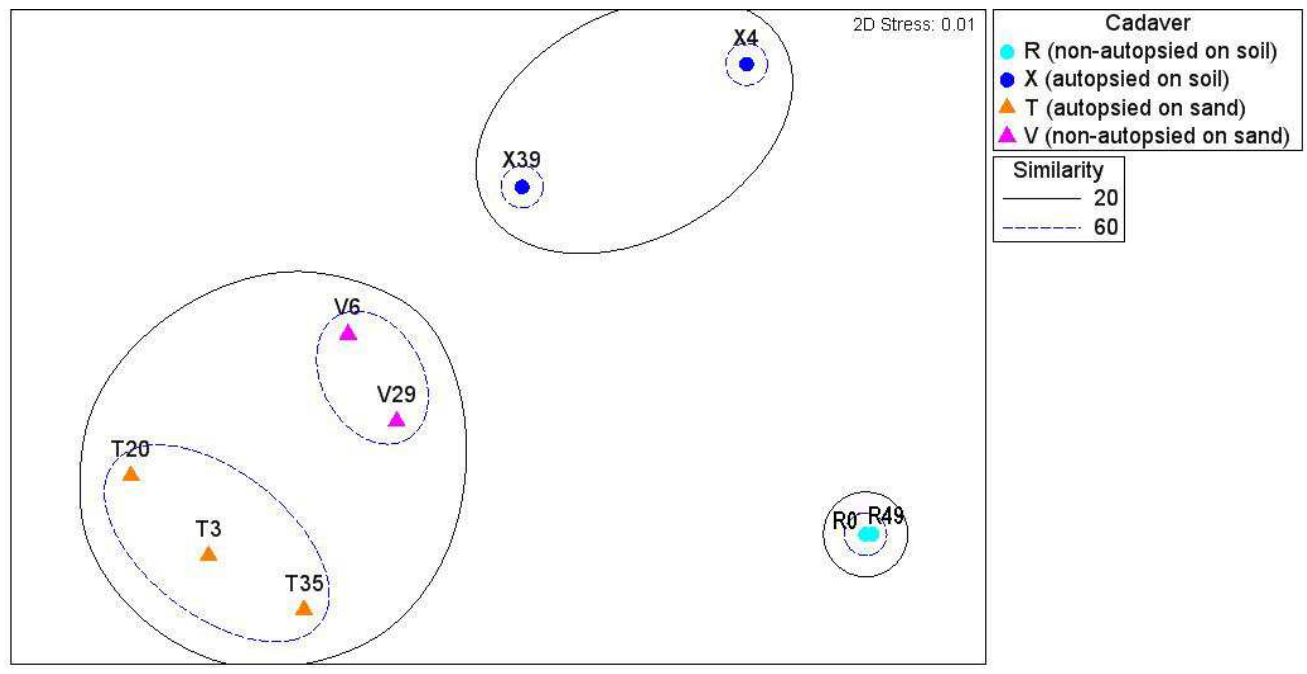

Figure 7.10 MDS plot of the Clostridium coccoides group specific DGGE profile data from selected soil and sand samples collected from under various cadavers. The Bray-Curtis similarity index was used on square root transformed data.

\subsection{The Clostridium perfringens group}

The Clostridium perfringens group DGGE gel (Figure 7.11) was of reasonable quality, with profiles containing between one (in the $C$. perfringens profile) and seven bands (in the R42 (non-autopsied on soil) profile). The bands were clearly separated using a denaturing gradient of $30-50 \%$.

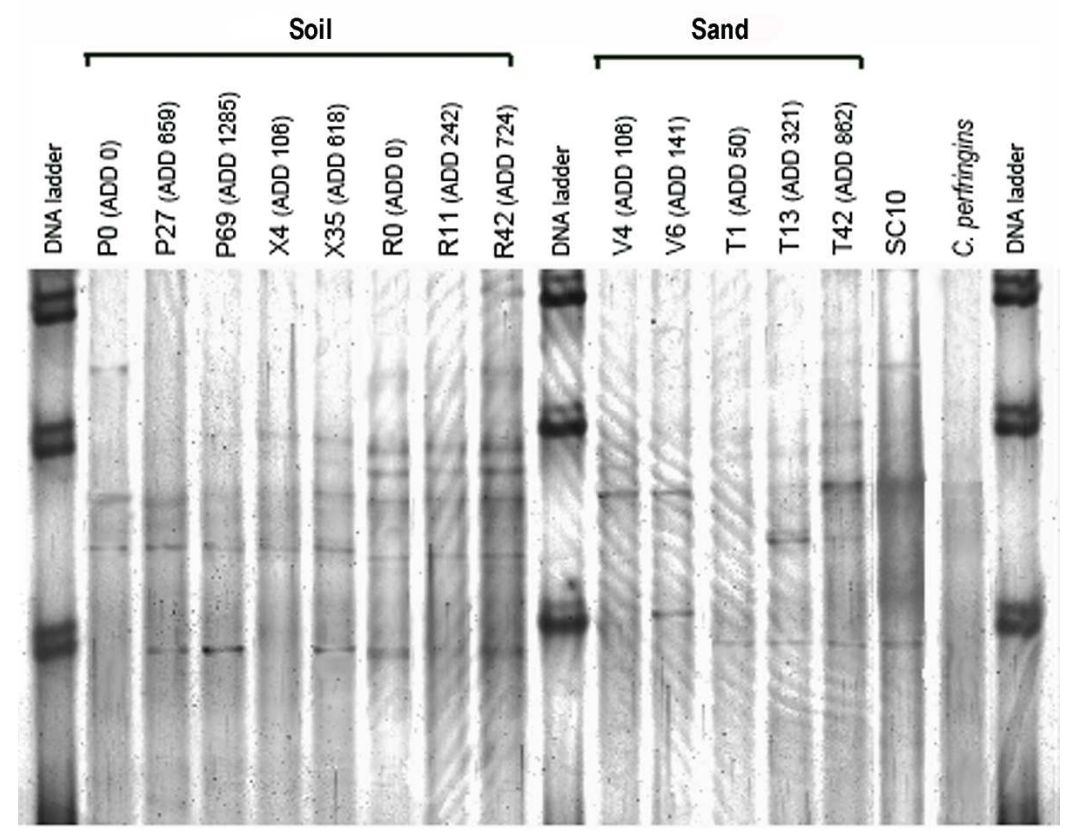

Figure 7.11 Photograph of the Clostridium perfringens group DGGE gel of profiles from selected sand and soil samples generated using -specific primers. 
The MDS plot (Figure 7.12) shows a minor differences in Clostridium perfringens group community constitution between profiles derived from soil and sand. The samples collected from under some cadavers (R-non-autopsied, soil; T-autopsied, sand; V-nonautopsied, sand) clustered closely according to the cadaver they were derived from, whereas the others (P-non-autopsied, soil; X-autopsied, soil) were more spread. There did not appear to be any association between samples collected from different cadavers at similar time points. There were a number of bands which were specific to a particular cadaver, but there were none that appeared specifically associated with either autopsied or non-autopsied cadavers, sand or soil samples or a particular stage of decomposition. The soil and sand profiles were significantly different overall $(p<0.01)$ but there was no significant difference between the control and decomposition derived samples, or between the different cadaver-derived samples.

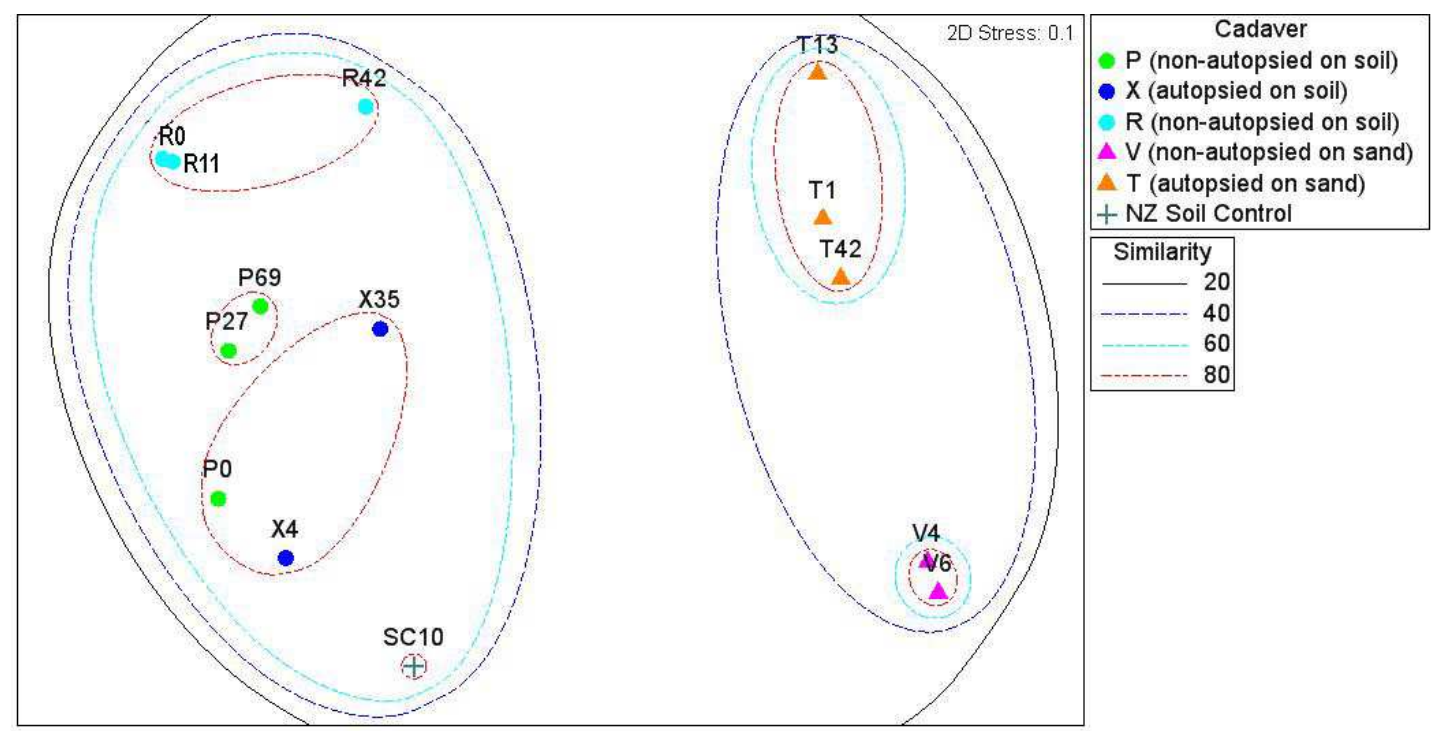

Figure 7.12 MDS plot of the Clostridium perfringens group specific DGGE profile data from selected soil and sand samples collected from under various cadavers. The Bray-Curtis similarity index was used on square root transformed data. 


\subsection{The Enterococcus group}

The Enterococcus group DGGE gel (Figure 7.13) was of good quality with clear, wellseparated bands in the centre of the 40-60\% gradient gel. The number of bands per profile ranged from zero (sample X4) to six (sample T24). The Enterococcus group was only detected in one soil sample (X4) in the group specific PCR (section 7.2.1.6) but no bands were detected in this sample. All the other samples were sand. The control Enterococcus faecalis produced a single band which appeared to be present in all profiles except X4 (autopsied on soil) and the two from cadaver Z (autopsied on sand).

The sample profiles from under cadaver $Z$ (autopsied on sand) are quite different to those from under cadavers $\mathrm{T}$ (autopsied on sand) and V (non-autopsied on sand). Two samples from cadaver $\mathrm{T}$ (T24 and T35) were more similar to the two samples from cadaver $\mathrm{V}$ than to others collected from under cadaver $\mathrm{T}$, with these four samples having less than $50 \%$ similarity to the other cadaver $\mathrm{T}$ samples. There were no bands that were found only in autopsied or only in non-autopsied cadaver sample profiles. There were also no bands that correlated to a particular stage of decomposition and were present in sample from more than one cadaver. There was no significant difference overall between profiles from autopsied and non-autopsied cadaver samples.

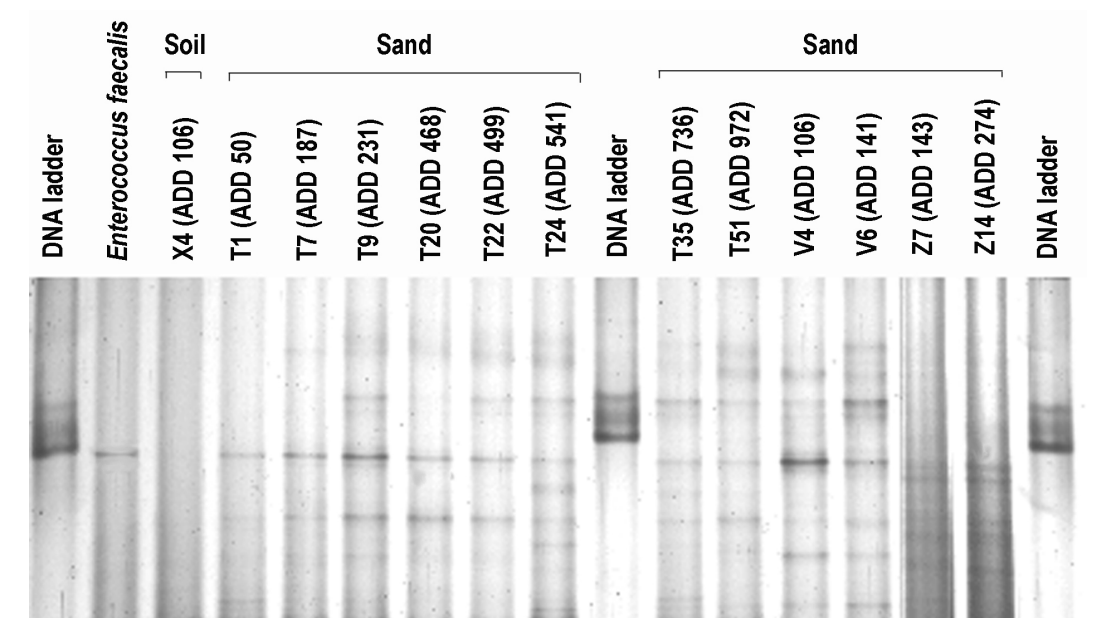

Figure 7.13 Photograph of the Enterococcus group-specific DGGE gel of profiles from selected sand and soil samples.The Lactobacillus group 
The DGGE analysis of the Lactobacillus group community profiles yielded a good quality gel with between six and nine bands per profile. All the samples analysed using DGGE were decomposition derived samples (as opposed to controls) from both soil and sand. The DGGE gel is shown in Figure 7.14. Three bands were present in all 14 sample profiles. Two further bands were present in all but one of the soil sample profiles (not in X4autopsied on soil) but were not present in the sand sample profiles. The lack of these bands probably accounts for the grouping of profile X4 with the sand sample profiles in Figure 7.15. Three early sand sample profiles (T1-ADD 50; Z4-ADD 97; V4-ADD 106) contained a band that was not present in any other samples. The Lactobacilli gasseri positive control contained two bands, both of which were present in all sample profiles.

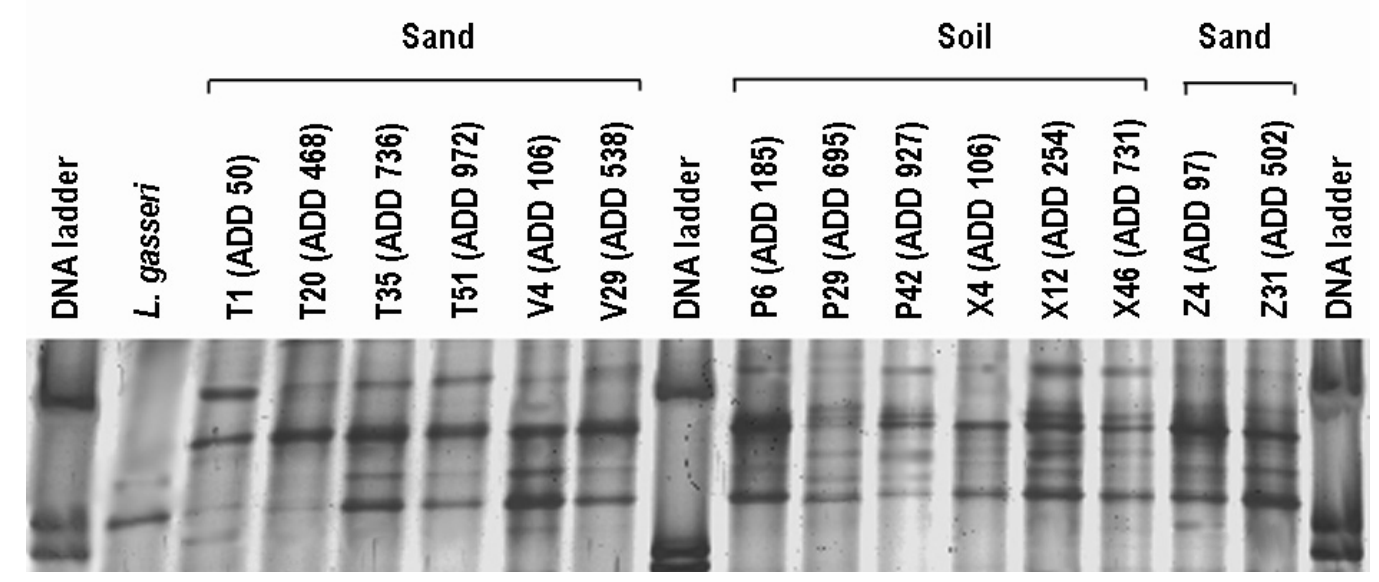

Figure 7.14 Photograph of DGGE gel of Lactobacilli communities from selected samples from under various cadavers.

There was a significant difference between the soil and sand sample profiles $(p<0.01)$ even though the similarity between the two groups was quite high. There were significant differences $(\mathrm{p}<0.1)$ between profiles from cadavers $\mathrm{P}$ (non-autopsied on soil) and $\mathrm{T}$ (autopsied on sand) and between cadavers X (autopsied on soil) and T, but the other groups were too small for significance to be assessed. There was no significant difference associated with autopsy. 


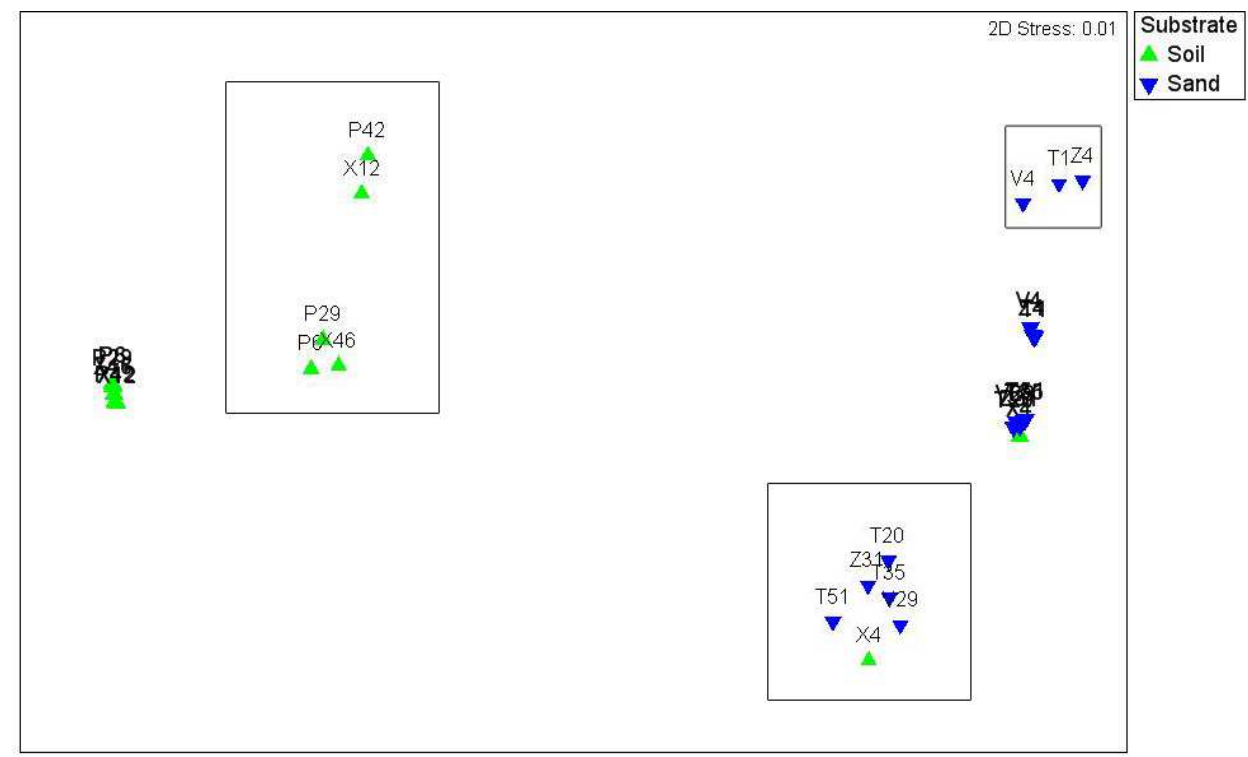

Figure 7.15 MDS analysis of DGGE profile data from the Lactobacillus group within selected soil and sand samples collected from under various cadavers. The boxes show a closer view of the clusters to enable distinction of the samples.

\subsubsection{Group-Specific Analysis Discussion}

\subsubsection{Actinobacteria}

Actinobacteria were detected using PCR in all cadaver decomposition samples and all soil controls. When further investigated using T-RFLP (section 7.2.3), higher diversity was found in the soil controls than in the soil decomposition samples, which in turn had higher diversity than the sand decomposition samples. This suggests that the decomposition event had a negative impact on the overall diversity of the Actinobacterial community in the soil. This may be caused by the severe changes in environmental conditions, such as the increase in $\mathrm{pH}$, that a decomposition event on soil causes. Most Actinobacteria favour a neutral pH (Niva et al., 2006), so the high pH may have created hostile conditions for some Actinobacterial species present in the soil. Previous studies have shown that long term organic and inorganic amendment of soils did not alter the level of diversity, but did change the Actinobacterial community structure (Piao et al., 2008). Heavy metal contaminated soils have been shown to have dominant Actinobacterial communities 
(Gremion et al., 2003; Nakatsu, 2005), and Actinobacterial species have been found in many extreme environments (Colquhoun et al., 1998; Priscu et al., 1999; Urzi et al., 2001), suggesting that at least some species are tolerant of foreign substances and harsh conditions that may inhibit the growth of other organisms. Introduction of carbon sources to poor soils (mine spoils) was found to stimulate the growth of certain Actinobacterial species (Hery et al., 2005), so it is possible that the decrease in diversity seen in response to the decomposition events could also be the result of some species that can utilise the nutrients released, outcompeting the other species.

Given the predominance of Actinobacteria in the human gut, it is perhaps surprising to see a reduction in diversity, with an expected influx of organisms from the cadaver into the underlying soil. The presence of T-RFs in profiles from the sand plot samples, however, suggests that some Actinobacterial species were likely to have derived from the cadaver itself, or possibly from the surrounding environment or colonising insects. The three TRFs that were found only in the sand plots may have been microflora released from the cadavers that were not able to survive in detectable quantities in the soil. This could be due to competition between these organisms and the existing soil microflora. This is highly likely given the differential survival rates of human pathogens seen in different soil types (Santamaria and Toranzos, 2003). Sandier soils are often associated with lower survival rates of pathogens but this may be related to moisture level (Guan and Holley, 2003). .

The presence of T-RFs specific to particular cadavers (as seen in samples from cadaver R (non-autopsied on soil) and cadaver T (autopsied on sand) may be caused by differences in individual cadaver Actinobacterial communities. The T-RF specific to cadaver $\mathrm{R}$, however, was only observed in sample R3 (ADD 85) which was very early on in decomposition, before this cadaver was seen to purge body fluids or enter active decay. It 
is possible that this was simply an artefact of the sampling method and heterogeneity of soil.

The relative simplicity of the Actinobacterial T-RFLP profiles when the group itself is known to be quite diverse suggested that the resolving power of the primer/enzyme combination may not have been optimal. This hypothesis was supported by using the MiCA Virtual Digest tool (http://mica.ibest.uidaho.edu/digest.php). The restriction enzyme used (MspI) was found to have limited resolving power, with the majority of organisms in the database returning expected T-RF sizes of 65 to $69 \mathrm{bp}$. All of the profiles contained high abundance T-RFs within this size range, suggesting that in retrospect, use of a different enzyme may have provided greater discrimination between the samples.

\subsubsection{The Atopobium group}

The Atopobium group was not detected in any of the control samples suggesting that the species which make up this group are not part of the normal soil community. The group's presence was detected in samples collected from under five of the six cadavers, with samples from under cadaver $\mathrm{R}$ all being negative. This is perhaps not unexpected, as cadaver $\mathrm{R}$ did not exhibit much abdominal drainage. The fact that these organisms were not detected in any of the control soil samples tested, could suggest that the presence of this group of organisms in a soil is indicative of a decomposition event. This raises the possibility of using organisms of this group as indicators of a site where a decomposition event has taken place. Forensic investigators do occasionally encounter crime scenes where it is thought that a decomposition event has occurred, but where the body has been moved to a second location (Dr S Cordiner, ESR Forensic Scientist, pers. comm.). The prominent NZ homicide of Wellington student Gavinn Dash was an example of such a scene. 
Testing for the presence of known decomposition related organisms could provide a simple confirmatory test of the occurrence of a decomposition event. More control soils would need to be tested to confirm that there was not just an absence of Atopobium in the control soils used in this study, and to determine whether faecal contamination of a soil would also give a positive Atopobium group result. It is possible that some species may be present in soil but in numbers too low to detect under normal soil conditions, but can proliferate under conditions such as those provided by a decomposition event.

The numbers of bands detected in the Atopobium community profiles were lower than might be expected, given that this group is a consistent member of the gut flora. The primers used were taken from a real-time PCR study (Rinttila et al., 2004) and the PCR product was relatively short $(120 \mathrm{bp})$. Having a shorter product is likely to decrease the number of nucleotide differences between amplicons from different organisms, reducing the difference in melting point and thus requiring a fine denaturing gradient, which is difficult to accurately prepare.

There were some differences between the soil and sand Atopobium profiles, but when plotted using MDS, samples did not clearly separate based on the substrate. The sample profiles did tend to cluster according to the cadaver they were derived from, and there were significant differences between some of the individual cadaver profile sets, suggesting that differences in each body's existing Atopobium communities were detected. Human gut microbial communities are known to differ from person to person, with the same general groups present at the phyla and class level, but with differences at the genera and species levels (Dethlefsen et al., 2007). The cadaver P profiles showed a greater variability over time than profiles from the other cadavers, which tended to have characteristic community patterns that persisted throughout the decomposition period. There is no obvious explanation for this and is likely to be caused by one or more of the numerous variables 
that could not be controlled for during decomposition of the different cadavers. It has been suggested that the Atopobium group diversifies with increasing host age (Harmsen et al., 2000) although this factor is unlikely to have influenced the results seen in this experiment.

The Atopobium group was detectable throughout the decomposition period. It was detected at the very first sampling of each of the autopsied cadavers and non-autopsied cadaver V (day 4, ADD 106). It was detected slightly later for cadaver P (non-autopsied on soil) in sample P8 (ADD 238), but persisted until cessation of sampling. It was detected in sample P69 which was collected 69 calendar days (ADD 1285) after cadaver placement. Because these organisms were detected in the earliest samples from most of the cadavers, it is likely that they begin to purge from the cadaver during the bloat stage when decomposition fluids first begin to be released.

The fact that members of the obligately anaerobic Atopobium group could be detected in the soil and sand samples after such a long period raises the possibility that these organisms are able to survive outside of the cadaver, in the decomposition environment. Enteric organism survival outside of the host depends on a number of environmental variables, many of which are not well defined, particularly in soil. Survival in water has been more extensively studied in relation to pathogen and faecal pollution indicators (Anderson et al., 2005). Survival times are likely to be dependant on factors such as moisture content, sunlight/UV exposure, temperature, soil type, nutrient status of the soil, existing microbial community composition, organic matter content and the type of organism (Guan and Holley, 2003). While little research has been performed on the survival of Atopobium species, faecal coliforms have been found to survive in soil for up to 55 days (McFarland, 2000). During the experimental sample collection period, it was noted that a distinctive layer formed at the interface between the cadaver/carcass and soil/sand. This layer of decomposition fluid was of a thick, viscous nature and was 
particularly pronounced on the soil. It is possible that this layer created a protected, perhaps anaerobic environment in which enteric organisms could survive. Pathogens are known to survive for long periods in harsh environments by forming biofilms (Huang et al., 2002) so it is possible that a similar situation is occurring during decomposition. Incorporation of a large quantity of organic matter into a soil system, such as in biosolids application, has been shown to increase overall microbial biomass, which leads to greater competition and higher die-off of pathogens (Epstein, 2002). However, if biosolids are not incorporated into the soil and left on the surface as a "cake" survival times are increased due to the more protected and pathogen-friendly environment within the sludge cake (Epstein, 2002). Pathogens have also been known to survive and be detected by coexisting within protozoa (Brown and Barker, 1999).

One further possibility that must be considered, is that rather than living organisms being detected in PCR, dead or non-viable cells are being detected or extracellular DNA is surviving in the decomposition environment (Hay et al., 2002; England and Trevors, 2003; England et al., 2004). Bacterial DNA has been recovered from air-dried soil samples dating back as far as 1914, suggesting that in simple systems, DNA is rapidly degraded by extracellular DNase activity in soil (Clark and Hirsch, 2008) or scavenged as a food source for other organisms. Factors such as soil type and organic matter content of soil are known to affect DNA survival. Naked DNA is able to bind with organic matter and clay particles and evade DNase and scavenger activity (England and Trevors, 2003). The increased potential enzyme activity seen under the cadavers for many of the enzymes assayed for (section 6.4.4) suggests that extracellular enzyme activity in general may have been upregulated in the decomposition environment, with DNases also likely to have been active. Enzymes, including DNases could themselves be deactivated by proteases, but the protease assay (section 6.4.4.8) did not show a determinable increase in this enzyme's activity. In 
addition, the environmental conditions created by a decomposition event, in particular the high $\mathrm{pH}$, seem likely to reduce the chances of DNA surviving for long periods, rather than promoting it. The fact that the overall bacterial communities were seen to change markedly but progressively over time using the T-RFLP and DGGE profiling also suggests that DNA was being degraded.

\subsubsection{The Bacteroides fragilis group}

Almost all of the decomposition derived samples tested positive for the presence of the Bacteroides fragilis group. This is not surprising given that the Bacteroidetes phylum is one of the two most abundant phyla in the gastrointestinal tract (Dethlefsen et al., 2007), with Bacteroides being the most commonly isolated genus from human faeces (Wilson, 2005). Even though Bacteroides species are not thought to be common soil inhabitants (Okabe and Shimazu, 2007), two control soils were found to be positive for the Bacteroides fragilis group. This suggests that there may have been contamination of the control site by faecal material at some recent time as Bacteroides have been shown to not survive for long outside of mammalian hosts (Kreader, 1998). A number of environmental factors such as temperature and predation by eukaryotic organisms affect how long Bacteroides can survive (Kreader, 1998; Okabe and Shimazu, 2007; Bell et al., 2009) but in water, which is by far the most studied environment in this context, their survival is usually measured in days (Bell et al., 2009). A recent project examining diversity within different land-use systems identified Bacteroides species within cotton field soil, but it was noted that the site was adjacent to livestock feedlots (Acosta-Martinez et al., 2008). It is also possible that a member of the normal soil flora, related to Bacteroides, whose $16 \mathrm{~S}$ DNA was also able to be amplified by the group specific primers.

The detection of the anaerobic Bacteroides group DNA in samples up to ADD 1285 post placement of cadaver P (non-autopsied on soil) could, like the detection of the Atopobium 
group in this sample, suggest the development of an anaerobic environment in the wet toplayer of the soil under the decomposing cadaver, or could, again, be the detection of nonviable cells or DNA that has persisted in the environment (Hay et al., 2002; England and Trevors, 2003; England et al., 2004). Because Bacteroides are the most aero-tolerant of all the anaerobes (Wilson, 2005), this was thought to be the group that was most likely to survive outside of the human body.

The DGGE analysis of the Bacteroides fragilis group yielded a number of bands in each profile (Figure 7.7). Visually, the profiles appeared quite similar, with a number of the bands present in all, or almost all profiles, but a significant difference between the sand and soil samples was detected. This is likely to be due to differences in band intensities rather than band presence or absence. There was no clear correlation between particular band intensities and treatment. In the MDS plot, samples clustered largely according to the cadaver they derived from, suggesting that individual cadaver Bacteroides community differences could be causing this. Variability in the Bacteroides species seen to purge from each cadaver is be expected given the variability seen between even closely-related individuals ( $\mathrm{Li}$ et al., 2009).

\subsubsection{The Bifidobacterium group}

The Bifidobacterium group was chosen for analysis because of its association with the gut and its likely absence in soil. This hypothesis supported because no Bifidobacterium group members were detected in the control soils. It was, however, detected in only four samples from three cadavers, all of which were early in the decomposition process (ADD50-321) and all of which derived from autopsied cadavers. This suggests that the invasive nature of the autopsy left exits in the cadaver that allowed egress of organisms into the 
surrounding environment that did not happen with simple purging of fluids from natural orifices. Autopsied cadavers are not ideal models for forensic decomposition cases, but the situation could arise where a victim has been severely wounded, giving a similar pathway for organisms out of the gut system.

The DGGE results showed very clear but simple Bifidobacterium profiles, suggesting that while members of this genus can be detected in some samples, it is not a dominant member of the decomposition-associated microbial community and is an unlikely candidate for forensic time since death interest.

\subsubsection{The Clostridium groups}

For this study, two major groups, the Clostridium perfringens (Cluster I) and the Clostridium coccoides (Cluster XIV) groups were looked at using group specific primers. Members of both groups were detected in samples from all six cadavers and in most control soil samples. This was expected, as Clostridium species from both groups are widely distributed in the environment (Matches et al., 1974; Wiegel et al., 2006) as well as being present within the human GIT (Wilson, 2005).

The quality of the DGGE gel results were somewhat disappointing. From the profiles that were obtained, very few bands were discernible, which was unexpected given the diversity within the Clostridium groups and the high number of samples that gave positive PCR results for these groups. Profiles tended to cluster according to the cadaver from which they were derived, suggesting that the cadaver derived organisms strongly influenced the Clostridium populations. The sand and soil sample profiles were significantly different, however, and the MDS plots showed this separation clearly, suggesting that the different environments that soil and sand offer and/or the soil's existing microflora had an impact on 
the composition of the communities. Because of the poor quality of the gels obtained for the Clostridium group specific DGGE analysis, the results need to interpreted with caution.

\subsubsection{The Enterococcus group}

Enterococci were detected in very few samples. They were detected in samples from under the three cadavers placed on sand, but in only one decomposition soil sample. This was an early sample from under cadaver X, which was autopsied (X4 - ADD 106). Enterococci were not detected in any of the soil control samples, suggesting that all the organisms detected derived from the cadavers themselves and that the organisms or their DNA were not able to survive in the soil environment, but could in the sand. This group was investigated because of its apparent high tolerance of unfavourable conditions and the fact that it had previously been found in soil. In this case it appears to not have survived, perhaps because of competition with the pre-existing microbial community (Morris and Blackwood, 2007). The lack of detection was a highly unexpected result, given that Enterococcus is used as a faecal indicator, has been found to survive for long periods in soils and is readily found in the environment (Aarestrup et al., 2002).

\subsubsection{The Lactobacillus group}

No members of the Lactobacillus group were detected in the control samples collected from the US. They were, however, detected in samples from under five of the six cadavers. They were not detected in samples collected from under cadaver R. This is not totally unexpected, as cadaver $\mathrm{R}$ displayed very slow decomposition and there was very little abdominal drainage even at ADD 797, which was the final sampling time-point for this cadaver. Very faint positive results for nested PCR were detected for three of the control soils from New Zealand. 
The DGGE profiles were dominated by strong bands common to all the profiles. There was some variability within the less densely stained bands, suggesting that the same Lactobacillus species were present and dominant in all the samples, but with some variability in the more minor community members. The extra band present in the earliest sample from under each of the cadavers on sand was interesting, as this is the only band which was found in all replicate cadaver samples of a given treatment (sand) at a given time-point. This band could be interesting for further investigation, but because it was not detected in the early soil decomposition samples, it is unlikely to be of forensic use. It is possible that the soil environment prevented detection of this band in the earliest soil decomposition samples.

\subsubsection{Evaluation of the Experimental Approach}

Overall, the group-specific DGGE results were not as good as originally anticipated. Despite repeated attempts, many of the gels were of poor quality with fewer bands in profiles than expected. The universal PCR DGGE profiles from previous chapters were of a much higher quality than the subsequent, more specific analyses. A number of factors may have influenced this. As mentioned earlier, the gel photographs were of particularly poor quality due to the need to rescan them for inclusion in this thesis. The original scans from which the banding patterns were analysed were much clearer with fewer artefacts obscuring the bands. Many of the specific primer pairs used were not designed specifically for DGGE analysis, but for real-time PCR assays and had not previously been assessed for their suitability for DGGE analysis of communities. It is possible that some of these primer pairs target regions of the $16 \mathrm{~S}$ gene that is highly conserved between related organisms, leading to a reduced estimate of diversity. This is more likely for the short products (Atopobium, Enterococcus and Clostridium perfringens at 120-140 bp) than for the longer products which would have covered multiple variable regions within the $16 \mathrm{~S}$ 
gene. These primers were taken from Rinttila et al.(2004) which did not give the position of the primer target sequences within the gene. In retrospect, designing custom primers using a programme to assess melting behaviour of some of the target strains to should have been performed. Because DGGE is successfully used to screen for single point mutations, however, even small variations in sequence should adequately affect the melting point of DNA from different organisms. Narrow denaturant gradients were tested with most of the specific PCR products, which would help separate similar sequences, but these gels seemed to be more prone to flaws in the forming of the gradient, resulting in blurry, non-distinct bands.

The quality of the gels is also likely to have affected the band analysis to some degree. The lack of separation of some of the banding patterns may have led to an underestimation of diversity present, with bands which had slightly different melting points being interpreted as bands at the same point in the gradient.

An option which was not explored at the time of this experimental work, was the construction of DNA ladders using a combination of organisms expected to be present in a sample. This would have enabled instant tentative identification of band species origin. In retrospect, this would have been particularly helpful given the failure of attempts to sequence from bands of interest (section 7.3).

Nested PCR was shown to be slightly more sensitive (as shown by the New Zealand soil control samples) than the standard PCR method used with it detecting the presence of target organisms in samples which were negative using standard PCR. This was not discovered until after the group-specific PCR amplifications had been completed and due to budgetary constraints, could not be repeated. If this method had been used for all samples, more positive results may have been detected. Hence, a negative PCR result in this experiment cannot be read as a complete absence of target organisms, rather as an 
indication that those organisms are not dominant members of the community. The original reasoning behind using the genomic DNA extracts for these PCRs rather than a nested PCR using amplified $16 \mathrm{~S}$ products, was that although $16 \mathrm{~S}$ primers are hoped to be close to universal, there are likely to be many organisms not amplified by these primers. A nested PCR would exclude these organisms. In retrospect, use of the nested approach may have been more appropriate.

\subsubsection{Overall conclusions of group-specific DGGE results}

Despite the quality of the gels not being as high as expected, some interesting findings can be noted.

As with some of the experimental results in Chapter 6, cadaver $\mathrm{R}$ returned quite different results to the other cadavers. Because cadaver R showed very slow decomposition with less abdominal drainage during active decay than the other cadavers, it was not necessarily surprising that groups of bacteria expected to derive from the gut were not detected in the soil under cadaver R. What was surprising is that this theory did not hold for all the groups of gut-derived organisms. Cadaver R samples were all negative for the Atopobium, Lactobacillus, Enterococcus and Bifidobacterium groups, but three isolated samples from the later stages of decomposition were positive for the Bacteroides fragilis group, and sample R49 contained a number of different bands in this profile. It is possible that these Bacteroides fragilis group organisms were already present in the soil, and were simply not detected in previous samplings due to non-uniform distribution in the soil or proliferated to a detectable level by that stage of the decomposition event. It is more likely, however, that detection of these organisms later in the sampling period, when cadaver $\mathrm{R}$ was starting to decay, that some gut flora were released into the underlying soil. Because the medical history of the individual prior to death is not known (other than the cause of death - renal 
failure, and the observation of a degree of wasting away prior to death), it is possible that medical treatment affected enteric bacterial groups differently. Bacteroides are known to have developed resistance to multiple antibiotics (Grisold et al., 2007) whereas Bifidobacterium and Lactobacillus species, for example, have more varying susceptibilities to antibiotics (Delgado et al., 2005). These results again highlight the difficulties associated with working with human cadavers and the many possible influences on decomposition that cannot be controlled for.

Although no significant differences were found between autopsied and non-autopsied cadavers when overall DGGE profiles were compared on each gel, it is possible that autopsy did affect which groups were detected in samples. Sample X4 (ADD 106; autopsied on soil) and sample Z7 (ADD 143; autopsied on sand) were the only samples in which all of the bacterial groups tested for were detected. X4 was the first sample collected after placement of cadaver X and Z7 was the second sample from under cadaver Z. The fact that both of these cadavers were autopsied raises the possibility that this allowed egress of a variety of organisms from the cadaver directly into the underlying substrate. The earliest sample collected from under the other autopsied cadaver (T1 ADD 50; on sand) gave positive results for all groups tested with the exception of Clostridium perfringens. It was positive for Bifidobacterium, which was only detected in four samples overall, all of which were from autopsied cadavers. The early samples collected under the three non-autopsied cadavers did not test positive for all groups, but the two earliest samples from under cadaver $\mathrm{V}$ (non-autopsied on sand) contained all but the Bifidobacterium group. This was a little surprising, given that by ADD 106, when sample V4 was collected, cadaver $\mathrm{V}$ did not exhibit heavy abdominal drainage, although some decomposition fluids were visible. The other non-autopsied cadavers had fewer groups detectable overall, but both of these were placed on soil. It is possible that many of the 
groups tested for are purged early in decomposition and that these could be more readily detectable in the sand samples, than in the soil samples which contain a pre-existing microbial population. In a simple community, less abundant organisms are more likely to be amplified during PCR than if those same organisms, at the same level of abundance were in a more diverse community. The invasive autopsy procedure may simply speed the release of these organisms, or allow egress of organisms into the underlying substrate that do not survive to be released through purging from natural orifices, as occurred in the nonautopsied cadavers.

With the exception of these early samples, and of the Bifidobacterium group, which was only detected in four samples (all early in the decomposition process), there did not appear to be any correlation between the detection of a particular group and the stage of decomposition. It seemed that if a group was detected in one sample from under a cadaver, it was detected in others throughout the sampling period, albeit sometimes sporadically. Enterococcus was detected only in early samples under cadavers V and Z (sand) and X (soil), but was detected throughout the sampling period for cadaver $\mathrm{T}$ (sand) and was not detected at all under cadavers $\mathrm{R}$ and $\mathrm{P}$ (on soil). The sporadic detection of these groups is likely to be a sampling and/or an extraction/PCR artefact rather than the fluctuation of the groups in relation to the decomposition process. Particularly early on in decomposition, fluids purge from the body and collect in puddles under wounds and natural orifices rather than saturating the entire sampling area. This would lead to isolated patches containing organisms. The sampling method used attempted to sample the entire area under the cadaver's torso, and all possible efforts were made to homogenise samples prior to subsampling, but it is likely that this affected results. As mentioned previously, a negative result for a group does not may it is absent from the sample site. The detection of organisms thought to derive from the cadaver itself throughout the entire decomposition 
period in both sand and soil (under cadaver X) suggests that either these organisms are able to survive for substantial periods in the environment created by decomposition, or that DNA is able to persist for those periods, if the cells themselves do not survive.

A number of bacterial groups that were detected under all the other cadavers were not detected under cadaver P. This is surprising given that this cadaver died suddenly with no obvious sickness prior to death that would have led to medical treatments such as antibiotic therapy that could affect the gut microflora. It did, however, decompose particularly quickly (section 6.4.1). One group that was not detected in any cadaver P derived samples was Clostridium perfringens. This is unusual given that this organism was detected in the nearby soil control at plot $O$. This group was detected more sporadically than the closely related Clostridium coccoides group, so this may be an artefact of the PCR assay itself. It may also, however, be that this group is out-competed by other organisms, reducing the likelihood of it being detected in samples, or is inhibited by a substance or condition associated with the decomposition process under this particular cadaver.

Another point worth noting is the detection of Lactobacillus and Bacteroides fragilis groups in control soil samples. These are largely considered to be enteric organisms and so would not necessarily be expected to be found in soil. Both have, however, been reported to be found in soil samples previously. It is also highly possible that contamination of the soil with faecal material has resulted in detection of these organisms. This is an important point to consider if any of these organisms were to be considered for forensic use, as preexisting populations could interfere with indicator species detection. Further refinement to the species level could circumvent this issue. Certain species, for example, are host specific (Aarestrup et al., 2002). Even if human specific species were used, however, contamination of sites by human biosolids is not unusual. Widespread testing of control 
soils would need to be conducted to determine the probability of encountering potential time since death biomarkers in soils unrelated to decomposition events.

For a number of bacterial groups, there were differences in detection of cadaver-derived organisms between the soil and sand samples, suggesting that the physical differences between the soil and sand environments, or perhaps the pre-existing soil microflora affected the survival of the cadaver-derived organisms. Also possible is that some aspect of the soil, such as the presence of humic acids, affected DNA extraction and/or amplification from soil samples, preventing detection of certain types of organism (MartinLaurent et al., 2001).

The overall aim of this part of the project was to investigate bacterial communities with a view to finding indicator groups or species particular to decomposition stages, or time since death. The only organisms found to be strongly associated with a particular stage of decomposition in this experiment was the Bifidobacterium group, with only early-stage decomposition samples found to contain this group. They were, however, also only found to be associated with autopsied cadavers, suggesting that they are unlikely to be of use as biomarkers in a forensic context. The group-specific DGGE approach, whilst theoretically being a good method for determining when particular groups can be detected and when particular phylotypes are present, the quality of the gels and the variability between the individual cadavers reduced the value of the exercise. The experiment did highlight, however, that individual variation in microflora can be detected in the decomposition environment and that this high individual variability must be taken into account when species level biomarkers are being considered. It also demonstrated that using higher taxonomic groupings as time-dependant biomarkers is unlikely to be of use forensically, as most of the groups tested in this experiment were found to be present at all stages of decomposition. 


\subsection{DGGE Band Excision}

Various bands of interest from four of the total bacterial community DGGE gels (pig carcass A, pig carcass D, cadaver $\mathrm{P}$ and cadaver V gels) and three of the simpler groupspecific DGGE gels (Lactobacillus, Enterococcus and Bifidobacteria gels) were excised in an attempt to isolate and sequence the DNA fragments contributing to the band. Bands were chosen that were present in many profiles or were prominent, but found exclusively in individual profiles.

\subsubsection{Methods}

Four band excision methods were tested.

\subsubsection{Silver stained band excision and reamplification}

Selected bands were carefully excised from the silver stained gels using sterile scalpel blades. Each gel slice was placed in a $0.2 \mathrm{~mL}$ microcentrifuge tube with $100 \mu \mathrm{L}$ diffusion buffer (0.5 $\mathrm{M}$ ammonium acetate, $10 \mathrm{mM}$ magnesium acetate, $1 \mathrm{mM}$ EDTA [pH 8.0], and $0.1 \% \mathrm{SDS}$ ) and incubated for $30 \mathrm{~min}$ at $50^{\circ} \mathrm{C}$ (Requena et al., 2002). The tubes were vortexed briefly and left at $4^{\circ} \mathrm{C}$ overnight. A $2 \mu \mathrm{L}$ aliquot of the supernatant was taken and used as the template for a PCR. The same PCR recipe and thermocycling regime were used as in the original amplifications (Tables 7.2 and 7.3) with the appropriate primers. A DGGE gel was then run as per the method described in section 3.4.4.

\subsubsection{Ethidium bromide stained band excision and reamplification}

Bands were also excised from gels stained with ethidium bromide rather than silver stain. Gels were run as per the method in section 3.4.4, but were immersed in ethidium bromide (10 mg/mL) for 15 minutes and visualised using ultra-violet (UV) light. Bands were excised quickly while exposed to UV light to prevent degradation of the DNA. Gel slices 
were then treated and the DNA reamplified as per the silver stained gel method above. A DGGE gel was then run as per the method described in section 3.4.4.

\subsubsection{Direct sequencing of kit extracted DNA}

Bands were excised with sterile scalpel blades, rinsed briefly with DNase/RNase free $\mathrm{H}_{2} \mathrm{O}$ and the DNA extracted using a Qiagen QIAquick Gel Extraction kit (Appendix A14). The DNA was eluted in $30 \mu \mathrm{L}$ for increased concentration. A $10 \mu \mathrm{L}$ aliquot was used directly for DNA sequencing using an ABI 3700 Genetic Analyser (Applied Biosystems) with a Big Dye Terminator sequencing kit (Applied Biosystems). The same forward primer was used as for the PCR amplification but without the DGGE GC clamp attached. Sequencing QIAquick Gel Extraction kit (Appendix A14). A $2 \mu \mathrm{L}$ aliquot of the eluted DNA was used as the template for a subsequent round of PCR using the same conditions and primers as for the first PCR (section 7.2.5.1). A DGGE gel was then run as per the method described in section 3.4.4.

\subsubsection{Results}

The DNA extracted from the selected bands was reamplified and re-run on another DGGE gel to ensure that a single, sharp band was present, to confirm that a single sequence was available for sequencing. Rather than the expected individual bands, however, each lane displayed the entire profile of the sample that the band was excised from. Figure 7.16 shows the original profile for sample P27 (ADD 659) aligned with the profile generated from the excised band, and the profile obtained when the corresponding band on the second gel was also excised and reamplified. The complete original profiles were obtained for every band excised including those taken from the simpler group-specific gels. Bands from higher and lower in the profiles were tested to determine whether this phenomenon was related to certain parts of the gradient. All bands gave similar results regardless of 
extraction method, site of the band within the gradient or gel staining method used. Direct sequencing of the DNA from the gel bands was unsuccessful due to a mixture of template sequences being present.

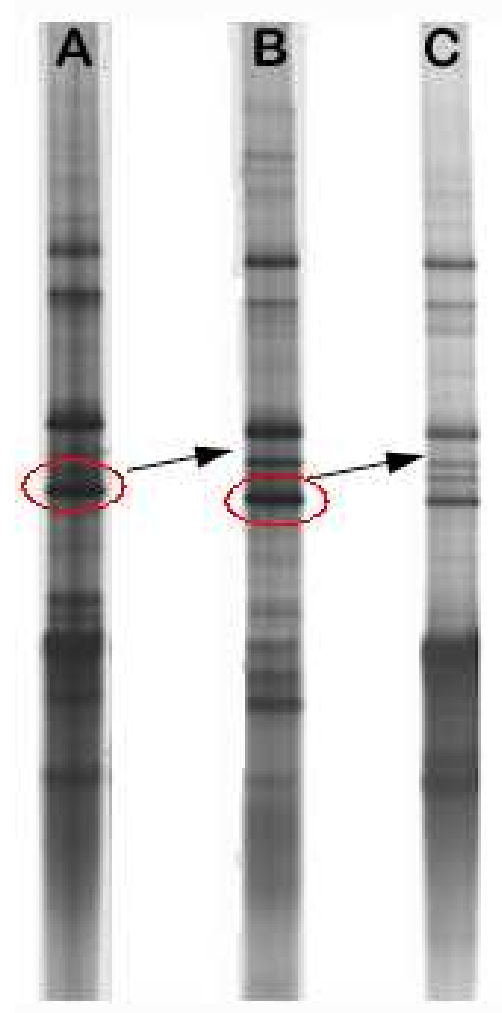

Figure 7.16 DGGE profiles of (A) sample P27 non-autopsied (cadaver on soil, ADD 659) produced using $16 S$ universal primers; (B) the profile produced by excising a band from A (circled), DNA eluted in diffusion buffer and reamplified using the same primers; $(C)$ the profile produced by excising the corresponding band from profile B, DNA extraction using the QIAquick gel extraction kit and reamplification with the same primers. These gels were silver stained.

\subsubsection{Discussion}

Many researchers have reported problems similar to those encountered in this study when attempting to sequence DNA from a band (Avrahami and Conrad, 2003; Kisand and Wikner, 2003) or when examining regions of a DGGE profile (Nikolausz et al., 2005). Comigration of different DNA fragments has previously been reported (Ferris et al., 1996; Sekiguchi et al., 2001; Erliconi, 2004; Gafan and Spratt, 2005) and incomplete band synthesis during PCR that leads to multiple bands for one template has also been reported 
(Janse et al., 2004). Nikolausz et al. (2005) concluded that "everything is everywhere" in a DGGE profile rather than in discrete bands. They also raised the theory that the banding pattern in a DGGE gel may not be simply the result of the separation of different amplicons according to their melting behaviour, but a consequence of complex interactions among different DNA structures. Single-stranded DNA generated during PCR has also been suggested as a cause of extra banding in DGGE gel excision (Jensen and Straus, 1993; Egert and Friedrich, 2003). The red staining seen on some of the original universal $16 \mathrm{~S}$ primer DGGE gels was also likely to have been caused by differential staining of ss-DNA. These bands often co-migrated with actual bands, and co-excision would be highly likely. Mung-bean nuclease treatment of the later gels, however, would have eliminated the ssDNA and so this is an unlikely cause of the banding pattern observed.

Some researchers have suggested that silver staining gels may impede successful reamplification from excised bands by causing damage to the DNA (Heuer and Smalla, 1997; Marschner, 2007). Many others, however, have successfully reamplified and sequenced DNA from silver stained gels (Caetano-Anollés and Trigiano, 1997; Mahmood et al., 2006; Dickie and Fitzjohn, 2007; Pandey et al., 2007; Thies et al., 2007; Delgado et al., 2008). Bands were not successfully purified from the ethidium bromide stained gels either, suggesting that the choice of stain was not the cause of the result.

The ability to simply and easily excise bands for sequencing was a major factor when deciding on the methods to be used in this study. The difficulties encountered in the running of the gels themselves and the inability to isolate amplicons, in this instance, using the band excision method, meant that this technique was not able to be used for band identification and more in depth analysis of the bacterial communities associated with decomposition. Cloning was subsequently used to obtain single amplicons for sequencing (Section 7.5). 


\subsection{Multi-digestion T-RFLP}

By generating multiple T-RFLP profiles for a sample using different restriction enzymes and comparing these data with predicted fragment lengths for sequences in a database such as the rRNA gene sequence database, predictions of potential phylogenetic placements can be made. This strategy has been used by a handful of researchers (Dickie and Fitzjohn, 2007; Pandey et al., 2007; Thies et al., 2007). The number of sequences available in online databases is growing exponentially as more and more sequence data from non-culturable organisms becomes available. Some known difficulties in this approach have been documented, including variation in the purine content of terminal fragments (Kaplan and Kitts, 2003) and differences in the mobility of fragments labelled with different fluorescent labels which can lead to sizing inaccuracies of up to 7 bp (Marsh, 2005). This approach was used to try to gain potential phylogenetic placements for peaks of interest without the expense of cloning and sequencing multiple clone libraries.

\subsubsection{Method}

Ten samples from each experiment (the two pig carcass experiments- Chapters 4 and 5, and the human cadaver experiment - Chapter 6) were chosen for analysis using multiple enzyme digestion. Samples were chosen because they had many clear peaks that were identifiable in a number of profiles or peaks that appeared only at certain times or in very few MspI T-RFLP profiles; covered all the decomposition carcasses/cadavers and some controls; and were collected from a wide range of timepoints (ADDs). A list was created of all the T-RFs present in all profiles, and the thirty samples which covered as many different T-RFs as possible were used. The samples chosen are listed in Table 7.17. For each sample, three separate T-RFLP analyses were performed using different restriction 
enzymes: AluI, HhaI and the previously used MspI (Table 7.16). The same protocol was used as in the initial digestions (section 3.4.3).

\begin{tabular}{|c|c|}
\hline Enzyme & Recognition site \\
\hline$M s p \mathrm{I}$ & $\mathrm{C}^{\wedge} \mathrm{CGG}$ \\
\hline$H h a \mathrm{I}$ & $\mathrm{GCG}^{\wedge} \mathrm{C}$ \\
\hline AluI & $\mathrm{AG}^{\wedge} \mathrm{CT}$ \\
\hline
\end{tabular}

Table 7.16 Restriction enzymes used and the sequences they recognise.

\begin{tabular}{|c|c|c|c|c|c|}
\hline \multicolumn{2}{|c|}{ First Pig Experiment } & \multicolumn{2}{c|}{ Second Pig Experiment } & \multicolumn{2}{c|}{ Human Cadaver Experiment } \\
\hline Sample & $A D D$ & Sample & $A D D$ & Sample & $A D D$ \\
\hline A9 (insects) & 148 & G9 (soil) & 127 & P16 (soil, non-autopsied) & 420 \\
A18 (insects) & 293 & G35 (soil) & 407 & P31 (soil, non-autopsied) & 730 \\
A32 (insects) & 521 & G48 (soil) & 542 & R18 (soil, non-autopsied) & 366 \\
B40 (no insects) & 637 & H11 (soil) & 158 & T1 (sand, autopsied) & 50 \\
C5 (insects) & 76 & H56 (soil) & 598 & T62 (sand, autopsied) & 1089 \\
D28 (no insects) & 465 & I15 (sand) & 208 & X15 (soil, autopsied) & 300 \\
D34 (no insects) & 550 & I33 (sand) & 384 & V22 (sand, non-autopsied) & 431 \\
D134 (no insects) & 1584 & J7 (sand) & 101 & V46 (sand, non-autopsied) & 731 \\
E46 (insects) & 712 & J56 (sand) & 598 & Z11 (sand, autopsied) & 216 \\
F16 (no insects) & 259 & K23 (soil control) & 297 & O42 (soil control) & 927 \\
\hline
\end{tabular}

Table 7.17 Samples chosen for multi-digestion T-RFLP analysis.

Virtual digests were performed using the Microbial Community Analysis (MiCA) 3 website (http://mica.ibest.uidaho.edu/news.php) to give a list of the theoretical peak sizes that every organism in the database would give if digested with each enzyme. This required the primers and enzyme used to be input and the database required to be chosen 
(Shyu et al., 2007). The Ribosomal Database Project II Release 9 Update 60 was used. This had 511,847 sequences within it at the time. An option to use only sequences $>1200$ was available but was not used in this case, so as to include as many potential identifications as possible. The virtual digest function allowed the user to set the sensitivity, allowing 0 to 10 mismatches within 0 to 15 bases from the $5^{\prime}$ end of the primer. The sensitivity was set at 4 and 7 for these analyses. From the 511,847 database entries, 92,551 entries contained both of these primer sequences.

A perl script (Appendix C4) was used to compare the T-RFLP data with predicted peak sizes in the virtual digest. The T-RFLP data from all three enzyme digests of each sample were combined to narrow down potential candidate species. The perl script searched each virtual digest dataset for entries which gave T-RFs of the size seen in the T-RFLP profiles. Entries which were identified in this way in all three virtual digest datasets were reported. The script allowed the user to set the tolerance for data matching. This was done because observed length and predicted length do not always match. Other investigators have reported that predicted T-RFs were anywhere from 0 to $7 \mathrm{bp}$ larger than observed lengths (Kitts 2001). The datasets were initially analysed in their entirety using all three enzyme data sets at a tolerance level of $\pm 1 \mathrm{bp}$. This resulted in a mean number of potential identifications of 1130 per sample. This number was greatly increased if the tolerance for data matching was increased. To reduce this number to a manageable level for interpretation, the analyses were run again using only T-RFs over $100 \mathrm{bp}$. This reduced the number of potential identifications per sample to between 600 and 3000 which was still too high to be manageable. The analyses were finally run using only the ten highest T-RFs in each profile. Results using tolerance levels from \pm 1 bp to \pm 7 bp were examined, and a level of $\pm 4 \mathrm{bp}$ using only the ten highest T-RFs was chosen. This level gave a mean number of matches of 180 per sample. 


\subsubsection{Results}

Results were collated from the perl script matches by recording all matches to known, classified organisms at the genus level. The majority of "hits" or closest matches were to uncultured organisms such as AB089046 uncultured Clostridiaceae bacterium Rs-C61 and a high proportion were to completely un-classified organisms, although the source was often included, such as AY493928 uncultured soil bacterium 537.

For each of the hits that gave classification as far as genus level, higher classification level data were obtained using the Ribosomal database. Many hits were not classified as far as genus level, but most of these tended to cluster around a few known organisms. For example, many Clostridiaceae bacterium hits would be accompanied by the more detailed Clostridium perfringens, Clostridium histolyticum, and Clostridium botulinum hits, so these are likely to be represented in the possible organism lists. The aim of this experiment was to identify potential bacterial groups that may have contributed to the T-RFs seen in the community T-RFLP profiles. While "uncultured Proteobacterium S134" may be a potential candidate, including such hits with little associated classification information did not contribute to meeting the aim.

The lists of potential matches for each of the ten T-RFs for each sample were combined and collated into peak size ranges. For example, the organism list for T-RFs of sizes 101 bp to $103 \mathrm{bp}$ from all of the samples were combined, as many samples contained T-RFs ranging from 100.8 to $103.3 \mathrm{bp}$. This led to $43 \mathrm{~T}-\mathrm{RF}$ size ranges that were investigated. These are listed in Table 7.18. 


\begin{tabular}{|c|c|c|}
\hline $\begin{array}{c}\text { T-RF Size } \\
\text { range }\end{array}$ & T-RF sizes & $\begin{array}{c}\text { Figure containing } \\
\text { results }\end{array}$ \\
\hline $50-200$ & $\begin{array}{c}50,101-103,105,107-113,116 / 117,121-123,125-132,141- \\
143,148 / 149,153,157,162,164,166,173,183,188-191\end{array}$ & Table 7.20 \\
\hline $200-300$ & $226-228,241,255-258,260-261,263-264,267-269,273,276$, & Table 7.21 \\
& $281,287,296$ & Table 7.22 \\
\hline $300-500$ & $364-366,396-399,403,418,420,423-425,435,445-451,453-$ \\
\hline
\end{tabular}

Table 7.18 The ten highest T-RFs in each of the 30 T-RFLP profiles chosen were combined to give a total of 46 T-RF size ranges.

A conservative 365 genera were identified as potential contributors to the 43 different TRF sizes. This translated as 121 different families, 55 orders, 25 classes and 17 phyla (Table 7.19). The orders identified as potential contributors to each T-RF size are shown in Tables 7.20, 7.21 and 7.22. 


\begin{tabular}{|c|c|}
\hline $\begin{array}{c}\text { Classification } \\
\text { Level }\end{array}$ & Potentially Contributing Organisms \\
\hline Phyla (16) & $\begin{array}{l}\text { Acidobacteria, Actinobacteria, Aquificae, Bacteroidetes, Chloroflexi, Cyanobacteria, Deferribacteres, Deinococcus-Thermus, Fibrobacteres, } \\
\text { Firmicutes, Fusobacteria, Nitrospira, Proteobacteria, Spirochaetes, Thermatogae, Thermodesulfobacteria, Verrucomicrobia. }\end{array}$ \\
\hline Classes (25) & $\begin{array}{c}\text { Acidobacteria, Actinobacteria, Alphaproteobacteria, Anaerolineae, Aquificae, Bacilli, Bacteroidetes, Betaproteobacteria, Chloroflexi, Clostridia, } \\
\text { Cyanobacteria, Deferribacteres, Deinococci, Deltaproteobacteria, Fibrobacteres, Fusobacteria, Gammaproteobacteria, Nitrospira, } \\
\text { Sphingobacteria, Spirochaetes, Thermodesulfobacteria, Thermatoga, Verrucomicrobia. }\end{array}$ \\
\hline Orders (55) & $\begin{array}{c}\text { Acidimicrobiales, Acidithiobacillales, Acidobacteriales, Actinomycetales, Aeromonadales, Alteromonadales, Aquificales, Bacillales, Bacteroidales, } \\
\text { Bifidobacteriales, Burkholderiales, Caldilineales, Caulobacterales, Chloroflexales, Chloroplast, Chromatiales, Clostridiales, Coriobacteriales, } \\
\text { Deferribacterales, Deinococcales, Desulfovibrionales, Desulfuromonales, Enterobacteriales, Fibrobacterales, Fusobacteriales, Halanaerobiales, } \\
\text { Hydrogenophilales, Kordiimonadales, Lactobacillales, Legionellales, Methylococcales, Neisseriales, Nitrospirales, Oceanospirillales, } \\
\text { Parvularculales, Pasteurellales, Pseudomonadales, Rhizobiales, Rhodobacterales, Rhodocyclales, Rhodospirillales, Rickettsiales, } \\
\text { Rubrobacterales, Salinisphaerales, Sphingobacteriales, Sphingomonadales, Spirochaetales, Syntrophobacterales, Thermoanaerobacterales, } \\
\text { Thermodesulfobacteriales, Thermotogales, Thiotrichales, Verrucomicrobiales, Vibrionales, Xanthomonadales }\end{array}$ \\
\hline Families (121) & 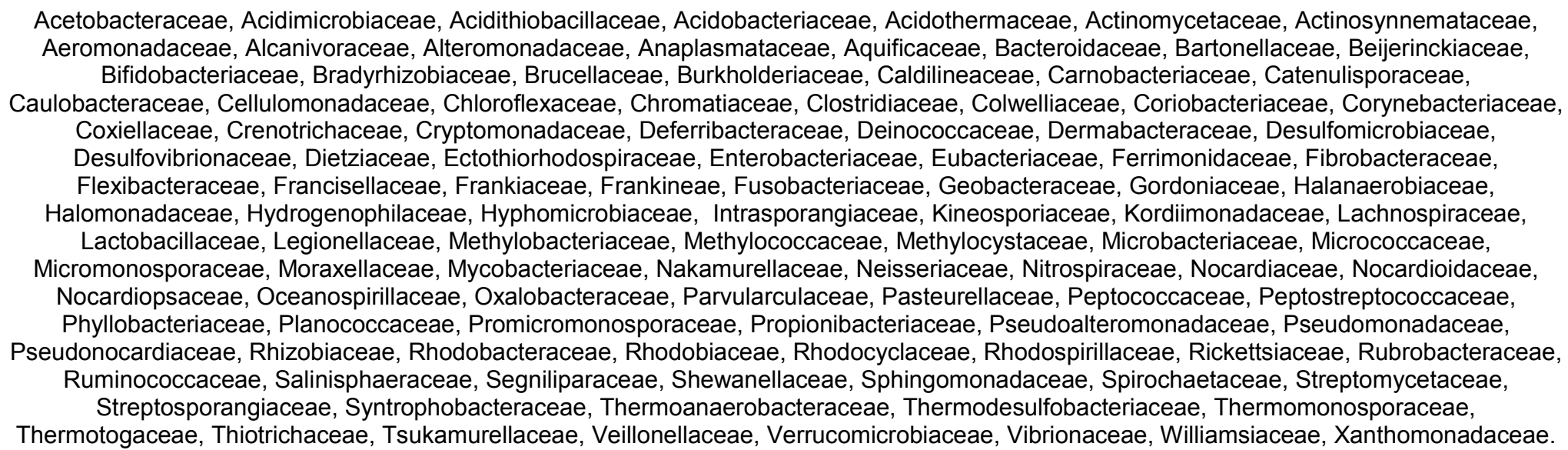 \\
\hline Genera (365) & See Appendix D2 \\
\hline
\end{tabular}

Table 7.19 Organisms identified as potentially contributing to the selected T-RFs, reported at phylum, class, order, and family levels. 


\begin{tabular}{|c|c|}
\hline T-RF size & Potentially Contributing Bacterial Orders \\
\hline 50 & $\begin{array}{c}\text { Actinomycetales, Actinomycetales, Bacteroidales, Clostridiales, Enterobacteriales, Nitrospirales, } \\
\text { Oceanospirillales, Pseudomonadales, Rhizobiales, Xanthomonadales }\end{array}$ \\
\hline $101-103$ & $\begin{array}{l}\text { Acidimicrobiales, Acidithiobacillales, Acidobacteriales, Actinomycetales, Alteromonadales, } \\
\text { Bifidobacteriales, Burkholderiales, Chloroflexale, Chromatiales, Clostridiales, Coriobacteriales, } \\
\text { Cyanobacteria, Methylococcales, Oceanospirillales, Pseudomonadales, Rhizobiales, } \\
\text { Rubrobacterales, Sphingobacteriales, Spirochaetales, Thermoanaerobacterales, Thiotrichales }\end{array}$ \\
\hline 105 & $\begin{array}{c}\text { Actinomycetales, Alteromonadales, Burkholderiales Clostridiales, Methylococcales, } \\
\text { Oceanospirillales, Pseudomonadales, Salinisphaerales Sphingobacteriales, } \\
\text { Thermoanaerobacterales }\end{array}$ \\
\hline $107-113$ & $\begin{array}{c}\text { Acidimicrobiales, Acidobacteriales, Actinomycetales, Alteromonadales, Burkholderiales, } \\
\text { Caulobacterales, Chloroflexale, Chromatiales, Clostridiales, Coriobacteriales, Cyanobacteria, } \\
\text { Deinococcales, Desulfovibrionales, Fibrobacterales, Fusobacteriales, Methylococcales, } \\
\text { Nitrospirales, Oceanospirillales, Pseudomonadales, Rhizobiales, Rhodobacterales, } \\
\text { Rhodospirillales, Rubrobacterales, Sphingobacteriales, Sphingomonadales, Spirochaetales, } \\
\text { Thermoanaerobacterales, Thermotogales, unclassified Clostridiales, unclassified } \\
\text { Cyanobacteria, Verrucomicrobiales }\end{array}$ \\
\hline $116 / 117$ & $\begin{array}{c}\text { Acidimicrobiales, Acidobacteriales, Actinomycetales, Alteromonadales, Caulobacterales, } \\
\text { Clostridiales, Cyanobacteria, Desulfovibrionales, Fusobacteriales, Nitrospirales, } \\
\text { Oceanospirillales, Pseudomonadales, Rhizobiales, Rhodobacterales, unclassified Clostridiales, } \\
\text { unclassified Cyanobacteria, Verrucomicrobiales, Xanthomonadales }\end{array}$ \\
\hline $121-123$ & $\begin{array}{c}\text { Actinomycetales, Actinomycetales, Aquificales, Clostridiales, Cyanobacteria, Deferribacterales, } \\
\text { Desulfovibrionales, Fusobacteriales, Pseudomonadales, Rhizobiales, Rhodospirillales, } \\
\text { Rubrobacterales, Thermoanaerobacterales, Thermodesulfobacteriales, Xanthomonadales }\end{array}$ \\
\hline $125-132$ & $\begin{array}{c}\text { Acidobacteriales, Actinomycetales, Clostridiales, Coriobacteriales, Deferribacterales, } \\
\text { Desulfovibrionales, Fusobacteriales, Lactobacillales, Legionellales, Pseudomonadales, } \\
\text { Rhizobiales, Rubrobacterales, Syntrophobacterales, Thermoanaerobacterales, } \\
\text { Xanthomonadales }\end{array}$ \\
\hline $141-143$ & $\begin{array}{c}\text { Acidobacteriales, Actinomycetales, Bacteroidales, Bifidobacteriales, Cyanobacteria, } \\
\text { Desulfuromonales, Nitrospirales, Xanthomonadales }\end{array}$ \\
\hline $148-149$ & Acidobacteriales, Actinomycetales, Clostridiales, Halanaerobiales, Rhizobiales, Vibrionales \\
\hline 153 & Acidobacteriales, Actinomycetales, Clostridiales \\
\hline 157 & Acidobacteriales, Caulobacterales, Pasteurellales, Pseudomonadales, Rhizobiales \\
\hline 162 & Acidobacteriales, Actinomycetales, Pseudomonadales \\
\hline 164 & Acidobacteriales, Actinomycetales, Alteromonadales, Clostridiales, Nitrospirales \\
\hline 166 & Acidobacteriales, Actinomycetales, Alteromonadales, Caldilineales, Clostridiales, Nitrospirales \\
\hline 173 & $\begin{array}{l}\text { Acidobacteriales, Actinomycetales, Alteromonadales, Caldilineales, Chloroflexales, } \\
\text { Clostridiales, Pasteurellales, Thermoanaerobacterales }\end{array}$ \\
\hline 183 & Acidobacteriales \\
\hline 188-191 & $\begin{array}{c}\text { Acidobacteriales, Bacteroidales, Burkholderiales, Clostridiales, Thiotrichales, unclassified } \\
\text { Oceanospirillales }\end{array}$ \\
\hline
\end{tabular}

Table 7.20 Bacterial orders that were found to be potential contributors to the T-RFS

$$
\text { of between } 50 \text { and } 200 \mathrm{bp} .
$$




\begin{tabular}{|c|c|}
\hline T-RF size & Potentially Contributing Bacterial Orders \\
\hline $226-228$ & Acidobacteriales \\
\hline 241 & Acidobacteriales, Actinomycetales, Clostridiales, Coriobacteriales, Fusobacteriales \\
\hline $255-258$ & Acidobacteriales, Acidobacteriales, Actinomycetales, Aquificales, Clostridiales, \\
Rhizobiales
\end{tabular}

Table 7.21 Bacterial orders that were found to be potential contributors to the T-RFs of between 200 and $300 \mathrm{bp}$.

A number of the phyla identified as potential contributors can practically be ruled out due to the low likelihood of them being present in the soil decomposition environment. Those phyla unlikely to be present are: the Thermatogae (strictly anaerobic thermophiles); the Aquificae (extremophiles); the Chloroflexi (anoxygenic phototrophs); the Thermodesulfobacteria (thermophilic sulphate-reducers); and the Deinococcus-Thermus (polyextremophiles).

Members of the remaining phyla (Acidobacteria, Actinobacteria, Bacteroidetes, Cyanobacteria, Deferribacteres, Firmicutes, Fusobacteria, Nitrospira, Proteobacteria, Spirochaetes and Verrucomicrobia) are more likely contributors to the T-RFs seen in this study because they are usually found in similar or contributing environments, such as soil or mammalian gastrointestinal tracts (Wilson, 2005; Treusch and Schleper, 2006). 


\begin{tabular}{|c|c|}
\hline T-RF Size & Potentially Contributing Bacterial Orders \\
\hline $364-366$ & $\begin{array}{c}\text { Burkholderiales, Caulobacterales, Chromatiales, Oceanospirillales, Parvularculales, } \\
\text { Rhizobiales, Rhodobacterales, Rhodocyclales, Vibrionales }\end{array}$ \\
\hline 396-399 & $\begin{array}{c}\text { Bacteroidales, Burkholderiales, Caulobacterales, Pseudomonadales, Rhizobiales, } \\
\text { Rhodobacterales, Rhodospirillales, Xanthomonadales }\end{array}$ \\
\hline 403 & Caulobacterales, Clostridiales, Rhizobiales, Rhodobacterales, Rhodospirillales \\
\hline 418 & Kordiimonadales, Nitrospirales, Rhodospirillales, Rickettsiales, Thiotrichales \\
\hline 420 & $\begin{array}{c}\text { Clostridiales, Kordiimonadales, Nitrospirales, Pseudomonadales, Rhodospirillales, } \\
\text { Rickettsiales, Thiotrichales }\end{array}$ \\
\hline $423-425$ & Clostridiales, Cyanobacteria, Methylococcales, Pseudomonadales, Xanthomonadales \\
\hline 435 & Actinomycetales, Aquificales, Chloroflexales, Pseudomonadales \\
\hline $445-451$ & $\begin{array}{l}\text { Acidithiobacillales, Acidobacteriales, Actinomycetales, Alteromonadales, Bacteroidales, } \\
\text { Burkholderiales, Chloroplast, Chromatiales, Clostridiales, Cyanobacteria, Enterobacteriales, } \\
\text { Fusobacteriales, Hydrogenophilales, Methylococcales, Pseudomonadales, Rhizobiales, } \\
\text { Sphingobacteriales, Thiotrichales, unclassified Oceanospirillales, Xanthomonadales }\end{array}$ \\
\hline $453-456$ & $\begin{array}{l}\text { Acidithiobacillales, Actinomycetales, Alteromonadales, Bacteroidales, Burkholderiales, } \\
\text { Chloroplast, Chromatiales, Clostridiales, Cyanobacteria, Enterobacteriales, } \\
\text { Hydrogenophilales, Legionellales, Methylococcales, Neisseriales, Pasteurellales, } \\
\text { Pseudomonadales, Rhizobiales, Sphingobacteriales, Thiotrichales, unclassified } \\
\text { Cyanobacteria, unclassified Oceanospirillales, Vibrionales, Xanthomonadales }\end{array}$ \\
\hline $457-463$ & $\begin{array}{c}\text { Acidithiobacillales, Aeromonadales, Alteromonadales, Bacteroidales, Burkholderiales, } \\
\text { Chloroplast, Chromatiales, Clostridiales, Cyanobacteria, Deferribacterales, } \\
\text { Desulfovibrionales, Enterobacteriales, Hydrogenophilales, Legionellales, Methylococcales, } \\
\text { Neisseriales, Oceanospirillales, Pasteurellales, Pseudomonadales, Rhizobiales, } \\
\text { Sphingobacteriales, Thermotogales, Thiotrichales, unclassified Cyanobacteria, Vibrionales, } \\
\text { Xanthomonadales }\end{array}$ \\
\hline 467-469 & $\begin{array}{c}\text { Actinomycetales, Alteromonadales, Chloroplast, Clostridiales, Deferribacterales, } \\
\text { Desulfovibrionales, Enterobacteriales, Legionellales, Pasteurellales, Sphingobacteriales, } \\
\text { Thermotogales, unclassified Oceanospirillales, Vibrionales }\end{array}$ \\
\hline $474-475$ & $\begin{array}{l}\text { Alteromonadales, Alteromonadales, Clostridiales, Enterobacteriales, Legionellales, } \\
\text { Pasteurellales, Vibrionales }\end{array}$ \\
\hline $479-481$ & Chloroflexales, Clostridiales, Enterobacteriales, Lactobacillales, Legionellales \\
\hline $483-486$ & Chloroflexales, Clostridiales, Enterobacteriales \\
\hline $495-500$ & Chloroflexales, Clostridiales, Chloroplast, Pseudomonadales, Vibrionales \\
\hline
\end{tabular}

Table 7.22 Bacterial orders that were found to be potential contributors to the T-RFs

of between 300 and 552 bp.

The lists of genera potentially contributing to particular T-RF sizes were also examined according to the substrate they came from (Table 7.23); from pig carcasses or human 
cadavers (Table 7.24); from decomposition events on soil or soil control samples (Table 7.25); whether pig carcasses were exposed to insect colonisation or not (Table 7.26); and whether cadavers were autopsied or not (Table 7.27). Orders present in one group but not in the corresponding group are bolded to highlight the differences.

\begin{tabular}{|c|c|}
\hline Treatment & Potentially Contributing Bacterial Orders \\
\hline \multirow{6}{*}{ Soil } & Acidimicrobiales, Acidithiobacillales, Acidobacteriales, Actinomycetales, \\
& Aeromonadales, Alteromonadales, Aquificales, Bacteroidales, Bifidobacteriales, \\
& Burkholderiales, Caldilineales, Caulobacterales, Chloroflexales, Chloroplast, \\
& Chromatiales, Clostridiales, Coriobacteriales, Cyanobacteria, Deferribacterales, \\
Deinococcales, Desulfovibrionales, Enterobacteriales, Fusobacteriales, \\
Hydrogenophilales, Lactobacillales, Legionellales, Methylococcales, Neisseriales, \\
Nitrospirales, Oceanospirillales, Parvularculales, Pasteurellales, Pseudomonadales, \\
Rhizobiales, Rhodobacterales, Rhodocyclales, Rhodospirillales, Rickettsiales, \\
Rubrobacterales, Sphingobacteriales, Sphingomonadales, Spirochaetales, \\
Thermoanaerobacterales, Thermodesulfobacteriales, Thermotogales, \\
Thiotrichales, unclassified Clostridia, unclassified Cyanobacteria, unclassified \\
Oceanospirillales, Verrucomicrobiales, Vibrionales, Xanthomonadales \\
\hline Acidimicrobiales, Acidobacteriales, Actinomycetales, Aeromonadales, \\
Alteromonadales, Aquificales, Bacillales, Bacteroidales, Bifidobacteriales, \\
Burkholderiales, Caldilineales, Caulobacterales, Chloroflexales, Chloroplast, \\
Chromatiales, Clostridiales, Coriobacteriales, Cyanobacteria, Deferribacterales, \\
Deinococcales, Desulfovibrionales, Enterobacteriales, Fusobacteriales, \\
Halanaerobiales, Hydrogenophilales, Kordiimonadales, Lactobacillales, \\
Legionellales, Methylococcales, Nitrospirales, Oceanospirillales, Parvularculales, \\
Pasteurellales, Pseudomonadales, Rhizobiales, Rhodobacterales, Rhodocyclales, \\
Rhodospirillales, Rickettsiales, Rubrobacterales, Salinisphaerales, \\
Sphingobacteriales, Sphingomonadales, Spirochaetales, Syntrophobacterales, \\
Thermoanaerobacterales, Thermotogales, Thiotrichales, unclassified Clostridia, \\
unclassified Cyanobacteria, unclassified Oceanospirillales, Verrucomicrobiales, \\
Vibrionales, Xanthomonadales
\end{tabular}

Table 7.23 Bacterial orders that were found to be potential contributors to the T-RFs from samples under pig carcasses and cadavers on soil and sand. 


\begin{tabular}{|c|c|}
\hline Treatment & Potentially Contributing Bacterial Orders \\
\hline Humans & $\begin{array}{c}\text { Acidimicrobiales, Acidithiobacillales, Acidobacteriales, Actinomycetales, } \\
\text { Alteromonadales, Aquificales, Bifidobacteriales, Burkholderiales, Caldilineales, } \\
\text { Caulobacterales, Chloroflexales, Chloroplast, Chromatiales, Clostridiales, } \\
\text { Coriobacteriales, Cyanobacteria, Deferribacterales, Desulfovibrionales, } \\
\text { Enterobacteriales, Fusobacteriales, Hydrogenophilales, Kordiimonadales, } \\
\text { Lactobacillales, Legionellales, Methylococcales, Neisseriales, Nitrospirales, } \\
\text { Oceanospirillales, Parvularculales, Pasteurellales, Pseudomonadales, Rhizobiales, } \\
\text { Rhodobacterales, Rhodocyclales, Rhodospirillales, Rickettsiales, Sphingobacteriales, } \\
\text { Spirochaetales, Syntrophobacterales, Thermoanaerobacterales, Thiotrichales, } \\
\text { Thermodesulfobacteriales, Thermotogales, Thiotrichales, unclassified Clostridia, } \\
\text { unclassified Cyanobacteria, unclassified Oceanospirillales, Verrucomicrobiales, } \\
\text { Vibrionales, Xanthomonadales }\end{array}$ \\
\hline Pigs & $\begin{array}{c}\text { Acidimicrobiales, Acidithiobacillales, Acidobacteriales, Actinomycetales, } \\
\text { Aeromonadales, Alteromonadales, Bacillales, Bacteroidales, Bifidobacteriales, } \\
\text { Burkholderiales, Caldilineales, Caulobacterales, Chloroflexales, Chloroplast, } \\
\text { Chromatiales, Clostridiales, Coriobacteriales, Cyanobacteria, Deferribacterales, } \\
\text { Deinococcales, Desulfovibrionales, Desulfuromonales, Enterobacteriales, } \\
\text { Fusobacteriales, Halanaerobiales, Hydrogenophilales, Lactobacillales, Legionellales, } \\
\text { Methylococcales, Neisseriales, Nitrospirales, Oceanospirillales, Parvularculales, } \\
\text { Pasteurellales, Pseudomonadales, Rhizobiales, Rhodobacterales, Rhodocyclales, } \\
\text { Rhodospirillales, Rickettsiales, Rubrobacterales, Salinisphaerales, } \\
\text { Sphingobacteriales, Sphingomonadales, Spirochaetales, Thermoanaerobacterales, } \\
\text { Thermotogales, Thiotrichales, unclassified Clostridia, unclassified Cyanobacteria, } \\
\text { unclassified Oceanospirillales, Verrucomicrobiales, Vibrionales, Xanthomonadales }\end{array}$ \\
\hline
\end{tabular}

Table 7.24 Bacterial orders that were found to be potential contributors to the T-RFs from samples under human cadavers and pig carcasses.

\begin{tabular}{|c|c|}
\hline Treatment & Potentially Contributing Bacterial Orders \\
\hline Soil Controls & $\begin{array}{l}\text { Acidimicrobiales, Acidithiobacillales, Acidobacteriales, Actinomycetales, } \\
\text { Alteromonadales, Bifidobacteriales, Burkholderiales, Caldilineales, } \\
\text { Caulobacterales, Chloroflexales, Chloroplast, Chromatiales, Clostridiales, } \\
\text { Coriobacteriales, Cyanobacteria, Deferribacterales, Deinococcales, } \\
\text { Desulfovibrionales, Enterobacteriales, Fibrobacterales, Fusobacteriales, } \\
\text { Hydrogenophilales, Legionellales, Methylococcales, Oceanospirillales, } \\
\text { Pasteurellales, Pseudomonadales, Rhizobiales, Rhodobacterales, } \\
\text { Rhodospirillales, Rubrobacterales, Sphingobacteriales, Sphingomonadales, } \\
\text { Spirochaetales, Thermoanaerobacterales, Thermotogales, Thiotrichales, } \\
\text { unclassified Cyanobacteria, unclassified Oceanospirillales, Vibrionales }\end{array}$ \\
\hline Soil Decomposition & $\begin{array}{c}\text { Acidimicrobiales, Acidithiobacillales, Acidobacteriales, Actinomycetales, } \\
\text { Aeromonadales, Alteromonadales, Aquificales, Bacteroidales, } \\
\text { Bifidobacteriales, Burkholderiales, Caldilineales, Caulobacterales, } \\
\text { Chloroflexales, Chloroplast, Chromatiales, Clostridiales, Coriobacteriales, } \\
\text { Cyanobacteria, Deferribacterales, Deinococcales, Desulfovibrionales, } \\
\text { Enterobacteriales, Fusobacteriales, Hydrogenophilales, Lactobacillales, } \\
\text { Legionellales, Methylococcales, Neisseriales, Nitrospirales, Oceanospirillales, } \\
\text { Parvularculales, Pasteurellales, Pseudomonadales, Rhizobiales, } \\
\text { Rhodobacterales, Rhodocyclales, Rhodospirillales, Rickettsiales, } \\
\text { Rubrobacterales, Sphingobacteriales, Sphingomonadales, Spirochaetales, } \\
\text { Thermoanaerobacterales, Thermodesulfobacteriales, Thermotogales, } \\
\text { Thiotrichales, unclassified Clostridia, unclassified Cyanobacteria, unclassified } \\
\text { Oceanospirillales, Verrucomicrobiales, Vibrionales, Xanthomonadales }\end{array}$ \\
\hline
\end{tabular}

Table 7.25 Bacterial orders that were found to be potential contributors to the T-RFs from samples under cadavers on soil and from soil control samples. 


\begin{tabular}{|c|c|}
\hline Treatment & Potentially Contributing Bacterial Orders \\
\hline Insects & $\begin{array}{l}\text { Acidimicrobiales, Acidobacteriales, Actinomycetales Alteromonadales, Bacillales, } \\
\text { Bacteroidales, Bifidobacteriales, Burkholderiales, Caulobacterales, Chloroflexales, } \\
\text { Chloroplast, Chromatiales, Clostridiales, Coriobacteriales, Cyanobacteria, } \\
\text { Deferribacterales, Desulfovibrionales, Desulfuromonales, Enterobacteriales, } \\
\text { Fusobacteriales, Legionellales, Methylococcales, Nitrospirales, Oceanospirillales, } \\
\text { Pasteurellales, Pseudomonadales, Rhizobiales, Rhodobacterales, Rubrobacterales, } \\
\text { Salinisphaerales, Sphingobacteriales, Thermoanaerobacterales, Thermotogales, } \\
\text { Thiotrichales, unclassified Clostridia, unclassified Cyanobacteria, unclassified } \\
\text { Oceanospirillales, Verrucomicrobiales, Vibrionales, Xanthomonadales }\end{array}$ \\
\hline No insects & $\begin{array}{l}\text { Acidithiobacillales, Acidobacteriales, Actinomycetales, Alteromonadales, Bacillales, } \\
\text { Bacteroidales, Burkholderiales, Caulobacterales, Chromatiales, Clostridiales, } \\
\text { Coriobacteriales, Cyanobacteria, Deinococcales, Desulfovibrionales, } \\
\text { Enterobacteriales, Fusobacteriales, Halanaerobiales, Hydrogenophilales, } \\
\text { Lactobacillales, Legionellales, Methylococcales, Nitrospirales, Oceanospirillales, } \\
\text { Parvularculales, Pseudomonadales, Rhizobiales, Rhodobacterales, Rhodocyclales, } \\
\text { Rubrobacterales, Salinisphaerales, Sphingobacteriales, Sphingomonadales, } \\
\text { Thermoanaerobacterales, Thiotrichales, unclassified Cyanobacteria, unclassified } \\
\text { Oceanospirillales, Verrucomicrobiales, Vibrionales, Xanthomonadales }\end{array}$ \\
\hline
\end{tabular}

Table 7.26 Bacterial orders that were found to be potential contributors to the T-RFs from samples under pig carcasses on sand and protected from or allowed to be colonised by insects.

\begin{tabular}{|c|c|}
\hline Treatment & Potentially Contributing Bacterial Orders \\
\hline \multirow{4}{*}{ Autopsied } & Acidimicrobiales, Acidithiobacillales, Acidobacteriales, Actinomycetales, \\
Alteromonadales, Aquificales, Bifidobacteriales, Burkholderiales, \\
Caldilineales, Caulobacterales, Chloroflexales, Chloroplast, Chromatiales, \\
Clostridiales, Coriobacteriales, Cyanobacteria, Deferribacterales, \\
Desulfovibrionales, Enterobacteriales, Fusobacteriales, Hydrogenophilales, \\
Kordiimonadales, Lactobacillales, Legionellales, Methylococcales, \\
Nitrospirales, Oceanospirillales, Pasteurellales, Pseudomonadales, \\
Rhizobiales, Rhodobacterales, Rhodospirillales, Rickettsiales, \\
Sphingobacteriales, Spirochaetales, Thermoanaerobacterales, \\
Thermodesulfobacteriales, Thiotrichales, unclassified Clostridia, \\
unclassified Cyanobacteria, unclassified Oceanospirillales, \\
Verrucomicrobiales, Vibrionales, Xanthomonadales
\end{tabular}

Table 7.27 Bacterial orders that were found to be potential contributors to the T-RFs from samples from under autopsied and non-autopsied cadavers. 
A few orders featured very strongly in the potential contributing organism lists. The Clostridiales, Pseudomonadales, Actinomycetales, Xanthomonadales and Rhizobiales all featured strongly for many T-RF sizes and in most samples. Of the $43 \mathrm{~T}-\mathrm{RF}$ size ranges, 29 had Clostridiales identified as potential contributors, 19 contained Pseudomonadales, 23 included Actinomycetales, 12 contained Xanthomonadales and 15 included Rhizobiales. There were many hits at each of these sizes to non-cultured or non-classified members of these groups, suggesting that they may be likely candidates for contributing many of the TRFs.

Xanthomonadales were identified as potential contributors in all insect associated decomposition plots, both soil and sand, for T-RFs of 12 different sizes. They were not, however, identified at potential contributors to any of the sizes in either of the soil control samples and were only identified as potential contributors in one of the three plots excluding insects.

The Ribosomal Database entries, in many cases, contain information about where the sequence was isolated from. In browsing through the potentially contributing organism lists, a noticeable number of the uncultured organisms of many different orders were sequenced from soil samples, rumen contents, sewage, anaerobic swine lagoons, faecal samples or were known human microflora. The summarised lists of potentially contributing orders did not show great differences between the different variables, e.g. the soil control and soil decomposition plots had largely the same organisms listed as potential contributors (Table 7.25). Differences were noticeable for some variables when the full lists of organisms were browsed for the source of the sequences. The vast majority of the soil control sample potential contributors, particularly the unclassified, uncultured organisms were derived from soil samples. Similarly, the decomposition plot sample list contained many organisms from rumen, sewage and faecal origins. 


\subsubsection{Discussion}

This technique for identifying organisms that could potentially be contributing to T-RFs in T-RFLP profiles is useful to give an idea of the families or orders of bacteria that are likely to be contributing. Because of the shortcomings of the database and of the T-RFLP method itself, it is unlikely that potential identification can be narrowed down to one or a few candidate species, unless multiple combinations of enzymes are used (rather than just three enzymes in separate digestions). Simplifying the profiles by comparing only the most dominant peaks also narrows the possible candidate species down, but can also eliminate realistic candidates. Results can also be influenced greatly by the sensitivity and tolerance levels set during the analysis. In this experiment, a less stringent approach was taken to try to identify the most likely contributing orders rather than trying to narrow identifications down to genera or species level.

There seemed to be some indication that the Xanthomonadales may be linked with the presence of insects. No species from this order were identified as potential contributors to the soil control sample T-RF sizes, and a single species was identified as a potential contributor to one T-RF size in an insect-free plot. The genera identified were Dokdonella, Dyella, Frateuria, Hydrocarboniphaga, Lysobacter, Pseudoxanthomonas, Rhodanobacter, Schineria, Steroidobacter, Xanthomonas, and Xylella. The Xanthomonadales group contains a number of pathogenic plant species that are transmitted via insects (Hopkins, 1989) and also a couple of genera, including Schineria that are associated with insect larvae. This suggests that the Xanthomonadales, if actually present within the bacterial community, may be contributed by insects.

The predictions of organisms likely to be contributing to particular T-RFs obtained using this multi-digestion restriction approach are compared with the actual contributing organisms identified from these samples using cloning and sequencing in section 7.5. 


\subsection{Cloning \& Sequencing}

Cloning and sequencing is a commonly employed technique for identification of species present within a mixed community (Zwolinski, 2007). Standard cloning and sequencing methods, however, are time consuming and costly and are not ideal for screening large quantities of samples.

\subsubsection{Aim}

The aim of this experiment was to evaluate a method which combines T-RFLP analysis with cloning and sequencing, and from the data generated, attempt to determine the organisms responsible for T-RFs seen in the earlier chapters and experiments.

\subsubsection{Method}

\subsubsection{Sample Selection}

Three timepoints were chosen for investigation: ADD 100, 200 and 500. The sample collected closest to these ADDs was selected from each set of decomposition samples. All three timepoints were investigated for the human cadaver experiment samples (Chapter 6) and the pig carcasses on soil and sand experiment samples (Chapter 5), but due to financial restrictions, only the ADD 100 was looked at from the pig carcasses on sand experiment (Chapter 4).

\subsubsection{Amplification of target DNA}

Fresh PCR product for each sample was generated using the genomic DNA extract as template. The primers F63 (5' AGG CCT AAC ACA TGC AAG TC 3') and R534 (5' ATT ACC GCG GCT GCT GG 3') were used with the PCR recipe and thermocycling regime as 
detailed in Tables 7.28 and 7.29. The reverse primer R534 was used because the primer pair used for T-RFLP profile generation (F63 and R1087) gave a product of $\sim 950 \mathrm{bp}$ which was too long for accurate sequencing. The Microbial Community Analysis (MiCA) 3 Primer Resolving Power tool (http://mica.ibest.uidaho.edu/primer.php) returned a resolving power of 151,981 for the primer pair F63/R534, out of a possible 511,847 sequences. Because many of the sequences within the database are short and do not necessarily cover both primer sites, this was considered to be reasonably good. This was also a higher resolving power than the T-RFLP F63/R1087 pair gave $(92,551)$.

\begin{tabular}{|c|c|}
\hline Component & Amount / Final Concentration \\
\hline Qiagen Taq Polymerase & $1.25 \mathrm{U}$ \\
PCR buffer & $1 \mathrm{x}$ \\
$\mathrm{MgCl}_{2}$ & $2 \mathrm{mM}$ \\
dNTPs & $200 \mu \mathrm{M}$ of each dNTP \\
F63 forward primer & $200 \mathrm{nM}$ \\
R534 reverse primer & $200 \mathrm{nM}$ \\
Template DNA & $20 \mathrm{ng}$ \\
$\mathrm{H}_{2} \mathrm{O}$ & To a total volume of $50 \mu \mathrm{L}$ \\
\hline
\end{tabular}

Table 7.28 PCR recipe for clone insert creation.

\begin{tabular}{|c|c|c|}
\hline & Time & Temperature \\
\hline Initial Denaturation & $3 \mathrm{~min}$ & $94^{\circ} \mathrm{C}$ \\
\hline 3 Step Cycling (x30) & & \\
Denaturation & $1 \mathrm{~min}$ & $94^{\circ} \mathrm{C}$ \\
Annealing & $45 \mathrm{sec}$ & $45^{\circ} \mathrm{C}$ \\
Extension & $1 \mathrm{~min}$ & $72^{\circ} \mathrm{C}$ \\
\hline Final Extension & $20 \mathrm{~min}$ & $72^{\circ} \mathrm{C}$ \\
\hline
\end{tabular}

Table 7.29 PCR thermocycling regime for clone insert creation. 


\subsubsection{Cloning}

Initially, the Invitrogen TOPO TA Cloning Kit for Sequencing was used as per the manufacturer's instructions. Control reactions were performed to test the transformation efficiency of the competent cells supplied with the kit. The TOP10 cells were not competent but viable, so an overnight culture of one vial $(50 \mu \mathrm{L})$ of cells was used to create new competent cells (Appendix A15). These cells routinely gave a transformation efficiency of $2 \times 10^{8}$. Because the TOPO TA Cloning Kit for Sequencing could only be purchased with competent cells included, a less expensive vector-only kit was purchased instead and used to generate all the clone libraries that had transformants sequenced. The Invitrogen TA Cloning Kit was used, which contains vector $\mathrm{pCR}^{{ }^{\circledR}} 2.1$ (Figure 7.17).

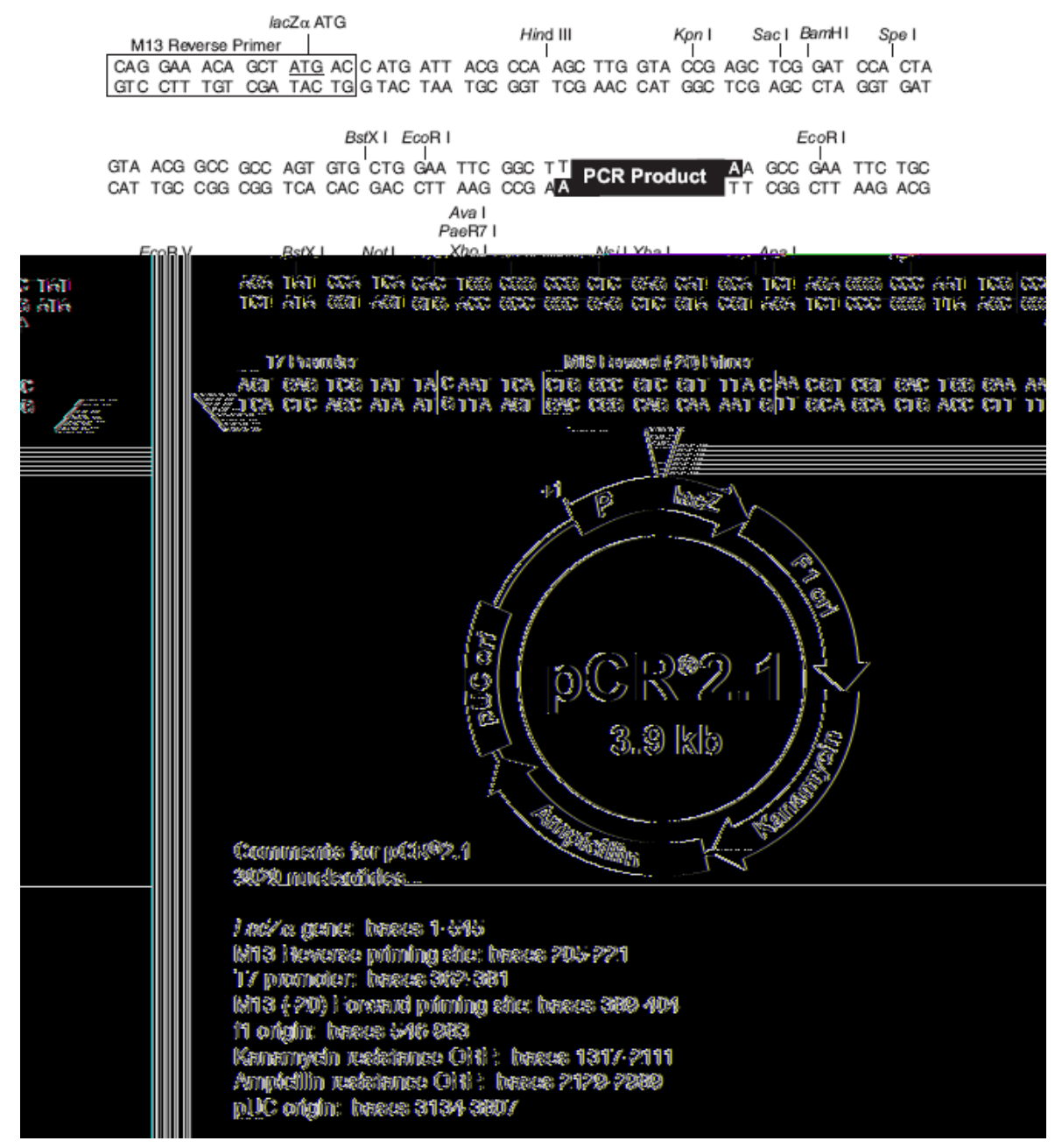

Figure 7.17 Map and details of vector pC $R^{\circledR} 2.1$ (from Invitrogen's TA Cloning Kit manual). 
Introducing the PCR product into the vector was performed as per the manufacturer's instructions. A vial of vector $\mathrm{pCR}^{\circledR} 2.1$ was centrifuged briefly to collect liquid at the bottom of the tube and the ligation reaction set up (Table 7.30) The volume of PCR product added varied depending on the concentration of DNA, but was usually 0.5 to $1 \mu \mathrm{L}$. A total of $6.4 \mathrm{ng}$ PCR products were added each time, as this gives the optimal 1:1 ratio of vector to insert. The reaction was incubated at $14^{\circ} \mathrm{C}$ overnight.

\begin{tabular}{|c|c|}
\hline COMPONENTS & VOLUME \\
\hline Fresh PCR product & $\mathrm{x} \mu \mathrm{L}$ \\
10x ligation buffer & $1 \mu \mathrm{L}$ \\
pCR2.1 vector $(25 \mathrm{ng} / \mu \mathrm{L})$ & $2 \mu \mathrm{L}$ \\
T4 DNA Ligase $(4.0$ Weiss units $)$ & $1 \mu \mathrm{L}$ \\
DNAse, RNase free $\mathrm{H}_{2} \mathrm{O}$ & To a total volume of $10 \mu \mathrm{L}$ \\
\hline
\end{tabular}

Table 7.30 Ligation reaction recipe.

A $2 \mu \mathrm{L}$ aliquot of the ligation reaction was transferred into a vial of competent E. coli and gently mixed. This was incubated on ice for 30 minutes. The reaction was then placed in a $42^{\circ} \mathrm{C}$ water bath for 30 seconds to heat-shock the cells, and the vial immediately transferred to ice. Room temperature LB broth $(250 \mu \mathrm{L})$ was added, the tube was tightly capped and shaken horizontally $(225 \mathrm{rpm})$ at $37^{\circ} \mathrm{C}$ for 1 hour. One $20 \mu \mathrm{L}$ aliquot, and one $50 \mu \mathrm{L}$ aliquot from each vial were spread on pre-warmed $\mathrm{LB}$ agar plates containing $1.6 \mathrm{mg}$ X-Gal and $100 \mu \mathrm{g} / \mathrm{mL}$ ampicillin. Because TOP10 E.coli do not contain the lac repressor, IPTG did not need to be added to the plates for blue/white screening. Plates were incubated overnight at $37^{\circ} \mathrm{C}$ and then shifted to $4^{\circ} \mathrm{C}$ for $2-3$ hours to allow colour development. 


\subsubsection{Screening of Clones}

To enable rapid and cost-effective screening of the clones, a version of the screening method described by Grant and Ogilvie (2004) was used.

Ninety-six colonies were randomly chosen from the clone library and patch-plated onto LB agar plates containing $100 \mu \mathrm{g} / \mathrm{mL}$ ampicillin and incubated overnight at $37^{\circ} \mathrm{C}$.

Growth from each colony was inoculated into one well of a deep-well 96-well plate containing $1 \mathrm{~mL}$ LB broth containing $100 \mu \mathrm{g} / \mathrm{mL}$ ampicillin. This was incubated overnight at $37^{\circ} \mathrm{C}$. For long-term storage of the clones, $500 \mu \mathrm{L}$ of overnight culture was mixed with $500 \mu \mathrm{L}$ of sterile $80 \%$ glycerol and frozen at $-80^{\circ} \mathrm{C}$.

Pooled samples were created for each row and column by taking $10 \mu \mathrm{L}$ from each well of the overnight culture plate and combining them in a separate strip of wells (see Figure 7.18).

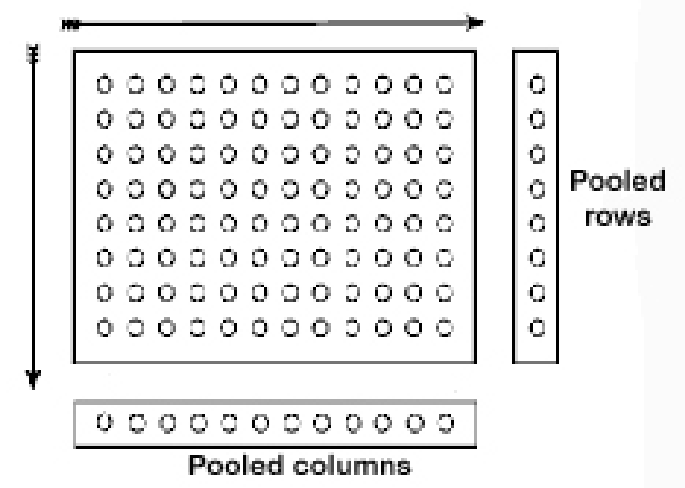

Figure 7.18 Pooled samples were created for each row and column by taking $10 \mu L$ from each well of the overnight culture plate and combining them in a separate strip of wells.

A PCR reaction was carried out using $3 \mu \mathrm{L}$ from each pooled sample as template, and the PCR recipe and protocol outlined in Tables 7.31 and 7.32. Primers M13 Forward (-20) (5' GTAAAACGACGGCCAG 3') and M13 Reverse (5' CAGGAAACAGCTATGAC 3') were used. Large scale amplification of Escherichia coli 16S DNA is avoided when 
priming sites on the vector are used. A DNA extraction step was not necessary as $E$. coli cells are lysed and plasmid inserts released within the first few cycles of the PCR.

\begin{tabular}{|c|c|}
\hline Component & Volume/Amount \\
\hline Qiagen Taq Polymerase & $1.25 \mathrm{U}$ \\
PCR buffer & $1 \mathrm{x}$ \\
$\mathrm{MgCl}_{2}$ & $2 \mathrm{mM}$ \\
dNTPs & $200 \mu \mathrm{M}$ of each dNTP \\
M13 Forward (-20) primer & $200 \mathrm{nM}$ \\
M13 Reverse primer & $200 \mathrm{nM}$ \\
Template DNA & $3 \mu \mathrm{L}$ pooled clones \\
DNase free $\mathrm{H}_{2} \mathrm{O}$ & To a total volume of $50 \mu \mathrm{L}$ \\
\hline
\end{tabular}

Table 7.31 Cloning PCR recipe.

\begin{tabular}{|c|c|c|}
\hline & Time & Temperature \\
\hline Initial Denaturation & $4 \mathrm{~min}$ & $94^{\circ} \mathrm{C}$ \\
\hline 3 Step Cycling (x30) & & \\
Denaturation & $1 \mathrm{~min}$ & $94^{\circ} \mathrm{C}$ \\
Annealing & $45 \mathrm{sec}$ & $45^{\circ} \mathrm{C}$ \\
Extension & $1.5 \mathrm{~min}$ & $72^{\circ} \mathrm{C}$ \\
\hline Final Extension & $20 \mathrm{~min}$ & $72^{\circ} \mathrm{C}$ \\
\hline
\end{tabular}

Table 7.32 PCR protocol for clone pool T-RFLP analysis.

The size and yield of the PCR products was determined by electrophoresis on a $2 \%$ agarose gel (Appendix A9). A $1 \mu \mathrm{L}$ sub-sample of the initial PCR product was used for a second round of PCR using fluorescently labelled FAM-F63 and HEX-R534 primers. The 
same PCR recipe (except the different primers) and thermocycling regime were used as in the previous step (Tables 7.31 and 7.32).

Amplification products were then purified using a QIAQuick PCR Purification Kit (Qiagen) and T-RFLP profiles were generated (section 3.4.3).

All T-RF peaks of 5000 fluorescence units or greater were included in the analysis, as the overall fluorescence of the pooled clone T-RFLP profiles were much higher than the profiles generated using individual samples. This could be because the pooled clone DNA mixture was much simpler, with a maximum of twelve different target sequences present. Including only peaks with fluorescence greater than 5000 units simplified the profiles to around the expected number of peaks ( 8 to 12$)$ per profile. These peaks were easily distinguishable by eye as having much greater fluorescence intensity than the background noise.

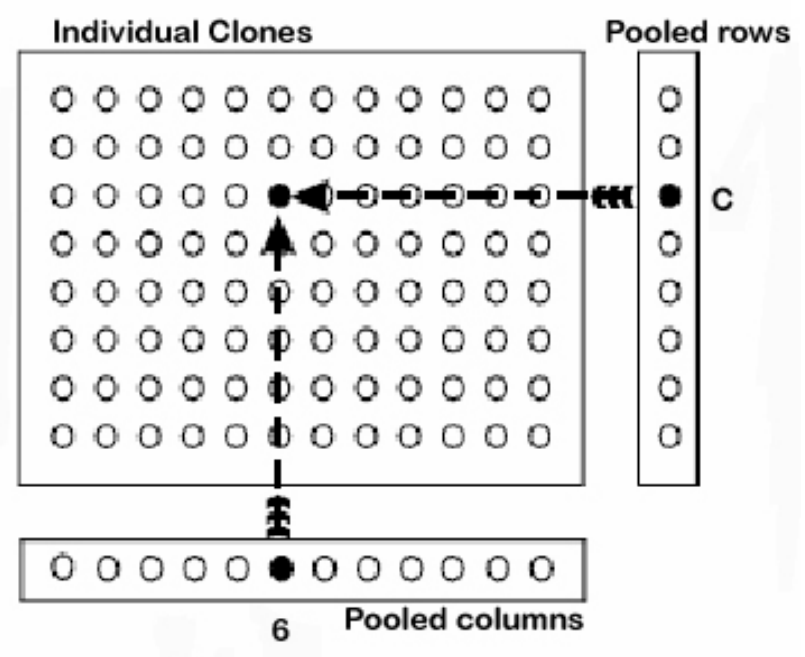

Figure 7.19 The well from which particular clone T-RFs originated could be determined by identifying that clone in both the corresponding row and column pooled clone T-RFLP profile.

By comparing the T-RFLP data from the pooled columns and rows, the wells from which particular clone T-RFs originated could be determined (Figure 7.19). This was automated using a perl script (written by J. Palmer) that identified corresponding column and row 
results and listed the T-RF sizes that could potentially be generated by the clone within each well (Appendix C4).

\subsubsection{Clone Choice for Sequencing}

The T-RFLP screening of the pooled clones allowed for tentative identification of the fragment length or T-RF each clone produced. The perl script allowed matching tolerances to be chosen and in this case, $\pm 1 \mathrm{bp}$ was used. In most cases, the number of potential TRFs (and hence clone types) that could potentially be contained within a given well was reduced to four or fewer possibilities although a number gave more, up to ten and some were reduced to no possible T-RF sizes. The screening method also gave a rough idea of the diversity contained within the clone library without the need to sequence the entire library. Twelve clones were chosen from each library for sequencing. Ideally, a greater number would have been sequenced for more informative results, but financial restrictions limited the number of sequencing reactions that could be performed. The aim was to sequence at least one representative of each common T-RF in each clone library. Multiple representatives were chosen from the most common T-RF sizes to determine whether one or multiple species were contributing to that peak. Less common phylotypes were also chosen for sequencing. Two libraries of similar time-points (V12 and Z11 - both cadavers on sand) had all 96 members of the clone library sequenced.

\subsubsection{DNA Sequencing}

Clones chosen for sequencing were taken from the glycerol stocks stored at $-80^{\circ} \mathrm{C}$. A sterile inoculating loop was used to dislodge a small quantity of each frozen culture without thawing the stocks. These were spread to obtain individual colonies on LB agar plates containing $100 \mathrm{mg} / \mathrm{mL}$ ampicillin and incubated overnight at $37^{\circ} \mathrm{C}$. Individual 
colonies were picked the next day and used as templates for PCR. The M13 primers and the PCR conditions and recipe outlined in Tables 7.31 and 7.32 were used. The size and yield of the PCR products were checked by electrophoresis on a $2 \%$ agarose gel (Appendix A9). A polyethylene glycol (PEG) 96-well plate cleanup method (Appendix A16) was used to remove excess PCR reagents before sequencing. An ABI 3700 Genetic Analyser (Applied Biosystems) was used to sequence the PCR products using a Big Dye Terminator sequencing kit (Applied Biosystems) and the $\mathrm{T} 7$ primer (5'GTAATACGACTCACTATAGGGC 3'). Sequencing was performed by the ESR Sequencing Lab facility.

\subsubsection{Sequence analysis}

Vector sequence was removed from the sequence data before checking for chimeras using the freely available programme Pintail, developed by Cardiff University's School of Biological Sciences [http://www.bioinformatics-toolkit.org/Pintail/index.html] (Ashelford et al., 2005), and the Chimera Check tool on the Ribosomal Database II version 8.1 website (Cole et al., 2009) [http://rdp8.cme.msu.edu/cgis/chimera.cgi]. These tools were both used as there is currently no definitive method for determining if a sequence is chimeric, so any sequences which had characteristics of chimeras were tested with both, and a decision made based on the information gained. Three sequences of questionable quality were excluded from further analysis. Each good quality sequence was then compared against the RDP database release 9.54 using the Sequence Match option (Cole et al., 2009). This tool reports similar sequences that exist within the database and determines the similarity between the submitted sequence and the database matches. The entire database was searched rather than taking the option of restricting the search to sequences greater than $1200 \mathrm{bp}$, to enable a more thorough search. The database entry with the greatest similarity was recorded for each sequence. 


\subsubsection{T-RFLP predictions vs. actual insert fragment sizes.}

By using the Virtual Digest function on the MiCA website (http://mica.ibest.uidaho.edu/digest.php), the T-RF that would be generated by every sequence in the database was determined. From this, the predicted size of the T-RF generated by the database entry most closely matching each cloned sequence was also determined. The actual T-RF size of the clone was also found by searching for the first MspI restriction site following the forward primer, within the cloned sequence. These values were then compared back to the size of fragments predicted to be from a given clone, by using the clone pooling method. A predicted T-RF could not be determined for some "closest matches" because the database sequence did not cover both the primer binding sites, so in these cases the next closest match was used to give an estimated T-RF size.

\subsubsection{Results}

Of the 600 clones chosen for sequencing, three were found to have no insert and ten were identified as possible chimeras, or of questionable quality and so were excluded from analysis. Good quality sequences could not be obtained for a remaining eight (after three sequencing attempts).

For the V12 library, $85 \%$ of cloned sequences matched their most-closely related match with a similarity score of over 0.8 (with 1.0 being an exact match). The sequences from the Z11 library all returned similarity scores of over 0.8 . For the remaining cloned sequences, all closest match scores were above 0.5 , and $80 \%$ were over 0.8 . 


\subsubsection{Clone identification}

Sequences representative of six of the 34 currently recognised Bacteria phyla (by the Ribosomal Database) were identified: Bacteroidetes, Firmicutes, Proteobacteria, Acidobacteria, Flavobacteria and Actinobacteria. The majority of cloned sequences were identified as belonging to the first three phyla listed. There were 23 orders, 42 families and 77 different genera identified as the closest match to the selected clones (Table 7.33). The orders of the closest matches obtained from each carcass and cadaver are shown in Table 7.34, and the orders identified from each time-point (early, mid or late) are shown in Table 7.35.

The most commonly returned closest match was to an unclassified Pseudomonad (bacterium 20RD; EF640582, S000893094) with 76 out of 579 clones (13\%) returning this as their closest match. This sequence itself was not able to give a predicted T-RF size due to a primer binding site not being included in the sequence, but the next closest match, in most cases, gave a predicted T-RF of $463 \mathrm{bp}$. This falls within a size region (457-463 bp) that gave multiple large T-RFs in many, if not most of the community T-RFLP profiles. This particular database entry was not returned as a potentially contributing organism for this size in the multi-digestion experiment (section 7.4), because of the primer site not being included in the sequence. The order Pseudomonadales was, however, identified as a potentially contributing organism at this size.

The 96 clone library sequenced from sample V12 (non-autopsied cadaver on sand) had 28 $\%$ of the sequences returning bacterium $20 \mathrm{RD}$ as the most similar match. More matches to it occurred with clones from the mid-decomposition time-point than the early or late timepoint samples, but did not appear to be specific to either substrate, a particular autopsy status or human cadavers/pig carcasses. 
Five out of the six phyla identified using the clone pooling and sequencing method were predicted as potential contributors to any of the T-RFs of interest (section 7.4). Some 10 out of 13 classes were also predicted, as were 17 out of 21 orders. Twenty-five out of 34 families were predicted but only 10 out of 58 were identified at the genus level. Those that were not predicted by the multi-digestion experiment are bolded in Table 7.33.

When examining the lists of bacterial orders that were detected in samples from the different cadavers and carcasses (Table 7.34), it becomes apparent that some bacterial orders were common to all, or nearly all decomposition events. Because so few clones were sequenced from each library, and because of the targeted method of choosing those clones, care must be taken when interpreting the absence of an order from a sample. Only one time-point was used to generate libraries from the pig carcass samples from the first experiment, so the absence of an order from these samples is certainly not suggestive of it not being present. Taking this into consideration, the Xanthomonadales were detected only in decomposition plots where the carcass or cadaver was colonised by insects, but were absent from the three plots where insects were excluded. The Sphingobacteriales were only detected in samples from under pig carcasses (on both soil and sand) and the Myxococcales and Rhodospirillales were detected only in samples from under human cadavers.

The Pseudomonadales were detected in at least one sample from every cadaver or carcass and the Flavobacteriales were found to be present in all except one cadaver (T, autopsied, on sand). The Clostridiales were identified in samples from all except one cadaver ( $R$, non-autopsied on soil), although were not detected in a number of the pig sample clone libraries. Some orders, such as the Lactobacillales, Oceanospirillales, Aeromonadales and Erysipelotrichales were present in libraries from only one cadaver or carcass, while the remaining orders were all detected in samples from multiple carcasses and cadavers. 
The Acidobacteriales were only detected in soil samples, as were the Actinomycetales, which were only detected in New Zealand soil samples (Table 7.35). There were no orders detected that were specific to a particular time-point, although the limited number of clones sequenced from each library reduces the likelihood of any such pattern being detected. 


\begin{tabular}{|c|c|}
\hline Classification Level & Organisms identified by Sequencing \\
\hline Phyla (6) & Acidobacteria, Actinobacteria, Bacteroidetes, Firmicutes, Flavobacteria, Proteobacteria \\
\hline Classes (13) & $\begin{array}{c}\text { Acidobacteria, Actinobacteria, Alphaproteobacteria, Bacilli, Bacteroidetes, Betaproteobacteria, Clostridia, Deltaproteobacteria, } \\
\text { Erysipelotrichi, Firmicutes, Flavobacteria, Gammaproteobacteria, Sphingobacteria }\end{array}$ \\
\hline Orders (21) & $\begin{array}{c}\text { Acidobacteriales, Actinomycetales, Aeromonadales, Bacillales, Bacteroidales, Burkholderiales, Caulobacterales, Clostridiales, } \\
\text { Enterobacteriales, Erysipelotrichales, Flavobacteriales, Lactobacillales, Myxococcales, Oceanospirillales, Pseudomonadales, } \\
\text { Rhizobiales, Rhodobacterales, Rhodospirillales, Sphingobacteriales, Sphingomonadales, Xanthomonadales }\end{array}$ \\
\hline Families (34) & $\begin{array}{c}\text { Acetobacteraceae, Acidobacteriaceae, Actinomycetaceae, Aeromonadaceae, Bacteroidaceae, Brucellaceae, Burkholderiaceae, } \\
\text { Caulobacteraceae, Clostridiaceae, Comamonadaceae, Crenotrichaceae, Cryomorphaceae, Enterobacteriaceae, } \\
\text { Enterococcaceae, Erysipelotrichaceae, Flavobacteriaceae, Gordoniaceae, Hyphomicrobiaceae, Lachnospiraceae, } \\
\text { Microbacteriaceae, Micrococcaceae, Moraxellaceae, Nannocystineae, Oceanospirillaceae, Oxalobacteraceae, } \\
\text { Peptococcaceae, Peptostreptococcaceae, Planococcaceae, Polyangiaceae, Porphyromonadaceae, Pseudomonadaceae, } \\
\text { Rhodobacteraceae, Sphingobacteriaceae, Xanthomonadaceae }\end{array}$ \\
\hline Genera (58) & $\begin{array}{c}\text { Acinetobacter, Aeromonas, Anaerovorax, Arthrobacter, Bacteroides, Brevundimonas, Burkholderia, Buttiauxella, } \\
\text { Chitinophaga, Chryseobacterium, Citrobacter, Collimonas, Comamonas, Cupriavidus, Desulfonispora, Duganella, Dyella, } \\
\text { Empedobacter, Enterobacter, Enterococcus, Erysipelothrix, Flavimonas, Flavobacterium, Fluviicola, Gordonia, } \\
\text { Helcococcus, Hyphomicrobium, Janthinobacterium, Klebsiella, Leucobacter, Marinospirillum, Morganella, Myroides, } \\
\text { Novosphingobium, Ochrobactrum, Paracoccus, Pedobacter, Pedomicrobium, Peptoniphilus, Peptostreptococcus, } \\
\text { Phenylobacterium, Proteiniphilum, Proteus, Providencia, Pseudochrobactrum, Pseudomonas, Psychrobacter, Raoultella, } \\
\text { Rhodobacter, Rhodoplanes, Schineria, Sedimentibacter, Sphingobacterium, Sphingopyxis, Sporanaerobacter, Stella, } \\
\text { Stenotrophomonas, Tissierella }\end{array}$ \\
\hline
\end{tabular}

Table 7.33 Bacteria identified as closest matches to cloned sequences grouped at the different taxonomic level. 


\begin{tabular}{|c|c|c|c|c|c|c|c|c|c|c|c|c|c|c|c|c|}
\hline \multirow[b]{2}{*}{ Bacterial Orders Identified } & \multicolumn{16}{|c|}{ Presence/Absence of Bacterial Orders in Samples from each Carcass/Cadaver } \\
\hline & $\begin{array}{c}\text { A } \\
\text { pig } \\
\text { sand } \\
\text { insects }\end{array}$ & $\begin{array}{c}\text { B } \\
\text { pig } \\
\text { sand } \\
\text { no } \\
\text { insects } \\
\end{array}$ & $\begin{array}{c}\mathrm{C} \\
\text { pig } \\
\text { sand } \\
\text { insects }\end{array}$ & $\begin{array}{c}\mathrm{D} \\
\text { pig } \\
\text { sand } \\
\text { no } \\
\text { insects } \\
\end{array}$ & $\begin{array}{c}\text { E } \\
\text { pig } \\
\text { sand } \\
\text { insects }\end{array}$ & $\begin{array}{c}\mathrm{F} \\
\text { pig } \\
\text { sand } \\
\text { no } \\
\text { insects } \\
\end{array}$ & $\begin{array}{c}\mathrm{G} \\
\text { pig } \\
\text { soil }\end{array}$ & $\begin{array}{c}\mathrm{H} \\
\text { pig } \\
\text { soil }\end{array}$ & $\begin{array}{c}\text { I } \\
\text { pig } \\
\text { sand }\end{array}$ & $\begin{array}{c}\mathrm{J} \\
\text { pig } \\
\text { sand }\end{array}$ & $\begin{array}{c}\mathrm{P} \\
\text { human } \\
\text { soil } \\
\text { no } \\
\text { autopsy } \\
\end{array}$ & $\begin{array}{c}\mathrm{R} \\
\text { human } \\
\text { soil } \\
\text { no } \\
\text { autopsy } \\
\end{array}$ & $\begin{array}{c}\mathrm{T} \\
\text { human } \\
\text { sand } \\
\text { autopsy }\end{array}$ & $\begin{array}{c}\mathrm{V} \\
\text { human } \\
\text { sand } \\
\text { no } \\
\text { autopsy } \\
\end{array}$ & $\begin{array}{c}\mathrm{X} \\
\text { human } \\
\text { soil } \\
\text { autopsy }\end{array}$ & $\begin{array}{c}\mathrm{Z} \\
\text { human } \\
\text { sand } \\
\text { autopsy }\end{array}$ \\
\hline Acidobacteriales & - & - & - & - & - & - & + & + & - & - & + & + & - & - & + & - \\
\hline Actinomycetales & - & - & - & - & - & - & + & + & - & - & - & - & - & - & - & - \\
\hline Aeromonadales & - & - & - & - & - & - & - & & - & - & - & - & - & - & - & + \\
\hline Bacillales & + & - & - & - & + & - & + & + & - & + & - & - & + & + & - & - \\
\hline Bacteroidales & - & - & - & - & - & - & - & + & + & - & - & - & - & + & - & - \\
\hline Burkholderiales & - & + & - & - & - & + & + & + & - & - & - & + & - & + & - & - \\
\hline Caulobacterales & - & + & - & - & - & - & - & + & - & - & - & + & - & + & - & - \\
\hline Clostridiales & - & - & - & - & - & + & + & - & + & + & + & - & + & + & + & + \\
\hline Enterobacteriales & - & - & + & + & + & - & - & - & - & - & + & + & + & + & + & + \\
\hline Erysipelotrichales & - & - & - & - & - & - & - & - & + & - & - & - & - & - & - & - \\
\hline Flavobacteriales & + & + & + & + & + & + & + & + & + & + & + & + & - & + & + & + \\
\hline Lactobacillales & - & - & - & - & - & - & - & - & - & - & - & - & + & - & - & - \\
\hline Myxococcales & - & - & - & - & - & - & - & - & - & - & + & + & - & - & - & - \\
\hline Oceanospirillales & - & - & - & - & - & - & - & - & - & + & - & - & - & - & - & - \\
\hline Pseudomonadales & + & + & + & + & + & + & + & + & + & + & + & + & + & + & + & + \\
\hline Rhizobiales & - & - & - & - & - & - & + & + & + & - & + & + & - & + & + & + \\
\hline Rhodobacterales & - & - & + & - & + & - & - & - & - & + & - & - & - & + & - & - \\
\hline Rhodospirillales & - & - & - & - & - & - & - & - & - & - & + & - & - & - & + & - \\
\hline Sphingobacteriales & - & + & - & + & + & - & + & + & - & - & - & - & - & - & - & - \\
\hline Sphingomonadales & - & - & - & - & - & - & - & - & - & - & - & + & - & - & + & - \\
\hline Unclassified Actinobacteria & - & - & - & - & - & - & - & - & - & - & + & - & - & - & - & - \\
\hline Unclassified Deltaproteobateria & - & - & - & - & - & - & + & - & - & - & + & + & - & - & - & - \\
\hline Xanthomonadales & + & - & + & - & + & - & + & + & + & + & + & - & + & - & - & + \\
\hline
\end{tabular}

Table 7.34 Bacterial orders identified as being present in the bacterial community associated with each pig carcass and cadaver. 


\begin{tabular}{|c|c|c|c|c|c|c|c|c|c|c|c|c|}
\hline \multirow[t]{2}{*}{ Bacterial Orders Identified } & \multicolumn{3}{|c|}{$\begin{array}{c}\text { Pig Carcasses on Soil } \\
(G \text { and } H)\end{array}$} & \multicolumn{3}{|c|}{$\begin{array}{c}\text { Pig Carcasses on Sand } \\
\text { (I and J) }\end{array}$} & \multicolumn{3}{|c|}{$\begin{array}{c}\text { Human Cadavers on } \\
\text { Soil }(P, R \text { and } X)\end{array}$} & \multicolumn{3}{|c|}{$\begin{array}{c}\text { Human Cadavers on } \\
\text { Sand }(T, V \text { and } Z)\end{array}$} \\
\hline & Early & Mid & Late & Early & Mid & Late & Early & $\overline{\mathrm{Mid}}$ & Late & Early & Mid & Late \\
\hline Acidobacteriales & + & - & - & - & - & - & + & + & + & - & - & - \\
\hline Actinomycetales & + & - & + & - & - & - & - & - & - & - & - & - \\
\hline Aeromonadales & - & - & - & - & - & - & - & - & - & - & + & - \\
\hline Bacillales & + & - & - & - & + & - & - & - & - & + & + & + \\
\hline Bacteroidales & + & - & - & - & + & + & - & + & + & - & + & + \\
\hline Burkholderiales & + & + & + & - & - & - & + & - & + & - & - & + \\
\hline Caulobacterales & + & - & - & - & - & - & + & - & - & - & + & - \\
\hline Clostridiales & - & + & - & + & + & + & + & + & + & + & + & + \\
\hline Enterobacteriales & - & - & - & - & - & - & + & - & + & + & + & - \\
\hline Erysipelotrichales & - & - & - & - & - & + & - & - & - & - & - & - \\
\hline Flavobacteriales & + & + & + & + & + & - & + & + & + & + & + & - \\
\hline Lactobacillales & - & - & - & - & - & - & - & - & - & - & + & - \\
\hline Myxococcales & - & - & - & - & - & - & + & + & + & - & - & - \\
\hline Oceanospirillales & - & - & - & - & - & + & - & - & - & - & - & - \\
\hline Pseudomonadales & + & + & + & + & + & + & + & + & + & + & + & + \\
\hline Rhizobiales & + & - & + & - & - & + & + & + & + & + & + & + \\
\hline Rhodobacterales & - & + & - & + & - & - & - & - & - & - & + & - \\
\hline Rhodospirillales & - & - & - & - & - & - & + & - & + & - & - & - \\
\hline Sphingobacteriales & + & - & + & - & - & - & - & - & - & - & - & - \\
\hline Sphingomonadales & - & - & - & - & - & - & - & + & + & - & - & - \\
\hline Unclassified Actinobacteria & - & - & - & - & - & - & - & - & + & - & - & - \\
\hline Unclassified Deltaproteobateria & + & - & - & - & - & - & + & - & + & - & - & - \\
\hline Xanthomonadales & + & + & - & + & - & - & + & - & + & + & + & + \\
\hline
\end{tabular}

Table 7.35 Bacterial orders identified as being present in the bacterial community associated with pig carcases on sand and soil, and with human cadavers on sand and soil. 


\subsubsection{Clone identification for entire libraries from samples V12 and Z11}

The relative frequencies of the bacterial orders detected in the two clone libraries (V12 and Z11) which were sequenced in full are shown in Figures 7.20 and 7.21. Despite both being samples from a similar stage of decomposition (ADD 254 and ADD 216) and both being from cadavers placed on sand, the libraries were markedly different. The Clostridiales were the most frequently detected order in both libraries, although together they represented $80 \%$ of the cloned sequences from the sample Z11 library and just $37 \%$ from the sample V12 library. The two libraries had almost equal numbers of the Enterobacteriales and both contained Pseudomonadales, although the V12 library was $30 \%$ Pseudomonadales and the Z11 library was just $1 \%$. The V12 library contained Xanthomonadales (15\%), Bacillales (3\%), Rhizobiales (2\%), Caulobacterales (1\%) and Rhodobacterales (1\%), none of which were detected in the Z11 library. The Z11 library did, however, contain Bacteroidales (3\%), Aeromonadales (1 \%) and Flavobacteriales (1 $\%)$, which were not present in the V12 library.

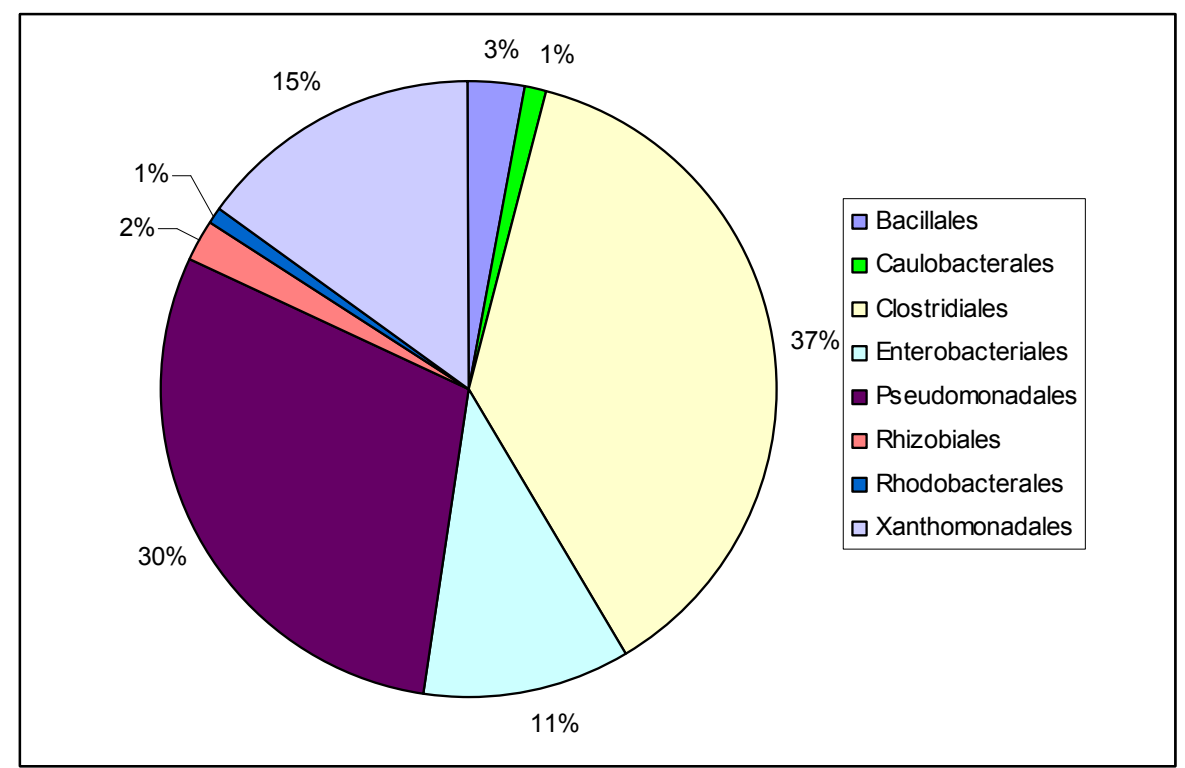

Figure 7.20 Relative frequencies of the different orders represented in the clone library generated from sample V12 (non-autopsied cadaver on sand). 


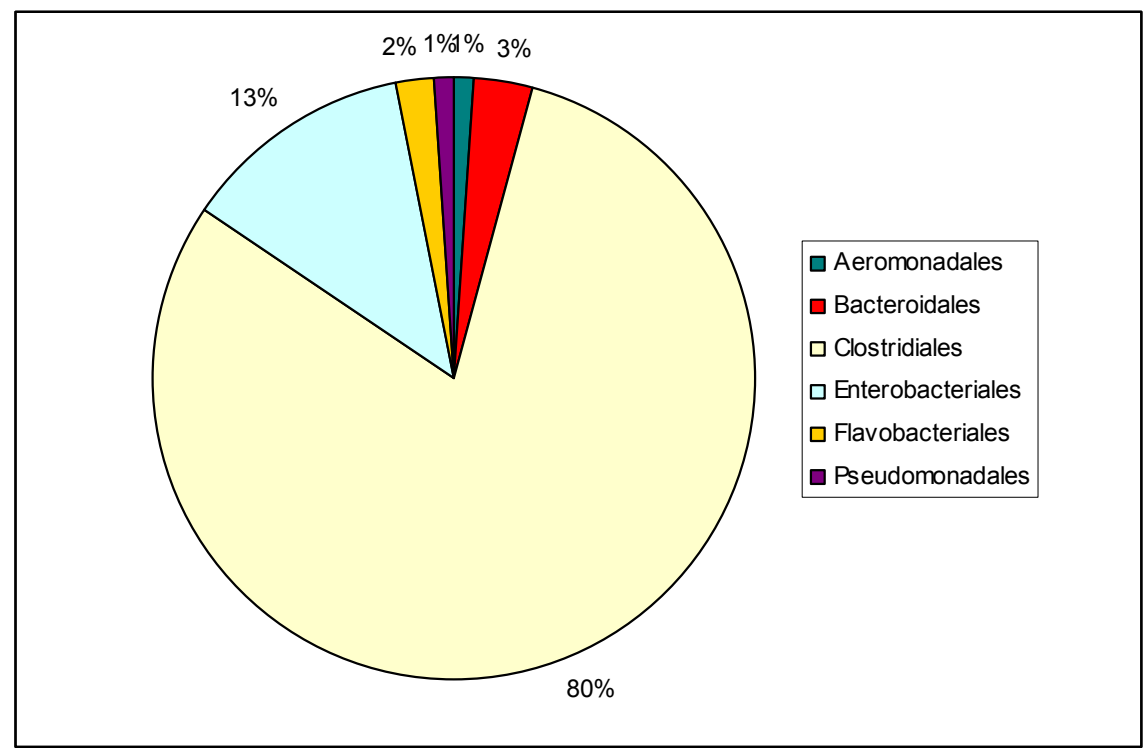

Figure 7.21 Relative frequencies of the different orders represented in the clone library generated from sample Z11 (autopsied cadaver on sand).

\subsection{T-RF identification}

For each cloned sequence, the expected T-RF size for the closest match was established using a virtual digest of all the organisms that the primer pair used could amplify in the Ribosomal Database (section 7.5.2.7). The T-RF size for the actual cloned sequence itself was also deduced by searching for the first $M s p$ I site following the F63 primer and calculating the length of the $5^{\prime}$ terminal fragment. Some $64 \%$ of the actual restriction fragment sizes matched (within $3 \mathrm{bp}$ ) those of the closest database match, with $35 \%$ of the closest matches not returning a predicted T-RF size because of insufficient sequence data. A further $1 \%$ returned completely different sizes to their closest match.

Only $59 \%$ of the actual restriction fragment sizes were successfully predicted by the clone pooling method using the parameters outlined in section 7.5.2.4. Subsequently, many TRFs of interest that were targeted for sequence identification did not have any contributing organisms identified despite selections being made in the hope of particular clones containing sequences generating those T-RFs. 
The organisms found to be associated with particular T-RF sizes are listed in Tables 7.36, 7.37 and 7.38. Orders that were identified as potentially contributing to that size in the multi-digestion experiment (section 7.4) are bolded. T-RF sizes that were not included in the multi-digestion experiment are italicised. Because the primers used for the initial PCR (F63/R534) generated a product of around $471 \mathrm{bp}$, there were a number of occasions when the predicted T-RF of the closest match was longer than the cloned sequence length, or below the $50 \mathrm{bp}$ accurate sizing threshold. The organisms found to be contributing to these sizes are included in the results as $<50 \mathrm{bp}$ and $>465 \mathrm{bp}$.

\begin{tabular}{|c|c|}
\hline T-RF size & Bacterial Orders \\
\hline$<50$ & $\begin{array}{l}\text { unclassified Actinobacteria, Actinomycetales, Lactobacillales, } \\
\text { Pseudomonadales, Rhizobiales, Sphingobacteriales }\end{array}$ \\
\hline $50-55$ & $\begin{array}{c}\text { Burkholderiales, Flavobacteriales, Lactobacillales, Pseudomonadales, } \\
\text { Sphingobacteriales }\end{array}$ \\
\hline $60-65$ & Acidobacteriales, Bacteroidales, Flavobacteriales, Xanthomonadales \\
\hline $80-85$ & Pseudomonadales, Rhizobiales \\
\hline $90-91$ & Flavobacteriales, Rhizobiales \\
\hline $101-103$ & Myxococcales \\
\hline 105 & Burkholderiales \\
\hline $107-113$ & $\begin{array}{c}\text { Acidobacteriales, Actinomycetales, Bacillales, Burkholderiales, } \\
\text { Clostridiales, Sphingobacteriales }\end{array}$ \\
\hline $115-117$ & $\begin{array}{c}\text { Acidobacteriales, Myxococcales, Rhizobiales, Rhodospirillales, } \\
\text { Sphingobacteriales, Sphingomonadales }\end{array}$ \\
\hline $121-123$ & Clostridiales \\
\hline $125-136$ & $\begin{array}{c}\text { Actinomycetales, Bacillales, Erysipelotrichales Pseudomonadales, } \\
\text { Rhizobiales, Xanthomonadales }\end{array}$ \\
\hline 141 & Bacillales \\
\hline 153 & Sphingobacteriales \\
\hline 157 & unclassified Deltaproteobacteria, Clostridiales, Xanthomonadales \\
\hline 166 & Acidobacteriales, Flavobacteriales \\
\hline 180 & Myxococcales \\
\hline 183 & Acidobacteriales \\
\hline $188-191$ & Flavobacteriales \\
\hline
\end{tabular}

Table 7.36 Bacterial orders identified by sequencing to be contributing T-RFs of sizes up to $200 \mathrm{bp}$. 


\begin{tabular}{|c|c|}
\hline T-RF size & Bacterial Orders \\
\hline $226-228$ & Acidobacteriales \\
\hline $241-6$ & Actinomycetales, Clostridiales \\
\hline 251 & Clostridiales \\
\hline $255-258$ & Acidobacteriales \\
\hline $260-261$ & Clostridiales, Flavobacteriales \\
\hline $263-264$ & Clostridiales, Pseudomonadales \\
\hline $267-269$ & Clostridiales, Pseudomonadales \\
\hline 296 & Xanthomonadales \\
\hline
\end{tabular}

Table 7.37 Bacterial orders identified by sequencing to be contributing T-RFs of sizes of between 200 and $300 \mathrm{bp}$.

\begin{tabular}{|c|c|}
\hline T-RF size & Bacterial Orders \\
\hline $226-228$ & Acidobacteriales \\
\hline $241-6$ & Actinomycetales, Clostridiales \\
\hline 251 & Clostridiales \\
\hline $255-258$ & Acidobacteriales \\
\hline $260-261$ & Clostridiales, Flavobacteriales \\
\hline $263-264$ & Clostridiales, Pseudomonadales \\
\hline $267-269$ & Clostridiales, Pseudomonadales \\
\hline 296 & Xanthomonadales \\
\hline
\end{tabular}

Table 7.38 Bacterial orders identified by sequencing to be contributing T-RFs of sizes greater than $300 \mathrm{bp}$.

A number of orders that were found to be represented in the clone libraries were not predicted by the multi-digestion digestion T-RFLP method (section 7.4). Particularly noticeable by their absence were the Bacillales, the Flavobacteriales and the Myxococcales which were represented by numerous cloned sequences. The Erysipelotrichales order was 
also not identified as a potential contributor in the multi-digestion experiment. All the other groups identified through cloning and sequencing were predicted, but not for the particular T-RF sizes that the sequences were associated with, for example, the Lactobacillales group was represented by cloned sequences which gave T-RFs in the 50-55 bp size range, but was not predicted to produce fragments of this length. The T-RF sizes predicted for the Lactobacillales were 125-132 and 479-481.

\subsubsection{Discussion}

\subsubsection{Experimental Approach}

The use of the pooled cloning T-RFLP method was an attempt to derive as much information about which organisms contribute to particular T-RFs seen in T-RFLP profiles while keeping the number of clones sequenced to a manageable and affordable minimum. Moeseneder et al. (2004) evaluated the use of T-RFLP for screening individual clones from an archaeal library and from this were able to phylogenetically identify all but one of the TRFs generated from individual clones in the complex community T-RFLP profiles. Grant and Ogilvie (2004) developed the method used in this experiment and concluded that it allows efficient identification of clones corresponding to a particular fragment in the fingerprint.

Because the primers used for the initial PCR (F63/R534) generated a product of around 471 $\mathrm{bp}$, there were many organisms for which the expected T-RF was longer than the sequence length. Because of this, T-RFs longer than $471 \mathrm{bp}$ in the original T-RFLP profiles (which used the F63-R1087 primer pair) would not have been distinguished. The limit for accurate sizing of T-RFs, however, was 500 bp so there was only a $30 \mathrm{bp}$ short-fall. 
Only $59 \%$ (314 out of 532 cloned sequences) gave similar T-RF sizes to the closest sequence match and to the T-RF sizes predicted for that clone based on the clone pooling method. The reason for this low percentage was investigated and it was found that if the match parameters of the perl script used to collate the column and row data (section 7.5.2.4) is relaxed (i.e. from \pm 1 to $\pm 3 \mathrm{bp}$ ), the accuracy of the method improved to $76 \%$. The original analysis from which the clones for sequencing were chosen was done using \pm 1 because there did not appear to be much drift occurring between profiles and by using a stricter matching parameter, fewer potential sizes were identified for each clone. Fewer potential sizes per clone appeared to make selecting clones of interest for sequencing more accurate. For example, if the T-RF size of interest was 261 and using \pm 1 bp matching gave an average of two potential sizes per clone, but using \pm 2 bp gave an average of five potential sizes per clone, choosing a clone containing a sequence that gives a $261 \mathrm{bp} T-\mathrm{RF}$ is more likely with the former matching parameter. Unfortunately, it appears that level of stringency often eliminated the correct prediction. The match parameter of \pm 1 bp was decided after looking at ten clone libraries with different levels of diversity indicated by the pooled clone profiles and the number of potential sizes reported at various match parameters between 1 and $5 \mathrm{bp}$.

Also detected as an issue in the pooling method, was the decision to use a cut-off value of 5000 fluorescence units. In some cases, the T-RF retrospectively found to be contributed by a certain clone was below this threshold level and so was not included in the analysis. This was a problem as some profiles contained more T-RFs than could be present based on the number of cloned sequences represented. The maximum number of different T-RFs should have been 12 for rows and 8 for columns. The 5000 fluorescence units restricted the number of T-RFs to 12 or fewer in most cases. The extra T-RFs are likely to be artefacts of the method, possibly incompletely digested PCR products (Lee et al., 2008b). 
The V12 and Z11 libraries were examined and compared back to their 20 column and row T-RFLP profiles. These particular datasets showed very few pseudo-T-RFs, but about half of those that did not seem to correlate with any sequences in the library were consistent with being partially digested sequences from within the clone mix. Every effort was made in this experiment to ensure that digestion was complete, by measuring and diluting the DNA prior to digestion to ensure correct ratios of DNA to enzyme and following the manufacturer's suggested incubation times and temperatures. Partial digestion, however, is a possible complicating factor. The other pseudo-TRFs may be artefacts generated during PCR or could be caused by handling errors such as transferring clones into the wrong well, or cross-contamination between nearby wells, which is suspected to have occurred in at least two cases in this study.

Overall, the T-RFLP clone screening method allowed targeted identification of organisms that contributed to T-RFs of interest. It emerged that the success of the method was very dependant on the analysis thresholds set. The tolerance level in matching fragments in column and row T-RFLP profiles affected the number of T-RFs that were predicted for a well to possibly contain. Some sequences identified generated T-RFs which fell outside the T-RFLP size standards, which could be avoided by careful selection of the restriction enzyme used. An important aspect was also setting sensible fluorescence thresholds for deciding which T-RFs were true/noise/artefacts.

\subsubsection{Bacterial identifications}

A total of twenty-three different bacterial orders were identified in the cloned sequences. Care must be taken in the interpretation of predominance of organisms based on their frequency in full clone libraries due to differences in $16 \mathrm{~S}$ operon copy number of up to 15 
between bacterial species (Madigan et al., 2000) and because of the targeted method in which clones were chosen for sequencing in this study.

\subsection{Pseudomonadales}

Although the targeted choice of clones for sequencing means that the results from this experiment cannot be interpreted quantitatively, the Pseudomonadales were very strongly represented with 126 of the 581 sequences (22\%) identified as likely Pseudomonadales. At least one Pseudomonadales sequence was identified from at least one sample from every carcass or cadaver. There were only five samples in which Pseudomonadales were not detected amongst the 12 clones sequenced from each (samples P8, P20, R11, T9 and V4 all cadavers, two autopsied, two not autopsied, two on soil, two on sand). The Pseudomonadaceae were also found to be contributing to 10 different T-RF sizes. This is not surprising, given that the Pseudomonadaceae are able to use a wide variety of organic compounds for carbon and energy sources and are very tolerant of varying environmental conditions (Borch et al., 1996; Mayr et al., 2003). Pseudomonas species are strongly involved in spoilage of meat, including pork products (Tryfinopoulou et al., 2002), fish (Jaspe et al., 1994) and milk (Tekoriene, 2008). They are also ubiquitous in the environment and are found in most soils and waters (Wang et al., 1999). Many Pseudomonadales exhibit anti-fungal activity, often through chitinase enzyme production (Macauley et al., 2007). The Pseudomonadaceae are strict aerobes, so their presence indicates that oxygen levels in the soil environment during a decomposition event must be adequate for their survival. This is in contrast to the finding in the group-specific DGGE experiments (section 7.2.5.2) that strictly anaerobic organisms were detectable in the soil environment. This suggests that there may be a mix of environmental niches with different oxygen levels beneath a decomposing cadaver. This is highly likely, as decomposition fluids do not purge uniformly from a cadaver but form puddles in the underlying substrate. 
The sampling method employed ensured collection of soil from within these puddles and also from less saturated areas under the cadaver. Because of their prevalence on meat products, it is likely that the Pseudomonadales are strongly involved in the degradation of the cadaver on the exposed surfaces.

The database entry S000893094 bacterium 20RD; EF640582 was identified as closest match to sequences derived from all three experiments, suggesting that this organism is common to humans and pigs. The RDP sequence was contributed by a study examining tetracycline resistance in a swine lagoon (Wilson, 2005), and was found to contain three tet resistance genes. Another isolate from the same study, S000893118 bacterium 20GR; EF640606 was also the closest match to 7 different clones but was identified as belonging to the Myroides genus (Flavobacteriales order).

The two families within the Pseudomonadales order represented in the sequences were Moraxellaceae and Pseudomonadaceae. The Moraxellaceae family comprises four genera, two of which (Moraxella and Acinetobacter) were identified in this study. The Pseudomonadaceae currently contains 10 genera of which only the Pseudomonas genera was detected. A number of unclassified Pseudomonadaceae were also identified, including those that most closely matched S000893094 bacterium 20RD; EF640582.

The cloned sequences which most closely matched database entry S000893094 bacterium 20RD; EF640582 seemed to display a considerable degree of diversity. Within the 76 sequences that hit on this database entry, there were seven different predicted T-RFs ranging from $437 \mathrm{bp}$ to having no restriction enzyme site within the sequence, which would give a T-RF of over $488 \mathrm{bp}$. Most gave T-RFs of 463 or $465 \mathrm{bp}$. The database entry gave an expected T-RF of $463 \mathrm{bp}$. The different length T-RFs were not associated with particular sources (pig or human), time-points or treatments. This diversity suggests that 
there were a number of closely related species present, rather than a single species that was detected many times.

The two pig carcasses on sand that were colonised by insects (I and J) during the second pig experiment (chapter 5), and two of the three from the first experiment (chapter 4) had predominantly Pseudomonadales identified from samples at all three time points. There was no predominance of this order evident for samples from the human cadavers on sand which showed a dominance by Clostridiales.

Thirteen of the Pseudomonadales sequences most closely matched Acinetobacter species. This genus is found on the skin of approximately $25 \%$ of humans (Towner, 2006) and an unknown proportion of pigs raising the possibility that skin microfloral species were detected. Acinetobacter are also, however, common in the wider environment, on foods and in soils (Cole et al., 2004). The sequences in this study most closely matched organisms from water (Hantsis-Zacharov and Halpern, 2007; Martin et al., 2007) but also human and cows milk (Wiegel et al., 2006).

\subsection{Clostridiales}

The Clostridiales are a hugely diverse order that contains an array of families which are incorrectly classified and not closely related to the core genus Clostridium. This leads to the order being very diverse morphologically and physiologically. The families that sequences from this study most closely matched were Clostridiaceae, Lachnospiraceae, Peptostreptococcaceae, Incertae Sedis groups XI and XIII. There were ten different genera identified (and a number of unclassified organisms) and Clostridium, Peptoniphilus, Tissierella, Peptostreptococcus and Helcococcus were the most common hits. Most of the sequences that were matched to derived from environments that had been impacted by 
animal products, such as wastewaters and swine manure pits (Whitehead and Cotta, 2004; Lefebvre et al., 2006) although some were also from environments not associated with animals, such as goldmines (Baker et al., 2003) and plants (Halpern et al., 2007). This suggests that the Clostridiales present in the decomposition plots may have been derived from both the carcasses and cadavers, but also from the soil and surrounding environment.

A total of seventy sequences (12\% of all sequences) were found to most closely match members of the Clostridiales order. While they were present in samples from all three time periods, some mid-point samples were strongly dominated by this order, particularly in the samples from under cadavers on sand ( $\mathrm{T}, \mathrm{V}$ and $\mathrm{Z}$ ) and cadaver $\mathrm{P}$ (non-autopsied on soil). The Clostridiales order was not identified in many pig carcass derived samples, with only six sequences in total being identified from all ten pig carcass decomposition sites. This is somewhat unexpected given that Clostridium and related organisms were found to be the second most prevalent organisms in porcine gastrointestinal tracts (Leser et al., 2002).

\subsection{Flavobacteriales}

The Flavobacteriales order was strongly represented in the sequenced clones, with 66 of the 581 sequences $(11 \%)$ from 21 different samples. The Flavobacteriales order belongs to the Bacteroidetes phyla, which is thought to often be under-represented in clone libraries (Kirchman, 2002), so it may be even more prevalent in decomposition samples. This order was not predicted as a potential contributor to any T-RFs in the multiple-digestion experiment even though both primers were found to successfully target Flavobacteriales organisms using the Ribosomal Database's probe match function (Cole et al., 2009). When the virtual digest files were examined, it was found that many Flavobacteriales species 
gave T-RF sizes of $35 \mathrm{bp}$ when digested with $M s p \mathrm{I}$ which is outside of the accurate T-RF sizing parameters (50-500 bp) and hence was not included in the analysis.

The most common genus found in the cloned Flavobacteriales sequences was Myroides. Members of this genus were found in samples collected from under all of the pigs that were colonised by insects, and from all of the human cadavers, except cadaver $\mathrm{T}$ (autopsied on sand). The known Myroides species are primarily associated with water samples and fly guts (Dharne et al., 2008a), which, coupled with the fact that this genus was only detected in decomposition events that were colonised by insects, suggests that this may have been the source of these organisms. Some of the closest Myroides matches were isolated from insects (Corby-Harris et al., 2007), but many were isolated from other sources, including a swine lagoon (Macauley et al., 2007), compost (Guo et al., 2007) and deep sea sediments (Zhang et al., 2008). The Flavobacteriales order was still detected in the samples from the carcasses not colonised by insects, but with Chryseobacterium and Flavobacterium being the two genera found. These genera have previously been associated with soil, cows milk and fresh water (Bernardet et al., 2006), but the closest matches in this study were from human gut samples (Ley et al., 2006), human blood (Vaneechoutte et al., 2007) and composts (Guo et al., 2007).

\subsection{Xanthomonadales}

A total of 45 sequences were matched to Xanthomonadales sequences from three genera (Shineria, Stenotrophomonas, Dyella, and unclassified Xanthomonadaceae). These organisms were found only in samples from cadavers and carcasses that were colonised with insects. Because only three samples that were from non-colonised carcasses had sequences cloned from them, care must be taken when interpreting this result, but these 
three genera do have a close association with insects. One species, which was the closest match to a number of sequences, Shineria larvae, has been isolated from maggot infested wounds (Roudiere et al., 2007), and to date has been primarily associated with larvae of the fly Wohlfahrtia magnificans, which causes myiasis in humans and animals (Toth et al., 2006). Because this fly species is not found in New Zealand (Dr Dallas Bishop, entomologist, Agriquality, pers. comm.), it is possible that this organism is also associated with other fly species that colonise dead tissue. One study, however, has found Schineria larvae in an aerobic batch reactor treating swine waste (Juteau et al., 2005) so it may be more widely distributed than previously thought. Schineria are known to grow well in alkaline conditions (Juteau et al., 2005) so may thrive in the higher soil pH created by the decomposition event.

\subsection{Enterobacteriales}

The Enterobacteriales are large group of organisms, found both in the wider environment and inhabiting the human gastrointestinal system. Most are Gram negative, facultatively anaerobic bacilli. Forty-one sequences were identified to be from eight different genera (Buttiauxella, Enterobacter, Citrobacter, Kliebsiella, Morganella, Proteus, Providencia, Raoultella plus two unclassified Enterobacteriales). These organisms were first identified from environmental samples (Harada et al., 1996; Lee et al., 2008a) and swine manure pits (Whitehead and Cotta, 2004; Li et al., 2008).

As with the Xanthomonadaceae and Myroides genus, organisms from the Providencia genus were found to be associated only with insect-colonised carcasses and cadavers. The closest hits to a number of clones were to members of this genus that were isolated from entomoparasitic (insect-infecting) nematodes (Somvanshi et al., 2006). The Providencia 
genus, however, also has member species that are associated with human and animal gastrointestinal tracts (Mohr O'Hara et al., 1999; Chander et al., 2006), and some of these, including Providencia alcalifaciens and Providencia rettgeri were also found to be closest hits to some clones. Providencia has also been associated with spoilage of bacon products, particularly of high pH (Garcia-Lopez et al., 1998).

\subsubsection{6 $\underline{\text { Rhizobiales }}$}

The Rhizobiales order contains but is not restricted to the nitrogen-fixing Rhizobia species. The Gram negative order currently contains 11 families which are primarily associated with soils and waters (Kersters et al., 2006). A total of 28 sequences belonging to this order were identified, which all fell within two families, Brucellaceae (genera Pseudochrobactrum and Ochrobactrum) and Hyphomicrobiaceae (Hyphomicrobium, Rhodoplanes and Pedomicrobium). Some sequences also matched unclassified Rhizobiales. The Brucellaceae family includes the pathogenic and zoonotic Brucella genus (Kersters et al., 2006). Ochrobactrum and Pseudochrobactrum species have also been associated with opportunistic pathogenesis in humans (Laura et al., 1996) and Ochrobactrum has also been found to be associated with human stomach microflora (Dharne et al., 2008b). The sequences which were most closely matched to in this study were derived from marine aquaculture biofilters (unpublished, RDP accession number AM403218), from deep water rice (Tripathi et al., 2006) and from sewage (unpublished, RDP accession number AY994315).

The Hyphomicrobiaceae family is traditionally associated with oligotrophic fresh-water systems (Kersters et al., 2006), but the sequences from this study most closely matched with sequences derived from soil (Kim et al., 2007a; Elshahed et al., 2008; Lesaulnier et al., 2008). There was a clear difference in the Rhizobiales families that were isolated from 
the soil and sand plots, with those from the soil plots most closely matching to the Hyphomicrobiaceae family and the few sand derived sequences which contained Rhizobiales, to the Brucellaceae family. One Brucellaceae sequence was found in a soil sample. This suggests that the Brucellaceae organisms may have derived from the carcasses/cadavers, although their sporadic appearance, particularly in soil, makes them unlikely candidates as time since death indicators.

\subsection{Bacteroidales}

Members of the Bacteroidales order are phenotypically and ecologically diverse. They are present in aquatic and terrestrial environments but are numerically important constituents of both animal and human oral cavities and intestinal microflora (Dick et al., 2005; Wilson, 2005).

. Fourteen sequences from this order were identified from under two pig carcasses $(\mathrm{H}$, on soil and I, on sand) and from under one cadaver (V, non-autopsied on sand). The two genera that the sequences most closely matched were Bacteroides and Proteiniphilum and were originally identified in studies examining faecal pollution of water, waste digesters (unpublished, RDP accession number EF586002) and swine-manure pits (Whittle et al., 2003) suggesting that these organisms were likely to have derived from the pig carcasses and cadavers rather than the environment. This presents the opportunity for further investigation into their use as time since death indicator species.

\subsection{Acidobacteriales}

Acidobacteria are ubiquitous and abundant members of soil bacterial communities (Jones et al., 2009), but little is known about their ecological importance because of difficulty in 
culturing this group. They are thought to be slow growing oligotrophs whose abundance in a soil is strongly negatively correlated with $\mathrm{pH}$. Because of the negative correlation between Acidobacteria and $\mathrm{pH}$, it was surprising to see 10 sequences from the Acidobacteriales order in this experiment, given the high $\mathrm{pH}$ that was produced by the decomposition events. It was only detected in the early stage samples from the NZ soil plots, but was identified in all three stages in the human cadaver decomposition plots in the USA. All of the cloned sequences that hit on Acidobacteria phyla members were to uncultured Acidobacteriales. The nearest hits were all from molecular studies examining the diversity of soil samples (Liles et al., 2003; Tringe et al., 2005; Elshahed et al., 2008; Jangid et al., 2008; Tarlera et al., 2008). Jones et al. (2009) found that as pH diverges from neutral, the diversity of Acidobacteria in soils decreases.

\subsection{Actinomycetales}

The Actinomycetales did not feature strongly in the sequences from this study despite being a large and diverse group of organisms likely to be present in the soil, human cadavers and environment surrounding the decomposition plots. Only three sequences from this order were identified and all three were from under the two pig carcasses on soil (in the second pig study chapter 5). No Actinomycetales were identified from soils from the US cadaver experiment (Chapter 6). It is unlikely that this order is not present on that soil type, so its non-detection is more likely to be the result of the small number of clones sequenced. The three genera identified were Leucobacter, Arthrobacter and Gordonia, all of which were identified as potential contributors to T-RFs in the multi-digestion experiment (section 7.4). The closest matches for the three sequences were isolated from a wastewater treatment plant (unpublished, RDP accession number DQ083486), diseased sea 
cucumbers (Deng et al., 2009) and soil (Kim et al., 2007b) respectively, indicating that the sequences detected could have come from both the pig carcasses and the soil.

\subsection{Erysipelotrichales}

A single cloned sequence (from sample I44 - pig carcass, sand, insect colonised, ADD 519) was identified as being most closely aligned to Erysipelothrix inopinata. This organism was isolated as a contaminant from a quality check on sterile broth (Verbarg et al., 2004), but is very closely related to the two other species in the Erysipelothrix genus. One of these, Erysipelothrix rhusiopathiae, is a pig pathogen which causes swine erysipelas. The other, Erysipelothrix tonsillarum, is an avirulent commensal organism isolated from the tonsils of healthy pigs (Stackebrandt et al., 2006). This organism is likely to be specific to pigs, and is unlikely to be related to a specific stage of decomposition so is of little interest forensically. It does, however, raise the possibility of detection of human specific organisms for determining if a decomposition event has occurred even if the body or carcass is no longer present, or whether an event involved a human or another species. Also potentially possible is the detection of human pathogens in decomposition sites, perhaps of use to investigators if a missing person was known to have a particular bacterial disease.

\subsection{Lactobacillales}

A single cloned sequence was identified as being most likely from the Lactobacillales order. This sequence, from sample T9 (cadaver T, sand, autopsied, ADD 231) was most closely aligned to Enterococcus dispar which was originally detected in pig blood following slaughter (Davila et al., 2006). This species is differentiated from other 
Enterococcus species because of its ability to grow at between 10-40 $\mathrm{C}$ (Collins et al., 1991). Enterococci are hardy organisms and can tolerate a wide variety of growth conditions including hypotonic, hypertonic, acidic and alkaline environments and they are resistant to bile salts, desiccation, detergents and many antimicrobial agents (Wilson, 2005). Enterococci are facultatively anaerobic organisms and are often used as indicator species for faecal contamination of water (Wheeler et al., 2002). The T9 sample was also one of just 13 samples (from four different cadavers) found to be positive for the presence of Enterococcus group species using the group specific DGGE analysis (section 7.2.5.2.6).

\subsection{Other orders}

Sphingobacteriales, Burkholderiales, Bacillales, Caulobacteriales, Rhodobacterales all detected sporadically between carcasses and cadavers.

There were 13 Sphingobacteriales sequences identified from three genera (Sphingobacterium, Pedobacter and Chitinophaga). These are strictly aerobic organisms from the Bacteroidetes phyla that have been isolated from a variety of sources including soil, clinical specimens, insect guts (Brinkmann et al., 2008), compost (Yoo et al., 2007) and sewage systems (Okabe et al., 2007). The Chitinophaga are chitinolytic environmental organisms (Sly et al., 1999; Kim et al., 2006).

The Burkholderiales (Proteobacteria) are environmentally derived aerobic Gram negative organisms from a variety of ecological niches, but the pathogenic species are the best studied (Kim et al., 2006). The closest matches in this study were from six different genera and all from soils (de Boer et al., 2001; Kim et al., 2006) except for one identified in fruit (Magalhaes et al., 2001). Three sand samples contained sequences most closely resembling those from Burkholderiales, with one of those samples collected under a pig carcass that 
was not allowed to be colonised by insects. This suggests that there may have been some contamination of the sand plots by surrounding soil, or that these organisms were introduced to the plot with or derived from the carcass it self. The pig carcasses were rinsed with a hose before placement in the colonisation-prevention cages, but adhering soil could have been inadvertently introduced on the pig's skin.

The Bacillales are a diverse group of Gram positive organisms associated with many diverse environments (Madigan et al., 2000). All of the Bacillales sequences matched most closely mainly to organisms associated with degradation of animal products (unpublished, RDP accession number DQ364588) and faecal sources (Enticknap et al., 2006), but two sequences were from soil (Abd El-Rahman et al., 2002; Zou et al., 2007). Bacillales were detected in both sand and soil samples making it likely that at least some were derived from the carcasses/cadavers.

The Caulobacteriales are Gram negative dimorphic prosthecate bacteria (have cellular appendages) that are usually associated with low nutrient systems, particularly water (Abraham et al., 2008) but have also been identified in sludge (Ryu et al., 2007) and soil (Yoon et al., 2007). Two genera, Brevundimonas and Phenylobacterium were detected in this study and the closest matches were isolated from soil (unpublished, RDP accession number EF601821), drinking water (Williams et al., 2004), sludge (unpublished, RDP accession number DQ168829) and swine-manure impacted soil (unpublished, RDP accession number EF471237).

The Rhodobacterales genera that were identified in this study were Paracoccus and Rhodobacter. These genera are metabolically versatile and are widely distributed in soil, 
sediment and activated sludge environments. They take part in bioconversion of organic and inorganic compounds (Liu et al., 2006).

\subsubsection{Full clone libraries - Samples V12 and Z11}

The two clone libraries that were sequenced in full (samples V12 and Z11 - both cadavers on sand, non-autopsied and autopsied respectively) were quite different in their composition. The most frequently detected order in both was the Clostridiales, but the dominance of this group was much more pronounced in the sample Z11 library with $80 \%$ of the sequences most closely matching this order. These two cadavers were reasonably similar, both were middle-aged females (42 and 54 years of age). Two differences were that cadaver $\mathrm{Z}$ was autopsied, while $\mathrm{V}$ was not, and that cadaver $\mathrm{Z}$ was larger at $100 \mathrm{~kg}$ compared with $55 \mathrm{~kg}$. Many Clostridium species are known to produce powerful lipolytic enzymes and have previously been associated with human decomposition under anaerobic conditions (Forbes, 2008). Cadaver Z had greater fat deposits than cadaver V, which may have favoured Clostridiales growth.

Because both of these cadavers decomposed on sand, it can be assumed that most of the bacterial species detected derived from either the cadaver itself or from colonising insects. The differences between these two cadavers most likely reflects the differences in natural microflora. Cadaver V had cancer listed as cause of death, the treatment of which prior to death may have affected her microflora, while cadaver $\mathrm{Z}$ died of unknown causes suddenly. Cadaver Z, with a BMI of 35.4 would be considered to be obese $(>30)$, which is known to affect gut microflora by increasing the proportion of Firmicutes, which includes the Clostridiales (Ley et al., 2005; Ley et al., 2006). Previous studies have found that the increase in Firmicutes is offset by a proportional decrease in Bacteroidetes (Ley et al., 2005), but this was not seen in this study. The Pseudomonadales and Xanthomonadales 
(both Proteobacteria) were the two orders which decreased in the V12 library. The differences between these two cadavers demonstrates how influential the body's own microflora is in which organisms are detected during the decomposition process and how this aspect must be considered in development of any future time since death estimation tool.

\subsubsection{Summary}

Many of the bacterial species identified using cloning and sequencing as being associated with decomposing cadavers are well known as organisms involved with the aerobic spoilage of meat, fish and milk (Garcia-Lopez et al., 1998; Holzapfel, 1998). This is a logical finding given that biologically speaking, carcasses and cadavers are composed of the same organic compounds as these food products. One big difference is that in most food products, the existing microflora would not be as extensive as with an entire carcass or cadaver. Contamination of meat can occur during processing, but properly processed carcasses can be regarded as sterile (Gill, 1998). The organisms usually associated with spoilage of meat (primarily Gram negative genera: Pseudomonas, Flavobacterium, Moraxella, Acinetobacter, Enterobacteriaceae, Vibrio, Aeromonas, Shewanella, Alteromonas, Alcaligenes, Psychrobacter) are widespread in abattoirs and meat processing plants and contaminate the meat products during processing and handling (Garcia-Lopez et al., 1998).

A recent paper examining the antimicrobial activity of maggots (Jaklic et al., 2008) found that Gram positive organisms are largely susceptible to the antimicrobial properties of maggot excreta/secreta, whereas most Gram negative species tested were not susceptible and were often found colonising maggots. Most of the species isolated from macerated maggots were related to Providencia, Proteus and Pseudomonas, all of which were 
identified as being associated with the decomposition events in this study. There was, however, a fairly even mix of Gram positive and negative organisms identified from the decomposition samples, indicating that this selective pressure was not strong in this environment.

An interesting observation was that many of the papers describing the research in which most closely matching sequences in the RDP database were found, were based around tetracycline resistance. Organisms from six different orders (Flavobacteriales, Bacteroidales, Xanthomonadales, Burkholderiales, Pseudomonadales and Clostridiales) were associated with four different studies examining the presences of tet genes in mixed bacterial samples from various environments (unpublished, RDP accession numbers EU442256 and EF471219) (Chee-Sanford et al., 2001; Macauley et al., 2007). The database sequence which returned the most hits in this study (S000893094 bacterium 20RD; EF640582) was from one of these studies (Macauley et al., 2007). Further Clostridiales sequences also most closely matched organisms from studies of vancomycin resistance (NZFSA, 2003) and some Pseudomonadales to a sulphonamide resistance (unpublished, RDP accession number EU370418). The matching of sequences to those from antibiotic resistance studies was not specific to just pig carcasses or just cadavers, and were not soil or sand sample specific. It was also not specific to particular cadavers who may have ingested the antibiotic tetracycline prior to death. While tetracycline is a commonly used antibiotic, including in conventional pig farming (Farrow et al., 1995) the pigs used in this study were certified organic, so should not have been exposed to this antibiotic. Human exposure to tetracycline is also high, with it being one of the most commonly prescribed antibiotics for over 50 years (Chopra and Roberts, 2001). Resistance to tetracycline is also common in both environmental and microfloral species, with most bacterial orders, both Gram positive and negative, containing species that have acquired resistance. The tet genes 
are often associated with conjugative and mobilisable genetic elements, as well as with multiple antibiotic resistance (Chopra and Roberts, 2001).

It is possible that antibiotic resistant organisms, particularly non-culturable environmental isolates are over-represented in the RDP database. Because the database is populate with sequences uploaded by researchers, those with a particular quirk, or phenotype of interest are likely to be studied more than unknown organisms of unknown significance. The number of unknown organism sequences, however, is ever-increasing with large-scale sequencing methods gaining popularity in environmental molecular ecology.

\subsection{Conclusions}

The aim of this chapter was to try to gain a more in-depth understanding of the process of decomposition and smaller-scale changes occurring within the greater bacterial communities, with the overall aim of identifying the bacterial species contributing to the peaks/bands seen in the profiling methods, in the hope that bacterial biomarkers for time since death could be identified. The information gained in this chapter is correlated with the community T-RFLP and biochemistry data from previous chapters, in chapter 8 .

\subsubsection{Experimental Approach}

Four different methods were used to explore the composition of the bacterial communities associated with decomposition. Three of these successfully contributed to gaining more of an understanding, while one method (sequencing from DGGE bands) did not work at all. A possible alternative to trying all these different approaches would have been to concentrate time and money on just one approach, allowing for more samples or T-RFs to have been investigated. In retrospect, it is fortuitous that multiple methods were trialled, as the method of choice would likely have been sequencing of the DGGE bands. 
The greatest difficulties were encountered with DGGE. Along with the inability to successfully excise bands for sequencing there were intermittent problems with clarity of the banding pattern, uneven staining, poor gradient formation and apparent low diversity with some of the primer choices. The DGGE method is also time-consuming compared with T-RFLP, and it seemed to be that pure luck dictated whether a gel would have good, clear band separation.

The multiple-digest method was successful in predicting many of the bacterial orders that were identified using cloning and sequencing. Many more orders were predicted than were actually found to be contributing to the T-RFs, but many of the predicted potentially contributing orders could be ruled out as unlikely to be associated with a decomposition event. This method is a cost-effective option for gaining a rough estimation of bacterial groups that may be contributing to T-RFs in a profile. It could be improved by increasing the number of restriction enzymes used, or by digesting products with multiple enzymes in the same reaction, which could narrow the number of potential contributors further.

Cloning and sequencing is a commonly used method for examining diversity within mixed bacterial communities. The clone pooling method described by Grant and Ogilvie (2004) was reasonably successful in allowing the targeted selection of key T-RFs for investigation. It became apparent that the correct selection of analysis parameters was important, but reasonable coverage of the T-RF sizes seen in the original profiles was obtained with the number of clones selected. The use of this method allowed identification of organisms contributing to different T-RFs for a fraction of the cost that sequencing entire libraries would have cost, and enabled somewhat targeted selection of T-RFs of interest as opposed to random clone selection, which probably would have resulted in lesser coverage of the 
different T-RF sizes. This method did restrict the quantitative analyses that could be performed using the data, but because cloning has associated biases, just how accurate quantification is using small libraries is open to debate.

\subsubsection{Insights into decomposition bacterial communities}

The experiments outlined in this chapter provided different insights into the bacterial communities.

The group specific PCR allowed groups of varying taxonomic level to be examined and resulted in the detection of a number of groups in various samples. Each of the bacterial groups examined was present in a different combination of samples but none except the Bifidobacteria group were correlated with particular stages of time-points during decomposition using presence/absence.

This experiment suggested that the bacterial community in the soil underlying a cadaver has a mixture of both soil or environmentally derived organisms, as well as enteric organisms from the cadaver itself, perhaps even with a dominance of enteric organisms. The reduction in diversity seen in the soil actinobacterial communities in response to the decomposition events, suggested that some members of the soil community could not compete effectively in the altered environmental conditions. The effect of the soil environment in comparison to the sand environment (which was much simpler and did not contain an existing microflora) was seen by the presence of some groups in both the soil and sand samples, but with different DGGE banding patterns depending on the substrate. Some groups, such as the Clostridium coccoides group were present in the soil control samples, but the effect of the addition of the cadaver derived species could not be determined due to poor resolution of the DGGE gels. Conversely, some groups (such as the Enterococcus group) were detected in sand, but not in soil, indicating that these 
organisms were perhaps not able to compete successfully and survive in the more complex soil environment.

The group specific DGGE did not detect any particular bands associated with specific stages of decomposition. This was complicated by the poor quality of the gels and the variability between cadavers. Many bands from many gels were found to be specific to a particular cadaver, indicating that the body's own highly variable combination of microfloral species was detectable. It seems that the higher group levels used in this experiment would not be suitable as indicators of time since death as those that were detected in samples from many different cadavers were almost ubiquitously present. When diversity within those groups was examined, however, it appeared that individual microfloral diversity was too great for any clear bio-indicators to be discerned.

The group specific DGGE detected the presence of some groups that were not detected by cloning or predicted by the multiple digest experiment. This is perhaps not surprising as many of the DGGE groups may have been present in very low numbers which would only have been detected by sequencing large numbers of clones. The virtual digest did contain members of these groups, suggesting that their omission was due to the limitations of the multiple digestion experiment (as discussed in section 7.4.3).

The cloning and sequencing approach showed slightly different findings to the groupspecific DGGE approach. Many of the bacteria associated with decomposition, as found in the substrate underlying the body, appear to derive from the substrate itself (i.e. soil) as well as the cadaver/carcass, and possibly from colonising insects. A mix of aerobic and anaerobic organisms could be detected in the underlying substrate, suggesting that a complex ecosystem is likely to exist, perhaps with pockets of different oxygen availabilities occurring. 
The sequencing experiment identified many bacterial orders which were associated with decomposition. Pseudomonadales, Clostridiales and Flavobacteriales were the closest matches to many sequences, suggesting that these groups may be important members of the decomposition environment and worth investigating further. These orders were also found to be contributing to multiple T-RFs and many T-RF sizes were found to be contributed to by multiple bacterial orders. Other orders, such as Burkholderiales, Rhizobiales and Sphingobacteriales were detected much more sporadically. No orders were associated specifically with a particular time-point, although this was not an expected outcome of this experiment, given that so few clones were sequenced from each sample.

This series of experiments confirmed that the interplay of bacterial organisms in the bacterial community underlying a cadaver is complex, with different groups responding to the decomposition event in different ways. The differences between the soil and sand plots suggest that in developing a time since death indicator system, various soil types would need to be investigated for their effects on the indicator organisms. These experimental results did not show a clear progression during the course of decomposition that the original whole community profiles did. The reasons behind this are explored further in chapter 8. None of the groups examined stood out as obvious contenders for biomarker contention although further investigation using better methods such as T-RFLP instead of DGGE may detect time-dependant changes in specific community structures. The cloning and sequencing experiment did identify other bacterial orders that are associated with decomposition which could be examined more closely. The ability to detect enteric organisms, which are not normally present in soil supports the feasibility of using microbes for time since death estimation, but more work is required to identify exactly which organisms, if any, are time-specific. 


\section{Chapter 8: Discussion}

The research outlined in this thesis is an examination of the bacterial communities associated with decomposition of humans (or model organism Sus scrofa) on soil and sand, with the goal of garnering a better understanding of the role bacteria play in a decomposition event. A broad investigative approach was taken to this research because scientific understanding of decomposition microbiology was very limited at the outset of this study. A large body of information has been amassed by using a number of soil chemistry measurements, enzyme assays and molecular fingerprinting and identification methods, coupled with observation of the decomposition process and of environmental variables. Combining these into a comprehensive picture of decomposition bacteriology was challenging. Key findings are outlined in this chapter and the results discussed in terms of the influencing factors and processes that may be occurring in the bacterial community associated with a decomposing human body. Time-lines of various carcass and cadaver decomposition events are included (Figures 8.1 to 8.4) to illustrate the interaction between physical and chemical changes during decomposition and bacterial community findings.

\subsection{Bacterial Community Complexity}

The total bacterial community associated with a decomposing carcass or cadaver appears to be reasonably complex, with a diverse array of organisms involved. Both the DGGE and T-RFLP profiling methods demonstrated this with substantial numbers of different TRFs and bands generated from samples on both soil and sand (a mean of 36 T-RFs per profile across all experiments). Some 58 different genera from six different phyla were identified as being present in the underlying bacterial community of at least one 
decomposition event. In some respects, this level of diversity was somewhat unexpected given the huge environmental perturbations that a decomposition event causes (discussed further in section 8.2) which could be expected to be unsuitable for survival of many bacterial species. The huge influx of different organic nutrients, however, is likely to promote an increase in overall microbial biomass, and may provide opportunities for organisms with different substrate utilisation abilities to compete successfully (discussed further in section 8.3).

Identification, via sequencing, also showed that the bacterial species detected had diverse nutritional requirements and environmental tolerances with aerobic organisms such as Pseudomonadales being detected in the same samples as obligately anaerobic bacteria such as Clostridiales and Sphingomonadales. Both Gram positive and negative organisms were found to be members of the bacterial communities. This suggests that the environment that is created by a decomposition event is not simple or homogeneous and may contain a range of microhabitats that allow such a diverse range of phenotypes to survive. This is quite possible, as decomposition fluids were not uniformly dispersed. Purged body fluids collected in pools around the body openings and a thick slime-like layer often formed at the interface between the carcass/cadaver and the underlying substrate, which could have provided the necessary conditions for anaerobe survival.

It is possible, however, that with the DNA-based profiling methods used in this study, nonviable organisms were being detected, falsely indicating that these organisms were surviving (England and Trevors, 2003; Levy-Booth et al., 2007). This is a possibility that must be considered when using DNA rather than RNA for community profiling. Even if these bacteria were surviving, the DNA-based methods cannot determine if those organisms are active members of the community or simply surviving, waiting for more suitable conditions for proliferation. It was felt, however, that the environmental 
conditions the non-viable cells and naked DNA would be exposed to during decomposition would prevent long-term survival. The T-RFLP profiles showed sequential community change as decomposition progressed, with individual T-RFs from early decomposition stages not being detected in later stages, which would suggest that residual DNA from dead organisms was being degraded.

\subsubsection{Sequential Community Change}

By using community "fingerprinting" methods T-RFLP and DGGE, and visualising the community changes over time using multi-dimensional scaling (MDS), the bacterial communities in soil and sand were found to change in a very sequential manner. Communities tended to undergo small composition changes between each sampling time, followed by occasional bigger shifts. There was almost always a shift after the first or second sampling, and this occurred in both soil and sand plots. This shift corresponded loosely with the beginning of the purging of fluids from the carcasses or cadavers (discussed further in section 8.3.1) although the ADD values differed for when these visual events occurred. Subsequent shifts did not correlate with particular ADDs or with obvious visual changes in the stage of decomposition. The same general pattern of sequential change occurred in all decomposition plots (less so for cadaver $\mathrm{R}$ which underwent particularly slow decomposition), with a few major community shifts early on in decomposition, followed by a series of smaller changes, as seen in the MDS analysis of the profile data. The same prominent T-RF size groups were identified in all the carcasses and cadavers, although the time-points when these appeared/disappeared differed. There were also multiple bacterial orders found to be contributing to each of these T-RFs so it is not surprising that these dominant T-RFs were common to all decomposition events. When all the less prominent T-RFs were considered, the replicate cadavers, in particular, did not 
show high similarity to each other in terms of overall community profile pattern. This suggests that individual microflora and body composition have an effect on the bacterial community composition.

\subsubsection{A mixing of communities}

The experiments on soil and sand and the identification of organisms through sequencing demonstrated that the members of the bacterial communities associated with a decomposition event are contributed by the soil (if present), the cadaver itself, and probably from colonising insects and the wider environment. Often, the source of the closest match to the sequences gave some clues as to their likely origin, for example, some organisms were associated with sewage sludge and pig slurries, which strongly suggested that they originated from the gut of the carcass/cadaver. Many of the bacterial orders identified in the sequencing exercise were found to be associated with multiple places of origin, for example, from insects as well as human and swine gastrointestinal tracts (Providencia spp.). Diverse orders such as the Clostridiales have members associated with both soil and the human and pig gastrointestinal tracts, so it is not possible to unequivocally state from where these organisms derived. The organisms found in sand samples from under the carcasses not colonised by insects, however, can be assumed to have originated from the carcasses themselves. Orders that had carcass-derived members were: Clostridiales, Pseudomonadales, Burkholderiales, Sphingobacteriales and Enterobacteriales. Burkholderiales are aerobic organisms most commonly associated with soils, so it is possible that these derived from soil adhering to the pig carcasses rather than the carcass itself. Similarly, Pseudomonadales are aerobic organisms not usually considered to be human or pig microflora, but are ubiquitous in the wider environment. These are likely to have been introduced by movement and handling of the pigs and from 
the non-sterile tubs and cages used. Elimination of all non-carcass organisms would have been impossible as sterilisation of the outside of the carcass would have destroyed the skin microflora, so the introduction of some organisms that are not truly microflora is preferable.

A number of organisms were found to be associated only with soil samples and came from the Actinomycetales, Acidobacteriales, Sphingomonadales and Myxococcales orders, and from the Deltaproteobacteria class. 


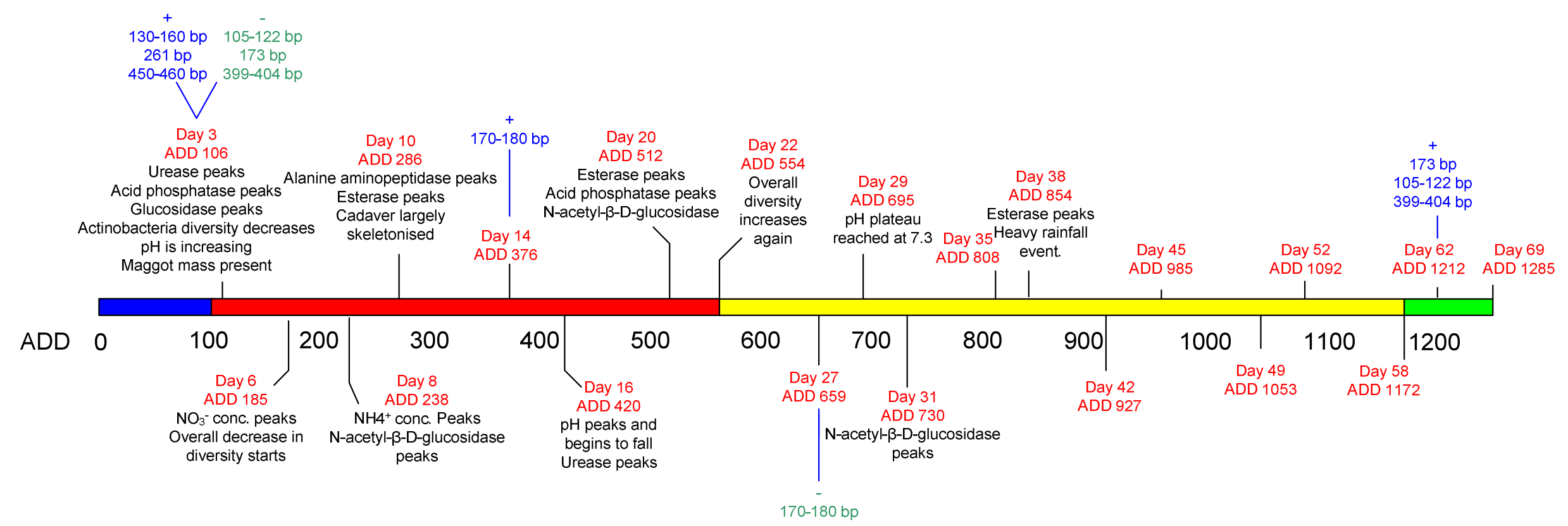

Figure 8.1 Time-line of events during decomposition of cadaver P (non-autopsied on soil). Changes in the timeline colour indicate community composition shifts determined by MDS analysis of T-RFLP data. Red text denotes sampling times. Black text details events related to decomposition. Blue text indicates prominent T-RF sizes that appear and/or disappear in T-RFLP profiles.

Possible identifications for prominent T-RFs from sequencing exercise - 130-160 bp: Actinomycetales, Bacillales, Clostridiales, Pseudomonadales, Rhizobiales, Sphingobacteriales, Xanthomonadales.261 bp: Clostridiales, Flavobacteriales. 450-460 bp: Burkholderiales, Clostridiales, Flavobacteriales, Pseudomonadales, Xanthomonadales. 105-122 bp: Burkholderiales, Acidobacteriales, Actinomycetales, Bacillales, Clostridiales, Sphingobacteriales, Myxococcales, Rhizobiales, Rhodospirillales, Sphingomonadales. 173: unknown organisms. 399-404 bp: Rhizobiales, Xanthomonadales, Rhodobacterales. 170-180: Myxococcales. 
Day 3 (ADD 43) Abdominal discolouration \& skin bubbling

Day 5 (ADD 76) Carcass fully bloated
$+\quad 103-109,117-118,147-149,155-158,240-250,364,389$

Day 9 (ADD 148) Large maggot mass present

$-117-118,364-366$ bp

Day 11 (ADD 182) Maggots reducing in numbers

Day 12 (ADD 193) Legs skeletonised and disarticulated

+240-250 bp - 104-109, 240-250 bp

Day 14 (ADD 225) Second maggot mass formed

Day 15 (ADD 242) Carcass and sand drier

Day 16 (ADD 259) Maggot mass migrating away from carcas

Day 18 (ADD 293)

$+60-65$ bp $\quad-481-499$ bp

Day 22 (ADD 361) Mostly skeletonised with a dry skin layer

400

Day 26 (ADD 433)

+ 87-92, 102-103 bp, 110-114, 143-146 bp, 206-208 bp

500

Dat 28 (ADD 465 ) Flies emerging from pupae

1500

Completely skeletonised with dry skin layer $-60-65$ bp
7 (ADD 114) Magots present. Tearing of abdominal skin
+50 . 124-126, 258-272, $457-462$ bp $-147-149,155-158,389,422$

Day 28 (ADD 424-429 bp, 469-479 bp
$A D D$

100

\section{Day 70}

Day 3 (ADD 43) Abdominal discolouration \& skin bubbling

Day 5 (ADD 76) Carcass fully bloated. Many fly eggs on face.
$+65-67,103-108,116-118,144,149,155-157,245-248$,

Day 7 (ADD 114) Mag, $(+24-426,447$

Day 9 (ADD 148) Large maggot mass around head

103-108, 116-118, 144, 149, 155-157, 424-426 bp Day 11 (ADD 182) Skin slippage when carcass moved
Day 12 (ADD 193) Large maggot mass around rear

Day 14 (ADD 225) Heavy abdominal drainage

Day 15 (ADD 242) Carcass and sand drier

$300 \quad \begin{aligned} & \text { Day } 18 \text { (ADD 293) } \\ & +93-96 \text { bp }\end{aligned}$

400

\section{Day $26($ ADD 433$)$
$+111-115,267-272,474-475$ bp}

500

Day 40 (ADD 637) Most soft tissue gone, sand drying

Day $50($ ADD 759)
$-111-115,267-272,474-475$ bp

${ }_{1400}$

1400

Day $115(\mathrm{ADD}, 140)$
$103-108,144,193,430-433$

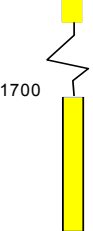

Day 3 (ADD 43) Abdominal discolouration

Day 5 (ADD 76) Carcass fully bloated

Day 7 (ADD 114) Skin slippage when carcass moved
$+65-67,103-109,116-118,144,147-149,155-158$

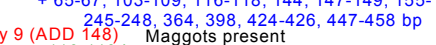

- $116-118$ bp

200

Day 12 (ADD 193) Legs skeletonised and disarticulated

Day 15 (ADD 242) + 260-262, 481, 459-462 bp - 103-109, 144, 149, 155-158, 424-426 bp

Day 16 (ADD 259) Heavy abdominal drainage

Day 18 (ADD 293) Large maggot mass

$+93-96 \mathrm{bp}$
400

?

800

Day 52 (ADD 788) Most soft tissue gone, sand drying

Day $115($ ADD 1440)
$+103-108,144,193,430-433$

700
Figure 8.2 Time-line of events

during decomposition of pig

carcasses $A, C$ and $E$ (on sand, with insect colonisation). Changes in the timeline colour indicate community composition shifts determined by $M D S$ analysis of T-RFLP data. Blue text indicates that those T-RF sizes appear in the T-RFLP profile and green denotes those that disappear. 
Possible identifications for prominent T-RFs from under carcases $A, C$ and $E$ (Figure 8.2) from sequencing exercise - 103-109 bp: Burkholderiales, Acidobacteriales, Actinomycetales, Bacillales, Clostridiales, Sphingobacteriales. 117-118 bp: unknown organisms. 147-149 bp: unknown organisms. 155-158 bp: Deltaproteobacteria, Clostridiales, Sphingobacteriales, Xanthomonadales. 240-250 bp: Actinomycetales, Clostridiales. 364 bp: Caulobacterales, Rhizobiales, Sphingomonadales. 389 bp Flavobacteriales. 398 bp: Rhizobiales, Xanthomonadales. 433 bp: Bacteroidales, Clostridiales, Pseudomonadales. 437 bp: Bacteroidales, Clostridiales,

Pseudomonadales, Xanthomonadales. 448-456 bp: Burkholderiales, Clostridiales, Flavobacteriales, Xanthomonadales. 481-499 bp: Bacillales, Clostridiales,

Deltaproteobacteria, Flavobacteriales, Pseudomonadales, Sphingobacteriales.

50 bp: Actinobacteria, Actinomycetales, Lactobacillales, Pseudomonadales, Rhizobiales, Sphingobacteriales. 124-126 bp: Actinomycetales, Bacillales, Pseudomonadales, Rhizobiales, Xanthomonadales. 258-272 bp: Acidobacteriales. Clostridiales, Pseudomonadales. 457-462 bp: Enterobacteriales, Flavobacteriales, Pseudomonadales, Xanthomonadales. 60-65 bp: Acidobacteriales, Bacteroidales, Flavobacteriales, Xanthomonadales.

206-208 bp: unknown organisms. 424-426 bp: Clostridiales. 87-92 bp: unknown organisms. 102-103 bp: Myxococcales. 110-114 bp: Acidobacteriales, Actinomycetales, Bacillales, Burkholderiales, Clostridiales, Sphingobacteriales. 143-146 bp: unknown organisms. 206-208 bp: unknown organisms. 424-429 bp: Clostridiales. 469-479 bp: Bacillales, Clostridiales, Deltaproteobacteria, Flavobacteriales, Pseudomonadales, Sphingobacteriales. 


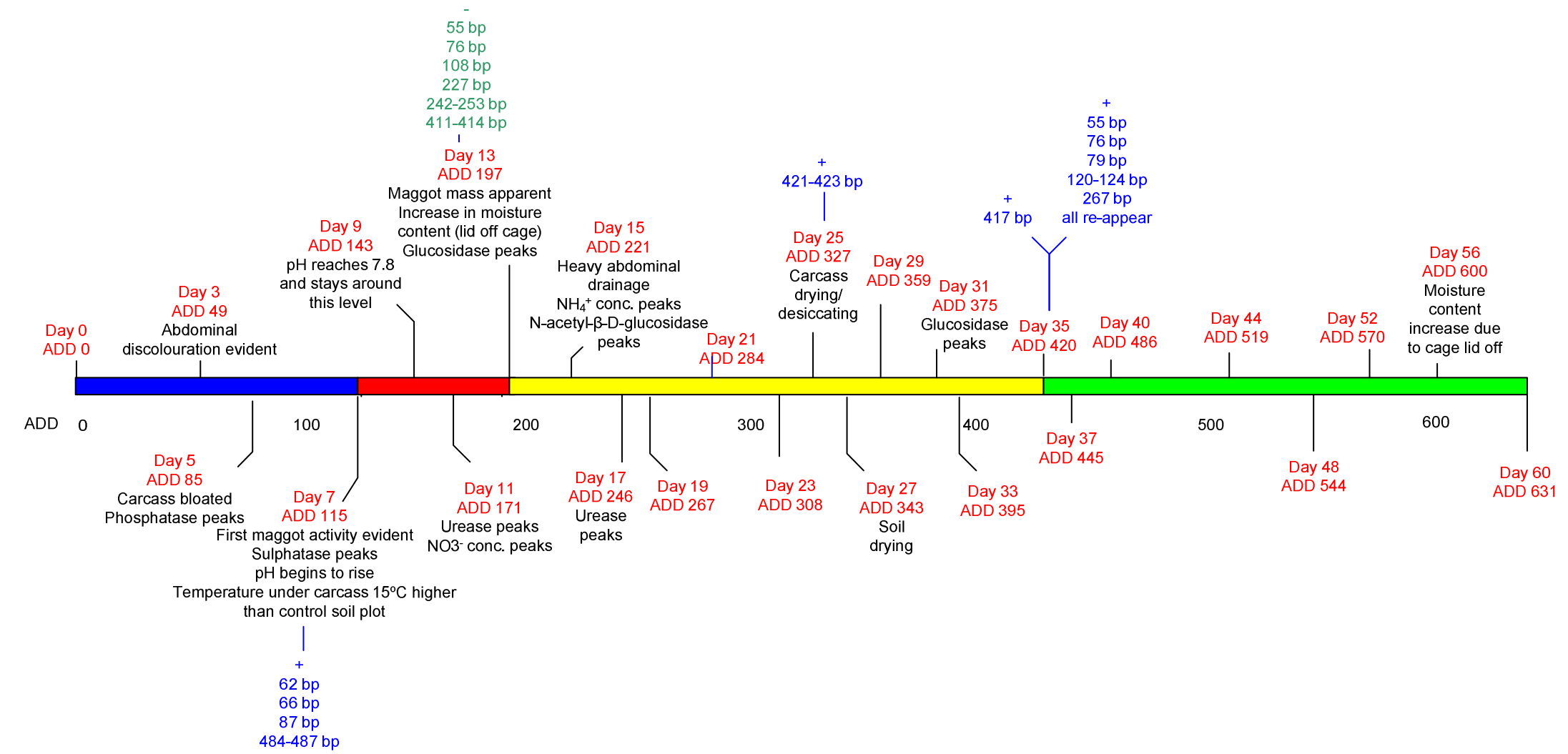

Figure 8.3 Time-line of events during decomposition of carcass $\boldsymbol{G}$ (on soil). Changes in the timeline colour indicate community composition shifts determined by MDS analysis of T-RFLP data. Red text denotes sampling times. Black text details events related to decomposition. Blue text indicates T-RF sizes that appear and/or disappear in T-RFLP profiles.

Possible identifications for prominent T-RFs from sequencing exercise - 62 bp: Acidobacteriales, Bacteroidales, Flavobacteriales, Xanthomonadales. 66 bp: unknown organisms. 87 bp: unknown organisms. 484-487 bp: Bacillales, Clostridiales, Deltaproteobacteria, Flavobacteriales, Pseudomonadales, Sphingobacteriales. 55 bp: Burkholderiales, Flavobacteriales, Lactobacillales, Pseudomonadales, Rhizobiales, Sphingobacteriales. 76 bp: unknown organisms. 108 bp: Acidobacteriales,

Actinomycetales, Bacillales, Burkholderiales, Clostridiales, Sphingobacteriales. 227 bp: Acidobacteriales. 242-253 bp: Actinomycetales, Clostridiales. $411-414$ bp: Rhodospiralles. 421-423 bp: unknown organisms. 417 bp: Xanthomonadales. 


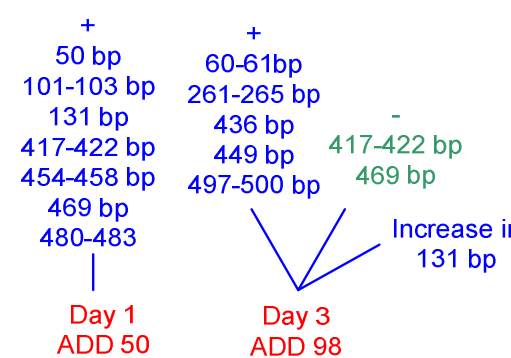

ADD $50 \quad$ ADD 98

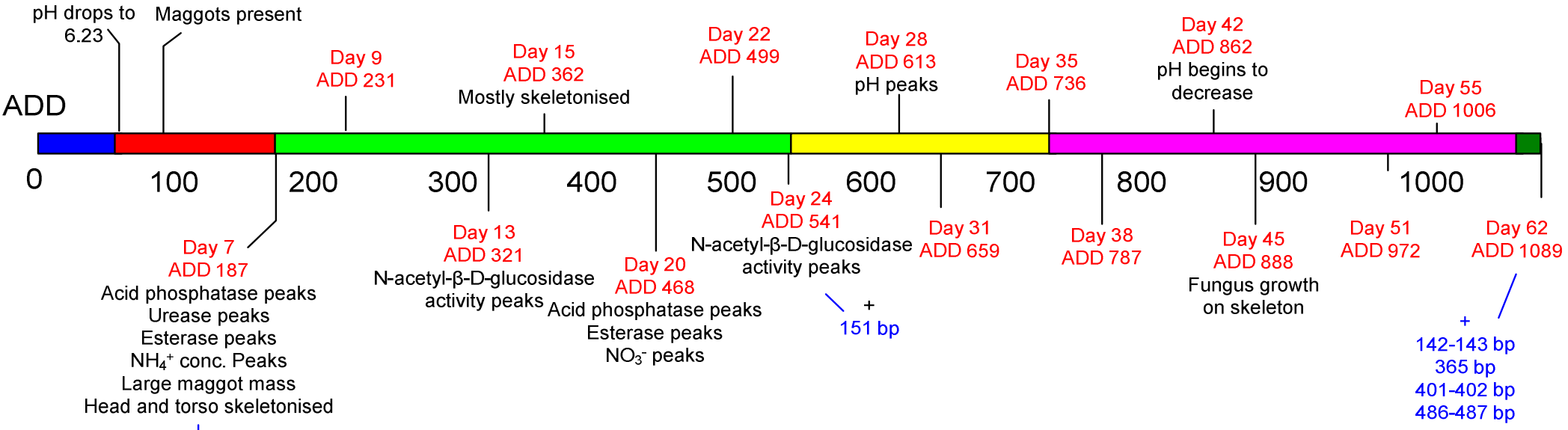

Head and torso skeletonised

486-487 bp

$$
\begin{gathered}
+ \\
257-259 \mathrm{bp} \\
460-461 \mathrm{bp}
\end{gathered}
$$

Figure 8.4 Time-line of events during decomposition of cadaver T (autopsied, on sand). Changes in the timeline colour indicate community composition shifts determined by MDS analysis of T-RFLP data.. Red text denotes sampling times. Black text details events related to decomposition. Blue text indicates T-RF sizes that appear and/or disappear in T-RFLP profiles.

Possible identifications for prominent T-RFs from sequencing exercise - 50 bp: Burkholderiales, Flavobacteriales, Lactobacillales, Pseudomonadales,

Sphingobacteriales. 101-103 bp: Myxococcales. 131 bp: Actinomycetales, Bacillales, Pseudomonadales, Rhizobiales, Xanthomonadales. $417-422$ bp: Xanthomonadales, Clostridiales. 454-458 bp: Burkholderiales, Clostridiales, Enterobacteriales, Pseudomonadales, Xanthomonadales. 469 bp: Bacillales, Clostridiales,

Deltaproteobacteria, Flavobacteriales, Pseudomonadales, Sphingobacteriales. 480-483 bp: Bacillales, Clostridiales, Deltaproteobacteria, Flavobacteriales, Pseudomonadales, Sphingobacteriales. 60-61 bp: Acidobacteriales, Bacteroidales, Flavobacteriales, Xanthomonadales. 261-265 bp: Clostridiales, Flavobacteriales, Pseudomonadales. 436 bp: Bacteroidales, Clostridiales, Pseudomonadales, Xanthomonadales. 449 bp: Flavobacteriales. $497-500$ bp: Bacillales, Clostridiales, Deltaproteobacteria, Flavobacteriales, Pseudomonadales, Sphingobacteriales. 257-259 bp: Acidobacteriales. 460-461 bp: Enterobacteriales, Flavobacteriales, Pseudomonadales, Xanthomonadales. 151 bp: unknown organisms. 142-143 bp: unknown organisms. 365 bp: Caulobacterales, Rhizobiales, Sphingomonadales. 401-402 bp: unknown organisms. 486-487 bp: Bacillales, Clostridiales, Deltaproteobacteria, Flavobacteriales, Pseudomonadales, Sphingobacteriales. 


\subsection{Physical Environment Changes}

Changes in the physical environment under a decomposing carcass were found to affect the composition of the bacterial community. Most environmentally derived bacterial species can tolerate moderate variability in environmental conditions, whereas many human derived organisms are more adapted to the conditions of the human body, which tend not to vary widely temporally or between individuals. A decomposition event results in a massive influx of organic substances, many of which alter the physical environment in which the bacterial community resides. Understanding some of these changes can help interpret the changes seen in the bacterial communities under the carcasses and cadavers in this study.

\subsection{1 pH}

An increase in the $\mathrm{pH}$ of the substrate underlying the decomposing carcasses and cadavers was common to all decomposition events. Some caused a rapid increase (such as cadaver P, Figure 6.11) while others caused a more gradual $\mathrm{pH}$ rise (e.g. cadaver R, Figure 6.11). Regardless of the initial $\mathrm{pH}$ of the substrate, all decomposition events caused the $\mathrm{pH}$ to rise to between 7.5 and 8.5. This is in contrast to other work that has found the initial $\mathrm{pH}$ of soil to greatly affect the final $\mathrm{pH}$ (Haslam and Tibbett, 2009). The $\mathrm{pH}$ rise is thought to be caused by the ammonification of protein to $\mathrm{NH}_{4}{ }^{+}-\mathrm{N}$ and the concentration of $\mathrm{NH}_{4}{ }^{+} \mathrm{N}$ tended to begin to increase around the same time as the $\mathrm{pH}$ began to increase.

For all the pig carcasses on sand, the $\mathrm{pH}$ rise began immediately, and was detectable from the first sampling day onwards (see Figure 8.2). For the carcasses on soil, the $\mathrm{pH}$ rise began at day 7 (ADD 187). Changes in the $\mathrm{pH}$ were evident a little earlier for the cadavers, with an increase evident from day 3 (ADD 106) onwards for cadaver P (nonautopsied on soil, Figure 8.1), and a few days later for the other cadavers on soil. 
The autopsied cadavers on sand caused an initial decrease in $\mathrm{pH}$, before it rose steadily to around the same level as seen in the soil sites. The acidification of the blood during the early decomposition phase is thought to be responsible for this drop, as the autopsied cadavers released a noticeable amount of blood immediately on placement. This decrease did not occur in the soil underlying carcass $X$ (autopsied), probably because of the buffering ability of the soil over that of the sand.

The $\mathrm{pH}$ was only seen to decrease after this rise in three of the cadavers $(\mathrm{P}, \mathrm{X}$ and $\mathrm{T})$ which were the first three cadavers placed, and therefore had the longest sampling periods (Figures 6.10 and 6.11). The beginning of the $\mathrm{pH}$ drop did not appear to coincide with any visual change in the cadavers, but did begin roughly when the $\mathrm{NH}_{4}{ }^{+}-\mathrm{N}$ concentrations in the soil also began to decrease, in most sites. The action of fermentative organisms (Clostridium, Rhizobium, Azotobacter, Nitrosomonas) may also contribute to the $\mathrm{pH}$ drop (Gill-King, 1997; Carter and Tibbett, 2008). Most studies into burial decomposition report an acidic shift after the initial rise in pH (Haslam and Tibbett, 2009). This was not seen in any of the decomposition events in this study, as the $\mathrm{pH}$ did not return to predecomposition levels, let alone to a more acidic $\mathrm{pH}$.

In general, most bacterial and fungal groups prefer a neutral $\mathrm{pH}$, but their tolerances can vary widely. Bacteria tend to predominate in soils with a $\mathrm{pH}$ slightly above 7 , whereas in soils with a $\mathrm{pH}$ below 5.5, fungi predominate due to the lack of bacterial competition (Stotzky, 1997). The decomposition event, therefore, should create conditions that favour bacterial growth rather than fungal, although quite heavy fungal growth was noted on the skin and bones of many of the carcasses and cadavers. The rise in $\mathrm{pH}$ is likely to favour the body derived organisms more than the soil flora, so some soil organisms may respond negatively to the $\mathrm{pH}$ rise. This may account for the loss of some profile T-RFs from the early soil site samples (e.g. cadaver P Figure 8.1). Many of the organisms identified in the 
sequencing exercise, such as the Clostridiales, Pseudomonadales and Enterococcales tolerate a wide $\mathrm{pH}$ range and so may outcompete those organisms which are more fastidious.

The increase in $\mathrm{pH}$ was a dramatic change to the soil environment and was likely a contributing factor to the demise of some organisms and the proliferation of others.

\subsubsection{Moisture Content}

The human body is around $72 \% \mathrm{H}_{2} \mathrm{O}$ (Schoeller, 1989), and pig carcasses about the same (Gnaedinger et al., 1963; Sheng and Huggins, 1979). An average human body of $75 \mathrm{~kg}$ could therefore be expected to release some $54 \mathrm{~L}$ of liquid into the surrounding environment as it decomposes. Microbes are strongly affected by the moisture levels in their environment, as this affects access to nutrients and oxygen. Low moisture levels inhibit microbial processes by lowering intracellular hydration and enzyme activity, and also by restricting substrate supply (Stark and Firestone, 1995). In soil, moisture content higher than about $50 \%$ of the water holding capacity of the soil leads to decreased aerobic bacterial survival because the dissolved oxygen is reduced to a level that can no longer support aerobic respiration.

The moisture content of the underlying substrates was measured in the second pig carcass experiment and the human cadaver experiment (Figures 5.5 and 6.8). Fluctuations in moisture content, some quite dramatic, were caused by rainfall events in both of these experiments. Short term, moderate rainfall events are unlikely to have long-term effects on the moisture content and oxygenation of soil, but very heavy or prolonged rainfall is likely to waterlog the soil. A very heavy rainfall event resulted in a dramatic but temporary increase in moisture content in the cadaver $\mathrm{P}$ site. In this case, the increase in moisture content did not correspond to any change in the visual state of the cadaver or to a 
shift in the bacterial community (Figure 8.1 ). The second pig carcass experiment also experienced a series of heavy rainfall events that led to some of the sites becoming waterlogged. The sand tubs, in particular, did not drain well and the maggots were drowned. Surprisingly, samples from these plots at the times when they were waterlogged contained predominantly aerobic organisms (as identified in the sequencing exercise). Flavobacteriales and Pseudomonadales were identified, whereas if the plots had very low oxygen levels, one would expect to find anaerobes such as Clostridiales predominating. This suggests that the impact of soil moisture on the bacterial community may not have been as strong as was expected or that these changes were not detected using the molecular methods.

\subsubsection{Mixing and movement by larvae}

The bacterial community, in many of the decomposition events, underwent a compositional shift at around the same time as the number of maggots present was at its greatest. It is possible that the presence of the maggots may have had an effect on the physical environment by moving and mixing the substrate. This was particularly noticeable in the pig carcass experiments where the carcasses were placed in tubs. Maggots in big masses move away from the carcass periodically to the edge of the mass to self-regulate their temperature (Dr Dallas Bishop, Forensic Entomologist, Agriquality, pers. comm.). This movement by the maggots shifted the position of the carcass itself, so it is likely to have some effect on the bacterial environment. One probable result is that the substrate becomes more aerated. This would encourage the proliferation of the aerobes associated with decomposition. The carcass with the largest maggot mass noted was

carcass A, which decomposed slightly faster than the two replicate carcasses. The bacterial orders identified by sequencing from the day 7 (ADD 114) sample, which had a 
growing maggot mass present, were primarily aerobic (Pseudomonadales and Flavobacteriales). A secondary effect may have been the reduction in the moisture content of the substrate. Maggot masses raise the temperature of the cadaver considerably, with differences of up to $13^{\circ} \mathrm{C}$ from the control soil temperature recorded in this study. The sand under the carcasses that had large maggot masses was visually drier than the sand under the non-colonised carcasses. These warmer, drier conditions are likely to have had some effect on the bacterial communities. The increased temperature would have been particularly favourable for the body derived flora, as these organisms are most comfortable at $37^{\circ} \mathrm{C}$, but a moderate increase in soil temperature can also stimulate soil microbial activity.

\subsection{Nutritional Environment Changes}

As a cadaver or carcass decomposes, a huge amount of organic material is released into the surrounding environment. This influx of nutrients is likely to have an effect on the bacterial population within the underlying substrate. Organisms which can utilise the substrates are able to proliferate, outcompeting those that cannot use the available nutrients as effectively. The by-products of these bacterial functions will then become available for a different microbe to use, setting up a succession of growth. The input of each nutrient, however, is limited, so as each is used up, the organisms utilising them cannot proliferate any longer and decline in numbers. The organisms themselves then become a food source for other organisms.

\subsubsection{Early fluid and gut microfloral purging}

The anaerobic gut microflora begin to proliferate immediately upon death as the human cells begin to autolyse (Gill-King, 1997). These organisms are able to enjoy a period of about 5 days within the carcass/cadaver (in average temperatures such as seen in the pig 
carcass experiments, see carcasses A, C and E timeline, Figure 8.2) until the gases they produce as a by-product start to force the liquidised tissue and accompanying organisms out into the environment. The early profiles from under carcasses on sand were diverse, with many prominent T-RFs present. A number of these organisms, however, do not seem to be able to survive in the external environment for long, with community profiles changing markedly, usually within one or two samplings (2-4 days). This was supported by the DGGE group-specific PCR results, where the Enterococcus and Bifidobacterium groups were detected in early samples, but not in the later samples. The environmental conditions (section 8.2) change dramatically around this time which may make the environment less hospitable to some organisms. The $\mathrm{pH}$ begins to rise at around the same time, caused by an influx of ammonium, which is produced by the ammonifying gut organisms beginning to breakdown muscle tissue inside the carcass during the bloat stage.

A shift in the bacterial community structure is also evident after the first few samplings under cadavers and carcasses on soil (see cadaver P and carcass G timelines, Figures 8.1 and 8.3). This initial shift, however, tended to be caused by the appearance of T-RFs; some the same as in the sand (gut microorganisms) and also some specific to the soil plots. This suggests that some soil bacteria are proliferating in response to the influx of nutrients into the soil. In the cadaver derived profiles, a number of T-RFs also disappear from the profiles early on, suggesting a negative response to the changed environment by some of the organisms. These T-RFs had possible identifications that included soil organisms such as: Burkholderiales, Acidobacteriales, Actinomycetales, Bacillales, Clostridiales, Myxococcales, Rhizobiales, Rhodospirillales and Rhodobacterales, as well as unknown organisms.

This early fluid that purges as the body bloats is likely to be nutrient rich, and appears to contain a diverse bacterial population that is released into the underlying substrate early on 
in the decomposition process, affecting the structure of the bacterial community in that substrate.

\subsubsection{Active decay and soft tissue degradation}

The active decay stage, when the insect colonisers are rapidly breaking down tissues, is likely to involve huge nutrient influxes, with many different organic compounds released into the underlying substrate.

One that was measured in this study was $\mathrm{NH}_{4}{ }^{+}-\mathrm{N}$, as this is a known breakdown product of muscle tissue, produced by the ammonifying gut flora. This $\mathrm{NH}_{4}{ }^{+}-\mathrm{N}$ can be used by ammonia oxidisers, which include the Nitrosomonas bacteria, the Crenarchaeota archaea and some fungal species (Leininger et al., 2006). Some heterotrophic bacteria are also able to use $\mathrm{NH}_{4}{ }^{+}-\mathrm{N}$ as a nitrogen source, and may be more active than the nitrifying Nitrosomonas in the perturbed decomposition environment. The nitrifiers are sensitive organisms and are also strict aerobes, so if the decomposition site was to turn anaerobic, this conversion pathway would stop. No Nitrosomonas sequences were identified in the sequencing exercise, although many potential heterotrophic ammonia users were, such as Pseudomonas, Bacillus and Clostridium (Bezbaruah, 1983). The nitrate $\left(\mathrm{NO}_{3}{ }^{-}\right)$results from this study were a little confusing, as in a normal soil system, the ammonium concentration would be seen to peak before the nitrate, as the nitrifiers convert some of the ammonium to nitrate. In many of the decomposition events, a spike in the concentration of $\mathrm{NO}_{3}{ }^{-} \mathrm{N}$ was seen before $\mathrm{NH}_{4}{ }^{+}-\mathrm{N}$ reached its maximum. It is possible that in the aerobic environment expected to be present in the early stages of decomposition, the nitrifiers are able to convert some of the $\mathrm{NH}_{4}{ }^{+}-\mathrm{N}$ to $\mathrm{NO}_{3}{ }^{-}-\mathrm{N}$, but as the environment becomes more anoxic and the $\mathrm{pH}$ and other environmental conditions change, the heterotrophic organisms take over and use the ammonium to create biomass. Additionally, the archaeal and fungal 
nitrifiers may be active at this time. The $\mathrm{NH}_{4}{ }^{+}-\mathrm{N}$ concentration was seen to decrease in most decomposition plots, suggesting that it was being used and converted to other forms rather than simply accumulating in the environment.

Other nutrients expected to be released in large quantities include the break down products of adipose tissue (fat). Lipid within the tissue is broken down by intrinsic lipases into triglycerides, and then fatty acids (Forbes, 2008). In an aerobic environment, these will then be converted to aldehydes and ketones by microbial action (Arpigny and Jaeger, 1999), and in an anaerobic environment, the free fatty acids will undergo further hydrolysis and hydrogenation, often caused by action of the Clostridiales (Sledzik and Micozzi, 1997). Clostridiales were present in many of the decomposition-derived samples tested, so are likely to have been able to utilise this nutrient source.

Carbohydrate decomposition is also likely to result in a large nutrient influx into the underlying substrate. Carbohydrates in the soft tissues can be converted to glucose by various organisms, which in turn can be oxidised completely by other organisms, or incompletely decomposed to form various organic acids and alcohols (Forbes, 2008).

\subsubsection{Insect larval growth and pupation}

The presence of insects in a decomposition event has many effects, including rapid degradation of the tissues and aeration of the underlying substrate by the maggot mass (section 8.2.3). Maggots are also known to secrete antimicrobial compounds, which may also impact on the bacterial community during decomposition (section 8.4.3). The presence of maggots and adult insects also introduces chitin into the decomposition site. Chitin is the second most abundant polymer on earth after cellulose (Duo-Chuan, 2006) and forms the structural component of the cell wall in fungi and is present in the exoskeletons and pupae of insects. The maggot cuticle (skin) is made of chitin, and because 
most maggots shed this three times as they grow before forming a chitin pupal casing, chitin is quite abundant where an insect mass is present. This polymer is likely to become a food source for specialised organisms that are able to degrade it. The Actinomycetes are known to have chitinolytic enzymes, and these organisms were found to be present in some decomposition sites.

The enzyme $\mathrm{N}$-acetyl- $\beta$-D-glucosaminidase was investigated in the second pig carcass and human decomposition experiments and was found to have elevated potential activity around the times when the maggot mass was at its greatest, with high activity often still possible right through until after the maggots had pupated. This increase in potential activity suggests that chitinolytic organisms were proliferating at this time. Because Actinomycetales were not detected in all decomposition sites, it is possible that other organisms with chitinase enzymes were active, or that fungal chitinases were responsible for most of the degradation.

\subsubsection{Microbial biomass die-off}

After the expected proliferation of organisms in response to the pulse of nutrients from the cadaver, there comes a time when that nutrient load has been exhausted. Organisms will proliferate when conditions and nutrients present suit their needs, but as soon as these are exhausted or change, and other organisms can out-compete them, they will die, becoming a new source of nutrients that other bacterial species can use. This may be what was occurring when new T-RFs appeared, for the first time, later in decomposition, after active decay had slowed and skeletonisation had occurred (Figure 8.2, day 115, ADD 1440). These organisms may have also been released from the cadaver during the active decay process, but not found the conditions favourable at the time and sat relatively dormant until conditions did suit them. 


\subsubsection{Recalcitrant tissue degradation}

As discussed in section, 8.3.4, T-RFs were seen to appear for the first time late in decomposition, some as late as 115 days (1440 ADDs) after death (see timeline for pig carcasses A, C and E - Figure 8.2 and also cadaver T, Figure 8.4 day 62 ADD 1089). It is possible that these T-RFs are from organisms that are able to degrade the recalcitrant tissues (hair, nails, connective tissue and bones) remaining after all the easily degraded soft tissues have gone.

Collagen is the most abundant protein in the human body, but it has a unique triple helix structure with strong inter- and intra-molecular bonds that make it difficult for organisms to degrade (Gill-King, 1997). Connective tissues contain high levels of collagen, and bones also contain collagen. Bone is degraded in soil partly by organisms with collagenase activity. Child et al. (1995) found that a number of different organisms isolated from bones buried in soil had collagenase activity. Pseudomonadales and Xanthomonadales were some of the most commonly isolated, as well as a number of fungal species. Both of these bacterial orders were identified as being potential contributors to the T-RF sizes that appeared for the first time towards the end of decomposition.

Hair is another recalcitrant tissue that can be degraded by organisms with keratinase activity. Many bacterial and fungal organisms produce keratinases, including members of the Xanthomonas and Bacillus genera (Riffel and Brandelli, 2006), both of which were seen in the decomposition sites.

Fungal growth was noted on many of the remains, often later on in the decomposition process, so may have played a role in degradation of recalcitrant polymers, perhaps providing a new, less complex nutritional source for bacterial species. Insects such as 
beetles and moths are also known to feed on the recalcitrant tissues (Dadour and Harvey, 2008), so may also convert these into more usable substrates for bacterial species. Fungi were not examined in this study due to resource constraints, but samples from under cadavers $\mathrm{P}$ and $\mathrm{R}$ were analysed for fungal community changes using T-RFLP and phospholipid fatty acid (PLFA) analysis by another student as part of an MSc project (Parkinson et al., 2009). The samples from this cadaver and cadaver $\mathrm{R}$ were chosen for analysis because they were both cadavers placed on soil, and had the longest sampling period, giving the student more samples to work with. These two cadavers did, however, have very different decomposition rates and were very mis-matched in terms of size, meaning they were not ideal replicates. The fungal data from cadaver $\mathrm{P}$ showed a difference in overall fungal community structure to the control soil samples but there were no successional or sequential changes evident over time. The PLFA data also showed a significant difference between the control and decomposition-derived samples, but, like the fungal T-RFLP data, showed no successional changes. For cadaver R, a difference was evident in overall community structure when compared with the control soil samples and a potential temporal trend were seen, with sample data clustering together in groupings of early (ADD 0-319), mid (ADD 366-603) and late (ADD 564-797) sampling times. The PLFA data showed less separation of the decomposition and control sample data although there was still a significant difference overall. This finding fits with the overall slower decomposition seen by cadaver R. Fungal growth was noted on the skin from the early stage (around ADD 171) onwards, and was quite extensive in the late samples, which may have contributed to the differences seen between the early, mid and late stages. 


\subsection{Variables affecting the bacteria present}

\subsubsection{Individual Microflora}

Every cadaver has its own unique microflora that will affect the composition of the bacterial community associated with it as it decomposes. These differences were apparent in both the pig carcass and cadaver studies. Whilst the pig carcasses were as close to replicates as possible, they were still individuals and had different genetic influences governing their microfloral structure even though their environment was the same. Each carcass had at least a few unique T-RFs, which could have been a result of microfloral differences. The human cadaver used in this study certainly would have had quite unique bacterial assemblages. These cadavers were of all different shapes and sizes, different ages and causes of death.

Two of the cadavers used were considered to be clinically obese. When the clone library sequences from one of these (cadaver $\mathrm{Z}$ - autopsied, on sand) were compared with those from a non-obese individual also on sand (cadaver $\mathrm{V}$ - non-autopsied), a marked difference could be seen in the community composition. The obese individual had a predominance of Clostridiales in their decomposition site, whereas the smaller cadaver had a more diverse group of organisms, including Pseudomonadales and Xanthomonadales. Obesity is thought to alter the gastrointestinal flora, increasing the proportion of Firmicutes, including the Clostridiales (Ley et al., 2005). This serves to demonstrate that individual flora is likely to influence which body derived organisms are detectable in soil.

Also, demonstrated in this study, was the effect that cause of death and treatment prior to death has on the microflora, and on the decomposition process. Some of the cadavers had cancer or other long term illnesses listed as cause of death, which is often preceded by a series of treatments, many of which have strong effects on the resident microbial 
community. Antimicrobials such as antibiotics would severely disrupt normal flora structure. It is also likely that normal eating habits are disrupted prior to death, which may further alter the microbes and the human body itself. A likely example of this was cadaver $\mathrm{R}$ who had renal failure listed as cause of death. This individual was very small and had likely undergone muscle wasting prior to death.

Also possible is that cadavers, particularly those that were autopsied, were washed with antimicrobial treatments in the morgue. Unfortunately it is unknown whether this occurred, but it is quite possible. This too would affect the bacteria associated with the cadaver, which could in turn impact on the bacterial community composition during decomposition.

\subsubsection{Autopsy}

The autopsied cadavers offered an insight into how serious injury could affect the decomposition microbiology. It is known that wounds allow insects greater access to the soft tissues, sometimes resulting in a more rapid decomposition. There were no striking differences between the autopsied and non-autopsied cadavers in terms of bacterial orders detected, biochemistry and enzyme activity or in the overall pattern of community change, but there were minor differences noted. The sand underneath the autopsied cadavers experienced a rapid, but transient decrease in $\mathrm{pH}$ in the earliest samples. This was likely to have been caused by a rapid influx of acidified blood into the sand. This $\mathrm{pH}$ drop was not detected in the soil plots, most likely due to the buffering ability of the soil. One bacterial group, the Bifidobacterium group, was only detected in samples derived from autopsied cadavers. This could be an indication that some gut microfloral species are able to survive briefly if allowed rapid efflux from the cadaver, but do not survive the decomposition

processes that occur internally prior to normal fluid purging of intact cadavers. The 
autopsied cadavers could be considered a comparison for forensic situations where decomposing individuals have had serious injuries such as gunshot wounds. It was unexpected that the autopsy process would have so little effect on the overall microbial degradation of the cadavers, although the variability between the human cadavers did make data interpretation more difficult.

\subsubsection{Presence of Insects}

The presence of insects has a huge effect on the decomposition process (as seen in Chapter 4). The non-colonised carcasses underwent a visually very slow, gradual decomposition. The carcasses went through the normal bloat stage and then transitioned slowly into a mummification process rather than an active decay. The bacterial community composition for the two treatments was very similar for the fresh and bloat stage samples, but diverged at the active decay/ mummification stage, with the colonised carcass samples appearing to change more between samplings than the non-colonised samples. This suggests that the decomposition products being liberated into the underlying sand change more frequently because of the entomological breakdown of the cadaver. The overall sequential change as shown by MDS analysis, however, was very similar between the colonised and noncolonised carcass plot. The same T-RFs were detected in both, suggesting that at least in the absence of soil microflora, the same bacterial species are responsible for decomposition.

Some T-RFs were present only in the insect colonised decomposition events, while some T-RFs were only present in those plots that did not have insects present. The identifications of bacterial orders present in the samples suggested that some of those seen exclusively with insect colonisation may have been contributed to the community by the insects themselves. It is also possible, however, that they may have been organisms released from the carcasses that were able to proliferate in the conditions caused by the 
presence of the insects, but not in the slightly different insect-free decomposition environment. Similarly, those T-RFs specific to the insect-free plots may have been suited to proliferation in this environment, but not the insect colonised one. Insect larvae are known to produce anti-microbial compounds (Chee-Sanford et al., 2001; Macauley et al., 2007) to protect themselves from infection. It is possible that the presence of these compounds would be high when maggot masses are large, and contribute to the decrease in diversity seen to occur around the same time as when the maggot mass peaks, in some decomposition events.

The bacterial orders that may have been introduced by the insects were the Xanthomonadales, Myroides (Flavobacteriales) and possibly Providencia (Enterobacteriales). These organisms are known to be associated with insect larvae and were only detected in the samples derived from colonised carcasses.

\subsubsection{Soil Environment and Microflora}

One of the enteric bacterial groups tested for, the Enterococcus group, was only detected in sand samples, suggesting that the soil environment was unfavourable for this group. The soil and sand environments are likely to be quite different, as the soil contains an existing microflora as well as a much more complex mixture of organic and inorganic components. It is quite possible that some organisms are able to survive better in the simpler sand system and others may be better suited to survival, or find protection within (e.g. attached to clay particles), the soil environment. This is an important finding because it highlights the potential for differences in survival of body derived organisms on different soil types. Development of a time since death tool that uses soil would require testing on a wide array of different soil types. The organisms present in a soil prior to the decomposition event can vary considerably based on the biotic and abiotic conditions, in 
particular the soil chemistry and plant types present. This is likely to affect which organisms are able to survive and thrive during the decomposition event. There are, however, many genera that are almost ubiquitous in soil, such as Arthrobacter, Bacillus and Pseudomonas (Killham, 1994). These are hardy organisms, and are likely to be detected in samples from decomposition events regardless of the soil type. Soil is an incredibly heterogeneous substrate that can vary considerably over very small distances (Macdonald et al., 2009). All soils have their own characteristics in terms of parent materials, chemistry, macro- and micro-flora, and so are likely to respond differently to a decomposition event. Soil type was not examined as a variable in this study due to resource limitations, so results from the two soils used cannot be assumed to hold for all soils.

In the decomposition events studied, the soil bacterial community did not revert back to the original community structure seen prior to the decomposition event, within the period sampled (up to 69 days, ADD 1285). The cadaver which was sampled for the longest period (cadaver P, Figure 8.1) did have T-RFs that were present in very early samples disappear and reappear at the very end of the sampling period ( $\sim 1200$ ADD). The pig carcasses on sand were sampled for 155 days (1792 ADD) but the sand community diversity remained high compared with the initial sterile conditions. Longer term studies with more carcasses or cadavers are required to establish how long the soil takes to revert to a pre-decomposition-like community structure, if ever. If these changes are relatively long lived, then their usefulness as an indicator that a decomposition event has occurred will be greater. 


\section{Chapter 9: Conclusions}

\subsection{The Bacteriology of Decomposition}

The bacterial community associated with a human cadaver decomposing on soil is diverse and complex, with organisms in the underlying soil deriving from a number of sources including: the soil itself, the cadaver, probably the colonising insects and the wider environment. The types of bacteria present, and the nutritional requirements and environmental tolerances that they exhibit are also diverse, with a variety of anaerobes and aerobes detected in the same samples. The bacterial community changes in a general sequential progression that is common across individual decomposition events for both human cadavers and pig carcasses. Abrupt shifts in the bacterial community composition, as opposed to small, gradual changes tend to occur in response to large physical changes in the carcass/cadaver as it progresses through decomposition, which are likely to cause large corresponding physical changes in the underlying substrate. Smaller, gradual community changes occur in between these more abrupt shifts. A large influx of cadaver microflora into the underlying substrate occurs early in decomposition, when body fluids begin to purge. Community changes also occur in response to major changes in the physical environment and nutritional availability, although the exact time that these occur is not uniform between different cadavers and carcasses. Changes in the $\mathrm{pH}$, moisture content and oxygen availability, and the physical mixing of the substrate by insect activity are all thought to have affected the bacterial community. As the carcass or cadaver decomposes, different compounds are released from the carcass, which allows the organisms that can utilise these as carbon sources, nitrogen/amino acids and electron acceptors, to proliferate. It is likely that as one group of organisms degrades a compound, the by-product will be 
able to be used by another microbial group, or once that group has exhausted its nutrient supply and died off, another will then degrade those dead cells, setting up some interdependence, and a succession, between organisms.

There is evidence that bacterial species released from the carcass/cadaver were able to survive for a period in the soil/sand environment. The survival of these often fastidious, organisms for sometimes prolonged periods is probably due to the alteration of environmental conditions in their favour. The observation that a slime-like layer formed at the interface of the carcass/cadaver and the underlying substrate, and the often high moisture content of the substrate suggested that anaerobic pockets were likely to exist. The heat generated by the insect larval and microbial activity would also have contributed to making conditions more like those that the body microfloral organisms were adapted to. As well as the organisms released from the cadaver or carcass into the underlying substrate in large quantities, there are also thought to be organisms released in smaller quantities that are able to exist autochthonously, waiting for conditions and nutrient availability to become favourable enough for proliferation. Some soil organisms are likely to do the same. The zymogenous soil organisms are those that can proliferate in response to simple carbon inputs, while the autochthonous population is often able to degrade complex and recalcitrant polymers, so may survive in low levels in the soil for some time until conditions become favourable for them.

Towards the end of the longer decomposition sampling periods, there was some evidence that the original soil organisms may return once the influx of organic materials has been exhausted. The overall community, however, remained perturbed for the duration of the experiments in this thesis, suggesting that these changes to the decomposition environment may be long lasting. 


\subsection{Pig Carcasses as Models for Studying Human Decomposition Microbiology}

One of the aims of this project was to evaluate the use of pig carcasses as models for human decomposition microbiology. Pigs are routinely used as models for humans in other fields of decomposition research due to their similar body composition, diet and manageable size. Previous studies examining the gut flora of pigs found that the dominant phyla were the same as those usually detected in humans (Leser et al., 2002) and results from this study support this finding. The same main bacterial orders were detected in the clone libraries of samples from both pig carcasses and human cadavers. The database entry which returned the most hits to sequences from clone libraries (S000893094 bacterium 20RD; EF640582) was identified in libraries from both pig carcass and cadaver plots.

The greatest advantage to using model organisms rather than actual human cadavers is the relative ease in obtaining carcasses that are very similar, which eliminates many potential sources of variation. If pig carcasses from the same farm are sourced, factors such as age, size, diet, general treatment and genetic makeup (to a degree) can be held constant, something that cannot be done when using human cadavers.

Some of the disadvantages experienced when working with human cadavers include:

- The study of cadavers is restricted to only a few facilities, which limits research to a few climates and geographical sites.

- Human bodies vary considerably in size and in composition, e.g. in muscle/fat ratio.

- Many human subjects are autopsied. 
- The cause of death varies in human subjects which can greatly impact on existing microflora. In some cases in this study, the cause of death was unknown or ambiguously listed as "natural causes".

- The antibiotic and antibacterial use by the person prior to death (or after death at the autopsy) is totally unknown. Antibiotics can perturb gut flora for up to two years post-treatment (Jernberg et al., 2007) and given the widespread use of antibiotics, many human cadavers are likely to be affected by this.

- Cadavers can only be used as they become available or stored frozen (which severely impacts the body's microflora).

- The time and treatment between death and arrival at facility can be highly variable.

- Human diet (and thus gut microflora) is very variable.

- Genetics can greatly affect microflora, and all human study participants are different genetically.

- The human cadavers donated vary in age, which also affects microflora.

- The physical lifting of even small cadavers requires two people which is not always practically possible in research.

The biochemistry results from the pig carcass and human cadaver experiments showed some striking similarities. The rise in soil/sand $\mathrm{pH}$ was seen in all decomposition events. Despite size differences between the human cadavers and pig carcasses, and differences in initial substrate $\mathrm{pH}$, it consistently rose to around $\mathrm{pH}$. This finding is supported by a recently published study on the effect of initial soil $\mathrm{pH}$ on decomposition of skeletal muscle (Haslam and Tibbett, 2009). The ammonium and nitrate concentrations also showed similarities, although there was some variability in when the concentrations of these were at their greatest. While the enzyme data was not particularly interpretable, the general trends and findings of those enzymes examined in both were the same. 
The bacterial orders identified in the pig decomposition events compared with the human decomposition events were very similar. There was, however, one organism associated with the pig carcasses that was closely related to a uniquely pig commensal organism, indicating that microfloral differences between the two are still detectable. The same general groups such as Pseudomonadales, Clostridiales, Flavobacteriales, Xanthomonadales and Enterobacteriales featured in the clone libraries from both human and pig decomposition. The same predominant T-RFs were also seen in both pig and human-derived T-RFLP profiles. There is likely to be more variation at lower taxonomic levels e.g. genus level, but at the order level, which was used in this study, the bacteriology of human and pig decomposition seems to be very similar.

Based on the advantages that pigs as model organisms have over human cadavers, the similar mix of bacterial orders that were identified and the overall similarities seen between chemistry, enzymatic and bacterial community results from the two, the use of pig carcasses for studying decomposition microbiology is recommended. Further studies with simultaneous decomposition of pig carcasses and human cadavers at the same site would, however, still be advantageous.

\subsection{Review of experimental approach}

\subsubsection{Experimental Design and Sampling}

Because of the novelty of this research, considerable thought was put into the experimental design. Previous work with soils containing decomposition by-products had demonstrated that applying molecular ecology tools to such samples was not always straightforward, so multiple methods and aspects of the bacterial community were tried. Some were successful and others were less informative. The set-up and sampling methods for the 
decomposition events was also carefully designed and improvements were made with each decomposition experiment.

One of the biggest challenges was to collect a representative sample. Efforts were made to collect soil or sand from as wide an area under the carcasses and cadavers as possible. Because fluids do not purge evenly over the substrate surface, localised pools of fluids were present, making it inevitable that some samples would contain more fluid than others. This was not so much of a problem in the black tubs used in the pig experiments, as once the insect larvae were present, they effectively homogenised and aerated the underlying substrate with their continual movement. This was also evident for the cadavers that had large maggot masses develop, but some had minimal insect larvae activity and subsequently little mixing of the substrate. While this sampling method was not ideal and likely affected the results to some extent, it was the best that could be devised.

Another issue throughout this research project was the cost of the molecular work and the inability to use replicates. Ideally, T-RFLP and DGGE analysis would have been performed in triplicate, but this would have significantly reduced the number of samples which could have been profiled with the available funding. A decision was made to not replicate these analyses, but to include replicate carcasses and cadavers, and to examine more time-points instead. Microbial biomass was originally intended to be measured at each sampling, but it became apparent early on that the method chosen was not appropriate for the experiment and was discontinued. This also allowed for more sequencing to be performed.

The running of three separate experiments in two different locations was also not ideal, with this introducing yet another variable to consider. It did, however, offer the advantage of allowing the experimental set-up and methodology to be improved as issues were 
discovered with each experiment. It also enabled the number of samples to be kept to a manageable level while still maximising the number of time-points examined.

\subsubsection{Laboratory Methods}

Whilst T-RFLP and DGGE are both sophisticated and popular tools for molecular based analysis of complex bacterial communities, both have their advantages and disadvantages. Both methods include a DNA extraction step and a PCR step, and so are subject to the biases associated with these steps (as discussed in section 2.3.1.1). These biases must be considered when drawing conclusions from data derived from these methods. PCR-based methods, however, remain to date, the most effective way of examining complex microbial communities.

Overall, the majority of the soil biochemistry and enzyme data was not particularly informative. There did not appear to be any real correlation between changes in potential enzyme activity and molecular data or with visual changes in the cadavers. Some of the enzyme results were difficult to explain, such as seeing nitrate peaks prior to ammonium peaks, even though the reverse is usually seen in soil after an influx of ammonium, due to conversion of ammonium into nitrate via nitrification (Dr T Speir, ESR Soil Scientist, pers. comm.). Similar results were seen by Stokes et al. (2009) in their study of the decomposition of buried skeletal tissue. These results basically highlight the difficulty of working with a complex organic system such as a decomposition event, where many different factors could be influencing the results, and where current understanding of the processes is limited. Better understanding of some of the results may have been gained if changes in the microbial biomass over time had been determined, but unfortunately this was not possible. Overall, the approach used was justified, with some methods clearly out- 
performing others. The use of these analyses, however, will allow future researchers of this topic to make more informed decisions about appropriate methodological approaches to studying human decomposition.

\subsection{Future research}

The novel, exploratory research presented in this thesis provides a solid starting point for further research. The potential for future research is strong, with new methods and techniques constantly being developed. A number of key areas and approaches that could be considered are discussed.

\subsubsection{Recommendations for further research}

This research was limited to the examination of the bacterial community associated with decomposition on soil. While bacteria appear to play an intrinsic role in the decomposition process, there are many other microbes that should also be examined, for example, the fungi and archaea. The fungal communities associated with two cadavers in this study were examined by an MSc student, but the results did not show any sequential changes. Visible fungal growth was apparent on many of the carcasses and cadavers and certain fungal species have been found associated with decomposition events (Carter and Tibbett, 2003). This suggests that further study is required.

A logical extension to the work already performed would be to examine more bacterial groups. Functional groups, such as those associated with the nitrogen cycle could be of interest, particularly given that ammonium and nitrate were liberated during the decomposition event. It would also be logical to further study the body microfloral groups, including those already touched on in this study. It was shown that cadaver- 
derived organisms could be detected in the soil throughout the decomposition period, and some were identified during sequencing. Study of organisms known to use particular substrates released during decomposition could also be targeted. Because the soil a body is found on can vary hugely in its composition and resident microbial community, concentration on the body microflora may be the most logical direction for development of a time since death estimation tool. This does not rule out the use of soil microbes, but study of more soil types in different environments and conditions would also be required.

If the molecular tools used in this study, such as T-RFLP and DGGE were used in future research, special consideration should be put into primer design and restriction enzyme choice. While the enzyme choice in this study was effective, greater discrimination between related bacterial orders could have been achieved with the use of multiple enzymes. This study used previously published primer sequences due to time and resource restraints, but future studies should look at developing the most effective primers for answering the research question.

Another potentially informative experiment would be to allow side-by-side decomposition of human cadavers and pig carcasses. This would allow for very effective comparisons to be made between the two, as many variables encountered in this study could be negated. This has previously been done, but only studied from an entomological perspective (Haskell et al., 2001).

While the enzyme assays used in this study were not particularly informative, there are many other biochemical tests which could be used to further our understanding of the decomposition process. Determining how microbial biomass and redox potential are affected would be logical next steps. Determining the amount of $\mathrm{NH}_{3}$ (ammonia) that 
volatilises during decomposition could also be interesting, as this would help piece together what is occurring in the nitrogen cycle.

\subsubsection{Alternative approaches}

While this research project was being conducted, molecular ecology methods underwent some exciting developments, with new technologies and ideas greatly enhancing study of complex microbial communities. Microbial ecology is a rapidly expanding field, with researchers developing new techniques to meet the requirements of the field (Kowalchuk et al., 2007). The rapid, high-throughput pyrosequencing method has revolutionised identification of organisms in complex communities such as soil (Roesch et al., 2007). Unfortunately this approach was not feasible at the outset of this study, as it would have been the logical and most desirable method for closely examining the complex decomposition community. Other sequencing approaches such as Serial Analysis of Ribosomal Sequence Tags (SARST) have also been developed in this time (Neufeld et al., 2004) and could have been useful techniques to investigate.

A recent development in T-RFLP technology is the ability to produce T-RFLP profiles for multiple target groups using different fluorescent tags. Singh et al. (2006) developed a multiplex T-RFLP method that used a multiplex PCR with different fluorophores attached to each primer set. Bacterial, fungal, archaeal and rhizobial groups were targeted. By combining multiple target groups into the one T-RFLP reaction, the amount of information generated from one sample is greatly enhanced, whilst keeping consumable costs down. Multiplex PCR requires more fine-tuning to get the primer sets working optimally together, but would definitely be advantageous when performing the type of exploratory research outlined in this thesis. 
Microarray technology has been available for some time, but until recently has not been hugely successful for examining diverse microbial communities (Gill et al., 2006; Yergeau et al., 2009). This technology has the disadvantage of only being able to detect taxa already described in databases. A comprehensive microarray chip PhyloChip (Brodie et al., 2006; DeSantis et al., 2007) has been used to assess soil communities and could potentially become a useful tool for further investigating decomposition microbiology.

RNA-based analysis of microbial communities is also a possible avenue for further investigation. By using RNA instead of DNA as a template for PCR, the active, metabolising members of the bacterial community can be examined without the dormant or dead members potentially being detected also (Josephson et al., 1993). The use of RNA for community analyses is gaining in popularity, but to date, DNA has been by far the more popular choice, due to the relative ease of working with DNA compared with RNA. Active populations have been shown to be sub-set of the residential community seen using DNA (Duineveld et al., 2001; Sharma et al., 2005). Shifts in community structure have been shown to be detectable earlier on using RNA (Morgan et al., 2002), but with continuous exposure to the same treatments that increase the biomass of the active population, the community structures using RNA and DNA can become more similar (Nakatsu, 2007). Sanchez et al. (2006) developed a form of T-RFLP that uses reverse transcriptase to analyse the active organisms only, using 16s rRNA. The use of RNA to examine the metabolising part of the microbial community would be a logical extension of the research outlined in this thesis.

High-throughput methods such as pyrosequencing are likely to play a major role in further decomposition-derived microbial community studies, but this type of approach is inappropriate as a routine forensic time since death determination tool. If biomarker microbial species that reliably come and go as decomposition progresses can be identified, 
then simple, rapid tests using specific DNA probes is a likely option. Techniques such as fluorescent in situ hybridisation (FISH), flow cytometry, or simple specific PCR could be used. An alternative future approach would be to concentrate on a smaller target population, for example the skin flora, and examine its role in decomposition.

An alternative approach to understanding complex microbial populations such as those involved in decomposition, is to use metagenomic (Gill et al., 2006) or metaproteomic methods (Maron et al., 2007). Metagenomics is the study of all the genes present within a microbial community, and metaproteomics, of the proteins. This approach can allow an assessment of the functionality of the community as a whole, avoid the DNA-based method problem of not knowing whether predicted genes are active (Verberkmoes et al., 2009) and can assess the functioning of a complex population as opposed to discerning which species are present.

Continuing advances in molecular and alternative methods for studying complex microbial communities make this an exciting time for researchers exploring these unknown ecosystems and hopefully these new methods will be applied to better understanding the decomposition microbial community in the near future.

\subsection{Forensic Application}

While the bacterial communities associated with a decomposing cadaver have been found to be complex, diverse and vary considerably between individuals, the research presented in this thesis indicates that the potential for developing a time-since death estimation tool using bacterial biomarkers certainly exists. With further research, particularly with the rapidly evolving molecular methods, bacterial groups, perhaps at the genera level, may be identified as being associated with particular stages of decomposition in most humans. 
The development of such a tool could dramatically enhance forensic death investigations by reducing the time and monetary costs required. The methods currently used, such as entomology, usually have high costs associated with them and so are used sparingly, usually only in cases where the time of death cannot be estimated using other types of evidence. A bacterial biomarker time since death method would also have a relatively rapid turnaround time, giving investigators more information to work with earlier on in an investigation. Such a method would also be able to be performed routinely in a typical forensic laboratory and would not require significant expertise to perform if it were based around basic molecular biological techniques. Theoretically, such a method could also reduce the reliance of time since death estimations on subjective opinions and the experience of the expert.

If time-dependant biomarkers cannot be found, development of other forensic tools is still a strong possibility. A test for determining if a decomposition event has occurred in a particular place (in the absence of the body) would be of use to forensic investigators in cases where bodies have been moved by perpetrators or by scavenging animals. Humanspecific strains of particular organisms such as Enterococcus could also establish whether that decomposition event was caused by a human body or other large mammal. By further developing an understanding the microbiology of decomposition, investigators may also be able to more effectively interprete crime scenes involving decomposing bodies.

Further research into understanding human decomposition also holds potential for other areas of science, including better understanding nutrient cycling and organic matter degradation and for discovering previously unknown organisms, some of which may have exploitable abilities, particularly in pollutant degradation or alternative fuel production. While this study has just scratched the surface of the complex world of microbes involved 
in human decomposition, it has opened a small window into the bacterial world and provided a beginning for future research to be based on. 


\section{References}

Aarestrup, F.M., Butaye, P. and Witte, W. (2002) Nonhuman Reservoirs of Enterococci. In: Gilmore, M.S. (Eds.) The Enterococci: Pathogenesis, Molecular Biology and Antibiotic Resistance. ASM Press, Washington, DC, USA.

Abd El-Rahman, H.A., Fritze, D., Sproeer, C. and Claus, D. (2002) Two novel psychrotolerant species, Bacillus psychrotolerans sp. nov. and Bacillus psychrodurans sp. nov., which contain ornithine in their cell walls. International Journal of Systematic and Evolutionary Microbiology 52: 2127-2133.

Abraham, W.-R., Macedo, A.J., Lunsdorf, H., Fischer, R., Pawelczyk, S., Smit, J. and Vancanney, M. (2008) Phylogeny by a polyphasic approach of the order Caulobacterales, proposal of Caulobacter mirabilis sp. nov., Phenylobacterium haematophilum sp. nov. and Phenylobacterium conjuntum sp. nov., and emendation of the genus Phenylobacterium. International Journal of Systematic and Evolutionary Microbiology 58: 1939-1949.

Acosta-Martinez, V., Dowd, S., Sun, Y. and Allen, V. (2008) Tag-encoded pyrosequencing analysis of bacterial diversity in a single soil type as affected by management and land use. Soil Biology \& Biochemistry 40: 2762-2770.

Amann, R.I., Ludwig, W. and Schleifer, K.H. (1995) Phylogenetic identification and in situ detection of individual microbial cells without cultivation. Microbiological Reviews 59: 143-169.

Amendt, J., Krettek, R., Niess, C., Zehner, R. and Bratzke, H. (2000) Forensic entomology in Germany. Forensic Science International 113: 309-314.

Amor, K.B. and Vaughan, E.E. (2006) Molecular ecology of the human intestinal microbiota. In: Ouwehand, A.C. and Vaughan, E.E. (Eds.) Gastrointestinal Microbiology. Taylor \& Francis, New York, pp. 124.

Anderson, G.S. (2001) Insect succession on carrion and its relationship to determining time since death. In: Byrd, J.H. and Castner, J.L. (Eds.) Forensic Entomology: The Utility of Arthropods in Legal Investigations. CRC Press, Boca Raton, Florida, pp. 143-176.

Anderson, K.L., Whitlock, J.E. and Harwood, V.J. (2005) Persistence and differential survival of fecal indicator bacteria in subtropical waters and sediments. Applied and Environmental Microbiology 71: 3041-3048.

Anderson, R.J., Groundwater, P.W., Huang, Y., James, A.L., Orenga, S., Rigby, A., Roger-Dalbert, C. and Perry, J.D. (2008) Synthesis and evaluation of novel chromogenic peptidase substrates based on 9(4'-aminophenyl)-10-methylacridinium salts as diagnostic tools in clinical bacteriology. Bioorganic \& Medicinal Chemistry Letters 18: 832-835.

Andersson, A.F., Lindberg, M., Jakobsson, H., Backhed, F., Nyren, P. and Engstrand, L. (2008) Comparative analysis of human gut microbiota by barcoded pyrosequencing. PLoS ONE 3: e2836.

Arnaldos, I., Romera, E., Garcia, M.D. and Luna, A. (2001) An initial study on the succession of sarcosaprophagous Diptera (Insecta) on carrion in the Southeastern Iberian peninsula. International Journal of Legal Medicine 114: 156-162.

Arpigny, J.L. and Jaeger, K.-E. (1999) Bacterial lipolytic enzymes: classification and properties. Biochemistry Journal 343: 177-183. 
Ashelford, K.E., Chuzhanova, N.A., Fry, J.C., Jones, A.J. and Weightman, A.J. (2005) At least 1 in 20 rRNA sequence records currently held in public repositories is estimated to contain substantial anomalies. Applied and Environmental Microbiology 71: 7724-7736.

Australian-Museum. (2003) Decomposition - What Happens to the Body After Death? [online] http://www.deathonline.net/decomposition/index.htm (19 April 2009)

Avaniss-Aghajani, E., Jones, K., Chapman, D. and Brunk, C. (1994) A molecular technique for identification of bacteria using small subunit ribosomal RNA sequences. Biotechniques 17: 144-146, 148-149.

Avis, P.G., Dickie, I.A. and Mueller, G.M. (2006) A 'dirty' business: testing the limitations of terminal restriction fragment length polymorphism (TRFLP) analysis of soil fungi. Molecular Ecology 15: 873-882.

Avrahami, S. and Conrad, R. (2003) Patterns of community change among ammonia oxidizers in meadow soils upon long-term incubation at different temperatures. Applied and Environmental Microbiology 69: 6152-6164.

Babapulle, C.J. and Jayasundera, N.P. (1993) Cellular changes and time since death. Medicine, Science and the Law 33: 213-222.

Baker, B.J., Moser, D.P., MacGregor, B.J., Fishbain, S., Wagner, M., Fry, N.K., Jackson, B., Speolstra, N., Loos, S., Takai, K., Lollar, B.S., Fredrickson, J., Balkwill, D., Onstott, T.C., Wimpee, C.F. and Stahl, D.A. (2003) Related assemblages of sulphate-reducing bacteria associated with ultradeep gold mines of South Africa and deep basalt aquifers of Washington State. Environmental Microbiology 5: 267-277.

Bartosch, S., Fite, A., Macfarlane, G.T. and McMurdo, M.E.T. (2004) Characterization of bacterial communities in feces from healthy elderly volunteers and hospitalised elderly patients by using Real-Time PCR and effects of antibiotic treatment on the fecal microbiota. Applied and Environmental Microbiology 70: 3575-3581.

Bass, W.M. (1997) Outdoor decomposition rates in Tennessee. In: Haglund, W.D. and Sorg, M.H. (Eds.) Forensic Taphonomy: the Postmortem Fate of Human Remains. CRC Press, Boca Raton, Florida, pp. 181-186.

Bass, W.M. and Jefferson, J. (2003) Death's Acre: Inside the Legendary 'Body Farm'. G.P. Putnam's Sons, New York.

Bell, A., Layton, A.C., McKay, L., Williams, D., Gentry, R. and Sayler, G.S. (2009) Factors influencing the persistence of fecal Bacteroides in stream water. Journal of Environmental Quality 38: 1224-1232.

Bernardet, J.-F., Hugo, C. and Bruun, B. (2006) The genera Chryseobacterium and Elizabethkingia. Prokaryotes 7: 638-676.

Bernhard, A.E. and Field, K.G. (2000) A PCR assay to discriminate human and ruminant feces on the basis of host differences in Bacteroides-Prevotella genes encoding $16 \mathrm{~S}$ rRNA. Applied and Environmental Microbiology 66: 4571-4574.

Bezbaruah, B. (1983) Ureolytic and nitrifying bacterial heterotrophs from acid soils. Journal of Biosciences 5: $267-278$.

Blackwood, C.B. and Buyer, J.S. (2007) Evaluating the physical capture nature method of terminal restriction fragment length polymorphism for comparison of soil microbial communities. Soil Biology \& Biochemistry 39: 590-599.

Blackwood, C.B., Oaks, A. and Buyer, J.S. (2005) Phylum- and class-specific PCR primers for general microbial community analysis. Applied and Environmental Microbiology 71: 6193-6198. 
Blakemore, L.C., Searle, P.L. and Daly, B.K. (1987) Methods for chemical analysis of soils. NZ Soil Bureau Scientific Report 80.

Bocaz-Beneventi, G., Tagliaro, F., Bortolotti, F., Manetto, G. and Havel, J. (2002) Capillary zone electrophoresis and artificial neural networks for estimation of the post-mortem interval (PMI) using electrolytes measurements in human vitreous humour. International Journal of Legal Medicine 116: 5-11.

Bock, J.H. and Norris, D.O. (1997) Forensic botany: An under-utilised resource. Journal of Forensic Sciences 42: 364-367.

Boot, R.G., Blommaart, E.F.C., Swart, E., Ghauharali-van der Vlugt, K., Bijl, N., Moe, C., Place, A. and Aerts, J.M.F.G. (2001) Identification of a novel acidic mammalian chitinase distinct from chitotriosidase. The Journal of Biological Chemistry 276: 6770-6778.

Borch, E., Kant-Muermans, M.-L. and Blixt, Y. (1996) Bacterial spoilage of meat and cured meat products. International Journal of Food Microbiology 33: 102-120.

Borresen, A.L., Hovig, E. and Brogger, A. (1988) Detection of base mutations in genomic DNA using denaturing gradient gel-electrophoresis (DGGE) followed by transfer and hybridization with genespecific probes. Mutation Research 202: 77-83.

Boutte, C., Grubisic, S., Balthasart, P. and Wilmotte, A. (2006) Testing of primers for the study of cyanobacterial diversity by DGGE. Journal of Microbiological Methods 65: 542-550.

Brinkmann, N., Martens, R. and Tebbe, C.C. (2008) Origin and diversity of metabolically active gut bacteria from laboratory-bred larvae of Manduca sexta (Sphingidae, Lepidoptera, Insecta). Applied and Environmental Microbiology doi:10.1128/AEM.01464-08.

Brodie, E.L., DeSantis, T.Z., Joyner, D.C., Baek, S.M., Larsen, J.T., Andersen, G.L., Hazen, T.C., Richardson, P.M., Herman, D.J., Tokunaga, T.K., Wan, J.M. and Firestone , M.K. (2006) Application of a high-density oligonucleotide microarray approach to study bacterial population dynamics during uranium reduction and reoxidation. Applied and Environmental Microbiology 72: 6288-6298.

Brown, M.R.W. and Barker, J. (1999) Unexplored reservoirs of pathogenic bacteria: protozoa and biofilms. Trends in Microbiology 7: 46-50.

Buckley, D.H., Graber, J.R. and Schmidt, T.M. (1998) Phylogenetic analysis of nonthermophilic members of the kingdom Crenarchaeota and their diversity and abundance in soil. Applied and Environmental Microbiology 64: 4333-4339.

Bull, H., Murray, P.G., Thomas, D., Fraser, A.M. and Nelson, P.N. (2002) Acid phosphatases. Journal of Clinical Pathology: Molecular Pathology 55: 65-72.

Burne, R.A. and Chen, Y.-Y.M. (2000) Bacterial ureases in infectious diseases. Microbes and Infection 2: 533-542.

Bussink, A.P., Speijer, D., Aerts, J.M.F.G. and Boot, R.G. (2007) Evolution of mammalian chitinase(-like) members of family 18 glycosyl hydrolases. Genetics 177: 959-970.

Caetano-Anollés, G. and Trigiano, R.N. (1997) Recovery of DNA amplification products from silver-stained polyacrylamide gels. In: White, B.A. (Eds.) PCR Cloning Protocols: From Molecular Cloning to Genetic Engineering. Humana Press Inc., New Jersey, USA., pp. 111-127.

Campobasso, C.P., Di Vella, G. and Introna, F. (2001) Factors affecting decomposition and Diptera colonization. Forensic Science International 120: 18-27. 
Cardinale, M., Brusetti, L., Quatrini, P., Borin, S., Puglia, A.M., Rizzi, A., Zanardini, E., Sorlini, C., Corselli, C. and Daffonchio, D. (2004) Comparison of different primer sets for use in automated ribosomal intergenic spacer analysis of complex bacterial communities. Applied and Environmental Microbiology 70: 6147-6156.

Carter, D.O. and Tibbett, M. (2002) Forensic taphonomy: The adaptation of the soil microbial decomposer community to soft tissue burial. Proceedings of the 33rd International Symposium on Archaeometry, Amsterdam: Institute for Geo- and Bioarchaeology, Vrije Universiteit.

Carter, D.O. and Tibbett, M. (2003) Taphonomic mycota: fungi with forensic potential. Journal of Forensic Sciences 48: 168-171.

Carter, D.O. and Tibbett, M. (2006) Microbial decomposition of skeletal muscle tissue (Ovis aries) in a sandy loam soil at different temperatures. Soil Biology \& Biochemistry 38: 1139-1145.

Carter, D.O. and Tibbett, M. (2008) Cadaver decomposition and soil: processes. In: Tibbett, M. and Carter, D.O. (Eds.) Soil Analysis in Forensic Taphonomy: Chemical and Biological Effects of Buried Human Remains. CRC Press, Boca Raton, Florida, pp. 29-51.

Carter, D.O., Yellowlees, D. and Tibbett, M. (2007) Cadaver decomposition in terrestrial ecosystems. Naturwissenschaften 94: 12-24.

Castellano, M.A., Villanueva, E.C. and von Frenckel, R. (1984) Estimating the date of dry bone remains. Journal of Forensic Sciences 29: 527-534.

Castignetti, D. and Hollocher, T.C. (1984) Heterotrophic nitrification among denitrifiers. Applied and Environmental Microbiology 47: 620-623.

Chander, Y., Goyal, S.M. and Gupta, S.C. (2006) Antimicrobial resistance of Providencia spp. isolated from animal manure. The Veterinary Journal 172: 188-191.

Chandler, D.P., Fredrickson, J.K. and Brockman, F.J. (1997) Effect of PCR template concentration on the composition and distribution of total community 16S rDNA clone libraries. Molecular Ecology 6: 475-482.

Chang, R. (2006) Chemistry. McGraw Hill Higher Education, New York.

Chee-Sanford, J.C., Aminov, R.I., Krapac, I.J., Garrigues-Jeanjean, N. and Mackie, R.I. (2001) Occurrence and diversity of tetracycline resistance genes in lagoons and groundwater underlying two swine production facilities. Applied and Environmental Microbiology 67: 1494-1502.

Chen, Y.-S., Yanagida, F. and Shinohara, T. (2005) Isolation and identification of lactic acid bacteria from soil using an enrichment procedure. Letters in Applied Microbiology 40: 195-200.

Child, A.M. (1995) Towards an Understanding of the Microbial Decomposition of Archaelogical Bone in the Burial Environment. Journal of Archaeological Science 22: 165-174.

Choi, J.-H., Lee, S.H., Fukushi, K. and Yamamoto, K. (2007) Comparison of sludge characteristics and PCR-DGGE based microbial diversity of nanofiltration and microfiltration membrane bioreactors. Chemosphere 67: 1543-1550.

Chopra, I. and Roberts, M. (2001) Tetracycline antibiotics: mode of action, applications, molecular biology and epidemiology of bacterial resistance. Microbiology and Molecular Biology Reviews 65: 232260.

Christensen, H., Hansen, M. and Sorensen, J. (1999) Counting and size classification of active soil bacteria by fluorescence in situ hybridization with an rRNA oligonucleotide probe. Applied and Environmental Microbiology 65: 1753-1761. 
Clark, I.M. and Hirsch, P.R. (2008) Survival of bacterial DNA and culturable bacteria in archived soils from the Rothamsted Broadbalk experiment. Soil Biology \& Biochemistry 40: 1090-1102.

Clark, M.A., Worrell, M.B. and Pless, J.E. (1997) Postmortem changes in soft tissues. In: Haglund, W.D. and Sorg, M.H. (Eds.) Forensic Taphonomy: The Postmortem Fate of Human Remains. CRC Press, Boca Raton, Florida, pp. 151-160.

Clarke, K.R. (1993) Non-parametric multivariate analyses of changes in community structure. Australian Journal of Ecology 18: 117-143.

Clarke, K.R. and Gorley, R.N. (2006) PRIMER v6: User Manual/Tutorial. PRIMER-E, Plymouth.

Clayton, R.A., Sutton, G., Hinkle, P.S., Bult, C. and Fields, C. (1995) Intraspecific variation in small-subunit rRNA sequences in GenBank: why single sequences may not adequately represent prokaryotic taxa. International Journal of Systematic Bacteriology 45: 595-599.

Coenye, T. and Vandamme, P. (2003) Intragenomic heterogeneity between multiple 16S ribosomal RNA operons in sequenced bacterial genomes. FEMS Microbiology Letters 228: 45-49.

Cole, A.C., Semmens, M.J. and LaPara, T.M. (2004) Stratification of activity and bacterial community structure in biofilms grown on membranes transferring oxygen. Applied and Environmental Microbiology 70: 1982-1989.

Cole, J.R., Wang, Q., Cardenas, E., Fish, J., Chai, B., Farris, R.J., Kulam-Syed-Mohideen, A.S., McGarrell, D.M., Marsh, T., Garrity, G.M. and Tiedje, J.M. (2009) The Ribosomal Database Project: improved alignments and new tools for rRNA analysis. Nucleic Acids Research 37: D141-D145.

Cole, S.T. and Girons, I.S. (1994) Bacterial genomics. FEMS Microbiology Reviews 14: 139-160.

Collins, M.D., Rodrigues, U.M., Pigott, N.E. and Facklam, R.R. (1991) Enterococcus dispar sp. nov., a new Enterococcus species from human sources. . Letters in Applied Microbiology 12: 95-98.

Colquhoun, J.A., Heald, S.C., Li, L., Tamaoka, J., Kato, C., Horikoshi, K. and Bull, A.T. (1998) Taxonomy and biotransformation activities of some deep-sea actinomycetes. . Extremophiles 2: 269-277.

Cools, D., Merckx, R., Vlassak, K. and Verhaegen, J. (2001) Survival of E. coli and Enterococcus spp. derived from pig slurry in soils of different textures. Applied Soil Ecology 17: 53-62.

Corby-Harris, V., Pontaroli, A.C., Shimkets, L.J., Bennetzen, J.L., Habel, K.E. and Promislow, D.E. (2007) Geographical distribution and diversity of bacteria associated with natural populations of Drosophila melanogaster. Applied and Environmental Microbiology 73: 3470-3479.

Dadour, I.R. and Harvey, M.L. (2008) The role of invertebrates in terrestrial decomposition: forensic applications. In: Tibbett, M. and Carter, D.O. (Eds.) Soil Analysis in Forensic Taphonomy:. CRC Press Inc., Boca Raton, Florida, pp. 109-122.

Davet, P. (2004) Microbial Ecology of the Soil and Plant Growth. Science Publishers Inc., Enfield, New Hampshire.

Davila, E., Zamora, L.M., Pla, M., Carretero, C. and Pares, D. (2006) Identification and antagonistic activity of lactic acid bacteria occurring in porcine blood from industrial slaughterhouses-a preliminary study. International Journal of Food Microbiology 107: 207-211.

Davis, M.E., Brown, D.C., Baker, A., Bos, K., Dirain, M.S., Halbrook, E., Johnson, Z.B., Maxwell, C. and Reherger, T. (2007) Effect of direct-fed microbial and antibiotic supplementation on gastrointestinal microflora, mucin histochemical characterization, and immune populations of weanling pigs. Livestock Science 108: 249-253. 
de Boer, W., Klein Gunnewiek, P.J.A., Kowalchuk, G.A. and van Veen, J.A. (2001) Growth of chitinolytic dune soil beta-subclass Proteobacteria in response to invading fungal hyphae. Applied and Environmental Microbiology 67: 3358-3362.

Delgado, S., Arroyo, R., Martin, R. and Rodriguez, J.M. (2008) PCR-DGGE assessment of the bacterial diversity of breast milk in women with lactational infectious mastitis. BMC Infectious Diseases 8: 51-58.

Delgado, S., Florez, A.B. and Mayo, B. (2005) Antibiotic susceptibility of Lactobacillus and Bifidobacterium species from the human gastrointestinal tract. Current Microbiology 50: 202-207.

Deng, H., He, C., Zhou, Z., Liu, C., Tan, K., Wang, N., Jiang, B., Gao, X. and Liu, W. (2009) Isolation and pathogenicity of pathogens from skin ulceration disease and viscera ejection syndrome of the sea cucumber Apostichopus japonicas. Aquaculture 287: 18-27.

DeSantis, T.Z., Brodie, E.L., Moberg, J.P., Zubieta, I.X., Piceno, Y.M. and Andersen, G.L. (2007) Highdensity universal 16S rRNA microarray analysis reveals broader diversity than typical clone library when sampling the environment. Microbial Ecology 53: 371-383.

Dethlefsen, L., McFall-Ngai, M. and Relman, D.A. (2007) An ecological and evolutionary perspective on human-microbe mutualism and disease. Nature 449: 811-818.

Dharne, M.S., Gupta, A.K., Rangrez, H.V., Ghate, H.V., Patole, M.S. and Shouche, Y.S. (2008a) Antibacterial activities of multi drug resistant Myroides odoratimus bacteria isolated from adult flesh flies (Diptera: Sarcophagidae) are independant of metallo beta-lactamase gene. Brazilian Journal of Microbiology 39: 397-404.

Dharne, M.S., Misra, S.P., Misra, V., Dwivedi, M., Patole, M.S. and Shouche, Y.S. (2008b) Isolation of urease-positive Ochrobactrum intermedium in the stomach of a non-ulcer dyspeptic patient from north India. Journal of Microbiology, Immunology and Infection 41: 183-186.

Dick, L.K., Bernhard, A.E., Brodeur, T.J., Santo Domingo, J.W., Simpson, J.M., Walters, S.P. and Field, K.G. (2005) Host distributions of uncultivated fecal Bacteroidales bacteria reveal genetic markers for fecal source identification. Applied and Environmental Microbiology 71: 3184-3191.

Dick, L.K. and Field, K.G. (2004) Rapid estimation of numbers of fecal Bacteroidetes by use of a quantitative PCR assay for 16S rRNA genes. Applied and Environmental Microbiology 70: 56955697.

Dickie, I.A. and Fitzjohn, R.G. (2007) Using terminal restriction fragment length polymorphism (T-RFLP) to identify mycorrhizal fungi: a methods review. Mycorrhiza 17: 259-270.

Drasar, B.S., Crowther, J.S., Goddard, P., Hawksworth, G., Hill, M.J., Peach, S. and Williams, R.E.O. (1973) The relation between diet and the gut microflora in man. Proceedings of the Nutrition Society: 4952 .

Duineveld, B.M., Kowalchuk, G.A., Keijzer, A., van Elsas, J.D. and van Veen, J.A. (2001) Analysis of bacterial communities in the rhizosphere of Chrysanthemum via denaturing gradient gel electrophoresis of PCR-amplified 16S rRNA as well as DNA fragments coding for 16S rRNA. Applied and Environmental Microbiology 67: 172-178.

Dumont, M.G. and Murrell, J.C. (2005) Stable isotope probing - linking microbial identity to function. Nature Reviews Microbiology 3: 499-504.

Dunbar, J.M., Ticknor, L.O. and Kuske, C.R. (2001) Phylogenetic specificity and reproducibility and new method for analysis of terminal restriction fragment profiles of 16S rRNA genes from bacterial communities. Applied and Environmental Microbiology 67: 190-197. 
Duo-Chuan, L. (2006) Review of fungal chitinases. Mycopathologia 161: 345-360.

Egert, M. and Friedrich, M.W. (2003) Formation of pseudo-terminal restriction fragments, a PCR-related bias affecting terminal restriction fragment length polymorphism analysis of microbial community structure. Applied and Environmental Microbiology 69: 2555-2562.

Elshahed, M.S., Youssef, N.H., Spain, A.M., Sheik, C., Najar, F.Z., Sukharnikov, L.O., Roe, B.A., Davis, J.P., Schloss, P.D., Bailey, V.L. and Krumholz, L.R. (2008) Novelty and uniqueness patterns of rare members of the soil biosphere. Applied and Environmental Microbiology 74: 5422-5428.

England, L.S. and Trevors, J.T. (2003) The microbial DNA cycle in soil. Rivista di Biologia/Biology Forum 96: 317-326.

England, L.S., Vincent, M.L., Trevors, J.T. and Holmes, S.B. (2004) Extraction, detection and persistence of extracellular DNA in forest litter microcosms. Molecular and Cellular Probes 18: 313-319.

Enticknap, J.J., Nonogaki, H., Place, A.R. and Hill, R.T. (2006) Microbial diversity associated with odor modification for production of fertilizers from chicken litter,. Applied and Environmental Microbiology 72: 4105-4114.

Epstein, E. (2002) Land Application of Sewage Sludge and Biosolids. CRC Press, Boca Raton, Florida.

Erliconi, D. (2004) PCR-DGGE fingerprinting: novel strategies for detection of microbes in food. Journal of Microbiological Methods 56: 297-314.

Farrow, J.A.E., Lawson, P.A., Hippe, H., Gauglitz, U. and Collins, M.D. (1995) Phylogenetic evidence that the gram-negative nonsporulating bacterium Tissierella (Bacteroides) praeacuta is a member of the Clostridium subphylum of the gram-positive bacteria and description of Tissierella creatinini sp. nov. International Journal of Systematic and Evolutionary Microbiology 45: 436-440.

Ferris, M.J., Muyzer, G. and Ward, D.M. (1996) Denaturing gradient gel electrophoresis profiles of 16S rRNA-defined populations inhabiting a hot spring microbial mat community. Applied and Environmental Microbiology 62: 340-346.

Ferris, M.J. and Ward, D.M. (1997) Seasonal distributions of dominant 16S rRNA-defined populations in a hot spring microbial mat examined by denaturing gradient gel electrophoresis. Applied and Environmental Microbiology 63: 1375-1381.

Fey, A. and Conrad, R. (2000) Effect of temperature on carbon and electron flow and on the archaeal community in methanogenic rice field soil. Applied and Environmental Microbiology 66: 47904797.

Fiedler, S. and Graw, M. (2003) Decomposition of buried corpses with special reference to the formation of adipocere. Naturwissenschaften 90: 291-300.

Fiedler, S., Schneckenberger, K. and Graw, M. (2004) Characterization of soils containing adipocere. Archives of Environmental Contamination and Toxicology 47: 561-568.

Flitters, N.E. (1963) Observations on the effect of hurricane "Carla" on insect activity. International Journal of Biometeorology 6: 1432-1254.

Florez, A.B. and Mayo, B. (2006) PCR-DGGE as a tool for characterising dominant microbial populations in the Spanish blue-veined Cabrales cheese. International Dairy Journal 16: 1205-1210.

Forbes, S.L. (2008) Decomposition chemistry in a burial environment. In: Tibbett, M. and Carter, D.O. (Eds.) Soil Analysis in Forensic Taphonomy: Chemical and Biological Effects of Buried Human Remains. CRC Press, Boca Raton, Florida, pp. 225-246. 
Forbes, S.L., Dent, B.B. and Stuart, B.H. (2005) The effect of soil type on adipocere formation. Forensic Science International 154: 35-43.

Forbes, S.L., Stuart, B.H., Dadour, I.R. and Dent, B.B. (2004) A preliminary investigation of the stages of adipocere formation. Journal of Forensic Sciences 49: 566-574.

Fournier, F., Felletier, D., Vigneault, C., Goyette, B. and Boivin, G. (2005) Effect of barometric pressure on flight initiation by Trichogamma pretiosum and Trichogamma evanescens (Hymenoptera: Trichogrammatidae). Environmental Entomology 34: 1534-1540.

Freeman, C., Liska, G., Ostle, N.J., Jones, S.E. and Lock, M.A. (1995) The use of fluorogenic substrates for measuring enzyme activity in peatlands. Plant and Soil 175: 147-152.

Frey, J.C., Angert, E.R. and Pell, A.N. (2006) Assessment of biases associated with profiling simple, model communities using terminal-restriction fragment length polymorphism-based analysis. Journal of Microbiological Methods 67: 9-19.

Frostegard, A., Courtois, S., Ramisse, V., Clerc, S., Bernillon, D., Le Gall, F., Jeannin, P., Nesme, X. and Simonet, P. (1999) Quantification of bias related to the extraction of DNA directly from soils. Applied and Environmental Microbiology 65: 5409-5420.

Gafan, G.P. and Spratt, D.A. (2005) Denaturing gradient gel electrophoresis gel expansion (DGGEGE) - An attempt to resolve the limitations of co-migration in the DGGE of complex polymicrobial communities. FEMS Microbiology Letters 253: 303-307.

Galloway, A. (1997) The process of decomposition: a model from the Arizona-Sonoran Desert. In: Haglund, W.D. and Sorg, M.H. (Eds.) Forensic Taphonomy: The Postmortem Fate of Human Remains. CRC Press, Inc., Boca Raton, Florida, pp. 139-150.

Gans, J., Woilinsky, M. and Dunbar, J. (2005) Computational improvements reveal great bacterial diversity and high metal toxicity in soil. Science 309: 1387-1390.

Gao, Z., Tseng, C., Pei, Z. and Blaser, M.J. (2007) Molecular analysis of human forearm superficial skin bacterial biota. Proceedings of the National Academy of Sciences 104: 2927-2932.

Garcia-Lopez, M.L., Prieto, M. and Otero, A. (1998) The physiological attributes of Gram-negative bacteria associated with spoilage of meat and meat products. In: Davies, A. and Board, R. (Eds.) The Microbiology of Meat and Poultry. Blackie Academic and Professional, London, pp. 1-34.

Gehrig-Fasel, J., Guisan, A. and Zimmerman, N.E. (2008) Evaluating thermal treeline indicators based on air and soil temperature using an air-to-soil temperature transfer model. Ecological Modelling 213: 345-355.

Gerberth, V.J. (1996) Estimating Time of Death. (Eds.) Practical Homicide Investigation: Tactics, Procedures and Forensic Techniques. CRC Press, Boca Raton, Florida.

Gianfreda, L. (2006) Enzyme activities in soil. In: Nannipieri, P. and Smalla, K. (Eds.) Nucleic acids and proteins in soil. Springer-Verlag, Berlin, pp. 257-311.

Gianfreda, L. and Bollag, J.-M. (1996) Influence of natural and anthropogenic factors on enzyme activity in soil. In: Stotzky, G. and Bollag, J.-M. (Eds.) Soil Biochemistry Volume 9. Marcel Dekker, New York, pp. 123-194.

Gill-King, H. (1997) Chemical and Ultrastructural Aspects of Decomposition. In: Haglund, W.D. and Sorg, M.H. (Eds.) Forensic Taphonomy: The Postmortem Fate of Human Remains. CRC Press, Boca Raton, Florida, pp. 93-104. 
Gill, C.O. (1998) Microbial contamination during slaughter and butchering of cattle, sheep and pigs. In: Davies, A. and Board, R. (Eds.) The Microbiology of Meat and Poultry. Blackie Academic and Professional, London.

Gill, S.R., Pop, M., Deboy, R.T., Eckburg, P.B., Turnbaugh, P.J. and Samuel, B.S. (2006) Metagenomic analysis of the human distal gut microbiome. . Science 312: 1355-1359.

Gnaedinger, R.H., Pearson, A.M., Reineke, E.P. and Hix, V.M. (1963) Body composition of market weight pigs. Journal of Animal Science 22: 495-500.

Goff, M.L. (2000) A Fly for the Prosecution: How Insect Evidence Helps Solve Crimes. Harvard University Press, Cambridge, Massachusetts.

Goff, M.L., Brown, W.A., Hewadikaram, K.A.A. and Omori, A.I. (1991) Effects of heroin in decomposing tissues on the development rate of Boettcherisca peregrina (Diptera: Sarcophagidae) and implications of this effect on estimation of postmortem intervals using arthropod development patterns. Journal of Forensic Sciences 36: 537-542.

Goff, M.L. and Lord, W.D. (2000) Entomotoxicology: Insects as toxicological indicators and the impact of drugs and toxins on insect development. In: Byrd, J.H. and Castner, J.L. (Eds.) Forensic Entomology: The Utility of Arthropods in Legal Investigations. CRC Press, Boca Raton, Florida, pp. 331-340.

Goff, M.L., Omori, A.I. and Goodbrod, J.R. (1989) Effect of cocaine in tissues on the rate of development of Boettcherisca peregrina (Diptera:Sarcophagidae). Journal of medical Entomology 26: 91-93.

Grant, A. and Ogilvie, L. (2004) Name that microbe: rapid identification of taxa responsible for individual fragments in fingerprints of microbial community structure. Molecular Ecology Notes 4: 133-136.

Gravinghoff, J. and Hutter, H.J. (1977) The activities of alanine aminopeptidase, leucine aminopeptidase, proline dipeptidase and prolyl dipeptidase in the mucosa of the small intestine. European Journal of Pediatrics 127: 1432-1076.

Greenberg, B. (1991) Flies as forensic indicators. Journal of Medical Entomology 28: 565-577.

Greenberg, B. and Kunich, J.C. (2002) Entomology \& the Law: Flies as Forensic Indicators. Cambridge University Press, Cambridge, UK.

Gremion, F., Chatzinotas, A. and Harms, H. (2003) Comparative 16S rDNA and 16S rRNA sequence analysis indicates that Actinobacteria might be a dominant part of the metabolically active bacteria in heavy metal-contaminated bulk and rhizosphere soil. Environmental Microbiology 5: 896-907.

Grisold, A.J., Wendelin, I., Presterl, E., Raggam, R.B., Masoud, L., Badura, A. and Marth, E. (2007) In vitro activity of ten antibiotics, including tigecycline, against Bacteroides species in Austria. European Journal of Clinical Microbiology and Infectious Disease 26: 525-527.

Guan, T.Y. and Holley, R.A. (2003) Pathogen survival in swine manure environments and transmission of human enteric illness - a review. Journal of Environmental Quality 32: 383-392.

Guarner, F. and Malagelada, J.-R. (2003) Gut flora in health and disease. Lancet 361: 512-519.

Guo, Y., Zhu, N., Zhu, S. and Deng, C.Y. (2007) Molecular phylogenetic diversity of bacteria and its spatial distribution in composts. Journal of Applied Microbiology 103: 1344-1354.

Halpern, M., Raats, D. and Lev-Yadun, S. (2007) Plant biological warfare: thorns inject pathogenic bacteria into herbivores. Environmental Microbiology 9: 584-592. 
Haneklaus, S., Bloem, E. and Schnug, E. (2007) Sulfur interactions in crop ecosystems. In: Hawkesford, M.J. and De Kok, L.J. (Eds.) Sulfur in plants: an ecological perspective (Plant Ecophysiology). Springer, Netherlands, pp. 17-58.

Hantsis-Zacharov, E. and Halpern, M. (2007) Culturable psychrotrophic bacterial communities in raw milk and their proteolytic and lipolytic traits. Applied and Environmental Microbiology 73: 7162-7168.

Hao, W.-L. and Lee, Y.-K. (2004) Microflora of the Gastrointestinal Tract In: Spencer, J.F.T. and Ragout de Spencer, A.L. (Eds.) Public Health Microbiology: Methods and Protocols. Humana Press, New Jersey.

Harada, H., Oyaizu, H. and Ishikawa, H. (1996) A consideration about the origin of aphid intracellular symbiont in connection with gut bacterial flora. Journal of General and Applied Microbiology 42: $17-26$.

Hardina, C.M. and Fujioka, R.S. (2006) Soil: the environmental source of Escherichia coli and enterococci in Hawaii's streams. Environmental Toxicology and Water Quality 6: 185-195.

Harmsen, H.J.M., Wildeboer-Veloo, A.C.M., Grijpstra, J., Knol, J., Degener, J.E. and Welling, G.W. (2000) Development of 16S rRNA-based probes for the Coriobacterium group and the Atopobium cluster and their application for enumeration of Coriobacteraceae in human feces from volunteers of different age groups. Applied and Environmental Microbiology 66: 4523-4527.

Harris, D. (1994) Analyses of DNA extracted from microbial communities. In: Ritz, K., Dighton, J. and Giller, K.E. (Eds.) Beyond the Biomass. John Wiley and Sons, New York, pp. 111-119.

Hartmann, M. and Widmer, F. (2008) Reliability for detecting composition and changes of microbial communities by T-RFLP genetic profiling. FEMS Microbiology Ecology 63: 249-260.

Haskell, N.H., Schoenly, K.G. and Hall, R.D. (2001) Testing reliability of animal models in research and training programs in forensic entomology, Part II, Final Report, Grant No. 97-IJ-CX-0046. U.S. National Institute of Justice, Washington, D.C.

Haslam, T.C.F. and Tibbett, M. (2009) Soils of contrasting $\mathrm{pH}$ affect the decomposition of buried mammalian (Ovis aries) skeletal muscle tissue. Journal of Forensic Science doi: 10.1111/j.15564029.2009.01070.x.

Hay, I., Morency, M.-J. and Seguin, A. (2002) Assessing the persistence of DNA in decomposing leaves of genetically modified poplar trees. Canadian Journal of Forest Research 32: 977-982.

Hayano, K. and Katami, A. (1977) Extraction of $\beta$-glucosidase activity from pea field soil. Soil Biology \& Biochemistry 9: 349-351.

Hayano, K. and Tubaki, K. (1985) Origin and properties of $\beta$-glucosidase activity of tomato-field soil. Soil Biology \& Biochemistry 17: 553-557.

Hebuterne, X. (2003) Gut changes attributes to ageing: effects on intestinal microflora. Current Opinion in Clinical Nutrition and Metabolic Care 6: 49-54.

Heilig, H.G.H., Zoetendal, E.G., Vaughan, E.E., Marteau, P., Akkermans, A.D.L. and de Vos, W.M. (2002) Molecular diversity of Lactobacillus spp. and other lactic acid bacteria in the human intestine as determined by specific amplification of $16 \mathrm{~S}$ ribosomal DNA Applied and Environmental Microbiology 68: 114-123.

Henssge, C. and Madea, B. (2007) Estimation of the time since death. Forensic Science International 165: 182-184. 
Hery, M., Herrera, A., Vogel, T.M., Normand, P. and Navarro, E. (2005) Effect of carbon and nitrogen input on the bacterial community structure of Neocaledonian nickel mine spoils. FEMS Microbiology Ecology 51: 333-340.

Heuer, H., Krsek, M., Baker, P., Smalla, K. and Wellington, E.M. (1997) Analysis of actinomycete communities by specific amplification of genes encoding 16S rRNA and gel-electrophoretic separation in denaturing gradients. Applied and Environmental Microbiology 63: 3233-3241.

Heuer, H. and Smalla, K. (1997) Application of denaturing gradient gel electrophoresis and temperature gradient gel electrophoresis for studying soil microbial communities. In: van Elsas, J.D., Trevors, J.T. and Wellington, E.M.H. (Eds.) Modern Soil Microbiology. Marcel Dekker, New York, pp. 353374.

Holdeman, L.V., Good, I.J. and Moore, W.E.C. (1976) Human fecal flora: variation in bacterial composition within individuals and a possible effect of emotional stress. Applied and Environmental Microbiology 31: 359-375.

Holzapfel, W.H. (1998) The Gram-positive bacteria associated with meat and meat products. In: Davies, A. and Board, R.G. (Eds.) The Microbiology of Meat and Poultry. Blackie Academic and Professional, London, pp. 35-84.

Hopkins, D.L. (1989) Xylella fastidiosa: xylem-limited bacterial pathogen of plants. Annual Review of Phytopathology 27: 271-290.

Hopkins, D.W. (2008) The role of soil organisms in terrestrial decomposition. In: Tibbett, M. and Carter, D.O. (Eds.) Soil Analysis in Forensic Taphonomy: Chemical and Biological Effects of Buried Human Remains. CRC Press, Boca Raton, Florida, pp. 53-66.

Hopkins, D.W., Wiltshire, P.E.J. and Turner, B.D. (2000) Microbial characteristics of soil from graves: an investigation at the interface of soil microbiology and forensic science. Applied Soil Ecology 14: 283-288.

Hornek, R., Pommerening-Röser, A., Koops, H.-P., Farnleiter, A.H., Kreuzinger, N., Kirschner, A. and Mach, R.L. (2006) Primers containing universal bases reduce multiple amo $A$ gene specific DGGE band patterns when analysing the diversity of beta-ammonia oxidizers in the environment. Journal of Microbiological Methods 66: 147-155.

Horswell, J., Cordiner, S.J., Maas, E.W., Martin, T.M., Sutherland, K.B., Speir, T.W., Nogales, B. and Osborn, A.M. (2002) Forensic comparison of soils by bacterial community DNA profiling. Journal of Forensic Sciences 47: 350-353.

Hovda, M.B., Sivertsvik, M., Lunestad, B.T., Lorentzen, G. and Rosnes, J.T. (2007) Characterisation of the dominant bacterial population in modified atmosphere packaged farmed halibut (Hippoglossus hippoglossus) based on 16S rDNA-DGGE. Food Microbiology 24: 362-371.

Huang, P.M., Bollag, J.-M. and Senesi, N. (2002) Interactions between soil particles and microorganisms: impact on the terrestrial ecosystem. John Wiley and Sons, New York.

Hugenholtz, P. and Goebel, B.M. (2001) The Polymerase Chain Reaction as a Tool to Investigate Microbial Diversity in Environmental Samples. (Eds.) Environmental Molecular Microbiology: Protocols and Applications. Horizon Scientific Press, Wyndmondham, U.K.

Jaklic, D., Lapanje, A., Zupancic, K., Smrke, D. and Gunde-Cimerman, N. (2008) Selective antimicrobial activity of maggots against pathogenic bacteria. Journal of Medical Microbiology 57: 617-625.

Janaway, R.C. (1996) The decay of buried human remains and their associated materials. In: Hunter, J., Roberts, C. and Martin, A. (Eds.) Studies in Crime: An Introduction to Forensic Archaeology. Batesford, London, pp. 58-85. 
Jangid, K., Williams, M.A., Franzluebbers, A.J., Sanderlin, J.S., Reeves, J.H., Jenkins, M.B., Endale, D.M., Coleman, D.C. and Whitman, W.B. (2008) Relative impacts of land-use, management intensity and fertilization upon soil microbial community structure in agricultural systems. Soil Biology \& Biochemistry 40: 2843-2853.

Janse, I., Bok, J. and Zwart, G. (2004) A simple remedy against artifactual double bands in denaturing gradient gel electrophoresis. Journal of Microbiological Methods 57: 279-281.

Jarvie, T. and Harkins, T. (2007) Metagenomics Analysis Using the Genome Sequencer FLX System. Biochemica 3: 4-6.

Jaspe, A., Palacios, P., Matias, P., Fernandez, L. and Sanjose, C. (1994) Proteinase activity of Pseudomonas fluorescens frown in cold milk supplemented with nitrogen and carbon sources. Journal of Dairy Science 77: 923-929.

Jawetz, E., Melnick, J.L. and Melnick, E.A. (1982) Review of Medical Microbiology. Lange Medical, Boston.

Jensen, M.A. and Straus, N. (1993) Effect of PCR conditions on the formation of heteroduplex and singlestranded DNA products in the amplification of bacterial ribosomal DNA spacer regions. PCR Methods and Applications 3: 186-194.

Jensen, S., Ovreas, L., Daae, F.L. and Torsvik, V. (1998) Diversity in methane enrichments from agricultural soil revealed by DGGE separation of PCR amplified 16S rDNA fragments. FEMS Microbiology Ecology 26: 17-26.

Jernberg, C., Lofmark, S., Edlund, C. and Jansson, J.K. (2007) Long-term ecological impacts of antibiotic administration on the human intestinal microbiota. The ISME Journal 1: 56-66.

Jernberg, C., Sullivan, A., Edlund, C. and Jansson, J.K. (2005) Monitoring of antibiotic-induced alterations in the human intestinal microflora and detection of probiotic strains by use of terminal restriction fragment length polymorphism. Applied and Environmental Microbiology 71: 501-506.

Jones, H.E. and Bowen, I.D. (1993) Acid phosphatase activity in the larval salivary glands of developing Drosophila melanogaster. Cell Biology International 17: 305-315.

Jones, R.T., Robeson, M.S., Lauber, C.L., Hamady, M., Knight, R. and Fierer, N. (2009) A comprehensive survey of soil acidobacterial diversity using pyrosequencing and clone library analysis. The ISME Journal 3: 442-453.

Jörgensen, R.G. (1995) Die quantitative Bestimmung der mikrobiellen Biomasse in Böden mit der Chloroform-Fumigations-Extraktions-Methode. Göttinger Bodenkundliche Berichte: 1-229.

Josephson, K.L., Gerba, C.P. and Pepper, I.L. (1993) Polymerase chain reaction detection of non-viable bacteria pathogens. Applied and Environmental Microbiology 59: 3513-3515.

Juteau, P., Tremblay, D., Villemur, R., Bisaillon, J.-G. and Beaudet, R. (2005) Analysis of the bacterial community inhabiting an aerobic thermophilic sequencing batch reactor (AT-SBR) treating swine waste. Environmental Biotechnology 66: 115-122.

Kaplan, C.W. and Kitts, C.L. (2003) Variation between observed and true terminal restriction fragment length is dependant on true TRF length and purine content. Journal of Microbiological Methods 54: 121-125.

Kersters, K., de Vos, P., Gillis, M., Swings, J., Van Damme, P. and Stackebrandt, E. (2006) Introduction to the Proteobacteria. Prokaryotes 5: 3-37.

Killham, K. (1994) Soil Ecology. Cambridge University Press, Cambridge, UK. 
Kim, H.B., Park, M.J., Yang, H.C., An, D.S., Jin, H.Z. and Yang, D.C. (2006) Burkholderia ginsengisoli sp. nov., a beta-glucosidase-producing bacterium isolated from soil of a ginseng field. International Journal of Systematic and Evolutionary Microbiology 56: 2529-2533.

Kim, J.-S., Sparovek, G., Longo, R.M., De Melo, W.J. and Crowley, D. (2007a) Bacterial diversity of terra preta and pristine forest soil from the Western Amazon. Soil Biology \& Biochemistry 39: 684-690.

Kim, J.O. and Weiser, J.N. (1999) The respiratory tract microflora and disease. In: Tannock, G.W. (Eds.) Medical Importance of the Normal Microflora. Kluwer Academic Publishers, London.

Kim, Y.H., Engesser, K.H. and Kim, S.J. (2007b) Physiological, numerical and molecular characterization of alkyl ether-utilizing rhodococci. Environmental Microbiology 9: 1497-1510.

Kirchman, D.L. (2002) The ecology of Cytophaga-Flavobacteria in aquatic environments. FEMS Microbiology Ecology 39: 91-100.

Kisand, V. and Wikner, J. (2003) Combining culture-dependant and -independant methodologies for estimation of richness of estuarine bacterioplankton consuming riverine dissolved organic matter. Applied and Environmental Microbiology 69: 3607-3616.

Kitts, C.L. (2001) Terminal restriction fragment patterns: A tool for comparing microbial communities and assessing community dynamics. Current Issues in Intestinal Microbiology 2: 17-25.

Klijn, A., Mercenier, A. and Arigoni, F. (2005) Lessons from the genomes of bifidobacteria. FEMS Microbiology Reviews 29: 491-509.

Kopczynski, E., Bateson, M. and Ward, D. (1994) Recognition of chimeric small-subunit ribosomal DNAs composed of genes from uncultivated microorganisms. Applied and Environmental Microbiology 60: 746-748.

Kowalchuk, G.A., Gerards, S. and Woldendorp, J.W. (1997) Detection and characterization of fungal infections of Ammophila arenaria (marram grass) roots by denaturing gradient gel electrophoresis of specifically amplified 18s rDNA. Applied and Environmental Microbiology 63: 3858-3865.

Kowalchuk, G.A., Speksnijder, A.C.L., Zhang, K. and Goodman, R.M.v.V., J. A. (2007) Finding the needles in the metagenome haystack. Microbial Ecology 53: 475-485.

Kramer, K.J. and Muthukrishnan, S. (1997) Insect chitinases: molecular biology and potential use as biopesticides. Insect Biochemistry and Molecular Biology 27: 887-900.

Kreader, C.A. (1998) Persistence of PCR-detectable Bacteroides distasonis from human feces in river water. Applied and Environmental Microbiology 64: 4103-4105.

Larsen, B. and Monif, G.R.G. (2001) Understanding the bacterial flora of the female genital tract. Clinical Infectious Diseases 32: 69-77.

Laura, D., De Socio, G., Frassanito, R. and Rotilio, D. (1996) Effects of atrazine on Ochrobactrum anthropi membrane fatty acids. Applied and Environmental Microbiology 62: 2644-2646.

Lee, A.H., Husseneder, C. and Hooper-Bui, L. (2008a) Culture-independent identification of gut bacteria in fourth-instar red imported fire ant, Solenopsis invicta Buren, larvae. Journal of Invertebrate Pathology 98: 20-33.

Lee, D.H., Zo, Y.G. and Kim, S.J. (1996) Nonradioactive method to study genetic profiles of natural bacterial communities by PCR-single-strand-conformation polymorphism. Applied and Environmental Microbiology 62: 3112-3120. 
Lee, Z.M.-P., Bussema, C. and Schmidt, T.M. (2008b) rrnDB: documenting the number of rRNA and tRNA genes in bacteria and archaea. Nucleic Acids Research doi:10.1093/nar/gkn689.

Leech, N.L., Barrett, K.C. and Morgan, G.A. (2005) SPSS for Intermediate Statistics: Use and Interpretation. Routledge, New York.

Lefebvre, O., Vasudevan, N., Thanasekaran, K., Moletta, R. and Godon, J.J. (2006) Microbial diversity in hypersaline wastewater: the example of tanneries. Extremophiles 10: 505-513.

Leininger, S., Urich, T., Schloter, M., Schwark, L., Nicol, G.W., Prosser, J.I., Schuster, S.C. and Schleper, C. (2006) Archaea predominate among ammonia-oxidizing prokaryotes in soils. Nature 442: 806-809.

Lerner, A., Shor, Y., Vinokurov, A., Okon, Y. and Jurkevitch, E. (2006) Can denaturing gradient gel electrophoresis (DGGE) analysis of amplified 16s rDNA of soil bacterial populations be used in forensic investigations? Soil Biology \& Biochemistry 38: 1188-1192.

Lesaulnier, C., Papamichail, D., McCorkle, S., Ollivier, B., Skiena, S., Taghavi, S., Zak, D. and van der Lelie, D. (2008) Elevated atmospheric CO2 affects soil microbial diversity associated with trembling aspen. Environmental Microbiology 10: 926-941.

Leser, T.D., Amenuvor, J.Z., Jensen, T.K., Lindecrona, R.H., Boye, M. and Moller, K. (2002) Cultureindependant analysis of gut bacteria: the pig gastrointestinal tract microbiota revisited. Applied and Environmental Microbiology 68: 673-690.

Levy-Booth, D.J., Campbell, R.G., Gulden, R.H., Hart, M.M., Powell, J.R., Klironomos, J.N., Pauls, K.P., Swanton, C.J., Trevors, J.T. and Dunfield, K.E. (2007) Cycling of extracellular DNA in the soil environment. Soil Biology \& Biochemistry 39: 2977-2991.

Ley, R.E., Backhed, F., Turnbaugh, P., Lozupone, C.A., Knight, R.D. and Gordon, J.I. (2005) Obesity alters gut microbial ecology. Proceedings of the National Academy of Sciences of the United States of America 102: 11070-11075.

Ley, R.E., Turnbaugh, P.J., Klein, S. and Gordon, J.I. (2006) Microbial ecology: human gut microbes associated with obesity. Nature 444: 1022-1023.

Li, M., Wang, B., Zhang, M., Rantalainen, M., Wang, S., Zhou, H., Zhang, Y., Shen, J., Pang, X., Zhang, M., Wei, H., Chen, Y., Lu, H., Zuo, J., Su, M., Qiu, Y., Jia, W., Xiao, C., Smith, L.M., Yang, S., Holmes, E., Tang, H., Zhao, G., Nicholson, J.K., Li, L. and Zhao, L. (2008) Symbiotic gut microbes modulate human metabolic phenotypes. Proceedings of the National Academy of Sciences 105: 2117-2122.

Li, M., Zhou, H., Hua, W., Wang, B., Wang, S., Zhao, G., Li, L., Zhao, L. and Pang, X. (2009) Molecular diversity of Bacteroides spp. in human fecal microbiota as determined by group-specific 16S rRNA gene clone library analysis. Systematic and Applied Microbiology 32: 193-200.

Li, M.Y., Zhou, G.H., Xu, X.L., Li, C.B. and Zhu, W.Y. (2006) Changes of bacterial diversity and main flora in chilled pork during storage using PCR-DGGE. Food Microbiology 23: 607-611.

Li, X. and Sarah, P. (2003) Arylsulfatase activity of soil microbial biomass along a Mediterranean-arid transect. Soil Biology \& Biochemistry 35: 925-934.

Liesack, W., Weylans, H. and Stackebrandt, E. (1991) Potential risks of gene amplification by PCR as determined by $16 \mathrm{~S}$ rDNA analysis of a mixed-culture of strict barophilic bacteria. Microbial Ecology 21: 191-198.

Liles, M.R., Manske, B.F., Bintrim, S.B., Handelsman, J. and Goodman, R.M. (2003) A census of rRNA genes and linked genomic sequences within a soil metagenomic library. Applied and Environmental Microbiology 69: 2684-2691. 
Liu, W.T., Marsh, T. and Forney, L.J. (1998) Determination of the microbial diversity of anaerobic-aerobic activated sludge by a novel molecular biological technique. Water Science and Technology 37: 417422 .

Liu, W.T., Marsh, T.L., Cheng, H. and Forney, L.J. (1997) Characterization of microbial diversity by determining terminal restriction fragment length polymorphisms of genes encoding 16S rRNA. Applied and Environmental Microbiology 63: 4516-4522.

Liu, X.Y., Wang, B.J., Jiang, C.Y. and Liu, S.J. (2006) Paracoccus sulfuroxidans sp. nov., a sulfur oxidizer from activated sludge. International Journal of Systematic and Evolutionary Microbiology 56: 26932695.

Lopes de Carvalho, L.M. and Linhares, A.X. (2001) Seasonality of insect succession and pig carcass decomposition in a natural forest area in Southeastern Brazil. Journal of Forensic Sciences 46: 604608.

Lueders, T. and Friedrich, M.W. (2003) Evaluation of PCR amplification bias by terminal restriction fragment length polymorphism analysis of small-subunit rRNA and mcrA genes by using defined template mixtures of methanogenic pure cultures and soil DNA extracts. Applied and Environmental Microbiology 69: 320-326.

Macauley, J.J., Adams, C.D. and Mormile, M.R. (2007) Diversity of tet resistance genes in tetracyclineresistant bacteria isolated from a swine lagoon with low antibiotic impact. Canadian Journal of Microbiology 53: 1307-1315.

Macdonald, C.A., Ang, A., Cordiner, S.J. and Horswell, J. (2009) Discrimination of soils at regional and local levels using bacterial and fungal T-RFLP profiling. Journal of Forensic Science in press.

Macfarlane, G.T., Allison, C., Gibson, S.A.W. and Cummings, J.H. (1988) Contribution of the microflora to proteolysis in the human large intestine. Journal of Applied Bacteriology 64: 37-46.

Madigan, M.T., Martinko, J.M. and Parker, J. (2000) Brock Biology of Microorganisms. Prentice Hall International, New Jersey.

Magalhaes, C.L., De Souza, E.M., Weber, O.B., Baldani, J.I., Dobereiner, J. and de Oliveira Pedrosa, F. (2001) 16S ribosomal DNA characterization of nitrogen-fixing bacteria isolated from banana (Musa spp.) and pineapple (Ananas comosus (L.) Merril). Applied and Environmental Microbiology 67: 2375-2379.

Mahmood, S., Freitag, T.E. and Prosser, J.I. (2006) Comparison of PCR primer-based strategies for characterization of ammonia oxidizer communities in environmental samples. . FEMS Microbiology Ecology 56: 482-493.

Maidak, B.L., Cole, J.R., Parker, C.T., Garrity, G.M., Larsen, N., Li, B., Lilburn, T.G., McCaughey, M.J., Olsen, G.J., Overbeek, R., Pramanik, S., Schmidt, T.M., Tiedje, J.M. and Woese, C.R. (1999) A new version of the RDP (Ribosomal Database Project). Nucleic Acids Research 27: 171-173.

Malgorn, Y. and Coquoz, R. (1999) DNA typing for identification of some species of Calliphoridae. An interest in forensic entomology. Forensic Science International 102: 111-119.

Mann, R.W., Bass, W.M. and Meadows, L. (1990) Time since death and decomposition of the human body: variables and observations in case and experimental field studies. Journal of Forensic Sciences 35: 103-111.

Maron, P.-A., Ranjard, L., Mougel, C. and Lemanceau, P. (2007) Metaproteomics: A new approach for studying functional microbial ecology. Microbial Ecology 53: 486-493. 
Marschner, P. (2007) Soil microbial community structure and function assessed by FAME, PLFA and DGGE - advantages and limitations. In: Varma, A. and Oelmuller, R. (Eds.) Soil Biology: Advanced Techniques in Soil Microbiology. Springer, Berlin Heidelberg, pp. 181-200.

Marsh, T. (1999) Terminal restriction fragment length polymorphism (T-RFLP): an emerging method for characterising diversity among homologous populations of amplification products. Current Opinion in Microbiology 2: 323-327.

Marsh, T., Liu, W.T., Forney, L.J. and Cheng, H. (1998) Beginning a molecular analysis of the eukaryal community in activated sludge. Water Science and Technology 37: 455-460.

Marsh, T.L. (2005) Culture-independent microbial community analysis with terminal restriction fragment length polymorphism. Methods in Enzymology 397: 308-329.

Martin-Laurent, F., Philippot, L., Hallet, S., Chaussod, R., Germon, J.C., Soulas, G. and Catroux, G. (2001) DNA Extraction from Soils: Old Bias for New Microbial Diversity Analysis Methods. Applied and Environmental Microbiology 67: 2354-2359.

Martin, R., Heilig, H.G.H.J., Zoetendal, E.G., E., J., Fernandez, L., Smidt, H. and Rodriguez, J.M. (2007) Cultivation-independent assessment of the bacterial diversity of breast milk among healthy women. Research in Microbiology 158: 31-37.

Marx, M.-C., Wood, M. and Jarvis, S.C. (2001) A microplate fluorimetric assay for the study of enzyme diversity in soils. Soil Biology \& Biochemistry 33: 1633-1640.

Matches, J.R., Liston, J. and Curran, D. (1974) Clostridium perfringens in the environment. Applied Microbiology 28: 655-660.

Matsuki, T., Watanabe, K., Fujimoto, J., Miyamoto, Y., Takada, T., Matsumoto, K., Oyaizu, H. and Tanaka, R. (2002) Development of 16S rRNA-gene-targeted group-specific primers for the detection and identification of predominant bacteria in human feces. Applied and Environmental Microbiology 68 .

Matsuki, T., Watanabe, K., Fujimoto, J., Takada, T. and Tanaka, R. (2004) Use of 16S rRNA gene-targeted group-specific primers for real-time PCR analysis of predominant bacteria in human feces. Applied and Environmental Microbiology 70: 7220-7228.

Mayr, D., Margesin, R., Klingsbichel, E., Hartungen, E., Jenewein, D., Schinner, F. and Mark, T.D. (2003) Rapid detection of meat spoilage by measuring volatile organic compounds by using proton transfer reaction mass spectrometry. Applied and Environmental Microbiology 69: 4697-4705.

McCartney, A.L. and Gibson, G.R. (2006) The Normal Microbiota of the Human Gastrointestinal Tract: History of Analysis, Succession, and Dietary Influences. In: Ouwehand, A.C. and Vaughan, E.E. (Eds.) Gastrointestinal Microbiology. Taylor \& Francis, New York, pp. 51-73.

McCartney, A.L., Wang, W. and Tannock, G.W. (1996) Molecular analysis of the composition of the bifidobacterial and lactobacillus microflora of humans. Applied and Environmental Microbiology 62: 4608-4613.

McClellan, J.B. and Garner, C.W. (1980) Purification and properties of human intestine alanine aminopeptidase. Biochimica et Biophysica Acta 613: 160-167.

McFarland, M.J. (2000) Biosolids engineering. McGraw-Hill Professional, New York.

Metcalfe, A.C., Krsek, M., Gooday, G.W., Prosser, J.I. and Wellington, E.M.H. (2002) Molecular analysis of a bacterial chitinolytic community in an upland pasture. Applied and Environmental Microbiology 68: 5042-5050. 
Micozzi, M.S. (1986) Experimental study of post mortem change under field conditions: Effects of freezing, thawing and mechanical injury. Journal of Forensic Sciences 31: 953-961.

Mille-Lindblom, C., Fischer, H. and Tranvik, L.J. (2006) Antagonism between bacteria and fungi: substrate competition and a possible tradeoff between fungal growth and tolerance towards bacteria. Oikos 113: $233-242$.

Miller, M., Palojarvi, A., Rangger, A., Reeslev, M. and Kjoller, A. (1998) The use of fluorogenic substrates to measure fungal presence and activity in soil. Applied and Environmental Microbiology 64: 613617.

Mobley, H.L.T. and Hausinger, R.P. (1989) Microbial ureases: significance, regulation, and molecular characterization. Microbiological Reviews 53: 85-108.

Moeseneder, M.M., Winter, C., Arrieta, J.M. and Herndl, G.J. (2001) Terminal-restriction fragment length polymorphism (T-RFLP) screening of a marine archaeal clone library to determine the different phylotypes. Journal of Microbiological Methods 44: 159-172.

Mohlenhoff, P., Muller, L., Gorbushina, A.A. and Petersen, K. (2001) Molecular approach to the characterisation of fungal communities: methods for DNA extraction, PCR amplification and DGGE analysis of painted art objects. FEMS Microbiology Letters 195: 169-173.

Mohr O'Hara, C., Steigerwalt, A.G., Green, D., McDowell, M., Hill, B.C., Brenner, D.J. and Miller, J.M. (1999) Isolation of Providencia heimbachae from human feces. Journal of Clinical Microbiology 37: 3048-3050.

More, M.I., Herrick, J.B., Silva, M.C., Ghiorse, W.C. and Madsen, E.L. (1994) Quantitative cell lysis of indigenous microorganisms and rapid extraction of microbial DNA from sediment. Applied and Environmental Microbiology 60: 1572-1580.

Morgan, C.A., Hudson, A., Konopka, A. and Nakatsu, C.H. (2002) Analysis of microbial activity in biomass-recycle reactors using denaturing gradient gel electrophoresis of 16S rDNA and 16S rRNA PCR products. Canadian Journal of Microbiology 48: 333-341.

Morris, S.J. and Blackwood, C.B. (2007) The ecology of soil organisms. In: Paul, E.A. (Eds.) Soil Microbiology, Ecology and Biochemistry. Academic Press, Oxford, UK, pp. 195-229.

Muyzer, G., Brinkhoff, T., Nubel, U., Santegoeds, C., Schaefer, H. and Wawer, C. (2004) Denaturing gradient gel electrophoresis (DGGE) in microbial ecology. In: Kowalchuk, G.A., De Bruijn, F.J., Head, I.M., Akkermans, A.D.L. and van Elsas, J.D. (Eds.) Molecular Microbial Ecology Manual. Kluwer Academic Press, Dordrecht, Netherlands, pp. 743-770.

Muyzer, G., de Waal, E.C. and Uitterlinden, A.G. (1993) Profiling of complex microbial populations by denaturing gradient gel electrophoresis analysis of polymerase chain reaction-amplified genes coding for 16S rRNA. Applied and Environmental Microbiology 59: 695-700.

Muyzer, G., Teske, A., Wirsen, C.O. and Jannasch, H.W. (1995) Phylogenetic relationships of Thiomicrospira species and their identification in deep-sea hydrothermal vent samples by denaturing gradient gel electrophoresis of $16 \mathrm{~S}$ rDNA fragments. Archives of Microbiology 164: $165-172$.

Myskowiak, J.B. and Doums, C. (2002) Effects of refrigeration on the biometry and development of Protophormia terraenovae (Robineau-Desvoidy) (Diptera: Calliphoridae) and its consequences in estimating post-mortem interval in forensic investigations. Forensic Science International 125: 25461.

Nakatsu, C.H. (2005) Soil microbial community responses to additions of organic carbon substrates and heavy metals $(\mathrm{Pb}$ and $\mathrm{Cr}$ ). Applied and Environmental Microbiology 71: 7679-7689. 
Nakatsu, C.H. (2007) Soil microbial community analysis using denaturing gradient gel electrophoresis. Soil Science Society of America Journal 71: 562-571.

Nannipieri, P., Kandeler, E. and Ruggiero, P. (2002) Enzyme Activities and Microbiological and Biochemical Processes in Soil In: Burns, R.G. and Dick, R.P. (Eds.) Enzymes in the Environment. Marcel Dekker Inc., New York, pp. 1-34.

Neufeld, J.D., Yu, Z., Lam, W. and Mohn, W.W. (2004) Serial analysis of ribosomal sequence tags (SARST): a high-throughput method for profiling complex microbial communities. Environmental Microbiology 6: 131-144.

Niemi, R.M. and Vepsalainen, M. (2005) Stability of the fluorogenic enzyme substrates and pH optima of enzyme activities in different Finnish soils. Journal of Microbiological Methods 60: 195-205.

Nikolausz, M., Sipos, R., Revesz, A., Szekely, A. and Marialigeti, K. (2005) Observation of bias associated with re-amplification of DNA isolated from denaturing gradient gels. FEMS Microbiology Letters 244: 385-390.

Niva, M., Hernesmaa, A., Haahtela, K., Salkinoja-Salonen, M., Sivonen, K. and Haukka, K. (2006) Actinobacterial communities of boreal forest soil and lake water are rich in mycobacteria. Boreal Environment Research 11: 45-53.

Nubel, U., Engelen, B., Felske, A., Snaidr, J., Wieshuber, A., Amann, R.I., Ludwig, W. and Backhaus, H. (1996) Sequence heterogeneities of genes encoding 16S rRNAs in Paenibacillus polymyxa detected by temperature gradient gel electrophoresis. Journal of Bacteriology 178: 5636-5643.

NZFSA (2003) Regulatory control of antibiotics to manage antibiotic resistance. Agricultural Compounds and Veterinary Medicines Group, New Zealand Food Safety Authority.

O'Brien, T.G. and Kuehner, A.C. (2007) Waxing grave about adipocere: soft tissue change in an aquatic context. Journal of Forensic Sciences 52: 294-301.

Okabe, S., Odagiri, M., Ito, T. and Satoh, H. (2007) Succession of sulfur-oxidizing bacteria in the microbial community on corroding concrete in sewer systems. Applied and Environmental Microbiology 73: 971-980.

Okabe, S. and Shimazu, Y. (2007) Persistence of host-specific Bacteroides-Prevotella 16S rRNA genetic markers in environmental waters: effects on temperature and salinity. Applied Microbiology and Biotechnology 76: 935-944.

Osborn, A.M., Moore, E.R. and Timmis, K.N. (2000) An evaluation of terminal-restriction fragment length polymorphism (T-RFLP) analysis for the study of microbial community structure and dynamics. Environmental Microbiology 2: 39-50.

Overdijk, B., Van Steijn, G.J. and Odds, F.C. (1996) Chitinase levels in guinea pig blood are increased after systemic infection with Aspergillus fumigatus. Glycobiology 6: 627-634.

Ovreas, L., Forney, L., Daae, F.L. and Torsvik, V. (1997) Distribution of bacterioplankton in meromictic Lake Saelenvannet, as determined by denaturing gradient gel electrophoresis of PCR-amplified gene fragments coding for 16S rRNA. Applied and Environmental Microbiology 63: 3367-3373.

Paget, E.L., Jocteur-Monrozier, L. and Simonet, P. (1992) Adsorption of DNA on clay minerals: protection against DNase I and influence on gene transfer. FEMS Microbiology Letters 97: 31-40.

Palmroth, M.R.T., Munster, U., Pichtel, J. and Puhakka, J.A. (2005) Metabolic responses of microbiota to diesel fuel addition in vegetated soil. Biodegradation 16: 91-101. 
Pandey, J., Ganesan, K. and Jain, R.K. (2007) Variations in T-RFLP profiles with differing chemistries of fluorescent dyes used for labeling the PCR primers. Journal of Microbiological Methods 68: 633638.

Parkinson, R.A. (2005) Forensic DNA Profiling of Bacterial Communities in Soil. School of Biological Sciences Victoria University of Wellington, New Zealand. M.Sc thesis.

Parkinson, R.A., Dias, K.-R., Horswell, J., Greenwood, P., Banning, N., Tibbett, M. and Vass, A.A. (2009) Microbial community analysis of human decomposition on soil. In: Ritz, K., Dawson, L.A. and Miller, D. (Eds.) Criminal and Environmental Soil Forensics. Springer, pp. 379-394.

Pascual, L.M., Daniele, M.B., Pajaro, C. and Barberis, L. (2006) Lactobacillus species isolated from the vagina: identification, hydrogen peroxide production and nonoxynol-9 resistance. Contraception 73 : 78-81.

Payne, J.A. (1965) A summer carrion study of the baby pig Sus scrofa Linnaeus. Ecology 46: 592-602.

Perry, W.L., Bass, W.M., Riggsby, W.S. and Sirotkin, K. (1988) The autodegradation of deoxyribonucleic acid (DNA) in human rib bone and its relationship to the time interval since death. Journal of Forensic Sciences 33: 144-153.

Piao, Z., Yang, L., Zhao, L. and Yin, S. (2008) Actinobacterial community structure in soils receiving longterm organic and inorganic amendments. Applied and Environmental Microbiology 74: 526-530.

Pickering, R.B. and Bachman , D.C. (1996) The Use of Forensic Anthropology. CRC Press, Inc., Boca Raton, Florida.

Priscu, J.C., Adams, E.E., Lyons, W.B., Voytek, M.A., Mogk, D.W., Brown, R.L., McKay, C.P., Takacs, C.D., Welch, K.A., Wolf, C.F., Kirshtein, J.D. and Avci, R. (1999) Geomicrobiology of subglacial ice above Lake Vostok, Antarctica. Science 286: 2141-2144.

Prosser, J.I. and Tough, A.J. (1991) Growth mechanisms and growth kinetics of filamentous microorganisms. Critical Reviews in Biotechnology 10: 253-274.

Qiu, X., Wu, L., Huang, H., McDonel, P.E., Palumbo, A.V., Tiedje, J.M. and Zhou, J. (2001) Evaluation of PCR-generated chimeras, mutations and heteroduplexes with 16S rRNA gene-based cloning. Applied and Environmental Microbiology 67: 880-887.

Querido, D. (1998) A preliminary investigation into postmortem changes in skinfold impedance during the early postmortem period in rats. Forensic Science International 96: 107-114.

Querido, D. (1999) A preliminary study of changes in scalp impedance during the early post-mortem period in rats. Forensic Science International 101: 123-130.

Ranjard, L., Poly, F., Combrisson, J., Richaume, A., Gourbiere, F., Thioulouse, J. and Nazaret, S. (2000) Heterogeneous cell density and genetic structure as bacterial pools associated with various soil microenvironments as determined by enumeration and DNA fingerprinting approach (RISA). Microbial Ecology 39: 263-272.

Rees, G.N., Baldwin, D.S., Watson, G.O., Perryman, S. and Nielsen, D.L. (2004) Ordination and significance testing of microbial community composition derived from terminal restriction fragment length polymorphisms: application of multivariate statistics. Antonie van Leeuwenhoek 86: 339347.

Requena, T., Burton, J., Matsuki, T., Munro, K., Simon, M.A., Tanaka, R., Watanabe, K. and Tannock, G.W. (2002) Identification, detection and enumeration of human Bifidobacterium species by PCR targeting the transaldolase gene. Applied and Environmental Microbiology 68: 2420-2427. 
Reysenbach, A., Giver, L., Wickham, G. and Pace, N. (1992) Differential amplification of rRNA genes by polymerase chain reaction. Applied and Environmental Microbiology 58: 3417-3418.

Riffel, A. and Brandelli, A. (2006) Keratinolytic bacteria isolated from feather waste. Brazilian Journal of Microbiology 37: 395-399.

Rinttila, T., Kassinen, A., Malinen, L., Krogius, L. and Palva, A. (2004) Development of an extensive set of $16 \mathrm{~S}$ rDNA-targeted primers for quantification of pathogenic and indigenous bacteria in faecal samples by real-time PCR. Journal of Applied Microbiology 97: 1166-1177.

Robinson, W. and Baker, F.C. (1939) The enzyme urease and the occurrence of ammonia in maggot-infected wounds. The Journal of Parasitology 25: 149-155.

Roesch, L.F.W., Fulthorpe, R.R., Riva, A., Casella, G., Hadwin, A.K.M., Kent, A.D., Daroub, S.H., Camargo, F.A.O., Farmerie, W.G. and Triplett, E.W. (2007) Pyrosequencing enumerates and contrasts soil microbial diversity. The ISME Journal 1: 283-290.

Roitberg, B.D., Sircom, J., Roitberg, C.A., van Alohen, J.J.M. and Mangel, M. (1993) Life expectancy and reproduction. Nature 364: 108.

Ronaghi, M. (2001) Pyrosequencing sheds light on DNA sequencing. Genome Research 11: 3-11.

Roth, R.R. and James, W.D. (1988) Microbial ecology of the skin. Annual Review of Microbiology 42: 441464.

Roudiere, L., Jean-Pierre, H., Comte, C., Zorgniotti, I., Marchandin, H. and Jumas-Bilak, E. (2007) Isolation of Schineria sp. from a man. Emerging Infectious Diseases 13: 659-660.

Roy, R.N. (1980) Fluorimetric assay of the activity of extracellular lipases of Pseudomonas fluorescens and Serratia marcescens. Journal of Applied Bacteriology 49: 265-271.

Ryu, S.H., Park, M., Lee, J.R., Yun, P.-Y. and Jeon, C.O. (2007) Brevundimonas aveniformis sp. nov., a stalked species isolated from activated sludge. International Journal of Systematic and Evolutionary Microbiology 57: 1561-1565.

Sanchez, J.I., Rossetti, L., Martinez, B., Rodriguez, A. and Giraffa, G. (2006) Application of reverse transcriptase PCR-based T-RFLP to perform semi-quantitative analysis of metabolically active bacteria in dairy fermentations. Journal of Microbiological Methods 65: 268-277.

Santamaria, J. and Toranzos, G.A. (2003) Enteric pathogens and soil: a short review. Intestinal Microbiology 6: 5-9.

Sardellio, M., Annunziata, I., Roma, G. and Ballabio, A. (2005) Sulfatases and sulfatase modifying factors: an exclusive and promiscuous relationship. Human Molecular Genetics 14: 3203-3217.

Satokari, R.M., Vaughan, E.E., Akkermans, A.D.L., Saarela, M. and de Vos, W.M. (2001) Bifidobacterial diversity in human feces detected by genus-specific PCR and denaturing gradient gel electrophoresis. Applied and Environmental Microbiology 67: 504-513.

Sawyer, W.R., Steup, D.R., Martin, B.S. and Forney, R.B. (1988) Cardiac blood pH as a possible indicator of postmortem interval. Journal of Forensic Sciences 33: 1439-1444.

Schlumbaum, A., Mauch, F., Vogeli, U. and Boller, T. (1986) Plant chitinases are potent inhibitors of fungal growth. Nature 324: 365-367.

Schmeisser, C., Steele, H. and Streit, W.R. (2007) Metagenomics, biotechnology with non-culturable microbes. Applied Microbiology and Technology 75: 955-962. 
Schmidt, J.K., Konig, B. and Reichl, U. (2007) Characterization of a three bacteria mixed culture in a chemostat: evaluation and application of a quantitative terminal-restriction fragment length polymorphism (T-RFLP) analysis for absolute and species specific cell enumeration. Biotechnology and Bioengineering 96: 738-756.

Schoeller, D.A. (1989) Changes in total body water with age. American Journal of Clinical Nutrition 50: 1176-1181.

Schrier, R.W., Wamg, W., Poole, B. and Mitra, A. (2004) Acute renal failure: definitions, diagnosis, pathogenesis, and therapy. Journal of Clinical Investigation 114: 5-14.

Schwieger, F. and Tebbe, C.C. (1998) A new approach to utilize PCR-single-strand-conformation polymorphism for $16 \mathrm{~S}$ rRNA gene-based microbial community analysis. Applied and Environmental Microbiology 64: 4870-4876.

Searle, P. (1984) The Berthelot or indophenol reaction and its use in the analytical chemistry of nitrogen. Analyst 109: 549-568.

Searle, P.L. and Speir, T.W. (1976) An automated colorimetric method for the determination of urease activity in soil and plant material. Communications in Soil Science and Plant Analysis 7: 365-374.

Sekiguchi, H., Tomioka, N., Nakahara, T. and Uchiyama, H. (2001) A single band does not always represent single bacterial strains in denaturing gradient gel electrophoresis analysis. Biotechnology Letters 23: 1205-1208.

Sharma, S., Aneja, M.K., Mayer, J., Munch, J.C. and Schloter, M. (2005) Characterization of bacterial community structure in rhizosphere soil of grain legumes. Microbial Ecology 49: 407-415.

Sheffield, V.C., Cox, D.R., Lerman, L.S. and Myers, R.M. (1989) Attachment of a 40-base-pair G+C-rich sequence (GC-clamp) to genomic DNA fragments by the polymerase chain reaction results in improved detection of single-base changes. Proceedings of the National Academy of Sciences 86: 232-236.

Sheng, H.-P. and Huggins, R.A. (1979) A review of body composition studies with emphasis on total body water and fat. American Journal of Clinical Nutrition 32: 630-649.

Shyu, C., Soule, T., Bent, S.J., Foster, J.A. and Forney, L.J. (2007) MiCA: A web-based tool for the analysis of microbial communities based on terminal-restriction fragment length polymorphisms of $16 \mathrm{~S}$ and 18S rRNA genes. Microbial Ecology 53: 562-570.

Simpson, J.M., McCracken, V.J., White, B.A., Gaskins, H.R. and Mackie, R.I. (1999) Application of denaturant gradient gel electrophoresis for the analysis of the porcine gastrointestinal microbiota. Journal of Microbiological Methods 36: 167-179.

Singh, B.K., Nazaries, L., Munro, S., Anderson, I.C. and Campbell, C.D. (2006) Use of multiplex terminal restriction fragment length polymorphism for rapid and simultaneous analysis of different components of the soil microbial community. Applied and Environmental Microbiology 72: 72787285 .

Singh, G.J.P. and Vardanis, A. (1984) Chitinases in the house-fly, Musca domestica: Pattern of activity in the life cycle and preliminary characterization. Insect Biochemistry 14: 215-218.

Skujins, J.J. and McLaren, A.D. (1969) Persistence of enzymatic activities in stored and geologically preserved soils. Enzymologia 34: 213-225.

Sledzik, P.S. and Micozzi, M.S. (1997) Autopsied, embalmed, and preserved human remains: Distinguishing features in forensic and historic contexts. In: Haglund, W.D. and Sorg, M.H. (Eds.) Forensic 
Taphonomy: The Postmortem Fate of Human Remains. CRC Press Inc., Boca Raton, FL., pp. 483496.

Sly, L.I., Taghavi, M. and Fegan, M. (1999) Phylogenetic position of Chitinophaga pinensis in the Flexibacter-Bacteroides-Cytophaga phylum. International Journal of Systematic Bacteriology 49: 479-481.

Smeeton, W.M.I., Koelmeyer, T.D., Holloway, B.A. and Singh, P. (1984) Insects associated with exposed human corpses in Auckland, New Zealand. Medicine, Science and the Law 24: 167-174.

Somvanshi, V.S., Lang, E., Staubler, B., Sproer, C., Schumann, P., Ganguly, S., Saxena, A.K. and Stackebrandt, E. (2006) Providencia vermicola sp. nov., isolated from infective juveniles of the entomopathogenic nematode Steinernema thermophilum. International Journal of Systematic and Evolutionary Microbiology 56: 629-633.

Speir, T.W. and Ross, D.F. (1978) Soil phosphatases and sulphatases. In: Burns, R.G. (Eds.) Soil Enzymes. Academic Press, New York, pp. 198-250.

Speir, T.W. and Ross, D.J. (1990) Temporal stability of enzymes in a peatland soil profile. Soil Biology \& Biochemistry 22: 1003-1005.

Speir, T.W., van Schaik, A.P., Llloyd-Jones, A.R. and Kettles, H.A. (2003) Temporal response of soil biochemical properties in a pastoral soil after cultivation following high application rates of undigested sewage sludge. Biology and Fertility of Soils 38: 377-385.

Stackebrandt, E. and Goebel, B.M. (1994) Taxonomic note: A place for DNA-DNA reassociation and 16S rRNA sequence analysis in the present species definition in bacteriology. International Journal of Systematic Bacteriology 44: 846-849.

Stackebrandt, E., Reboli, A.C. and Farrar, W.E. (2006) The Genus Erysipelothrix. Prokaryotes 4: 492-510.

Stark, J.M. and Firestone, M.K. (1995) Mechanisms for soil moisture effects on activity of nitrifying bacteria. Applied and Environmental Microbiology 61: 218-221.

Stokes, K.L., Forbes, S.L., Benninger, L.A., Carter, D.O. and Tibbett, M. (2009) Decomposition studies using animal models in contrasting environments: evidence from temporal changes in soil chemistry and microbial activity. In: Ritz, K., Dawson, L.A. and Miller, D. (Eds.) Criminal and Environmental Soil Forensics. Springer, pp. 357-377.

Stotzky, G. (1997) Soil as an environment for environmental life. In: van Elsas, J.D., Trevors, J.T. and Wellington, E.M. (Eds.) Modern Soil Microbiology. Marcel Dekker, New York.

Suzuki, M., Rappe, M.S. and Giovannoni, S.J. (1998) Kinetic bias in estimates of coastal picoplankton community structure obtained by measurements of small-subunit rRNA gene PCR amplicon length heterogeneity. Applied and Environmental Microbiology 64: 4522-4529.

Suzuki, M.T. and Giovannoni, S.J. (1996) Bias caused by template annealing in the amplification of mixtures of 16S rRNA genes by PCR. Applied and Environmental Microbiology 62: 625-630.

Svensson, A., Wendel, O. and B.A.J., F. (1981) Techniques of Crime Scene Investigation. Elsevier North Holland, Inc.

Swift, B., Lauder, I., Black, S. and Norris, J. (2001) An estimation of the post-mortem interval in human skeletal remains: a radionuclide and trace element approach. Forensic Science International 117: 7387.

Tannock, G.W. (1994) Normal Microflora: An Introduction to Microbes Inhabiting the Human Body. Springer. 
Tarlera, S., Jangid, K., Ivester, A.H., Whitman, W.B. and A, W.M. (2008) Microbial community succession and bacterial diversity in soils during 77,000 years of ecosystem development. FEMS Microbiology Ecology 64: 129-140.

Tekoriene, R. (2008) Distribution of the genus Pseudomonas bacteria in oil-polluted soil, water, polymeric materials, plant remnants and food products. Ekologija 54: 143-148.

Teske, A., Wawer, C., Muyzer, G. and Ramsing, N.B. (1996) Distribution of sulfate-reducing bacteria in a stratified fjord (Mariager Fjord, Denmark) as evaluated by most-probable number counts and denaturing gradient gel electrophoresis of PCR-amplified ribosomal DNA fragments. Applied and Environmental Microbiology 62: 1405-1415.

Thies, F.L., König, W. and König, B. (2007) Rapid characterization of the normal and disturbed vaginal microbiota by application of $16 \mathrm{~S}$ rRNA gene terminal RFLP fingerprinting. Journal of Medical Microbiology 56: 755-761.

Thies, J. (2007a) Soil microbial community analysis using terminal restriction fragment length polymorphisms. Soil Science Society of America Journal 71: 579-591.

Thies, J.E. (2007b) Molecular methods for studying soil ecology. In: Paul, E.A. (Eds.) Soil Microbiology, Ecology and Biochemistry. Elsevier.

Thirup, L., Johnsen, K. and Winding, A. (2001) Succession of indigenous Pseudomonas spp. and actinomycetes on barley roots affected by the antagonistic strain Pseudomonas fluorescens DR54 and the fungicide Imazalil. Applied and Environmental Microbiology 67: 1147-1153.

Thorn, G. (1997) The fungi in soil. In: van Elsas, J.D., Trevors, J.T. and Wellington, E.M. (Eds.) Modern Soil Microbiology. Marcel Dekker, New York.

Thornton, J.I. (1986) Forensic soil characterisation. Forensic Science Progress 1: 3-35.

Tjoelker, L.W., Gosting, L., Frey, S., Hunter, C.L., Trong, H.L., Steiner, B., Brammer, H. and Gray, P.W. (2000) Structural and functional definition of the human chitinase chitin-binding domain. . Journal of Biological Chemistry 275: 514-520.

Tokuda, G., Saito, H. and Watanabe, H. (2007) A digestive beta-glucosidase from the salivary glands of the termite, Neotermes koshunensis (Shiraki): distribution, characterization and isolation of its precursor cDNA by 5'- and 3'-RACE amplifications with degenerate primers. Insect Biochemistry and Molecular Biology 32: 1681-1698.

Torsvik, V., Goksoyr, J. and Daae, F.L. (1990) High diversity in DNA of soil bacteria. Applied and Environmental Microbiology 56: 782-787.

Tortora, G. and Grabowski, S. (2000) Principles of Anatomy and Physiology. Wiley Inc., New York.

Toth, E.M., Hell, E., Kovacs, G., Borsodi, A.K. and Marialigeti, K. (2006) Bacteria isolated from the different developmental stages and larval organs of the obligate parasitic fly, Wohlfahrtia magnifica (Diptera: Sarcophagidae). Microbial Ecology 51: 13-21.

Towner, K. (2006) The Genus Acinetobacter. Prokaryotes 6: 746-758.

Treusch, A.H. and Schleper, C. (2006) The microbial soil flora: novel approaches for accessing the phylogenetic and physiological diversity of prokaryotes. In: Konig, H. and Varma, A. (Eds.) Intestinal Microorganisms of Soil Invertebrates. Springer-Verlag, Berlin Heidelberg.

Tringe, S.G., von Mering, C., Kobayashi, A., Salamov, A.A., Chen, K., Chang, H.W., Podar, M., Short, J.M., Mathur, E.J., Detter, J.C., Bork, P., Hugenholtz, P. and Rubin, E.M. (2005) Comparative metagenomics of microbial communities. Science 308: 554-557. 
Tripathi, A.K., Verma, S.C., Chowdhury, S.P., Lebuhn, M., Gattinger, A. and Schloter, M. (2006) Ochrobactrum oryzae sp. nov., an endophytic bacterial species isolated from deep-water rice in India. International Journal of Systematic and Evolutionary Microbiology 56: 1677-1680.

Tryfinopoulou, P., Tsakalidou, E. and Nychas, G.-J.E. (2002) Characterization of Pseudomonas spp. associated with spoilage of gilt-head sea bream stored under various conditions. Applied and Environmental Microbiology 68: 65-72.

Turner, B.D. and Wiltshire, P.E.J. (1999) Experimental validation of forensic evidence: a study of the decomposition of buried pigs in a heavy clay soil. Forensic Science International 101: 113-122.

Urzi, C., Brusetti, L., Salamone, P., Sorlini, C., Stackebrandt, E. and Daffonchio, D. (2001) Biodiversity of Geodermatophilaceae isolated from altered stones and monuments in the Mediterranean basin. Environmental Microbiology 3: 471-479.

Van de Peer, Y., Chapelle, S. and De Wachter, R. (1996) A quantitative map of nucleotide substitution rates in bacterial rRNA. Nucleic Acids Research 24: 3381-3391.

van der Maarel, M.J., Artz, R.R., Haanstra, R. and Forney, L.J. (1998) Association of marine archaea with the digestive tracts of two marine fish species. Applied and Environmental Microbiology 64: 28942898.

Vaneechoutte, M., Kampfer, P., De Baere, T., Avesani, V., Janssens, M. and Wauters, G. (2007) Chryseobacterium hominis sp. nov., to accommodate clinical isolates biochemically similar to CDC groups II-h and II-c. International Journal of Systematic and Evolutionary Microbiology 57: 26232628.

Varel, V.H., Robinson, I.M. and Pond, W.G. (1987) Effect of Dietary Copper Sulfate, Aureo SP250, or Clinoptilolite on Ureolytic Bacteria Found in the Pig Large Intestine. Applied and Environmental Microbiology 53: 2009-2012.

Vass, A.A. (2001) Beyond the grave - understanding human decomposition. Microbiology Today 28: 190192.

Vass, A.A., Barshick, S.A., Sega, G., Caton, J., Skeen, J.T., Love, J.C. and Synstelien, J.A. (2002) Decomposition chemistry of human remains: a new methodology for determining the postmortem interval. Journal of Forensic Sciences 47: 542-553.

Vass, A.A., Bass, W.M., Wolt, J.D., Foss, J.E. and Ammons, J.T. (1992) Time since death determinations of human cadavers using soil solution. Journal of Forensic Sciences 37: 1236-1253.

Vass, A.A., Smith, R.R., Thompson, C.V., Burnett, M.N., Dulgerian, N. and Eckenrode, B.A. (2008) Odor analysis of decomposing buried human remains. Journal of Forensic Sciences 53: 384-391.

Vass, A.A., Smith, R.R., Thompson, C.V., Burnett, M.N., Wolf, D.A., Synstelien, J.A., Dulgerian, N. and Eckenrode, B.A. (2004) Decompositional odor analysis database. Journal of Forensic Sciences 49: 760-769.

Ventura, M., Canchaya, C., Tauch, A., Chandra, G., Fitzgerald, G.F., Chater, K.F. and van Sinderen, D. (2007) Genomics of Actinobacteria: tracing the evolutionary history of an ancient phylum. Microbiology and Molecular Biology Reviews 71: 495-548.

Vepsalainen, M., Kukkonen, S., Vestberg, M., Sirvio, H. and Maarit Niemi, R. (2001) Application of soil enzyme activity test kit in a field experiment. Soil Biology \& Biochemistry 33: 1665-1672.

Verbarg, S., Rheims, H., Emus, S., Fruhling, A., Kroppenstedt, R.M., Stackebrandt, E. and Schumann, P. (2004) Erysipelothrix inopinata sp. nov., isolated in the course of sterile filtration of vegetable 
peptone broth, and description of Erysipelotrichaceae fam. nov. International Journal of Systematic and Evolutionary Microbiology 54: 221-225.

Verberkmoes, N.C., Russell, A.L., Shah, M., Godzik, A., Rosenquist, M., Halfvarson, J., Lefsrud, M.G., Apajalahti, J., Tysk, C., Hettich, R. and Jansson, J.K. (2009) Shotgun metaproteomics of the human distal gut microbiota. The ISME Journal 3: 179-189.

Vitali, B., Pugliese, C., Biagi, E., Candela, M., Turroni, S., Bellen, G., Donders, G.G.G. and Brigidi, P. (2007) Dynamics of vaginal bacterial communities in women developing bacterial vaginosis, candidiasis or no infection, analyzed by PCR-denaturing gradient gel electrophoresis and real-time PCR. Applied and Environmental Microbiology 73: 5731-5741.

von Witzengerode, F., Gobel, U.B. and Stackebrandt, E. (1997) Determination of microbial diversity in environmental samples: pitfalls of PCR-based rRNA analysis. FEMS Microbiology Reviews 21: 213-229.

Walter, J. (2008) Ecological role of Lactobacilli in the gastrointestinal tract: implications for fundamental and biomedical research. Applied and Environmental Microbiology 74: 4985-4996.

Walter, J., Hertel, C., Tannock, G.W., Lis, C.M., Munro, K. and Hammes, W.P. (2001) Detection of Lactobacillus, Pediococcus, Leuconostoc, and Weissella species in human feces by using groupspecific PCR primers and denaturing gradient gel electrophoresis. Applied and Environmental Microbiology 67: 2578-2585.

Wang, M., Ahrne, S., Jeppsson, B. and Molin, G. (2005) Comparison of bacterial diversity along the human intestinal tract by direct cloning and sequencing of $16 \mathrm{~S}$ rRNA genes. FEMS Microbiology Ecology 54: $219-231$

Wang, M., Karlsson, C., Olsson, C., Adlerberth, I., Wold, A.E., Strachan, D.P., Matricardi, P.M., Aberg, N., Perkin, M.R., Tripodi, S., Coates, A.R., Hesselmar, B., Saalman, R., Molin, G. and Ahrne, S. (2007) Reduced diversity in the early fecal microbiota of infants with atopic eczema. Journal of Allergy and Clinical Immunology: 129-134.

Wang, S.-L., Yieh, T.-C. and Shih, I.-L. (1999) Production of antifungal compounds by Pseudomonas aeruginosa K-187 using shrimp and crab shell powder as a carbon source. Enzyme and Microbial Technology 25: 142-148.

Wang, X., Heazlewood, S.P., Krause, D.O. and Florin, T.H.J. (2003) Molecular characterization of the microbial species that colonize human ileal and colonic mucosa by using $16 \mathrm{~S}$ rDNA sequence analysis. Journal of Applied Microbiology 95: 508-520.

Ward, D.M., Bateson, M.M., Weller, R. and Ruff-Roberts, A.L. (1992) Ribosomal RNA analysis of microorganisms as they occur in nature. In: Marshall, K.C. (Eds.) Advances in Microbial Ecology. Plenum Press, New York, pp. 219-286.

Weaver, A.R., Kissel, D.E., Chen, F., West, L.T., Adkins, W., Rickman, D. and Luvall, J.C. (2004) Mapping soil $\mathrm{pH}$ buffering capacity of selected fields in the coastal plain. Soil Science Society of America Journal 68: 662-668.

Wheeler, A.L., Hartel, P.G., Godfrey, D.G., Hill, J.L. and Segars, W.I. (2002) Potential of Enterococcus faecalis as a human fecal indicator for microbial source tracking. Journal of Environmental Quality 31: 1286-1293.

Whitehead, T.R. and Cotta, M.A. (2004) Isolation and identification of hyper-ammonia producing bacteria from swine manure storage pits. Current Microbiology 48: 20-26. 
Whittle, G., Whitehead, T.R., Hamburger, N., Shoemaker, N.B., Cotta, M.A. and Salyers, A.A. (2003) Identification of a new ribosomal protection type of tetracycline resistance gene, tet(36), from swine manure pits. Applied and Environmental Microbiology 69: 4151-4158.

Widmer, F., Hartmann, M., Frey, B. and Kolliker, R. (2006) A novel strategy to extract specific phylogenetic sequence information from community T-RFLP. Journal of Microbiological Methods 66: 512-520.

Wiegel, J., Tanner, R. and Rainey, F.A. (2006) An introduction to the Family Clostridiaceae. Prokaryotes 4: 654-678.

Williams, M.M., Domingo, J.W.S., Meckes, M.C., Kelty, C.A. and Rochon, H.S. (2004) Phylogenetic diversity of drinking water bacteria in a distribution system simulator. Journal of Applied Microbiology 96: 954-964.

Wilson, M. (2005) Microbial Inhabitants of Humans: Their ecology and role in health and disease. Cambridge University Press, Cambridge, UK.

Wilson, M., McNab, R. and Henderson, B. (2002) Bacterial Disease Mechanisms: An Introduction to Cellular Microbiology. Cambridge University Press, Cambridge, UK.

Winogradsky, S. (1949) Methode dans la microbiologie du sol. Masson et Cie, Paris, France.

Wittmann, C., Suominen, K.P. and Salkinoja-Salonen, M.S. (2000) Evaluation of ecological disturbance and intrinsic bioremediation potential of pulp mill-contaminated lake sediment using key enzymes as probes. Environmental Pollution 107: 255-261.

Wood, M. (1995) Environmental Soil Biology. Chapman \& Hall, Glasgow, UK.

Woodmansey, E.J., McMurdo, M.E.T., Macfarlane, G.T. and Macfarlane, S. (2004) Comparison of compositions and metabolic activities of fecal microbiotas in young adults and in antibiotic-treated and non-antibiotic-treated elderly subjects. Applied and Environmental Microbiology 70: 61136122.

Wozny, M.A., Bryant, M.P., Holdeman, L.V. and Moore, W.E.C. (1977) Urease assay and urease-producing species of anaerobes in the bovine rumen and human feces. Applied and Environmental Microbiology 33: 1097-1104.

Wu, Y., Adam, R., Williams, S.A. and Bianco, A.E. (1996) Chitinase genes expressed by infective larvae of the filarial nematodes, Acanthocheilonema vitae and Onchocerca volvulus. Molecular and Biochemical Parasitology 75: 207-219.

Yang, C.H. and Crowley, D.E. (2000) Rhizosphere microbial community structure in relation to root location and plant iron nutritional status. Applied and Environmental Microbiology 66: 345-351.

Yap, W.H., Zhang, Z. and Wang, Y. (1999) Distinct types of rRNA operons exist in the genome of the actinomycete Thermomonospora chromogena and evidence for horizontal transfer of an entire rRNA operon. Journal of Bacteriology 181: 5201-5209.

Yergeau, E., Filion, M., Vujanovic, V. and St-Arnaud, M. (2005) A PCR-denaturing gradient gel electrophoresis approach to assess Fusarium diversity in asparagus. Journal of Microbiological Methods 60: 143-154.

Yergeau, E., Schoondermark-Stolk, S.A., Brodie, E.L., Dejean, S., DeSantis, T.Z., Goncalves, O., Piceno, Y.M., Andersen, G.L. and Kowalchuk, G.A. (2009) Environmental microarray analyses of Antarctic soil microbial communities. The ISME Journal 3: 340-351.

Yin, H.C. (2006) A histochemical study of the distribution of phosphatase in plant tissues. New Phytologist 44: 191-195. 
Yoo, S.-H., Weon, H.-Y., Jang, H.-B., Kim, B.-Y., Kwon, S.-W., Go, S.-J. and Stackebrandt, E. (2007) Sphingobacterium composti sp. nov. isolated from cotton-waste composts. International Journal of Systematic and Evolutionary Microbiology 57: 1590-1593.

Yoon, J.-H., Kang, S.-J., Lee, J.-S., Oh, H.W. and Oh, T.-K. (2007) Brevundimonas lenta sp. nov., isolated from soil. International Journal of Systematic and Evolutionary Microbiology 57: 2236-2240.

Zhang, X.Y., Zhang, Y.J., Chen, X.L., Qin, Q.L., Zhao, D.L., Li, T.G., Dang, H.Y. and Zhang, Y.Z. (2008) Myroides profundi sp. nov. isolated from deep-sea sediment of the southern Okinawa Trough. FEMS Microbiology Letters 287: 108-112.

Zoetendal, E.G., Akkermans, A.D.L., Akkermans-van Vliet, W.M., de Visser, J.A.G.M. and De Vos, W.M. (2001) The host genotype affects the bacterial community in the human gastrointestinal tract. Microbial Ecology in Health and Disease 13: 129-134.

Zou, C.S., Mo, M.H., Gu, Y.Q., Zhou, J.P. and Zhang, K.Q. (2007) Possible contributions of volatileproducing bacteria to soil fungistasis. Soil Biology \& Biochemistry 39: 2371-2379.

Zwolinski, M.D. (2007) DNA Sequencing: Strategies for Soil Microbiology. Soil Science Society of America Journal 71: 592-600. 


\section{Appendices (on CD)}

Appendix A: Method Protocols

Appendix B: DGGE 16S whole community gel photos

Appendix C: Perl Scripts

Appendix D: Species Lists

Appendix E: Pig Carcass Decomposition Photos 